\title{
The Silicon-Hydrogen Exchange Reaction: A Catalytic $\sigma$-Bond Metathesis Approach to the Enantioselective Synthesis of Enol Silanes
}

\author{
Hui Zhou, ${ }^{\dagger}$ Han Yong Bae, ${ }^{\dagger, \dagger}$ Markus Leutzsch, ${ }^{\dagger}$ Jennifer L. Kennemur, ${ }^{\dagger}$ Diane Bécart, ${ }^{\dagger}$ and Benjamin List ${ }^{* \dagger}$ \\ ${ }^{\dagger}$ Max-Planck-Institut für Kohlenforschung, Kaiser-Wilhelm-Platz 1, D-45470 Mülheim an der Ruhr, Germany \\ ${ }^{\ddagger}$ Department of Chemistry, Sungkyunkwan University, 16419, Suwon, Korea.
}

*Email: list@kofo.mpg.de

\section{Content}

1. General considerations

2. Preparation and characterization of imidodiphosphorimidates (IDPis)

3. Development of suitable reaction conditions and catalyst identification

4. The preparation and characterization of cyclic ketones

5. Substrate scope for the deprotosilylation of ketones $\mathbf{1}$ with allylsilane reagents $\mathbf{2 a}$ or $\mathbf{2 b}$

6. Gram scale reaction and derivatizations of the enol silane $\mathbf{3 a}$

7. Formal synthesis of iloprost

8. Development of a catalytic asymmetric protodesilylation of racemic enol silanes 


\section{General considerations}

\section{Chemicals}

Unless otherwise indicated, starting materials were obtained from Sigma-Aldrich, ABCR-GmbH, TCI, or Acros Co. Ltd. Moreover, commercially available reagents were used without additional purification. Ketone 1a was obtained from commercial suppliers and used after chromatographic purification and recrystallization. Ketones $\mathbf{1 c}-\mathbf{1 o}^{1}, \mathbf{1 s}^{2}, \mathbf{1} \mathbf{u}^{3}, \mathbf{1} \mathbf{v}^{4}, \mathbf{1} \mathbf{w}^{3}$ and $\mathbf{1 a c} \mathbf{c}^{5}$ were synthesized according to literature procedure. The allyl(tert-butyl)dimethylsilane 2a was obtained from commercial suppliers, and used after distillation. Triethyl(2-methylallyl)silane $\mathbf{2} \mathbf{b}^{6}$ was synthesized according to literature procedures. The chiral imidodiphosphorimidate acids (IDPis) $\mathbf{4 a}-\mathbf{4} \mathbf{f}^{7}$ were synthesized according to literature procedures.

\section{Solvents}

Solvents (Et $2 \mathrm{O}$, THF, 1,4-Dioxane, Cyclohexane, $\mathrm{CH}_{2} \mathrm{Cl}_{2}, \mathrm{CHCl}_{3}$, Benzene and Toluene) were dried by distillation from an appropriate drying agent in the technical department of the Max-Planck-Institut für Kohlenforschung and received in Schlenk flasks under argon. In addition, more solvents (MTBE, $\mathrm{CH}_{3} \mathrm{CN}$ and Mesitylene) were purchased from commercial suppliers and dried over molecular sieves.

\section{Inert Gas}

Dry argon was purchased from Air Liquide with $>99.5 \%$ purity.

\section{Thin Layer Chromatography}

Thin-layer chromatography (TLC) was performed using silica gel pre-coated plastic sheets (Polygram SIL G/UV 254 , 0.2 mm, with fluorescent indicator; Macherey-Nagel) which was visualized with a UV lamp (254 nm) and/or phosphomolybdic acid (PMA). PMA stain: PMA (20 g) in EtOH (200 mL).

\section{Column Chromatography}

Column chromatography (CC) was carried out using Merck silica gel (60 А, 230-400 mesh, particle size 0.040-0.063 mm) using technical grade solvents. Elution was accelerated using compressed argon. All reported yields, unless otherwise specified, refer to spectroscopically and chromatographically pure compounds.

\section{Nomenclature}

Nomenclature follows the suggestions proposed by the computer program ChemBioDraw (12.0.3.1216) of CBD/cambridgesoft.

\section{Nuclear Magnetic Resonance Spectroscopy}

${ }^{1} \mathrm{H},{ }^{13} \mathrm{C},{ }^{19} \mathrm{~F},{ }^{31} \mathrm{P}$ Nuclear magnetic resonance (NMR) spectra for compound characterization were recorded on Bruker AVIII-500 MHz, NMR spectrometer in a suitable deuterated solvent. The solvent employed and the respective measuring frequency are indicated for each experiment. Chemical shifts are reported with tetramethylsilane (TMS) serving as a universal reference of all nuclides. The resonance multiplicity is described as s (singlet), $\mathrm{d}$ (doublet), $\mathrm{t}$ (triplet), q (quadruplet), m (multiplet), and b (broad). All spectra were recorded at $298 \mathrm{~K}$, processed with MestReNova 10.0.2 suits of program, and coupling constants are reported as observed. The residual deuterated solvent signal relative to tetramethylsilane was used as the internal reference in ${ }^{1} \mathrm{H} \mathrm{NMR} \mathrm{spectra}\left(\mathrm{e} . \mathrm{g}\right.$. $\mathrm{CDCl}{ }_{3}=7.26$ ppm, 
$\left.\mathrm{CD}_{2} \mathrm{Cl}_{2}=5.32 \mathrm{ppm}\right){ }^{8}$ Signals are reported as follows: chemical shift $\delta$ in ppm (multiplicity, coupling constant $J$ in $\mathrm{Hz}$, number of protons). All X-nuclei spectra were acquired proton decoupled unless otherwise noted.

Kinetic NMR measurements were performed at a Bruker AVIII- 300MHz WB NMR spectrometer. The temperature of low temperature experiments was calibrated against a $4 \% \mathrm{MeOH}$ in MeOD- $d_{4}$ sample. ${ }^{9}$ Further details are mentioned in the corresponding section.

\section{Mass Spectrometry}

Electrospray ionization (ESI) mass spectrometry was conducted on a Bruker ESQ 3000 spectrometer. High resolution mass spectra were determined on a Bruker APEX III FTMS (7 T magnet). The ionization method and mode of detection employed is indicated for the respective experiment and all masses are reported in atomic units per elementary charge $(\mathrm{m} / \mathrm{z})$ with an intensity normalized to the most intense peak.

\section{Specific Rotations}

Specific rotations $\left([\boldsymbol{\alpha}]_{\mathbf{D}}^{\mathbf{T}}\right)$ were measured with a Rudolph RA Autopol IV Automatic Polarimeter at the indicated temperature with a sodium lamp (sodium D line, $\lambda=589 \mathrm{~nm}$ ). Measurements were performed in an acid resistant $1 \mathrm{~mL}$ cell $(50 \mathrm{~mm}$ length) with concentrations $(\mathrm{g} /(100 \mathrm{~mL}))$ reported in the corresponding solvent.

\section{High Performance Liquid Chromatography}

High performance liquid chromatography (HPLC) was performed on a Shimadzu LC-20AD liquid chromatograph SIL-20AC auto sampler, CMB-20A using Daicel columns with a chiral stationary phase. All solvents used were HPLC-grade solvents purchased from Sigma-Aldrich. The column employed and the respective solvent mixture are indicated for each experiment.

\section{Gas Chromatography}

Gas chromatography (GC) analyses on a chiral stationary phase were performed on HP 6890 and 5890 series instruments (split-mode capillary injection system, flame ionization detector (FID), hydrogen carrier gas). The conditions employed are described in detail for the individual experiments.

\section{Abbreviations}

e.r. $=$ enantiomeric ratio, $\mathrm{TLC}=$ thin layer chromatography, $\mathrm{THF}=$ tetrahydrofuran, $\mathrm{MTBE}=$ methyl tert-butyl ether, $\mathrm{CH}_{3} \mathrm{CN}=$ acetonitrile, Mesitylene = 1,3,5-trimethylbenzene, TBS $=\mathrm{SiMe}_{2}{ }^{t} \mathrm{Bu}, \mathrm{TMS}=\mathrm{SiMe}_{3}, \mathrm{TES}=$ triethylsilyl, $\mathrm{Tf}=\mathrm{SO}_{2} \mathrm{CF}_{3}, \mathrm{MOM}=$ methoxymethyl ether. 


\section{Preparation and characterization of imidodiphosphorimidates (IDPis) ${ }^{7}$}


$(S, S)-I D P i \mathbf{4 a - 4 f}$

\section{Representative procedure for 3,3'-disubstituted BINOL synthesis by Suzuki coupling and the subsequent hydrolysis:}

\section{(S)-3,3'-bis(9,9-diethyl-9H-fluoren-3-yl)-[1,1'-binaphthalene]-2,2'-diol}



In a flame dried 2-neck round-bottom flask with a condenser, $(S)-2,2$ '-(2,2'-bis(methoxymethoxy)-1,1'binaphthyl-3,3'-diyl)bis(4,4,5,5-tetramethyl-1,3,2-dioxaborolane) (500 mg, $0.8 \mathrm{mmol}, 1.0$ equiv.), 2'bromospiro[cyclobutane-1,9'-fluorene] (524 mg, 2.3 equiv.), and tetrakis(triphenylphosphine)palladium (92 $\mathrm{mg}, 0.08 \mathrm{mmol}, 0.1$ equiv.) were dissolved in 1,4-dioxane $(10 \mathrm{~mL})$. After degassing the reaction mixture with argon for 10-15 min, a degassed aqueous solution of $\mathrm{K}_{2} \mathrm{CO}_{3}(2.0 \mathrm{M}, 3 \mathrm{~mL})$ was added. The resultant mixture was then transferred into a microwave reactor and stirred at $90{ }^{\circ} \mathrm{C}$ for $2 \mathrm{~h}$. After cooling the reaction system to room temperature, the mixture was further purified by column chromatography to afford $(S)$ 2',2'"-(2,2'-bis(methoxymethoxy)-[1,1'-binaphthalene]-3,3'-diyl)dispiro[cyclobutane-1,9'-fluorene]. A solution of this material and Amberlyst ${ }^{\circledR}-15$ (Hydrogen form) in THF/MeOH (1:1) was then stirred at $90{ }^{\circ} \mathrm{C}$ for $12 \mathrm{~h}$. After completion of the reaction (as monitored by TLC analysis), Amberlyst ${ }^{\circledR}-15$ was removed by filtration, and the mixture was concentrated under reduced pressure. Finally, the crude mixture was purified by column chromatography (Ethyl acetate/hexanes 1:20 to 1:4) to afford the title compound as a white/off white solid (overall yield of the two steps: $75 \%$ ).

${ }^{1} \mathrm{H}$ NMR $\left(501 \mathrm{MHz}, \mathrm{CD}_{2} \mathrm{Cl}_{2}\right) \delta 8.13(\mathrm{~s}, 1 \mathrm{H}), 7.99(\mathrm{dd}, J=8.2,1.2 \mathrm{~Hz}, 1 \mathrm{H}), 7.89-7.83(\mathrm{~m}, 1 \mathrm{H}), 7.81-7.77(\mathrm{~m}, 1 \mathrm{H}), 7.76-7.71(\mathrm{~m}, 2 \mathrm{H})$, $7.43(\mathrm{ddd}, J=8.1,6.8,1.3 \mathrm{~Hz}, 1 \mathrm{H}), 7.40-7.32(\mathrm{~m}, 4 \mathrm{H}), 7.25(\mathrm{dd}, J=8.5,1.1 \mathrm{~Hz}, 1 \mathrm{H}), 5.55(\mathrm{~s}, 1 \mathrm{H}), 2.17-2.01(\mathrm{~m}, 4 \mathrm{H}), 0.38(\mathrm{td}, J=$ 7.4, $1.5 \mathrm{~Hz}, 6 \mathrm{H})$.

${ }^{13} \mathrm{C}$ NMR $\left(126 \mathrm{MHz}, \mathrm{CD}_{2} \mathrm{Cl}_{2}\right) \delta 153.1,153.0,150.7,139.3,139.2,137.2,133.5,131.6,131.5,130.0,128.9,128.8,128.1,127.5$, 127.4, 124.6, 124.6, 124.5, 123.1, 119.9, 119.8, 113.2, 52.4, 33.7, 33.6, 17.3.

$\mathrm{R} f=0.28($ Ethyl acetate/hexanes $=1: 9)$.

ESI-HRMS $(\mathrm{m} / z)$ : calculated for $\mathrm{C}_{54} \mathrm{H}_{45} \mathrm{O}_{2}\left([\mathrm{M}-\mathrm{H}]^{-}\right)$: 725.3425 , found: 725.3424 .

m.p. $=220.2-224 \cdot 8^{\circ} \mathrm{C}$.

$[\boldsymbol{\alpha}]_{\mathbf{D}}^{\mathbf{2 5}}=85.2\left(c 0.14, \mathrm{CHCl}_{3}\right)$.

(S)-3,3'-di(triphenylen-2-yl)-[1,1'-binaphthalene]-2,2'-diol 




In a flame dried 2-neck round-bottom flask with a condenser, (S)-2,2'-(2,2'-bis(methoxymethoxy)1,1'binaphthyl-3,3'-diyl)bis(4,4,-5,5-tetramethyl-1,3,2-dioxaborolane) (1.1 g, $1.8 \mathrm{mmol}, 1.0$ equiv.), 2 bromophenanthrene (1.1 g, 2.3 equiv.), and tetrakis(triphenylphosphine)palladium (208 mg, $0.08 \mathrm{mmol}, 0.1$ equiv.) were dissolved in 1,4-dioxane $(22 \mathrm{~mL})$. After degassing the reaction mixture with argon for 10-15 min, a degassed aqueous solution of $\mathrm{K}_{2} \mathrm{CO}_{3}(2.0 \mathrm{M}, 6.8 \mathrm{~mL})$ was added. The resultant mixture was then transferred into a microwave reactor and stirred at $90{ }^{\circ} \mathrm{C}$ for $2 \mathrm{~h}$. After cooling the reaction to room temperature, the mixture was further purified by column chromatography to afford $(S)-2,2^{\prime}-\left(2,2^{\prime}-\right.$ bis(methoxymethoxy)-[1,1'-binaphthalene]-3,3'-diyl)ditriphenylene. A solution of this material and Amberlyst $^{\circledR}-15$ (Hydrogen form) in THF/MeOH (1:1) was then stirred at $90{ }^{\circ} \mathrm{C}$ for $12 \mathrm{~h}$. After the completion of the reaction (as monitored by TLC analysis), Amberlyst ${ }^{\circledR}-15$ was removed by filtration, and the mixture was concentrated under reduced pressure. Finally, the crude mixture was purified by column chromatography (Ethyl acetate/hexanes 1:20 to 1:4) to afford the title compound as a white/off white solid (overall yield of the two steps: $85 \%$ ).

${ }^{1} \mathrm{H}$ NMR $\left(501 \mathrm{MHz}, \mathrm{CD}_{2} \mathrm{Cl}_{2}\right) \delta 9.08(\mathrm{~d}, J=1.7 \mathrm{~Hz}, 2 \mathrm{H}), 8.81-8.76(\mathrm{~m}, 4 \mathrm{H}), 8.72(\mathrm{ddd}, J=9.5,7.5,3.2 \mathrm{~Hz}, 6 \mathrm{H}), 8.28(\mathrm{~s}, 2 \mathrm{H}), 8.09(\mathrm{dd}$, $J=8.5,1.8 \mathrm{~Hz}, 2 \mathrm{H}), 8.05(\mathrm{dd}, J=8.2,1.2 \mathrm{~Hz}, 2 \mathrm{H}), 7.70(\mathrm{~m}, J=8 \mathrm{H}), 7.47(\mathrm{ddd}, J=8.1,6.7,1.3 \mathrm{~Hz}, 2 \mathrm{H}), 7.41(\mathrm{ddd}, J=8.3,6.8,1.4$ $\mathrm{Hz}, 2 \mathrm{H}), 7.34(\mathrm{dd}, J=8.4,1.1 \mathrm{~Hz}, 2 \mathrm{H}), 5.69(\mathrm{~s}, 2 \mathrm{H})$.

${ }^{13} \mathrm{C}$ NMR $\left(126 \mathrm{MHz}, \mathrm{CD}_{2} \mathrm{Cl}_{2}\right) \delta 151.0,136.9,133.7,132.2,131.0,130.4,130.3,130.2,130.1,129.9,129.5,129.2,129.0,127.9,127.8$, $127.8,124.9,124.8,124.7,123.8,123.8,123.8,113.1$.

$\mathrm{R} f=0.23$ (Ethyl acetate/hexanes $=1: 4)$.

ESI-HRMS $(m / z)$ : calculated for $\mathrm{C}_{56} \mathrm{H}_{33} \mathrm{O}_{2}\left([\mathrm{M}-\mathrm{H}]^{-}\right)$: 737.2486, found: 737.2497.

m.p. $=286.3-288.1^{\circ} \mathrm{C}$.

$[\boldsymbol{\alpha}]_{\mathbf{D}}^{25}=88.3\left(c 0.29, \mathrm{CHCl}_{3}\right)$.

\section{(S)-3,3'-bis(3',5'-di-tert-butyl-[1,1'-biphenyl]-4-yl)-[1,1'-binaphthalene]-2,2'-diol}



In a flame dried 2-neck round-bottom flask with a condenser, $(S)$-2,2'-(2,2'-bis(methoxymethoxy)1,1'binaphthyl-3,3'-diyl)bis(4,4,-5,5-tetramethyl-1,3,2-dioxaborolane) (500 mg, $0.8 \mathrm{mmol}, 1.0$ equiv.), 4'-bromo-3,5-di-tert-butyl-1,1'-biphenyl $\quad(635 \quad \mathrm{mg}, \quad 2.3 \quad$ equiv. $), \quad$ and tetrakis(triphenylphosphine)palladium (92 mg, $0.08 \mathrm{mmol}, 0.1$ equiv.) were dissolved in 1,4-dioxane $(10 \mathrm{ml})$. After degassing the reaction mixture under argon for 10-15 min, a degassed aqueous solution of $\mathrm{K}_{2} \mathrm{CO}_{3}(2.0 \mathrm{M}, 3 \mathrm{~mL})$ was added. The resultant mixture was then transferred into a microwave reactor and stirred at $90{ }^{\circ} \mathrm{C}$ for $2 \mathrm{~h}$. After cooling the reaction to room temperature, the mixture was further purified by column chromatography to afford $(S)$-3,3'-bis(3',5'-di-tert-butyl-[1,1'-biphenyl]-4yl)-2,2'-bis(methoxymethoxy)-1,1'-binaphthalene. A solution of this material and Amberlyst ${ }^{\circledR}-15$ (Hydrogen form) in THF/MeOH $(1: 1)$ was then stirred at $90{ }^{\circ} \mathrm{C}$ for $12 \mathrm{~h}$. After the completion of the reaction (as monitored by TLC analysis), Amberlyst ${ }^{\circledR}-15$ was removed by filtration, and the mixture was concentrated under reduced pressure. Finally, the crude mixture was purified by column chromatography (ethyl acetate /hexanes 1:20 to 1:4) to afford the title compound as a white/off white solid (overall yield of thes two steps: $75 \%$ ).

${ }^{1} \mathrm{H}$ NMR $\left(501 \mathrm{MHz}, \mathrm{CD}_{2} \mathrm{Cl}_{2}\right) \delta 8.02(\mathrm{~s}, 2 \mathrm{H}), 7.90(\mathrm{~d}, J=8.1 \mathrm{~Hz}, 2 \mathrm{H}), 7.77-7.73(\mathrm{~m}, 4 \mathrm{H}), 7.68-7.64(\mathrm{~m}, 4 \mathrm{H}), 7.43(\mathrm{~d}, J=1.8 \mathrm{~Hz}, 4 \mathrm{H})$, $7.39(\mathrm{t}, J=1.8 \mathrm{~Hz}, 2 \mathrm{H}), 7.34(\mathrm{~d}, J=1.4 \mathrm{~Hz}, 2 \mathrm{H}), 7.26(\mathrm{~d}, J=1.5 \mathrm{~Hz}, 2 \mathrm{H}), 7.15(\mathrm{dd}, J=8.4,1.1 \mathrm{~Hz}, 2 \mathrm{H}), 5.42(\mathrm{~s}, 2 \mathrm{H}), 1.31(\mathrm{~s}, 36 \mathrm{H})$.

${ }^{13} \mathrm{C}$ NMR $\left(126 \mathrm{MHz}, \mathrm{CD}_{2} \mathrm{Cl}_{2}\right) \delta 151.4,150.4,141.8,140.1,136.3,133.1,131.3,130.5,129.9,129.6,128.5,127.4,127.3,124.3,124.2$, 121.7, 121.6, 112.5, 34.9, 31.3. 
$\mathrm{R} f=0.49$ (Ethyl acetate/hexanes $=1: 9)$.

ESI-HRMS (m/z): calculated for $\mathrm{C}_{60} \mathrm{H}_{61} \mathrm{O}_{2}\left([\mathrm{M}-\mathrm{H}]^{-}\right)$: 813.4677 , found: 813.4682.

m.p. $=175.3-178.5^{\circ} \mathrm{C}$.

$[\boldsymbol{\alpha}]_{\mathbf{D}}^{\mathbf{2 5}}=40.0\left(c 0.17, \mathrm{CHCl}_{3}\right)$.

The synthesis of phosphorimidoyl trichlorides $R_{\mathrm{f}}-\mathbf{1}-5$.



The syntheses of ((trifluormethyl)sulfonyl)phosphorimidoyl trichloride ${ }^{10} \mathrm{R}_{\mathrm{f}} \mathbf{- 1}$, ((perfluoroethyl)sulfonyl)phosphorimidoyl trichloride ${ }^{11}$ $\mathrm{R}_{\mathrm{f}} \mathbf{2}$ have been described previously. ((perfluorohexyl)sulfonyl)phosphorimidoyl trichloride $\mathrm{R}_{\mathrm{f}} \mathbf{3}$ and ((perfluorooctyl)sulfonyl)phosphorimidoyl trichloride $\mathrm{R}_{\mathrm{f}} \mathbf{- 4}$ were synthesized as reported in the literature ${ }^{6}$. ((perfluorophenyl)sulfonyl)phosphorimidoyl trichloride $\mathrm{R}_{\mathrm{f}} \mathbf{- 5}$ was synthesized according to the procedure reported in the literature ${ }^{12}$.

The synthesis of ((perfluoronaphthalen-2-yl)sulfonyl)phosphorimidoyl trichloride $\mathrm{R}_{\mathrm{f}}-6$



Step 1. According to a known procedure ${ }^{13}$ : octafluoronaphthalene (5.4 g, 20 mmol, 1 equiv.) was dispersed in a mixture of ethylene glycol $(15 \mathrm{~mL})$ and anhydrous $\mathrm{N}, \mathrm{N}$ dimethylformamide $(30 \mathrm{~mL})$. A yellow solution of sodium hydrosulphide hydrate $(2.2 \mathrm{~g}$, $40 \mathrm{mmol}$, 2 equiv. in ethylene glycol $(15 \mathrm{ml})$ and anhydrous $\mathrm{N}, \mathrm{N}$-dimethylformamide $(30 \mathrm{ml})$ was slowly added over 10 min keeping the reaction temperature below $5{ }^{\circ} \mathrm{C}$. The reaction temperature was raised to $20{ }^{\circ} \mathrm{C}$ and the reaction were monitored by TLC (hexanes as eluent). After complete consumption of the starting material ( 90 min), the orange mixture was acidified $\left(2 \mathrm{M} \mathrm{H}_{2} \mathrm{SO}_{4}\right)$ extracted with ether, dried over anhydrous $\mathrm{Na}_{2} \mathrm{SO}_{4}$ and the crude brown solid (3.6 g, $12.5 \mathrm{mmol}, 63 \%$ crude yield) was used without purification for the next step.

Step 2. According to a known procedure ${ }^{14}$ : N-chlorosuccinimide (5.6 g, 42 mmol, 4 equiv.) was added to a mixture of $\mathrm{HCl}: \mathrm{MeCN}$ (2 $\mathrm{M}, 1: 5,12 \mathrm{~mL})$ and then the mixture was cooled to $0{ }^{\circ} \mathrm{C}$. The 1,3,4,5,6,7,8-heptafluoronaphthalene-2-thiol (3 $\mathrm{g}, 10.5 \mathrm{mmol}, 1$ equiv.) was added portion wise using a spatula into the mixture. Temperature of the reaction mixture was kept below $15{ }^{\circ} \mathrm{C}$ for 30 min. The cooling bath was removed and it was allowed to warm up to room temperature. Once the reaction mixture became orange homogeneous solution ( 15 min, consumption of starting materials was confirmed by TLC: $20 \%$ EtOAc in hexanes), it was diluted with ethyl acetate $(100 \mathrm{~mL})$. The organic layer was washed with copious amount of aq. $\mathrm{NaCl}(5 \mathrm{x} 50 \mathrm{~mL})$ to remove excess NCS and dried over anhydrous $\mathrm{Na}_{2} \mathrm{SO}_{4}$, concentrated in vacuo to get the desired product as brown solid (3.2 $\mathrm{g}, 9.1 \mathrm{mmol}, 86 \%$ crude yield). The crude product was used for the next step without purification. 
Step 3. According to a known procedure ${ }^{15}$ : 1,3,4,5,6,7,8-Heptafluoronaphthalene-2-sulfonyl chloride (3.2 g, 9.1 mmol, 1 equiv.) was dissolved in newly-distilled THF and then the mixture was cooled to $-10{ }^{\circ} \mathrm{C} .0 .5 \mathrm{M}$ ammonia solution in dioxane $(\sim 30 \mathrm{~mL}, 14.6 \mathrm{mmol}$, 1.6 equiv.) was added dropwise to get a dark brown solution. The cooling bath was removed and it was allowed to attain room temperature. The reaction mixture was stirred for another $3 \mathrm{~h}$ (consumption of starting materials was confirmed by TLC: 30\% EtOAc in hexanes). The volatiles were removed via vacuum and the crude product was directly subjected to silica gel flash column chromatography using 25\% EtOAc in hexanes as eluents. The pure desired product was isolated as yellow solid (1.8 g, 5.4 mmol, 59\% yield). Yield over three steps were $32 \%$ from octafluoronaphthalene.

Step 4. In a flame dried pre-weighed Schlenk flask $(25 \mathrm{~mL})$ under Ar equipped with a magnetic stirring bar, solid $\mathrm{PCl}_{5}(0.951 \mathrm{~g}, 4.57$ mmol, 1.3 equiv.) was added and weighed directly inside the Schlenk flask. 1,3,4,5,6,7,8-heptafluoronaphthalene-2-sulfonamide (1.17 $\mathrm{g}, 3.15 \mathrm{mmol}, 1.0$ equiv.) were placed. $0.5 \mathrm{~mL}$ of dry toluene was added and the mixture was heated to $110{ }^{\circ} \mathrm{C}$ under Ar. The yellow solution was stirred for $30 \mathrm{~min}$ and then volatiles were carefully removed at $300 \mathrm{mbar}$ until bubbling disappeared. The liquid mixture was then heated to $130{ }^{\circ} \mathrm{C}$ for $1 \mathrm{~h}$ at 10 mbar to remove the excess amount of $\mathrm{PCl}_{5}$ by sublimation. $\mathrm{PCl}_{5}$ that condensed in the top part of the Schlenk flask was removed by heating with hot gun $\left(100^{\circ} \mathrm{C}\right.$ for $\left.15 \mathrm{~min}\right)$. The mixture was then cooled to room temperature and the remaining traces of $\mathrm{HCl}$ were removed in vacuo to afford the desired product $\mathrm{R}_{\mathrm{f}}-\mathbf{6}$ as a light brown solid (1.61 $\mathrm{g}, 98 \%$ ).

Note: ${ }^{13} \mathrm{C}$ NMR spectrum could not be obtained due to very low signal intensity.

((perfluoronaphthalen-2-yl)sulfonyl)phosphorimidoyl trichloride $\mathrm{R}_{\mathrm{f}}-6$



${ }^{19} \mathrm{~F}$ NMR $\left(471 \mathrm{MHz}, \mathrm{CDCl}_{3}\right) \delta-111.3(\mathrm{dd}, J=77.7,19.0 \mathrm{~Hz}),-134.9(\mathrm{~m}),-140.2--140.4(\mathrm{~m}),-144.4--$ $144.5(\mathrm{~m}),-145.6(\mathrm{dt}, J=58.9,19.1 \mathrm{~Hz}),-147.8(\mathrm{t}, J=19.2 \mathrm{~Hz}),-152.7--152.8(\mathrm{~m})$. (Decoupling artifacts are visible around the $19 \mathrm{~F}-$ decoupled signals.)

${ }^{31} \mathrm{P}$ NMR $\left(203 \mathrm{MHz}, \mathrm{CDCl}_{3}\right) \delta 8.1$.

CI-HRMS ( $m / z)$ : calculated for $\mathrm{C}_{10} \mathrm{H}_{4} \mathrm{~N}_{2} \mathrm{O}_{2} \mathrm{~F}_{7} \mathrm{Cl}_{3} \mathrm{~S}_{1} \mathrm{P}_{1}\left(\left[\mathrm{M}+\mathrm{NH}_{4}\right]^{+}\right)$: 484.8685, found: 484.8686 .

General procedure for the preparation of compounds 4a-4f. To a mixture of the 3,3'-disubstituted BINOL (2.1 equiv.) and various (substituted sulfonyl)phosphorimidoyl trichlorides (2.1 equiv.) in toluene ( $0.1 \mathrm{M})$ was added diisopropylethylamine (16 equiv.) at room temperature under an argon atmosphere. After stirring for 10-30 min, HMDS (1.0 equiv.) was added. After an additional 10 min at room temperature, the reaction mixture was sealed and heated to $120^{\circ} \mathrm{C}$ for $2-3 \mathrm{~d}$. The mixture was diluted with ethyl acetate and insoluble solid was filtered off through a short pad of Celite. Then the solvent was removed under reduced pressure and the crude residue was purified by column chromatography on silica gel using ethyl acetate/hexanes mixtures. After evaporation of solvent, the collected solid was stirred in a biphasic solution (DCM/6 N HCl) for $15 \mathrm{~min}$ and extracted with DCM. Azeotropic removal of water using toluene gave catalysts 4a-4f as white solids in their acidic form (yield: 50-65\%).

$(S, S)$-IDPi-4a:



${ }^{1} \mathrm{H}$ NMR $\left(501 \mathrm{MHz}, \mathrm{CD}_{2} \mathrm{Cl}_{2}\right) \delta 8.16(\mathrm{~s}, 2 \mathrm{H}), 8.10(\mathrm{dd}, J=12.3,8.3 \mathrm{~Hz}, 4 \mathrm{H}), 7.91(\mathrm{ddd}, J$ $=8.1,6.7,1.1 \mathrm{~Hz}, 2 \mathrm{H}), 7.89-7.84(\mathrm{~m}, 4 \mathrm{H}), 7.81(\mathrm{~d}, J=7.5 \mathrm{~Hz}, 2 \mathrm{H}), 7.75(\mathrm{ddd}, J=8.3$, $6.8,1.3 \mathrm{~Hz}, 2 \mathrm{H}), 7.67(\mathrm{dt}, J=7.7,0.9 \mathrm{~Hz}, 2 \mathrm{H}), 7.63(\mathrm{ddd}, J=8.2,6.4,1.5 \mathrm{~Hz}, 2 \mathrm{H}), 7.59$ $-7.53(\mathrm{~m}, 4 \mathrm{H}), 7.49-7.39(\mathrm{~m}, 6 \mathrm{H}), 7.35-7.17(\mathrm{~m}, 11 \mathrm{H}), 6.54(\mathrm{~d}, J=8.0 \mathrm{~Hz}, 2 \mathrm{H}), 6.47(\mathrm{dd}$, $J=7.9,1.6 \mathrm{~Hz}, 2 \mathrm{H}), 6.34(\mathrm{dd}, J=8.0,1.7 \mathrm{~Hz}, 2 \mathrm{H}), 2.83-2.73(\mathrm{~m}, 2 \mathrm{H}), 2.70-2.53(\mathrm{~m}, 9 \mathrm{H})$, $2.44-2.20(\mathrm{~m}, 13 \mathrm{H}), 2.20-2.10(\mathrm{~m}, 2 \mathrm{H})$.

${ }^{13} \mathrm{C}$ NMR (126 MHz, $\left.\mathrm{CD}_{2} \mathrm{Cl}_{2}\right) \delta 153.0,152.6,152.4,151.9,143.8(\mathrm{t}, J=5.2 \mathrm{~Hz}), 142.8(\mathrm{t}$, $J=4.7 \mathrm{~Hz}), 139.5,138.7,138.6,138.5,135.1,134.5,134.4,133.7,132.2,131.8,131.8$, $131.7,131.6,131.4,129.3,129.0,128.7,128.6,127.9,127.6,127.3,127.0,126.9,126.8$, $126.7,126.6,126.6,123.7,123.4,122.5,122.4,121.9,119.6,119.0,118.6,118.0,52.0$, $51.9,33.2,32.9,32.8,32.5,16.8,16.7$. 
${ }^{19} \mathrm{~F}$ NMR $\left(471 \mathrm{MHz}, \mathrm{CD}_{2} \mathrm{Cl}_{2}\right) \delta-79.3,-115.8,-116.1$.

${ }^{31} \mathrm{P}$ NMR $\left(203 \mathrm{MHz}, \mathrm{CD}_{2} \mathrm{Cl}_{2}\right) \delta-17.8$.

$\mathrm{R} f=0.27($ Ethyl acetate/hexanes $=1: 4)$.

ESI-HRMS ( $m / z)$ : calculated for $\mathrm{C}_{108} \mathrm{H}_{72} \mathrm{~F}_{10} \mathrm{~N}_{3} \mathrm{O}_{8} \mathrm{P}_{2} \mathrm{~S}_{2}\left([\mathrm{M}-\mathrm{H}]^{-}\right)$: 1854.4082 , found: 1854.4098 .

m.p. $=284.4-289.5^{\circ} \mathrm{C}$.

$[\boldsymbol{\alpha}]_{\mathbf{D}}^{\mathbf{2 5}}=205.0\left(c 0.12, \mathrm{CHCl}_{3}\right)$

$(S, S)-I D P i-4 b:$



${ }^{1} \mathrm{H}$ NMR $\left(501 \mathrm{MHz}, \mathrm{CD}_{2} \mathrm{Cl}_{2}\right) \delta 8.16(\mathrm{~s}, 2 \mathrm{H}), 8.10(\mathrm{~d}, J=8.3 \mathrm{~Hz}, 2 \mathrm{H}), 8.07-8.02(\mathrm{~m}$, 2H), 7.92 (ddd, $J=8.1,6.7,1.1 \mathrm{~Hz}, 2 \mathrm{H}), 7.89-7.84(\mathrm{~m}, 4 \mathrm{H}), 7.79-7.74(\mathrm{~m}, 4 \mathrm{H}), 7.67-$ $7.59(\mathrm{~m}, 5 \mathrm{H}), 7.56(\mathrm{~d}, J=7.3 \mathrm{~Hz}, 2 \mathrm{H}), 7.51(\mathrm{~d}, J=1.6 \mathrm{~Hz}, 2 \mathrm{H}), 7.47(\mathrm{~d}, J=7.9 \mathrm{~Hz}, 2 \mathrm{H})$, $7.42-7.37(\mathrm{~m}, 2 \mathrm{H}), 7.33-7.14(\mathrm{~m}, 11 \mathrm{H}), 7.11(\mathrm{~s}, 2 \mathrm{H}), 6.75(\mathrm{dd}, J=7.9,1.7 \mathrm{~Hz}, 2 \mathrm{H})$, $6.63(\mathrm{~d}, J=8.0 \mathrm{~Hz}, 2 \mathrm{H}), 6.32(\mathrm{dd}, J=8.0,1.7 \mathrm{~Hz}, 2 \mathrm{H}), 5.21-4.88(\mathrm{~m}, 1 \mathrm{H}), 2.81-2.71$ $(\mathrm{m}, 2 \mathrm{H}), 2.65(\mathrm{ddd}, J=11.2,9.5,7.2 \mathrm{~Hz}, 2 \mathrm{H}), 2.62-2.50(\mathrm{~m}, 7 \mathrm{H}), 2.41-2.25(\mathrm{~m}, 10 \mathrm{H})$, $2.25-2.03(\mathrm{~m}, 7 \mathrm{H})$.

${ }^{13} \mathrm{C}$ NMR $\left(126 \mathrm{MHz}, \mathrm{CD}_{2} \mathrm{Cl}_{2}\right) \delta 153.0,152.6,152.4,152.0,143.9,142.8,139.5,138.7$, $138.7,138.5,135.1,134.3,134.2,133.4,132.2$, 131.9, 131.8, 131.6, 131.5, 131.3, 129.5, $129.1,128.7,128.4,127.9,127.5,127.3,127.0,126.8,126.7,126.7,126.5,126.4,123.7$, $123.4,123.3,122.4,122.4,122.0,119.4,119.0,118.5,118.0,51.9,51.9,33.2,33.0$,

$32.7,32.4,16.8,16.6$.

${ }^{19} \mathrm{~F}$ NMR $\left(471 \mathrm{MHz}, \mathrm{CD}_{2} \mathrm{Cl}_{2}\right) \delta-81.1(\mathrm{t}, J=9.9 \mathrm{~Hz}),-111.7(\mathrm{dt}, J=129.3 \mathrm{~Hz}, J=15.0 \mathrm{~Hz}),-120.3(\mathrm{dd}, J=23.3,13.6 \mathrm{~Hz}),-121.7-$ $-122.0(\mathrm{~m}),-123.0(\mathrm{q}, J=13.8 \mathrm{~Hz}),-126.4(\mathrm{t}, J=14.8 \mathrm{~Hz})$.

${ }^{31} \mathrm{P}$ NMR $\left(203 \mathrm{MHz}, \mathrm{CD}_{2} \mathrm{Cl}_{2}\right) \delta-17.2$.

$\mathrm{R} f=0.42($ Ethyl acetate/hexanes $=1: 4)$.

ESI-HRMS ( $m / z)$ : calculated for $\mathrm{C}_{116} \mathrm{H}_{72} \mathrm{~N}_{3} \mathrm{O}_{8} \mathrm{P}_{2} \mathrm{~S}_{2} \mathrm{~F}_{26}\left([\mathrm{M}-\mathrm{H}]^{-}\right)$: 2254.3826, found: 2254.3813 .

m.p. $=228.7-233 \cdot 1^{\circ} \mathrm{C}$.

$[\boldsymbol{\alpha}]_{\mathbf{D}}^{25}=168.0\left(c 0.10, \mathrm{CHCl}_{3}\right)$

$(S, S)-I D P i-4 c:$



${ }^{1} \mathrm{H}$ NMR (501 MHz, $\left.\mathrm{CD}_{2} \mathrm{Cl}_{2}\right) \delta 8.07(\mathrm{~s}, 2 \mathrm{H}), 8.01(\mathrm{~d}, J=8.2 \mathrm{~Hz}, 2 \mathrm{H}), 7.95(\mathrm{dd}, J=8.3$, $1.2 \mathrm{~Hz}, 2 \mathrm{H}), 7.82$ (ddd, $J=8.1,6.7,1.2 \mathrm{~Hz}, 2 \mathrm{H}), 7.79-7.73(\mathrm{~m}, 4 \mathrm{H}), 7.70-7.63(\mathrm{~m}, 4 \mathrm{H})$, $7.58-7.50(\mathrm{~m}, 4 \mathrm{H}), 7.47(\mathrm{dd}, J=7.5,1.0 \mathrm{~Hz}, 2 \mathrm{H}), 7.43(\mathrm{~d}, J=1.6 \mathrm{~Hz}, 2 \mathrm{H}), 7.38(\mathrm{~d}, J=$ $8.0 \mathrm{~Hz}, 2 \mathrm{H}), 7.32-7.29(\mathrm{~m}, 4 \mathrm{H}), 7.22(\mathrm{~d}, J=7.4 \mathrm{~Hz}, 2 \mathrm{H}), 7.13$ (ddtd, $J=35.5,18.1,7.5$, $1.2 \mathrm{~Hz}, 9 \mathrm{H}), 7.02(\mathrm{~s}, 2 \mathrm{H}), 6.66(\mathrm{dd}, J=7.9,1.6 \mathrm{~Hz}, 2 \mathrm{H}), 6.54(\mathrm{~d}, J=8.0 \mathrm{~Hz}, 2 \mathrm{H}), 6.24$ $(\mathrm{dd}, J=8.0,1.7 \mathrm{~Hz}, 2 \mathrm{H}), 2.67(\mathrm{q}, J=9.8 \mathrm{~Hz}, 2 \mathrm{H}), 2.60-2.42(\mathrm{~m}, 8 \mathrm{H}), 2.31-1.98(\mathrm{~m}, 14 \mathrm{H})$.

${ }^{13} \mathrm{C} \mathrm{NMR}\left(126 \mathrm{MHz}, \mathrm{CD}_{2} \mathrm{Cl}_{2}\right) \delta 153.0,152.6,152.4,152.1,143.9(\mathrm{t}, J=4.7 \mathrm{~Hz}), 142.8$ (t, $J=4.7 \mathrm{~Hz}), 139.5,138.7,138.7,138.5,135.1,134.3,134.2,133.4,132.2$, 131.9, $131.8,131.6,131.5,131.2,129.5,129.1,128.7,128.4,127.9,127.5,127.3,127.0,126.8$, $126.7,126.7,126.5,126.4,123.7,123.4,123.3,122.4,122.3,122.0,119.4,119.0,118.5$, $118.1,53.8,53.6,53.4,53.2,53.0,51.9,51.9,33.2,33.0,32.7,32.4,16.7,16.6$.

${ }^{19} \mathrm{~F}$ NMR $\left(471 \mathrm{MHz}, \mathrm{CD}_{2} \mathrm{Cl}_{2}\right) \delta-81.1(\mathrm{t}, J=10.0 \mathrm{~Hz}),-111.7(\mathrm{qt}, J=16.0 \mathrm{~Hz}, J=14.4 \mathrm{~Hz}),-120.3,-121.6,-122.1,-122.9,-126.3$. 
${ }^{31} \mathrm{P}$ NMR (203 MHz, $\left.\mathrm{CD}_{2} \mathrm{Cl}_{2}\right) \delta-17.2$.

$\mathrm{R} f=0.51($ Ethyl acetate/hexanes $=1: 4)$.

ESI-HRMS ( $m / z)$ : calculated for $\mathrm{C}_{120} \mathrm{H}_{72} \mathrm{~F}_{34} \mathrm{~N}_{3} \mathrm{O}_{8} \mathrm{P}_{2} \mathrm{~S}_{2}\left([\mathrm{M}-\mathrm{H}]^{-}\right)$: 2454.3699, found: 2454.3705 .

m.p. $=242.3-243.3^{\circ} \mathrm{C}$.

$[\boldsymbol{\alpha}]_{\mathbf{D}}^{\mathbf{2 5}}=147.7\left(c 0.13, \mathrm{CHCl}_{3}\right)$.

LC-MS (50 mm YMC TriArt Bio C4, 3.0 mm i.D., 0.1\% TFA/MeCN 15:85, $1.0 \mathrm{~mL} / \mathrm{min}, 5.0 \mathrm{MPa}, 308 \mathrm{~K}): 254 \mathrm{~nm}: t_{R}=5.63 \mathrm{~min}, 98 \%$ $\left([\mathrm{M}-\mathrm{H}]^{-}\right): 2454$.

$(S, S)-I D P i-4 d:$



${ }^{1} \mathrm{H}$ NMR (501 MHz, $\left.\mathrm{CD}_{2} \mathrm{Cl}_{2}\right) \delta 8.17(\mathrm{~s}, 2 \mathrm{H}), 8.08(\mathrm{~d}, J=8.2 \mathrm{~Hz}, 2 \mathrm{H}), 8.03(\mathrm{~d}, J=8.2 \mathrm{~Hz}$, 2H), 7.84-7.79 (m, 4H), $7.74(\mathrm{~d}, J=7.5 \mathrm{~Hz}, 2 \mathrm{H}), 7.70(\mathrm{~d}, J=1.7 \mathrm{~Hz}, 2 \mathrm{H}), 7.68-7.58(\mathrm{~m}$, $8 \mathrm{H}), 7.45$ (dt, $J=7.2,1.6 \mathrm{~Hz}, 4 \mathrm{H}), 7.37$ (ddd, $J=8.1,6.7,1.3 \mathrm{~Hz}, 2 \mathrm{H}), 7.33-7.21$ (m, $10 \mathrm{H}), 7.21-7.13(\mathrm{~m}, 4 \mathrm{H}), 6.95(\mathrm{~d}, J=8.0 \mathrm{~Hz}, 2 \mathrm{H}), 6.73(\mathrm{dd}, J=8.0,1.7 \mathrm{~Hz}, 2 \mathrm{H}), 6.56$ $(\mathrm{dd}, J=8.0,1.6 \mathrm{~Hz}, 2 \mathrm{H}), 4.02(\mathrm{~d}, J=29.6 \mathrm{~Hz}, 1 \mathrm{H}), 3.62(\mathrm{~d}, J=24.7 \mathrm{~Hz}, 2 \mathrm{H}), 2.99-2.86$ $(\mathrm{m}, 2 \mathrm{H}), 2.66(\mathrm{dd}, J=7.2,4.1 \mathrm{~Hz}, 2 \mathrm{H}), 2.53(\mathrm{q}, J=8.3 \mathrm{~Hz}, 4 \mathrm{H}), 2.40-2.11(\mathrm{~m}, 5 \mathrm{H}), 1.61$ (s, 3H), $1.40(\mathrm{~s}, 2 \mathrm{H}), 1.28(\mathrm{~s}, 4 \mathrm{H})$.

${ }^{13} \mathrm{C}$ NMR $\left(126 \mathrm{MHz}, \mathrm{CD}_{2} \mathrm{Cl}_{2}\right) \delta 152.9,152.3,152.1,152.1,143.9(\mathrm{t}, J=4.5 \mathrm{~Hz}), 143.0$ $(\mathrm{t}, J=4.5 \mathrm{~Hz}), 139.1,138.6,138.6,138.4,135.0,134.6,133.9,133.7,131.9,131.7$, $131.7,131.5,131.4,131.1,129.2,128.7,128.4,127.7,127.4,127.3,126.9,126.8,126.8$, $126.7,126.7,126.6,126.5,124.0,123.2,123.1,122.4,122.4,119.5,119.3,118.9,118.3$, $51.9,51.6,33.3,33.2,32.9,32.6,16.7,16.7$.

${ }^{19} \mathrm{~F}$ NMR $\left(471 \mathrm{MHz}, \mathrm{CD}_{2} \mathrm{Cl}_{2}\right) \delta-136.4(\mathrm{~d}, J=22.2 \mathrm{~Hz}),-146.7,-160.0(\mathrm{t}, J=20.3 \mathrm{~Hz})$.

${ }^{31} \mathrm{P}$ NMR (203 MHz, $\left.\mathrm{CD}_{2} \mathrm{Cl}_{2}\right) \delta-15.6$.

$\mathrm{R} f=0.54($ Ethyl acetate/hexanes $=1: 4)$.

EI-HRMS ( $m / z)$ : calculated for $\mathrm{C}_{116} \mathrm{H}_{72} \mathrm{~F}_{10} \mathrm{~N}_{3} \mathrm{O}_{8} \mathrm{P}_{2} \mathrm{~S}_{2}\left([\mathrm{M}-\mathrm{H}]^{-}\right)$: 1950.4082 , found: 1950.4082 .

m.p. $=288.9-291.6^{\circ} \mathrm{C}$.

$[\boldsymbol{\alpha}]_{\mathbf{D}}^{\mathbf{2 5}}=382.0\left(c 0.10, \mathrm{CHCl}_{3}\right)$

LC-MS (50 mm Zorbax SB300-C8, $3.5 \mu \mathrm{m}, 4.6 \mathrm{~mm}$ i.D., 0.1\% TFA/MeCN 15:85, $1.0 \mathrm{~mL} / \mathrm{min}, 5.0 \mathrm{MPa}, 308 \mathrm{~K}): 254 \mathrm{~nm}: t_{R}=3.78$ $\min , 96 \%\left([\mathrm{M}-\mathrm{H}]^{-}\right): 1950$.

$(S, S)-I D P i-4 \mathbf{e}:$



${ }^{1} \mathrm{H}$ NMR $\left(501 \mathrm{MHz}, \mathrm{CD}_{2} \mathrm{Cl}_{2}\right) \delta 8.95(\mathrm{~d}, J=1.7 \mathrm{~Hz}, 2 \mathrm{H}), 8.78(\mathrm{dd}, J=8.4,4.8 \mathrm{~Hz}, 4 \mathrm{H})$, $8.61-8.56(\mathrm{~m}, 2 \mathrm{H}), 8.50-8.40(\mathrm{~m}, 10 \mathrm{H}), 8.19(\mathrm{dd}, J=8.4,1.4 \mathrm{~Hz}, 2 \mathrm{H}), 8.11-8.06$ $(\mathrm{m}, 4 \mathrm{H}), 8.06-8.00(\mathrm{~m}, 6 \mathrm{H}), 8.00-7.91(\mathrm{~m}, 4 \mathrm{H}), 7.82(\mathrm{ddd}, J=8.4,7.0,1.1 \mathrm{~Hz}$, $2 \mathrm{H}), 7.74(\mathrm{~d}, J=8.5 \mathrm{~Hz}, 2 \mathrm{H}), 7.72-7.66(\mathrm{~m}, 6 \mathrm{H}), 7.63(\mathrm{~d}, J=8.6 \mathrm{~Hz}, 6 \mathrm{H}), 7.58-$ $7.41(\mathrm{~m}, 16 \mathrm{H}), 6.58(\mathrm{~d}, J=8.7 \mathrm{~Hz}, 2 \mathrm{H}), 6.15(\mathrm{dd}, J=8.5,1.8 \mathrm{~Hz}, 2 \mathrm{H}), 5.69(\mathrm{~d}, J=$ $8.5 \mathrm{~Hz}, 2 \mathrm{H})$.

${ }^{13} \mathrm{C}$ NMR $\left(126 \mathrm{MHz}, \mathrm{CD}_{2} \mathrm{Cl}_{2}\right) \delta 143.5,143.1,134.6,132.3,132.3,132.1,131.7,131.6$, $130.2,129.9,129.7,129.4,129.4,129.3,129.3,129.3,129.2,129.1,129.1,128.8$, $128.5,128.0,127.7,127.6,127.4,127.3,127.2,127.2,127.04,126.8,126.8,126.8$, $126.6,126.4,124.9,124.4,123.7,123.6,123.4,123.4,123.3,123.2,123.2,122.9$, $122.8,122.80,122.4,122.0$. 
${ }^{19} \mathrm{~F}$ NMR $\left(471 \mathrm{MHz}, \mathrm{CD}_{2} \mathrm{Cl}_{2}\right) \delta-79.3$.

${ }^{31} \mathrm{P}$ NMR (203 MHz, $\left.\mathrm{CD}_{2} \mathrm{Cl}_{2}\right) \delta-14.5$.

$\mathrm{R} f=0.35$ (Ethyl acetate/hexanes $=2: 3)$.

ESI-HRMS ( $m / z)$ : calculated for $\mathrm{C}_{114} \mathrm{H}_{64} \mathrm{~F}_{6} \mathrm{~N}_{3} \mathrm{O}_{8} \mathrm{P}_{2} \mathrm{~S}_{2}\left([\mathrm{M}-\mathrm{H}]^{-}\right)$: 1842.3520 ; found: 1842.3538 .

m.p. $=389.4-395.5^{\circ} \mathrm{C}$.

$[\boldsymbol{\alpha}]_{\mathbf{D}}^{25}=197.3\left(c 0.22, \mathrm{CHCl}_{3}\right)$

The data were in accordance with the reported literature ${ }^{16}$.

$(S, S)-I D P i-4 f:$



${ }^{1} \mathrm{H}$ NMR $\left(501 \mathrm{MHz}, \mathrm{CD}_{2} \mathrm{Cl}_{2}\right) \delta 8.17-8.11(\mathrm{~m}, 2 \mathrm{H}), 7.89(\mathrm{~s}, 2 \mathrm{H})$, $7.80(\mathrm{~d}, J=7.0 \mathrm{~Hz}, 4 \mathrm{H}), 7.65(\mathrm{ddd}, J=8.1,6.5,1.5 \mathrm{~Hz}, 2 \mathrm{H})$, $7.44-7.35(\mathrm{~m}, 7 \mathrm{H}), 7.22(\mathrm{~d}, J=6.4 \mathrm{~Hz}, 10 \mathrm{H}), 7.19(\mathrm{t}, J=1.8$ $\mathrm{Hz}, 2 \mathrm{H}), 7.16(\mathrm{~d}, J=1.8 \mathrm{~Hz}, 6 \mathrm{H}), 7.12(\mathrm{t}, J=1.8 \mathrm{~Hz}, 2 \mathrm{H}), 6.95$ $(\mathrm{d}, J=1.8 \mathrm{~Hz}, 4 \mathrm{H}), 6.93-6.86(\mathrm{~m}, 4 \mathrm{H}), 6.73(\mathrm{~d}, J=8.4 \mathrm{~Hz}, 4 \mathrm{H})$, $4.89(\mathrm{~s}, 1 \mathrm{H}), 1.13(\mathrm{~s}, 36 \mathrm{H}), 0.99(\mathrm{~s}, 36 \mathrm{H})$.

${ }^{13} \mathrm{C}$ NMR $\left(126 \mathrm{MHz}, \mathrm{CD}_{2} \mathrm{Cl}_{2}\right) \delta 150.9,150.8,143.7,143.7(\mathrm{t}, J$ $=5.0 \mathrm{~Hz}), 143.4(\mathrm{t}, J=5.0 \mathrm{~Hz}), 141.5,141.5,141.1,140.0$, $139.6,134.4,133.9,133.7,133.4,132.4,131.8,131.7,131.2$, $131.0,131.0,129.5,129.5,129.0,128.2,127.1,127.1,126.8$, $126.7,126.4,126.3,123.4,122.3,121.3,121.2,121.1,34.7$, $34.6,31.0,29.7$.

${ }^{19} \mathrm{~F}$ NMR $\left(471 \mathrm{MHz}, \mathrm{CD}_{2} \mathrm{Cl}_{2}\right) \delta-112.2(\mathrm{dd}, J=76.0,17.8 \mathrm{~Hz}),-133.7(\mathrm{~d}, J=18.5 \mathrm{~Hz}),-140.8(\mathrm{dt}, J=77.3,17.3 \mathrm{~Hz}),-145.2(\mathrm{dt}, J=$ $59.0,16.5 \mathrm{~Hz}),-146.8(\mathrm{dt}, J=58.6,18.7 \mathrm{~Hz}),-149.8(\mathrm{t}, J=18.7 \mathrm{~Hz}),-154.5(\mathrm{~d}, J=20.0 \mathrm{~Hz})$.

${ }^{31} \mathrm{P}$ NMR $\left(203 \mathrm{MHz}, \mathrm{CD}_{2} \mathrm{Cl}_{2}\right) \delta-10.1$.

$\mathrm{R} f=0.8($ Ethyl acetate/hexanes $=1: 4)$.

ESI-HRMS (m/z): calculated for $\mathrm{C}_{140} \mathrm{H}_{120} \mathrm{~F}_{14} \mathrm{~N}_{3} \mathrm{O}_{8} \mathrm{P}_{2} \mathrm{~S}_{2}\left([\mathrm{M}-\mathrm{H}]^{-}\right)$: 2362.7774, found: 2362.7775.

m.p. $=193.8-195.2^{\circ} \mathrm{C}$.

$[\boldsymbol{\alpha}]_{\mathbf{D}}^{25}=241.8\left(c 0.11, \mathrm{CHCl}_{3}\right)$

\section{Development of suitable reaction conditions and catalyst identification.}

\section{General procedure for the optimization of the catalytic silylation of ketones with allylsilanes.}

Allyl silane $\mathbf{2 a}$ or $\mathbf{2 b}(0.05 \mathrm{mmol}, 2.0$ equiv.) was placed in a flame-dried Schlenk flask, which was equipped with a teflon-coated magnetic stirring bar. IDPi $\mathbf{4 a}-\mathbf{4 f}(0.01$ equiv.) and solvent (0.08 $\mathrm{M}, 0.3 \mathrm{~mL})$ were added, and the resultant solution was stirred for 5 min at $25^{\circ} \mathrm{C}$. Ketone $1 \mathrm{a}(4.4 \mathrm{mg}, 0.025 \mathrm{mmol}, 1.0$ equiv.) was slowly added and the reaction mixture was stirred for an additional $12 \mathrm{~h}$. After the ketone was fully consumed, as monitored by TLC, the reaction mixture was treated with triethylamine. Volatiles were removed in vacuo and the yield of 3a was determined by NMR analysis with nitrobenzene as internal standard. The enantiomeric ratio was determined by HPLC after purification by prep. TLC.

Table S1. Initial screening with commonly used Brønsted acid catalysts: 
<smiles>C=CC[C+](C)(C)CCCCC</smiles>

$1 \mathrm{a}$

$2 a$ $\underset{\text { Toluene, } \mathrm{rt}, 12 \mathrm{~h}}{\stackrel{\text { Acid catalyst }(1 \mathrm{~mol} \%)}{\longrightarrow}}$<smiles>O=[Sb](O)C1=CCC(c2ccccc2)CC1</smiles>

3a<smiles>[R]c1cc2ccccc2c2c1OP(=O)(O)Oc1c([R])cc3ccccc3c1-2</smiles>

(S)-Phosphoric Acid
$\mathrm{R}=\mathrm{Ph}$

No Reaction



(S)-Imidodiphosphoric Acid $R=$ 2-isopropyl-5-methylphenyl

No Reaction



(R)-Disulfonimide $\mathrm{R}=3,5-\left(\mathrm{CF}_{3}\right)_{2}-\mathrm{C}_{6} \mathrm{H}_{3}$

No Reaction



(S,S)-Imidodiphosphorimidate $\mathbf{R}=\mathbf{P h}$

Full Conv.

Table S2. Screening of different IDPi catalysts:<smiles>C=CC[C+]1CC(=O)CCC1c1ccccc1</smiles>

1a

2a

<smiles>PC1CC=C(C[SeH3-])CC1</smiles>

$3 a$



(S,S)-IDPi 4a-4f



$\mathrm{R}_{f}=\mathrm{C}_{2} \mathrm{~F}_{5}(4 \mathrm{a}) \mathrm{R}_{f}=n-\mathrm{C}_{6} \mathrm{~F}_{13}(4 \mathrm{~b})$

$\mathrm{R}_{f}=n-\mathrm{C}_{8} \mathrm{~F}_{17}(4 \mathrm{c}) \quad \mathrm{R}_{f}=\mathrm{C}_{6} \mathrm{~F}_{5}(4 \mathrm{~d})$<smiles>Cc1ccc2c(ccc3ccccc32)c1</smiles>

$\mathrm{R}_{f}=\mathrm{CF}_{3}(\mathbf{4 e})$<smiles>Cc1ccc(-c2cc(C(C)(C)C)cc(C(C)(C)C)c2)cc1</smiles>

$\mathrm{R}_{f}=\mathrm{C}_{10} \mathrm{~F}_{7}(\mathbf{4 f})$

\begin{tabular}{cccc}
\hline entry $^{a}$ & catalyst & yield $(\%)^{b}$ & e.r. $^{c}$ \\
\hline 1 & $\mathbf{4 a}$ & 95 & $82: 18$ \\
2 & $\mathbf{4 b}$ & 99 & $85.5: 14.5$ \\
3 & $\mathbf{4 c}$ & 99 & $88: 12$ \\
4 & $\mathbf{4 d}$ & 99 & $80: 20$ \\
5 & $\mathbf{4 e}$ & 80 & $80: 20$ \\
6 & $\mathbf{4 f}$ & 99 & $75: 25$ \\
\hline
\end{tabular}

${ }^{a}$ Reactions were conducted with ketone $1 \mathbf{a}(0.025 \mathrm{mmol})$, allyl silane $\mathbf{2 a}\left(2.0\right.$ equiv.), and catalyst $\mathbf{4 a}-\mathbf{4 f}(1.0 \mathrm{~mol} \%)$ in toluene at rt. ${ }^{b}$ All yields were determined by crude ${ }^{1} \mathrm{H}$ NMR analysis with nitrobenzene as internal standard. ${ }^{c}$ The enantiomeric ratio (e.r.) was determined by HPLC analysis.

Table S3. Screening of different solvents: 




1a 2 a $3 a$

\begin{tabular}{cccc}
\hline entry $^{a}$ & solvent & yield $(\%)^{b}$ & e.r. ${ }^{c}$ \\
\hline 1 & $\mathrm{Et}_{2} \mathrm{O}$ & 99 & $60: 40$ \\
2 & $\mathrm{THF}$ & $\mathrm{NR}$ & -- \\
3 & $\mathrm{MTBE}$ & $\mathrm{NR}$ & -- \\
4 & $\mathrm{CH}_{3} \mathrm{CN}$ & $\mathrm{NR}$ & -- \\
5 & $\mathrm{CH}_{2} \mathrm{Cl}_{2}$ & 99 & $68: 32$ \\
6 & $\mathrm{CHCl}_{3}$ & 99 & $70: 30$ \\
7 & $\mathrm{Cyclohexane}$ & 99 & $70: 30$ \\
8 & Benzene & 99 & $80: 20$ \\
9 & Mesitylene & 99 & $78: 22$ \\
10 & Toluene & 99 & $82: 18$ \\
11 & $1,4-$-Dioxane & 99 & $87: 13$ \\
\hline
\end{tabular}

${ }^{a}$ Reactions were conducted with ketone $\mathbf{1 a}(0.025 \mathrm{mmol})$, silane source $\mathbf{2 a}\left(2.0\right.$ equiv.), and catalyst $\mathbf{4 a}(1.0 \mathrm{~mol} \%)$ at $25^{\circ} \mathrm{C} .{ }^{b} \mathrm{All}$ yields were determined by crude ${ }^{1} \mathrm{H}$ NMR analysis with nitrobenzene as internal standard. ${ }^{c}$ The enantiomeric ratio (e.r.) was determined by HPLC analysis.

Table S4. Screening of different silicon sources:



1a



$2 b$



2c



2d



$2 e$



$2 f$

99\%, $87: 13$ e.r. $\quad 77 \%, 64: 36$ e.r. $\quad 99 \%, 87: 13$ e.r. $\quad 10 \%, 53.5: 46.5$ e.r. $\quad$ No Reaction

only Mukaiyama

aldol product was observed

Notes: 1. the reaction time with $\mathbf{2} \boldsymbol{b}$ was 7 h. 2. compared to $\mathbf{2 c}$, silicon source $2 \boldsymbol{a}$ was commercially available, thus it was chosen as optimal agent for the next screening.

Table S5. Screening of the reaction temperature: 




${ }^{a}$ Reactions were conducted with ketone $1 \mathbf{a}(0.025 \mathrm{mmol}), \mathbf{2 a}(2.0$ equiv. $)$, and catalyst $\mathbf{4 c}(1.0 \mathrm{~mol} \%)$ in combined solvent $($ toluene/dioxane $=2: 1)$ at $25^{\circ} \mathrm{C} .{ }^{b}$ All yields were determined from crude NMR analysis with newly distilled nitrobenzene as internal standard. ${ }^{c}$ The enantiomeric ratio (e.r.) was determined by HPLC analysis. ${ }^{d}$ with sole dioxane as solvent.

\section{The preparation and characterization of cyclic ketones}



The 4-aryl substituted ketones were prepared according to the methods reported in the literature with minor modifications. $\mathbf{1 c}, \mathbf{1 e}, \mathbf{1 j}$, 1k and $\mathbf{1 n}$ are known compounds and the spectra are in accordance with the reported structures. ${ }^{1}$

Step 1: To a flame dried two-neck round-bottom flask with a condenser, magnesium turnings $(0.60 \mathrm{~g}, 49.6 \mathrm{mmol})$ and a small portion of iodine as initiator was added. After the first $5 \mathrm{~mL}$ of $\mathrm{R}^{1}-\mathrm{Br}(23.5 \mathrm{mmol})$ solution in THF (40 mL) was added in one portion, the suspension was heated till the color changed from dark brown to clear (which indicates the formation of the Grignard reagent). Then the remaining solution of $\mathrm{R}^{1}-\mathrm{Br}$ was slowly added via a funnel and the reaction mixture was heated to reflux for $2 \mathrm{~h}$. After the flask was cooled to room temperature, a solution of 1,4-cyclohexanedione monoethylene ketal (3.50 g, $22.4 \mathrm{mmol}) \mathrm{in}$ THF (40 mL) was added dropwise, and the reaction mixture was heated to reflux for $12 \mathrm{~h}$. The mixture was treated with a saturated aqueous $\mathrm{NH}_{4} \mathrm{Cl}$ solution $(50$ $\mathrm{mL})$, and the resultant mixture was extracted with ethyl acetate $(3 \times 50 \mathrm{~mL})$. The organic layers were dried $\left(\mathrm{MgSO}_{4}\right)$, filtered, and concentrated under reduced pressure to give the crude product. This material was purified directly by column chromatography on silica gel to afford the desired alcohols.

Step 2: To a solution of alcohols $(15.0 \mathrm{mmol})$ in pyridine $(85 \mathrm{~mL})$ at $0{ }^{\circ} \mathrm{C}$ was added DBU $(1,8$-diazabicyclo[5.4.0]undec-7-ene) $(4.5$ $\mathrm{mL}, 30.0 \mathrm{mmol})$ followed by $\mathrm{POCl}_{3}(2.7 \mathrm{~mL}, 29.5 \mathrm{mmol})$ dropwise. The resultant orange solution was stirred at room temperature for $1 \mathrm{~h}$ and at $80{ }^{\circ} \mathrm{C}$ for 90 min during which time the orange color darkened. The solution was cooled to $0{ }^{\circ} \mathrm{C}$ and carefully treated with ethyl acetate $(100 \mathrm{~mL})$ and $\mathrm{H}_{2} \mathrm{O}(100 \mathrm{~mL})$. The organic phase was washed with $\mathrm{H}_{2} \mathrm{O}$ and brine, dried $\left(\mathrm{MgSO}_{4}\right)$, and evaporated in vacuo to give the alkenes as orange-brown oil. The crude products were purified by column chromatography on silica gel to afford the desired pure alkenes.

Step 3: The pure alkenes from the above reactions were dissolved in 1:1 MeOH/ethyl acetate (25 mL) and Palladium (10\% on carbon, $0.3 \mathrm{~g}$ ) was added. The mixture was stirred for $5 \mathrm{~h}$ under hydrogen $(4 \mathrm{MPa})$ and filtered through a pad of Celite ${ }^{\circledR}$. The solvent was 
removed under reduced pressure to afford the crude ketals. The crude products were purified directly by column chromatography on silica gel to afford the desired pure ketals.

Step 4: The ketals were dissolved in a mixture of THF, water, and concentrated sulfuric acid (4:2:1, $70 \mathrm{~mL})$. The mixture was stirred for $90 \mathrm{~min}$, diluted with brine $(50 \mathrm{~mL})$, and extracted with ethyl acetate $(3 \times 50 \mathrm{~mL})$. The combined organic extracts were dried $\left(\mathrm{MgSO}_{4}\right)$, filtered, and concentrated. Column chromatography of the residue over silica gel gave the desired pure 4-substituted cyclohexanones.

\section{4-(4-chlorophenyl)cyclohexan-1-one (1d)}

${ }^{1} \mathrm{H}$ NMR $\left(501 \mathrm{MHz}, \mathrm{CDCl}_{3}\right) \delta 7.22(\mathrm{~d}, J=8.4 \mathrm{~Hz}, 2 \mathrm{H}), 7.10(\mathrm{~d}, J=8.4 \mathrm{~Hz}, 2 \mathrm{H}), 2.94(\mathrm{tt}, J=12.1,3.4 \mathrm{~Hz}, 1 \mathrm{H}), 2.46-2.39(\mathrm{~m}$, $4 \mathrm{H}), 2.18-2.06(\mathrm{~m}, 2 \mathrm{H}), 1.91-1.79(\mathrm{~m}, 2 \mathrm{H})$.

${ }^{13} \mathrm{C} \mathrm{NMR}\left(126 \mathrm{MHz}, \mathrm{CDCl}_{3}\right) \delta 210.6,143.2,132.2,128.7,128.0,42.2,41.2,33.9$.

$\mathrm{R} f=0.35($ Ethyl acetate/hexanes $=1: 4)$.

EI-HRMS $(\mathrm{m} / \mathrm{z})$ : calculated for $\mathrm{C}_{12} \mathrm{H}_{13} \mathrm{O}_{1} \mathrm{Cl}_{1}\left[\mathrm{M}^{+\bullet}\right]$ : 208.0649, found: 208.0651 .

m.p. $=66.2-68.4^{\circ} \mathrm{C}$.

\section{4-(4-(trifluoromethyl)phenyl)cyclohexan-1-one (1f)}

(1)

${ }^{1} \mathrm{H} \mathrm{NMR}\left(501 \mathrm{MHz}, \mathrm{CDCl}_{3}\right) \delta 7.51(\mathrm{~d}, J=8.1 \mathrm{~Hz}, 2 \mathrm{H}), 7.29(\mathrm{~d}, J=8.0 \mathrm{~Hz}, 2 \mathrm{H}), 3.03(\mathrm{tt}, J=12.1,3.4 \mathrm{~Hz}, 1 \mathrm{H}), 2.51-2.40(\mathrm{~m}$, $4 \mathrm{H}), 2.21-2.10(\mathrm{~m}, 2 \mathrm{H}), 1.95-1.81(\mathrm{~m}, 2 \mathrm{H})$.

${ }^{13} \mathrm{C}$ NMR $\left(126 \mathrm{MHz}, \mathrm{CDCl}_{3}\right) \delta 210.3,148.7,128.9(\mathrm{q}, J=32.6 \mathrm{~Hz}), 127.0,125.5(\mathrm{q}, J=3.8 \mathrm{~Hz}), 124.1(\mathrm{app} \mathrm{d}, J=271.8 \mathrm{~Hz})$, $42.6,41.1,33.7$.

${ }^{19} \mathrm{~F}$ NMR $\left(471 \mathrm{MHz}, \mathrm{CDCl}_{3}\right) \delta-64.4$

$\mathrm{R} f=0.29($ Ethyl acetate/ hexanes $=1: 4)$.

EI-HRMS (m/z): calculated for $\mathrm{C}_{13} \mathrm{H}_{13} \mathrm{O}_{1} \mathrm{~F}_{3}\left[\mathrm{M}^{+\bullet}\right]: 242.0913$, found: 242.0915 .

m.p. $=45.6-46.9^{\circ} \mathrm{C}$.

\section{4-(3-(tert-butyl)phenyl)cyclohexan-1-one (1g)}



${ }^{1} \mathrm{H}$ NMR (501 MHz, $\left.\mathrm{CDCl}_{3}\right) \delta$ 7.20-7.18 (m, 3H), 7.01-6.96 (m, 1H), 3.00-2.90 (m, 1H), 2.51-2.39 (m, 4H), $2.17(\mathrm{ddt}, J$ $=14.7,5.9,2.4 \mathrm{~Hz}, 2 \mathrm{H}), 1.96-1.83(\mathrm{~m}, 2 \mathrm{H}), 1.26(\mathrm{~s}, 9 \mathrm{H})$.

${ }^{13} \mathrm{C}$ NMR $\left(126 \mathrm{MHz}, \mathrm{CDCl}_{3}\right) \delta 211.3,151.4,144.4,128.2,123.8,123.5,123.5,43.1,41.4,34.7,34.1,31.4$.

$\mathrm{R} f=0.50$ (Ethyl acetate/hexanes $=1: 4)$.

EI-HRMS $(\mathrm{m} / z)$ : calculated for $\mathrm{C}_{16} \mathrm{H}_{22} \mathrm{O}_{1}\left[\mathrm{M}^{+\bullet}\right]: 230.1665$, found: 230.1667 .

m.p. $=52.2-53.8^{\circ} \mathrm{C}$.

\section{4-(3-chlorophenyl)cyclohexan-1-one (1h)}


1

${ }^{1} \mathrm{H}$ NMR (501 MHz, $\left.\mathrm{CDCl}_{3}\right) \delta 7.07(\mathrm{~s}, 4 \mathrm{H}), 2.92(\mathrm{tt}, J=12.1,3.5 \mathrm{~Hz}, 1 \mathrm{H}), 2.45(\mathrm{~d}, J=1.0 \mathrm{~Hz}, 4 \mathrm{H}), 2.13$ (dddd, $J=13.7$, $6.4,5.2,3.2 \mathrm{~Hz}, 2 \mathrm{H}), 1.96-1.77(\mathrm{~m}, 2 \mathrm{H})$.

${ }^{13} \mathrm{C}$ NMR (126 MHz, $\left.\mathrm{CDCl}_{3}\right) \delta 211.3,151.4,144.4,128.2,123.9,123.6,123.5,43.1,41.5,34.7,34.1,31.4$.

$\mathrm{R} f=0.36($ Ethyl acetate/hexanes $=1: 4)$.

ESI-HRMS $(\mathrm{m} / \mathrm{z})$ : calculated for $\mathrm{C}_{13} \mathrm{H}_{16} \mathrm{O}_{1} \mathrm{Na}_{1}\left([\mathrm{M}+\mathrm{Na}]^{+}\right)$: 211.1093 , found: 211.1095 .

m.p. $=58.9-62.3^{\circ} \mathrm{C}$.

\section{4-(3-(trifluoromethyl)phenyl)cyclohexan-1-one (1i)}



${ }^{1} \mathrm{H}$ NMR (501 MHz, $\left.\mathrm{CDCl}_{3}\right) \delta 7.43(\mathrm{dt}, J=3.8,2.5 \mathrm{~Hz}, 2 \mathrm{H}), 7.40-7.33(\mathrm{~m}, 2 \mathrm{H}), 3.08-2.98(\mathrm{~m}, 1 \mathrm{H}), 2.49-2.42(\mathrm{~m}, 4 \mathrm{H})$, $2.23-2.12(\mathrm{~m}, 2 \mathrm{H}), 1.96-1.83(\mathrm{~m}, 2 \mathrm{H})$.

${ }^{13} \mathrm{C}$ NMR $\left(126 \mathrm{MHz}, \mathrm{CDCl}_{3}\right) \delta 210.3,145.6,130.8$ (app d, $\left.J=33.2 \mathrm{~Hz}\right), 130.0,129.0,124.1$ (app d, $\left.J=275.1 \mathrm{~Hz}\right), 123.5$ (q, $J=3.1 \mathrm{~Hz}), 42.6,41.1,33.7$.

$\mathrm{R} f=0.30$ (Ethyl acetate/hexanes $=1: 4)$.

EI-HRMS $(m / z)$ : calculated for $\mathrm{C}_{13} \mathrm{H}_{13} \mathrm{O}_{1} \mathrm{~F}_{3}\left[\mathrm{M}^{+\bullet}\right]: 242.0913$, found: 242.0916 .

m.p. $=71.5-72.5^{\circ} \mathrm{C}$.

\section{4-(3,5-difluorophenyl)cyclohexan-1-one (11)}



${ }^{1} \mathrm{H}$ NMR $\left(501 \mathrm{MHz}, \mathrm{CDCl}_{3}\right) \delta 6.73-6.65(\mathrm{~m}, 2 \mathrm{H}), 6.61(\mathrm{tt}, J=8.9,2.3 \mathrm{~Hz}, 1 \mathrm{H}), 2.95(\mathrm{tt}, J=12.1,3.4 \mathrm{~Hz}, 1 \mathrm{H}), 2.49-2.40$ (m, 4H), 2.21-2.09 (m, 2H), 1.89-1.75 (m, 2H).

${ }^{13} \mathrm{C}$ NMR $\left(126 \mathrm{MHz}, \mathrm{CDCl}_{3}\right) \delta 210.1,164.1-162.2(\mathrm{dd}, J=248.5 \mathrm{~Hz}, J=12.6 \mathrm{~Hz}), 148.7$ (t, $\left.J=8.6 \mathrm{~Hz}\right), 109.6-109.5$ $(\mathrm{dd}, J=19.6 \mathrm{~Hz}, J=5.5 \mathrm{~Hz}), 102.0(\mathrm{t}, J=37.9 \mathrm{~Hz}), 42.4(\mathrm{t}, J=2.1 \mathrm{~Hz}), 41.0,33.5$.

$\mathrm{R} f=0.36$ (Ethyl acetate/hexanes $=1: 4)$.

EI-HRMS $(\mathrm{m} / z)$ : calculated for $\mathrm{C}_{12} \mathrm{H}_{12} \mathrm{O}_{1} \mathrm{~F}_{2}\left[\mathrm{M}^{+*}\right]$ : 210.0851, found: 210.0853 .

m.p. $=76.8-80.6^{\circ} \mathrm{C}$.

\section{4-(naphthalen-1-yl)cyclohexan-1-one (1m)}



${ }^{1} \mathrm{H}$ NMR $\left(501 \mathrm{MHz}, \mathrm{CDCl}_{3}\right) \delta 8.08(\mathrm{~d}, J=8.5 \mathrm{~Hz}, 1 \mathrm{H}), 7.85-7.78(\mathrm{~m}, 1 \mathrm{H}), 7.68(\mathrm{dt}, J=8.2,1.1 \mathrm{~Hz}, 1 \mathrm{H}), 7.49(\mathrm{ddd}, J=$ $8.5,6.8,1.5 \mathrm{~Hz}, 1 \mathrm{H}), 7.44(\mathrm{ddd}, J=8.1,6.8,1.2 \mathrm{~Hz}, 1 \mathrm{H}), 7.38(\mathrm{dd}, J=8.2,7.2 \mathrm{~Hz}, 1 \mathrm{H}), 7.31(\mathrm{dd}, J=7.2,1.2 \mathrm{~Hz}, 1 \mathrm{H})$, 3.77 (tt, $J=12.0,3.2 \mathrm{~Hz}, 1 \mathrm{H}), 2.67-2.43(\mathrm{~m}, 4 \mathrm{H}), 2.32(\mathrm{ddq}, J=13.6,6.0,3.1 \mathrm{~Hz}, 2 \mathrm{H}), 1.99$ (tdd, $J=13.3,12.0,4.6 \mathrm{~Hz}$, $2 \mathrm{H})$.

${ }^{13} \mathrm{C}$ NMR $\left(126 \mathrm{MHz}, \mathrm{CDCl}_{3}\right) \delta 211.0,140.5,134.0,131.2,129.2,127.1,126.1,125.6,125.6,122.7,122.2,41.7,37.6$, 33.4 .

$\mathrm{R} f=0.39$ (Ethyl acetate/hexanes $=1: 4)$.

EI-HRMS $(\mathrm{m} / \mathrm{z})$ : calculated for $\mathrm{C}_{16} \mathrm{H}_{16} \mathrm{O}_{1}\left[\mathrm{M}^{+\bullet}\right]$ : 224.1196, found: 224.1200 .

m.p. $=71.1-73.8^{\circ} \mathrm{C}$. 
4,4'-disubstituted cyclohexanone $1 \mathrm{~s}$ was prepared according to a literature report with minor revisions ${ }^{2}$



Step 1: To a $25 \mathrm{~mL}$ round-bottom flask with a condenser, 2-phenylpropanal (20 mmol, $2 \mathrm{~g}, 1.0$ equiv.), but-3-en-2-one (30 mmol, 2.4 $\mathrm{mL}, 1.5$ equiv.), p-toluenesulfonic acid monohydrate ( $4 \mathrm{mmol}, 0.76 \mathrm{~g}, 0.2$ equiv.), and toluene $(6 \mathrm{~mL})$ were added subsequently. Then the reaction mixture was stirred in an oil bath at $80^{\circ} \mathrm{C}$ for $12 \mathrm{~h}$ till completion (as monitored by TLC). After the reaction mixture cooled down to room temperature, the solvent was removed in vacuo. Then $1 \mathrm{M}$ aqueous sodium hydroxide solution was added to the residue and the mixture extracted with ethyl acetate $(3 \times 10 \mathrm{~mL})$. The combined organic phase was washed by brine, dried over $\mathrm{NaSO}_{4}$, and filtered. The crude product was purified by column chromatography on silica gel to afford the desired pure unsaturated ketone.

Step 2: To a solution of the aforementioned unsaturated ketone $(9.1 \mathrm{mmol}, 1.7 \mathrm{~g})$ in ethyl acetate $(17 \mathrm{~mL})$ at $\mathrm{rt}$ was added Palladium ( $10 \%$ on carbon, $0.3 \mathrm{~g}$ ). The resultant suspension was stirred for $5 \mathrm{~h}$ under hydrogen $\left(8 \mathrm{bar}\right.$ ) and filtered through a pad of Celite ${ }^{\circledR}$. The crude products were purified by column chromatography on silica gel to afford the desired pure ketone $\mathbf{1 s}$.

\section{4-methyl-4-phenylcyclohexan-1-one 1s}

$\underbrace{\mathrm{O}}_{\mathrm{Ph}}$

${ }^{1} \mathrm{H}$ NMR $\left(501 \mathrm{MHz}, \mathrm{CDCl}_{3}\right) \delta$ 7.47-7.42 (m, 2H), 7.41-7.36 (m, 2H), 7.27-7.23 (m, 1H), 2.54-2.45 (m, 2H), 2.42-2.27 (m, $4 \mathrm{H}), 2.01-1.89(\mathrm{~m}, 2 \mathrm{H}), 1.33(\mathrm{~s}, 3 \mathrm{H})$.

${ }^{13} \mathrm{C}$ NMR (126 MHz, $\left.\mathrm{CDCl}_{3}\right) \delta 211.6,145.9,128.7,126.1,125.5,38.3,37.6,37.1,31.0$.

$\mathrm{R} f=0.50$ (Ethyl acetate/hexanes $=1: 4)$.

EI-HRMS $(\mathrm{m} / z)$ : calculated for $\mathrm{C}_{13} \mathrm{H}_{16} \mathrm{O}_{1}\left[\mathrm{M}^{+*}\right]$ : 188.1195 , found: 188.1199.

m.p. $=43.7-44.2^{\circ} \mathrm{C}$.

\section{Biscyclopentanone $1 \mathrm{u}$ and $1 \mathrm{w}$ were prepared according to reported methods ${ }^{3}$}



Step 1. To a solution of cis-bicyclo [3.3.0]octan-3,7-dione $(25 \mathrm{~g}, 181.06 \mathrm{mmol})$ in toluene $(300 \mathrm{~mL})$ was added 2,2-dimethyl-1,propanediol $(18.9 \mathrm{~g}, 181.06 \mathrm{mmol})$ and p-toluenesulfonic acid monohydrate $(5 \%)$. The resultant solution was stirred at room temperature 
overnight. After completion of the reaction (as monitored by TLC), the mixture was evaporated in vacuo and the crude residue was subjected to column chromatography to give the mono-protected ketone $\mathbf{1 w}$.

Step 2. The aforementioned mono-protected ketone $(5.19 \mathrm{~g}, 23.1 \mathrm{mmol})$ was dissolved in dry $\mathrm{MeOH}(80 \mathrm{~mL})$ and treated with $\mathrm{NaBH}_{4}$ portion wise (1.73 g, $45.8 \mathrm{mmol}, 2.0$ equiv.) at $0{ }^{\circ} \mathrm{C}$. The mixture was stirred for $4 \mathrm{~h}$ and then $0.1 \mathrm{M} \mathrm{NaOH}(100 \mathrm{~mL})$ was added while keeping the temperature in between $0-10{ }^{\circ} \mathrm{C}$. The aqueous layer was extracted with diethyl ether and the combined organic layers were dried over sodium sulfate, filtered, and concentrated to give 5',5'-dimethylhexahydro-1H-spiro[pentalene-2,2'-[1,3]dioxan]-5-ol as colorless crystals.

Step 3. To a solution of 5',5'-dimethylhexahydro-1H-spiro[pentalene-2,2'-[1,3]dioxan]-5-ol (5.83 g, $25.8 \mathrm{mmol})$, pyridine (4.62 mL, $4.56 \mathrm{~g}, 58.0 \mathrm{mmol})$ and DMAP $(90 \mathrm{mg}, 0.720 \mathrm{mmol})$ in $\mathrm{CH}_{2} \mathrm{Cl}_{2}(50 \mathrm{~mL})$ was added O-phenyl chlorothioformate (5.00 g, $\left.22.9 \mathrm{mmol}\right)$ and the reaction mixture was stirred for $4 \mathrm{~h}$. The mixture was then filtered through $\mathrm{SiO}_{2}$ with hexanes/ethyl acetate $(3: 1)$ and the filtrate was concentrated under reduced pressure to give O-(5,5-dimethylhexahydro-1'H-spiro[1,3-dioxane-2,2'-pentalen]-5'-yl)-O-phenyl thiocarbonate as a yellow solid, which was used without further purification.

Step 4. To a solution of O-(5,5-dimethylhexahydro-1'H-spiro[1,3-dioxane-2,2'-pentalen]-5'-yl) O-phenylthiocarbonate (25.8 mmol) in absolute toluene $(100 \mathrm{~mL}), \mathrm{AIBN}(50 \mathrm{mg}, 0.3 \mathrm{mmol})$ and $\mathrm{Bu}_{3} \mathrm{SnH}(7.69 \mathrm{~mL}, 8.46 \mathrm{mg}, 29.0$ mmol) were added. The mixture was degassed by passing a stream of dry Argon for $10 \mathrm{~min}$ through the stirred solution and stirred at $130{ }^{\circ} \mathrm{C}$ for $16 \mathrm{~h}$. The desired product 5,5-dimethylhexahydro-1'H-spiro[1,3-dioxane-2,2'-pentalene] was obtained after column chromatography with silica gel.

Step 5. p-Toluenesulfonic acid (219 mg, $1.15 \mathrm{mmol})$ was added to a solution of 5,5-dimethylhexahydro-1'H-spiro[1,3-dioxane-2,2'pentalene] $(19.0 \mathrm{mmol})$ in acetone $(300 \mathrm{~mL})$. The resultant reaction mixture was stirred at $\mathrm{rt}$ for $60 \mathrm{~h}$. After the reaction was completed, the solvent was removed through evaporation and the reaction residue was purified by column chromatography to give the desired ketone $\mathbf{1 u}$.

\section{(3aR,6aS)-hexahydropentalen-2(1H)-one $1 \mathrm{u}$}



${ }^{1} \mathrm{H}$ NMR $\left(501 \mathrm{MHz}, \mathrm{CD}_{2} \mathrm{Cl}_{2}\right) \delta 2.78-2.64(\mathrm{~m}, 2 \mathrm{H}), 2.54-2.38(\mathrm{~m}, 2 \mathrm{H}), 2.06-1.90(\mathrm{~m}, 4 \mathrm{H}), 1.79(\mathrm{tdd}, J=13.6,7.6,6.1$ $\mathrm{Hz}, 1 \mathrm{H}), 1.71-1.60(\mathrm{~m}, 1 \mathrm{H}), 1.43(\mathrm{dtd}, J=12.6,7.3,4.9 \mathrm{~Hz}, 2 \mathrm{H})$.

${ }^{13} \mathrm{C}$ NMR $\left(126 \mathrm{MHz}, \mathrm{CD}_{2} \mathrm{Cl}_{2}\right) \delta 220.4,44.6,39.7,33.4,22.5$.

$\mathrm{R} f=0.19$ (Ethyl acetate/hexanes $=1: 9)$.

EI-HRMS $(m / z)$ : calculated for $\mathrm{C}_{8} \mathrm{H}_{12} \mathrm{O}_{1}\left[\mathrm{M}^{+\bullet}\right]$ : 124.0883 , found: 124.0885 .

\section{$(3 a R, 6 a S)-5 ', 5 '$-dimethyltetrahydro-1H-spiro[pentalene-2,2'-[1,3]dioxan]-5(3H)-one $1 \mathrm{w}$}



${ }^{1} \mathrm{H}$ NMR $\left(501 \mathrm{MHz}, \mathrm{CDCl}_{3}\right) \delta 3.42(\mathrm{~d}, J=1.0 \mathrm{~Hz}, 2 \mathrm{H}), 3.38(\mathrm{~d}, J=1.0 \mathrm{~Hz}, 2 \mathrm{H}), 2.82-2.71(\mathrm{~m}, 2 \mathrm{H}), 2.45-2.35(\mathrm{~m}, 2 \mathrm{H})$, 2.26-2.18 (m, 2H), 2.14-2.06 (m, 2H), 1.79-1.71 (m, 2H), 0.89 (s, 6H).

${ }^{13} \mathrm{C}$ NMR $\left(126 \mathrm{MHz}, \mathrm{CDCl}_{3}\right) \delta 220.1,109.5,72.2,72.1,44.5,41.2,36.8,30.1,22.4$.

$\mathrm{R} f=0.11$ (Ethyl acetate/hexanes $=1: 9)$.

EI-HRMS $(m / z)$ : calculated for $\mathrm{C}_{13} \mathrm{H}_{20} \mathrm{O}_{3}\left[\mathrm{M}^{+}\right]$: 224.1407, found: 224.1407.

m.p. $=38.4-42.5^{\circ} \mathrm{C}$. 
Cyclobutanone $1 v$ was prepared according to a literature method with minor modification ${ }^{4}$



Step 1. To a stirred suspension of freshly-prepared $\mathrm{Zn}-\mathrm{Cu}$ couple (1.60 g, $25 \mathrm{mmol})$ and styrene (10 mmol) in dry Et ${ }_{2} \mathrm{O}(20 \mathrm{~mL})$ was added a solution of trichloroacetic chloride $(2.2 \mathrm{~mL}, 20 \mathrm{mmol})$ and phosphorus oxychloride $(1.9 \mathrm{~mL}, 20 \mathrm{mmol}) \mathrm{in} \mathrm{Et}_{2} \mathrm{O}(10 \mathrm{~mL}) \mathrm{dropwise}$ through an addition funnel, after $2 \mathrm{~h}$ at reflux. The mixture was cooled to room temperature and then filtered through a pad of Celite. The residue was extracted with $\mathrm{Et}_{2} \mathrm{O}(3 \times 15 \mathrm{~mL})$. The organic phase was concentrated under reduced pressure to give a crude product, which was carefully purified through column chromatography with silica gel.

Step 2. The solution of the obtained product from the above-mentioned step in acetic acid (10 mL) was added dropwise to a vigorously stirred suspension of zinc dust $(2.6 \mathrm{~g}, 40 \mathrm{mmol})$ in acetic acid $(8 \mathrm{~mL})$ at $0{ }^{\circ} \mathrm{C}$. After the addition, the reaction mixture was heated to 70 ${ }^{\circ} \mathrm{C}$ for $2 \mathrm{~h}$. The mixture was allowed to cool to room temperature and then evacuated to remove most of the acetic acid. The residue was dissolved in $\mathrm{Et}_{2} \mathrm{O}(20 \mathrm{~mL})$ and then poured into a separation funnel containing water $(20 \mathrm{~mL})$ and $\mathrm{Et}_{2} \mathrm{O}(20 \mathrm{~mL})$. The organic layer was washed with water $(3 \times 10 \mathrm{~mL})$, saturated sodium bicarbonate solution $(2 \times 10 \mathrm{~mL})$, brine $(50 \mathrm{~mL})$ and dried over $\mathrm{MgSO}_{4}$. The solution was then filtered and concentrated, followed by purification by flash chromatography to afford the desired pure cyclobutanone $\mathbf{1 v}$ as a light yellow liquid. (Notes: the mono-chlorine substituted 3-ph cyclobutanone byproduct from the first step, which is inseparable from the desired product, can be further reduced with fine Zn powder, thus furnishing the desired product that can be used without purification)

\section{3-phenylcyclobutan-1-one 1v}



${ }^{1} \mathrm{H}$ NMR $\left(501 \mathrm{MHz}, \mathrm{CD}_{2} \mathrm{Cl}_{2}\right) \delta 7.43-7.32(\mathrm{~m}, 4 \mathrm{H}), 7.31-7.25(\mathrm{~m}, 1 \mathrm{H}), 3.72(\mathrm{ddd}, J=16.4,9.1,7.5 \mathrm{~Hz}, 1 \mathrm{H}), 3.55-$ $3.45(\mathrm{~m}, 2 \mathrm{H}), 3.32-3.22(\mathrm{~m}, 2 \mathrm{H})$.

${ }^{13} \mathrm{C}$ NMR $\left(126 \mathrm{MHz}, \mathrm{CD}_{2} \mathrm{Cl}_{2}\right) \delta 206.2,143.9,128.6,126.5,126.4,54.6,28.4$.

$\mathrm{R} f=0.22($ Ethyl acetate/hexanes $=1: 9)$.

EI-HRMS $(\mathrm{m} / \mathrm{z})$ : calculated for $\mathrm{C}_{10} \mathrm{H}_{10} \mathrm{O}_{1}\left[\mathrm{M}^{+\bullet}\right]:$ 146.0726, found: 146.0726 .

3-phenyl cyclopentanone 1ac was prepared according to a literature method 5



To a stirred solution of $[\mathrm{Rh}(\mathrm{cod}) \mathrm{Cl}]_{2}(1 \mathrm{~mol} \%)$ and $\mathrm{PhB}(\mathrm{OH})_{2}(1.37 \mathrm{~g}, 11.3 \mathrm{mmol})$ in degassed aqueous $1,4-$ dioxane was added a solution of 2-cyclopentene-1-one $(0.63 \mathrm{~mL}, 7.5 \mathrm{mmol})$ in aqueous 1,4-dioxane and degassed $\mathrm{Et}_{3} \mathrm{~N}$ (1.0 equiv.). The resulting solution was stirred at $50{ }^{\circ} \mathrm{C}$ for $6 \mathrm{~h}$, then cooled to rt and concentrated under reduced pressure. The crude product was purified via column chromatography and the desired brown oil 1ac was obtained.

rac-3-phenylcyclohexan-1-one (1ac)



${ }^{1} \mathrm{H}$ NMR $\left(501 \mathrm{MHz}, \mathrm{CDCl}_{3}\right) \delta 7.28(\mathrm{dd}, J=8.3,6.9 \mathrm{~Hz}, 2 \mathrm{H}), 7.21-7.14(\mathrm{~m}, 3 \mathrm{H}), 3.36(\mathrm{tt}, J=11.0,6.5 \mathrm{~Hz}, 1 \mathrm{H}), 2.65-2.55$ $(\mathrm{m}, 1 \mathrm{H}), 2.45-2.33(\mathrm{~m}, 2 \mathrm{H}), 2.33-2.17(\mathrm{~m}, 2 \mathrm{H}), 1.92(\mathrm{dtd}, J=12.5,11.0,10.5,8.7 \mathrm{~Hz}, 1 \mathrm{H})$.

${ }^{13} \mathrm{C}$ NMR $\left(126 \mathrm{MHz}, \mathrm{CDCl}_{3}\right) \delta 218.4,143.0,128.6,126.7,126.7,45.8,42.2,38.8,31.2$.

$\mathrm{R} f=0.16$ (Ethyl acetate/hexanes $=1: 9)$. 
EI-HRMS $(m / z)$ : calculated for $\mathrm{C}_{11} \mathrm{H}_{12} \mathrm{O}_{1}\left[\mathrm{M}^{+\bullet}\right]$ : 160.0883 , found: 160.0884 .

\section{Substrate scope for the deprotosilylation of ketones 1 with allylsilane reagents $2 \mathrm{a}$ or $2 \mathrm{~b}$}



$1 a-1 w$



2b



$-20^{\circ} \mathrm{C}, 1-5 \mathrm{~d}$



$3 a-3 w$

\section{General procedure for the catalytic desymmetrization of ketones with allylsilanes.}

Allyl silane 2a or $\mathbf{2 b}(80 \mu \mathrm{L}, 0.4 \mathrm{mmol}, 2.0$ equiv.) was placed in a flame-dried Schlenk flask, equipped with a teflon-coated magnetic stirring bar. IDPi 4a-4f (0.01 equiv.) and a solvent mixture of toluene with 1,4-dioxane $(2: 1 v / v 0.08 \mathrm{M}, 2.4 \mathrm{~mL})$ were added at $25^{\circ} \mathrm{C}$ and stirred for $30 \mathrm{~min}$. The resultant mixture was cooled to $-20{ }^{\circ} \mathrm{C}$ for $10 \mathrm{~min}$, and ketones $\mathbf{1 a}-\mathbf{1 w}(0.2 \mathrm{mmol}, 1.0$ equiv.) were slowly added. Then the reaction was stirred for $1-5 \mathrm{~d}$ at $-20^{\circ} \mathrm{C}$. After the ketone was fully consumed as monitored by TLC, the reaction was treated with one drop of triethylamine. All volatiles were removed in vacuo and the crude residue was purified by column chromatography with silica gel to afford the desired enol silanes $\mathbf{3 a}-\mathbf{3 w}$.

\section{Analytical data of products}

\section{(R)-tert-butyldimethyl((1,2,3,6-tetrahydro-[1,1'-biphenyl]-4-yl)oxy)silane 3a}



The reaction was performed according to the general procedure and catalyzed by IDPi 4c.

The titled product was purified by column chromatography with hexanes as eluent to afford $\mathbf{3 a}$ as a colorless oil (57.1 mg, $99 \%$ yield).

${ }^{1} \mathrm{H}$ NMR (501 MHz, $\left.\mathrm{CD}_{2} \mathrm{Cl}_{2}\right) \delta$ 7.31-7.22 (m, 4H), 7.20-7.15 (m, 1H), 4.96-4.91 (m, 1H), 2.75 (ddt, J = 16.5, 8.2, 3.1 Hz, $1 \mathrm{H}), 2.31-2.12(\mathrm{~m}, 3 \mathrm{H}), 2.09-2.00(\mathrm{~m}, 1 \mathrm{H}), 1.93(\mathrm{dddt}, J=8.9,4.2,2.9,1.6 \mathrm{~Hz}, 1 \mathrm{H}), 1.90-1.79(\mathrm{~m}, 1 \mathrm{H}), 0.93(\mathrm{~s}, 9 \mathrm{H}), 0.16$ $(\mathrm{s}, 3 \mathrm{H}), 0.15(\mathrm{~s}, 3 \mathrm{H})$.

${ }^{13} \mathrm{C}$ NMR $\left(126 \mathrm{MHz}, \mathrm{CD}_{2} \mathrm{Cl}_{2}\right) \delta 151.3,147.7,129.0,127.6,126.7,104.3,40.8,32.8,31.0,31.0,26.3,18.7,-3.8,-4.0$.

$\mathrm{R} f=0.36$ (Hexanes).

EI-HRMS $(m / z)$ : calculated for $\mathrm{C}_{18} \mathrm{H}_{28} \mathrm{O}_{1} \mathrm{Si}_{1}\left[\mathrm{M}^{+\bullet}\right]: 288.1904$, found: 288.1903 .

HPLC (OJ-3R, Acetonitrile: Water $=70: 30,1.0 \mathrm{~mL} / \mathrm{min}, 298 \mathrm{~K}, 224 \mathrm{~nm}): t_{\mathrm{R} 1}=24.1 \mathrm{~min}, t_{\mathrm{R} 2}=22.9 \mathrm{~min}$, e.r. $=97: 3$.

$[\boldsymbol{\alpha}]_{\mathbf{D}}^{\mathbf{2 5}}=29.0\left(c 0.49, \mathrm{CHCl}_{3}\right)$.

\section{(R)-tert-butyldimethyl((4'-methyl-1,2,3,6-tetrahydro-[1,1'-biphenyl]-4-yl)oxy)silane 3b}



The reaction was performed according to the general procedure and catalyzed by IDPi 4c.

The titled product was purified by column chromatography with hexanes as eluent to afford $\mathbf{3 b}$ as a white solid (54.4 mg, $90 \%$ yield).

${ }^{1} \mathrm{H}$ NMR $\left(501 \mathrm{MHz}, \mathrm{CD}_{2} \mathrm{Cl}_{2}\right) \delta$ 6.99-6.91 (m, 4H), 4.80-4.73 (m, 1H), 2.59-2.51 (m, 1H), 2.15 (s, 3H), 2.08 (dddd, J=13.0, 9.9, 4.4, 2.1 Hz, 2H), 2.03-1.95 (m, 1H), 1.93-1.84 (m, 1H), 1.79-1.72 (m, 1H), 1.72-1.61 (m, 1H), $0.78(\mathrm{~s}, 9 \mathrm{H}), 0.16(\mathrm{~s}$, $3 \mathrm{H}), 0.16(\mathrm{~s}, 3 \mathrm{H})$.

${ }^{13} \mathrm{C}$ NMR $\left(126 \mathrm{MHz}, \mathrm{CD}_{2} \mathrm{Cl}_{2}\right) \delta 151.0,144.4,136.0,129.5,127.3,104.2,40.2,32.7,30.9,30.8,26.1,21.3,18.5,-4.0,-4.1$. 
$\mathrm{R} f=0.61($ Ethyl acetate/hexanes $=1: 20)$.

EI-HRMS (m/z): calculated for $\mathrm{C}_{19} \mathrm{H}_{30} \mathrm{O}_{1} \mathrm{Si}_{1}\left[\mathrm{M}^{+\bullet}\right]$ : 302.2060, found: 302.2055 .

HPLC (OJ-3R, Acetonitrile: Water $=70: 30,1.0 \mathrm{~mL} / \mathrm{min}, 298 \mathrm{~K}, 220 \mathrm{~nm}): t_{\mathrm{R} 1}=20.2 \mathrm{~min}, t_{\mathrm{R} 2}=18.2 \mathrm{~min}$, e.r. $=97: 3$.

$[\boldsymbol{\alpha}]_{\mathbf{D}}^{25}=31.3\left(c 0.53, \mathrm{CH}_{3} \mathrm{CN}\right)$.

m.p. $=31.9-35.2^{\circ} \mathrm{C}$.

\section{(R)-tert-butyl((4'-methoxy-1,2,3,6-tetrahydro-[1,1'-biphenyl]-4-yl)oxy)dimethylsilane 3c}

OTBS The reaction was performed according to the general procedure and catalyzed by IDPi 4c.

OMe

The titled product was purified by column chromatography with hexanes as eluent to afford $\mathbf{3 c}$ as a colorless oil (59.9 mg, $94 \%$ yield).

${ }^{1} \mathrm{H}$ NMR (501 MHz, $\left.\mathrm{CD}_{2} \mathrm{Cl}_{2}\right) \delta 7.04-6.94(\mathrm{~m}, 2 \mathrm{H}), 6.71-6.62(\mathrm{~m}, 2 \mathrm{H}), 4.79-4.73(\mathrm{~m}, 1 \mathrm{H}), 3.61(\mathrm{~s}, 3 \mathrm{H}), 2.55(\mathrm{ddq}, J=13.0$, $8.1,3.1 \mathrm{~Hz}, 1 \mathrm{H}), 2.14-2.02(\mathrm{~m}, 2 \mathrm{H}), 1.98$ (dddt, $J=16.5,10.2,3.8,2.0 \mathrm{~Hz}, 1 \mathrm{H}), 1.93-1.84(\mathrm{~m}, 1 \mathrm{H}), 1.75(\mathrm{dddd}, J=13.3$, 5.8, 4.6, $2.8 \mathrm{~Hz}, 1 \mathrm{H}), 1.70-1.59(\mathrm{~m}, 1 \mathrm{H}), 0.78(\mathrm{~s}, 9 \mathrm{H}), 0.00(\mathrm{~s}, 3 \mathrm{H}), 0.00(\mathrm{~s}, 3 \mathrm{H})$.

${ }^{13} \mathrm{C}$ NMR $\left(126 \mathrm{MHz}, \mathrm{CD}_{2} \mathrm{Cl}_{2}\right) \delta 157.9,150.4,138.9,127.6,113.6,103.6,55.1,39.1,32.1,30.4,30.2,25.5,17.9,-4.6,-4.7$.

$\mathrm{R} f=0.50($ Ethyl acetate/hexanes $=1: 20)$.

EI-HRMS (m/z): calculated for $\mathrm{C}_{19} \mathrm{H}_{30} \mathrm{O}_{2} \mathrm{Si}_{1}\left[\mathrm{M}^{+\bullet}\right]:$ 318.2010, found: 318.2004.

HPLC (AD-3R, Acetonitrile: Water = 70:30, $1.0 \mathrm{~mL} / \mathrm{min}, 298 \mathrm{~K}, 220 \mathrm{~nm}): t_{\mathrm{R} 1}=15.1 \mathrm{~min}, t_{\mathrm{R} 2}=14.4 \mathrm{~min}$, e.r. $=96: 4$.

$[\boldsymbol{\alpha}]_{\mathbf{D}}^{25}=27.4\left(c 0.46, \mathrm{CH}_{3} \mathrm{CN}\right)$.

\section{(R)-tert-butyl((4'-chloro-1,2,3,6-tetrahydro-[1,1'-biphenyl]-4-yl)oxy)dimethylsilane 3d}



The reaction was performed according to the general procedure and catalyzed by IDPi 4c.

The titled product was purified by column chromatography with hexanes as eluent to afford 3d as a white solid (63.9 mg, $99 \%$ yield).

${ }^{1} \mathrm{H}$ NMR (501 MHz, $\left.\mathrm{CD}_{2} \mathrm{Cl}_{2}\right) \delta$ 7.15-7.06 (m, 2H), 7.06-6.97 (m, 2H), 4.81-4.70 (m, 1H), 2.59 (ddq, J = 14.3, 8.2, $2.9 \mathrm{~Hz}$, $1 \mathrm{H}), 2.15-2.03(\mathrm{~m}, 2 \mathrm{H}), 2.03-1.93(\mathrm{~m}, 1 \mathrm{H}), 1.93-1.82(\mathrm{~m}, 1 \mathrm{H}), 1.76(\mathrm{dddt}, J=10.6,5.9,2.9,1.5 \mathrm{~Hz}, 1 \mathrm{H}), 1.67(\mathrm{dddd}, J=$ $12.8,11.6,10.6,5.6 \mathrm{~Hz}, 1 \mathrm{H}), 0.78(\mathrm{~s}, 9 \mathrm{H}), 0.00(\mathrm{~s}, 3 \mathrm{H}), 0.00(\mathrm{~s}, 3 \mathrm{H})$.

${ }^{13} \mathrm{C}$ NMR $\left(126 \mathrm{MHz}, \mathrm{CD}_{2} \mathrm{Cl}_{2}\right) \delta 151.1,146.0,131.9,128.9,128.8,103.8,40.0,32.4,30.7,30.6,26.1,18.5,-4.0,-4.2$.

$\mathrm{R} f=0.53$ (Ethyl acetate/hexanes $=1: 20)$.

EI-HRMS (m/z): calculated for $\mathrm{C}_{18} \mathrm{H}_{27} \mathrm{O}_{1} \mathrm{Cl}_{1} \mathrm{Si}_{1}\left[\mathrm{M}^{+\bullet}\right]: 322.1514$, found: 322.1509 .

HPLC (OJ-3R, Acetonitrile: Water $=65: 35,1.0 \mathrm{~mL} / \mathrm{min}, 298 \mathrm{~K}, 220 \mathrm{~nm}): t_{\mathrm{R} 1}=25.2 \mathrm{~min}, t_{\mathrm{R} 2}=23.9 \mathrm{~min}$, e.r. $=94.5: 5.5$.

$[\boldsymbol{\alpha}]_{\mathbf{D}}^{25}=24.7\left(c 0.68, \mathrm{CHCl}_{3}\right)$

m.p. $=41.0-41.8^{\circ} \mathrm{C}$.

\section{(R)-tert-butyl((4'-fluoro-1,2,3,6-tetrahydro-[1,1'-biphenyl]-4-yl)oxy)dimethylsilane 3e}


OTBS The reaction was performed according to the general procedure and catalyzed by IDPi $\mathbf{4 c}$.

The titled product was purified by column chromatography with hexanes as eluent to afford $\mathbf{3 e}$ as a colorless oil (60.7 mg, 99\% yield).

${ }^{1} \mathrm{H}$ NMR (501 MHz, $\left.\mathrm{CD}_{2} \mathrm{Cl}_{2}\right) \delta$ 7.09-7.02 (m, 2H), 6.86-6.79 (m, 2H), 4.80-4.73 (m, 1H), 2.60 (ddt, $J=14.6,8.2,3.0 \mathrm{~Hz}$, $1 \mathrm{H}), 2.16-2.03(\mathrm{~m}, 2 \mathrm{H}), 2.03-1.94(\mathrm{~m}, 1 \mathrm{H}), 1.92-1.83(\mathrm{~m}, 1 \mathrm{H}), 1.76(\mathrm{ddtd}, J=11.8,5.8,2.8,1.7 \mathrm{~Hz}, 1 \mathrm{H}), 1.67(\mathrm{dddd}, J=$ 12.7, 11.6, 10.6, $5.6 \mathrm{~Hz}, 1 \mathrm{H}), 0.78(\mathrm{~s}, 9 \mathrm{H}), 0.00(\mathrm{~s}, 3 \mathrm{H}), 0.00(\mathrm{~s}, 3 \mathrm{H})$.

${ }^{13} \mathrm{C}$ NMR $\left(126 \mathrm{MHz}, \mathrm{CD}_{2} \mathrm{Cl}_{2}\right) \delta 162.0(\mathrm{~d}, J=252.14 \mathrm{~Hz}), 151.3,143.3(\mathrm{~d}, J=3.2 \mathrm{~Hz}), 128.2(\mathrm{~d}, J=7.7 \mathrm{~Hz}), 114.7(\mathrm{~d}, J=$ $21.1 \mathrm{~Hz}), 103.3,39.2,32.0,30.2,30.0,25.4,17.8,-4.6,-4.7$.

${ }^{19} \mathrm{~F}$ NMR $\left(471 \mathrm{MHz}, \mathrm{CD}_{2} \mathrm{Cl}_{2}\right) \delta-118.4$.

$\mathrm{R} f=0.53($ Ethyl acetate/hexanes $=1: 20)$.

EI-HRMS ( $\mathrm{m} / \mathrm{z})$ : calculated for $\mathrm{C}_{18} \mathrm{H}_{27} \mathrm{O}_{1} \mathrm{Si}_{1} \mathrm{~F}_{1}\left[\mathrm{M}^{+*}\right]$ : 306.1810, found: 306.1805 .

HPLC (OJ-3R, Acetonitrile: Water $=60: 40,1.0 \mathrm{~mL} / \mathrm{min}, 298 \mathrm{~K}, 220 \mathrm{~nm}): t_{\mathrm{R} 1}=36.8 \mathrm{~min}, t_{\mathrm{R} 2}=33.2 \mathrm{~min}$, e.r. $=94: 6$.

$[\boldsymbol{\alpha}]_{\mathbf{D}}^{25}=26.7\left(c 0.42, \mathrm{CH}_{3} \mathrm{CN}\right)$.

\section{(R)-tert-butyldimethyl((4'-(trifluoromethyl)-1,2,3,6-tetrahydro-[1,1'-biphenyl]-4-yl)oxy)silane 3f}



The reaction was performed according to the general procedure and catalyzed by IDPi $4 \mathbf{c}$.

The titled product was purified by column chromatography with hexanes as eluent to afford $\mathbf{3 f}$ as a white solid (69.2 $\mathrm{mg}, 97 \%$ yield).

${ }^{1} \mathrm{H}$ NMR $\left(501 \mathrm{MHz}, \mathrm{CD}_{2} \mathrm{Cl}_{2}\right) \delta 7.56(\mathrm{~d}, J=8.1 \mathrm{~Hz}, 2 \mathrm{H}), 7.38(\mathrm{~d}, J=8.0 \mathrm{~Hz}, 2 \mathrm{H}), 4.93(\mathrm{dt}, J=4.6,1.9 \mathrm{~Hz}, 1 \mathrm{H}), 2.88-2.79$ $(\mathrm{m}, 1 \mathrm{H}), 2.34-2.14(\mathrm{~m}, 3 \mathrm{H}), 2.09-2.01(\mathrm{~m}, 1 \mathrm{H}), 1.98-1.92(\mathrm{~m}, 1 \mathrm{H}), 1.87$ (dddd, $J=12.8,11.6,10.7,5.7 \mathrm{~Hz}, 1 \mathrm{H}), 0.93(\mathrm{~s}$, $9 \mathrm{H}), 0.16(\mathrm{~s}, 3 \mathrm{H}), 0.15(\mathrm{~s}, 3 \mathrm{H})$.

${ }^{13} \mathrm{C}$ NMR $\left(126 \mathrm{MHz}, \mathrm{CD}_{2} \mathrm{Cl}_{2}\right) \delta 151.9,151.3,128.7(\operatorname{app~d}, J=32.0 \mathrm{~Hz}), 128.1,125.9(\mathrm{q}, J=3.8 \mathrm{~Hz}), 125.2(\operatorname{app} \mathrm{d}, J=$ $271.4 \mathrm{~Hz}), 103.9,40.6,32.4,30.6,30.6,26.2,18.6,-3.8,-4.0$.

${ }^{19} \mathrm{~F}$ NMR $\left(471 \mathrm{MHz}, \mathrm{CD}_{2} \mathrm{Cl}_{2}\right) \delta-62.6$.

$\mathrm{R} f=0.64($ Ethyl acetate/hexanes $=1: 20)$.

EI-HRMS (m/z): calculated for $\mathrm{C}_{19} \mathrm{H}_{27} \mathrm{O}_{1} \mathrm{Si}_{1} \mathrm{~F}_{3}\left[\mathrm{M}^{+\bullet}\right]$ : 356.1778, found: 356.1777.

HPLC (AD-3R, Acetonitrile: Water $=60: 40,1.0 \mathrm{~mL} / \mathrm{min}, 298 \mathrm{~K}, 237 \mathrm{~nm}): t_{\mathrm{R} 1}=25.1 \mathrm{~min}, t_{\mathrm{R} 2}=23.3 \mathrm{~min}$, e.r. $=96: 4$.

$[\alpha]_{\mathbf{D}}^{25}=23.4\left(c 0.46, \mathrm{CH}_{3} \mathrm{CN}\right)$.

m.p. $=57.4-58.7^{\circ} \mathrm{C}$.

\section{(R)-tert-butyl((3'-(tert-butyl)-1,2,3,6-tetrahydro-[1,1'-biphenyl]-4-yl)oxy)dimethylsilane 3g}



The reaction was performed according to the general procedure and catalyzed by IDPi $\mathbf{4 c}$.

The titled product was purified by column chromatography with hexanes as eluent to afford $\mathbf{3 g}$ as a colorless oil (68.2 $\mathrm{mg}$, $99 \%$ yield).

${ }^{1} \mathrm{H}$ NMR $\left(501 \mathrm{MHz}, \mathrm{CD}_{2} \mathrm{Cl}_{2}\right) \delta 7.10(\mathrm{q}, J=1.5 \mathrm{~Hz}, 1 \mathrm{H}), 7.08-7.03(\mathrm{~m}, 2 \mathrm{H}), 6.88(\mathrm{ddd}, J=7.4,2.8,1.7 \mathrm{~Hz}, 1 \mathrm{H}), 4.77(\mathrm{dd}$, $J=4.1,1.9 \mathrm{~Hz}, 1 \mathrm{H}), 2.56(\mathrm{dtd}, J=11.4,5.1,3.0 \mathrm{~Hz}, 1 \mathrm{H}), 2.15-1.96(\mathrm{~m}, 3 \mathrm{H}), 1.95-1.84(\mathrm{~m}, 1 \mathrm{H}), 1.81-1.64(\mathrm{~m}, 2 \mathrm{H}), 1.15$ $(\mathrm{s}, 9 \mathrm{H}), 0.78(\mathrm{~s}, 9 \mathrm{H}), 0.00(\mathrm{~s}, 3 \mathrm{H}), 0.00(\mathrm{~s}, 3 \mathrm{H})$.

${ }^{13} \mathrm{C}$ NMR $\left(126 \mathrm{MHz}, \mathrm{CD}_{2} \mathrm{Cl}_{2}\right) \delta 151.7,151.1,147.1,128.5,124.6,124.3,123.5,104.2,41.0,35.1,32.8,31.8,30.9,26.1,18.5,-4.0,-$ 4.1.

$\mathrm{R} f=0.53($ Ethyl acetate/hexanes $=1: 20)$ 
EI-HRMS (m/z): calculated for $\mathrm{C}_{22} \mathrm{H}_{36} \mathrm{O}_{1} \mathrm{Si}_{1}\left[\mathrm{M}^{+\bullet}\right]: 344.2530$, found: 344.2525 .

HPLC (OD-3R, Acetonitrile: Water $=65: 35,1.0 \mathrm{~mL} / \mathrm{min}, 298 \mathrm{~K}, 220 \mathrm{~nm}): t_{\mathrm{R} 1}=23.3 \mathrm{~min}, t_{\mathrm{R} 2}=24.7 \mathrm{~min}$, e.r. $=96: 4$.

$[\boldsymbol{\alpha}]_{\mathbf{D}}^{25}=28.1\left(c 0.54, \mathrm{CH}_{3} \mathrm{CN}\right)$.

\section{(R)-tert-butyl((3'-chloro-1,2,3,6-tetrahydro-[1,1'-biphenyl]-4-yl)oxy)dimethylsilane 3h}



The reaction was performed according to the general procedure and catalyzed by IDPi 4c.

The titled product was purified by column chromatography with hexanes as eluent to afford $\mathbf{3 h}$ as a colorless oil (54.9 mg, $85 \%$ yield).

${ }^{1} \mathrm{H}$ NMR (501 MHz, $\left.\mathrm{CD}_{2} \mathrm{Cl}_{2}\right) \delta$ 7.11-7.04 (m, 2H), 7.04-6.95 (m, 2H), $4.77(\mathrm{dt}, J=4.6,1.8 \mathrm{~Hz}, 1 \mathrm{H}), 2.60(\mathrm{ddq}, J=14.5$, 8.1, 3.0 Hz, 1H), 2.17-1.95 (m, 3H), 1.94-1.85 (m, 1H), $1.78(\mathrm{ddtd}, J=13.6,5.9,2.9,1.8 \mathrm{~Hz}, 1 \mathrm{H}), 1.68(\mathrm{dddd}, J=12.8$, 11.7, 10.7, 5.6 Hz, 1H), 0.78 (s, 9H), 0.00 (s, 3H), 0.00 (s, 3H).

${ }^{13} \mathrm{C}$ NMR $\left(126 \mathrm{MHz}, \mathrm{CD}_{2} \mathrm{Cl}_{2}\right) \delta 151.3,149.8,134.7,130.4,127.8,126.8,126.1,104.0,40.5,32.5,30.8,30.7,26.3,18.7,-3.8,-3.9$.

$\mathrm{R} f=0.61($ Ethyl acetate/hexanes $=1: 20)$.

EI-HRMS $(m / z)$ : calculated for $\mathrm{C}_{18} \mathrm{H}_{27} \mathrm{O}_{1} \mathrm{Cl}_{1} \mathrm{Si}_{1}\left[\mathrm{M}^{+\bullet}\right]: 322.1514$, found: 322.1509 .

HPLC (AD-3R, Acetonitrile: Water =60:40, $1.0 \mathrm{~mL} / \mathrm{min}, 298 \mathrm{~K}, 220 \mathrm{~nm}): t_{\mathrm{R} 1}=33.3 \mathrm{~min}, t_{\mathrm{R} 2}=30.0 \mathrm{~min}$, e.r. $=98: 2$.

$[\boldsymbol{\alpha}]_{\mathbf{D}}^{25}=25.7\left(c 0.42, \mathrm{CH}_{3} \mathrm{CN}\right)$.

\section{(R)-tert-butyldimethyl((3'-(trifluoromethyl)-1,2,3,6-tetrahydro-[1,1'-biphenyl]-4-yl)oxy)silane 3i}

\footnotetext{
OTBS The reaction was performed according to the general procedure and catalyzed by IDPi 4c.
The titled product was purified by column chromatography with hexanes as eluent to afford $\mathbf{3 i}$ as a colorless oil (69.2 mg, $97 \%$ yield).

${ }^{1} \mathrm{H}$ NMR $\left(501 \mathrm{MHz}, \mathrm{CD}_{2} \mathrm{Cl}_{2}\right) \delta 7.36(\mathrm{dq}, J=1.9,1.0 \mathrm{~Hz}, 1 \mathrm{H}), 7.33-7.24(\mathrm{~m}, 3 \mathrm{H}), 4.78(\mathrm{dt}, J=4.5,1.8 \mathrm{~Hz}, 1 \mathrm{H}), 2.69(\mathrm{dtd}$, $J=14.8,5.1,3.0 \mathrm{~Hz}, 1 \mathrm{H}), 2.21-1.98(\mathrm{~m}, 3 \mathrm{H}), 1.94-1.86(\mathrm{~m}, 1 \mathrm{H}), 1.80(\mathrm{ddtd}, J=12.1,6.2,3.0,2.0 \mathrm{~Hz}, 1 \mathrm{H}), 1.72(\mathrm{dddd}$, $\left.\mathrm{CF}_{3} \quad J=12.8,11.5,10.5,5.6 \mathrm{~Hz}, 1 \mathrm{H}\right), 0.78(\mathrm{~s}, 9 \mathrm{H}), 0.01(\mathrm{~s}, 3 \mathrm{H}), 0.00(\mathrm{~s}, 3 \mathrm{H})$.

${ }^{13} \mathrm{C} \mathrm{NMR}\left(126 \mathrm{MHz}, \mathrm{CD}_{2} \mathrm{Cl}_{2}\right) \delta 151.1,148.4,131.1(\mathrm{q}, J=1.1 \mathrm{~Hz}), 130.8(\mathrm{q}, J=31.7 \mathrm{~Hz}), 129.4,124.2(\mathrm{q}, J=273.1 \mathrm{~Hz}), 123.4(\mathrm{q}, J$ $=3.7 \mathrm{~Hz}), 123.3(\mathrm{q}, J=3.9 \mathrm{~Hz}), 103.7,40.4,32.3,30.6,30.5,26.1,18.5,-4.1,-4.2$.

${ }^{19} \mathrm{~F}$ NMR $\left(471 \mathrm{MHz}, \mathrm{CD}_{2} \mathrm{Cl}_{2}\right) \delta-62.8$.

$\mathrm{R} f=0.61($ Ethyl acetate/hexanes $=1: 20)$.

EI-HRMS (m/z): calculated for $\mathrm{C}_{19} \mathrm{H}_{27} \mathrm{O}_{1} \mathrm{Si}_{1} \mathrm{~F}_{3}\left[\mathrm{M}^{+\bullet}\right]$ : 356.1778 , found: 356.1774 .

HPLC (OJ-3R, Acetonitrile: Water $=60: 40,1.0 \mathrm{~mL} / \mathrm{min}, 298 \mathrm{~K}, 244 \mathrm{~nm}): t_{\mathrm{R} 1}=22.1 \mathrm{~min}, t_{\mathrm{R} 2}=23.9 \mathrm{~min}$, e.r. $=97: 3$.

$[\boldsymbol{\alpha}]_{\mathbf{D}}^{25}=22.4\left(c 0.34, \mathrm{CH}_{3} \mathrm{CN}\right)$.

\section{(R)-tert-butyldimethyl((2'-methyl-1,2,3,6-tetrahydro-[1,1'-biphenyl]-4-yl)oxy)silane 3j}



The reaction was performed according to the general procedure and catalyzed by IDPi $\mathbf{4 c}$.

The titled product was purified by column chromatography with hexanes as eluent to afford $\mathbf{3 j}$ as a colorless oil (55.7 mg, $92 \%$ yield).

${ }^{1} \mathrm{H}$ NMR $\left(501 \mathrm{MHz}, \mathrm{CD}_{2} \mathrm{Cl}_{2}\right) \delta 7.22(\mathrm{dd}, J=7.8,1.4 \mathrm{~Hz}, 1 \mathrm{H}), 7.18-7.11(\mathrm{~m}, 2 \mathrm{H}), 7.07(\mathrm{td}, J=7.3,1.5 \mathrm{~Hz}, 1 \mathrm{H}), 4.95(\mathrm{dt}, J=$ $4.8,1.9 \mathrm{~Hz}, 1 \mathrm{H}), 3.03-2.92(\mathrm{~m}, 1 \mathrm{H}), 2.35(\mathrm{~s}, 3 \mathrm{H}), 2.32-2.18(\mathrm{~m}, 2 \mathrm{H}), 2.17-2.02(\mathrm{~m}, 2 \mathrm{H}), 1.94-1.81(\mathrm{~m}, 2 \mathrm{H}), 0.95(\mathrm{~s}, 9 \mathrm{H})$, $0.18(\mathrm{~s}, 3 \mathrm{H}), 0.17(\mathrm{~s}, 3 \mathrm{H})$. 
${ }^{13} \mathrm{C}$ NMR $\left(126 \mathrm{MHz}, \mathrm{CD}_{2} \mathrm{Cl}_{2}\right) \delta 151.0,145.4,136.1,130.7,126.7,126.2,125.9,104.3,36.2,31.7,31.1,30.2,26.1,19.7,18.5,-4.0,-$ 4.1.

$\mathrm{R} f=0.56($ Ethyl acetate/hexanes $=1: 20)$.

EI-HRMS ( $\mathrm{m} / \mathrm{z})$ : calculated for $\mathrm{C}_{19} \mathrm{H}_{30} \mathrm{O}_{1} \mathrm{Si1}\left[\mathrm{M}^{+\cdot}\right]: 302.2060$, found: 302.2055 .

HPLC (OD-3R, Acetonitrile: Water $=65: 35,1.0 \mathrm{~mL} / \mathrm{min}, 298 \mathrm{~K}, 220 \mathrm{~nm}): t_{\mathrm{R} 1}=20.8 \mathrm{~min}, t_{\mathrm{R} 2}=19.9$ min, e.r. $=96: 4$.

$[\boldsymbol{\alpha}]_{\mathbf{D}}^{25}=24.8\left(c 0.42, \mathrm{CH}_{3} \mathrm{CN}\right)$.

(R)-tert-butyl((3',5'-dimethyl-1,2,3,6-tetrahydro-[1,1'-biphenyl]-4-yl)oxy)dimethylsilane 3k



The reaction was performed according to the general procedure and catalyzed by IDPi $\mathbf{4 c}$.

The titled product was purified by column chromatography with hexanes as eluent to afford $\mathbf{3 k}$ as a colorless oil (59.5 $\mathrm{mg}$, 94\% yield).

${ }^{1} \mathrm{H}$ NMR $\left(501 \mathrm{MHz}, \mathrm{CD}_{2} \mathrm{Cl}_{2}\right) \delta 6.84(\mathrm{~d}, J=9.0 \mathrm{~Hz}, 3 \mathrm{H}), 4.93(\mathrm{dt}, J=4.7,2.4 \mathrm{~Hz}, 1 \mathrm{H}), 2.67(\mathrm{ddq}, J=10.7,8.3,3.1 \mathrm{~Hz}$, $1 \mathrm{H}), 2.29(\mathrm{~s}, 6 \mathrm{H}), 2.27-2.12(\mathrm{~m}, 3 \mathrm{H}), 2.09-2.00(\mathrm{~m}, 1 \mathrm{H}), 1.93-1.87(\mathrm{~m}, 1 \mathrm{H}), 1.83(\mathrm{ddt}, J=12.8,7.0,5.2 \mathrm{~Hz}, 1 \mathrm{H}), 0.95(\mathrm{~s}$, $9 \mathrm{H}), 0.17$ (s, 3H), $0.16(\mathrm{~s}, 3 \mathrm{H})$.

${ }^{13} \mathrm{C}$ NMR $\left(126 \mathrm{MHz}, \mathrm{CD}_{2} \mathrm{Cl}_{2}\right) \delta 150.4,146.7,137.6,127.5,124.7,103.6,39.9,32.0,30.3,30.2,25.5,21.0,17.9,-4.6,-4.7$.

$\mathrm{R} f=0.20$ (Hexanes).

EI-HRMS ( $m / z)$ : calculated for $\mathrm{C}_{20} \mathrm{H}_{32} \mathrm{O}_{1} \mathrm{Si}_{1}\left[\mathrm{M}^{+*}\right]: 316.2217$, found: 316.2214 .

HPLC (OJ-3R, Acetonitrile: Water $=70: 30,1.0 \mathrm{~mL} / \mathrm{min}, 298 \mathrm{~K}, 220 \mathrm{~nm}): t_{\mathrm{R} 1}=11.6 \mathrm{~min}, t_{\mathrm{R} 2}=12.3 \mathrm{~min}$, e.r. $=97: 3$.

$[\boldsymbol{\alpha}]_{\mathbf{D}}^{25}=32.8\left(c 0.5, \mathrm{CHCl}_{3}\right)$.

\section{(R)-tert-butyl((3',5'-difluoro-1,2,3,6-tetrahydro-[1,1'-biphenyl]-4-yl)oxy)dimethylsilane 3l}



The reaction was performed according to the general procedure and catalyzed by IDPi $\mathbf{4 c}$.

The titled product was purified by column chromatography with hexanes as eluent to afford $\mathbf{3 1}$ as a colorless oil (53.9 $\mathrm{mg}$, $83 \%$ yield).

${ }^{1} \mathrm{H}$ NMR $\left(501 \mathrm{MHz}, \mathrm{CD}_{2} \mathrm{Cl}_{2}\right) \delta 6.84-6.74(\mathrm{~m}, 2 \mathrm{H}), 6.65(\mathrm{tt}, J=9.1,2.4 \mathrm{~Hz}, 1 \mathrm{H}), 4.91(\mathrm{dt}, J=5.1,2.3 \mathrm{~Hz}, 1 \mathrm{H}), 2.82-2.72$ (m, 1H), 2.33-2.09 (m, 3H), 2.09-1.99 (m, 1H), 1.94 (ddtd, $J=13.6,6.0,3.0,1.9 \mathrm{~Hz}, 1 \mathrm{H}), 1.81$ (dddd, $J=12.9,11.5$, $10.5,5.7 \mathrm{~Hz}, 1 \mathrm{H}), 0.93(\mathrm{~s}, 9 \mathrm{H}), 0.16(\mathrm{~s}, 3 \mathrm{H}), 0.15(\mathrm{~s}, 3 \mathrm{H})$.

${ }^{13} \mathrm{C}$ NMR $\left(126 \mathrm{MHz}, \mathrm{CD}_{2} \mathrm{Cl}_{2}\right) \delta 164.7-162.8(\mathrm{dd}, J=247.4 \mathrm{~Hz}, J=13.1 \mathrm{~Hz}), 151.8(\mathrm{t}, J=8.4 \mathrm{~Hz}), 151.2,110.5-110.4(\mathrm{dd}, J=19.2$ $\mathrm{Hz}, J=5.4 \mathrm{~Hz}), 103.7,101.9(\mathrm{t}, J=25.6 \mathrm{~Hz}), 101.7,40.5(\mathrm{t}, J=2.0 \mathrm{~Hz}), 32.2,30.5,26.2,18.7,-3.8,-3.9$.

${ }^{19} \mathrm{~F}$ NMR $\left(471 \mathrm{MHz}, \mathrm{CD}_{2} \mathrm{Cl}_{2}\right) \delta-111.4$.

$\mathrm{R} f=0.20$ (Hexanes).

EI-HRMS ( $m / z)$ : calculated for $\mathrm{C}_{18} \mathrm{H}_{26} \mathrm{O}_{1} \mathrm{Si}_{1} \mathrm{~F}_{2}\left[\mathrm{M}^{+*}\right]$ : 324.1716 , found: 324.1710 .

HPLC (OJ-3R, Acetonitrile: Water $=60: 40,1.0 \mathrm{~mL} / \mathrm{min}, 298 \mathrm{~K}, 220 \mathrm{~nm}): t_{\mathrm{R} 1}=19.0 \mathrm{~min}, t_{\mathrm{R} 2}=20.7 \mathrm{~min}$, e.r. $=95: 5$.

$[\alpha]_{\mathbf{D}}^{25}=29.2\left(c 0.5, \mathrm{CHCl}_{3}\right)$.

\section{(R)-tert-butyldimethyl((4-(naphthalen-1-yl)cyclohex-1-en-1-yl)oxy)silane 3m}

The reaction was performed according to the general procedure and catalyzed by IDPi $\mathbf{4 c}$. 




The titled product was purified by column chromatography with hexanes as eluent to afford $\mathbf{3 m}$ as a colorless oil (67.0 mg, $99 \%$ yield).

${ }^{1} \mathrm{H}$ NMR $\left(501 \mathrm{MHz}, \mathrm{CD}_{2} \mathrm{Cl}_{2}\right) \delta 7.95(\mathrm{~d}, J=8.4 \mathrm{~Hz}, 1 \mathrm{H}), 7.67(\mathrm{dd}, J=8.1,1.4 \mathrm{~Hz}, 1 \mathrm{H}), 7.52(\mathrm{dd}, J=6.8,2.5 \mathrm{~Hz}, 1 \mathrm{H}), 7.36$ $-7.20(\mathrm{~m}, 4 \mathrm{H}), 4.84-4.77(\mathrm{~m}, 1 \mathrm{H}), 3.41(\mathrm{tt}, J=9.5,4.7 \mathrm{~Hz}, 1 \mathrm{H}), 2.31-2.23(\mathrm{~m}, 1 \mathrm{H}), 2.22-2.03(\mathrm{~m}, 2 \mathrm{H}), 1.97-1.80(\mathrm{~m}, 3 \mathrm{H})$, $0.79-0.75(\mathrm{~m}, 9 \mathrm{H}), 0.01(\mathrm{~s}, 3 \mathrm{H}), 0.00(\mathrm{~s}, 3 \mathrm{H})$.

${ }^{13} \mathrm{C}$ NMR $\left(126 \mathrm{MHz}, \mathrm{CD}_{2} \mathrm{Cl}_{2}\right) \delta 151.1,143.1,134.5,132.1,129.4,126.9,126.3,126.2,125.8,123.8,123.0,104.3,35.4,32.1,31.0$, $30.4,26.1,18.5,-4.0,-4.1$.

$\mathrm{R} f=0.47($ Ethyl acetate/hexanes $=1: 20)$.

EI-HRMS ( $m / z)$ : calculated for $\mathrm{C}_{22} \mathrm{H}_{30} \mathrm{O}_{1} \mathrm{Si}_{1}\left[\mathrm{M}^{+\bullet}\right]: 338.2060$, found: 338.2060 .

$\operatorname{HPLC}(\mathrm{OD}-3 \mathrm{R}$, Acetonitrile: Water $=65: 35,1.0 \mathrm{~mL} / \mathrm{min}, 298 \mathrm{~K}, 220 \mathrm{~nm}): t_{\mathrm{R} 1}=56.9 \mathrm{~min}, t_{\mathrm{R} 2}=60.8 \mathrm{~min}$, e.r. $=96: 4$.

$[\boldsymbol{\alpha}]_{\mathbf{D}}^{\mathbf{2 5}}=2.3\left(c 0.44, \mathrm{CH}_{3} \mathrm{CN}\right)$.

\section{(R)-tert-butyldimethyl((4-(naphthalen-2-yl)cyclohex-1-en-1-yl)oxy)silane 3n}



The reaction was performed according to the general procedure and catalyzed by IDPi 4c.

The titled product was purified by column chromatography with hexanes as eluent to afford $\mathbf{3 n}$ as a white solid (65.7 mg, $97 \%$ yield).

${ }^{1} \mathrm{H}$ NMR (501 MHz, $\left.\mathrm{CD}_{2} \mathrm{Cl}_{2}\right) \delta 7.81(\mathrm{tdd}, J=8.9,8.2,3.0,1.8 \mathrm{~Hz}, 3 \mathrm{H}), 7.69(\mathrm{dd}, J=1.7,0.8 \mathrm{~Hz}, 1 \mathrm{H}), 7.50-7.37(\mathrm{~m}, 3 \mathrm{H})$, $4.99(\mathrm{dd}, J=4.0,2.2 \mathrm{~Hz}, 1 \mathrm{H}), 3.00-2.88(\mathrm{~m}, 1 \mathrm{H}), 2.42-2.23(\mathrm{~m}, 3 \mathrm{H}), 2.15-1.90(\mathrm{~m}, 3 \mathrm{H}), 0.96(\mathrm{~s}, 9 \mathrm{H}), 0.01(\mathrm{~s}, 3 \mathrm{H}), 0.00(\mathrm{~s}$, $3 \mathrm{H})$.

${ }^{13} \mathrm{C}$ NMR $\left(126 \mathrm{MHz}, \mathrm{CD}_{2} \mathrm{Cl}_{2}\right) \delta 150.5,144.3,133.6,132.2,127.7,127.5,127.4,126.0,125.8,125.1,124.7,103.5,40.0$, $31.8,30.1,30.1,25.5,17.9,-4.6,-4.7$.

$\mathrm{R} f=0.47$ (Ethyl acetate/hexanes $=1: 20)$.

EI-HRMS (m/z): calculated for $\mathrm{C}_{22} \mathrm{H}_{30} \mathrm{O}_{1} \mathrm{Si}_{1}\left[\mathrm{M}^{+\bullet}\right]: 338.2060$, found: 338.2057 .

$\mathrm{HPLC}\left(\mathrm{OJ}-3, \mathrm{CO}_{2} / 2-\mathrm{Propanol}=90: 10,1.0 \mathrm{~mL} / \mathrm{min}, 323 \mathrm{~K}, 220 \mathrm{~nm}\right): t_{\mathrm{R} 1}=8.1 \mathrm{~min}, t_{\mathrm{R} 2}=8.6 \mathrm{~min}$, e.r. $=95.5: 4.5$.

$[\boldsymbol{\alpha}]_{\mathbf{D}}^{25}=30.5\left(c 0.40, \mathrm{CH}_{3} \mathrm{CN}\right)$.

m.p. $=42.9-43.1^{\circ} \mathrm{C}$.

\section{(R)-tert-butyldimethyl((1,2,3,6-tetrahydro-[1,1':4',1'"-terphenyl]-4-yl)oxy)silane 3o}



The reaction was performed according to the general procedure and catalyzed by IDPi $\mathbf{4 c}$.

The titled product was purified by column chromatography with hexanes as eluent to afford $\mathbf{3 o}$ as a white solid (66.4 mg, $91 \%$ yield)

${ }^{1} \mathrm{H}$ NMR (501 MHz, $\left.\mathrm{CD}_{2} \mathrm{Cl}_{2}\right) \delta$ 7.45-7.39 (m, 2H), 7.39-7.34 (m, 2H), 7.26 (dd, J = 8.4, 7.0 Hz, 2H), 7.18-7.12 (m, 3H), 4.81 $-4.76(\mathrm{~m}, 1 \mathrm{H}), 2.64(\mathrm{tdd}, J=11.3,5.2,3.0 \mathrm{~Hz}, 1 \mathrm{H}), 2.19-2.00(\mathrm{~m}, 3 \mathrm{H}), 1.94-1.86(\mathrm{~m}, 1 \mathrm{H}), 1.84-1.77$ (m, $1 \mathrm{H}), 1.72(\mathrm{dddd}, J$ $=12.8,11.7,10.7,5.6 \mathrm{~Hz}, 1 \mathrm{H}), 0.78(\mathrm{~s}, 9 \mathrm{H}), 0.00(\mathrm{~s}, 3 \mathrm{H}), 0.00(\mathrm{~s}, 3 \mathrm{H})$.

${ }^{13} \mathrm{C}$ NMR $\left(126 \mathrm{MHz}, \mathrm{CD}_{2} \mathrm{Cl}_{2}\right) \delta 150.7,146.2,141.1,138.9,128.9,127.5,127.2,127.1,127.0,103.6,39.8,32.1,30.4,30.3,25.6,18.1$, $-4.4,-4.6$.

$\mathrm{R} f=0.43($ Ethyl acetate/hexanes $=1: 20)$.

EI-HRMS ( $m / z)$ : calculated for $\mathrm{C}_{24} \mathrm{H}_{32} \mathrm{O}_{1} \mathrm{Si}_{1}\left[\mathrm{M}^{+\bullet}\right]: 364.2217$, found: 364.2214 .

HPLC (Amycoat RP, Acetonitrile/MeOH/Water $=45: 45: 10,1.0 \mathrm{~mL} / \mathrm{min}, 298 \mathrm{~K}, 220 \mathrm{~nm}$ ): $t_{\mathrm{R} 1}=5.3 \mathrm{~min}, t_{\mathrm{R} 2}=4.5 \mathrm{~min}$, e.r. $=96: 4$. 
$[\boldsymbol{\alpha}]_{\mathbf{D}}^{25}=38.2\left(c 0.65, \mathrm{CH}_{3} \mathrm{CN}\right)$.

m.p. $=49.6-50.7^{\circ} \mathrm{C}$.

\section{(R)-tert-butyl((4-(tert-butyl)cyclohex-1-en-1-yl)oxy)dimethylsilane 3p}

OTBS The reaction was performed according to the general procedure and catalyzed by IDPi $\mathbf{4 d}$ at room temperature.



The titled product was purified by column chromatography with hexanes as eluent to afford $\mathbf{3 p}$ as a colorless oil (51.6 $\mathrm{mg}$, $96 \%$ yield).

${ }^{1} \mathrm{H}$ NMR $\left(501 \mathrm{MHz}, \mathrm{CD}_{2} \mathrm{Cl}_{2}\right) \delta 4.70(\mathrm{dt}, J=5.8,2.1 \mathrm{~Hz}, 1 \mathrm{H}), 2.03-1.81(\mathrm{~m}, 3 \mathrm{H}), 1.74-1.61(\mathrm{~m}, 2 \mathrm{H}), 1.19-1.02(\mathrm{~m}, 2 \mathrm{H}), 0.77$ $(\mathrm{d}, J=21.2 \mathrm{~Hz}, 18 \mathrm{H}), 0.00(\mathrm{~s}, 3 \mathrm{H}), 0.00(\mathrm{~s}, 3 \mathrm{H})$.

${ }^{13} \mathrm{C}$ NMR $\left(126 \mathrm{MHz}, \mathrm{CD}_{2} \mathrm{Cl}_{2}\right) \delta 151.3,104.7,44.9,32.8,31.8,27.9,26.3,25.9,25.2,18.7,-3.8,-3.9$.

$\mathrm{R} f=0.42$ (pentane).

EI-HRMS $(m / z)$ : calculated for $\mathrm{C}_{16} \mathrm{H}_{32} \mathrm{O}_{1} \mathrm{Si}_{1}\left[\mathrm{M}^{+*}\right]: 268.2217$, found: 268.2215 .

HPLC (OJ-3R, MeOH: Water $=75: 25,1.0 \mathrm{~mL} / \mathrm{min}, 298 \mathrm{~K}, 214 \mathrm{~nm}): t_{\mathrm{R} 1}=11.9 \mathrm{~min}, t_{\mathrm{R} 2}=12.1 \mathrm{~min}$, e.r. $=97: 3$.

$[\alpha]_{\mathbf{D}}^{25}=32.3\left(c 0.60, \mathrm{CHCl}_{3}\right)$.

\section{$(R)-\left(\left[1,1^{\prime}\right.\right.$-bi(cyclohexan)]-3-en-4-yloxy)(tert-butyl)dimethylsilane 3q}

OTBS The reaction was performed according to the general procedure and catalyzed by IDPi $\mathbf{4 b}$ for $2 \mathrm{~d}$.



The titled product was purified by column chromatography with hexanes as eluent to afford $\mathbf{3 q}$ as a colorless oil (51.8 $\mathrm{mg}$, $88 \%$ yield).

${ }^{1} \mathrm{H}$ NMR $\left(501 \mathrm{MHz}, \mathrm{CD}_{2} \mathrm{Cl}_{2}\right) \delta 4.70(\mathrm{dt}, J=4.9,2.1 \mathrm{~Hz}, 1 \mathrm{H}), 2.00-1.78(\mathrm{~m}, 3 \mathrm{H}), 1.72-1.46(\mathrm{~m}, 7 \mathrm{H}), 1.23-0.97(\mathrm{~m}, 6 \mathrm{H}), 0.93$ $-0.81(\mathrm{~m}, 2 \mathrm{H}), 0.80(\mathrm{~s}, 9 \mathrm{H}), 0.00(\mathrm{~s}, 3 \mathrm{H}), 0.00(\mathrm{~s}, 3 \mathrm{H})$.

${ }^{13} \mathrm{C}$ NMR $\left(126 \mathrm{MHz}, \mathrm{CD}_{2} \mathrm{Cl}_{2}\right) \delta 151.1,104.3,42.8,39.8,31.1,30.9,30.9,28.0,27.4,27.4,27.1,26.1,18.5,-4.0,-4.2$.

$\mathrm{R} f=0.40$ (pentane).

EI-HRMS (m/z): calculated for $\mathrm{C}_{18} \mathrm{H}_{34} \mathrm{O}_{1} \mathrm{Si}_{1}\left[\mathrm{M}^{+*}\right]: 294.2373$, found: 294.2369 .

HPLC (OJ-3R, Acetonitrile: Water $=70: 30,1.0 \mathrm{~mL} / \mathrm{min}, 298 \mathrm{~K}, 220 \mathrm{~nm}): t_{\mathrm{R} 1}=11.7 \mathrm{~min}, t_{\mathrm{R} 2}=12.3 \mathrm{~min}$, e.r. $=92: 8$.

$[\boldsymbol{\alpha}]_{\mathbf{D}}^{25}=31.6\left(c 0.38, \mathrm{CHCl}_{3}\right)$.

\section{(R)-((4-benzylcyclohex-1-en-1-yl)oxy)(tert-butyl)dimethylsilane 3r}



The reaction was performed according to the general procedure and catalyzed by IDPi $\mathbf{4 b}$.

The titled product was purified by column chromatography with hexanes as eluent to afford $\mathbf{3 r}$ as a colorless oil (55.7 mg, $92 \%$ yield).

${ }^{1} \mathrm{H}$ NMR (501 MHz, $\mathrm{CD}_{2} \mathrm{Cl}_{2}$ ) $\delta 7.15(\mathrm{dd}, J=8.3,6.5 \mathrm{~Hz}, 2 \mathrm{H}), 7.09-7.00(\mathrm{~m}, 3 \mathrm{H}), 4.72-4.63(\mathrm{~m}, 1 \mathrm{H}), 2.44(\mathrm{dd}, J=6.7,4.3$ $\mathrm{Hz}, 2 \mathrm{H}), 1.99-1.80(\mathrm{~m}, 3 \mathrm{H}), 1.64$ (tq, $J=12.8,5.6,4.3 \mathrm{~Hz}, 3 \mathrm{H}), 1.23$ (ddd, $J=9.9,6.4,2.5 \mathrm{~Hz}, 1 \mathrm{H}), 0.79$ (s, 9H), 0.00 (s, $3 \mathrm{H}),-0.01(\mathrm{~s}, 3 \mathrm{H})$.

${ }^{13} \mathrm{C}$ NMR $\left(126 \mathrm{MHz}, \mathrm{CD}_{2} \mathrm{Cl}_{2}\right) \delta 151.2,142.1,129.9,128.9,126.5,103.9,43.1,36.4,31.0,30.3,29.6,26.3,18.7,-3.8,-3.9$.

$\mathrm{R} f=0.60$ (Hexanes).

EI-HRMS ( $m / z)$ : calculated for $\mathrm{C}_{19} \mathrm{H}_{30} \mathrm{O}_{1} \mathrm{Si}_{1}\left[\mathrm{M}^{+*}\right]: 302.2066$, found: 302.2068 .

HPLC (OJ-3R, Acetonitrile: Water $=70: 30,1.0 \mathrm{~mL} / \mathrm{min}, 298 \mathrm{~K}, 259 \mathrm{~nm}): t_{\mathrm{R} 1}=13.5 \mathrm{~min}, t_{\mathrm{R} 2}=12.7 \mathrm{~min}$, e.r. $=92: 8$. 
$[\boldsymbol{\alpha}]_{\mathbf{D}}^{25}=6.4\left(c 0.50, \mathrm{CHCl}_{3}\right)$.

\section{(R)-tert-butyldimethyl((1-methyl-1,2,3,6-tetrahydro-[1,1'-biphenyl]-4-yl)oxy)silane 3s}

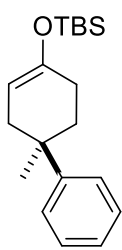

The reaction was performed according to the general procedure and catalyzed by IDPi $4 \mathbf{a}$.

The titled product was purified by column chromatography with hexanes as eluent to afford $\mathbf{3 s}$ as a colorless oil (50.8 mg, $80 \%$ yield).

${ }^{1} \mathrm{H}$ NMR $\left(501 \mathrm{MHz}, \mathrm{CD}_{2} \mathrm{Cl}_{2}\right) \delta 7.41-7.33(\mathrm{~m}, 2 \mathrm{H}), 7.29(\mathrm{dd}, J=8.5,7.0 \mathrm{~Hz}, 2 \mathrm{H}), 7.20-7.12(\mathrm{~m}, 1 \mathrm{H}), 4.90(\mathrm{t}, J=4.1 \mathrm{~Hz}$, $1 \mathrm{H}), 2.50$ (ddt, $J=16.9,4.1,2.0 \mathrm{~Hz}, 1 \mathrm{H}), 2.21-2.11(\mathrm{~m}, 1 \mathrm{H}), 2.05-1.92(\mathrm{~m}, 2 \mathrm{H}), 1.85-1.63(\mathrm{~m}, 2 \mathrm{H}), 1.25$ (s, 3H), $0.89-0.82$ $(\mathrm{m}, 9 \mathrm{H}), 0.07(\mathrm{~s}, 3 \mathrm{H}), 0.01(\mathrm{~s}, 3 \mathrm{H})$.

${ }^{13} \mathrm{C}$ NMR $\left(126 \mathrm{MHz}, \mathrm{CD}_{2} \mathrm{Cl}_{2}\right) \delta 150.8,149.4,128.7,126.7,126.2,103.8,37.1,36.7,36.1,29.7,28.5,26.2,18.6,-3.9,-4.0$.

$\mathrm{R} f=0.62$ (Hexanes).

EI-HRMS $(m / z)$ : calculated for $\mathrm{C}_{19} \mathrm{H}_{30} \mathrm{O}_{1} \mathrm{Si}_{1}\left[\mathrm{M}^{+\bullet}\right]: 302.2060$, found: 302.2056 .

HPLC (OJ-3R, Acetonitrile: Water $=70: 30,1.0 \mathrm{~mL} / \mathrm{min}, 298 \mathrm{~K}, 220 \mathrm{~nm}): t_{\mathrm{R} 1}=17.0 \mathrm{~min}, t_{\mathrm{R} 2}=21.6 \mathrm{~min}$, e.r. $=94: 6$.

$[\boldsymbol{\alpha}]_{\mathbf{D}}^{25}=-7.2\left(c 0.25, \mathrm{CHCl}_{3}\right)$.

\section{(R)-4-((tert-butyldimethylsilyl)oxy)cyclohex-3-ene-1-carboxylate 3t}

OTBS The reaction was performed according to the general procedure and catalyzed by IDPi 4c.

The titled product was purified by column chromatography with hexanes as eluent to afford $3 \mathrm{t}$ as a colorless oil (52.3 mg,
$92 \%$ yield). $3.1 \mathrm{~Hz}, 1 \mathrm{H}), 2.28-2.18(\mathrm{~m}, 2 \mathrm{H}), 2.17-1.93(\mathrm{~m}, 3 \mathrm{H}), 1.83-1.66(\mathrm{~m}, 1 \mathrm{H}), 1.23(\mathrm{t}, J=7.1 \mathrm{~Hz}, 3 \mathrm{H}), 0.91(\mathrm{~s}, 9 \mathrm{H}), 0.12(\mathrm{~s}, 6 \mathrm{H})$.

${ }^{13} \mathrm{C}$ NMR $\left(126 \mathrm{MHz}, \mathrm{CD}_{2} \mathrm{Cl}_{2}\right) \delta 175.7,150.5,102.6,60.5,39.5,29.3,26.7,25.9,25.8,18.2,14.4,-4.4,-4.4$.

$\mathrm{R} f=0.16$ (Hexanes).

EI-HRMS (m/z): calculated for $\mathrm{C}_{15} \mathrm{H}_{28} \mathrm{O}_{3} \mathrm{Si}_{1}\left[\mathrm{M}^{+\bullet}\right]: 284.1802$, found: 284.1798.

HPLC (AD-3R, Acetonitrile: Water $=50: 50,1.0 \mathrm{~mL} / \mathrm{min}, 298 \mathrm{~K}, 220 \mathrm{~nm}): t_{\mathrm{R} 1}=38.5 \mathrm{~min}, t_{\mathrm{R} 2}=32.4 \mathrm{~min}$, e.r. $=98: 2$.

$[\boldsymbol{\alpha}]_{\mathbf{D}}^{\mathbf{2 5}}=17.2\left(c 0.51, \mathrm{CHCl}_{3}\right)$

(((3aS,6aS)-1,3a,4,5,6,6a-hexahydropentalen-2-yl)oxy)triethylsilane 3u



The reaction was performed according to the general procedure and catalyzed by IDPi $4 \mathbf{e}$.

The titled product was purified by column chromatography with hexanes as eluent to afford $\mathbf{3 u}$ as a colorless oil (46.7 mg, $98 \%$ yield).

${ }^{1} \mathrm{H}$ NMR $\left(501 \mathrm{MHz}, \mathrm{CD}_{2} \mathrm{Cl}_{2}\right) \delta 4.51(\mathrm{t}, J=2.0 \mathrm{~Hz}, 1 \mathrm{H}), 3.09(\mathrm{dq}, J=5.2,2.5 \mathrm{~Hz}, 1 \mathrm{H}), 2.67-2.55(\mathrm{~m}, 2 \mathrm{H}), 1.96(\mathrm{ddd}, J=$ $13.0,2.9,1.8 \mathrm{~Hz}, 1 \mathrm{H}), 1.80-1.70(\mathrm{~m}, 1 \mathrm{H}), 1.69-1.54(\mathrm{~m}, 2 \mathrm{H}), 1.53-1.45(\mathrm{~m}, 1 \mathrm{H}), 1.43-1.33(\mathrm{~m}, 2 \mathrm{H}), 1.01(\mathrm{t}, J=8.0 \mathrm{~Hz}, 9 \mathrm{H}), 0.71(\mathrm{q}$, $J=8.1 \mathrm{~Hz}, 6 \mathrm{H})$.

${ }^{13} \mathrm{C}$ NMR $\left(126 \mathrm{MHz}, \mathrm{CD}_{2} \mathrm{Cl}_{2}\right) \delta 153.4,106.8,46.5,41.8,38.1,35.6,33.4,25.1,6.4,4.7$.

$\mathrm{Rf}=0.51$ (Hexanes)

EI-HRMS $(m / z)$ : calculated for $\mathrm{C}_{14} \mathrm{H}_{26} \mathrm{O}_{1} \mathrm{Si}_{1}\left[\mathrm{M}^{+\bullet}\right]: 238.1747$, found: 238.1748 .

HPLC (AD-3R, Acetonitrile: Water = 50:50, $1.0 \mathrm{~mL} / \mathrm{min}, 298 \mathrm{~K}, 215 \mathrm{~nm}): t_{\mathrm{R} 1}=37.1 \mathrm{~min}, t_{\mathrm{R} 2}=39.2 \mathrm{~min}$, e.r. $=95: 5$. 
$[\boldsymbol{\alpha}]_{\mathbf{D}}^{25}=-11.1\left(c 0.74, \mathrm{CH}_{3} \mathrm{CN}\right)$.

\section{(R)-tert-butyldimethyl((3-phenylcyclobut-1-en-1-yl)oxy)silane 3v}

OTBS The reaction was performed according to the general procedure with 8 equiv. 2a catalyzed by IDPi 4e with sole dioxane as solvent at room temperature for $12 \mathrm{~h}$.

The titled product was purified by column chromatography with hexanes as eluent to afford $\mathbf{3 v}$ as a colorless oil (50.0 $\mathrm{mg}$, $96 \%$ yield).

${ }^{1} \mathrm{H}$ NMR (501 MHz, $\left.\mathrm{CD}_{2} \mathrm{Cl}_{2}\right) \delta$ 7.29-7.25 (m, 4H), $7.17(\mathrm{td}, \mathrm{J}=5.4,2.6 \mathrm{~Hz}, 1 \mathrm{H}), 4.85(\mathrm{~d}, \mathrm{~J}=0.9 \mathrm{~Hz}, 1 \mathrm{H}), 3.54(\mathrm{dt}, \mathrm{J}=4.6$, $1.2 \mathrm{~Hz}, 1 \mathrm{H}), 3.09(\mathrm{dd}, \mathrm{J}=12.9,4.6 \mathrm{~Hz}, 1 \mathrm{H}), 2.34(\mathrm{dd}, \mathrm{J}=12.8,1.6 \mathrm{~Hz}, 1 \mathrm{H}), 0.96(\mathrm{~s}, 9 \mathrm{H}), 0.23(\mathrm{~s}, 3 \mathrm{H}), 0.22(\mathrm{~s}, 3 \mathrm{H})$.

${ }^{13} \mathrm{C}$ NMR $\left(126 \mathrm{MHz}, \mathrm{CD}_{2} \mathrm{Cl}_{2}\right) \delta 149.9,145.0,128.1,126.5,125.9,106.0,44.2,36.9,25.4,18.0,-4.9$.

$\mathrm{R} f=0.55$ (Hexanes).

EI-HRMS $(m / z)$ : calculated for $\mathrm{C}_{16} \mathrm{H}_{24} \mathrm{OSi}_{1}\left[\mathrm{M}^{+\bullet}\right]: 260.1588$, found: 260.1591 .

HPLC (OJ-3R, Acetonitrile: Water $=60: 40,1.0 \mathrm{~mL} / \mathrm{min}, 298 \mathrm{~K}, 220 \mathrm{~nm}): t_{\mathrm{R} 1}=24.6 \mathrm{~min}, t_{\mathrm{R} 2}=18.8 \mathrm{~min}$, e.r. $=89: 11$.

$[\boldsymbol{\alpha}]_{\mathbf{D}}^{25}=3.0\left(c 0.41, \mathrm{CH}_{3} \mathrm{CN}\right)$.

\section{Gram scale reaction and derivatizations of the enol silane 3a}

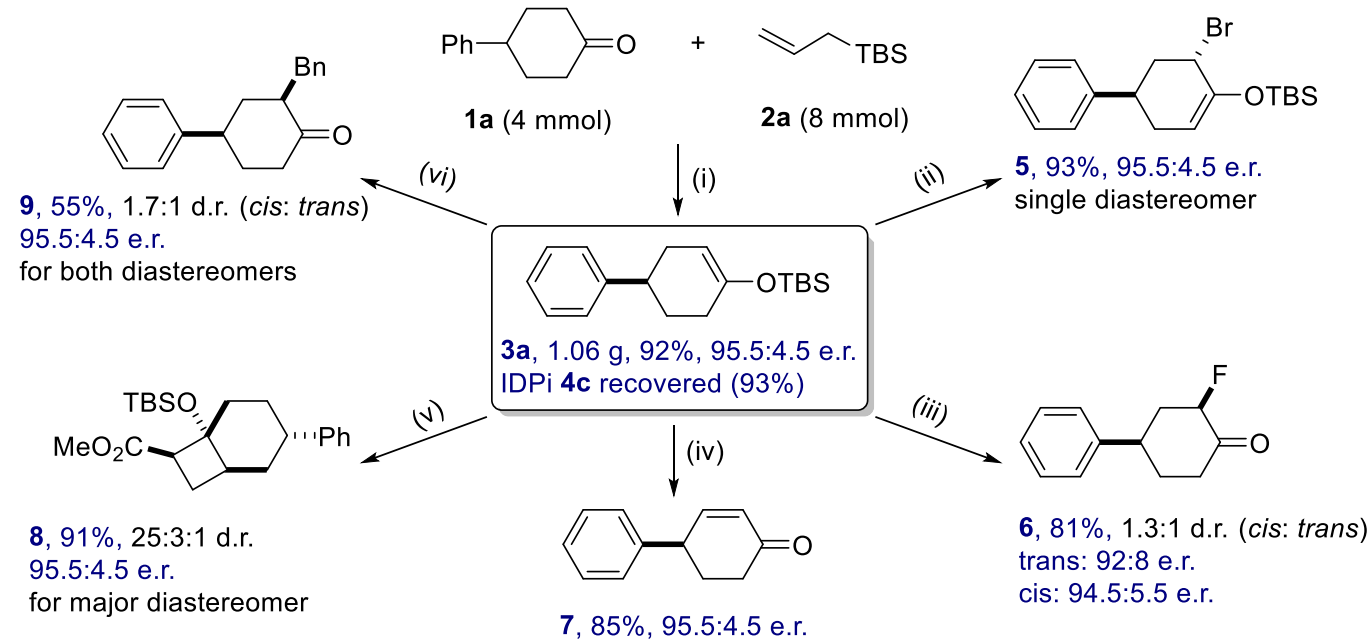

i. Gram scale catalytic deprotosilylative desymmetrization of ketone 1a with allylsilane 2 a.

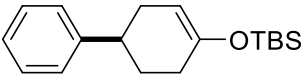

Allyl(tert-butyl)dimethylsilane 2a (1.4 mL, $7.0 \mathrm{mmol}, 2.0$ equiv.) was added to a flame-dried Schlenk flask, equipped with a teflon-coated magnetic stirring bar. IDPi $4 \mathbf{c}(86.0 \mathrm{mg}, 0.035 \mathrm{mmol}, 0.01$ equiv.) in combined solvent (toluene/dioxane $=2: 1,0.08 \mathrm{M}, 43.7 \mathrm{~mL}$ ) weas added at $25^{\circ} \mathrm{C}$ and stirred for $1 \mathrm{~h}$. The reaction mixture was cooled to $-20^{\circ} \mathrm{C}$ for $15 \mathrm{~min}$, and ketone 1a $\left(609.8 \mathrm{mg}, 3.5 \mathrm{mmol}, 1.0\right.$ equiv.) was slowly added, then the resultant mixture was stirred at $-20{ }^{\circ} \mathrm{C}$. After the ketone 1a was fully consumed which was monitored by TLC, the reaction was quenched by three drops of triethylamine through pipet. Organic volatiles were evaporated in vacuo and the crude mixture was purified by the silica column to afford the desired enol silane 3a (1.06 g, 92\% yield, 95.5:4.5 e.r.), and the recycled IDPi catalyst $\mathbf{4 c}$ was collected ( $80 \mathrm{mg}, 93 \%)$.

\section{ii. Preparation of $(((1 R, 3 S)$-3-bromo-1,2,3,6-tetrahydro-[1,1'-biphenyl]-4-yl)oxy $)(t e r t$-butyl)dimethylsilane 5}




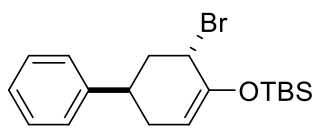

To a solution of compound $3 \mathbf{a}(57.7 \mathrm{mg}, 0.2 \mathrm{mmol})$ in $\mathrm{THF}(0.1 \mathrm{M})$, N-Bromosuccinimide was added at $0{ }^{\circ} \mathrm{C}$.

The reaction mixture was stirred for $1.5 \mathrm{~h}$ at this temperature. After the reaction was completed (monitored by

TLC), the reaction mixture was quenched with water $(1 \mathrm{~mL})$ and extracted with ethyl acetate $(3 \times 5 \mathrm{~mL})$. The organic layers were combined, dried over anhydrous $\mathrm{Na}_{2} \mathrm{SO}_{4}$ and filtered through celite. The volatile components were removed under reduced pressure, and purified by column chromatography (hexanes) affording the brominated enol silane $\mathbf{5}$ as a colorless oil (68.3 $\mathrm{mg}$, $93 \%$ yield).

${ }^{1} \mathrm{H}$ NMR (501 MHz, $\left.\mathrm{CD}_{2} \mathrm{Cl}_{2}\right) \delta$ 7.14-7.09 (m, 2H), 7.08-7.04 (m, 2H), 7.04-6.99 (m, 1H), $4.87(\mathrm{dd}, J=5.6,2.6 \mathrm{~Hz}, 1 \mathrm{H}), 4.53-4.46(\mathrm{~m}$, $1 \mathrm{H}), 3.18-3.01(\mathrm{~m}, 1 \mathrm{H}), 2.29-2.21(\mathrm{~m}, 1 \mathrm{H}), 2.21-2.10(\mathrm{~m}, 3 \mathrm{H}), 0.77$ (s, 9H), $0.00(\mathrm{~s}, 3 \mathrm{H}), 0.00(\mathrm{~s}, 3 \mathrm{H})$.

${ }^{13} \mathrm{C}$ NMR $\left(126 \mathrm{MHz}, \mathrm{CD}_{2} \mathrm{Cl}_{2}\right) \delta 149.3,145.0,128.4,127.0,126.4,107.1,51.8,39.8,35.2,32.2,25.4,18.0,-4.7,-5.1$.

$\mathrm{R} f=0.24$ (Hexanes).

ESI-HRMS $(\mathrm{m} / \mathrm{z})$ : calculated for $\mathrm{C}_{18} \mathrm{H}_{28} \mathrm{O}_{1} \mathrm{Br}_{1} \mathrm{Si}_{1}\left([\mathrm{M}+\mathrm{H}]^{+}\right)$: 367.1087, found: 367.1085 .

HPLC (OJ-3R, Acetonitrile: Water $=80: 20,1.0 \mathrm{~mL} / \mathrm{min}, 298 \mathrm{~K}, 254 \mathrm{~nm}): t_{\mathrm{R} 1}=5.6 \mathrm{~min}, t_{\mathrm{R} 2}=8.1 \mathrm{~min}$, e.r. $=$ 95:5.

$[\boldsymbol{\alpha}]_{\mathbf{D}}^{25}=-12.8\left(c 0.42, \mathrm{CH}_{3} \mathrm{CN}\right)$.

\section{iii. Preparation of $(2 R, 4 R)$-2-fluoro-4-phenylcyclohexan-1-one 6}

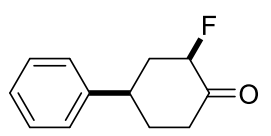

According to a known procedure with minor modification ${ }^{17}$

To a solution of compound $3 \mathbf{a}$ (57.7 mg, $0.2 \mathrm{mmol})$ in $\mathrm{CH}_{3} \mathrm{CN}(0.06 \mathrm{M})$, selectfluor (1.1 equiv.) was added at rt.

The reaction mixture was stirred for $1 \mathrm{~h}$ at this temperature. After the reaction was completed (monitored by TLC), the reaction mixture quenched with water $(1 \mathrm{~mL})$ and extracted with ethyl acetate $(3 \times 5 \mathrm{~mL})$. The organic layers were combined, dried over $\mathrm{Na}_{2} \mathrm{SO}_{4}$ and filtered through celite. The volatile components were removed under reduced pressure, and purified by column chromatography (ethyl acetate/hexanes 1:9) affording the fluorinated cyclic ketone $\mathbf{6}$ as a colorless oil (31.1 mg, 81\% yield).

There are two diastereomers formed, d.r. $=1.3: 1$, for the major product:

${ }^{1} \mathrm{H}$ NMR $\left(500 \mathrm{MHz}, \mathrm{CD}_{2} \mathrm{Cl}_{2}\right) \delta$ 7.36-7.30 (m, 2H), 7.30-7.21 (m, 3H), 5.10 (dddd, $\left.J=48.3,12.4,6.7,0.7 \mathrm{~Hz}, 1 \mathrm{H}\right), 3.18(\mathrm{ddt}, J=12.7$, $9.2,1.9 \mathrm{~Hz}, 1 \mathrm{H}), 2.62(\mathrm{dddt}, J=12.2,6.5,4.3,3.2 \mathrm{~Hz}, 1 \mathrm{H}), 2.59-2.51(\mathrm{~m}, 2 \mathrm{H}), 2.27-2.16(\mathrm{~m}, 1 \mathrm{H}), 2.10(\mathrm{qd}, J=12.4,9.7 \mathrm{~Hz}, 1 \mathrm{H})$, $1.96-1.80(\mathrm{~m}, 1 \mathrm{H})$.

${ }^{13} \mathrm{C}$ NMR $\left(126 \mathrm{MHz}, \mathrm{CD}_{2} \mathrm{Cl}_{2}\right) \delta 204.5-204.3$ (d, $\left.J=14.2 \mathrm{~Hz}\right), 142.9$ (d, $\left.J=1.2 \mathrm{~Hz}\right), 128.6,126.9,126.6,92.6-91.0(\mathrm{~d}, J=191.2 \mathrm{~Hz})$, $41.2-41.1(\mathrm{~d}, J=9.1 \mathrm{~Hz}), 40.7-40.6(\mathrm{~d}, J=18.3 \mathrm{~Hz}), 39.4,34.2-34.2(\mathrm{~d}, J=1.2 \mathrm{~Hz})$.

${ }^{19} \mathrm{~F}$ NMR $\left(470 \mathrm{MHz}, \mathrm{CD}_{2} \mathrm{Cl}_{2}\right) \delta-189.4$.

$\mathrm{R} f=0.24$ (Ethyl acetate/hexanes $=1: 20)$.

ESI-HRMS $(m / z)$ : calculated for $\mathrm{C}_{12} \mathrm{H}_{13} \mathrm{O}_{1} \mathrm{~F}_{1}\left[\mathrm{M}^{+}\right]$: 192.0945 , found: 192.0947.

HPLC $(\mathrm{AD}-3, n$-heptane $/ i-\mathrm{PrOH}=99: 1,1.0 \mathrm{~mL} / \mathrm{min}, 298 \mathrm{~K}, 254 \mathrm{~nm})$ : for major diastereomer, $t_{\mathrm{R} 1}=15.0 \mathrm{~min}, t_{\mathrm{R} 2}=18.5 \mathrm{~min}$, e.r. $=$ 91.5:8.5. for minor diastereomer, $t_{\mathrm{R} 1}=9.0 \mathrm{~min}, t_{\mathrm{R} 2}=5.9 \mathrm{~min}$, e.r. $=94.5: 5.5$.

$[\alpha]_{\mathbf{D}}^{25}=18.3\left(c 0.48, \mathrm{CHCl}_{3}\right)$. (For the major diastereomer)

Optimal rotation data of trans-product was reported. lit $^{18}$

$[\boldsymbol{\alpha}]_{\mathbf{D}}=36.9\left(c 1.04, \mathrm{CHCl}_{3}\right)$.

\section{iv. Preparation of $(R)$-2,3-dihydro-[1,1'-biphenyl]-4(1H)-one 7}


According to a known procedure with minor modification ${ }^{19}$

To a solution of compound $3 \mathbf{a}(57.7 \mathrm{mg}, 0.2 \mathrm{mmol})$ in $\mathrm{CH}_{3} \mathrm{CN}(0.1 \mathrm{M}), \mathrm{Pd}(\mathrm{OAc})_{2}(49.4 \mathrm{mg}, 0.22 \mathrm{mmol}, 1.1$ equiv.)

was slowly added at $0{ }^{\circ} \mathrm{C}$. The reaction mixture was stirred for $12 \mathrm{~h}$ at this temperature. After the reaction was completed (monitored by TLC), the reaction mixture was quenched with water $(1 \mathrm{~mL})$ and extracted with ethyl acetate $(3 \times 5 \mathrm{~mL})$. The organic layers were combined, dried over $\mathrm{Na}_{2} \mathrm{SO}_{4}$ and filtered through celite. The volatile components were removed under reduced pressure, and purified by column chromatography (ethyl acetate/hexanes 1:9) affording the aldehyde 7 as a white solid (29.2 $\mathrm{mg}, 85 \%$ yield).

${ }^{1} \mathrm{H}$ NMR $\left(501 \mathrm{MHz}, \mathrm{CD}_{2} \mathrm{Cl}_{2}\right) \delta 7.33-7.25(\mathrm{~m}, 2 \mathrm{H}), 7.21-7.11(\mathrm{~m}, 3 \mathrm{H}), 6.90(\mathrm{ddd}, J=10.2,2.8,1.4 \mathrm{~Hz}, 1 \mathrm{H}), 6.02(\mathrm{dd}, J=10.2,2.6 \mathrm{~Hz}$, $1 \mathrm{H}), 3.65(\mathrm{ddt}, J=9.9,5.2,2.7 \mathrm{~Hz}, 1 \mathrm{H}), 2.46-2.32(\mathrm{~m}, 2 \mathrm{H}), 2.26(\mathrm{dqd}, J=13.3,4.9,1.4 \mathrm{~Hz}, 1 \mathrm{H}), 1.95(\mathrm{dddd}, J=13.4,11.1,9.5,5.5$ $\mathrm{Hz}, 1 \mathrm{H})$.

${ }^{13} \mathrm{C}$ NMR $\left(126 \mathrm{MHz}, \mathrm{CD}_{2} \mathrm{Cl}_{2}\right) \delta 198.6,152.7,143.2,129.7,128.7,127.5,126.8,42.6,36.9,32.5$.

$\mathrm{R} f=0.12($ Ethyl acetate/hexanes $=1: 9)$.

ESI-HRMS $(\mathrm{m} / \mathrm{z})$ : calculated for $\mathrm{C}_{12} \mathrm{H}_{13} \mathrm{O}_{1}\left([\mathrm{M}+\mathrm{H}]^{+}\right): 173.0960$, found: 173.0963 .

HPLC (OJ-3, $n$-heptane $/ i-\mathrm{PrOH}=99: 1,1.0 \mathrm{~mL} / \mathrm{min}, 298 \mathrm{~K}, 220 \mathrm{~nm}): t_{\mathrm{R} 1}=15.8 \mathrm{~min}, t_{\mathrm{R} 2}=12.2 \mathrm{~min}$, e.r. $=$ 95.5:4.5.

$[\boldsymbol{\alpha}]_{\mathbf{D}}^{25}=179.5\left(c 0.28, \mathrm{C}_{6} \mathrm{H}_{6}\right)$

And the absolute configuration of unsaturated ketone $\mathbf{7}$ was determined to be $\boldsymbol{R}$ by comparing the optical rotation value with the reported value. $\mathrm{Lit}^{20}$

$[\boldsymbol{\alpha}]_{\mathbf{D}}^{25}=195.0\left(c 1.0, \mathrm{C}_{6} \mathrm{H}_{6}\right)$.

The configuration of the enolsilane product 3a could be deduced by analogy to be $\boldsymbol{R}$.

\section{v. Preparation of methyl (1S,3S,6R,7R)-6-((tert-butyldimethylsilyl)oxy)-3 phenylbicyclo[4.2.0]octane-7-carboxylate 8}

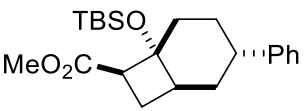

According to a known procedure with minor modification ${ }^{21}$

To a solution of compound 3a $(57.7 \mathrm{mg}, 0.2 \mathrm{mmol})$ in DCM $(0.1 \mathrm{M})$, methyl acrylat (2 equiv.) and diethylaluminium chloride $\left(0.2\right.$ equiv.) was added at $-78^{\circ} \mathrm{C}$. The reaction mixture was stirred for $4 \mathrm{~h}$ at this temperature. After the reaction completed (monitored by TLC), the reaction mixture was quenched with water $(1 \mathrm{~mL})$ and extracted with ethyl acetate $(3 \times 5$ $\mathrm{mL}$ ). The organic layers were combined and dried over $\mathrm{Na}_{2} \mathrm{SO}_{4}$ and filtered through celite. The volatile components were removed under reduced pressure and purified by column chromatography (ethyl acetate/hexanes 1:20) affording the bridged product 8 as a colorless oil (68.2 mg, 91\% yield).

There were three diastereomers formed, d.r. $=25: 6: 1$, for the major product:

${ }^{1} \mathrm{H}$ NMR (501 MHz, $\left.\mathrm{CD}_{2} \mathrm{Cl}_{2}\right) \delta 7.13-7.07(\mathrm{~m}, 2 \mathrm{H}), 7.04-6.97(\mathrm{~m}, 3 \mathrm{H}), 3.49(\mathrm{~s}, 3 \mathrm{H}), 2.80(\mathrm{dd}, J=10.1,8.3 \mathrm{~Hz}, 1 \mathrm{H}), 2.46(\mathrm{td}, J=5.5$, $3.0 \mathrm{~Hz}, 1 \mathrm{H}), 2.24(\mathrm{tq}, J=5.0,1.9 \mathrm{~Hz}, 1 \mathrm{H}), 1.76-1.55(\mathrm{~m}, 4 \mathrm{H}), 1.52-1.39(\mathrm{~m}, 4 \mathrm{H}), 0.74(\mathrm{~s}, 9 \mathrm{H}), 0.00(\mathrm{~s}, 3 \mathrm{H}), 0.00(\mathrm{~s}, 3 \mathrm{H})$.

${ }^{13} \mathrm{C}$ NMR $\left(126 \mathrm{MHz}, \mathrm{CD}_{2} \mathrm{Cl}_{2}\right) \delta 172.3,147.4,128.3,128.2,126.8,125.8,75.5,53.8,53.6,53.4,53.1,52.9,50.9,49.5,41.8,39.8,32.5$, $32.3,27.9,25.4,19.2,17.8,-3.1$.

$\mathrm{R} f=0.45$ (Ethyl acetate/hexanes $=1: 20)$

ESI-HRMS $(\mathrm{m} / z)$ : calculated for $\mathrm{C}_{22} \mathrm{H}_{34} \mathrm{O}_{3} \mathrm{Si}_{1} \mathrm{Na}_{1}\left([\mathrm{M}+\mathrm{Na}]^{+}\right)$: 397.2169 , found: 397.2167.

HPLC (OJ-3R, Acetonitrile: Water $=60: 40,1.0 \mathrm{~mL} / \mathrm{min}, 298 \mathrm{~K}, 220 \mathrm{~nm}): t_{\mathrm{R} 1}=21.5 \mathrm{~min}, t_{\mathrm{R} 2}=20.2$ min, e.r. $=95: 5$ (for major diastereomer).

$[\boldsymbol{\alpha}]_{\mathbf{D}}^{25}=39.6\left(c 0.50, \mathrm{CH}_{3} \mathrm{CN}\right)$. (For the major diastereomer)

\section{vi. Preparation of $(2 S, 4 R)$-2-benzyl-4-phenylcyclohexan-1-one 9}




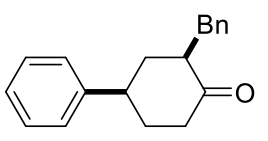

According to a known procedure with minor modification ${ }^{22}$

To a solution of compound $3 \mathbf{a}(57.7 \mathrm{mg}, 0.2 \mathrm{mmol})$ in $\mathrm{DCM}(0.1 \mathrm{M}), \mathrm{ZnCl}_{2}$ (1.0 equiv.) and $\mathrm{BnBr}(3 \mathrm{equiv}$.) was added at $\mathrm{rt}$. The reaction mixture was stirred for $4 \mathrm{~h}$ at this temperature. After the reaction completed (monitored by TLC), the reaction mixture quenched with water $(1 \mathrm{~mL})$ and extracted with ethyl acetate $(3 \times 5 \mathrm{~mL})$. The organic layers were combined and dried over $\mathrm{Na}_{2} \mathrm{SO}_{4}$, then filtered through celite. The volatile components were removed under vacuo and purified by column chromatography (ethyl acetate/hexanes 1:10) affording the ketone $\mathbf{9}$ as a white solid (29.1 mg, 55\% yield).

There are two diastereomers formed, d.r. $=1.7: 1$, for the major product:

${ }^{1} \mathrm{H}$ NMR $\left(501 \mathrm{MHz}, \mathrm{CD}_{2} \mathrm{Cl}_{2}\right) \delta 7.22-7.13(\mathrm{~m}, 4 \mathrm{H}), 7.13-7.05(\mathrm{~m}, 6 \mathrm{H}), 3.52(\mathrm{~s}, 1 \mathrm{H}), 3.18(\mathrm{dd}, J=14.1,4.8 \mathrm{~Hz}, 1 \mathrm{H}), 3.01-2.91(\mathrm{~m}, 1 \mathrm{H})$, $2.69(\mathrm{~d}, J=5.0 \mathrm{~Hz}, 1 \mathrm{H}), 2.48(\mathrm{ddd}, J=13.8,6.0,1.2 \mathrm{~Hz}, 1 \mathrm{H}), 2.39$ (ddd, $J=13.7,4.4,2.5 \mathrm{~Hz}, 1 \mathrm{H}), 2.31(\mathrm{dd}, J=14.1,8.7 \mathrm{~Hz}, 1 \mathrm{H})$, 2.16-2.08 (m, 1H), 2.05 (dd, $J=13.1,5.5 \mathrm{~Hz}, 1 \mathrm{H}), 1.84(\mathrm{dd}, J=13.1,4.4 \mathrm{~Hz}, 1 \mathrm{H})$.

${ }^{13} \mathrm{C}$ NMR $\left(126 \mathrm{MHz}, \mathrm{CDCl}_{3}\right) \delta 211.5,144.4,140.0,129.0,128.4,128.2,126.6,126.4,125.9,51.7,43.3,41.7,40.4,35.1,35.0$.

$\mathrm{R} f=0.22($ Ethyl acetate/hexanes $=1: 9)$.

EI-HRMS $(m / z)$ : calculated for $\mathrm{C}_{19} \mathrm{H}_{20} \mathrm{O}_{1}\left[\mathrm{M}^{+\bullet}\right]: 264.1508$, found: 264.1508 .

HPLC (AD-3, $n$-heptane/ $i$-PrOH $=99: 1,1.0 \mathrm{~mL} / \mathrm{min}, 298 \mathrm{~K}, 220 \mathrm{~nm})$ : for major diastereomer, $t_{\mathrm{R} 1}=10.0 \mathrm{~min}, t_{\mathrm{R} 2}=10.9 \mathrm{~min}$, e.r. $=$ 94.5:5.5. for minor diastereomer, $t_{\mathrm{R} 1}=13.3 \mathrm{~min}, t_{\mathrm{R} 2}=14.7 \mathrm{~min}$, e.r. $=94: 6$.

$[\boldsymbol{\alpha}]_{\mathbf{D}}^{25}=15.5\left(c 0.21, \mathrm{CHCl}_{3}\right)$. (For the major diastereomer)

For the minor product:

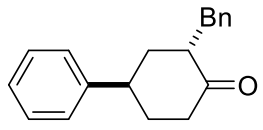

${ }^{1} \mathrm{H}$ NMR $\left(500 \mathrm{MHz}, \mathrm{CDCl}_{3}\right) \delta 7.34-7.27(\mathrm{~m}, 4 \mathrm{H}), 7.24-7.17(\mathrm{~m}, 6 \mathrm{H}), 3.25(\mathrm{tt}, J=10.3,4.0 \mathrm{~Hz}, 1 \mathrm{H}), 3.12(\mathrm{dd}, J=$ $13.0,5.2 \mathrm{~Hz}, 1 \mathrm{H}), 2.84(\mathrm{dd}, J=13.0,9.6 \mathrm{~Hz}, 1 \mathrm{H}), 2.84-2.75(\mathrm{~m}, 1 \mathrm{H}), 2.66(\mathrm{ddd}, J=14.9,11.4,5.9 \mathrm{~Hz}, 1 \mathrm{H}), 2.46$ $(\mathrm{dtd}, J=14.9,4.8,1.3 \mathrm{~Hz}, 1 \mathrm{H}), 2.27-2.18(\mathrm{~m}, 1 \mathrm{H}), 2.18-2.05(\mathrm{~m}, 1 \mathrm{H}), 2.09(\mathrm{ddd}, J=13.8,10.3,5.2 \mathrm{~Hz}, 1 \mathrm{H}), 1.95$ (dtd, $J=13.8,4.4,4.0,2.2 \mathrm{~Hz}, 1 \mathrm{H})$.

${ }^{13} \mathrm{C} \mathrm{NMR}\left(126 \mathrm{MHz}, \mathrm{CDCl}_{3}\right) \delta 213.3,144.1,138.8,128.9,128.6,128.6,126.7,126.5,126.5,50.6,38.6,37.1,36.8,36.6,33.0$.

Recycling of catalyst $4 \mathrm{c}$ recovered from the gram-scale reaction:

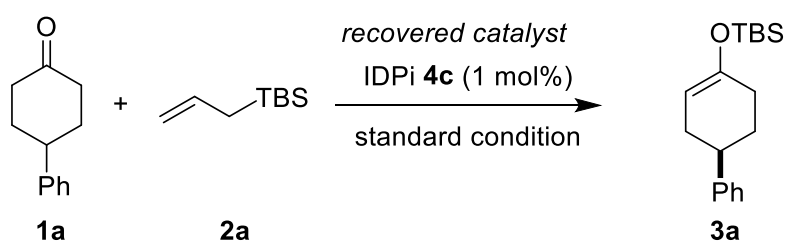

$99 \%, 97: 3$ er

Allyl silane 2a (80 $\mu \mathrm{L}, 0.4 \mathrm{mmol}, 2.0$ equiv.) was placed in a flame-dried Schlenk flask, equipped with a Teflon-coated magnetic stirring bar. Recovered IDPi 4c (0.01 equiv.) and solvent mixture toluene with 1,4-dioxane $(2: 1 v / v 0.08 \mathrm{M}, 2.4 \mathrm{~mL})$ were added at $25^{\circ} \mathrm{C}$ and stirred for $30 \mathrm{~min}$. The resultant mixture was cooled to $-20{ }^{\circ} \mathrm{C}$ for $10 \mathrm{~min}$, and ketones $1 \mathrm{a}(0.2 \mathrm{mmol}, 1.0$ equiv.) was slowly added. After stirring at $-20{ }^{\circ} \mathrm{C}$ for $2 \mathrm{~d}$, till the ketone was fully consumed monitored by TLC, the reaction was quenched with one drop of triethylamine added via pipet. Organic volatiles were evaporated in vacuo and the crude residue was purified by column chromatography with silica gel to afford the desired enol silanes 3a (99\%, 97:3 e.r.).

\section{Formal synthesis of iloprost ${ }^{23}$}




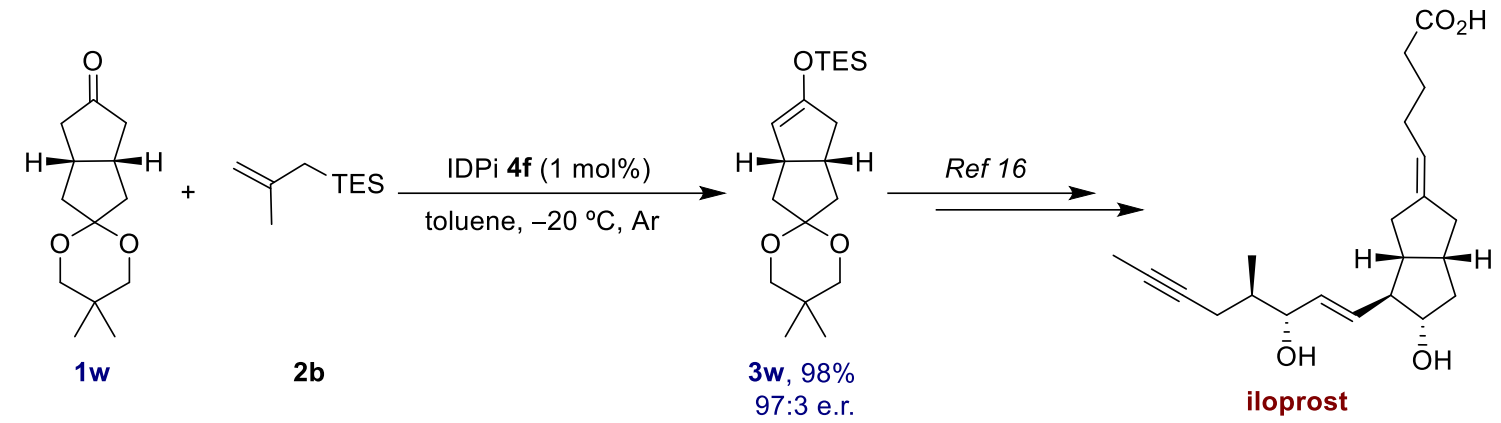

Triethyl(2-methylallyl)silane $\mathbf{2 b}(79 \mu \mathrm{L}, 0.4 \mathrm{mmol}, 2.0$ equiv.) was placed in a flame-dried Schlenk flask, equipped with a teflon-coated magnetic stirring bar. IDPi $4 \mathbf{f}\left(0.01\right.$ equiv.) in solvent mixture toluene $(0.08 \mathrm{M}, 2.4 \mathrm{~mL})$ was added at $25^{\circ} \mathrm{C}$ and stirred for 10 min. The reaction mixture was cooled to $-20^{\circ} \mathrm{C}$ for $10 \mathrm{~min}$, and ketone $\mathbf{1 w}(0.2 \mathrm{mmol}, 1.0$ equiv.) was slowly added. The resultant solution was stirred at $-20^{\circ} \mathrm{C}$. After the ketone was fully consumed monitored by TLC, the reaction was quenched by one drop of trimethylamine through pipet. Organic volatiles were evaporated in vacuo and the crude residue was purified by column chromatography with silica gel to afford the desired enol silane $\mathbf{3 w}$ as light yellow oil (66.4 $\mathrm{mg}, 98 \%, 97: 3 \mathrm{er})$.

\section{(((3aR, 6aS)-5',5'-dimethyl-3,3a,4,6a-tetrahydro-1H-spiro[pentalene-2,2'-[1,3]dioxan]-5-yl)oxy)trimethylsilane 3w}

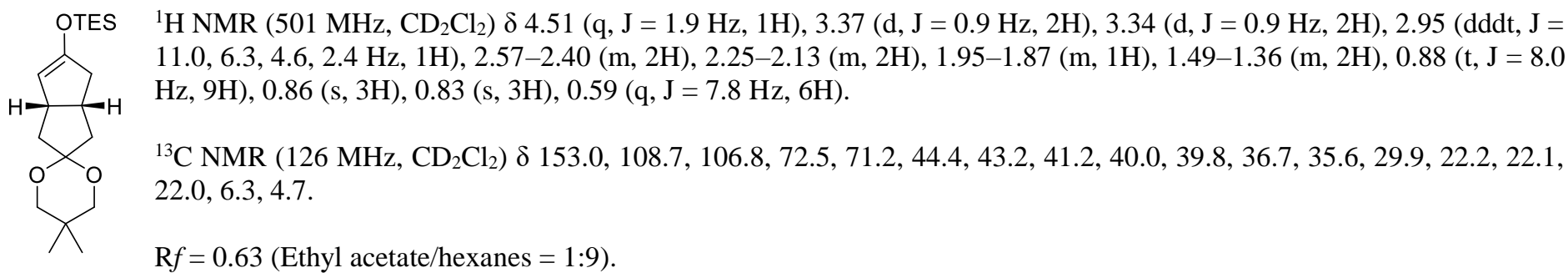

EI-HRMS $(m / z)$ : calculated for $\mathrm{C}_{19} \mathrm{H}_{35} \mathrm{O}_{3} \mathrm{Si}_{1}\left([\mathrm{M}+\mathrm{H}]^{+}\right): 339.2350$, found: 339.2345 .

HPLC (OJ-3R, Acetonitrile: Water $=70: 30,1.0 \mathrm{~mL} / \mathrm{min}, 298 \mathrm{~K}, 220 \mathrm{~nm}): t_{\mathrm{R} 1}=7.0 \mathrm{~min}, t_{\mathrm{R} 2}=6.6 \mathrm{~min}$, e.r. $=97: 3$.

$[\boldsymbol{\alpha}]_{\mathbf{D}}^{\mathbf{2 5}}=8.0\left(c 0.10, \mathrm{CH}_{3} \mathrm{CN}\right) .[\boldsymbol{\alpha}]_{\mathbf{D}}^{\mathbf{2 0}}=7.3(c 0.10, \mathrm{THF})$.

And the absolute configuration of the titled product was confirmed to be $\mathbf{3 a R}, \mathbf{6 a S}$ according to the reported data.lit ${ }^{23}$ $[\boldsymbol{\alpha}]_{\mathbf{D}}^{\mathbf{2 0}}=2.1(c 9.8, \mathrm{THF})$.

\section{Development of a catalytic asymmetric protodesilylation of racemic enol silanes.}

General procedure for the optimization of catalytic kinetic resolution of racemic silyl enol ethers with different proton sources.

Racemic silyl enol ether 3x (0.2 mmol, 1.0 equiv.) was placed in a flame-dried Schlenk flask with a teflon-coated magnetic stirring bar. IDPi $4 \mathbf{c}-\mathbf{d}$ ( 0.01 equiv.) in toluene $(0.1 \mathrm{M}, 2 \mathrm{~mL})$ was added at $25^{\circ} \mathrm{C}$, after $5 \mathrm{~min}$, the reaction mixture was cooled to a certain temperature for $10 \mathrm{~min}$, then proton source ( 0.5 equiv., with $\mathrm{H}_{2} \mathrm{O}: 0.25$ equiv.) was added. After calibration with triphenylmethane as internal standard, the resultant solution was stirred at this temperature for proper time and monitored by GC or NMR analysis. Then the reaction was quenched by one drop of triethylamine through pipet. Organic volatiles were evaporated under vacuo and the crude residue was purified by prep. TLC, and the enantiomeric ratio (e.r.) was determined by HPLC. 


\begin{tabular}{cccccc}
$( \pm)-\mathbf{3} \mathbf{x}$ & & & & \\
\hline
\end{tabular}

${ }^{a}$ Unless otherwise noted, reactions were performed with racemic silyl enol ether $3 \mathbf{x}(0.1 \mathrm{mmol})$, proton source ( 0.5 equiv., with $\mathrm{H}_{2} \mathrm{O}$ : 0.25 equiv. $)$, and catalyst $(1 \mathrm{~mol} \%)$ in toluene $(0.2 \mathrm{~mL}, 0.5 \mathrm{M})$ under argon at $0{ }^{\circ} \mathrm{C}$ for $24 \mathrm{~h} .{ }^{b}$ Conversion was determined by GC analysis. ${ }^{c}$ The enantiomeric ratio (e.r.) was determined by HPLC analysis. ${ }^{d}$ The selectivity factor $(s)$ was determined by the equation: $s=k_{\text {rel }}($ fast $/ \operatorname{slow})=\ln [(1-C)(1-e e)] / \ln [(1-C)(1+e e)]$ (where $e e$ is the enantiomeric excess of the remained starting material $3 \mathbf{x}$ and $C$ is the conversion). ${ }^{e}$ Reaction at $-30{ }^{\circ} \mathrm{C}$ for $12-72 \mathrm{~h} .{ }^{f} \mathrm{Reaction}$ at $60{ }^{\circ} \mathrm{C}$ for $24 \mathrm{~h}$. TMP $=2,4,6$-trimethylphenol. $\mathrm{BCA}=$ biphenyl carboxylic acid.

\section{Scope of the catalytic asymmetric protodesilylative kinetic resolution of racemic enol silanes.}

General procedure for the optimization of catalytic kinetic resolution of racemic silyl enol ethers with different proton sources.

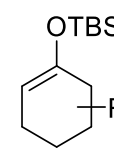

$( \pm)-3$

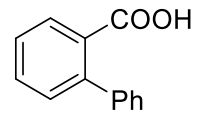

10 (0.5 equiv.)

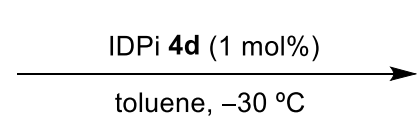

toluene, $-30^{\circ} \mathrm{C}$

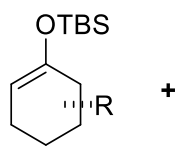

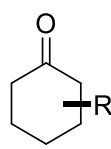

1

Racemic silyl enol ether (0.2 mmol, 1.0 equiv.) was placed in a flame-dried Schlenk flask equipped with a teflon-coated magnetic stirring bar. IDPi $4 \mathbf{d}$ (0.01 equiv.) in toluene $(0.1 \mathrm{M}, 2 \mathrm{~mL})$ was added at $25^{\circ} \mathrm{C}$ and stirred for 5 min. The reaction mixture was cooled to $-30{ }^{\circ} \mathrm{C}$ or $-60{ }^{\circ} \mathrm{C}$ for $10 \mathrm{~min}$, then 2-biphenylcarboxylic acid 10 (0.1M solution in toluene) was added. The resultant solution was stirred at $-30^{\circ} \mathrm{C}$ or $-60{ }^{\circ} \mathrm{C}$ for proper time and monitored by GC or NMR analysis (GC calibrations for all substrates were conducted with triphenylmethane as internal standard, and NMR yields were determined with triphenylmethane as internal standard). When the conversion of the racemic enol silane was around 50\%, the reaction was quenched by one drop of triethylamine through pipet. Organic volatiles were evaporated under reduced pressure and the crude product was purified by column chromatography (starting with sole hexanes with eluent to obtain the recovered silyl enol ethers, then with ethyl acetate : hexanes $=1: 20-1: 10$ as eluent to get the ketone byproducts) or prep. TLC, then the enantiomeric ratio was determined by HPLC analysis.

\section{Analytical data of products}

\section{(S)-tert-butyldimethyl((1,2,3,6-tetrahydro-[1,1'-biphenyl]-4-yl)oxy)silane 3a}

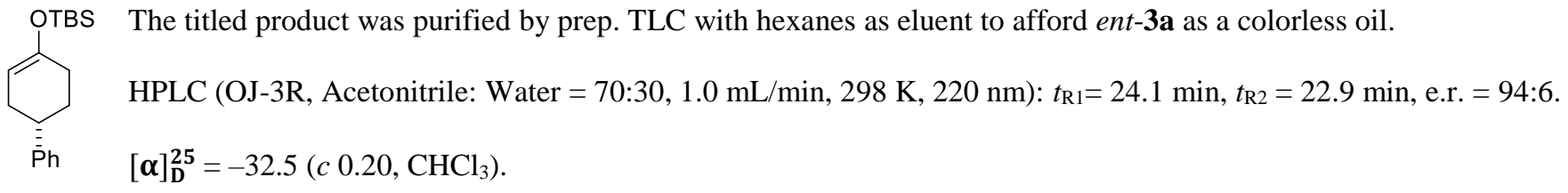


OTBS The titled product was purified by prep. TLC with hexanes as eluent to afford $\mathbf{3 x}$ as a colorless oil.

${ }^{1} \mathrm{H}$ NMR $\left(501 \mathrm{MHz}, \mathrm{CD}_{2} \mathrm{Cl}_{2}\right) \delta 4.82(\mathrm{dt}, J=5.7,2.1 \mathrm{~Hz}, 1 \mathrm{H}), 2.13-1.89(\mathrm{~m}, 3 \mathrm{H}), 1.82-1.69(\mathrm{~m}, 2 \mathrm{H}), 1.52-1.44(\mathrm{~m}, 1 \mathrm{H})$, $1.34-1.18(\mathrm{~m}, 2 \mathrm{H}), 0.91(\mathrm{~s}, 9 \mathrm{H}), 0.89$ (dd, $J=6.8,5.1 \mathrm{~Hz}, 6 \mathrm{H}), 0.12$ (s, 3H), 0.11 (s, 3H).

${ }^{13} \mathrm{C} \mathrm{NMR}\left(126 \mathrm{MHz}, \mathrm{CD}_{2} \mathrm{Cl}_{2}\right) \delta 151.3,104.4,40.9,32.8,31.0,28.0,27.3,26.2,20.6,20.4,18.6,-3.8,-4.0$.

$\mathrm{R} f=0.54$ (Hexanes).

EI-HRMS $(m / z)$ : calculated for $\mathrm{C}_{15} \mathrm{H}_{30} \mathrm{O}_{1} \mathrm{Si}_{1}\left[\mathrm{M}^{+\bullet}\right]$ : 254.2060 , found: 254.2057.

HPLC (OJ-3R, MeOH: Water $=80: 20,1.0 \mathrm{~mL} / \mathrm{min}, 298 \mathrm{~K}, 220 \mathrm{~nm}): t_{\mathrm{R} 1}=15.3 \mathrm{~min}, t_{\mathrm{R} 2}=14.2 \mathrm{~min}$, e.r. $=97: 3$.

$[\boldsymbol{\alpha}]_{\mathbf{D}}^{\mathbf{2 5}}=-40.7\left(c 0.28, \mathrm{CHCl}_{3}\right)$

(S)-tert-butyldimethyl((4-ethylcyclohex-1-en-1-yl)oxy)silane 3y

OTBS The titled product was purified by column chromatography (sole hexanes to ethyl acetate : hexanes =1:20) to afford $\mathbf{3 y}$ as a<smiles>CC[C@H]1CC=C(C)CC1</smiles>
colorless oil.

${ }^{1} \mathrm{H}$ NMR $\left(501 \mathrm{MHz}, \mathrm{CD}_{2} \mathrm{Cl}_{2}\right) \delta 4.81(\mathrm{dt}, J=5.1,2.5 \mathrm{~Hz}, 1 \mathrm{H}), 2.13-2.02(\mathrm{~m}, 2 \mathrm{H}), 1.99-1.91(\mathrm{~m}, 1 \mathrm{H}), 1.80-1.72(\mathrm{~m}, 1 \mathrm{H})$, $1.69-1.60(\mathrm{~m}, 1 \mathrm{H}), 1.41-1.32(\mathrm{~m}, 1 \mathrm{H}), 1.32-1.22(\mathrm{~m}, 3 \mathrm{H}), 0.91(\mathrm{~d}, J=5.1 \mathrm{~Hz}, 12 \mathrm{H}), 0.12(\mathrm{~s}, 6 \mathrm{H})$.

${ }^{13} \mathrm{C}$ NMR $\left(126 \mathrm{MHz}, \mathrm{CD}_{2} \mathrm{Cl} 2\right) \delta 151.3,104.3,36.1,30.8,30.5,29.8,29.4,26.3,18.7,12.3,-3.8,-3.9$.

$\mathrm{R} f=0.62$ (Hexanes).

EI-HRMS $(\mathrm{m} / z)$ : calculated for $\mathrm{C}_{14} \mathrm{H}_{28} \mathrm{O}_{1} \mathrm{Si}_{1}\left[\mathrm{M}^{+\bullet}\right]: 240.4559$, found: 240.4559 .

HPLC (OJ-3R, MeOH: Water = 80:20, $1.0 \mathrm{~mL} / \mathrm{min}, 298 \mathrm{~K}, 220 \mathrm{~nm}): t_{\mathrm{R} 1}=14.3 \mathrm{~min}, t_{\mathrm{R} 2}=13.4 \mathrm{~min}$, e.r. $=98: 2$.

$[\boldsymbol{\alpha}]_{\mathbf{D}}^{25}=-46.7\left(c 0.30, \mathrm{CH}_{3} \mathrm{CN}\right)$

(S)-tert-butyldimethyl((4-methylcyclohex-1-en-1-yl)oxy)silane 3z

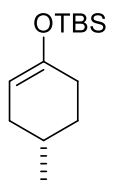

The titled product was purified by column chromatography (sole hexanes to ethyl acetate : hexanes $=1: 20$ ) to afford $\mathbf{3 z}$ as a colorless oil.

${ }^{1} \mathrm{H}$ NMR $\left(501 \mathrm{MHz}, \mathrm{CD}_{2} \mathrm{Cl}_{2}\right) \delta 4.81-4.77(\mathrm{~m}, 1 \mathrm{H}), 2.13-2.01(\mathrm{~m}, 2 \mathrm{H}), 1.98-1.89(\mathrm{~m}, 1 \mathrm{H}), 1.73-1.55(\mathrm{~m}, 3 \mathrm{H}), 1.36-1.23$ $(\mathrm{m}, 1 \mathrm{H}), 0.94(\mathrm{~d}, J=6.4 \mathrm{~Hz}, 3 \mathrm{H}), 0.91(\mathrm{~s}, 9 \mathrm{H}), 0.11(\mathrm{~s}, 6 \mathrm{H})$.

${ }^{13} \mathrm{C}$ NMR $\left(126 \mathrm{MHz}, \mathrm{CD}_{2} \mathrm{Cl}_{2}\right) \delta 151.1,104.2,33.0,32.0,30.4,29.2,26.2,21.8,18.6,-3.8,-3.9$.

$\mathrm{R} f=0.58$ (Hexanes).

EI-HRMS ( $m / z)$ : calculated for $\mathrm{C}_{13} \mathrm{H}_{26} \mathrm{O}_{1} \mathrm{Si}_{1}\left[\mathrm{M}^{+\bullet}\right]$ : 226.1747, found: 226.1743 .

HPLC (OJ-3R, MeOH: Water $=75: 25,1.0 \mathrm{~mL} / \mathrm{min}, 298 \mathrm{~K}, 220 \mathrm{~nm}): t_{\mathrm{R} 1}=23.0 \mathrm{~min}, t_{\mathrm{R} 2}=21.3 \mathrm{~min}$, e.r. $=91: 9$.

$[\boldsymbol{\alpha}]_{\mathbf{D}}^{\mathbf{2 5}}=-10.0\left(c 0.30, \mathrm{CH}_{3} \mathrm{CN}\right)$.

\section{(S)-ethyl-4-((tert-butyldimethylsilyl)oxy)cyclohex-3-ene-1-carboxylate 3t}

OTBS The titled product was purified by column chromatography (sole hexanes to ethyl acetate : hexanes = $1: 20$ ) to afford ent-3t as a colorless oil.

HPLC (AD-3R, Acetonitrile: Water = 50:50, $1.0 \mathrm{~mL} / \mathrm{min}, 298 \mathrm{~K}, 220 \mathrm{~nm}): t_{\mathrm{R} 1}=39.3 \mathrm{~min}, t_{\mathrm{R} 2}=31.8 \mathrm{~min}$, e.r. $=99: 1$.

$\overline{\mathrm{C}}_{2} \mathrm{Et}$ 


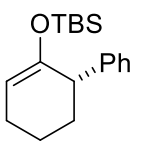

The titled product was purified by column chromatography (sole hexanes to ethyl acetate : hexanes $=1: 20$ ) to afford 3aa as a colorless oil.

${ }^{1} \mathrm{H}$ NMR (501 MHz, $\left.\mathrm{CD}_{2} \mathrm{Cl}_{2}\right) \delta$ 7.28-7.23 (m, 2H), 7.22-7.18 (m, 2H), 7.18-7.13 (m, 1H), $5.04(\mathrm{td}, J=4.0,1.2 \mathrm{~Hz}, 1 \mathrm{H})$, $3.35(\mathrm{ddd}, J=6.4,4.5,2.1 \mathrm{~Hz}, 1 \mathrm{H}), 2.21-2.05(\mathrm{~m}, 2 \mathrm{H}), 2.05-1.97(\mathrm{~m}, 1 \mathrm{H}), 1.71-1.63(\mathrm{~m}, 1 \mathrm{H}), 1.62-1.55(\mathrm{~m}, 1 \mathrm{H}), 1.51-1.43(\mathrm{~m}, 1 \mathrm{H})$, $0.67(\mathrm{~s}, 9 \mathrm{H}), 0.07(\mathrm{~s}, 3 \mathrm{H}), 0.00(\mathrm{~s}, 3 \mathrm{H})$.

${ }^{13} \mathrm{C}$ NMR $\left(126 \mathrm{MHz}, \mathrm{CD}_{2} \mathrm{Cl}_{2}\right) \delta$ 150.9, 144.6, 128.3, 127.8, 125.7, 105.3, 46.2, 33.2, 25.1, 24.1, 19.7, 17.6, -4.8, -5.1.

$\mathrm{R} f=0.41$ (Hexanes).

ESI-HRMS $(m / z)$ : calculated for $\mathrm{C}_{18} \mathrm{H}_{29} \mathrm{O}_{1} \mathrm{Si}_{1}\left([\mathrm{M}+\mathrm{H}]^{+}\right)$: 289.1982, found: 289.1977 .

HPLC (OD-3R, Acetonitrile: Water $=50: 50,1.0 \mathrm{~mL} / \mathrm{min}, 298 \mathrm{~K}, 220 \mathrm{~nm}): t_{\mathrm{R} 1}=78.7 \mathrm{~min}, t_{\mathrm{R} 2}=72.8 \mathrm{~min}$, e.r. $=99: 1$.

$[\boldsymbol{\alpha}]_{\mathbf{D}}^{25}=41.3\left(c=0.16, \mathrm{CH}_{3} \mathrm{CN}\right)$.

(R)-2-phenylcyclohexan-1-one 1aa<smiles>O=C1CCCCC1Pc1ccccc1</smiles>

HPLC (OD-3, $n$-heptane $/ i-\operatorname{PrOH}=98: 2,1.0 \mathrm{~mL} / \mathrm{min}, 298 \mathrm{~K}, 220 \mathrm{~nm}): t_{\mathrm{R} 1}=8.1 \mathrm{~min}, t_{\mathrm{R} 2}=7.3 \mathrm{~min}$, e.r. $=17: 83$.

\section{(S)-([1,1'-bi(cyclohexan)]-2-en-2-yloxy)(tert-butyl)dimethylsilane 3ab}

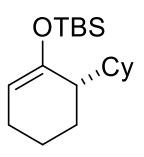

The titled product was purified by column chromatography (sole hexanes to ethyl acetate : hexanes $=1: 20$ ) to afford 3ab as a colorless oil.

${ }^{1} \mathrm{H}$ NMR $\left(501 \mathrm{MHz}, \mathrm{CD}_{2} \mathrm{Cl}_{2}\right) \delta 4.70(\mathrm{ddd}, J=4.9,3.4,1.6 \mathrm{~Hz}, 1 \mathrm{H}), 1.94-1.86(\mathrm{~m}, 1 \mathrm{H}), 1.82(\mathrm{dtd}, J=7.5,3.5,1.9 \mathrm{~Hz}$, $2 \mathrm{H}), 1.70(\mathrm{~d}, J=3.7 \mathrm{~Hz}, 1 \mathrm{H}), 1.64-1.54(\mathrm{~m}, 2 \mathrm{H}), 1.53-1.46(\mathrm{~m}, 3 \mathrm{H}), 1.37-1.22(\mathrm{~m}, 4 \mathrm{H}), 1.16-0.96(\mathrm{~m}, 4 \mathrm{H}), 0.87(\mathrm{dd}, J=12.2,3.5$ $\mathrm{Hz}, 1 \mathrm{H}), 0.79(\mathrm{~s}, 9 \mathrm{H}), 0.00(\mathrm{~s}, 3 \mathrm{H}), 0.00(\mathrm{~s}, 3 \mathrm{H})$.

${ }^{13} \mathrm{C}$ NMR (126 MHz, $\left.\mathrm{CD}_{2} \mathrm{Cl}_{2}\right) \delta$ 152.3, 104.2, 44.2, 38.5, 31.2, 27.6, 27.3, 26.9, 26.8, 25.5, 24.3, 24.1, 21.9, 17.9, -4.7, -5.0.

$\mathrm{R} f=0.71$ (Hexanes).

ESI-HRMS $(\mathrm{m} / \mathrm{z})$ : calculated for $\mathrm{C}_{18} \mathrm{H}_{35} \mathrm{O}_{1} \mathrm{Si}_{1}\left([\mathrm{M}+\mathrm{H}]^{+}\right)$: 295.2451, found: 295.2452 .

HPLC (OJ-3R, MeOH: Water = 80:20,1.0 mL/min, $298 \mathrm{~K}, 220 \mathrm{~nm}): t_{\mathrm{R} 1}=15.2 \mathrm{~min}, t_{\mathrm{R} 2}=13.7 \mathrm{~min}$, e.r. $=$ 99.9:0.1.

$[\boldsymbol{\alpha}]_{\mathbf{D}}^{25}=-30.5\left(c 0.28, \mathrm{CH}_{3} \mathrm{CN}\right)$.

$(R)-[1,1 '-b i(c y c l o h e x a n)]-2-o n e 1 a b$<smiles>O=C1CCCCC1C1CCCCC1</smiles>

$\mathrm{GC}\left(30.0 \mathrm{~m}\right.$ BGB 176 , injection temperature: $220^{\circ} \mathrm{C}, 120^{\circ} \mathrm{C}, 0.5$ bar $\left.\mathrm{H}_{2}\right): t_{\mathrm{R} 1}=54.6 \mathrm{~min}, t_{\mathrm{R} 2}=52.6 \mathrm{~min}$, e.r. $=15: 85$.

\section{(S)-tert-butyldimethyl((4-methylcyclohex-1-en-1-yl)oxy)silane 3ac}

\footnotetext{
OTBS The titled product was purified by column chromatography (sole hexanes to ethyl acetate : hexanes $=1: 20$ ) to afford 3ac as a light yellow liquid. For this compound, $s=\ln [(1-C)(1-e e)] / \ln [(1-C)(1+e e)]\left(C=e e_{3 \mathrm{ac}} / e e_{3 \mathrm{ac}}+e e_{1 \mathrm{ac}}\right)$.

"Ph ${ }^{1} \mathrm{H}$ NMR (501 MHz, $\left.\mathrm{CD}_{2} \mathrm{Cl}_{2}\right) \delta 7.12-7.05(\mathrm{~m}, 4 \mathrm{H}), 7.01-6.96(\mathrm{~m}, 1 \mathrm{H}), 4.49(\mathrm{p}, J=2.0 \mathrm{~Hz}, 1 \mathrm{H}), 3.28(\mathrm{tt}, J=9.1,6.9 \mathrm{~Hz}$, $1 \mathrm{H}), 2.59-2.45(\mathrm{~m}, 2 \mathrm{H}), 2.29-2.12(\mathrm{~m}, 2 \mathrm{H}), 0.76(\mathrm{~s}, 9 \mathrm{H}), 0.00(\mathrm{~s}, 3 \mathrm{H}), 0.00(\mathrm{~s}, 3 \mathrm{H})$.
}

${ }^{13} \mathrm{C}$ NMR $\left(126 \mathrm{MHz}, \mathrm{CD}_{2} \mathrm{Cl}_{2}\right) \delta 153.6,147.3,128.2,126.7,125.7,101.4,41.8,41.4,37.5,25.4,17.9,-4.8,-4.9$. 
$\mathrm{R} f=0.35$ (Hexanes).

ESI-HRMS (m/z): calculated for $\mathrm{C}_{17} \mathrm{H}_{27} \mathrm{O}_{1} \mathrm{Si}_{1}\left([\mathrm{M}+\mathrm{H}]^{+}\right)$: 275.1826, found: 275.1826.

HPLC (OJ-3R, Acetonitrile: Water $=80: 20,1.0 \mathrm{~mL} / \mathrm{min}, 298 \mathrm{~K}, 220 \mathrm{~nm}): t_{\mathrm{R} 1}=6.0 \mathrm{~min}, t_{\mathrm{R} 2}=6.3 \mathrm{~min}$, e.r. $=95: 5$.

$[\boldsymbol{\alpha}]_{\mathbf{D}}^{25}=-6.7\left(c 0.30, \mathrm{CH}_{3} \mathrm{CN}\right)$.

(S)-3-phenylcyclopentan-1-one 1ac

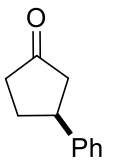

HPLC (AS-3, $n$-heptane $/ i-\mathrm{PrOH}=99: 1,1.0 \mathrm{~mL} / \mathrm{min}, 298 \mathrm{~K}, 220 \mathrm{~nm}$ ): $t_{\mathrm{R} 1}=21.2 \mathrm{~min}, t_{\mathrm{R} 2}=18.6 \mathrm{~min}$, e.r. $=15: 85$.

\section{tert-butyldimethylsilyl [1,1'-biphenyl]-2-carboxylate}

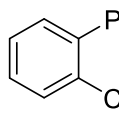

The titled product was purified by prep. TLC with hexanes as eluent:

$0.59(\mathrm{~s}, 9 \mathrm{H}), 0.00(\mathrm{~s}, 6 \mathrm{H})$.

${ }^{13} \mathrm{C}$ NMR $\left(126 \mathrm{MHz}, \mathrm{CD}_{2} \mathrm{Cl}_{2}\right) \delta 168.6,142.2,141.7,132.6,130.9,130.8,130.0,128.5,128.1,127.2,127.0,25.2,17.4,-5.3$.

$\mathrm{R} f=0.51($ Ethyl acetate/hexanes $=1: 20)$.

ESI-HRMS ( $\mathrm{m} / \mathrm{z})$ : calculated for $\left([\mathrm{M}+\mathrm{Na}]^{+}\right)$: 335.1438 , found: 335.1436.

\section{Limitations of the method:}

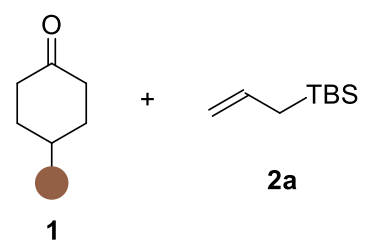

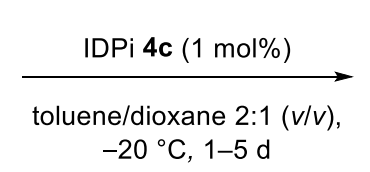

toluene/dioxane $2: 1(\mathrm{v} / \mathrm{v})$
$-20^{\circ} \mathrm{C}, 1-5 \mathrm{~d}$

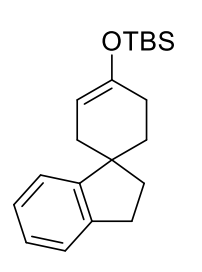

$67: 33$ e.r.
$75 \%$

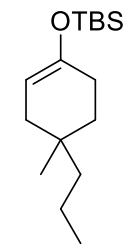

$99 \%$
$66: 34$ e.r.

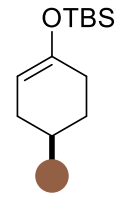

$(R)-3$

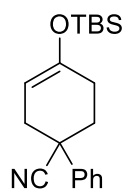

$10 \%$ $58: 42$ e.r.

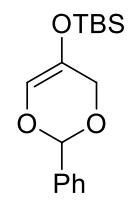

N.R.

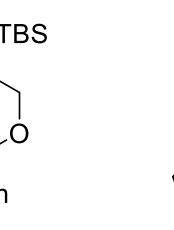

N.R.<smiles></smiles>

$99 \%$ $76: 24$ e.r.

\section{Mechanistic study}

Procedure of reactivity comparison between $\mathrm{Tf}_{2} \mathrm{NH}$ (Bistriflimide) and confined IDPi catalysts $4 \mathbf{c}$ : 


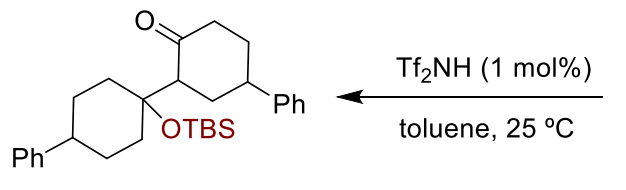

11

homo-aldol adduct

$12 \mathrm{~h},>99 \%$
$\prod_{\mathrm{Ph}}^{\mathrm{O}}$

1a
$+\gamma^{\mathrm{TBS}}$

$2 a$

$\mathrm{Tf}_{2} \mathrm{NH}(1 \mathrm{~mol} \%)$

$\mathrm{Et}_{3} \mathrm{~N}(1 \mathrm{~mol} \%)$

toluene, $25^{\circ} \mathrm{C}$

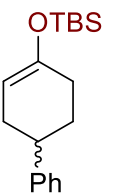

rac-3a

$1.5 \mathrm{~h}, 65 \%$

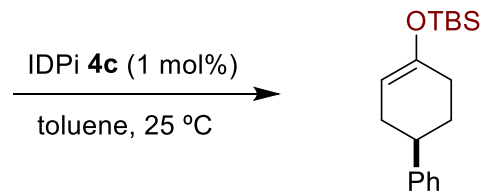

3a

silyl enol ether

12 h, $99 \%$, 88:12 er

Reaction with IDPi: Allyl(tert-butyl)dimethylsilane 2a ( $80 \mu \mathrm{L}, 0.4 \mathrm{mmol}, 2.0$ equiv.) was placed in a flame-dried Schlenk flask, equipped with a teflon-coated magnetic stirring bar. IDPi $4 \mathbf{c}\left(0.01\right.$ equiv.) and toluene $(0.08 \mathrm{M}, 2.4 \mathrm{~mL})$ were added at $25^{\circ} \mathrm{C}$ and stirred for $5 \mathrm{~min}$, ketone 1a was added afterwards, then the resultant solution was stirred at this temperature for $12 \mathrm{~h}$. After the ketone was fully consumed which was monitored by TLC, the reaction was quenched by one drop of triethylamine through pipet. Organic volatiles were evaporated in vacuo and the crude residue was purified by silica column chromatography to afford the desired enol silane 3a in quantitative yield with 88:12 e.r.

Reaction with $\mathrm{Tf}_{2} \mathrm{NH}$ : Allyl(tert-butyl)dimethylsilane $\mathbf{2 a}(80 \mu \mathrm{L}, 0.4 \mathrm{mmol}, 2.0$ equiv.) and ketone 1a was placed in a flame-dried Schlenk flask, equipped with a teflon-coated magnetic stirring bar. $\mathrm{Tf}_{2} \mathrm{NH}(0.01$ equiv. $)$ and toluene $(0.08 \mathrm{M}, 2.4 \mathrm{~mL})$ were added at $25{ }^{\circ} \mathrm{C}$ and stirred for $12 \mathrm{~h}$. After the ketone was fully consumed which was monitored by TLC, the reaction was quenched by one drop of triethylamine through pipet. Then the components of the crude reaction solution were verified by GC-MS and HRMS, which was a mixture of homo-aldol adducts 11 in quantitative yield without formation of the enol silane 3a. [see the GC-MS (ESI) spectrum below] (for the reaction at $-20^{\circ} \mathrm{C}$, after $24 \mathrm{~h}$, the total conversion of $\mathbf{1 a}$ is $\sim 40 \%$, only $\sim 5 \% \mathbf{3 a}$ was observed, homo-aldol adducts were still the major products, which indicates the enol silane can be the intermediate for aldol reaction)

Notes: small amount of Hosomi-Sakurai product may also formed as inseparable mixture with homo-aldol products.

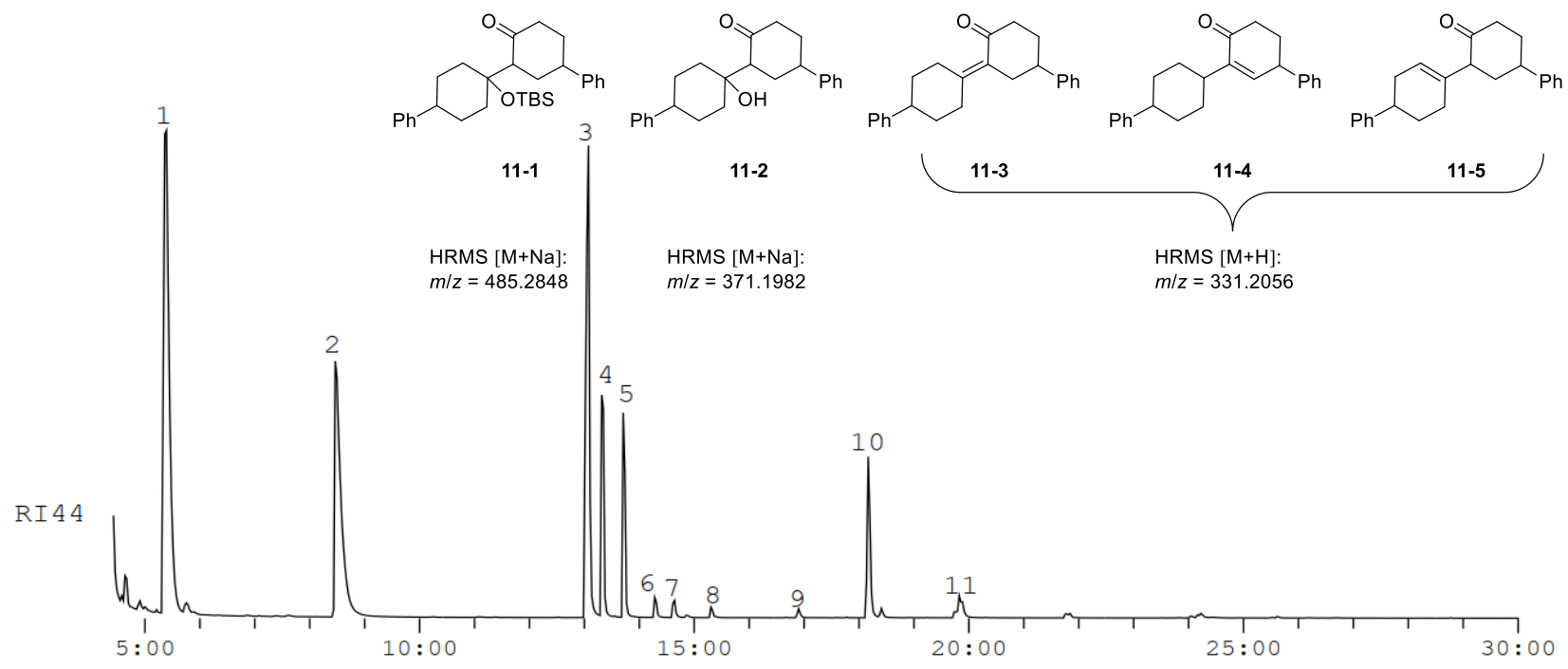


Figure S1. ESI-MS spectrum of the reaction mixture catalyzed by $\mathrm{Tf}_{2} \mathrm{NH}$. 11-5 was isolatable and the structure was confirmed by NMR analysis.

\section{4-(2-oxo-5-phenylcyclohexyl)-1,2,3,6-tetrahydro-1,1'-biphenyl 11-5}<smiles>O=C1CCC(c2ccccc2)CC1C1=CCC(c2ccccc2)CC1</smiles>

The titled product was purified by column chromatography with ethyl acetate/hexanes $=1: 4$ as eluent to afford 11-5 as a white solid.

${ }^{1} \mathrm{H}$ NMR $\left(501 \mathrm{MHz}, \mathrm{CDCl}_{3}\right) \delta 7.27-7.14(\mathrm{~m}, 10 \mathrm{H}), 5.53-5.43(\mathrm{~m}, 1 \mathrm{H}), 3.11(\mathrm{tdd}, J=12.2,8.8,3.4 \mathrm{~Hz}, 2 \mathrm{H})$, 2.72 (dddt, $J=25.2,12.3,5.2,2.8 \mathrm{~Hz}, 1 \mathrm{H}), 2.53-2.40(\mathrm{~m}, 2 \mathrm{H}), 2.17(\mathrm{ddtd}, J=18.3,11.1,5.5,2.7 \mathrm{~Hz}, 4 \mathrm{H})$, $2.07-1.84(\mathrm{~m}, 5 \mathrm{H}), 1.84-1.63(\mathrm{~m}, 1 \mathrm{H})$.

${ }^{13} \mathrm{C}$ NMR $\left(126 \mathrm{MHz}, \mathrm{CDCl}_{3}\right) \delta 210.4,147.2,147.1,144.7,135.6,135.4,128.6,128.4,128.4,128.3,126.9,126.8,126.7,126.7,126.6$, 126.0, 126.0, 124.1, 123.9, 58.3, 57.8, 43.5, 43.4, 42.0, 41.9, 40.2, 40.0, 39.9, 39.6, 34.5, 34.4, 33.7, 33.4, 30.0, 29.8, 28.1, 27.5.

$\mathrm{R} f=0.31($ Ethyl acetate/hexanes $=1: 4)$.

ESI-HRMS $(\mathrm{m} / \mathrm{z})$ : calculated for $\mathrm{C}_{24} \mathrm{H}_{27} \mathrm{O}_{1}\left([\mathrm{M}+\mathrm{H}]^{+}\right): 331.2056$, found: 331.2056.

Reaction with $\mathrm{Tf}_{2} \mathrm{NH}$ and $\mathrm{Et}_{3} \mathrm{~N}$ : Allyl(tert-butyl)dimethylsilane 2a (60 $\mu \mathrm{L}, 0.3 \mathrm{mmol}, 1.5$ equiv.) and ketone 1a (35 mg, $0.2 \mathrm{mmol}, 1.0$ equiv.) was placed in a flame-dried Schlenk flask, equipped with a teflon-coated magnetic stirring bar. $\mathrm{Tf}_{2} \mathrm{NH}(0.01$ equiv. $), \mathrm{Et}_{3} \mathrm{~N}(0.01$ equiv. $)$, and toluene $(0.1 \mathrm{M}, 2.0 \mathrm{~mL})$ were added stepwise at $25^{\circ} \mathrm{C}$ and stirred for $1.5 \mathrm{~h}$, then the reaction was quenched by triethylamine and the solvent was removed by vacuum. The yield of the desired racemic enol silane $\mathbf{3 a}$ was determined to be $65 \%$ with nitrobenzene as internal standard via the ${ }^{1} \mathrm{H}$ NMR analysis attached below (Figure S2).

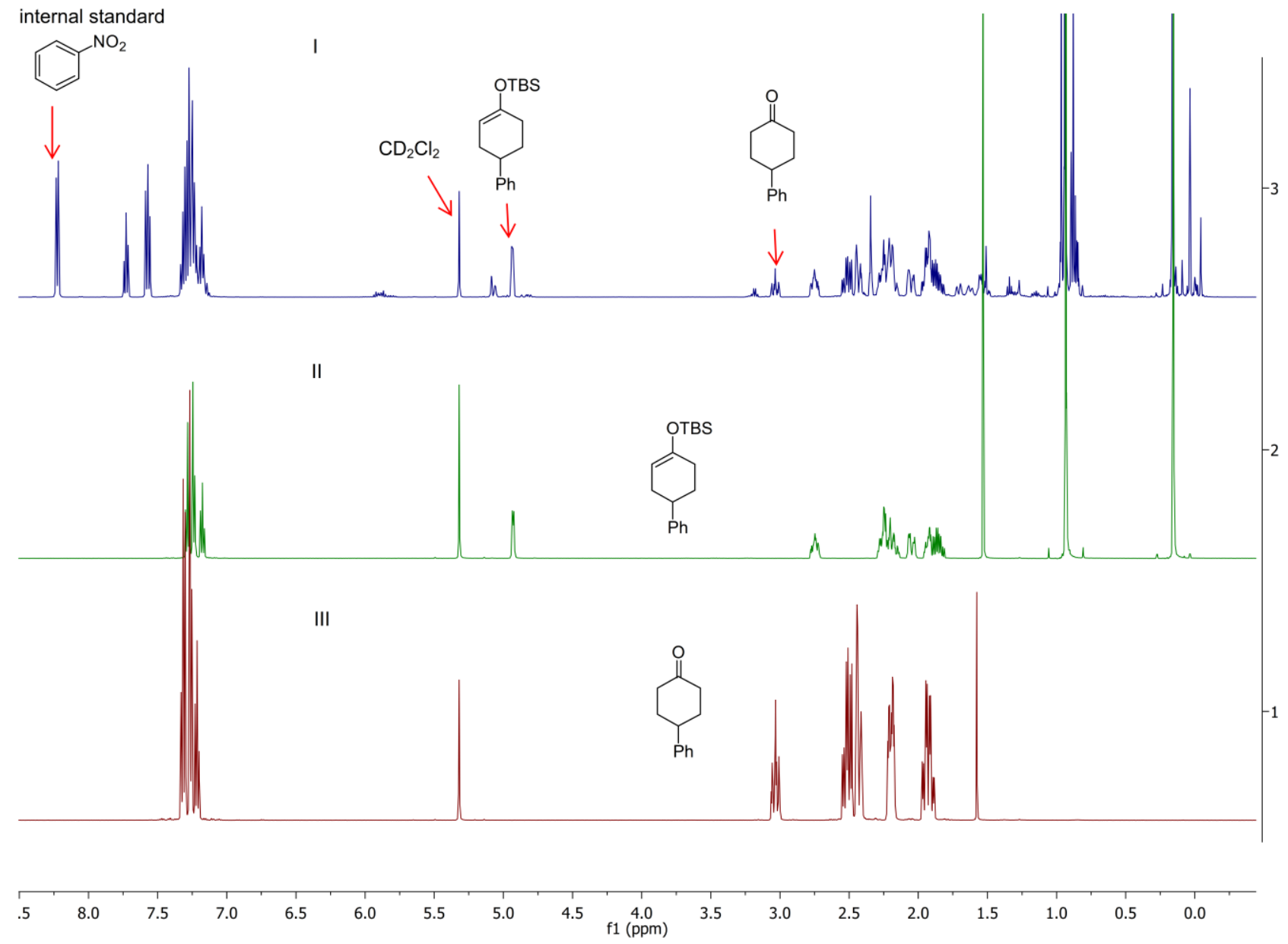


Figure S2. ${ }^{1} \mathrm{H}$ NMR studies of the reaction catalyzed by $\mathrm{Tf}_{2} \mathrm{NH}$ and $\mathrm{Et}_{3} \mathrm{~N}$. (I). Crude ${ }^{1} \mathrm{H}$ NMR analysis of the reaction with nitrobenzene as internal standard in $\mathrm{CD}_{2} \mathrm{Cl}_{2}$. (II); ${ }^{1} \mathrm{H} \mathrm{NMR}$ of the desired product 3a in $\mathrm{CD}_{2} \mathrm{Cl}_{2}$; (III). ${ }^{1} \mathrm{H}$ NMR of the starting material $\mathbf{1 a}$ in $\mathrm{CD}_{2} \mathrm{Cl}_{2}$.

Notes: with pre-mixed $\mathrm{Tf}_{2} \mathrm{NH}$ and $\mathrm{Et}{ }_{3} \mathrm{~N}$, no reaction was detected. Separate addition of the acid and amine is indispensable for the promotion of enol silylation.

\section{Kinetic studies}

When studying the reaction in a toluene- $d_{8} /$ dioxane- $d_{8}=2: 1$ mixture, a significant dormant period was observed (Figure S3).

Procedure: allyl(tert-butyl)dimethylsilane $2 \mathbf{a}(20 \mu \mathrm{L}, 0.1 \mathrm{mmol}, 2.0$ equiv.) was placed in a flame-dried Schlenk flask, equipped with a teflon-coated magnetic stirring bar. IDPi $4 \mathbf{c}\left(0.01\right.$ equiv.) and toluene- $\mathrm{d}_{8} /$ dioxane- $\mathrm{d}_{8}=2: 1(0.08 \mathrm{M}, 0.6 \mathrm{~mL})$ were added at $25{ }^{\circ} \mathrm{C}$ and stirred for $5 \mathrm{~min}$. The reaction mixture was cooled to $-20{ }^{\circ} \mathrm{C}$ for $10 \mathrm{~min}$, ketone $1 \mathrm{a}(8.7 \mathrm{mg}, 0.05 \mathrm{mmol}, 1.0$ equiv. $)$ was added afterwards, then the resultant solution was transferred to a $5 \mathrm{~mm}$ NMR tube and reaction was performed at this temperature for $3 \mathrm{~d}$.
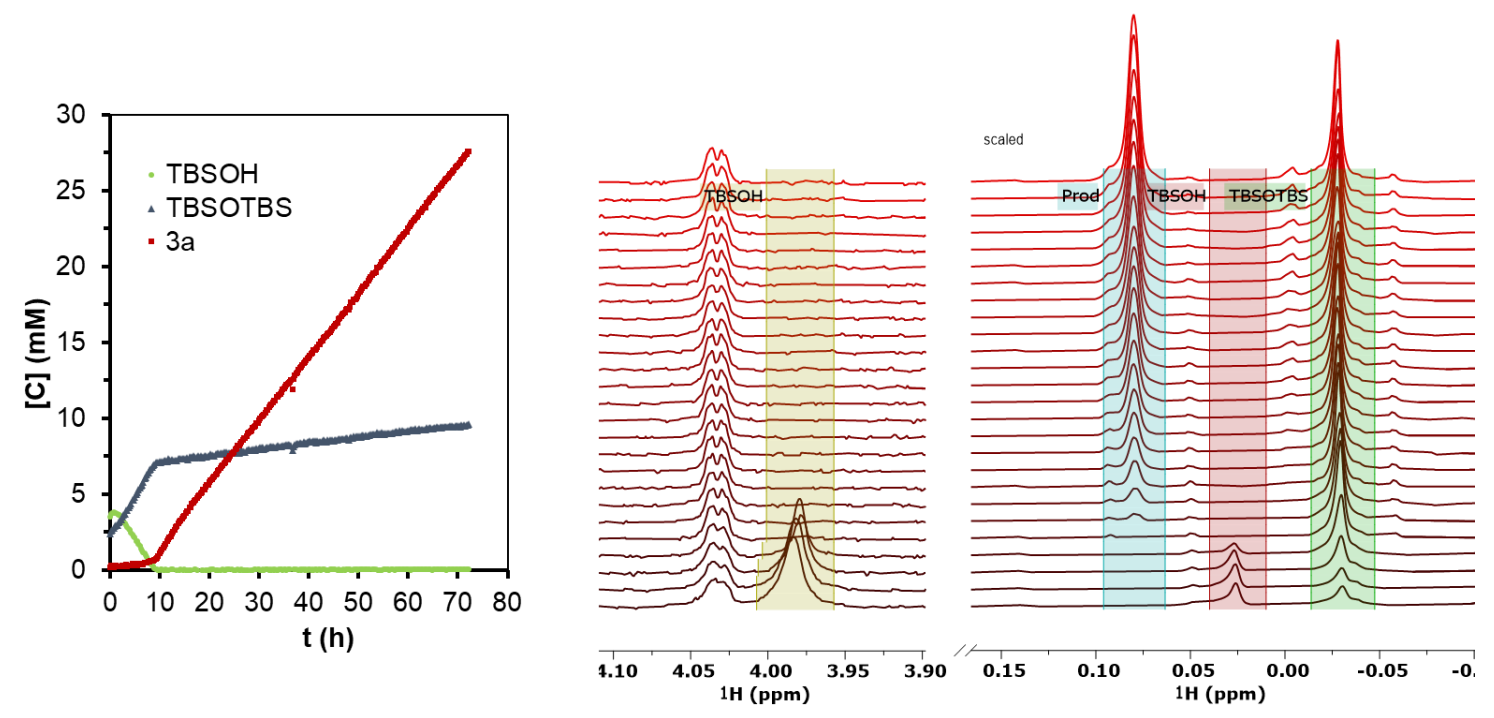

Figure S3. left: Conversion plot showing the initial formation of TBSOH and TBSOTBS; right: ${ }^{1} \mathrm{H}$ NMR spectra showing regions of interest taken at different time points during the reaction.

Conclusion: during the first $10 \mathrm{~h}$ of NMR monitoring of the reaction, no product formation was observed. Instead, only the conversion of TBSOH accompanied by the formation of TBSOTBS. Till all the TBSOH was consumed fully, the formation of the desired product starts to be observed. In sharp contrast, the reaction in toluene- $\mathrm{d}_{8}$ had a $\sim 0.5 \mathrm{~h}$ dormant period, which was detected via NMR studies.

Determination of reaction order of IDPi catalyst $\mathbf{4 c}$.

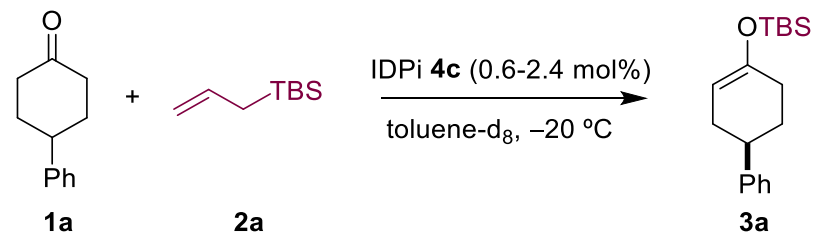

The catalyst order was determined by time normalization analysis reported by the Bures group. ${ }^{24-29}$ When comparing conversion plots normalized to different catalyst orders (Figure S4), the curves were found to overlap nicely when a first order with respect to the catalyst 
was used, implying no significant influence from off-cycle equilibria or synergistic effects. The model reaction was conducted with 0.6 , 1.2 and $2.4 \mathrm{~mol} \%$ of catalyst $\mathbf{4 c}$ under standard reaction conditions.
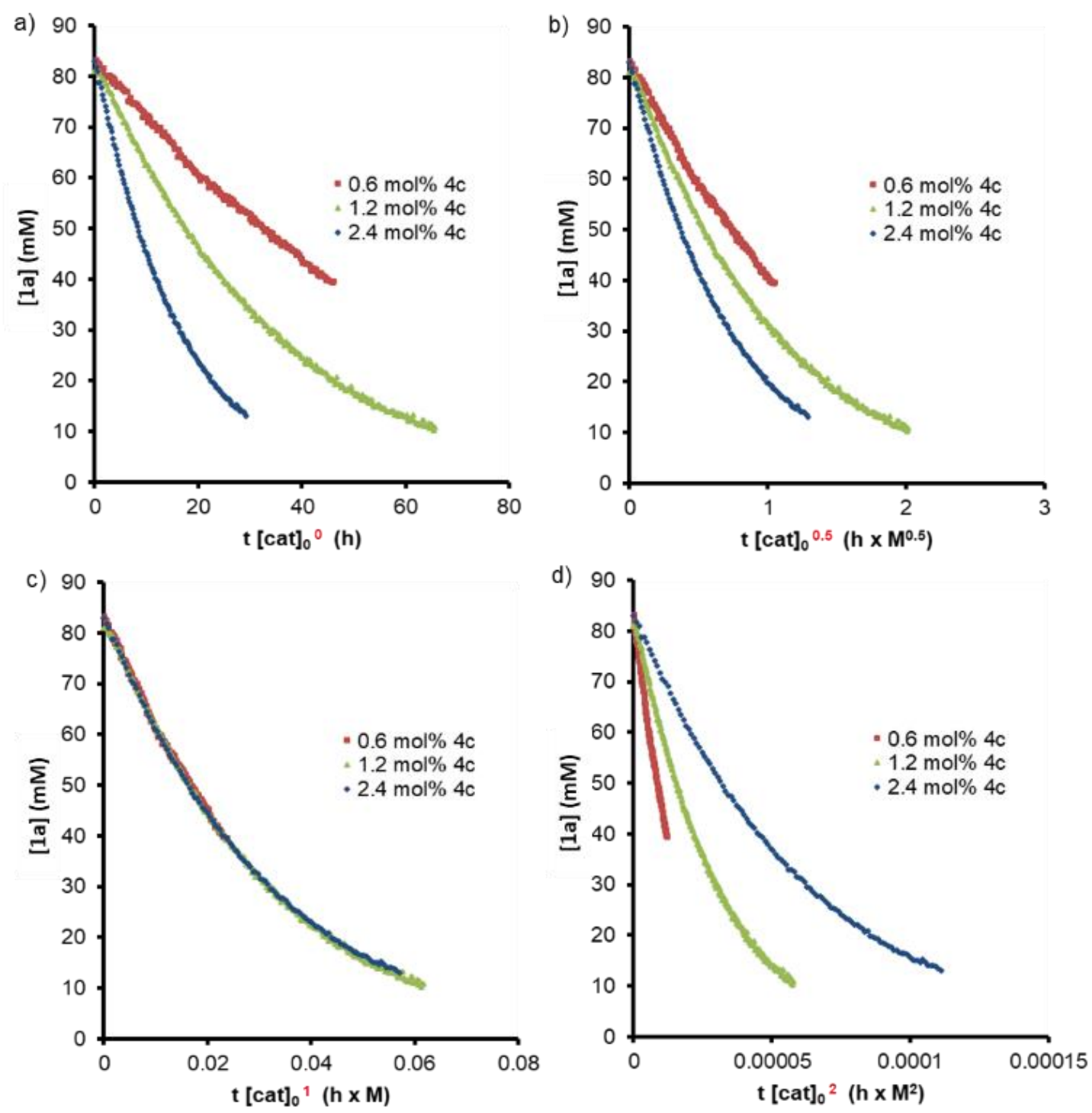

Figure S4. Conversion plots obtained from NMR measurements with time scales normalized to different catalyst orders: a) zero-order reaction; b) half-order reaction; c) first-order reaction and d) second-order reaction.

\section{Determination of reaction order of ketone $1 \mathbf{a}$ and allylsilane $\mathbf{2 a}$}

The order of the ketone 1a was also determined by variable time normalisation analysis (VTNA). Reaction plots normalized to different reaction order of ketone can be found in Figure S5. By comparing the outcome of different orders, the reactions were found to in accordance with the zeroth order in ketone 1a (Figure S5).

Note: we have observed this zeroth order dependence of aldehyde in the cyanosilylation of aldehyde catalyzed by disulfonimide. ${ }^{30}$ 

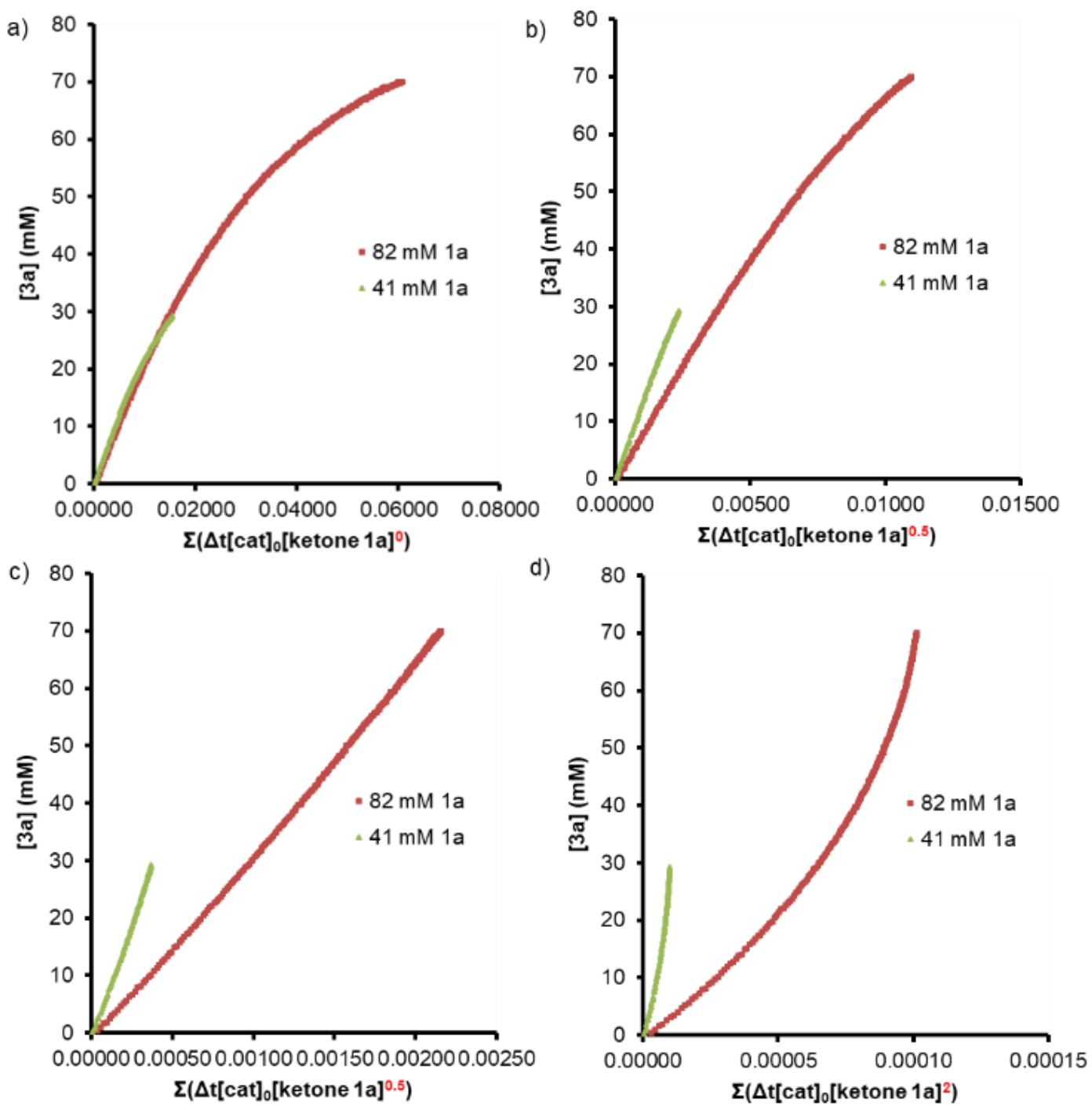

Figure S5. Conversion plots obtained from NMR measurements with time scales normalized to the catalyst concentration and different orders of ketone 1a: a) zero-order reaction; b) half-order reaction; c) first-order reaction and d) second-order reaction.

The reaction order with respect to the silane source was also determined by VTNA. Concentration plots obtained from NMR normalized to different orders of silane 2a can be found in Figure S6. 

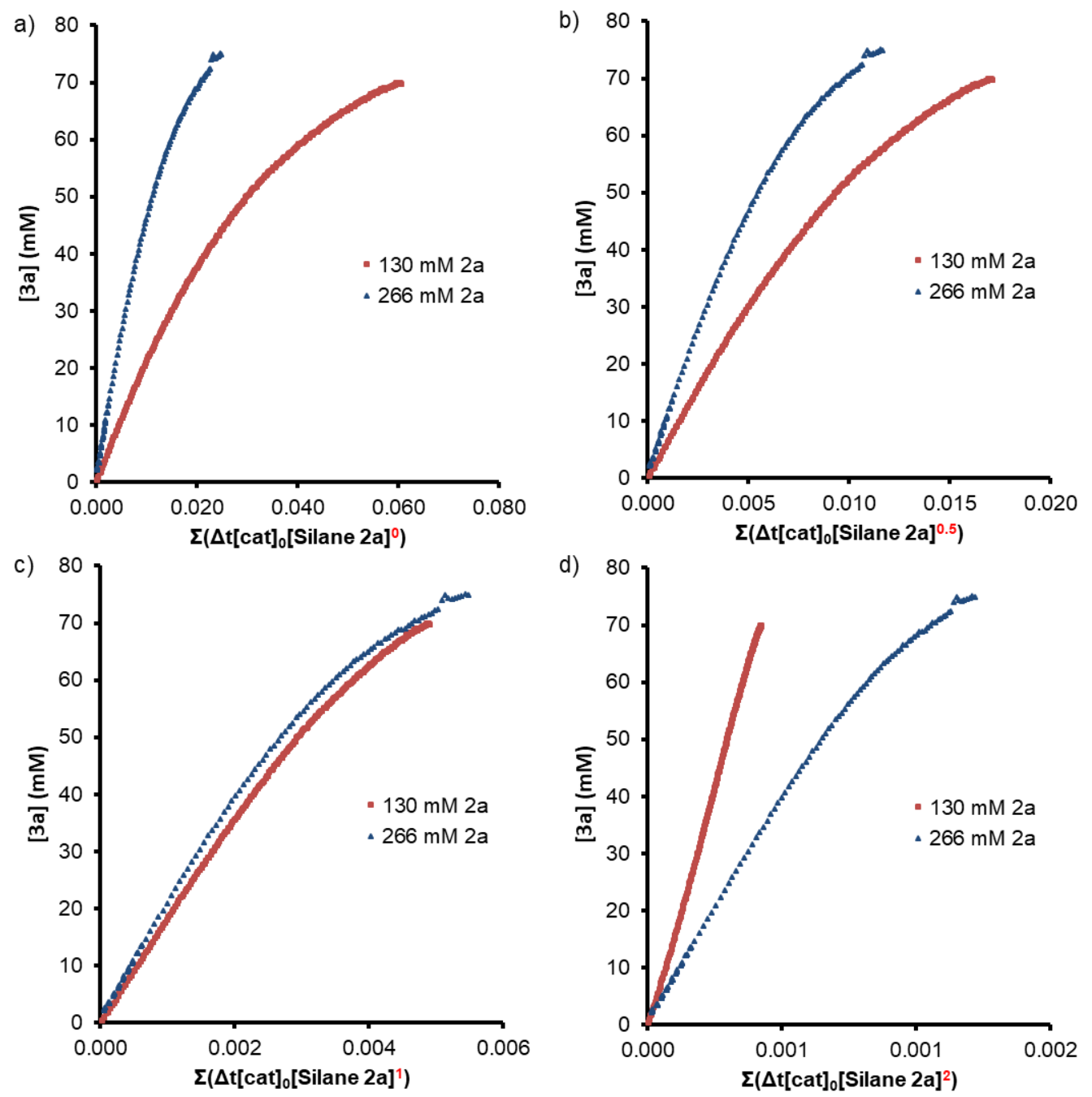

Figure S6. Product concentrations obtained from NMR measurements with time scales normalized to the catalyst concentration and different orders of ketone 1aa: a) zero-order reaction; b) half-order reaction; c) first-order reaction and d) second-order reaction.

The best overlap was found, when a first order dependence in silane concentration was assumed (Figure S6c).

Taking all the measurements into account, this data supports the hypothesis that the silylation of the catalyst is the overall rate determining step of the reaction.

\section{Study of racemization process of unsymmetric ketones}<smiles>[R]C1CCC=C([SeH2])C1</smiles>

$( \pm)-3$<smiles>O=C(O)c1ccccc1-c1ccccc1</smiles>

10 (0.5 equiv.)

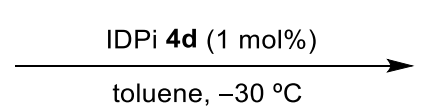

toluene, $-30^{\circ} \mathrm{C}$

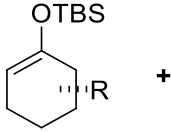<smiles>O=C1CCCCC1</smiles>

1

partially racemize

During the substrate screening of the catalytic asymmetric protodesilylative kinetic resolution of racemic enol silanes with 2-biphenyl carboxylic acid, we found that under the reaction conditions the chiral ketone byproducts partially racemize. This may due to the tautomerization between ketone and enol, and also caused by the kinetic equilibrium among enol silane regiomers and the relevant ketone. 
In order to verify the tautomerization, reactions of both enantiomers of 2-phenylcyclohexanone with IDPi $\mathbf{4 d}$ were conducted, and the $e e$ was checked by HPLC analysis.
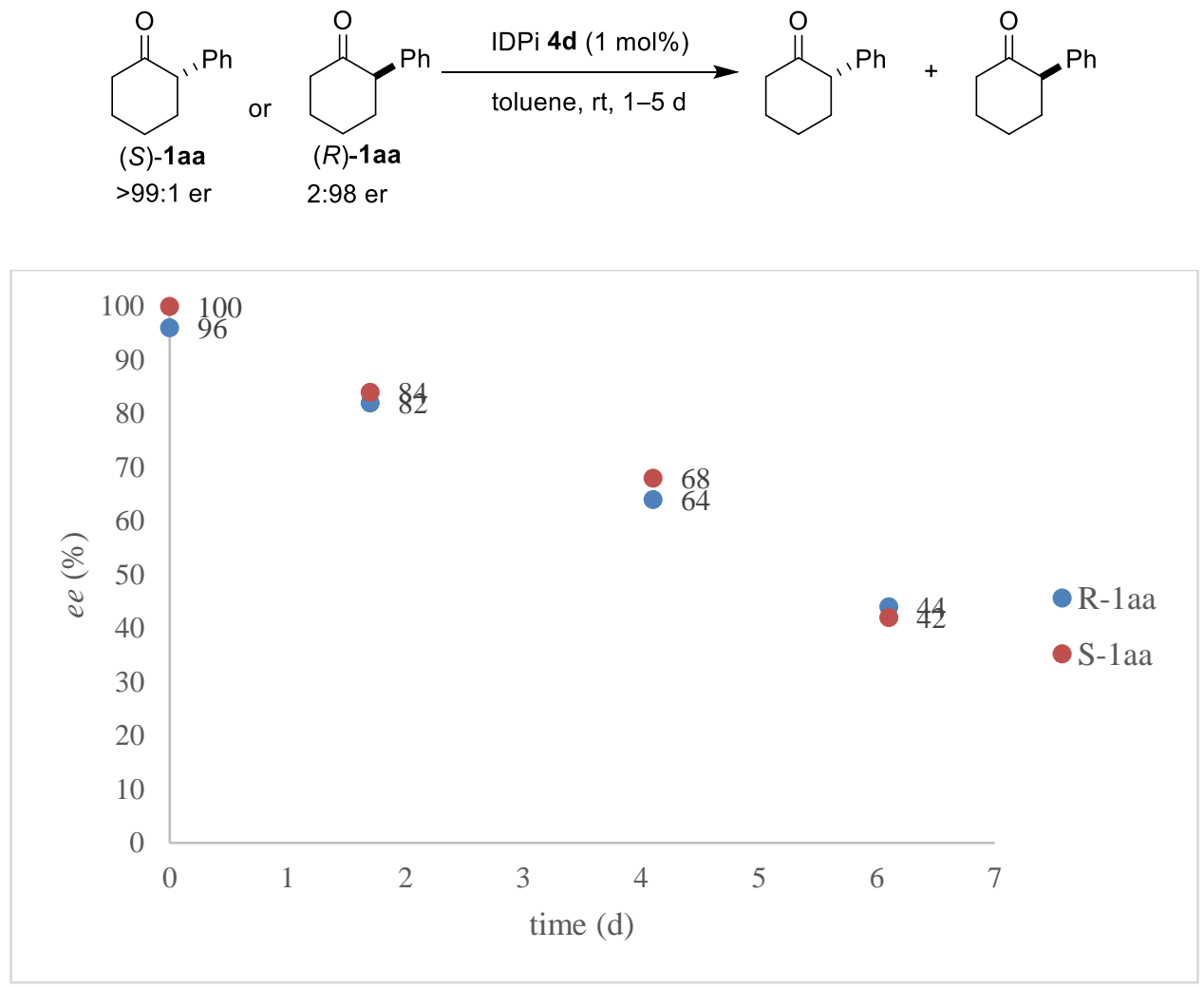

Figure S7. Study of keto-enol tautomerization

With the data in hand, we confirmed one reason is tautomerization, which causes ketone racemization. However, under lower temperature, this tautomerization effect could be slower.

Since the rate of tautomerization is quite slow, there may be another reason. In order to verify our hypothesis, the kinetic resolution between racemic enolsilane 3aa and biphenyl carboxylic acid $\mathbf{1 0}$ under standard condition were performed in toluene- $\mathrm{d}_{8}$, and the reaction was monitored by reaction progress kinetic analysis (Figure S8).

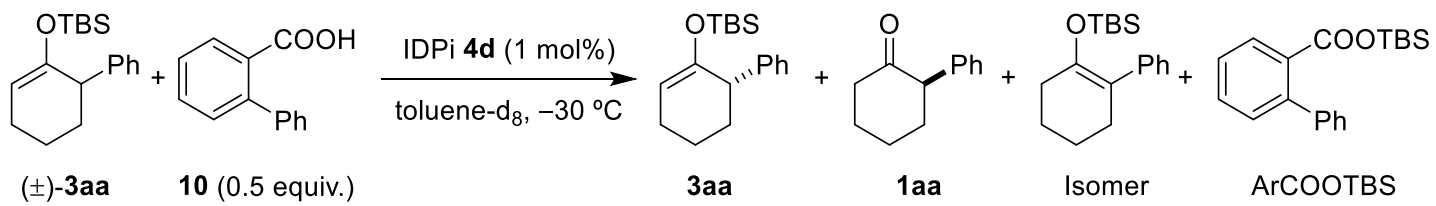




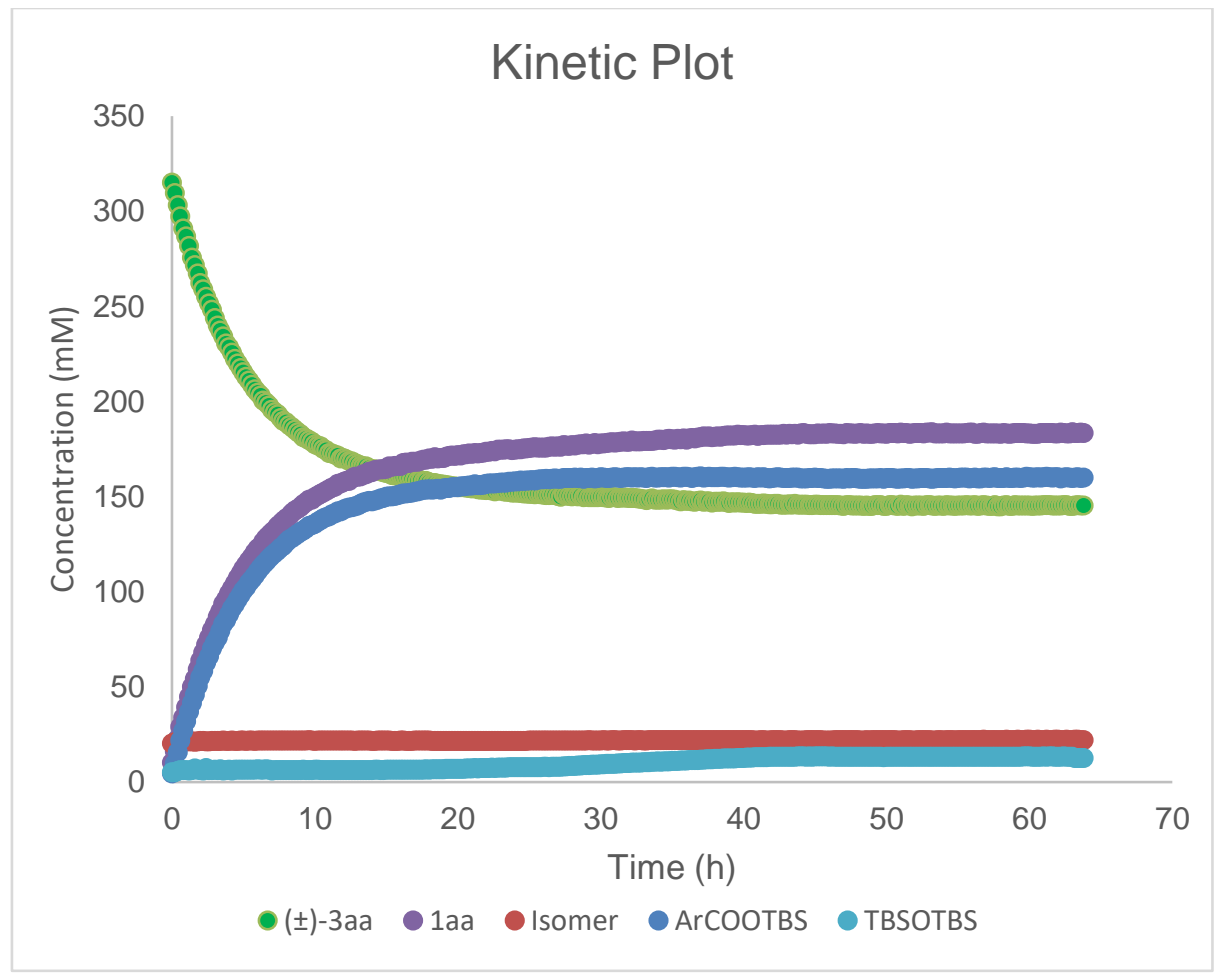

Figure S8. Reaction progress kinetic analysis of the kinetic resolution of racemic enol silane 3aa and acid 10

From the kinetic plot, the racemic enolsilane 3aa keep decreasing, accompanied by increasing of the ketone 1aa. It is quite clear that there is equilibrium between 3aa and 1aa in the reaction system, which possibly cause the racemization of 1aa. The starting racemic enolsilane 3aa was a mixture of kinetic product and thermodynamic product (isomer) (95:5), and the ratio of isomer remains the same throughout the entire reaction process. To 2-substituted enol silane, there presumed to be no equilibrium between the kinetic product and the thermodynamic product under acidic atmosphere. ArCOOTBS keeps increasing till the conversion was $\sim 50 \%$, after that, TBSOTBS starts to be observed from the hydrolysis of 3aa with less reactive adventitious water. Finally, the reaction end up with $\sim 55 \%$ conversion.

\section{${ }^{31} P$ NMR analysis of the reaction solution:}

The hypothesis that the silylation of the catalyst is the rate-determining step can be further confirmed by ${ }^{31} \mathrm{P}$ NMR spectra taken at different time points (Figure S9). At the beginning of the reaction, only the protonated form of the catalyst is observed $\left(\delta_{31 P}=-16.9\right.$ ppm). After $12 \mathrm{~h}$, two new signals at -11.3 and $-20.0 \mathrm{ppm}$ with a characteristic coupling ${ }^{1} J_{\mathrm{PP}}=134 \mathrm{~Hz}$ appeared. At early stage of the reaction, most of the catalyst remained in the protonated form and when it started to be silylated, the enolsilane product was immediately generated. At a later stage, the amount of ketone presumed to be prominent in terms of rate limiting factors, while the silylated catalyst can also be observed in the reaction mixture. If the silylation of the ketone turned out to be the rate determining step instead of the catalyst silylation, we would expect to observe the only presence of the Cat-TBS in the reaction. In contrary, both of the Cat-TBS (silylated catalyst) and Cat-H (initial protonated catalyst) exist till the end of the reaction. 

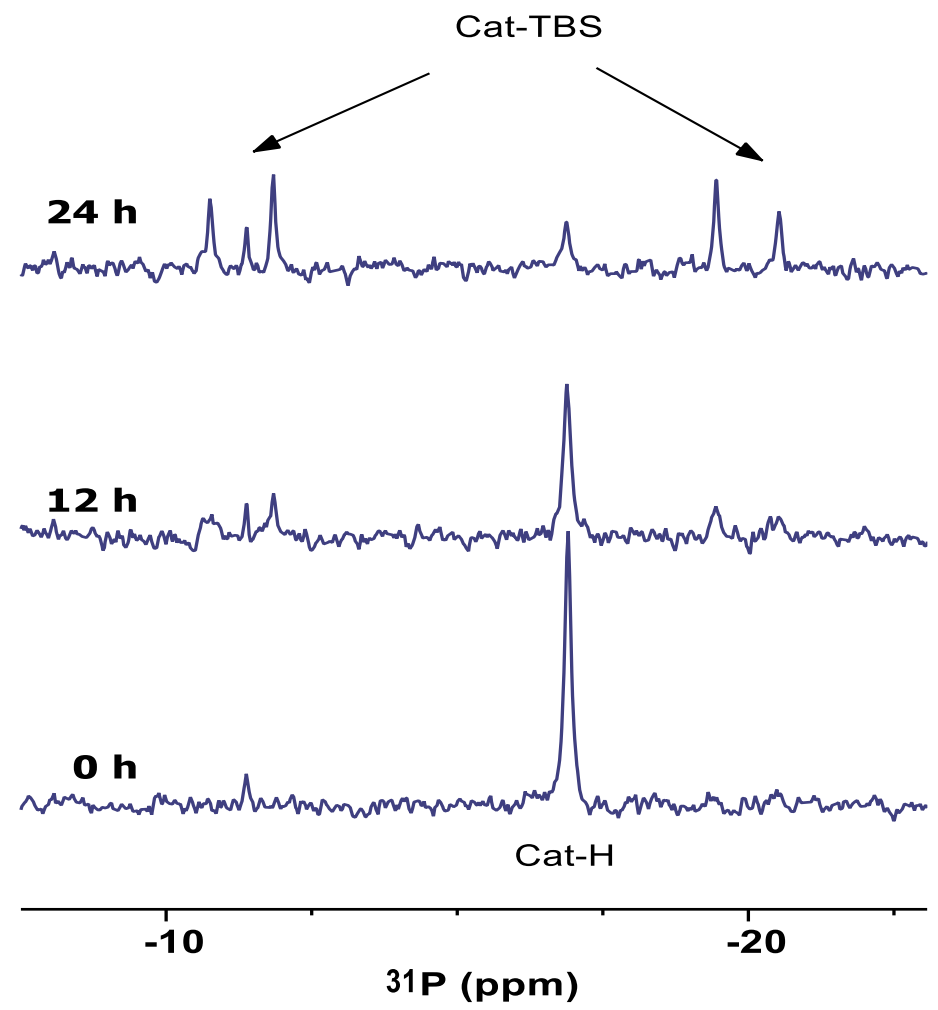

Figure S9. ${ }^{31} \mathrm{P}$ NMR spectra taken at different time points showing the different catalyst species presenting in the entire reaction process.

The ESI-MS spectrum acquired from the mixture of IDPi 4d and BCA showed below:

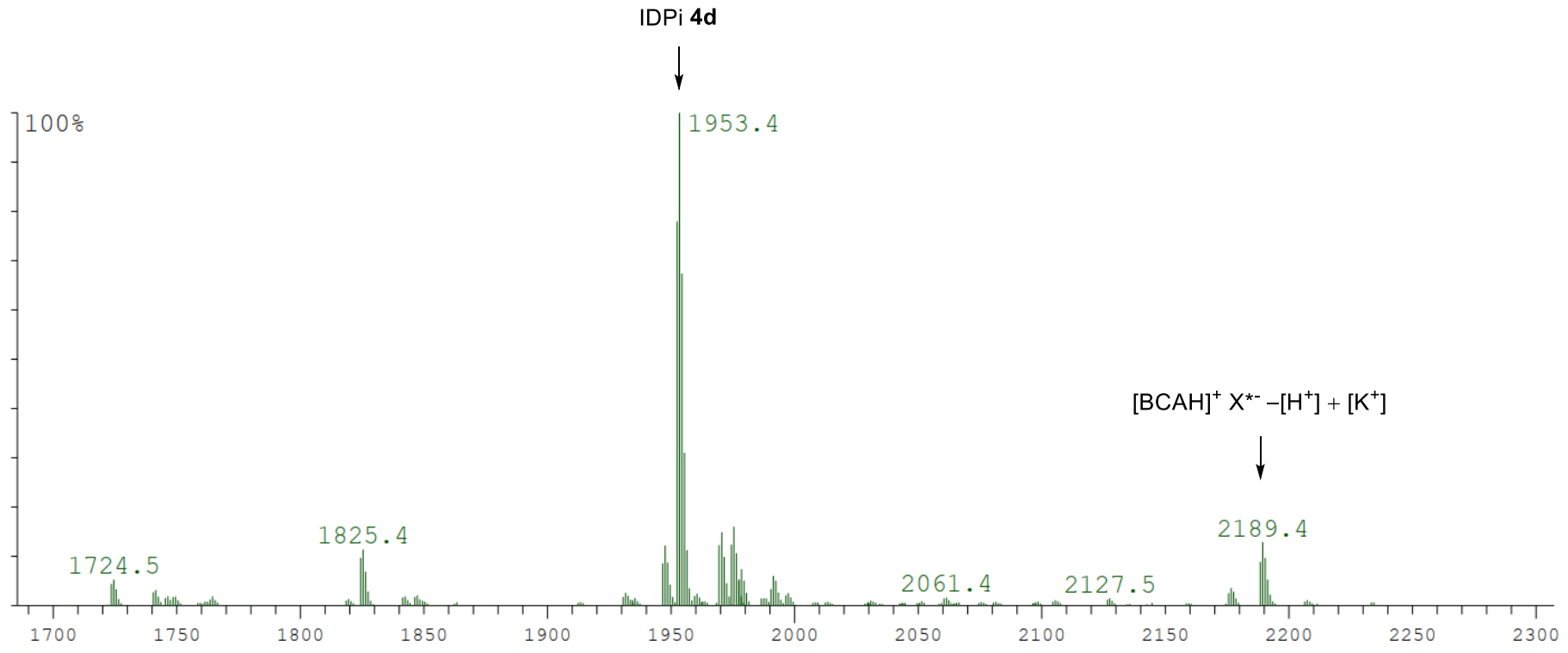

Figure S10. Detection of catalyst species of the kinetic resolution. 


\section{Characterization and study of dynamic behavior of catalyst 4c-TBS}

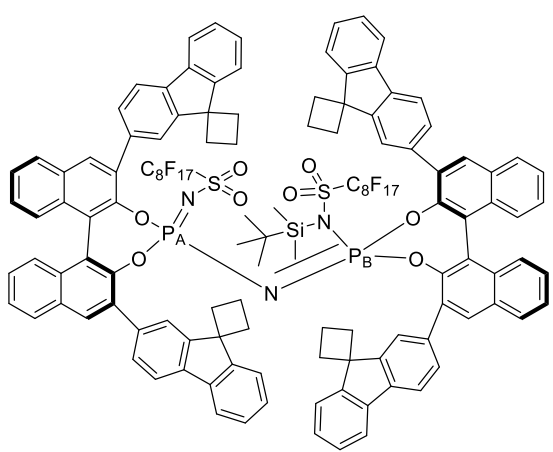

4c-N-TBS

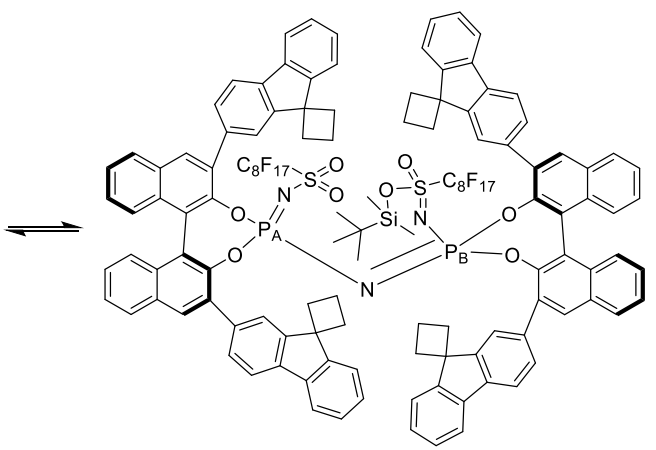

4c-O-TBS
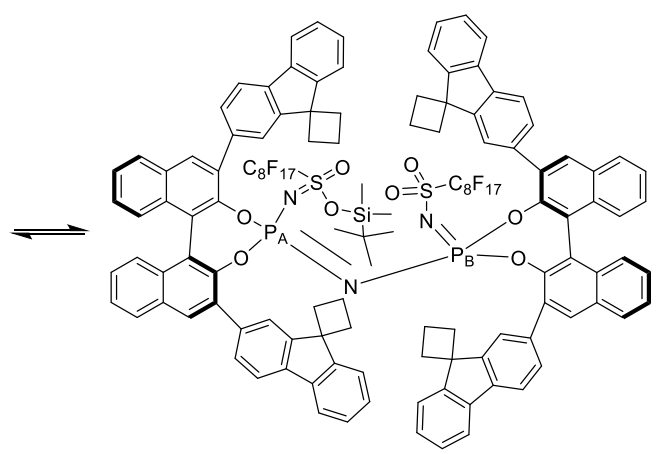

4c-O-TBS

${ }^{1} \mathrm{H}$ NMR in Toluene-d $\mathrm{d}_{8}$ :

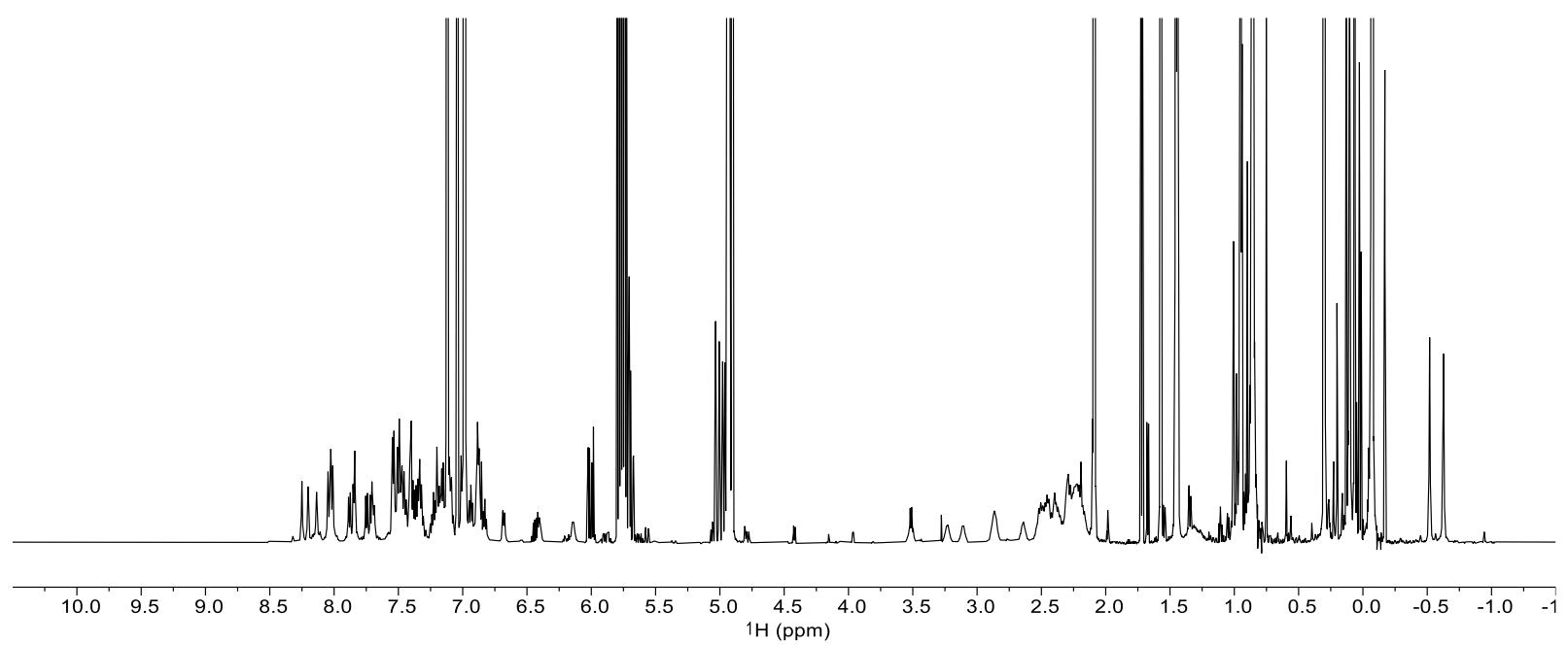

${ }^{31} \mathrm{P}$ NMR spectrum of the activated catalyst TBS-4c:

$31 \mathrm{P},-1 \mathrm{D}$

iี

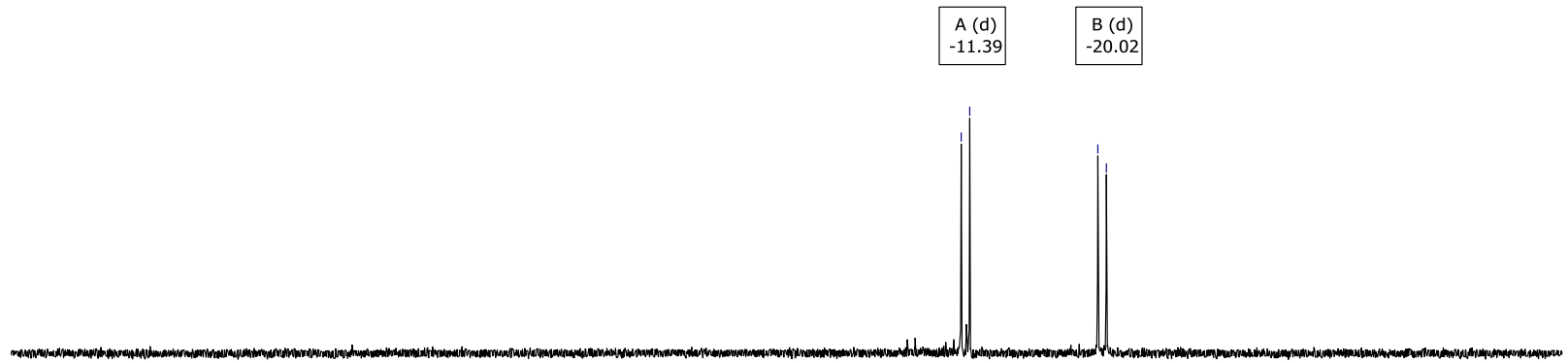

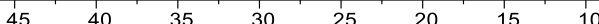

0
$31 \mathrm{P}(\mathrm{ppm})$

${ }^{1} \mathrm{H}-{ }^{31} \mathrm{P}$ HMBC NMR spectrum of the activated catalyst $4 \mathbf{c}$-TBS showing long range coupling of the protons at position 4 in the BINOLbackbone to the $31 \mathrm{P}$ in the core of the catalyst. 


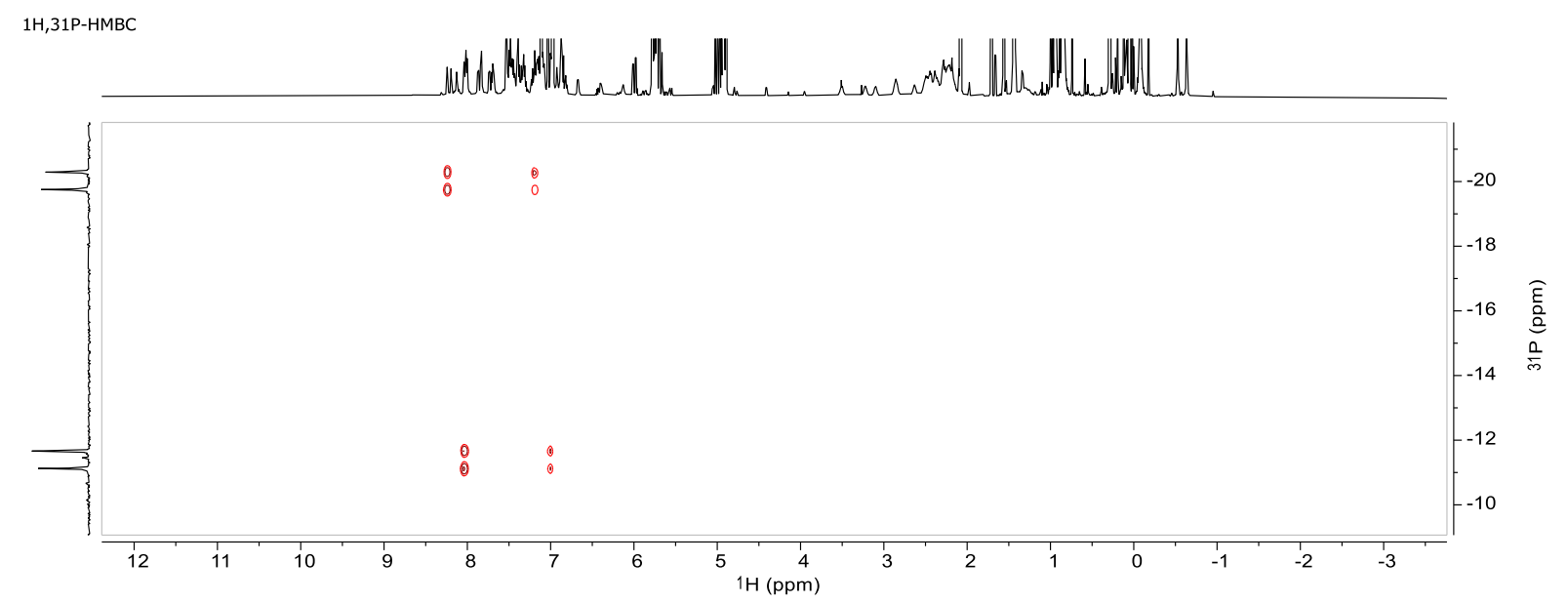

${ }^{29} \mathrm{Si}$ INEPT NMR spectrum of the activated catalyst TBS-4c showing multiple species obtained from the activation of the catalyst. The signal attributed to the silylated catalyst appears at $54.9 \mathrm{ppm}$.

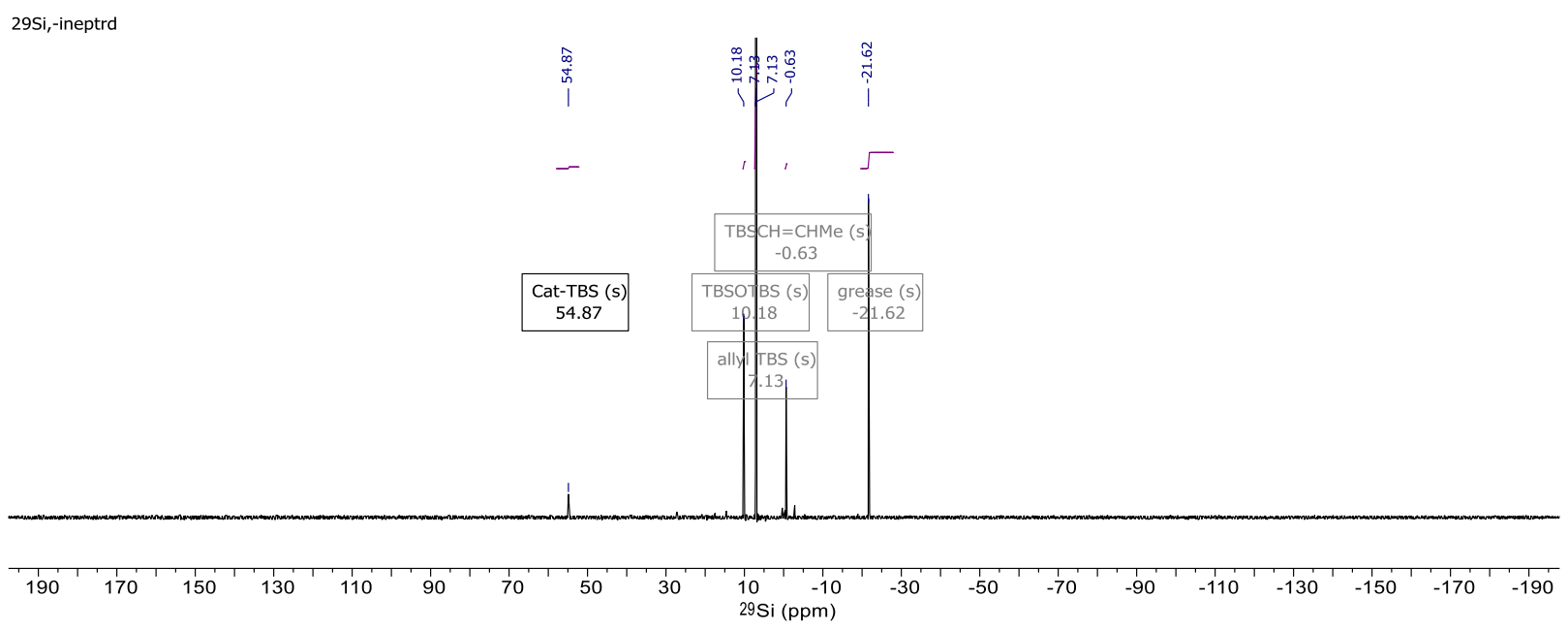

From ${ }^{1} \mathrm{H}-{ }^{29} \mathrm{Si}$ HMBC NMR spectrum of the activated catalyst $4 \mathbf{c}$-TBS, the correlation of the TBS moiety to the ${ }^{29} \mathrm{Si}$ signal appears at $54.9 \mathrm{ppm}$. Other signals in the mixture can be assigned to the side products from the silylation process and grease. 
$1 \mathrm{H}, 29 \mathrm{Si}-\mathrm{HMBC}$

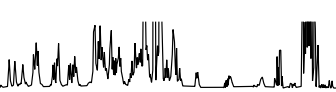

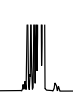

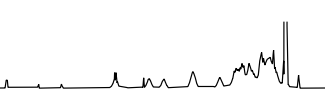

illlelllw



${ }^{1}$ H EASY ROESY NMR spectrum of the activated catalyst 4c-TBS showing only an excerpt of the aromatic region. The red boxes show the chemical exchange between the 4 and 4' positions of both sites of the catalyst. The spectrum was acquired with a spin-lock time of $300 \mathrm{~ms}$.

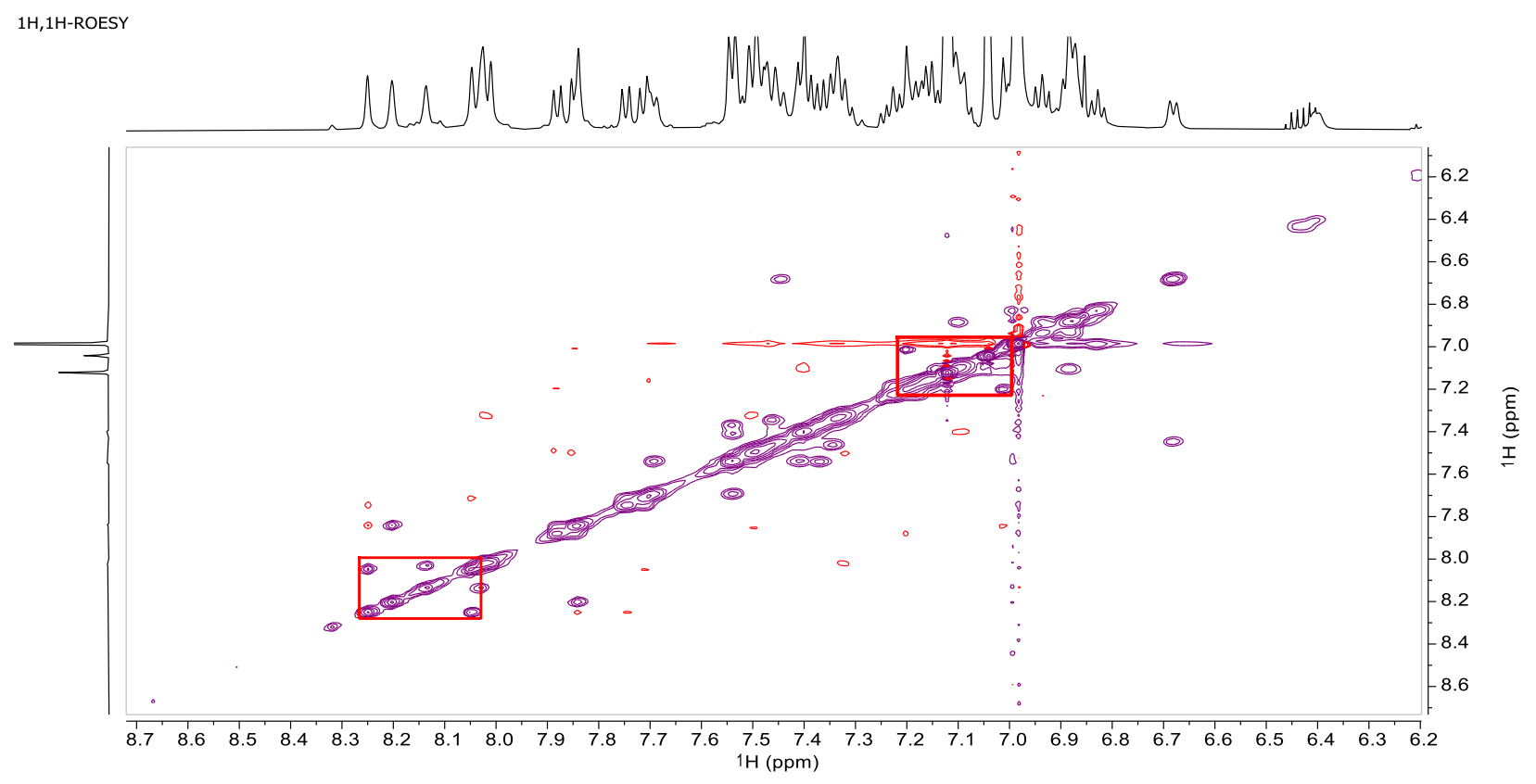

${ }^{1}$ H EASY ROESY NMR spectrum of the activated catalyst 4c-TBS showing the signals of the TBS group attached to the catalyst.

The red box shows the chemical exchange between the diastereotopic Si-Me groups. 


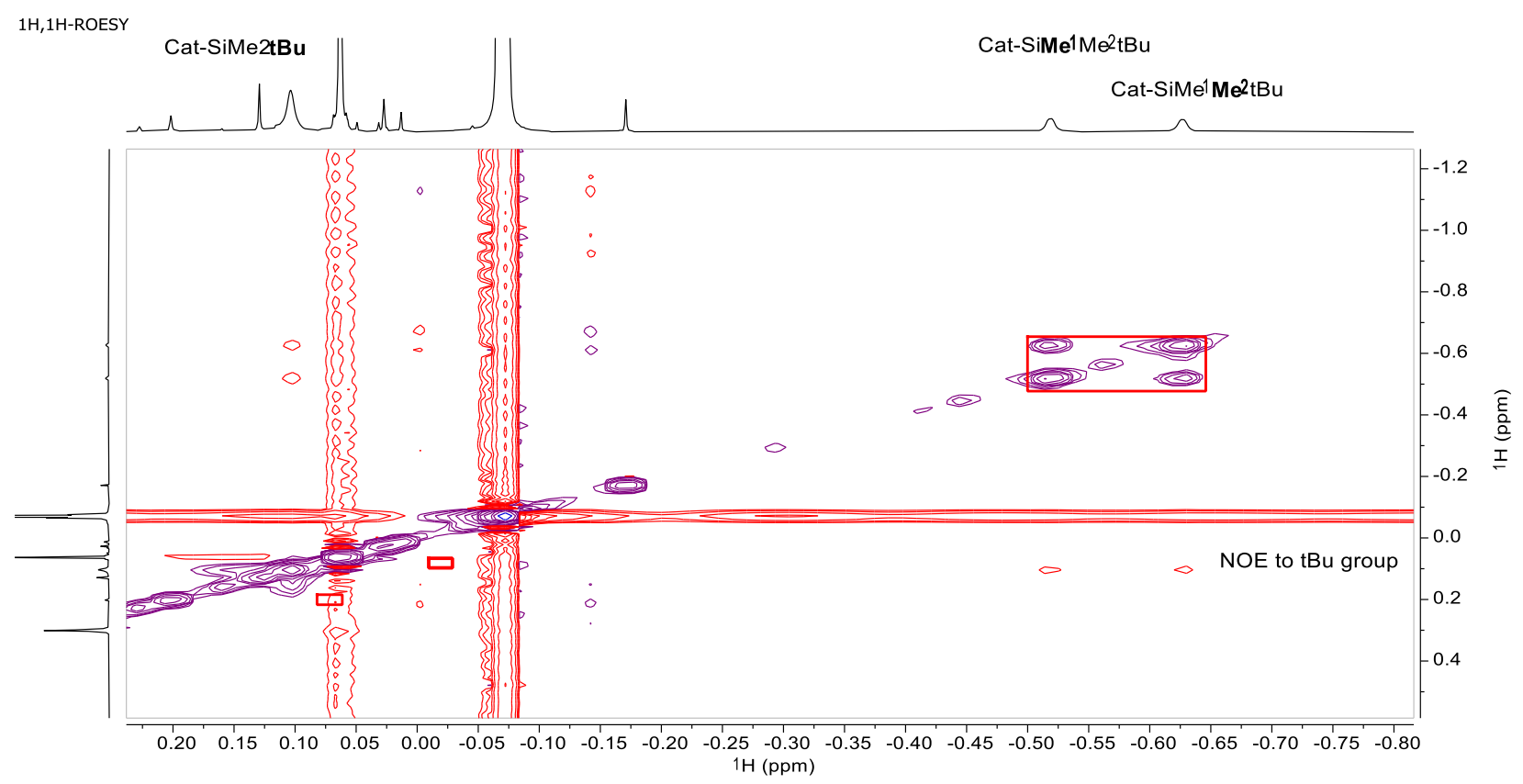

The spectrum was acquired with a spin-lock time of $300 \mathrm{~ms}$. The exchange peaks in the ROESY show that there is an internal silicon transfer within the catalyst observable at a $0.3 \mathrm{~s}$ timescale (see the scheme below), which is much quicker than the reaction rates observed during the reaction.
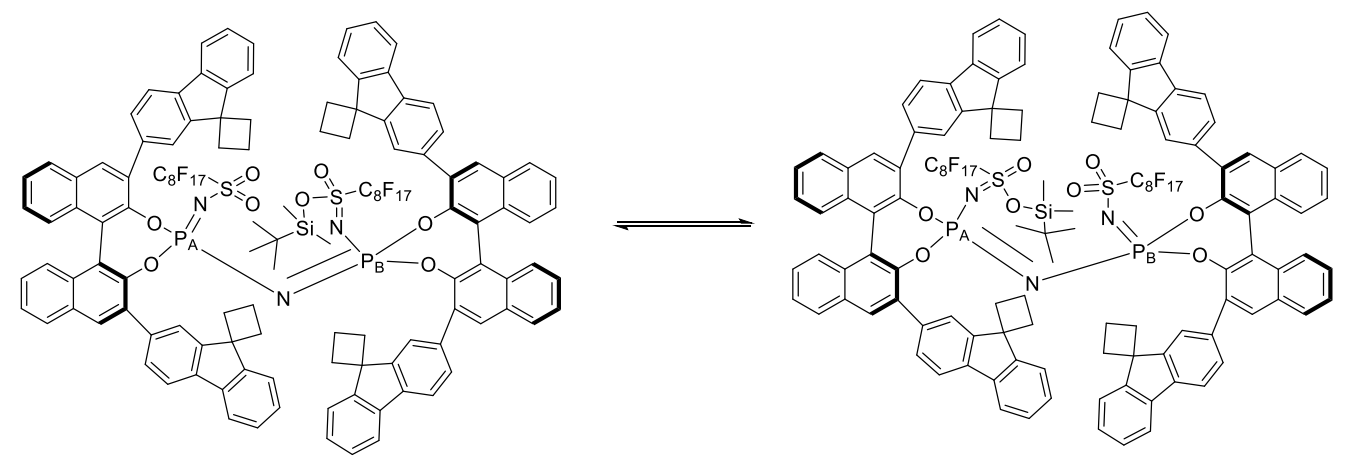


\section{References}

1. Zhou, L., Liu, X., Ji, J., Zhang, Y., Hu, X., Lin, L., Feng, X. Enantioselective Baeyer-Villiger Oxidation: Desymmetrization of Meso Cyclic Ketones and Kinetic Resolution of Racemic 2-Arylcyclohexanones. J. Am. Chem. Soc. 2012, 134, 17023-17026.

2. Bokka, A., Mao, J. X., Hartung, J., Martinez, S. R., Simanis, J. A., Nam, K., Jeon, J., Shen, X. Asymmetric Synthesis of Remote Quaternary Centers by Copper-Catalyzed Desymmetrization: An Enantioselective Total Synthesis of (+)-Mesembrine. Org. Lett. 2018, 20, 5158-5162.

3. Pachali, S., Hofmann, C., Rapp, G., Schobert, R., Baro, A., Frey, W., Laschat, S. Stereoselective Synthesis of (2E, 4Z)-Dienamides Employing (triphenylphosphoranylidene)Ketene. Eur. J. Org. Chem. 2009, 2009, 2828-2835.

4. Malkov, A. V., Friscourt, F., Bell, M., Swarbrick, M.E., Kočovský, P. Enantioselective Baeyer-Villiger Oxidation Catalyzed by Palladium(II) Complexes with Chiral P,N-Ligands. J. Org. Chem. 2008, 73, 3996-4003.

5. Cosgrove, S. C., Plane, J. M. C., Marsden, S. P. Radical-Mediated Direct C-H Amination of Arenes with Secondary Amines. Chem. Sci. 2018, 9, 6647-6652.

6. Gatzenmeier, T., Turberg, M., Yepes, D., Xie, Y., Neese, F., Bistoni, G., List, B. Scalable and Highly Diastereo-and Enantioselective Catalytic Diels-Alder Reaction of $\alpha$, $\beta$-Unsaturated Methyl Esters. J. Am. Chem. Soc. 2018, 140, $12671-12676$.

7. Kaib, P. S. J., Schreyer, L., Lee, S., Properzi, R., List, B. Extremely Active Organocatalysts Enable a Highly Enantioselective Addition of Allyltrimethylsilane to Aldehydes. Angew. Chem. Int. Ed. 2016, 55, 13200-13203.

8. Fulmer, G.R., Miller, A.J., Sherden, N.H., Gottlieb, H.E., Nudelman, A., Stoltz, B.M., Bercaw, J.E., Goldberg, K.I. NMR Chemical Shifts of Trace Impurities: Common Laboratory Solvents, Organics, and Gases in Deuterated Solvents Relevant to the Organometallic Chemist. Organometallics, 2010, 29, 2176-2179.

9. Hoffman, R. E. Standardization of Chemical Shifts of TMS and Solvent Signals in NMR Solvents. Magn. Reson. Chem, 2006, 44, 606-616.

10. Lee, S., Kaib, P. S. J., List, B. N-Triflylphosphorimidoyl Trichloride: A Versatile Reagent for the Synthesis of Strong Chiral Brønsted Acids. Synlett 2017, 12, 1478-1480.

11. Liu, L., Kim, H., Xie, Y., Farès, C., Kaib, P. S. J., Goddard, R., List, B. Catalytic Asymmetric [4+2]-Cycloaddition of Dienes with Aldehydes. J. Am. Chem. Soc. 2017, 139, 13656-13659.

12. Lee, S., Bae, H. Y., List, B. Can a Ketone Be More Reactive than an Aldehyde? Catalytic Asymmetric Synthesis of Substituted Tetrahydrofurans. Angew. Chem. Int. Ed. 2018, 57, 12162-12166.

13. Brooke, G. M. Partially Fluorinated Heterocyclic Compounds. Part 26 [1]. An Investigation into the Mode of Cyclisation in the Reaction of Lithium 1, 3, 4, 5, 6, 7, 8-Heptafluoro-2-naphthalenethiolate with Dimethyl Acetylenedicarboxylate. J. Fluor. Chem. 1989, 43, 393-403.

14. Nishiguchi, A., Maeda, K., Miki, S. Sulfonyl Chloride Formation from Thiol Derivatives by N-chlorosuccinimide Mediated Oxidation. Synthesis 2006, 2006, 4131-4134.

15. Shimizu, M., Kikuchi, J., Kondoh, A., Terada, M. Chiral Brønsted acid-catalyzed intramolecular SN 2' reaction for enantioselective construction of a quaternary stereogenic center. Chem. Sci. 2018, 9, 5747-5757.

16. Ouyang, J., Kennemur, J. L., De, C. K., Farès, C., List, B. Strong and Confined Acids Enable a Catalytic Asymmetric Nazarov Cyclization of Simple Divinyl Ketones. J. Am. Chem. Soc. 2019, 141, 3414-3418.

17. Gao, R., Yi, C. S. Catalytic Formation of Silyl Enol Ethers and Its Applications for Aldol-Type Condensation and Aminomethylation Reactions. ACS. Catal. 2011, 1, 544-547.

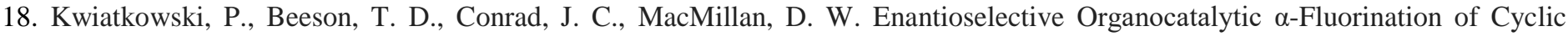
Ketones. J. Am. Chem. Soc. 2011, 133, 1738-1741.

19. Kato, M., Watanabe, M., Tooyama, Y., Vogler, B., Yoshikoshi, A. Efficient and Convenient Syntheses of $(R)-(-)-C r y p t o n e ~ a n d ~(S)$ 
-(-)-4-Isopropenyl-2-cyclohexen-1-one. Synthesis 1992, 11, 1055-1057.

20. Aoki, K., Nakajima, M., Tomioka, K., Koga, K. Reinvestigation on the Optical Purities of Optically Active Trimethylsilyl Enol Ethers of 4-Substituted Cyclohexanones. Chem. Pharm. Bull. 1993, 41, 994-996.

21. Takasu, K., Nagao, S., Ihara, M. Synthesis of Medium-Sized Cyclic $\gamma$-Haloketones by Radical Mediated Ring-Opening Reaction of Lewis Acid Catalyzed (2+2)-Cycloaddition Product. Tetrahedron. Lett. 2005, 46, 1005-1008.

22. Kad, G. L., Singh, V., Khurana, A., Chauhary, S., Singh, J. Regioselective $\alpha$-Alkylation of Silyl Enolates Using a Mild Catalyst$\mathrm{ZnCl}_{2}$ Doped on Acidic Alumina. Synth. Commun. 1999, 29, 3439-3442.

23. Chandrasekhar, S., Sridhar, C., Srihari, P. A Carbohydrate Approach for the Formal Total Synthesis of the Prostacyclin Analogue (16S)-Iloprost. Tetrahedron: Asymmetry. 2012, 23, 388-394.

24. Lutz, V., Baro, A., Fischer, P., Laschat, S. Synthesis of Functionalized Hydropentalenes by an Asymmetric Deprotonation/Alkylation Strategy. Eur. J. Org. Chem. 2010, 2010, 1149-1157.

25. Nielsen, C. D. T., Burés, J. Visual Kinetic Analysis. Chem. Sci. 2019, 10, 348-353.

26. Burés, J. A Simple Graphical Method to Determine the Order in Catalyst. Angew. Chem Int. Ed. 2016, 55, $2028-2031$.

27. Burés, J. What Is the Order of a Reaction? Top. Catal. 2017, 60, 631-633.

28. Burés, J., Armstrong, A., Blackmond, D. G. Kinetic Correlation between Aldehyde/Enamine Stereoisomers in Reactions between Aldehydes with $\alpha$-Stereocenters and Chiral Pyrrolidine-Based Catalysts. Chem. Sci. 2012, 3, $1273-1277$.

29. Burés, J. Variable Time Normalization Analysis: General Graphical Elucidation of Reaction Orders from Concentration Profiles. Angew. Chem. Int. Ed. 2016, 55, 16084-16087.

30. Zhang, Z., Klussmann, M., List, B. Kinetic Study of Disulfonimide Catalyzed Cyanosilylation of Aldehyde Using a Method of Progress Rates. Synlett 2020, DOI: 10.1055/s-0040-1707129. 


\section{Copies of NMR spectra}

\section{(S)-3, 3'-bis(9,9-diethyl-9H-fluoren-3-yl)-[1,1'-binaphthalene]-2,2'-diol:}

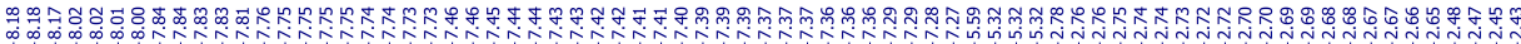

\begin{tabular}{|ll|}
\hline \multicolumn{1}{|c|}{ Parameter } & \multicolumn{1}{c|}{ Value } \\
Solvent & $\mathrm{CD} 2 \mathrm{Cl} 2$ \\
Temperature & 297.9 \\
Spectrometer Frequency & 500.81 \\
Nucleus & $1 \mathrm{H}$ \\
\hline
\end{tabular}

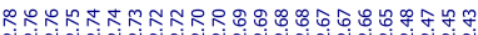



\begin{tabular}{|ll|}
\hline \multicolumn{1}{|c}{ Parameter } & \multicolumn{1}{c|}{ Value } \\
Solvent & $\mathrm{CD} 2 \mathrm{Cl} 2$ \\
Temperature & 298.0 \\
Spectrometer Frequency & 125.94 \\
Nucleus & $13 \mathrm{C}$ \\
\hline
\end{tabular}

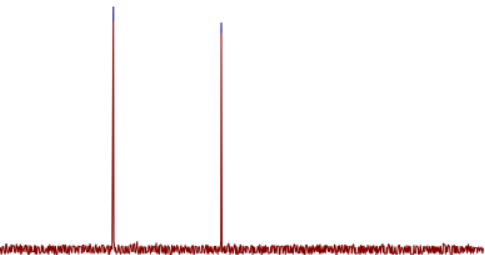


(S)-3,3'-di(triphenylen-2-yl)-[1,1'-binaphthalene]-2,2'-diol:
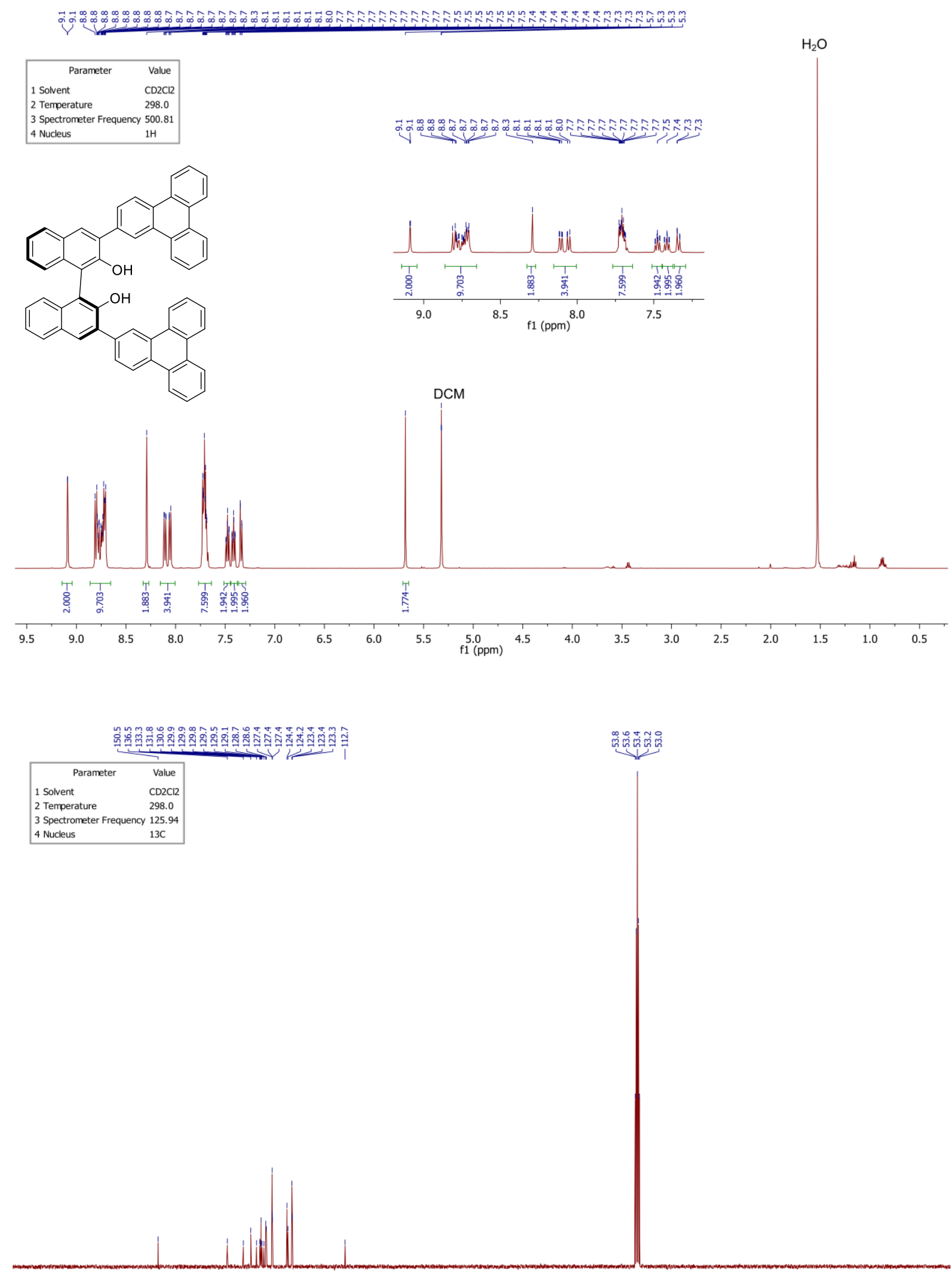

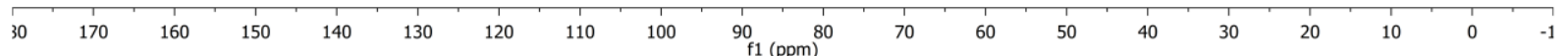


(S)-3,3'-bis(3',5'-di-tert-butyl-[1,1'-biphenyl]-4-yl)-[1,1'-binaphthalene]-2,2' -diol:

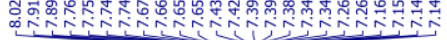

\begin{tabular}{|ll|}
\hline \multicolumn{1}{|c|}{ Parameter } & \multicolumn{1}{c|}{ Value } \\
1 Solvent & $\mathrm{CD} 2 \mathrm{Cl} 2$ \\
2 Temperature & 297.8 \\
3 Spectrometer Frequency & 500.81 \\
4 Nucleus & $1 \mathrm{H}$ \\
\hline
\end{tabular}

(Bu

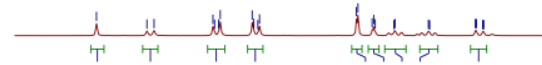

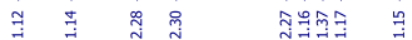

$\begin{array}{lllllllllllllll}8.2 & 8.1 & 8.0 & 7.9 & 7.8 & 7.7 & 7.6 & 7.5 & 7.4 & 7.3 & 7.2 & 7.1 & 7.0\end{array}$

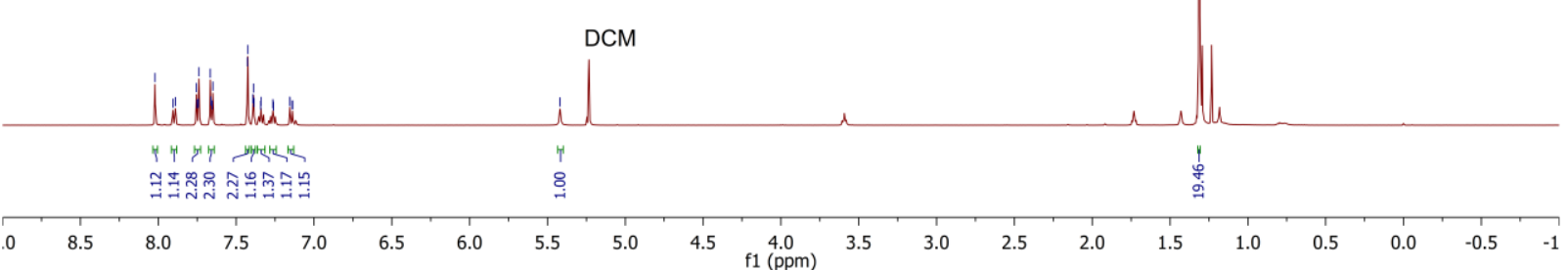

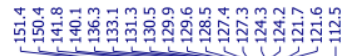

\begin{tabular}{|ll|}
\hline \multicolumn{1}{|c|}{ Parameter } & \multicolumn{1}{c|}{ Value } \\
Solvent & CD2C12 \\
Temperature & 298.0 \\
Spectrometer Frequency & 125.94 \\
Nucleus & $13 \mathrm{C}$ \\
\hline
\end{tabular}

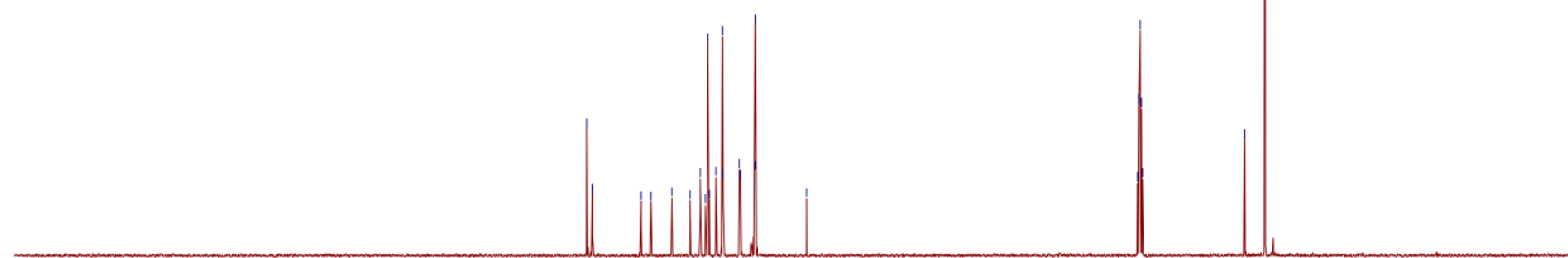

$\begin{array}{llllllllllllllllllllllllllllll}250 & 240 & 230 & 220 & 210 & 200 & 190 & 180 & 170 & 160 & 150 & 140 & 130 & 120 & 110 & 100 & 90 & 80 & 70 & 60 & 50 & 40 & 30 & 20 & 10 & 0 & -10 & -20\end{array}$ 


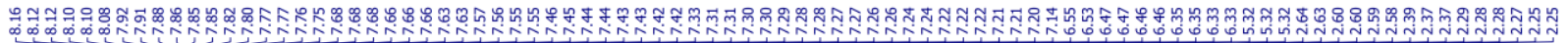



\begin{tabular}{|ll|}
\hline \multicolumn{1}{|c|}{ Parameter } & \multicolumn{1}{c|}{ Value } \\
Solvent & CD2C12 \\
Temperature & 298.0 \\
Spectrometer Frequency & 125.94 \\
Nucleus & $13 \mathrm{C}$ \\
\hline
\end{tabular}

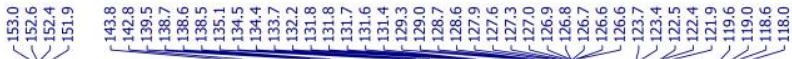
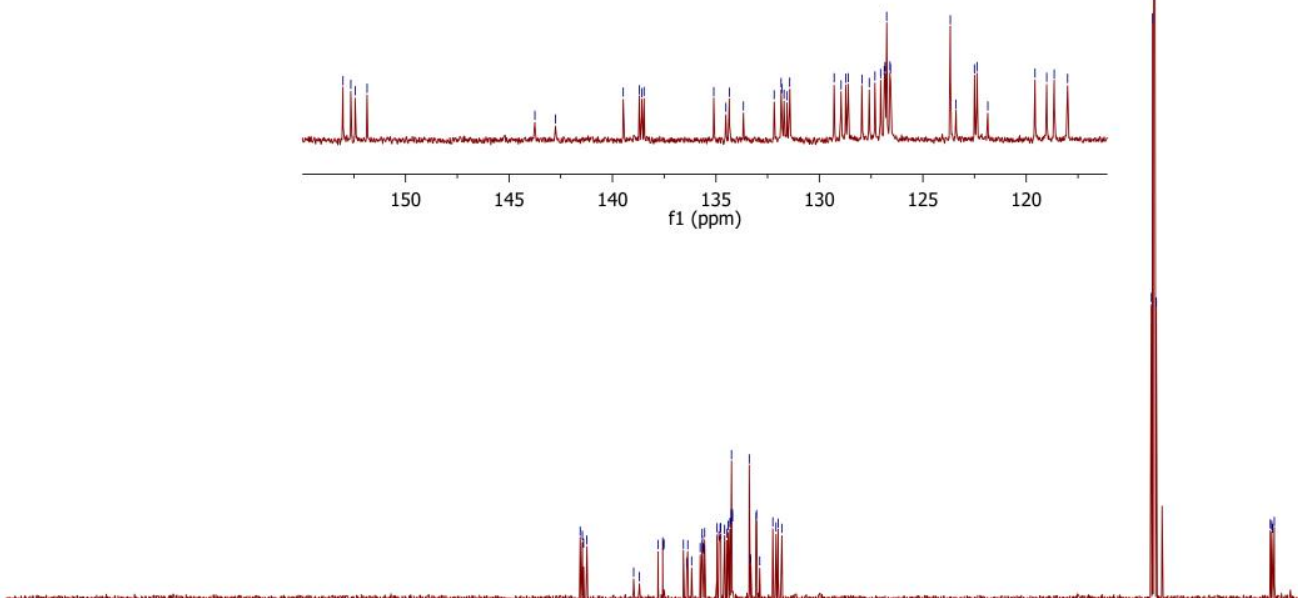

$\begin{array}{llllllllllllllllllllllllllllllllllll}250 & 240 & 230 & 220 & 210 & 200 & 190 & 180 & 170 & 160 & 150 & 140 & 130 & 120 & 110 & 100 & 90 & 80 & 70 & 60 & 50 & 40 & 30 & 20 & 10 & 0 & -10 & -20\end{array}$ 


\begin{tabular}{|ll|}
\hline \multicolumn{1}{|c}{ Parameter } & \multicolumn{1}{c|}{ Value } \\
Solvent & CD2Cl2 \\
Temperature & 298.0 \\
Spectrometer Frequency & 471.21 \\
Nucleus & $19 \mathrm{~F}$ \\
\hline
\end{tabular}

i



$\begin{array}{llllllllllllllllllllllllllllll}240 & 220 & 200 & 180 & 160 & 140 & 120 & 100 & 80 & 60 & 40 & 20 & 0 & -20 & -40 & -60 & -80 & -100 & -120 & -140 & -160 & -180 & -200 & -220 & -240 & -260 & -280 & -300 & -320 & -34\end{array}$ f1 (ppm)

\begin{tabular}{|ll|}
\hline \multicolumn{1}{|c|}{ Parameter } & \multicolumn{1}{c|}{ Value } \\
Solvent & CD2C12 \\
Temperature & 298.0 \\
Spectrometer Frequency & 202.73 \\
Nucleus & $31 \mathrm{P}$ \\
\hline
\end{tabular}
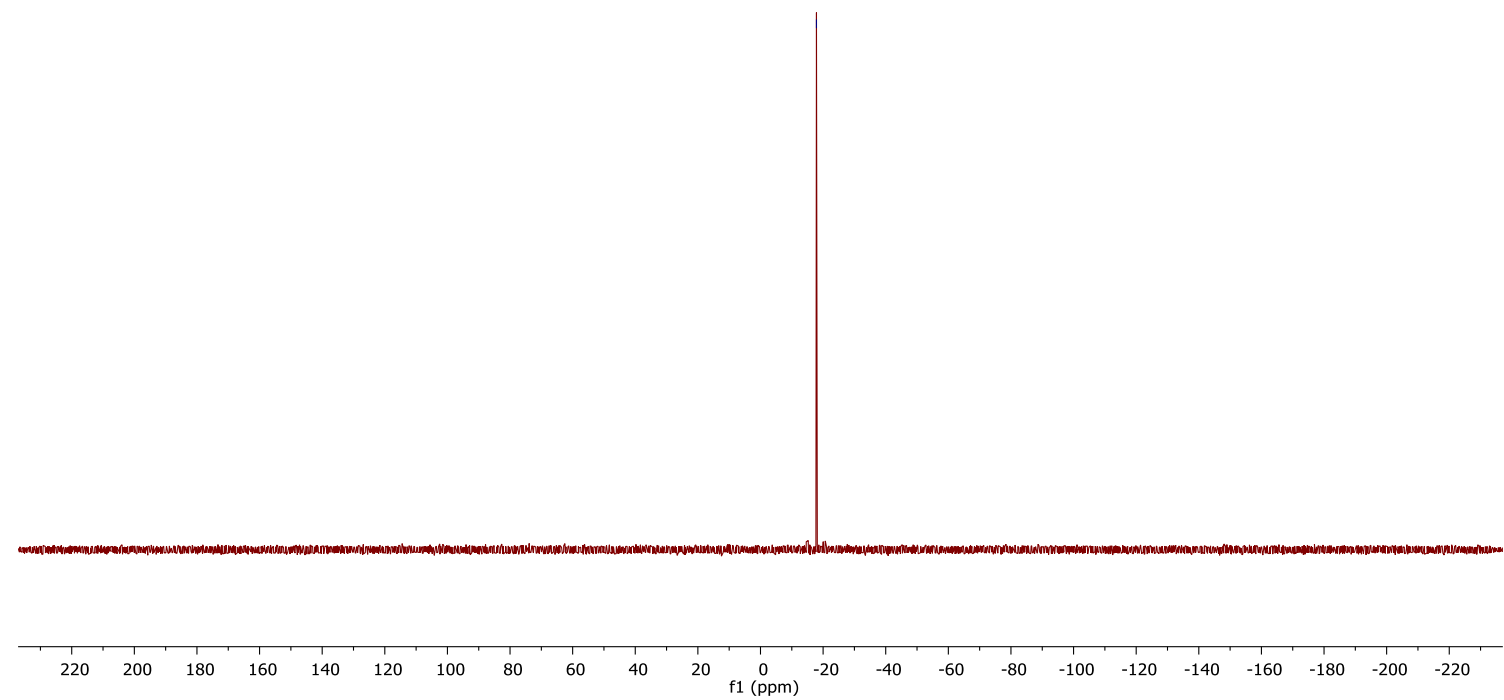










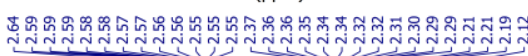



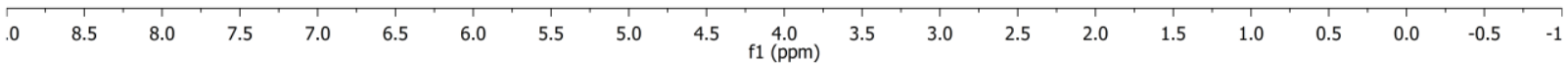

\begin{tabular}{|lc|}
\hline \multicolumn{1}{|c|}{ Parameter } & Value \\
Solvent & CD2C12 \\
Temperature & 298.0 \\
Spectrometer Frequency & 125.94 \\
Nucleus & $13 \mathrm{C}$ \\
\hline
\end{tabular}

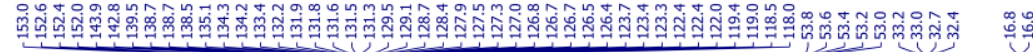



\begin{tabular}{|c|c|c|c|c|c|c|c|}
\hline 155 & 150 & 145 & 140 & $\begin{array}{r}1 \\
135 \\
\text { ppm) }\end{array}$ & 130 & 125 & 120 \\
\hline
\end{tabular}

$250 \quad 240$

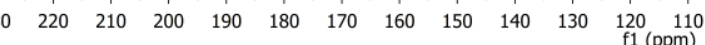

120110 

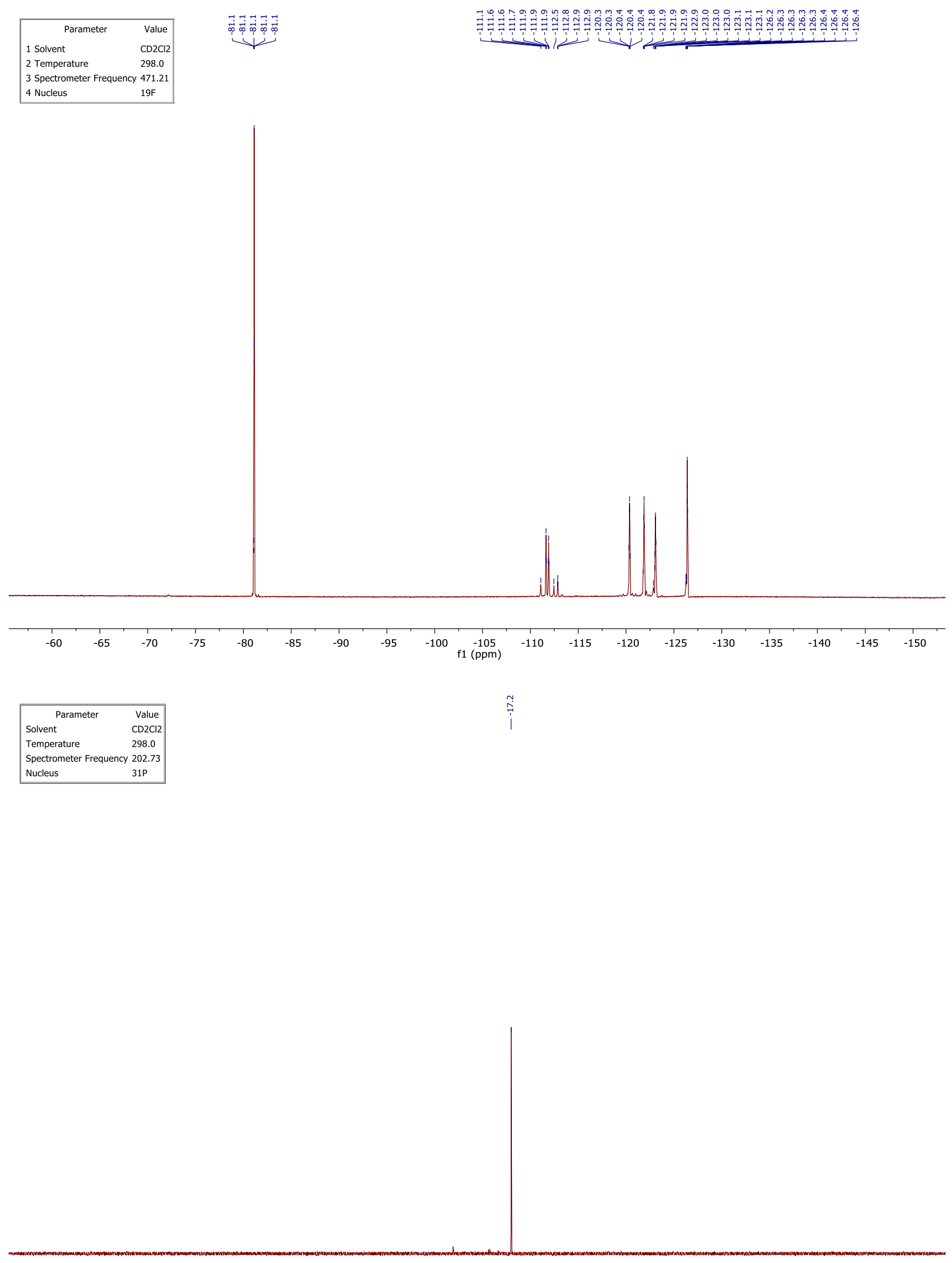

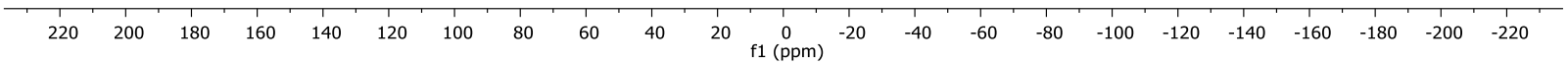


$(S, S)-I D P i-4 c:$

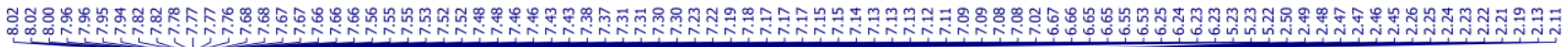
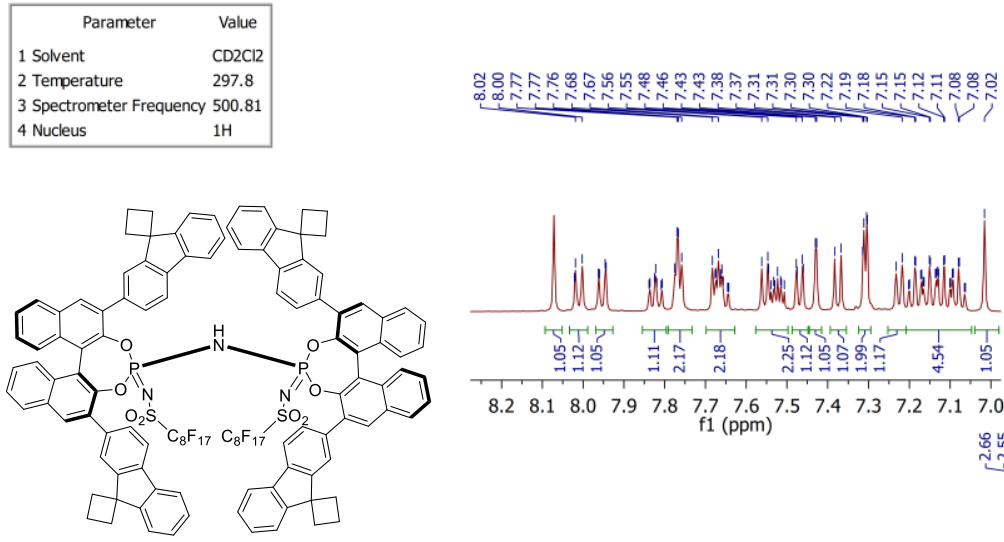

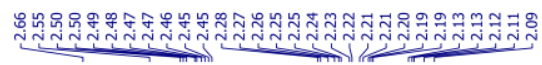

$4 \mathrm{c}$

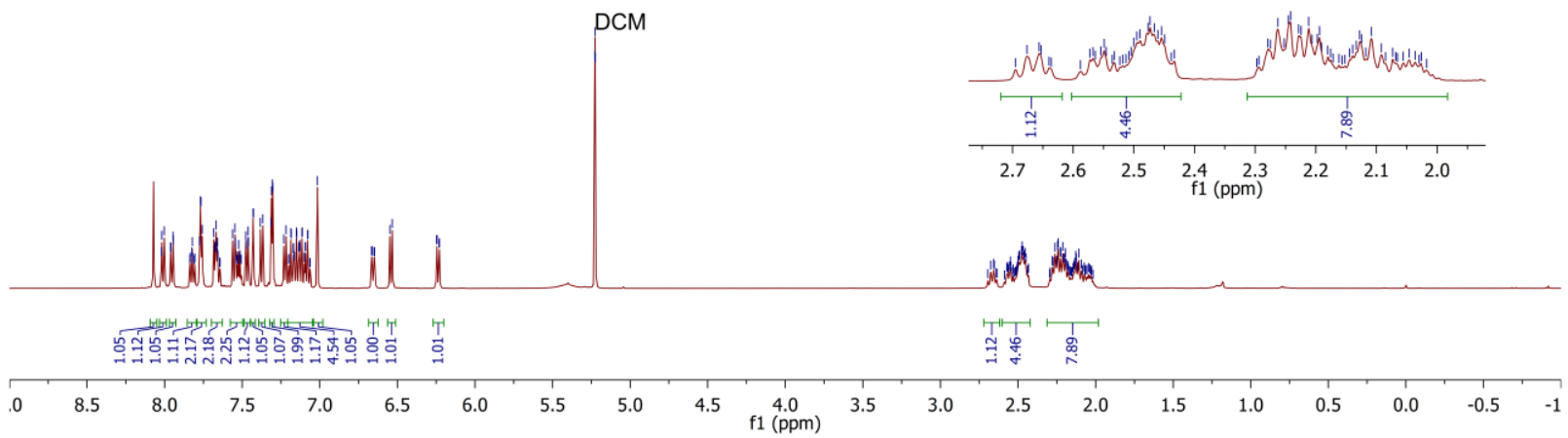

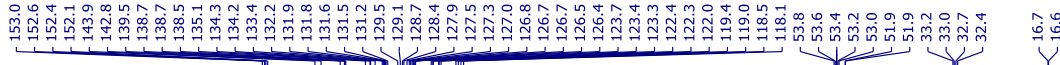

\begin{tabular}{|c|c|}
\hline Parameter & Value \\
\hline Solvent & $\mathrm{CD} 2 \mathrm{Cl}$ \\
\hline Temperature & 298.0 \\
\hline Spectrometer Frequen & 125.9 \\
\hline ucleus & $13 \mathrm{C}$ \\
\hline
\end{tabular}

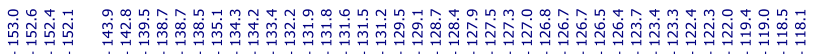

il
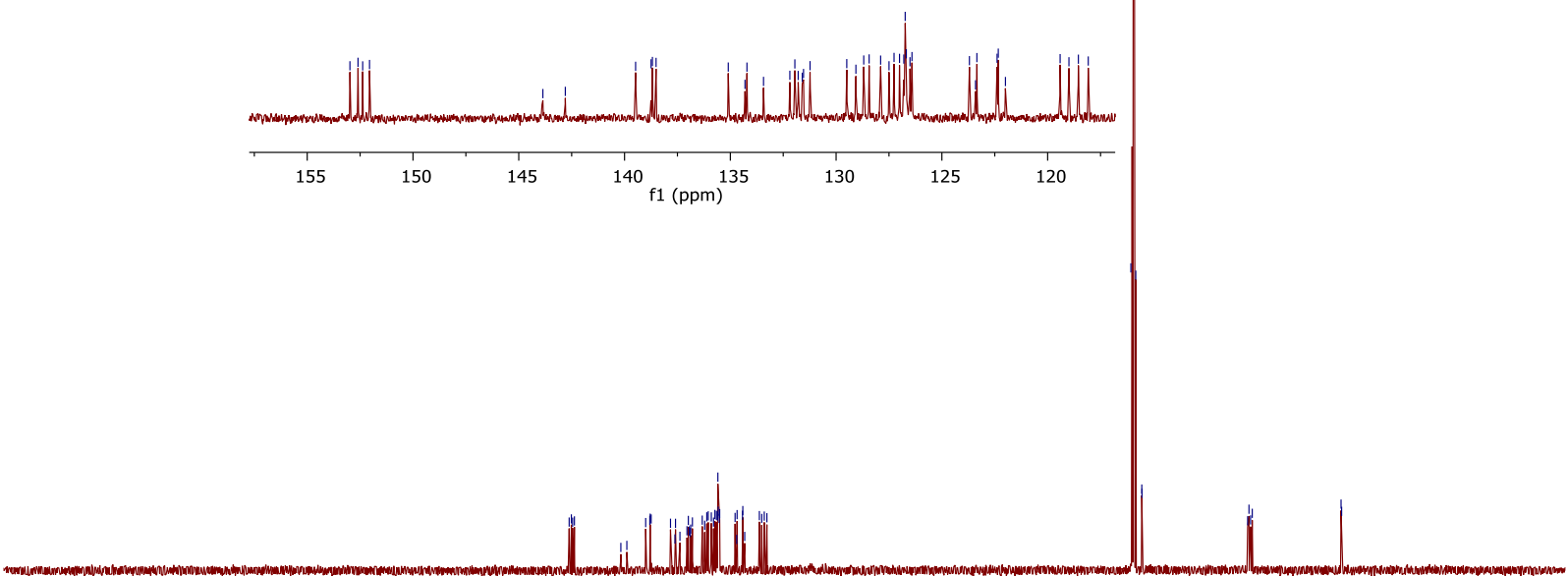

$\begin{array}{lllllllllllllllllllllllllllll}250 & 240 & 230 & 220 & 210 & 200 & 190 & 180 & 170 & 160 & 150 & 140 & 130 & \begin{array}{c}120 \\ \mathrm{f} 1(\mathrm{ppm})\end{array} & 100 & 90 & 80 & 70 & 60 & 50 & 40 & 30 & 20 & 10 & 0 & -10 & -20\end{array}$ 


\begin{tabular}{|ll|}
\hline \multicolumn{1}{|c|}{ Parameter } & Value \\
Solvent & $\mathrm{CD} 2 \mathrm{Cl} 2$ \\
Temperature & 298.0 \\
Spectrometer Frequency & 471.21 \\
Nucleus & $19 \mathrm{~F}$ \\
\hline
\end{tabular}

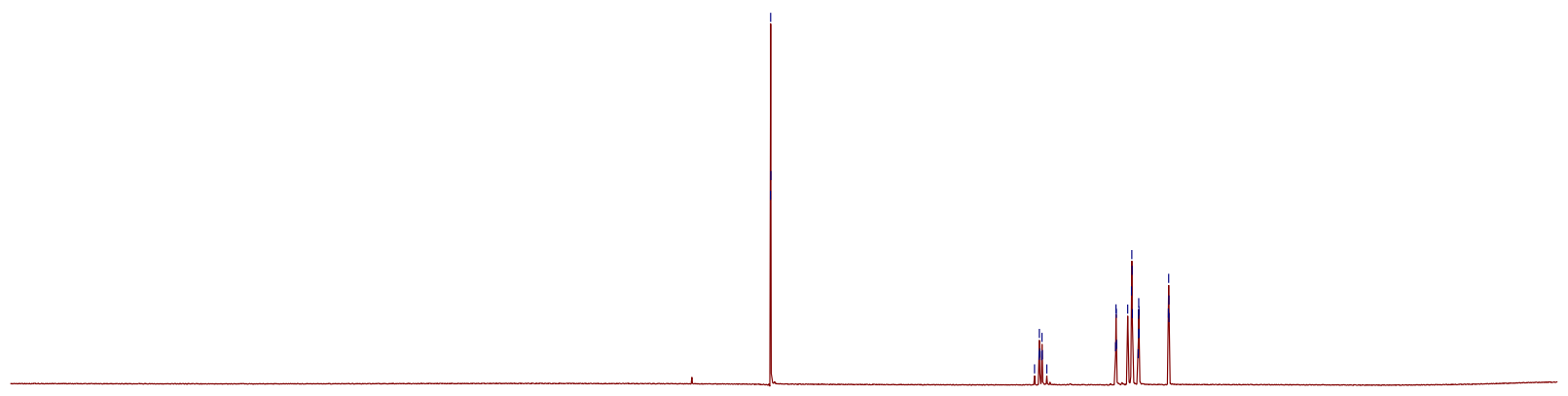



\begin{tabular}{|c|c|}
\hline Parameter & Value \\
\hline Solvent & $\mathrm{CD} 2 \mathrm{Cl} 2$ \\
\hline Temperature & 298.0 \\
\hline Spectrometer Frequen & 202.73 \\
\hline Nucleus & $31 \mathrm{P}$ \\
\hline
\end{tabular}
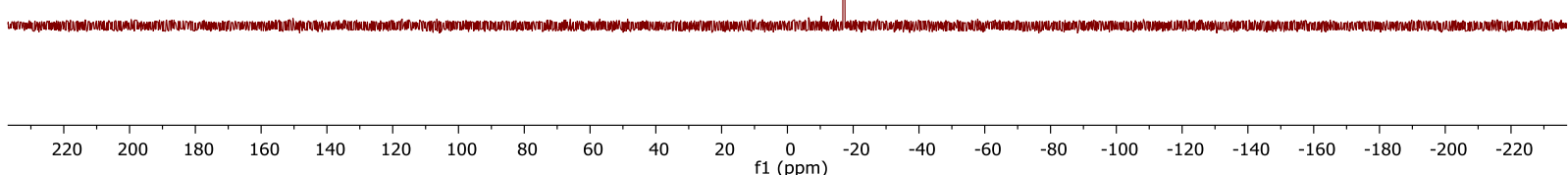


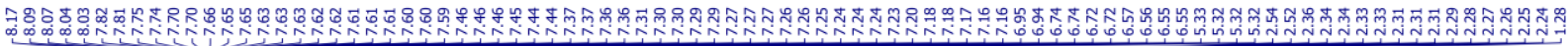

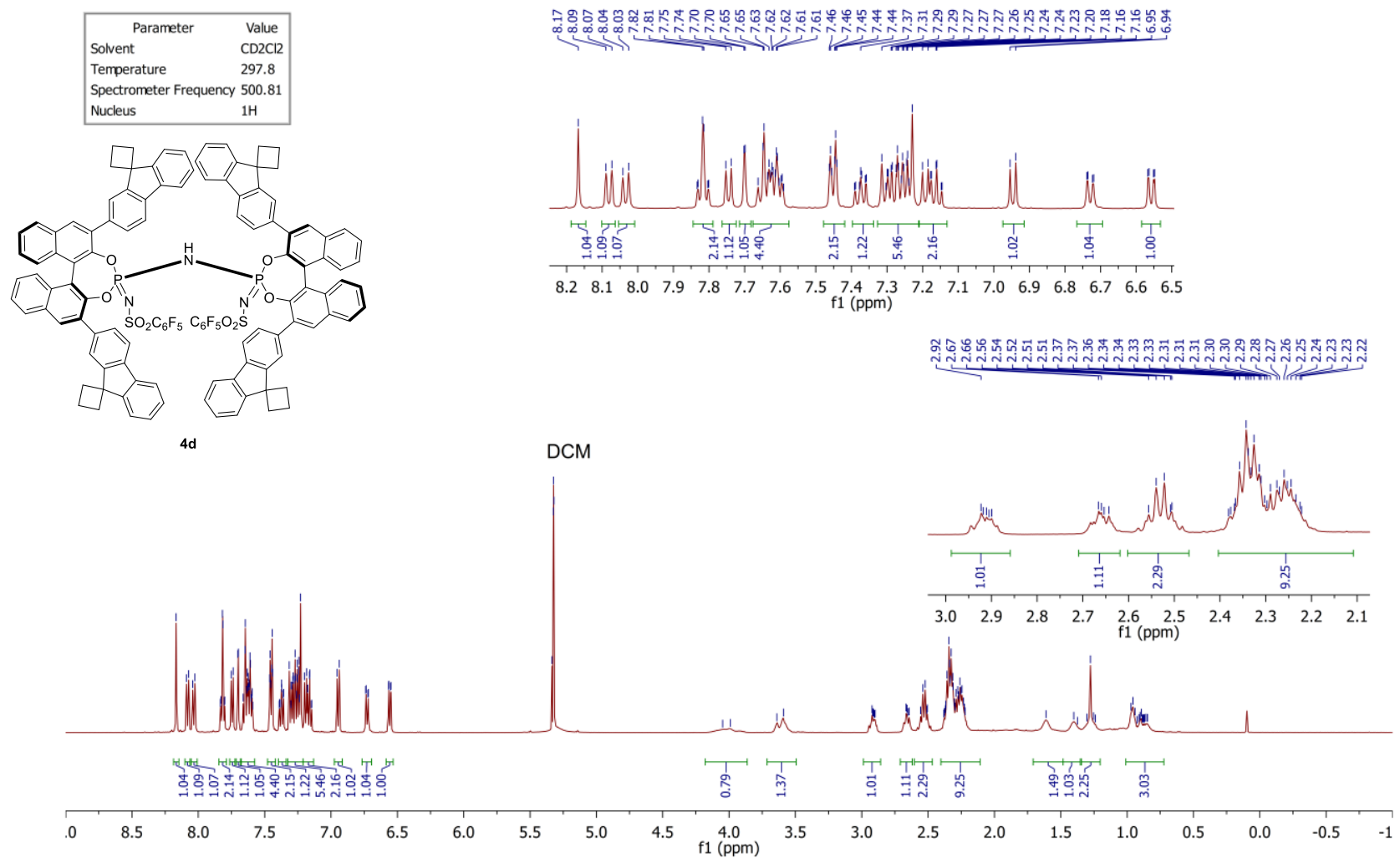

\begin{tabular}{|ll|}
\hline \multicolumn{1}{|c|}{ Parameter } & \multicolumn{1}{c|}{ Value } \\
Solvent & CD2C12 \\
Temperature & 298.0 \\
Spectrometer Frequency & 125.94 \\
Nucleus & $13 \mathrm{C}$ \\
\hline
\end{tabular}
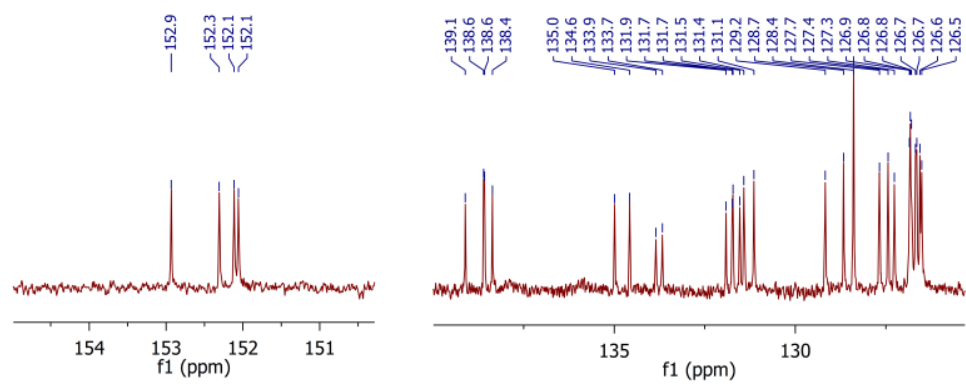

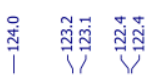
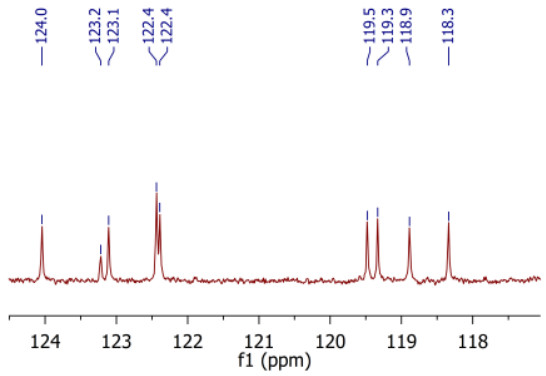

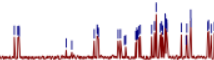

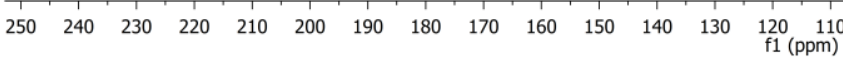




\begin{tabular}{|ll|}
\hline \multicolumn{1}{|c|}{ Parameter } & \multicolumn{1}{c|}{ Value } \\
Solvent & $\mathrm{CD} 2 \mathrm{Cl} 2$ \\
Temperature & 298.0 \\
Spectrometer Frequency & 471.21 \\
Nucleus & $19 \mathrm{~F}$ \\
\hline
\end{tabular}

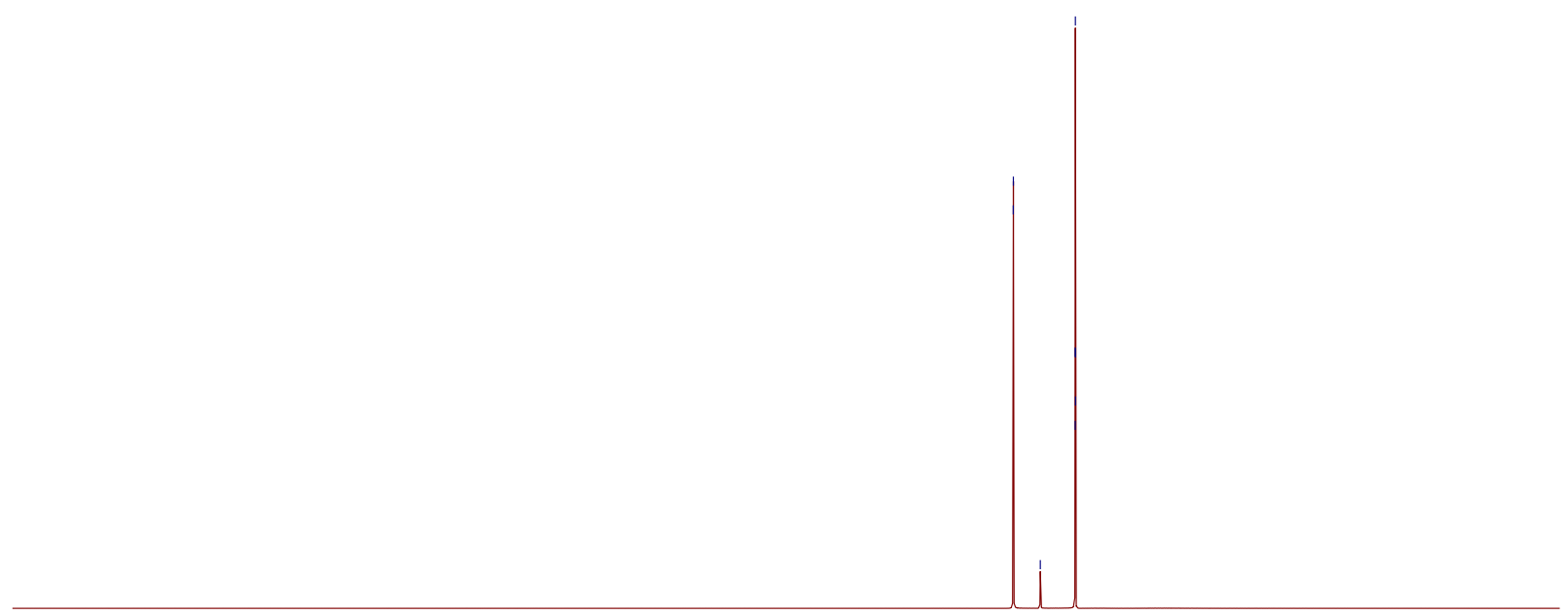

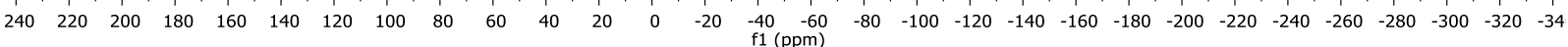

\begin{tabular}{|c|c|}
\hline Parameter & Value \\
\hline Solvent & $\mathrm{CD} 2 \mathrm{Cl} 2$ \\
\hline Temperature & 298.0 \\
\hline Spectrometer Frequenc & 202.73 \\
\hline Nucleus & $31 \mathrm{P}$ \\
\hline
\end{tabular}




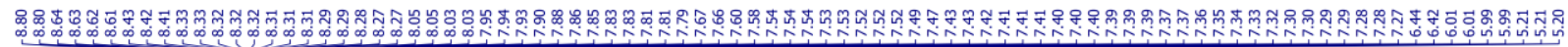

\begin{tabular}{|lc|}
\hline \multicolumn{1}{|c|}{ Parameter } & \multicolumn{1}{c|}{ Value } \\
1 Solvent & $\mathrm{CD} 2 \mathrm{Cl} 2$ \\
2 Temperature & 298.0 \\
3 Spectrometer Frequency & 500.81 \\
4 Nucleus & $1 \mathrm{H}$ \\
\hline
\end{tabular}
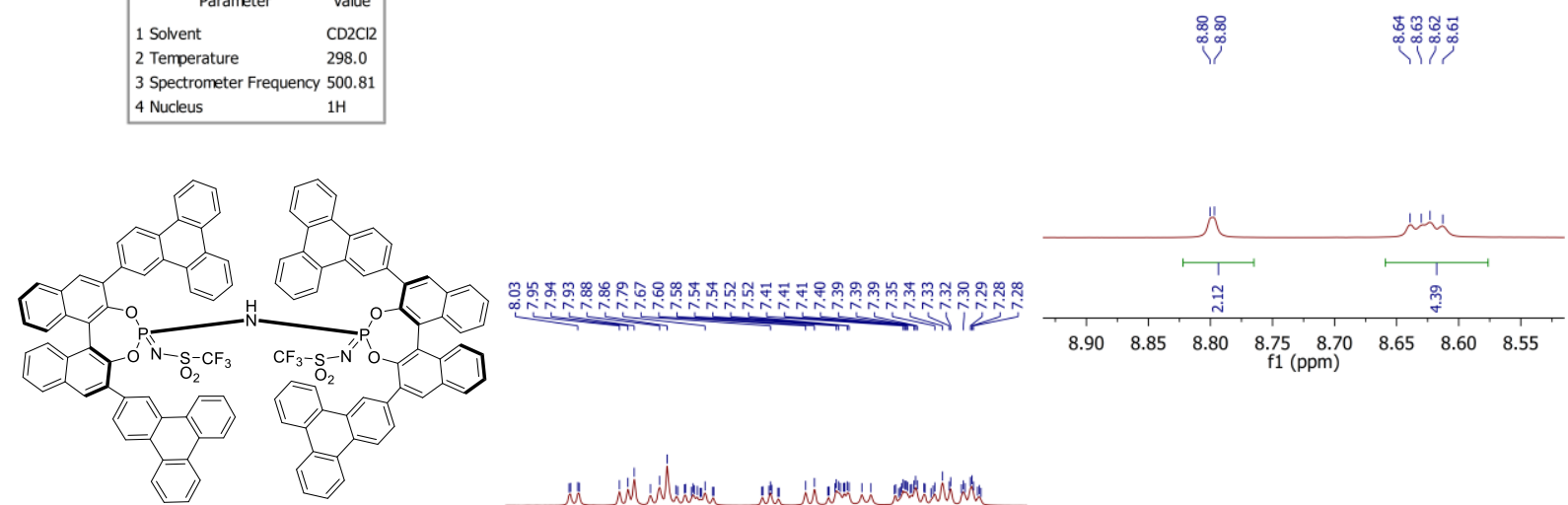

$4 \mathrm{e}$

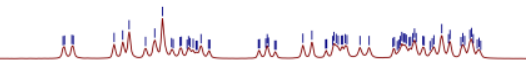



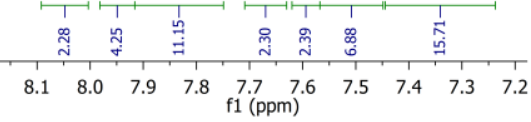
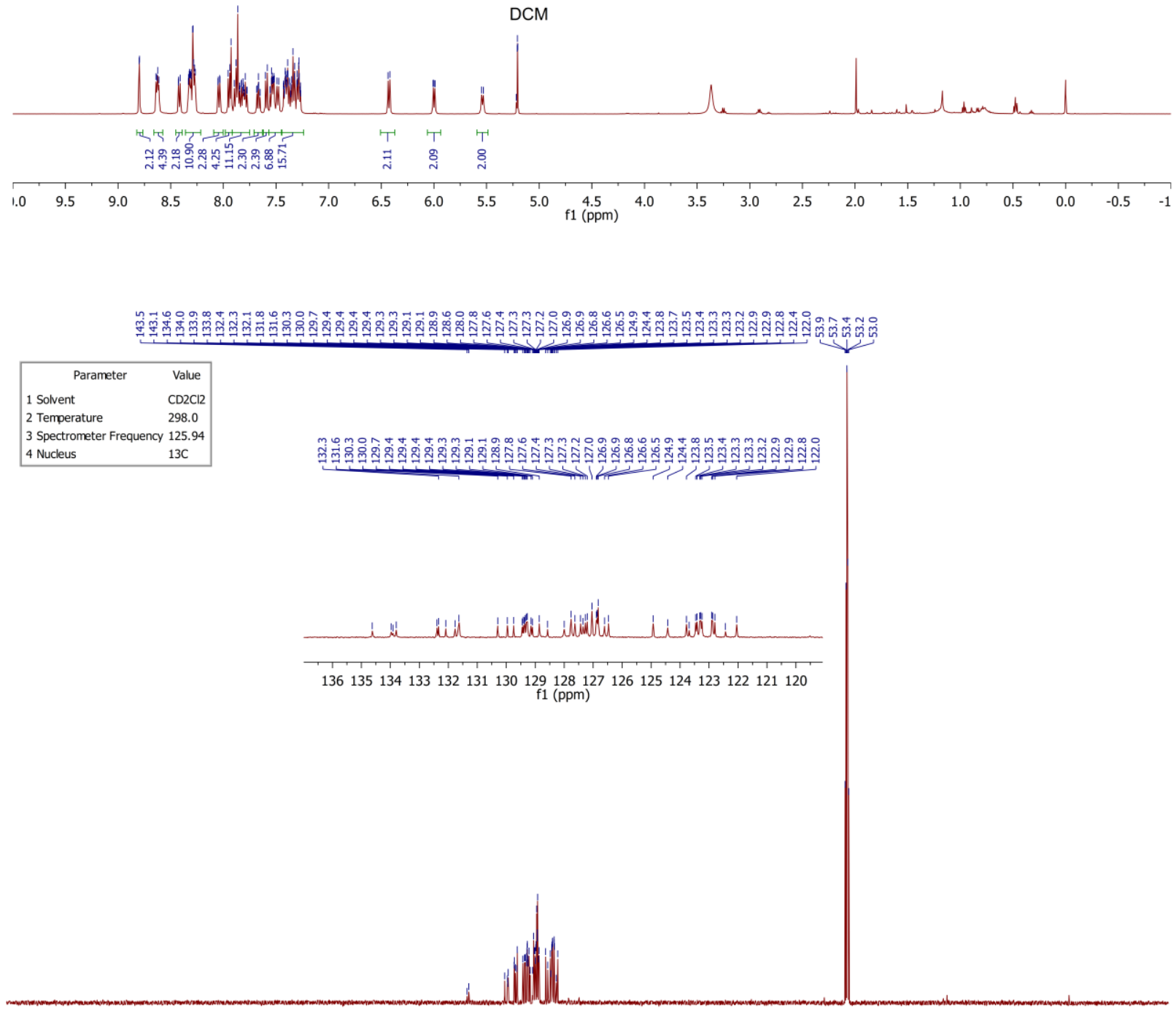

$\begin{array}{rlllllllllllllllllllllllllllllllll}250 & 240 & 230 & 220 & 210 & 200 & 190 & 180 & 170 & 160 & 150 & 140 & 130 & 120 & 110 & 100 & 90 & 80 & 70 & 60 & 50 & 40 & 30 & 20 & 10 & 0 & -10 & -20\end{array}$ 


\begin{tabular}{|lc|}
\hline \multicolumn{1}{|c|}{ Parameter } & Value \\
1 Solvent & $\mathrm{CD} 2 \mathrm{Cl} 2$ \\
2 Temperature & 298.0 \\
3 Spectrometer Frequency & 471.21 \\
4 Nucleus & $19 \mathrm{~F}$ \\
\hline
\end{tabular}

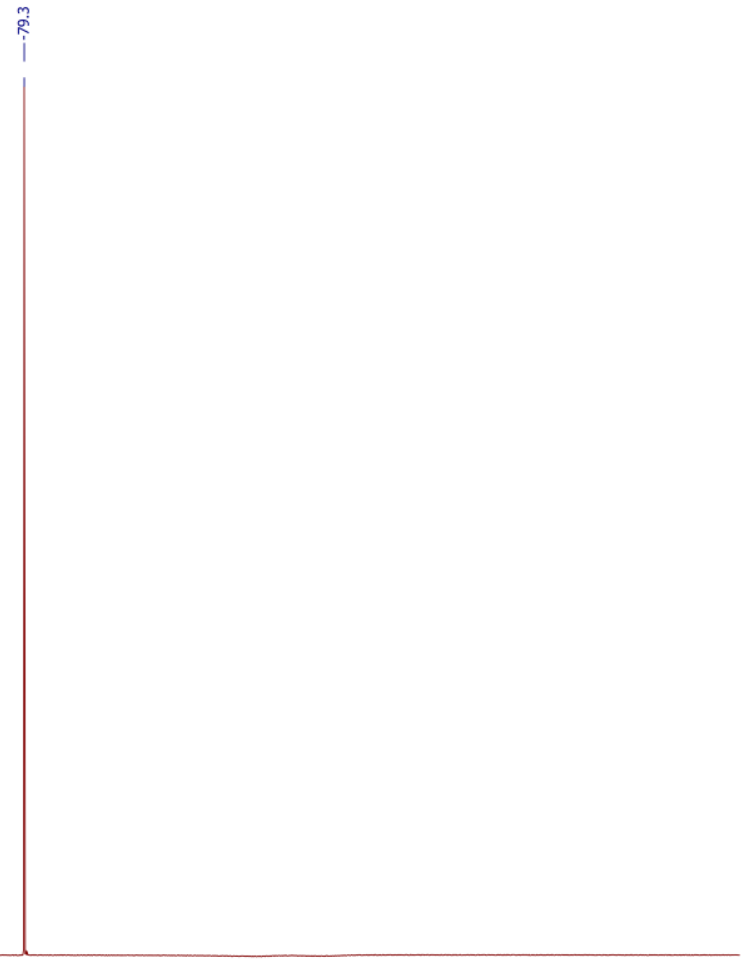

$\begin{array}{llllllllllllllllllllllllllllllll}240 & 220 & 200 & 180 & 160 & 140 & 120 & 100 & 80 & 60 & 40 & 20 & 0 & -20 & -40 & -60 & -80 & -100 & -120 & -140 & -160 & -180 & -200 & -220 & -240 & -260 & -280 & -300 & -320 & -341\end{array}$

\begin{tabular}{|lc|}
\hline \multicolumn{1}{|c|}{ Parameter } & \multicolumn{1}{c|}{ Value } \\
1 Solvent & $\mathrm{CD} 2 \mathrm{Cl2}$ \\
2 Temperature & 298.0 \\
3 Spectrometer Frequency 202.73 \\
4 Nucleus & $31 \mathrm{P}$ \\
\hline
\end{tabular}

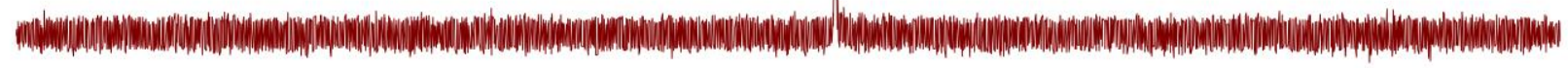

$\begin{array}{llllllllllllllllllllllll}220 & 200 & 180 & 160 & 140 & 120 & 100 & 80 & 60 & 40 & 20 & \begin{array}{c}0 \\ \mathrm{f} 1(\mathrm{ppm})\end{array} & -20 & -40 & -60 & -80 & -100 & -120 & -140 & -160 & -180 & -200 & -220\end{array}$ 
((perfluoronaphthalen-2-yl)sulfonyl)phosphorimidoyl trichloride $\mathrm{R}_{\mathrm{f}}-6$ :

\begin{tabular}{|lc|}
\hline \multicolumn{1}{|c}{ Parameter } & Value \\
1 Solvent & CDCl3 \\
2 Temperature & 298.0 \\
3 Spectrometer Frequency & 471.21 \\
4 Nucleus & $19 \mathrm{~F}$ \\
\hline
\end{tabular}
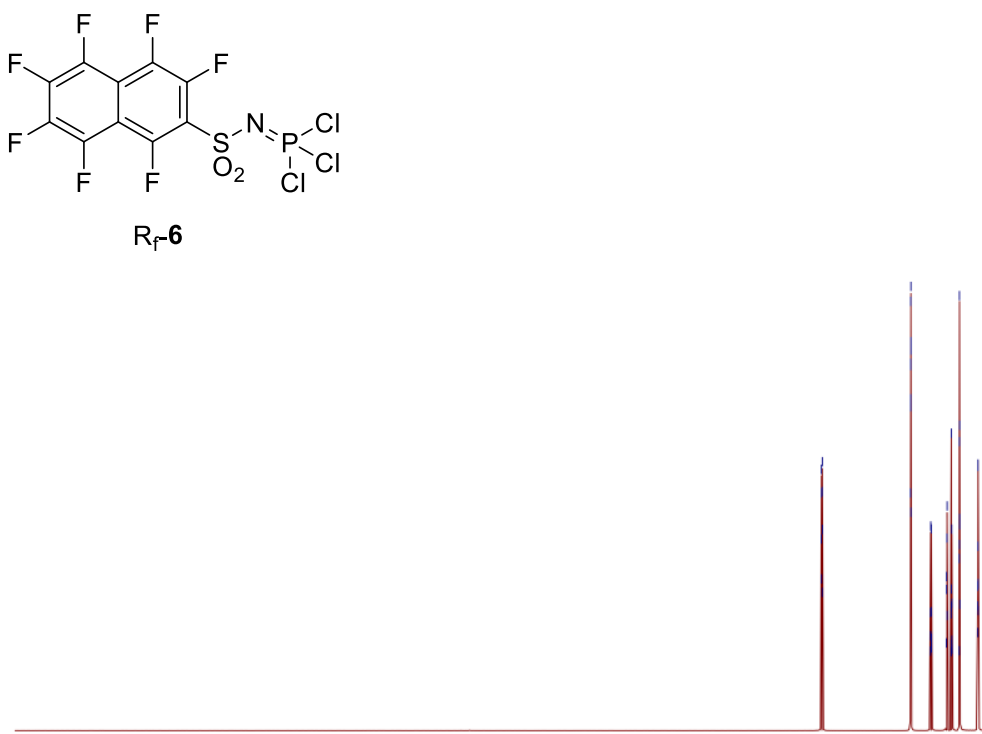

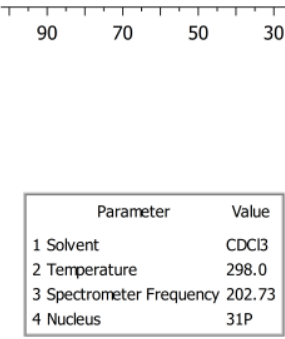

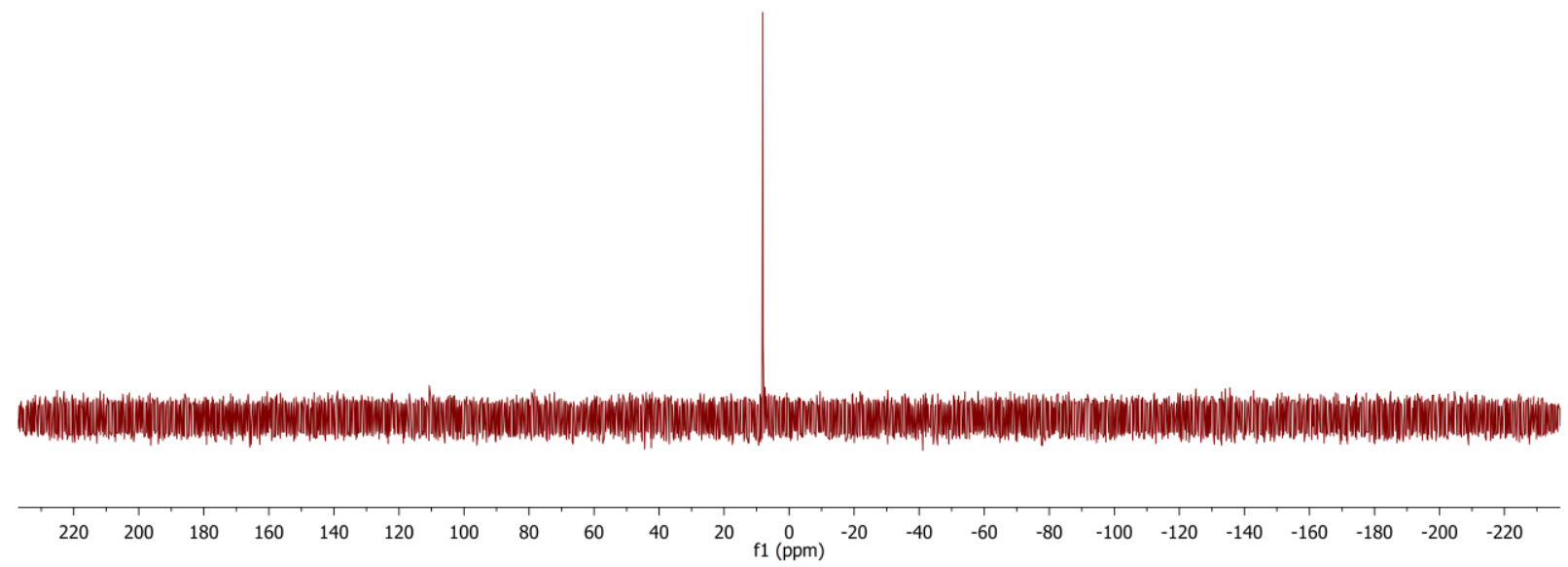




\section{$(S, S)$-IDPi-4f:}

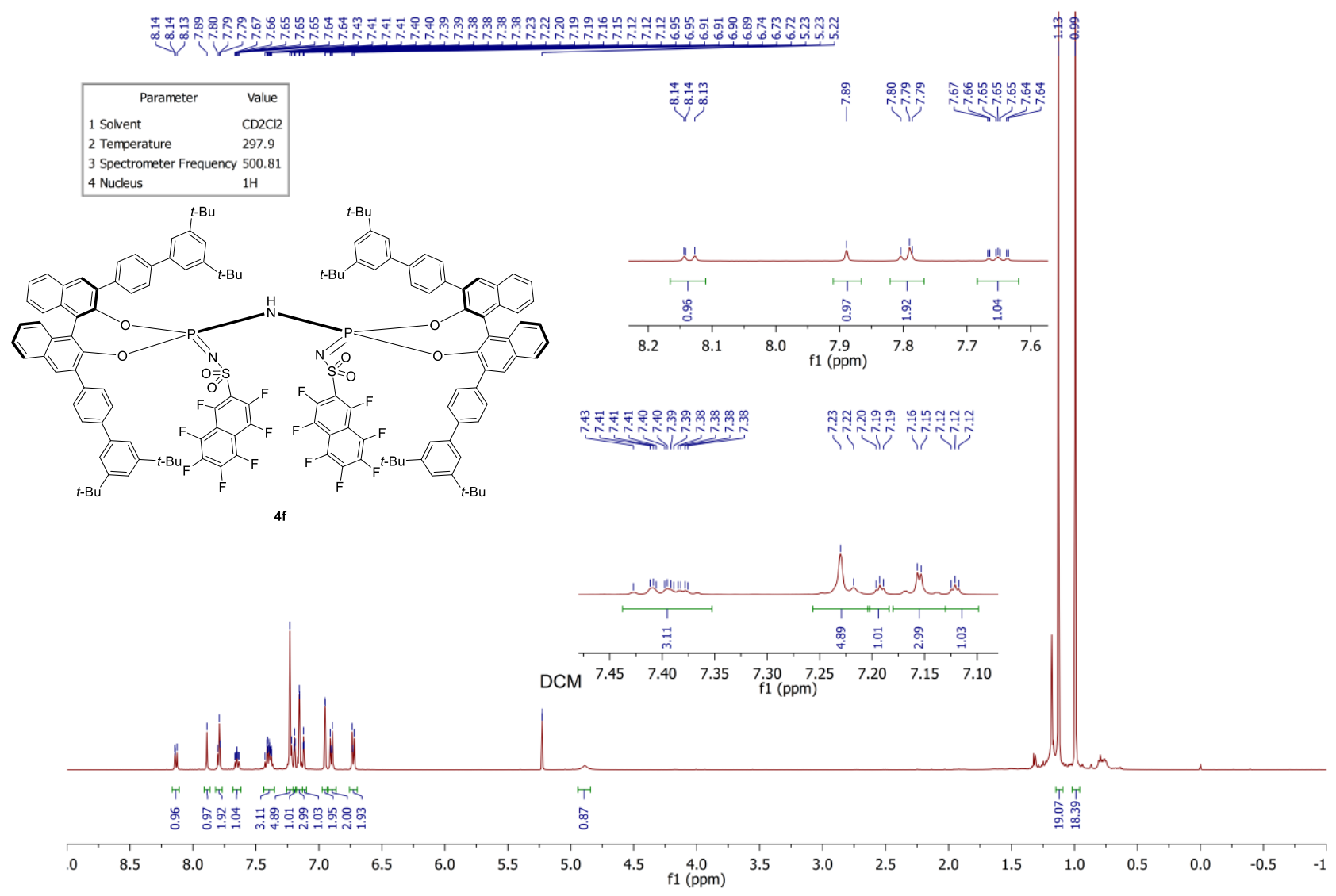

\begin{tabular}{|ll|}
\hline \multicolumn{1}{|c|}{ Parameter } & \multicolumn{1}{c|}{ Value } \\
1 Solvent & CD2C12 \\
2 Temperature & 298.0 \\
3 Spectrometer Frequency & 125.94 \\
4 Nucleus & $13 \mathrm{C}$ \\
\hline
\end{tabular}

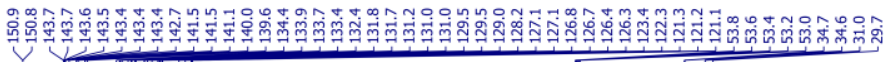

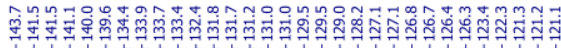

रा।

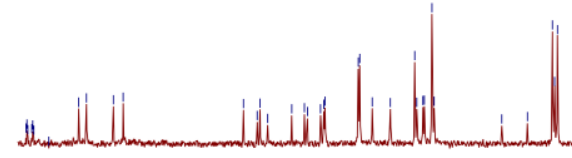

$\begin{array}{lllllllllllllll}144 & 142 & 140 & 138 & 136 & 134 & 132 & 130 & 128 & 126 & 124 & 122 & 120\end{array}$

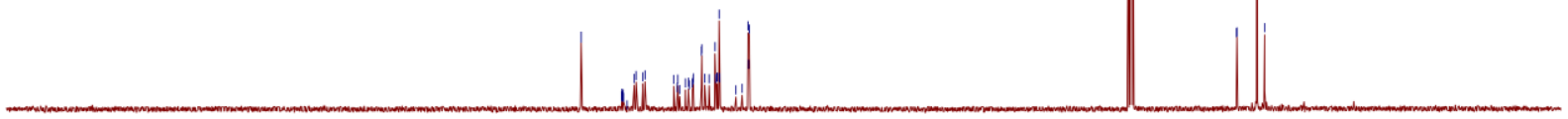

$\begin{array}{llllllllllllllllllllllllllllllll}250 & 240 & 230 & 220 & 210 & 200 & 190 & 180 & 170 & 160 & 150 & 140 & 130 & 120 & 110 & 100 & 90 & 80 & 70 & 60 & 50 & 40 & 30 & 20 & 10 & 0 & -10 & -20\end{array}$ soo 


\begin{tabular}{|lc|}
\hline \multicolumn{1}{|c}{ Parameter } & \multicolumn{1}{c|}{ Value } \\
1 Solvent & $\mathrm{CD} 2 \mathrm{Cl} 2$ \\
2 Temperature & 298.0 \\
3 Spectrometer Frequency & 471.21 \\
4 Nucleus & $19 \mathrm{~F}$ \\
\hline
\end{tabular}

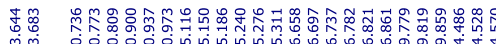

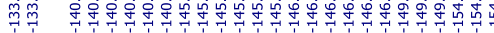

3 Spectrometer Frequency 471.2

$Y$ v V V

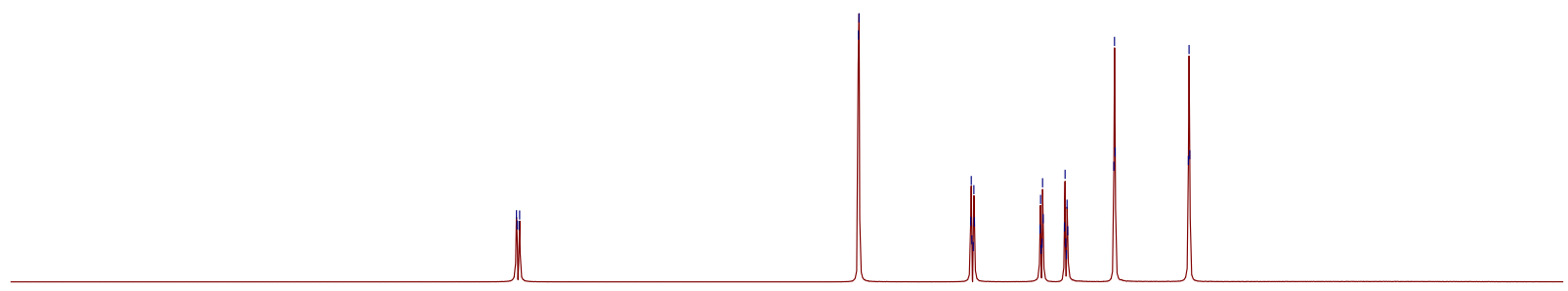

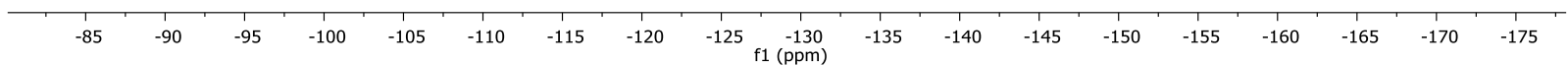

\begin{tabular}{|lc|}
\hline \multicolumn{1}{|c|}{ Parameter } & \multicolumn{1}{c|}{ Value } \\
1 Solvent & $\mathrm{CD} 2 \mathrm{Cl} 2$ \\
2 Temperature & 298.0 \\
3 Spectrometer Frequency & 202.73 \\
4 Nucleus & $31 \mathrm{P}$ \\
\hline
\end{tabular}

3 Spectrometer Frequency 202.73

4 Nucleus $\quad 31 \mathrm{P}$

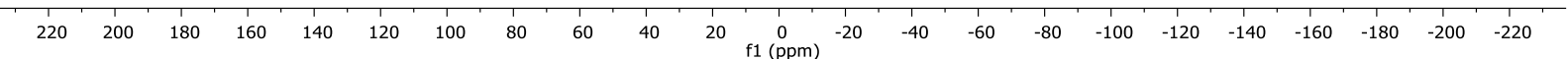


4-(4-chlorophenyl)cyclohexan-1-one 1d:
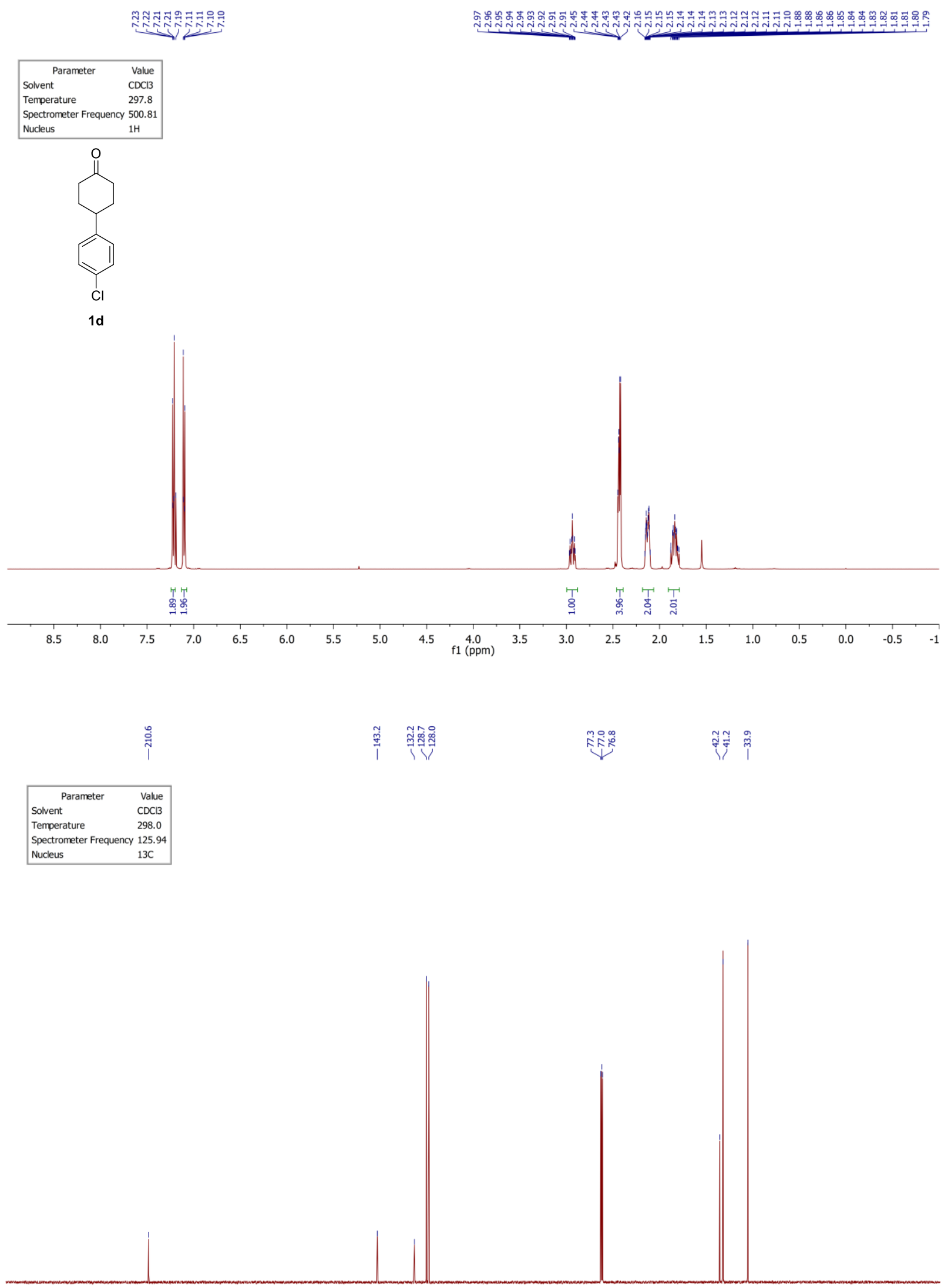

$\begin{array}{lllllllllllllllllllllllllllll}250 & 240 & 230 & 220 & 210 & 200 & 190 & 180 & 170 & 160 & 150 & 140 & 130 & 120 & 110 & 100 & 90 & 80 & 70 & 60 & 50 & 40 & 30 & 20 & 10 & 0 & -10 & -20\end{array}$ ग00 
4-(4-(trifluoromethyl)phenyl)cyclohexan-1-one 1f:

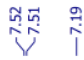

\begin{tabular}{|lc|}
\hline \multicolumn{1}{|c|}{ Parameter } & Value \\
Solvent & CDCl3 \\
Temperature & 297.9 \\
Spectrometer Frequency & 500.81 \\
Nucleus & $1 \mathrm{H}$ \\
\hline
\end{tabular}

Nucleus $1 \mathrm{H}$

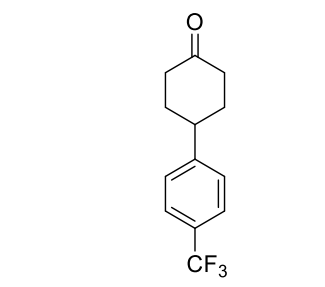

$1 \mathrm{f}$

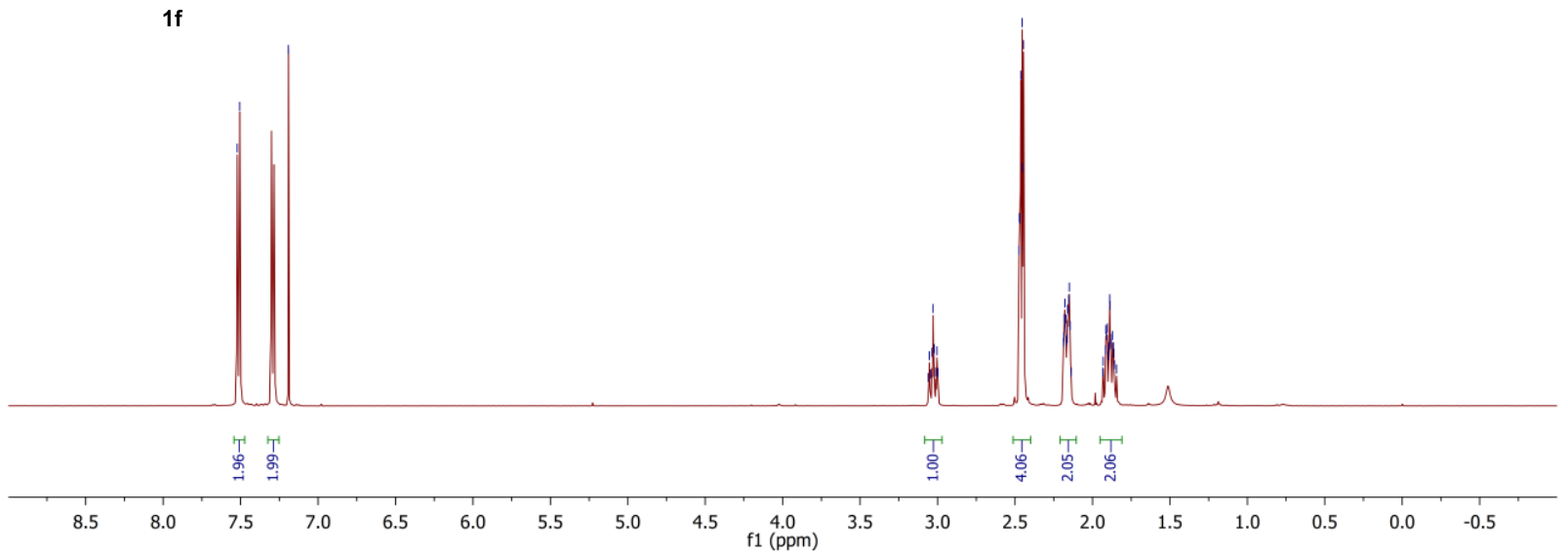

竞

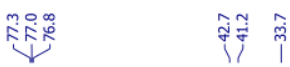

\begin{tabular}{|ll|}
\hline \multicolumn{1}{|c}{ Parameter } & \multicolumn{1}{c|}{ Value } \\
Solvent & CDCl3 \\
Temperature & 298.0 \\
Spectrometer Frequency & 125.94 \\
Nucleus & $13 \mathrm{C}$ \\
\hline
\end{tabular}

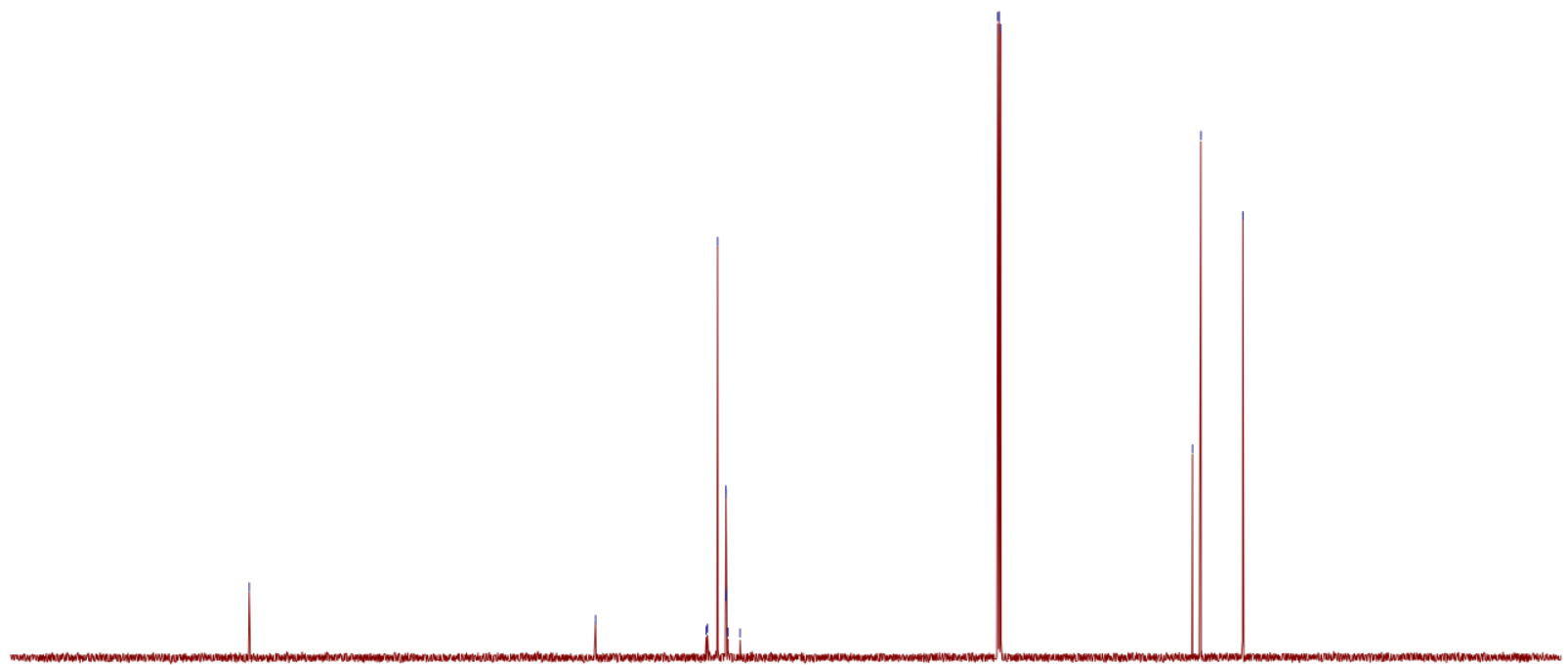

$\begin{array}{llllllllllllllllllllllllllll}250 & 240 & 230 & 220 & 210 & 200 & 190 & 180 & 170 & 160 & 150 & 140 & 130 & \begin{array}{l}120 \\ \mathrm{f} 1(\mathrm{ppm})\end{array} & 100 & 90 & 80 & 70 & 60 & 50 & 40 & 30 & 20 & 10 & 0 & -10 & -20\end{array}$ 


\begin{tabular}{|ll|}
\hline \multicolumn{1}{|c|}{ Parameter } & \multicolumn{1}{c|}{ Value } \\
Solvent & CD2Cl2 \\
Temperature & 297.9 \\
Spectrometer Frequency & 471.21 \\
Nucleus & $19 \mathrm{~F}$ \\
\hline
\end{tabular}

\section{4-(3-(tert-butyl)phenyl)cyclohexan-1-one 1g:}

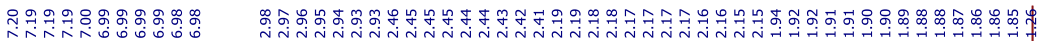

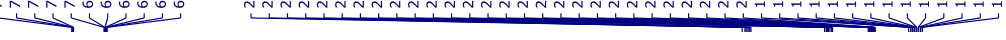

\begin{tabular}{|c|c|}
\hline Parameter & Value \\
\hline Solvent & $\mathrm{CDCl} 3$ \\
\hline Temperature & 297.9 \\
\hline Spectrometer Fr & y 500.81 \\
\hline Nucleus & $1 \mathrm{H}$ \\
\hline
\end{tabular}

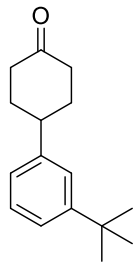

$1 \mathrm{~g}$

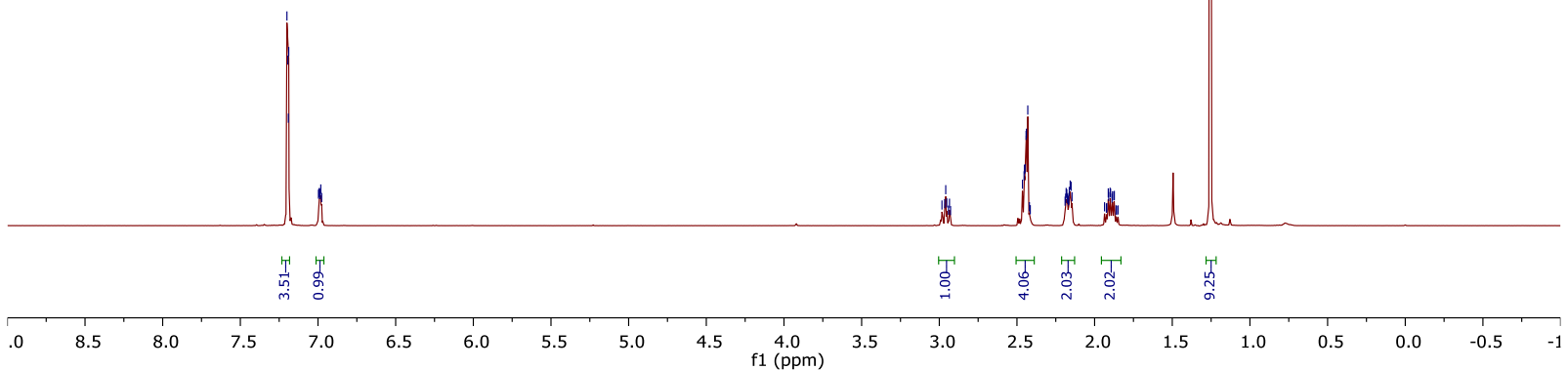




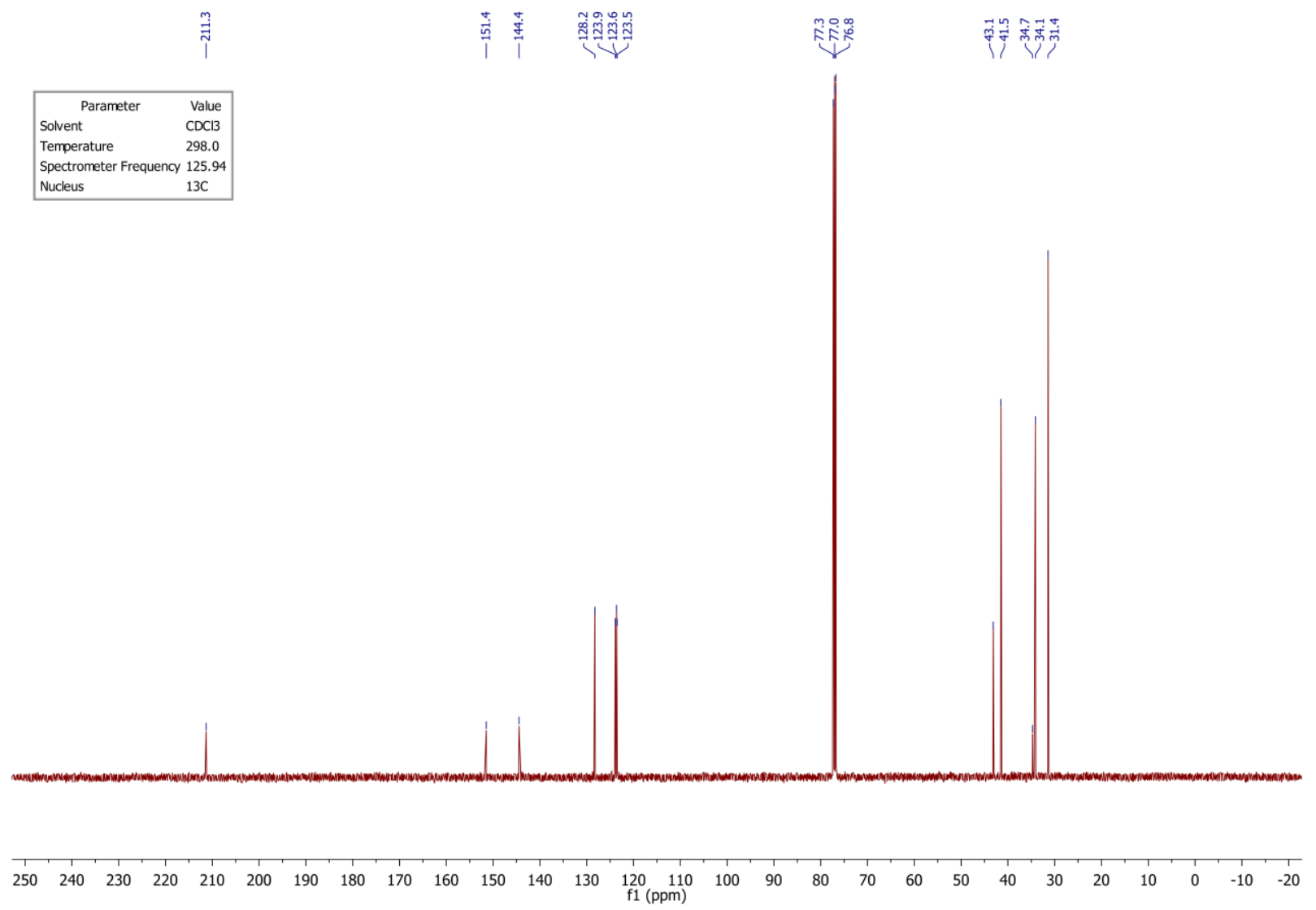

\section{4-(3-chlorophenyl)cyclohexan-1-one 1h:}

\begin{tabular}{|lr|}
\hline \multicolumn{1}{|c|}{ Parameter } & Value \\
Solvent & CDC13 \\
Temperature & 297.9 \\
Spectrometer Frequency & 500.81 \\
Nucleus & $1 \mathrm{H}$ \\
\hline
\end{tabular}



1h

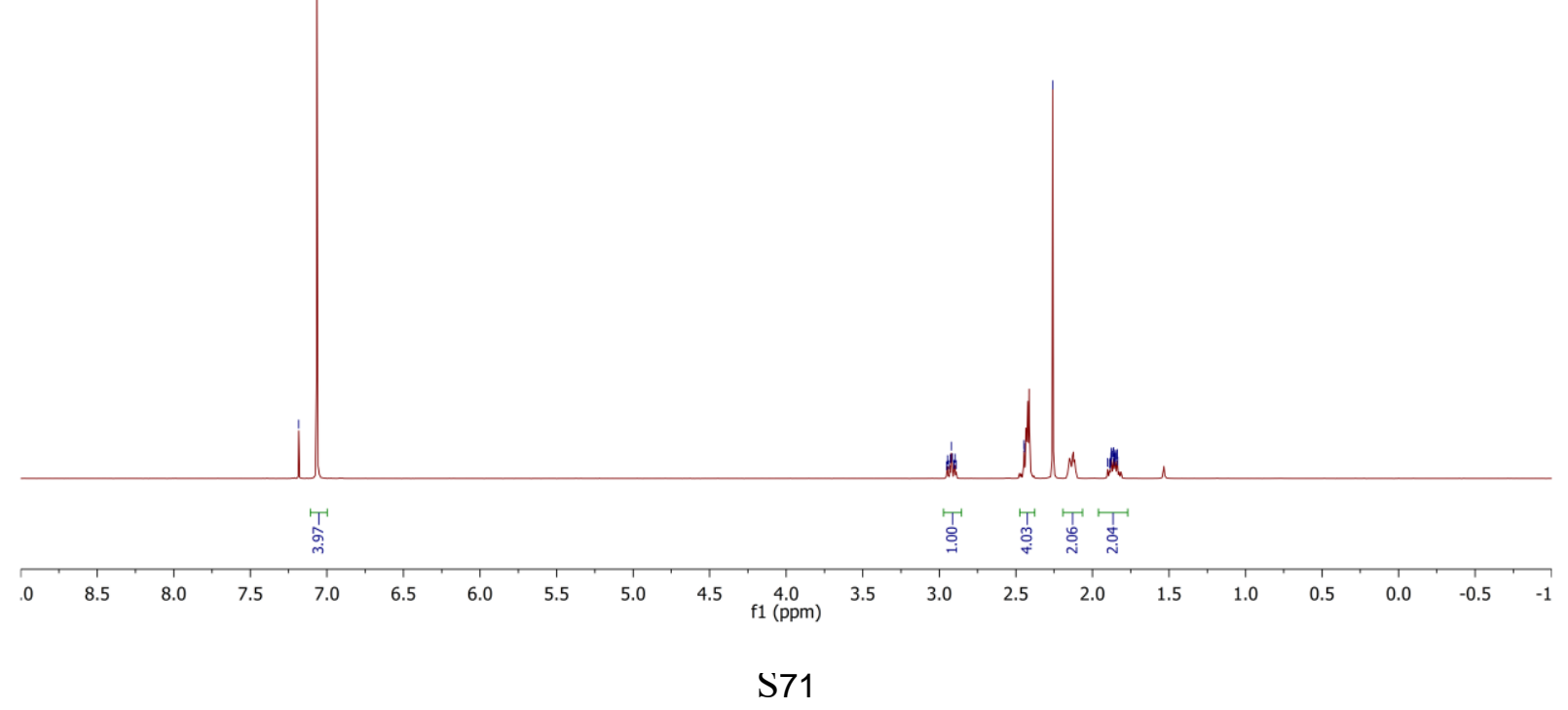



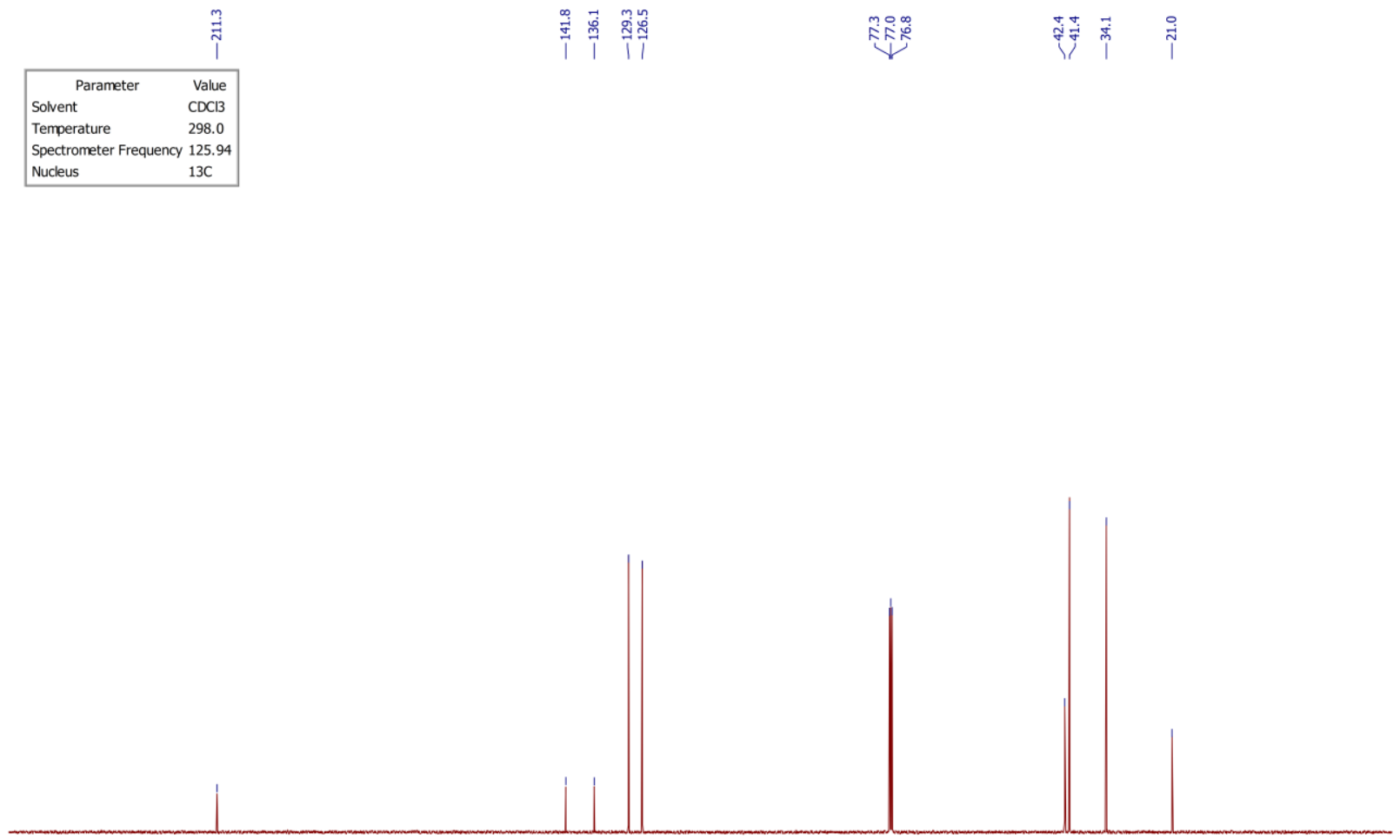

$\begin{array}{llllllllllllllllllllllllllllllllllllll}250 & 240 & 230 & 220 & 210 & 200 & 190 & 180 & 170 & 160 & 150 & 140 & 130 & 120 & 110 & 100 & 90 & 80 & 70 & 60 & 50 & 40 & 30 & 20 & 10 & 0 & -10 & -20\end{array}$

\section{4-(3-(trifluoromethyl)phenyl)cyclohexan-1-one 1i:}
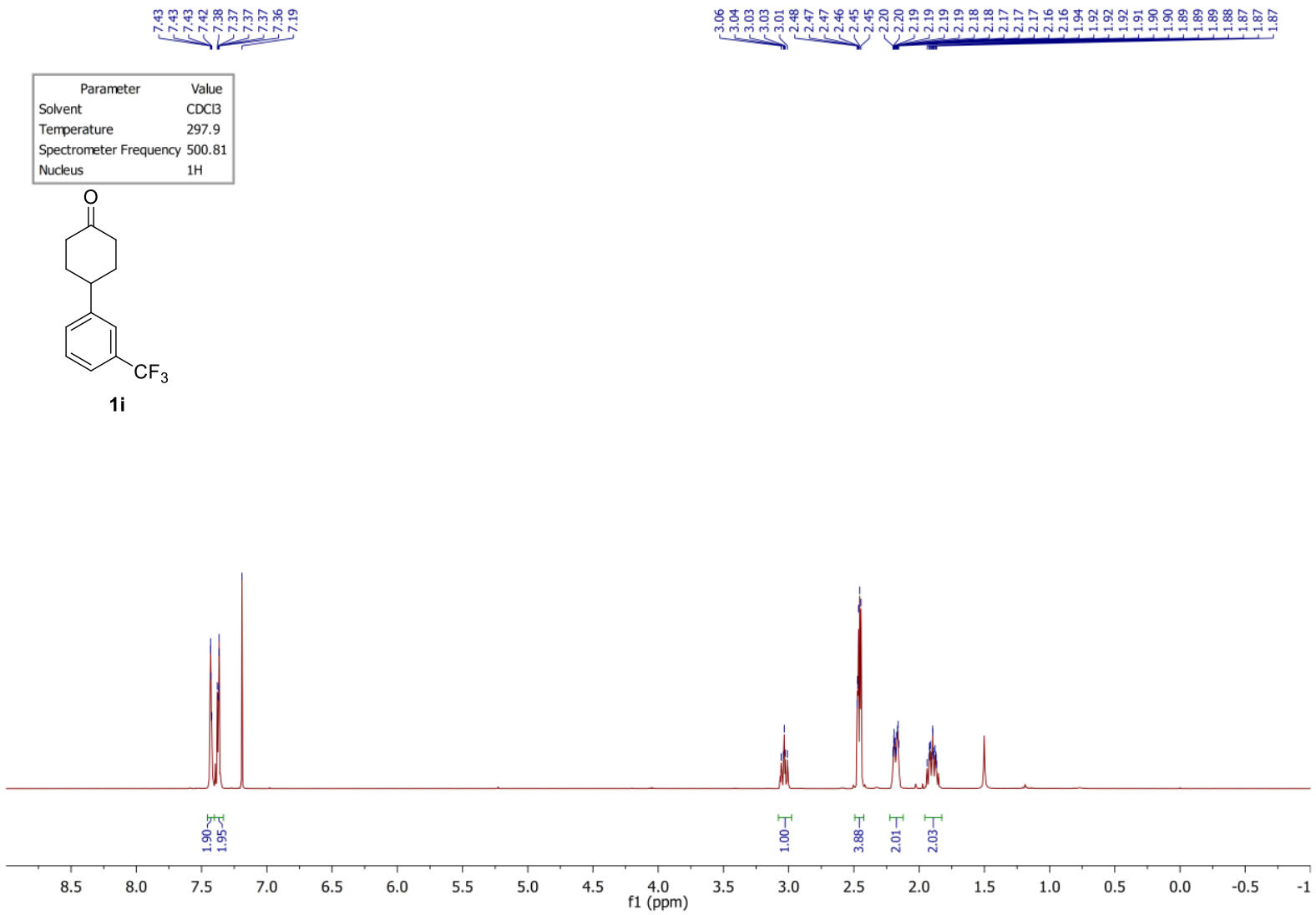


\begin{tabular}{|lc|}
\hline \multicolumn{1}{|c|}{ Parameter } & Value \\
Solvent & $\mathrm{CDC13}$ \\
Temperature & 298.0 \\
Spectrometer Frequency & 125.94 \\
Nucleus & $13 \mathrm{C}$ \\
\hline
\end{tabular}

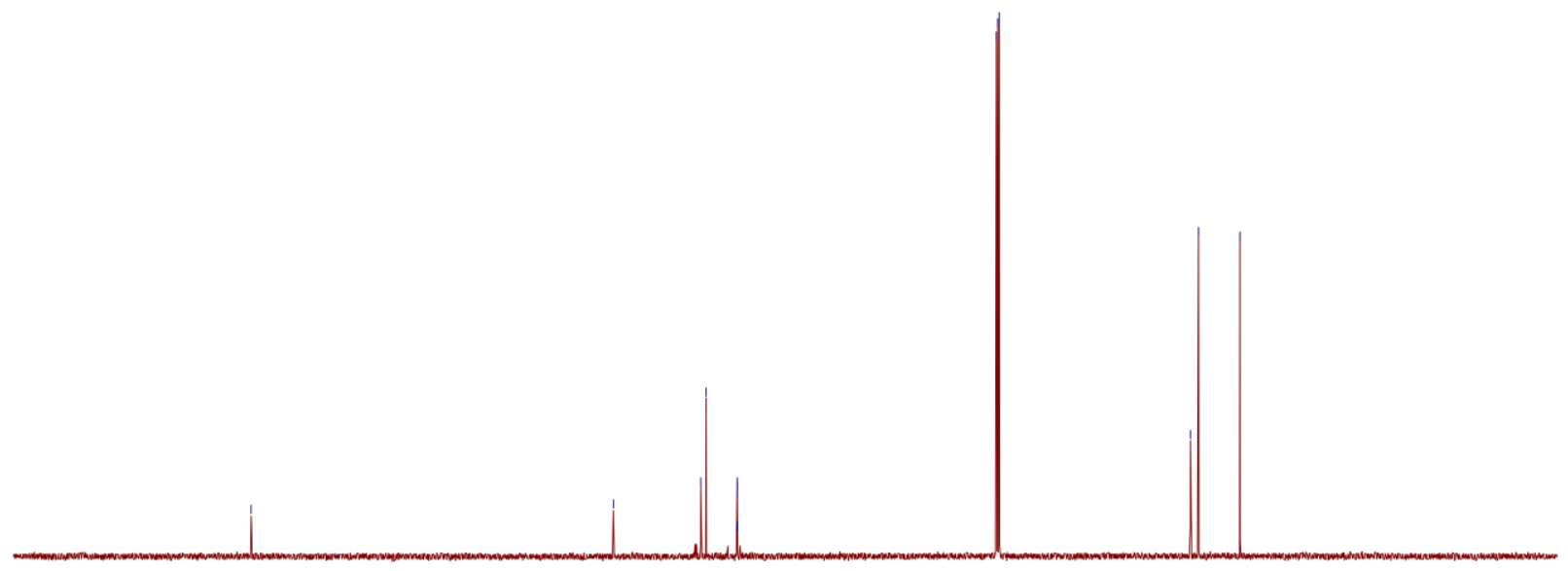

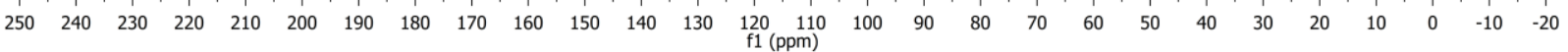

\begin{tabular}{|lc|}
\hline \multicolumn{1}{|c|}{ Parameter } & Value \\
Solvent & CD2C12 \\
Temperature & 298.0 \\
Spectrometer Frequency & 471.21 \\
Nucleus & $19 \mathrm{~F}$ \\
\hline
\end{tabular}

Nucleus

$19 \mathrm{~F}$

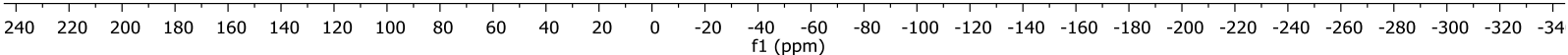


4-(3,5-difluorophenyl)cyclohexan-1-one 11:

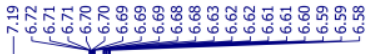

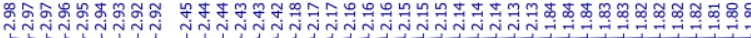

\begin{tabular}{|lc|}
\hline \multicolumn{1}{|c}{ Parameter } & Value \\
Solvent & CDC13 \\
Temperature & 297.9 \\
Spectrometer Frequency & 500.81 \\
Nucleus & $1 \mathrm{H}$ \\
\hline
\end{tabular}

Nucleus

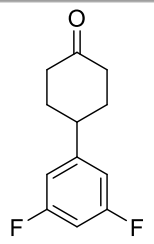

11
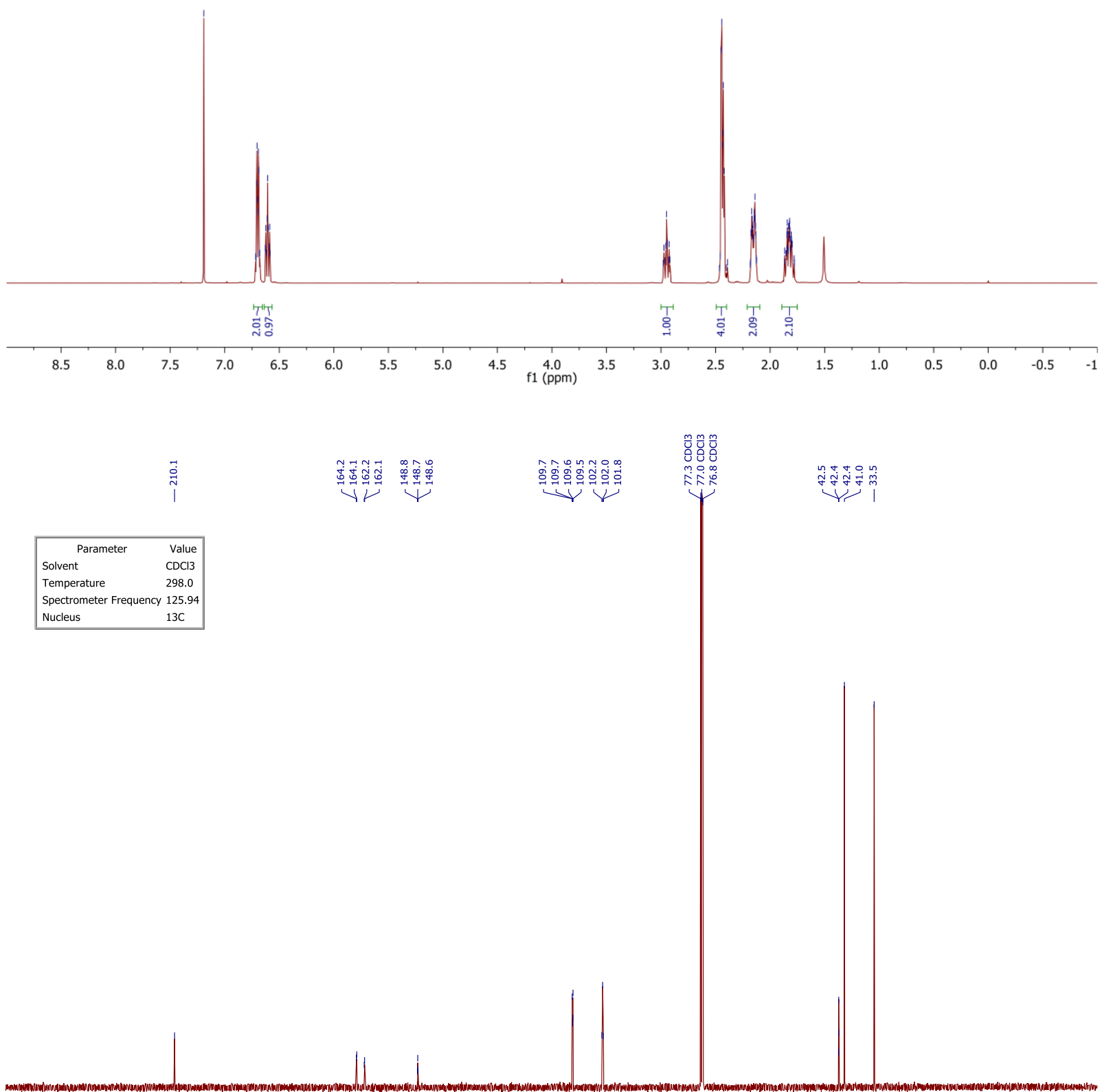

$\begin{array}{lllllllllllllllllllllllllllllllll}250 & 240 & 230 & 220 & 210 & 200 & 190 & 180 & 170 & 160 & 150 & 140 & 130 & 120 & 110 & 100 & 90 & 80 & 70 & 60 & 50 & 40 & 30 & 20 & 10 & 0 & -10 & -20\end{array}$ 


\begin{tabular}{|ll|}
\hline \multicolumn{1}{|c|}{ Parameter } & Value \\
Solvent & $\mathrm{CDCl3}$ \\
Temperature & 298.0 \\
Spectrometer Frequency & 471.21 \\
Nucleus & $19 \mathrm{~F}$ \\
\hline
\end{tabular}

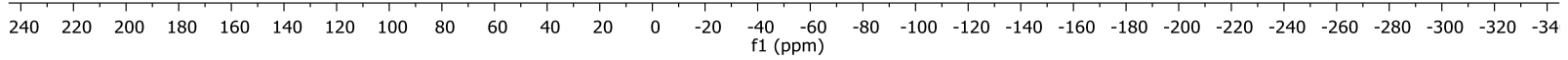

\section{4-(naphthalen-1-yl)cyclohexan-1-one 1m:}

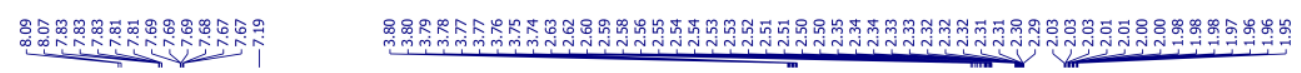

\begin{tabular}{|lr|}
\hline \multicolumn{1}{|c}{ Parameter } & Value \\
Solvent & CDCI3 \\
Temperature & 297.8 \\
Spectrometer Frequency & 500.81 \\
Nucleus & $1 \mathrm{H}$ \\
\hline
\end{tabular}

$1 \mathrm{H}$

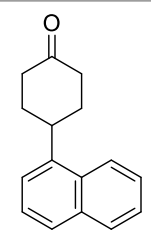

$1 \mathrm{~m}$

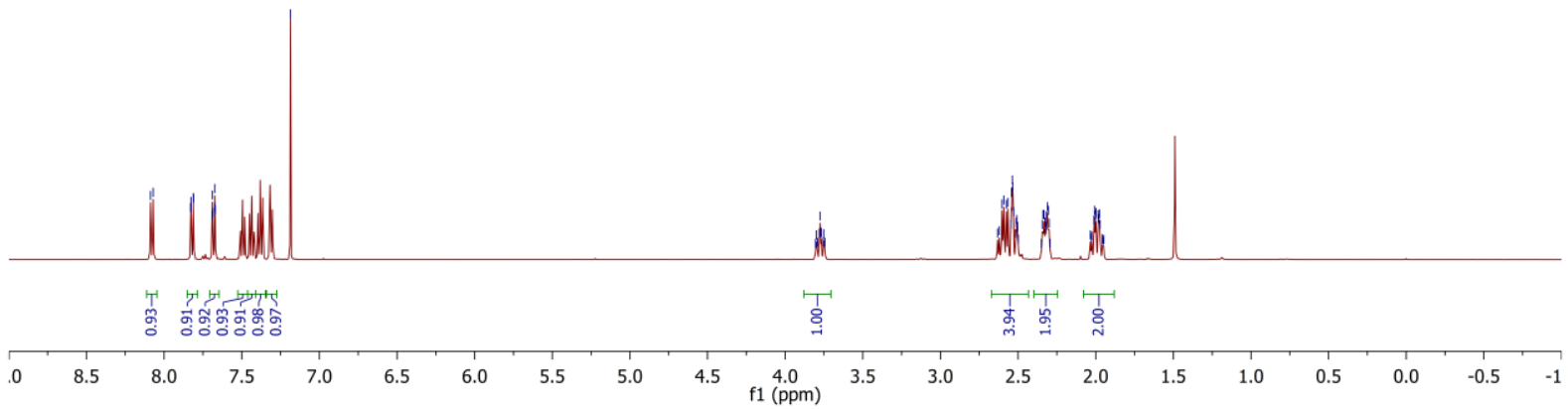




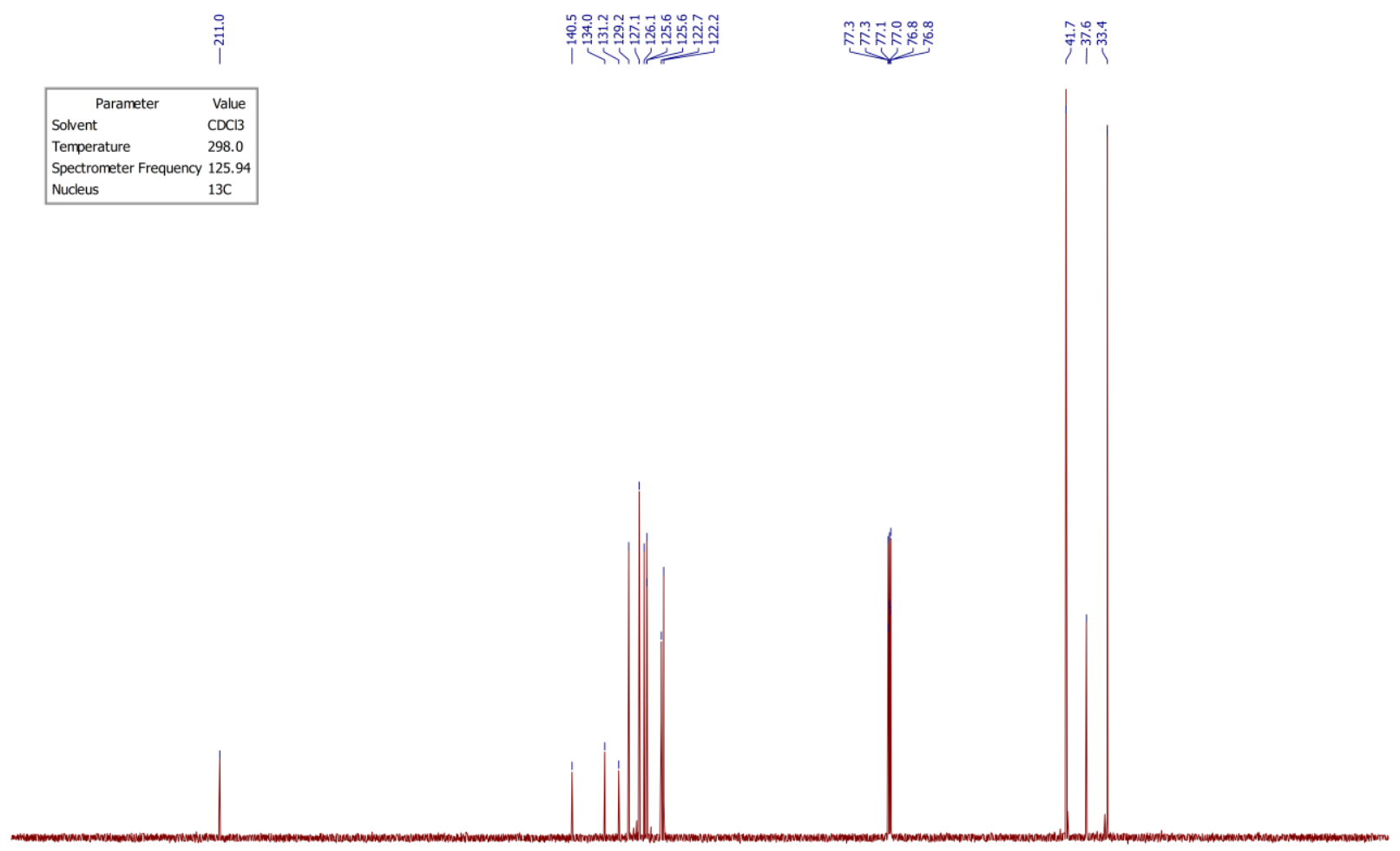

$\begin{array}{rlllllllllllllllllllllllllll}250 & 240 & 230 & 220 & 210 & 200 & 190 & 180 & 170 & 160 & 150 & 140 & 130 & 120 & 110 & 100 & 90 & 80 & 70 & 60 & 50 & 40 & 30 & 20 & 10 & 0 & -10 & -20\end{array}$

4-methyl-4-phenylcyclohexan-1-one 1s:

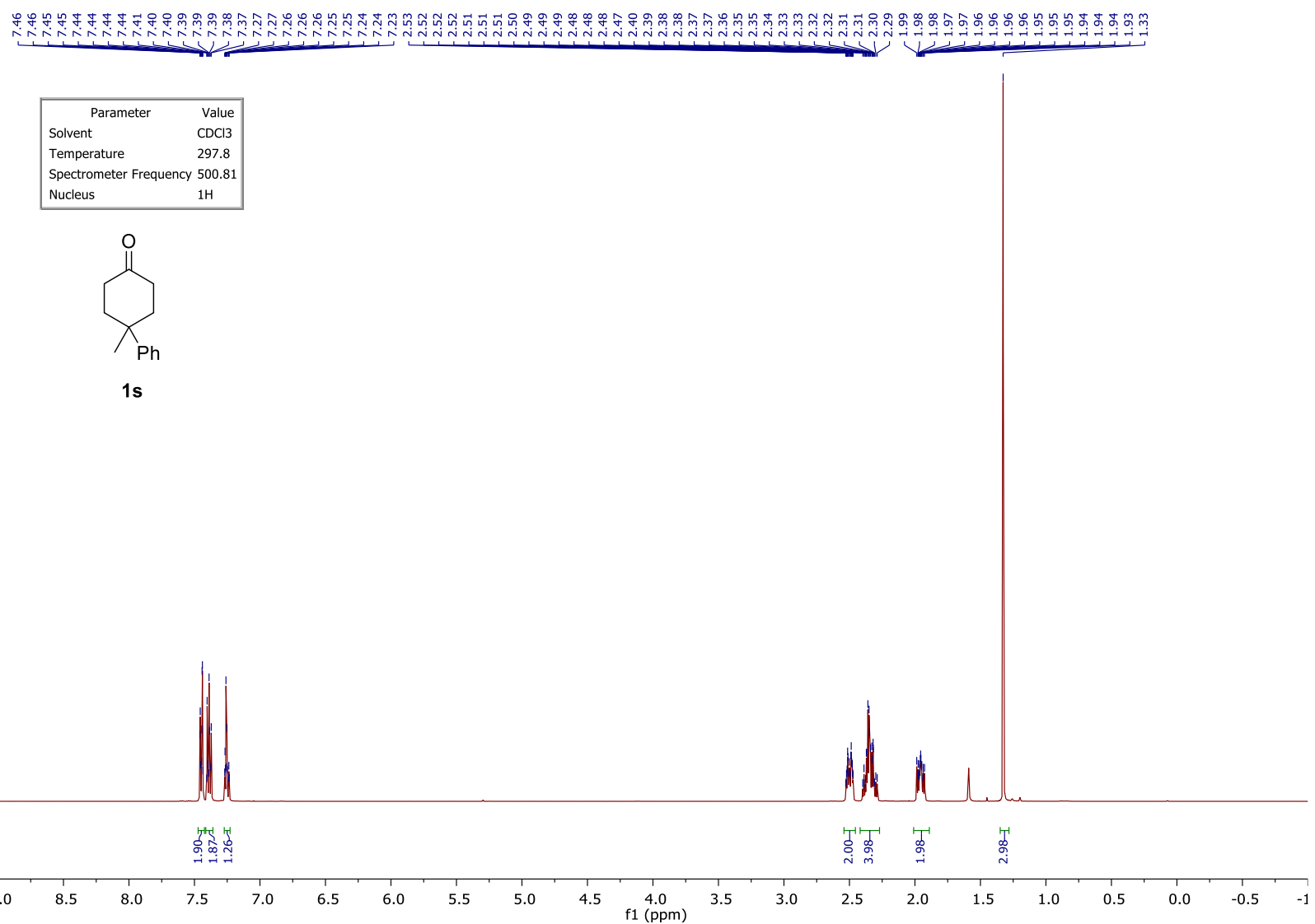




\begin{tabular}{|lc|}
\hline \multicolumn{1}{|c|}{ Parameter } & Value \\
Solvent & $\mathrm{CDC} 13$ \\
Temperature & 298.0 \\
Spectrometer Frequency & 125.94 \\
Nucleus & $13 \mathrm{C}$ \\
\hline
\end{tabular}

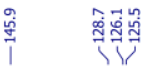

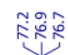

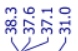

Nucleus $13 \mathrm{C}$

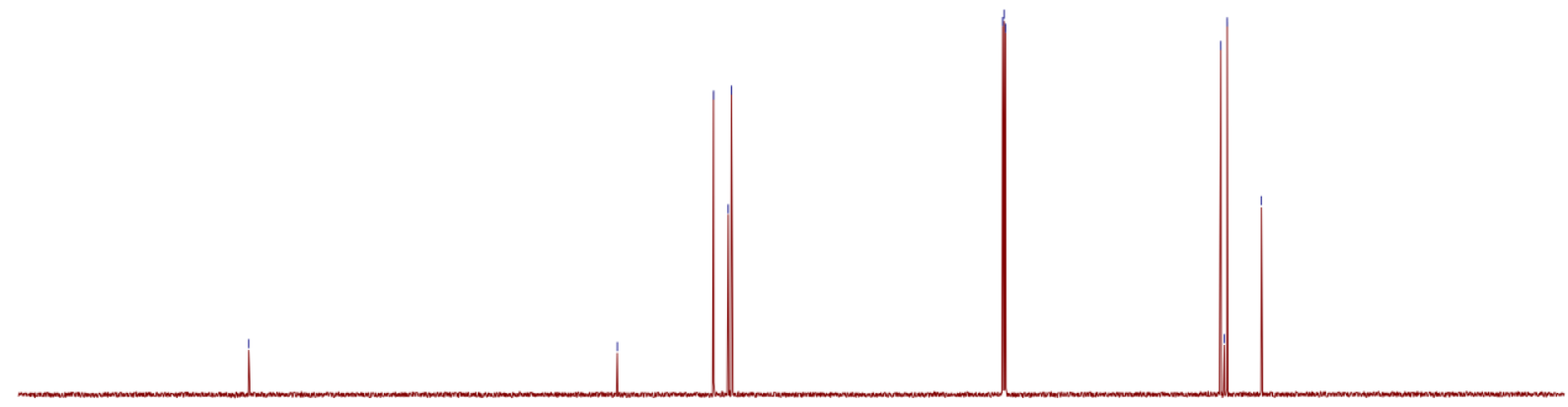

$\begin{array}{llllllllllllllllllllllllllllllllllllll}250 & 240 & 230 & 220 & 210 & 200 & 190 & 180 & 170 & 160 & 150 & 140 & 130 & 120 & 110 & 100 & 90 & 80 & 70 & 60 & 50 & 40 & 30 & 20 & 10 & 0 & -10 & -20\end{array}$

\section{(3aR,6aS)-hexahydropentalen-2(1H)-one $1 \mathrm{u}$ :}

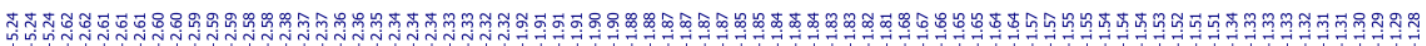

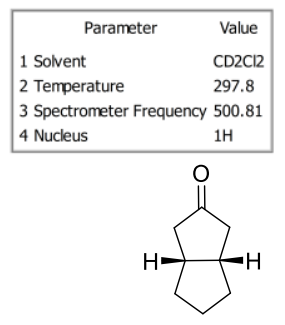

$1 \mathrm{u}$

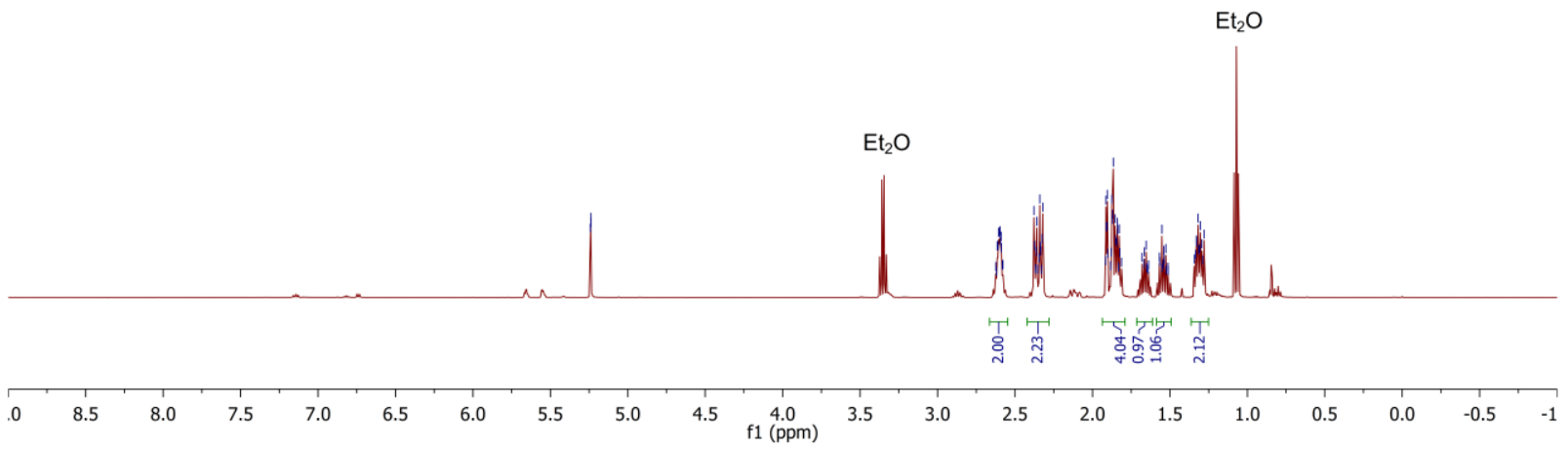



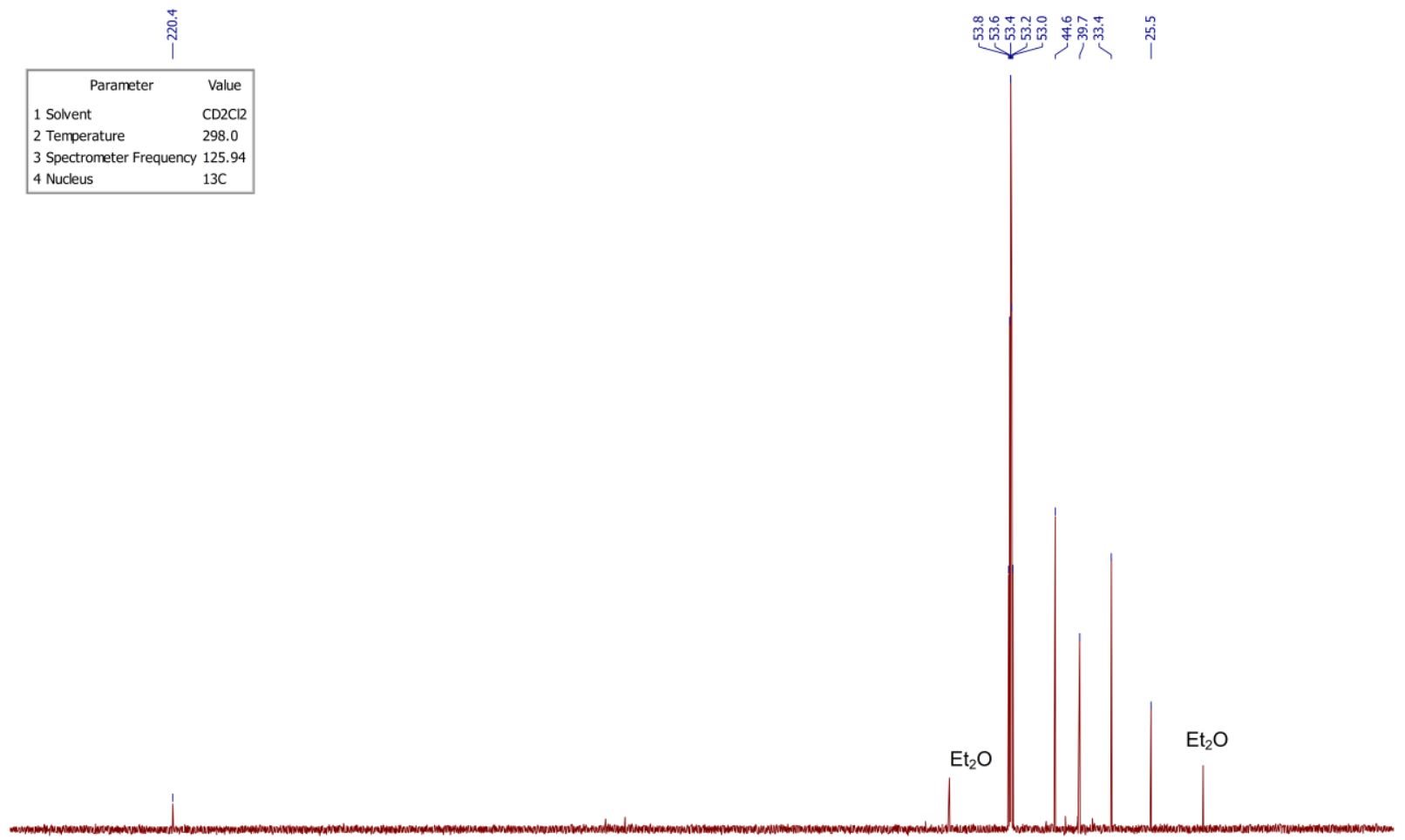

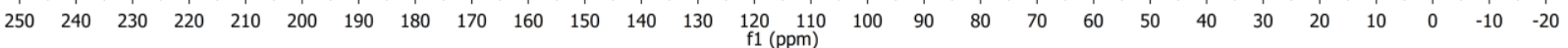

(3aR,6aS)-5',5'-dimethyltetrahydro-1H-spiro[pentalene-2,2'-[1,3]dioxan]-5(3H)-one 1w:
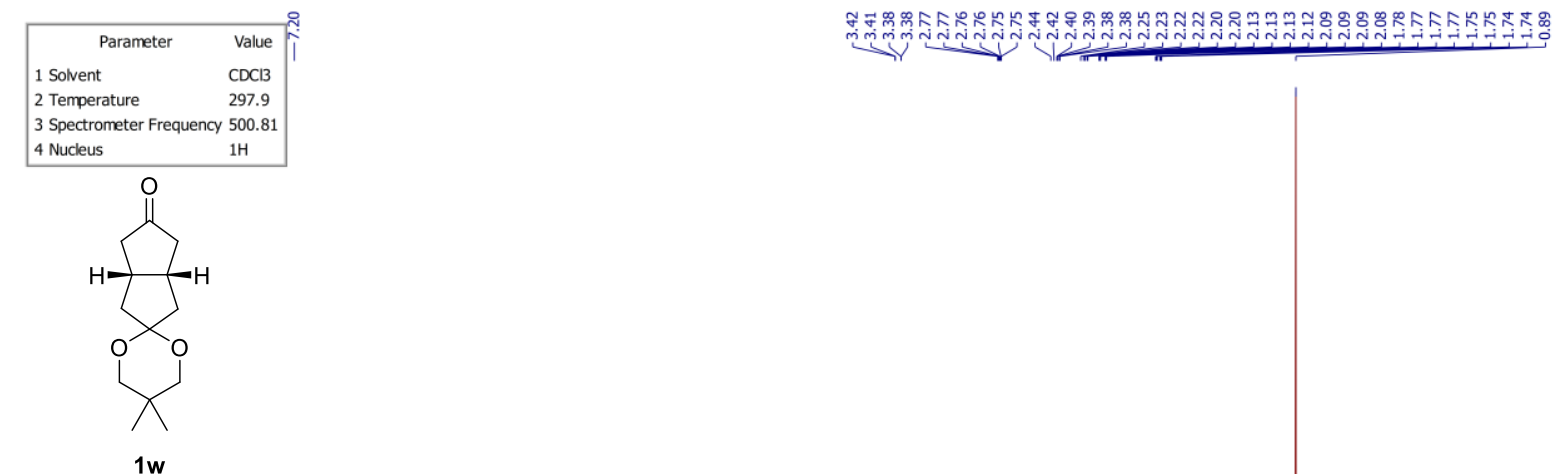

$1 w$

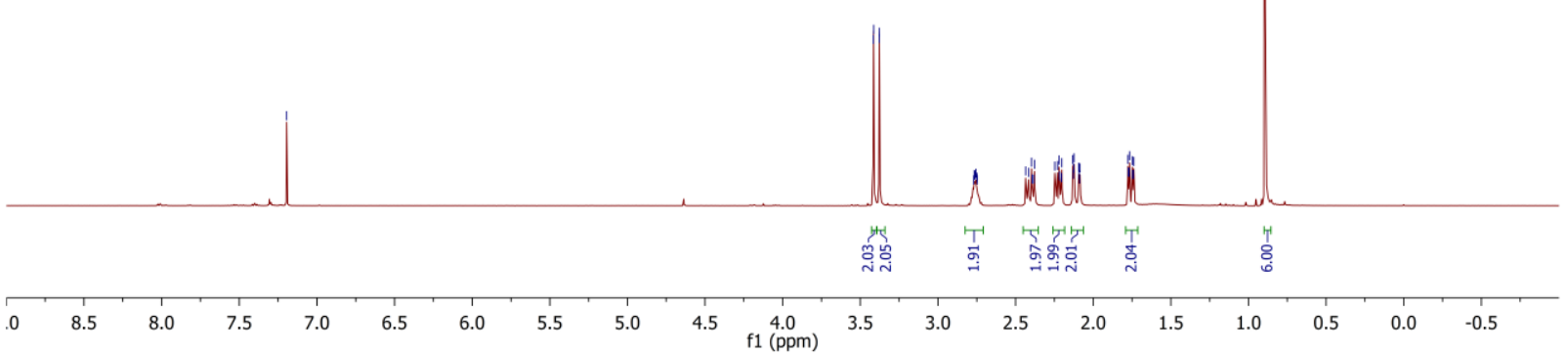




\begin{tabular}{|lc|}
\multicolumn{1}{c|}{} & \multicolumn{1}{c|}{$\overrightarrow{\text { I }}$} \\
\hline \multicolumn{1}{c|}{ Parameter } & Value \\
1 Solvent & CDC13 \\
2 Temperature & 298.0 \\
3 Spectrometer Frequency 125.94 \\
4 Nucleus & $13 \mathrm{C}$ \\
\hline
\end{tabular}

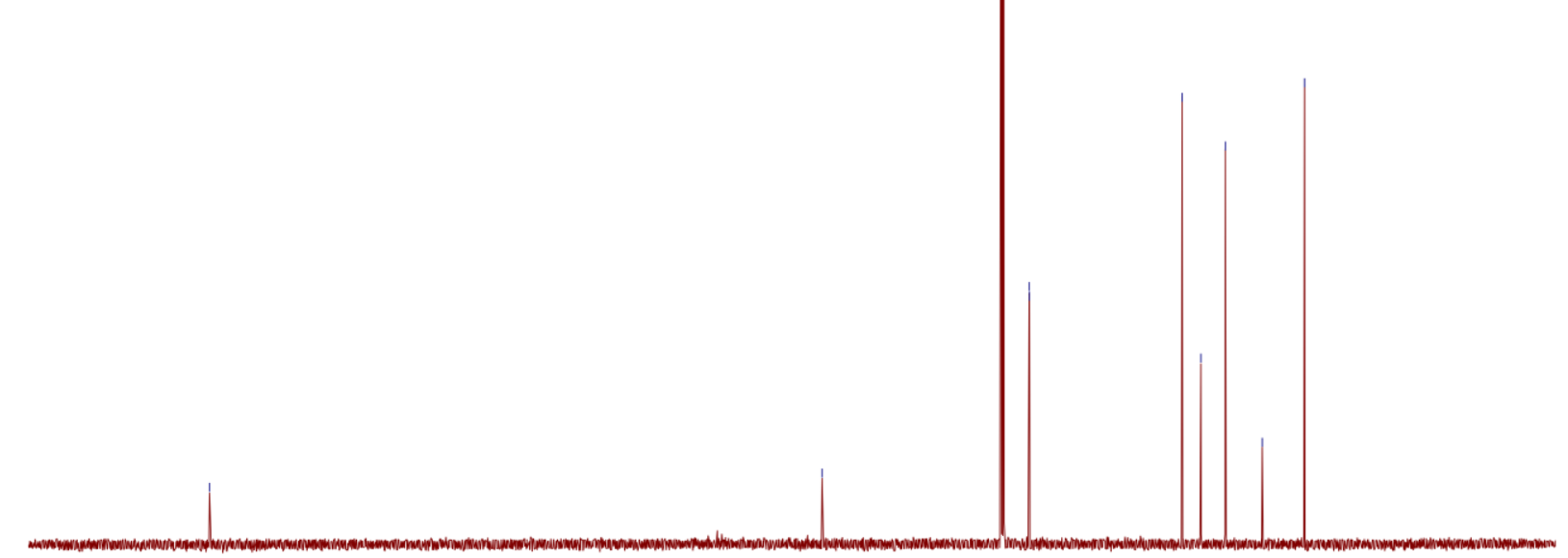

$\begin{array}{llllllllllllllllllllllllllll}250 & 240 & 230 & 220 & 210 & 200 & 190 & 180 & 170 & 160 & 150 & 140 & 130 & \begin{array}{l}\text { f1 } \\ \text { (ppm) }\end{array} & 100 & 90 & 80 & 70 & 60 & 50 & 40 & 30 & 20 & 10 & 0 & -10 & -20\end{array}$

\section{3-phenylcyclobutan-1-one 1v:}

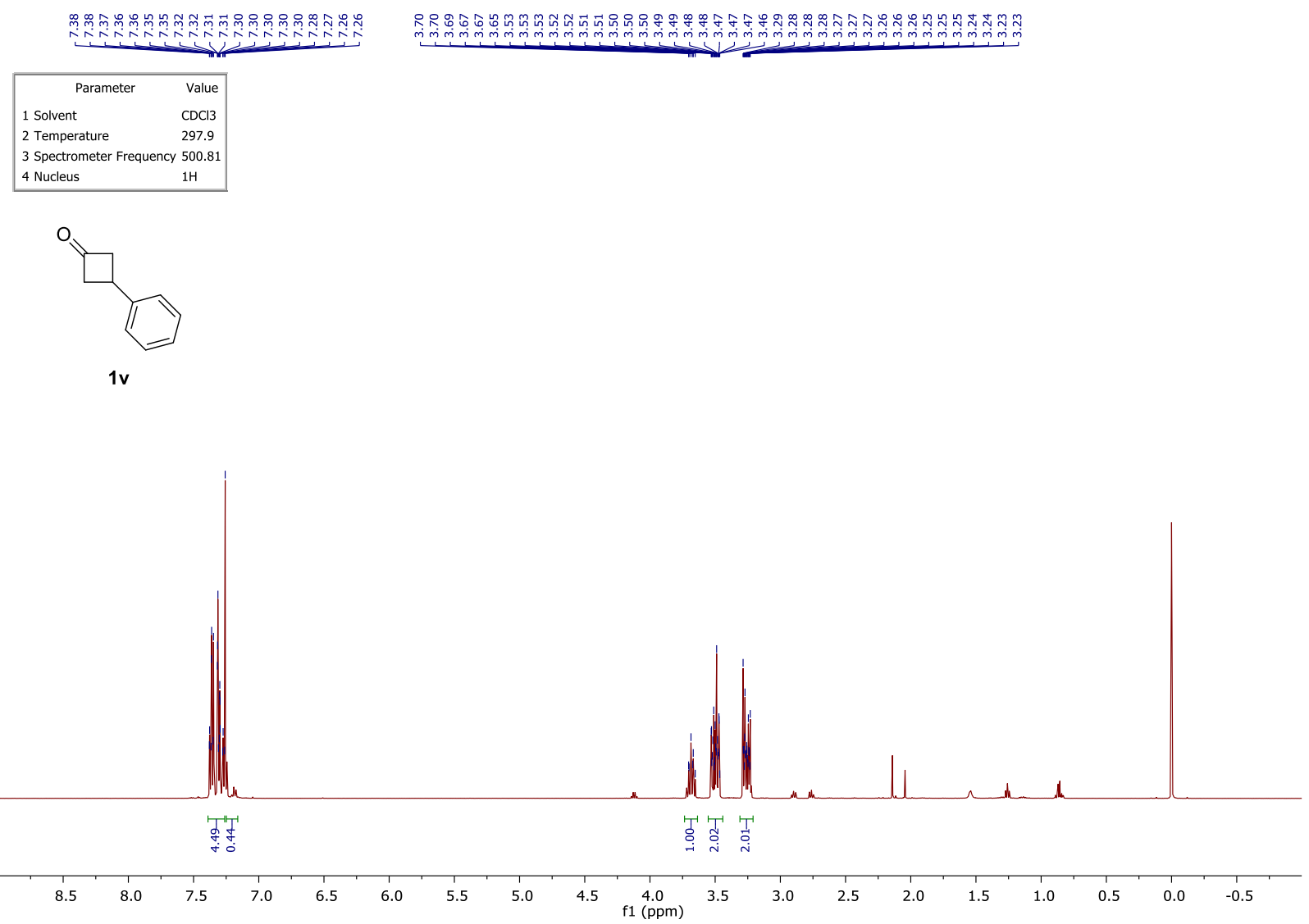



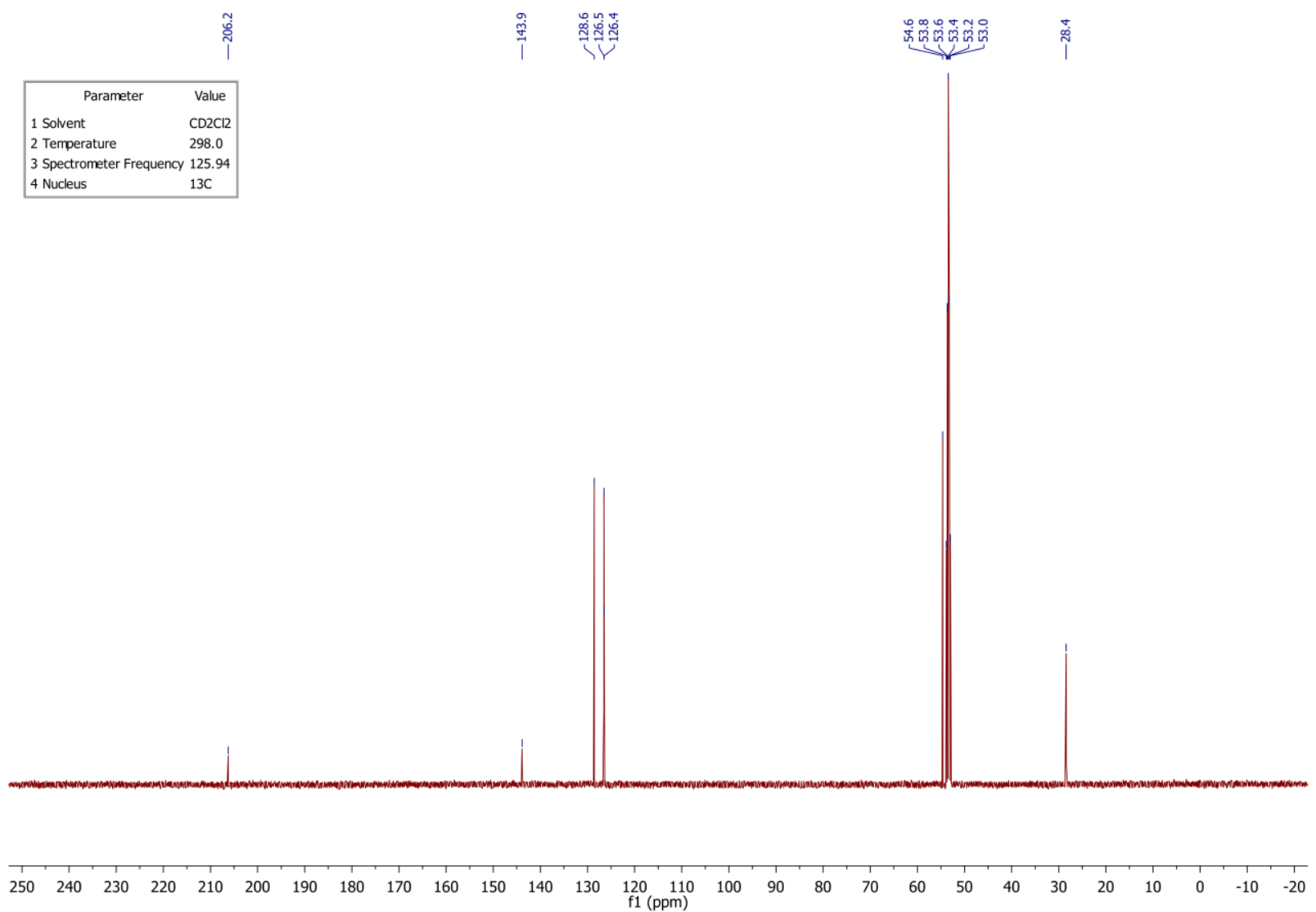

rac-3-phenylcyclohexan-1-one 1ac:

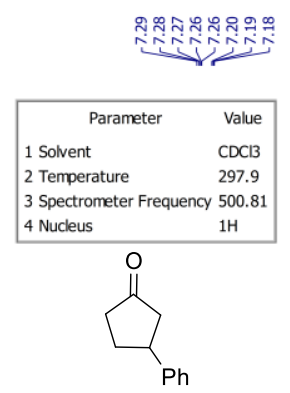

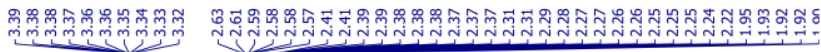

1 ac

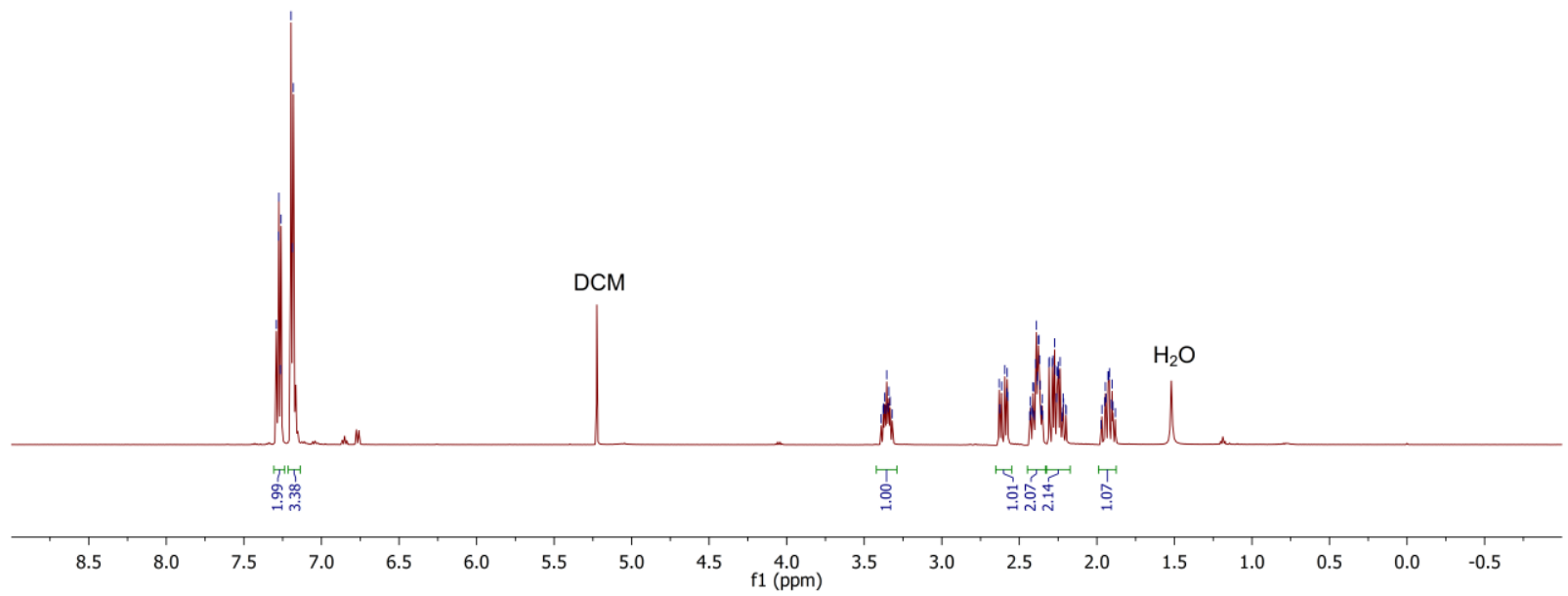



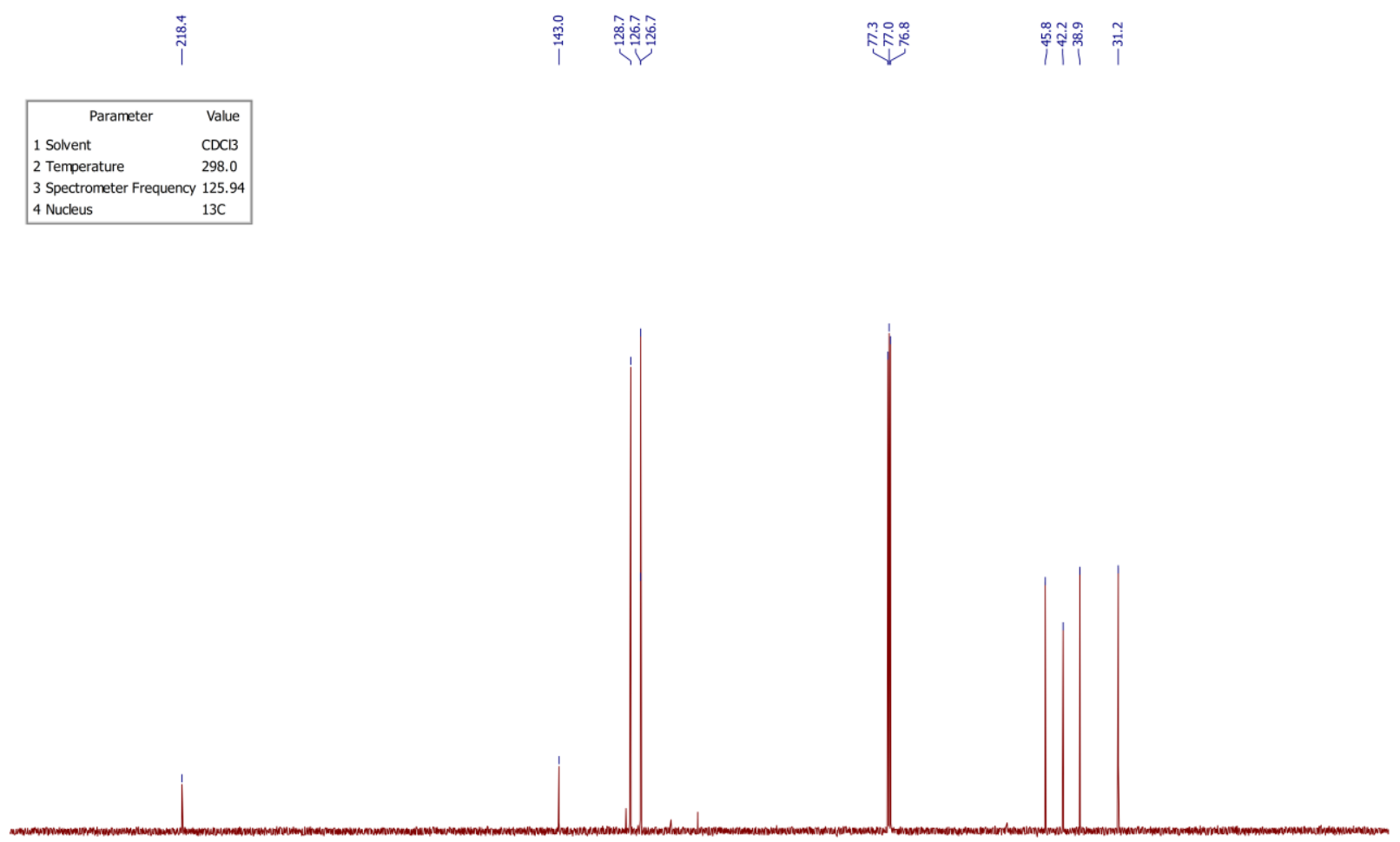

\begin{tabular}{rllllllllllllllllllllllllllll}
\hline 250 & 240 & 230 & 220 & 210 & 200 & 190 & 180 & 170 & 160 & 150 & 140 & 130 & 120 & 110 & 100 & 90 & 80 & 70 & 60 & 50 & 40 & 30 & 20 & 10 & 0 & -10 & -20
\end{tabular}

(R)-tert-butyldimethyl((1,2,3,6-tetrahydro-[1,1'-biphenyl]-4-yl)oxy)silane 3a:

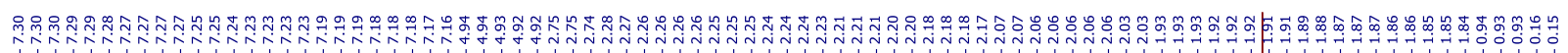

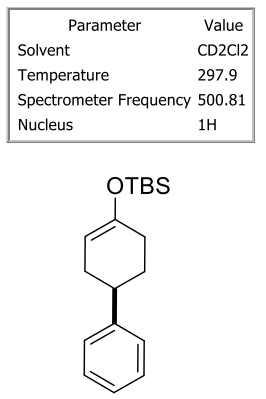

$3 \mathbf{a}$

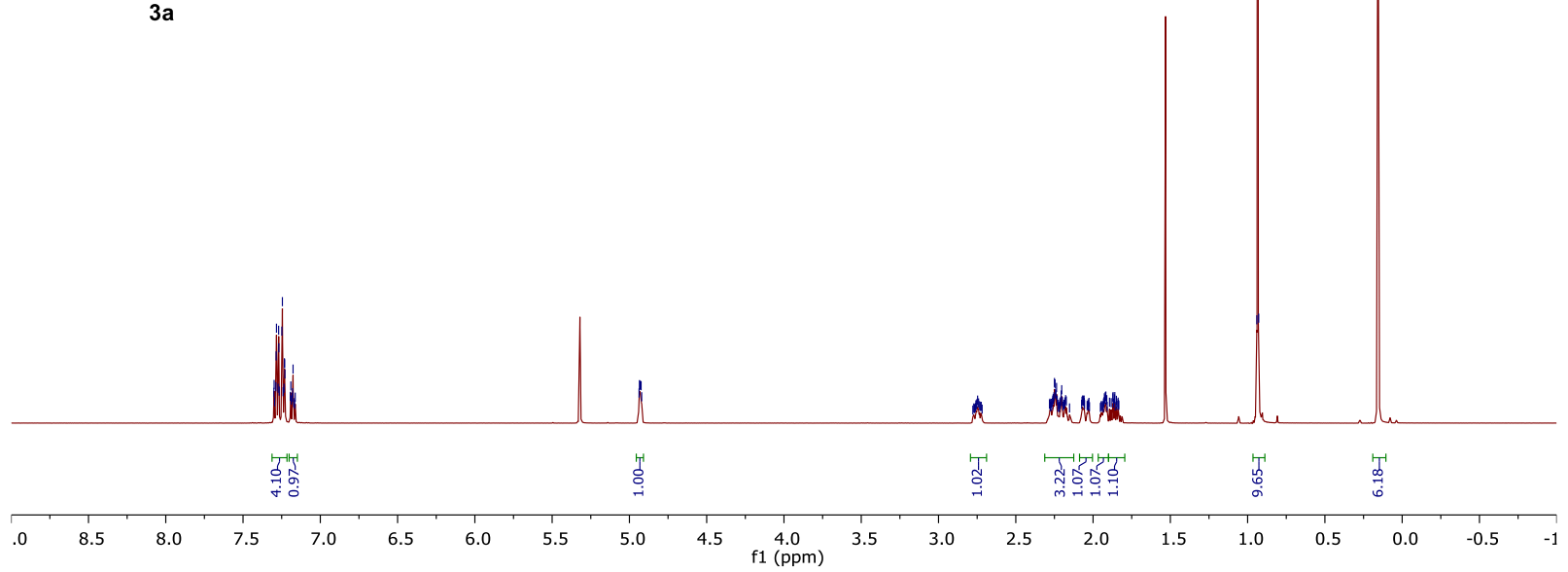




\begin{tabular}{|ll|}
\hline \multicolumn{1}{|c|}{ Parameter } & \multicolumn{1}{c|}{ Value } \\
Solvent & $\mathrm{CD} 2 \mathrm{C12}$ \\
Temperature & 298.0 \\
Spectrometer Frequency & 125.94 \\
Nucleus & $13 \mathrm{C}$ \\
\hline
\end{tabular}

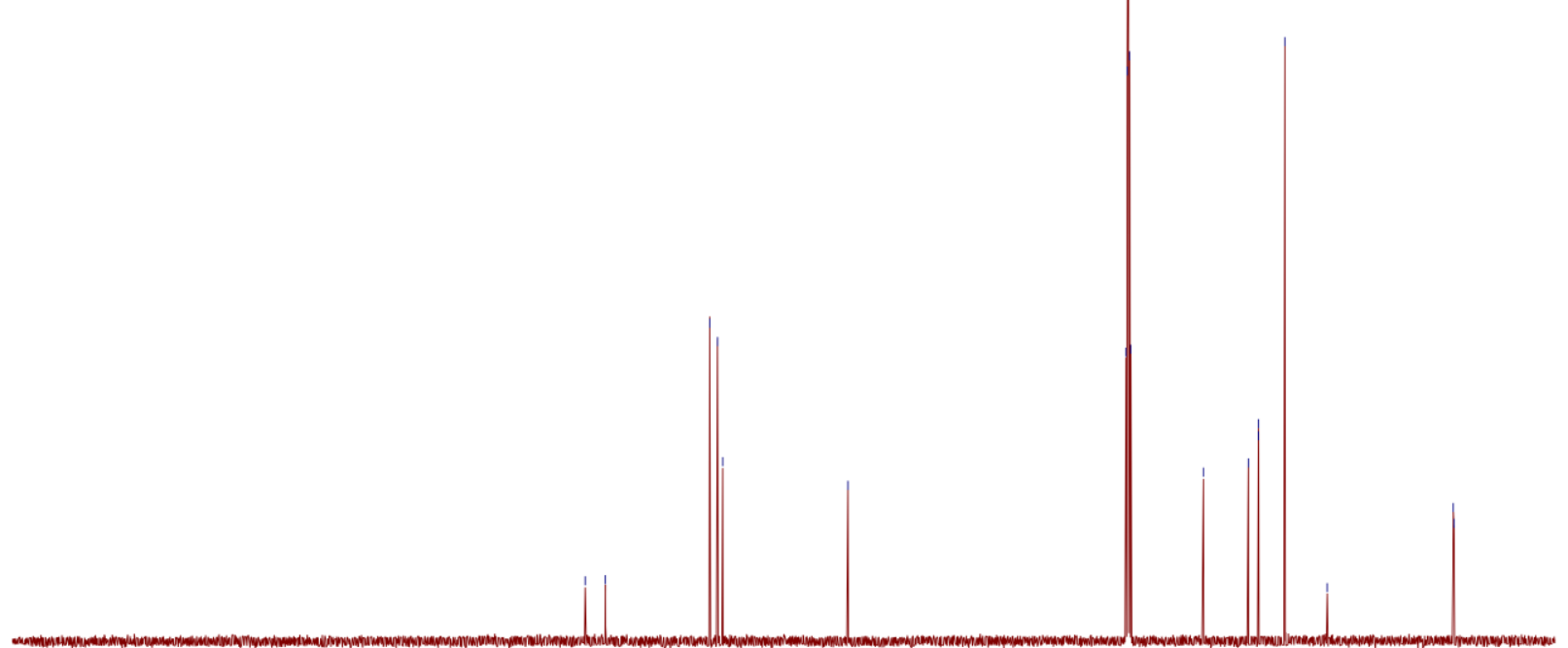

$\begin{array}{llllllllllllllllllllllllllll}250 & 240 & 230 & 220 & 210 & 200 & 190 & 180 & 170 & 160 & 150 & 140 & 130 & \begin{array}{l}120 \\ \mathrm{f} 1(\mathrm{ppm})\end{array} & 100 & 90 & 80 & 70 & 60 & 50 & 40 & 30 & 20 & 10 & 0 & -10 & -20\end{array}$

(R)-tert-butyldimethyl((4'-methyl-1,2,3,6-tetrahydro-[1,1'-biphenyl]-4-yl)oxy)silane 3b:

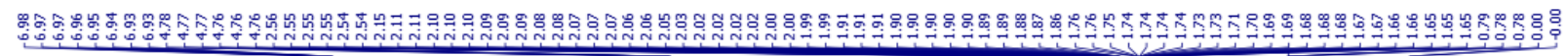

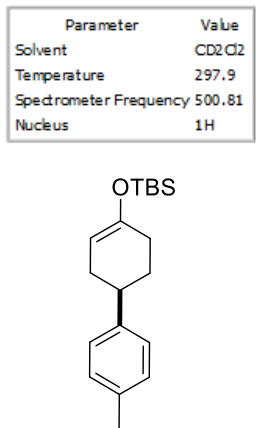

3b

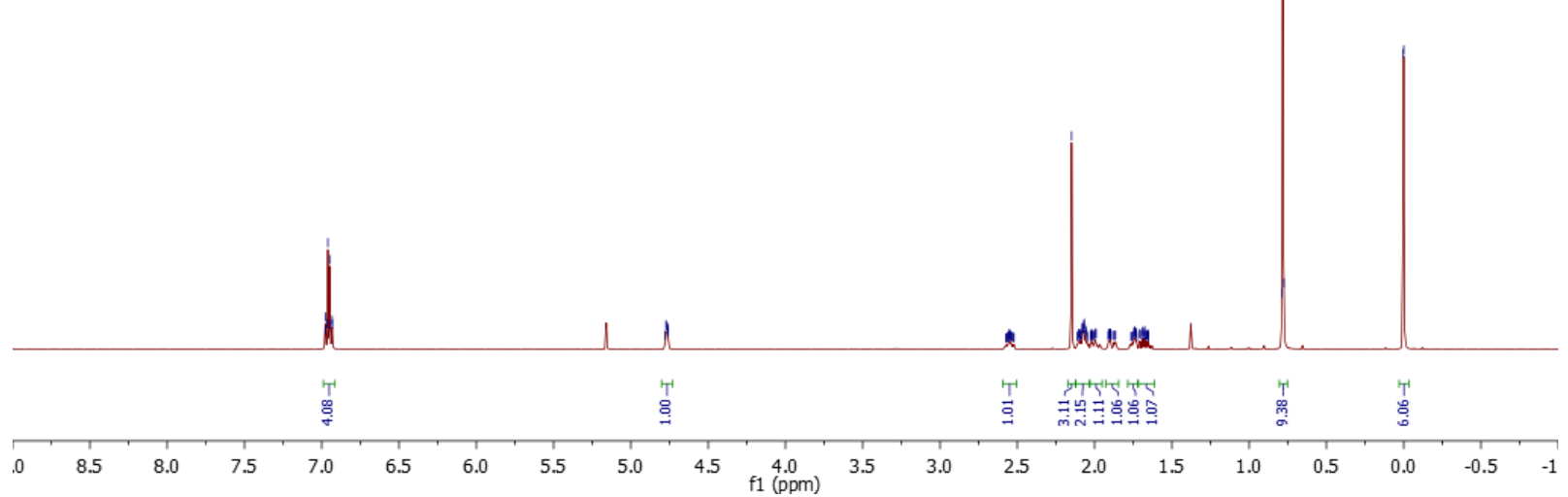




\begin{tabular}{|ll|}
\hline \multicolumn{1}{|c|}{ Parameter } & \multicolumn{1}{c|}{ Value } \\
Solvent & CD2C12 \\
Temperature & 298.0 \\
Spectrometer Frequency & 125.94 \\
Nucleus & $13 \mathrm{C}$ \\
\hline
\end{tabular}

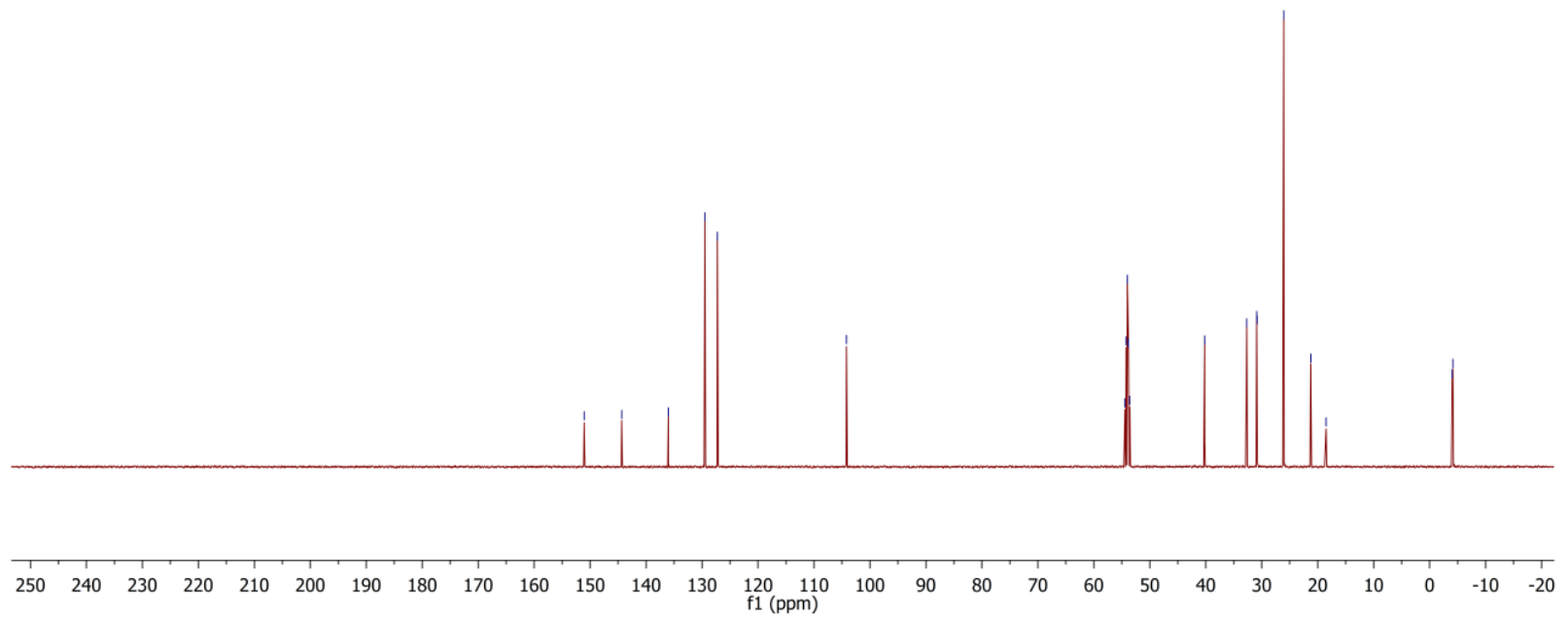

(R)-tert-butyl((4'-methoxy-1,2,3,6-tetrahydro-[1,1'-biphenyl]-4-yl)oxy)dimethylsilane 3c:

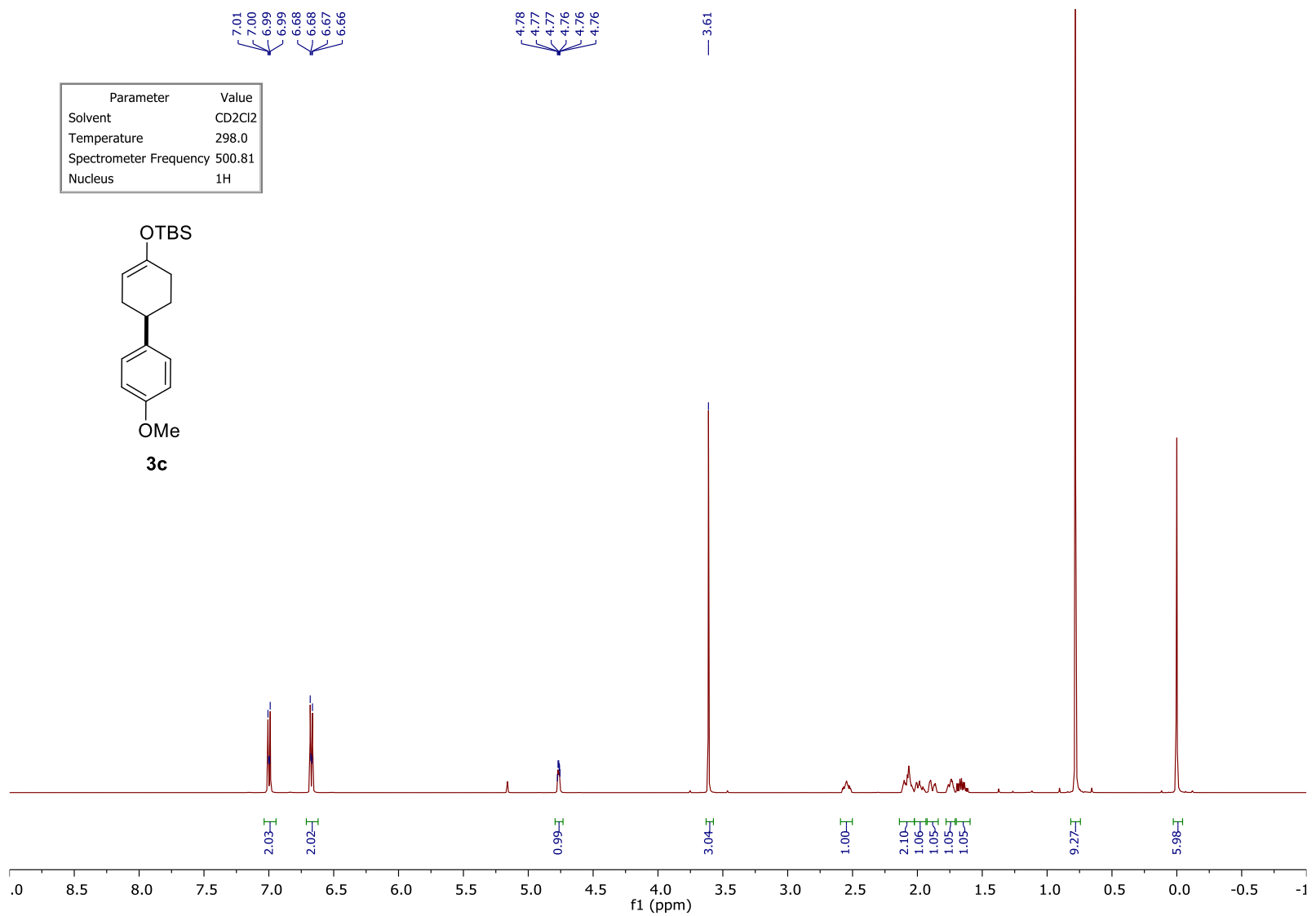




\begin{tabular}{|ll|}
\hline \multicolumn{1}{|c|}{ Parameter } & \multicolumn{1}{c|}{ Value } \\
Solvent & CD2C12 \\
Temperature & 298.0 \\
Spectrometer Frequency & 125.94 \\
Nucleus & $13 \mathrm{C}$ \\
\hline
\end{tabular}

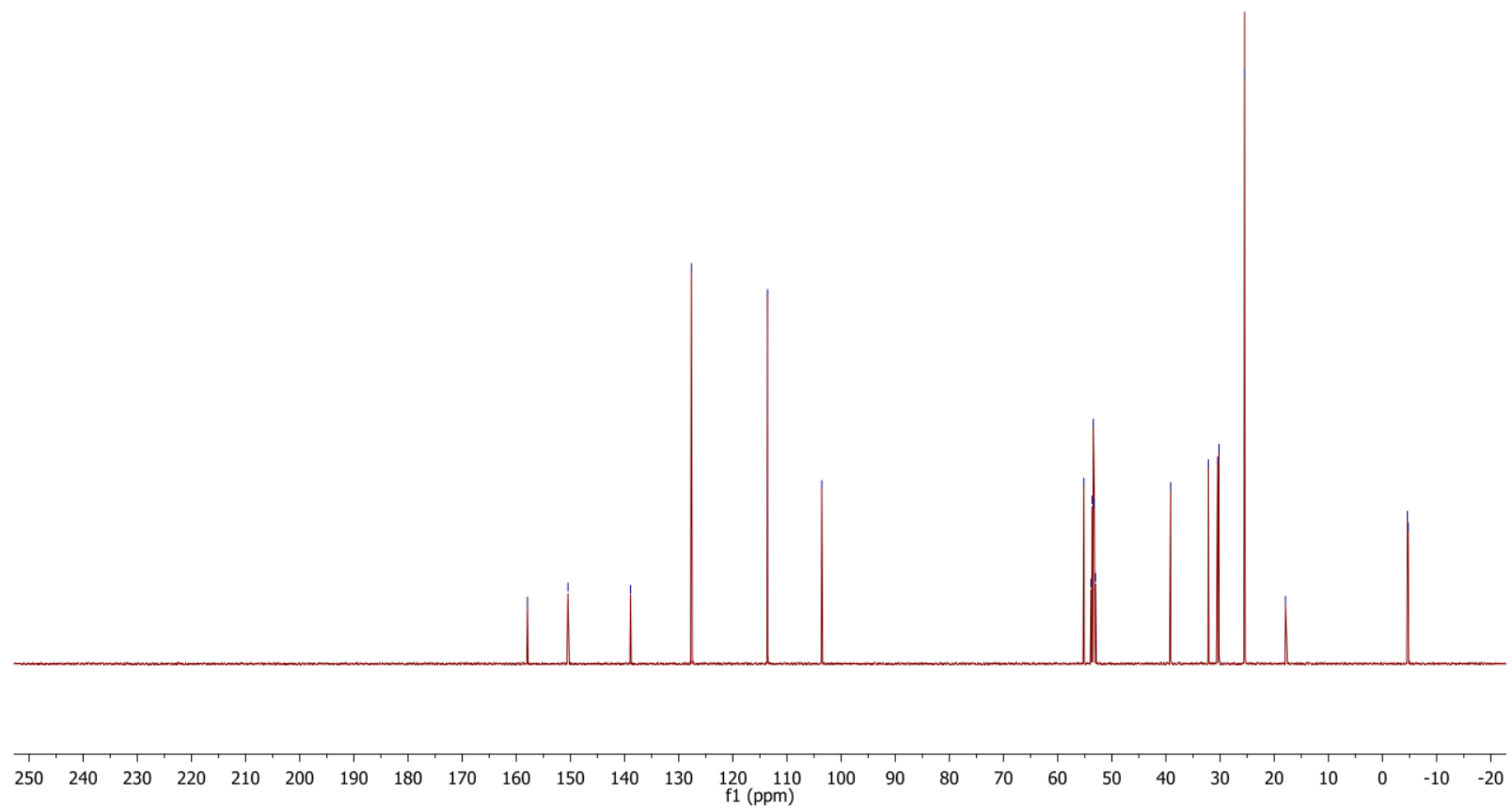

(R)-tert-butyl((4'-chloro-1,2,3,6-tetrahydro-[1,1'-biphenyl]-4-yl)oxy)dimethylsilane 3d:

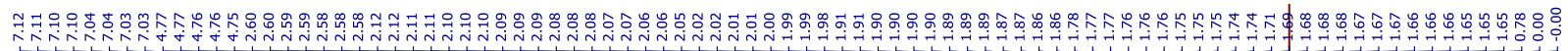

\begin{tabular}{|ll|}
\hline \multicolumn{1}{|c}{ Parameter } & \multicolumn{1}{c|}{ Value } \\
Solvent & CD2Cl2 \\
Temperature & 297.9 \\
Spectrometer Frequency & 500.81 \\
Nucleus & $1 \mathrm{H}$ \\
\hline
\end{tabular}

Nucleus

$1 \mathrm{H}$<smiles>Clc1ccc(C2CC=C(O[SeH2])CC2)cc1</smiles>

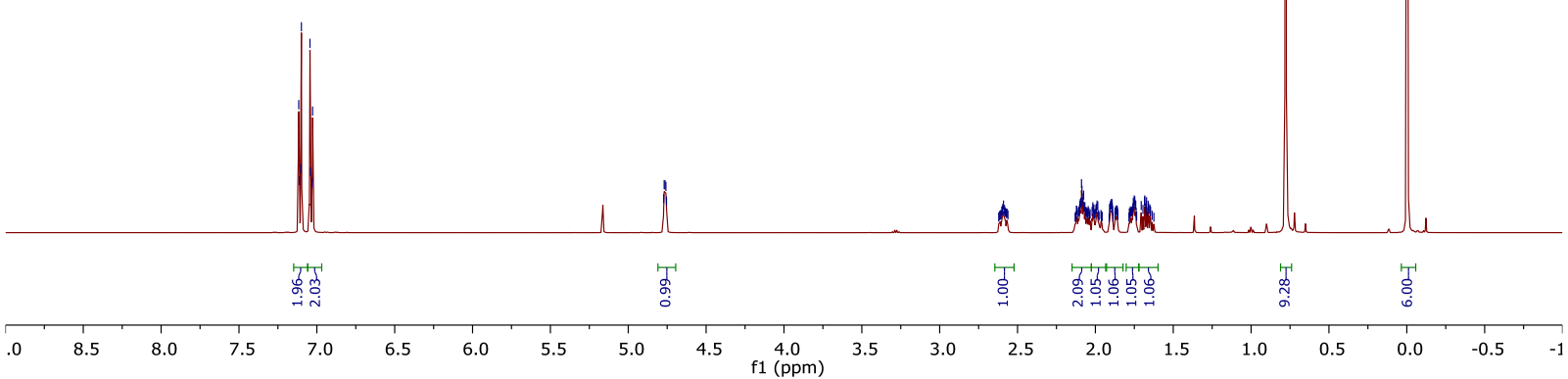




\begin{tabular}{|ll|}
\hline \multicolumn{1}{|c|}{ Parameter } & \multicolumn{1}{c|}{ Value } \\
Solvent & CD2C12 \\
Temperature & 298.0 \\
Spectrometer Frequency & 125.94 \\
Nucleus & $13 \mathrm{C}$ \\
\hline
\end{tabular}

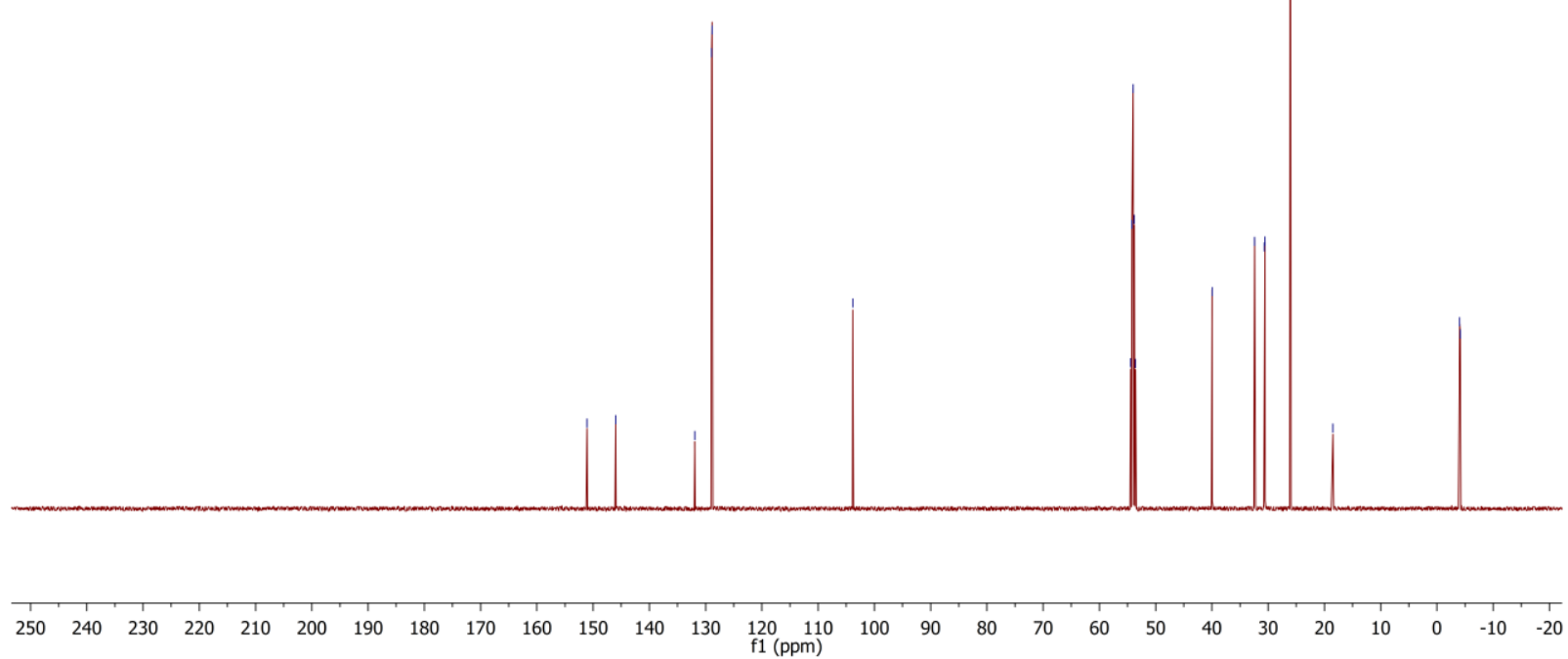

(R)-tert-butyl((4'-fluoro-1,2,3,6-tetrahydro-[1,1'-biphenyl]-4-yl)oxy)dimethylsilane 3e:

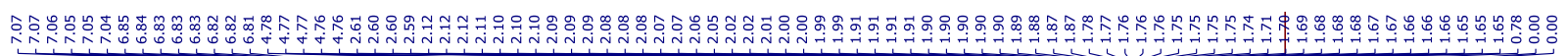

\begin{tabular}{|ll|}
\hline \multicolumn{1}{|c}{ Parameter } & \multicolumn{1}{c}{ Value } \\
Solvent & CD2Cl2 \\
Temperature & 297.9 \\
Spectrometer Frequency & 500.81 \\
Nucleus & $1 \mathrm{H}$ \\
\hline
\end{tabular}

Nucleus

$1 \mathrm{H}$

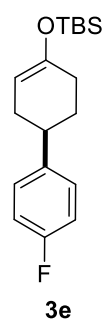

$3 e$

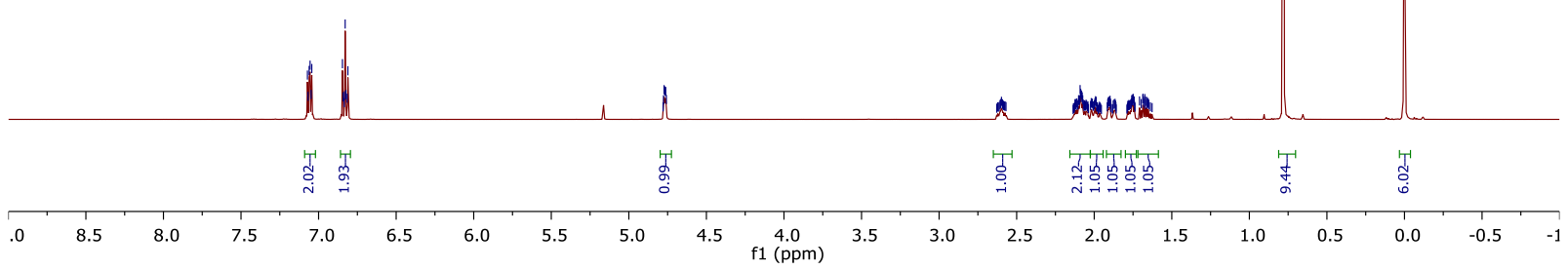



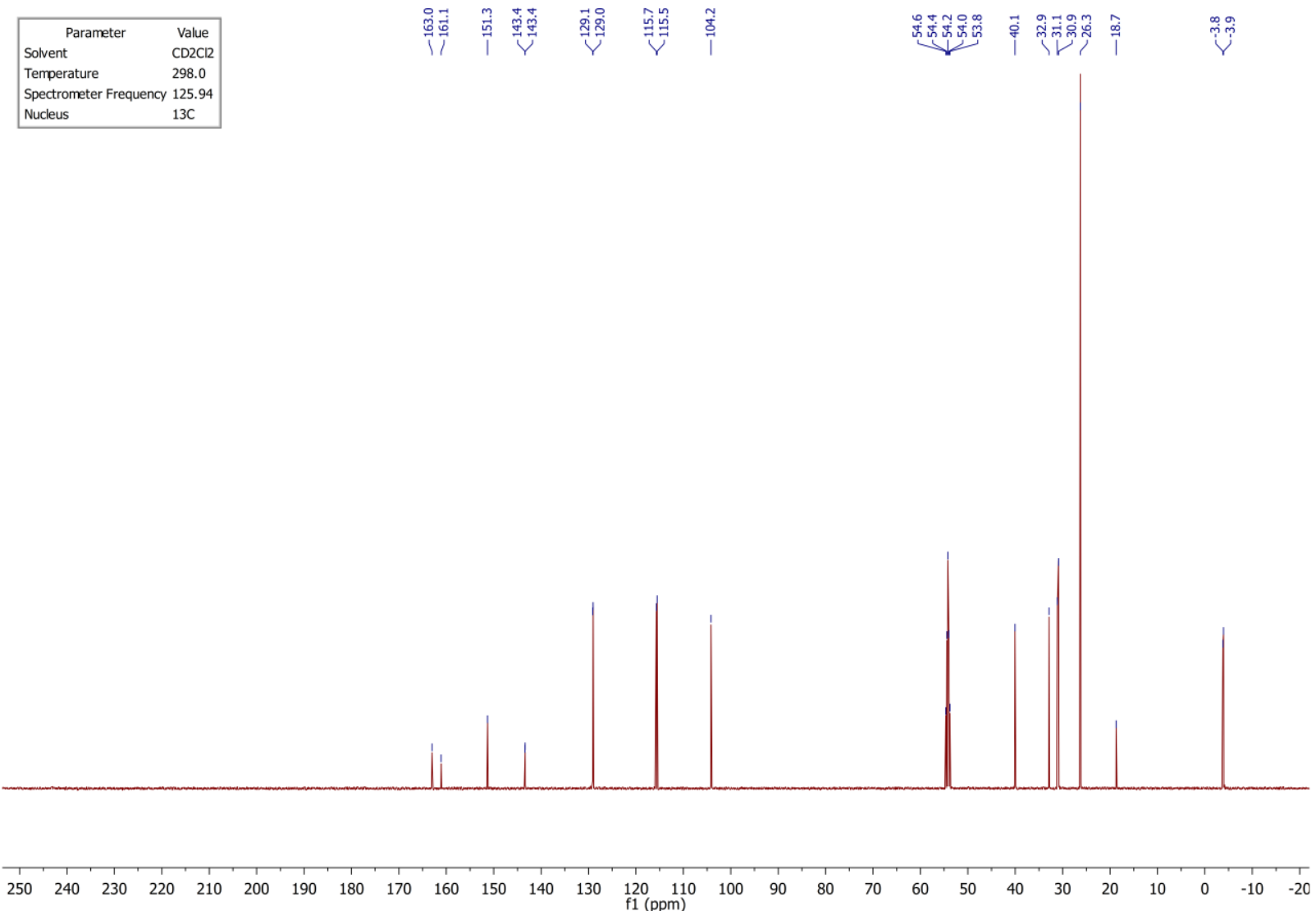

\begin{tabular}{|lc|}
\hline \multicolumn{1}{c}{ Parameter } & Value \\
Solvent & CD2Cl2 \\
Temperature & 298.0 \\
Spectrometer Frequency & 471.21 \\
Nucleus & $19 \mathrm{~F}$ \\
\hline
\end{tabular}


(R)-tert-butyldimethyl((4'-(trifluoromethyl)-1,2,3,6-tetrahydro-[1,1'-biphenyl]-4-yl)oxy)silane 3f:

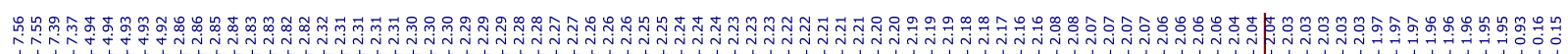

\begin{tabular}{|c|c|}
\hline Parameter & Value \\
\hline Solvent & $\mathrm{CD} 2 \mathrm{Cl} 2$ \\
\hline Temperature & 297.9 \\
\hline \multirow{2}{*}{\multicolumn{2}{|c|}{ Spectrometer Frequency 500.81}} \\
\hline & $1 \mathrm{H}$ \\
\hline
\end{tabular}

OTBS



$3 f$
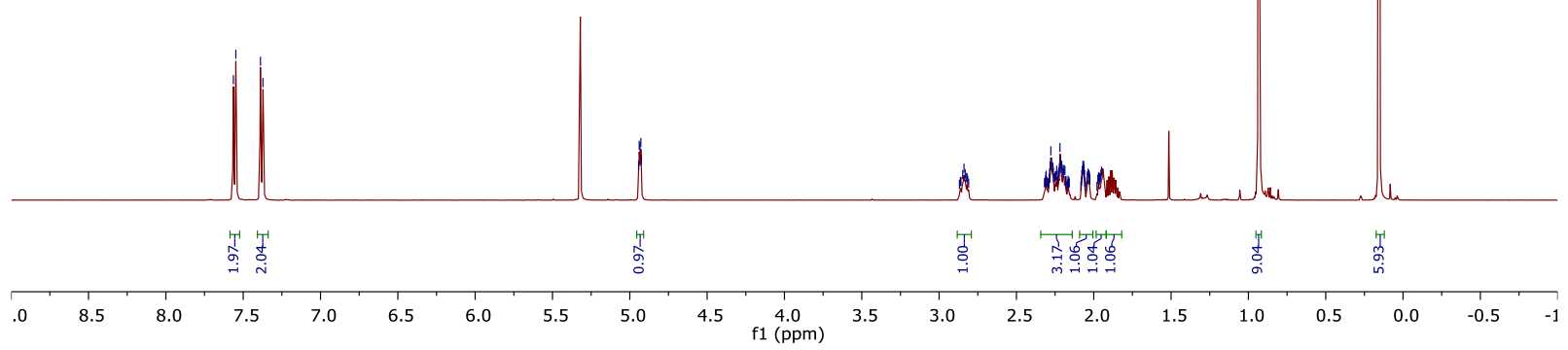

\begin{tabular}{|ll|}
\hline \multicolumn{1}{|c|}{ Parameter } & \multicolumn{1}{c|}{ Value } \\
Solvent & $\mathrm{CD} 2 \mathrm{Cl} 2$ \\
Temperature & 298.0 \\
Spectrometer Frequency & 125.94 \\
Nucleus & $13 \mathrm{C}$ \\
\hline
\end{tabular}

जี่

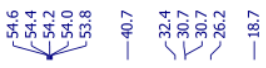

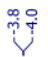

Nucleus $3 \mathrm{C}$ 


\begin{tabular}{|lc|}
\hline \multicolumn{1}{|c|}{ Parameter } & \multicolumn{1}{c|}{ Value } \\
Solvent & CD2CI2 \\
Temperature & 298.0 \\
Spectrometer Frequency & 471.21 \\
Nucleus & $19 \mathrm{~F}$ \\
\hline
\end{tabular}

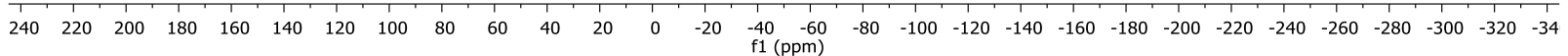
(R)-tert-butyl((3'-(tert-butyl)-1,2,3,6-tetrahydro-[1,1'-biphenyl]-4-yl)oxy)dimethylsilane 3g:

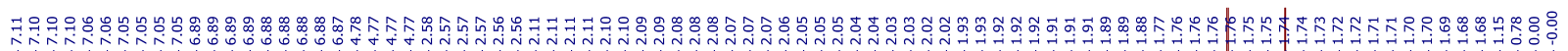

\begin{tabular}{|lc|}
\hline \multicolumn{1}{|c}{ Parameter } & Value \\
Solvent & CD2Cl2 \\
Temperature & 298.0 \\
Spectrometer Frequency & 500.81 \\
Nucleus & $1 \mathrm{H}$ \\
\hline
\end{tabular}

Nucleus

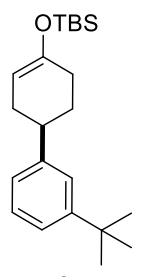

$3 g$

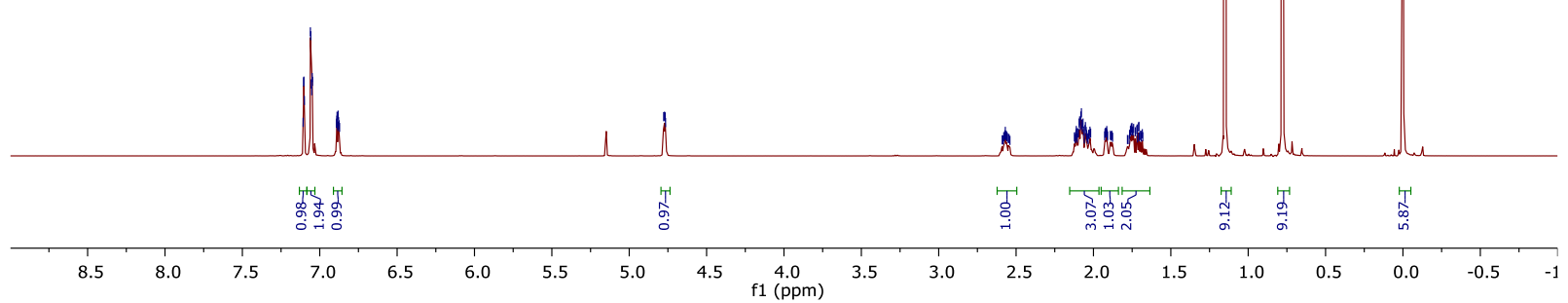




\begin{tabular}{|ll|}
\hline \multicolumn{1}{|c|}{ Parameter } & \multicolumn{1}{c|}{ Value } \\
Solvent & CD2C12 \\
Temperature & 298.0 \\
Spectrometer Frequency & 125.94 \\
Nucleus & $13 \mathrm{C}$ \\
\hline
\end{tabular}

\section{(R)-tert-butyl((3'-chloro-1,2,3,6-tetrahydro-[1,1'-biphenyl]-4-yl)oxy)dimethylsilane 3h:}

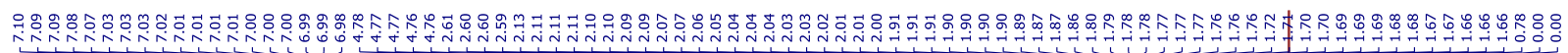

\begin{tabular}{|ll|}
\hline \multicolumn{1}{|c}{ Parameter } & Value \\
Solvent & CD2Cl2 \\
Temperature & 297.9 \\
Spectrometer Frequency & 500.81 \\
Nucleus & $1 \mathrm{H}$ \\
\hline
\end{tabular}

OTBS<smiles>COc1cccc(C2CC=C(C)CC2)c1</smiles>

3h

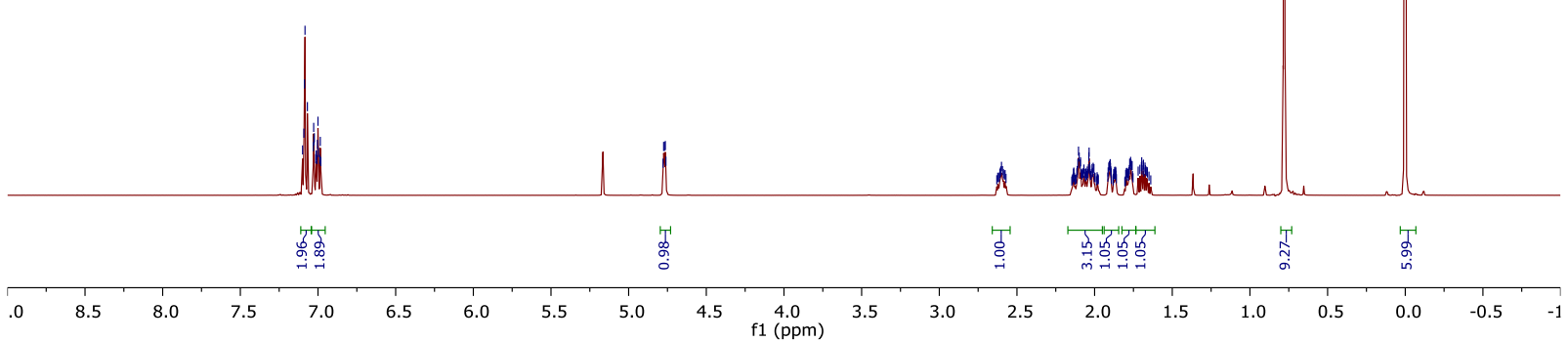




\begin{tabular}{|ll|}
\hline \multicolumn{1}{|c|}{ Parameter } & \multicolumn{1}{c|}{ Value } \\
Solvent & CD2C12 \\
Temperature & 298.0 \\
Spectrometer Frequency & 125.94 \\
Nucleus & $13 \mathrm{C}$ \\
\hline
\end{tabular}

(R)-tert-butyldimethyl((3'-(trifluoromethyl)-1,2,3,6-tetrahydro-[1,1'-biphenyl]-4-yl)oxy)silane 3i:

\begin{tabular}{|ll|}
\hline \multicolumn{1}{|c|}{ Parameter } & \multicolumn{1}{c|}{ Value } \\
Solvent & CD2C12 \\
Temperature & 297.9 \\
Spectrometer Frequency & 500.81 \\
Nucleus & $1 \mathrm{H}$ \\
\hline
\end{tabular}

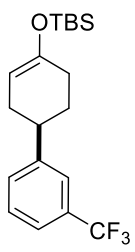

$3 \mathbf{i}$

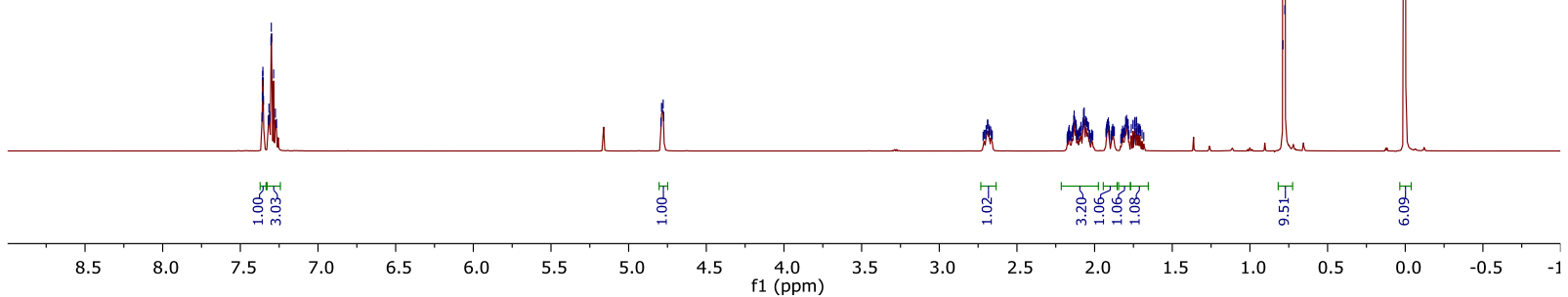




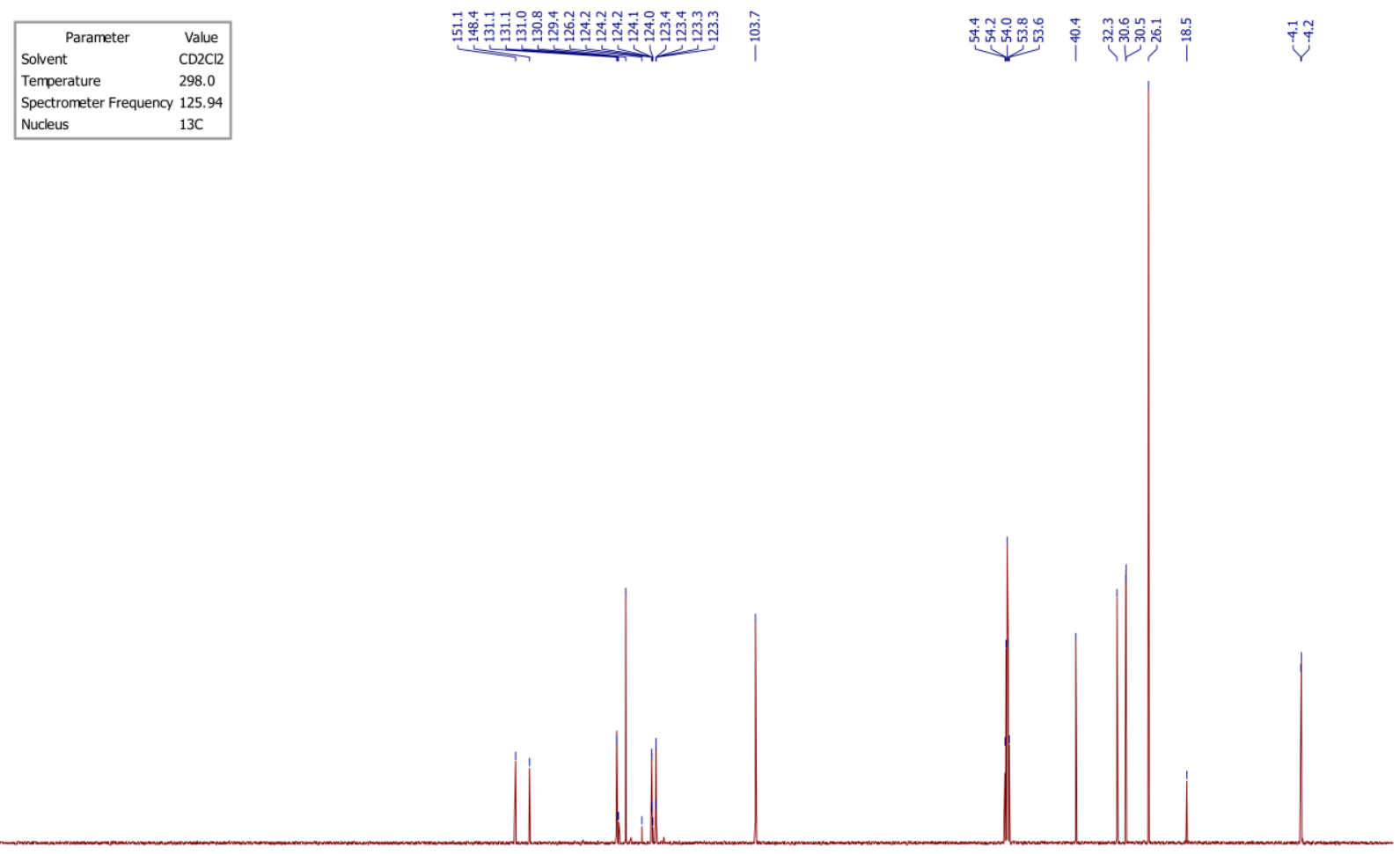

$\begin{array}{llllllllllllllllllllllllllll}250 & 240 & 230 & 220 & 210 & 200 & 190 & 180 & 170 & 160 & 150 & 140 & 130 & 120 & 110 & 100 & 90 & 80 & 70 & 60 & 50 & 40 & 30 & 20 & 10 & 0 & -10 & -20\end{array}$

\begin{tabular}{|ll|}
\hline \multicolumn{1}{|c|}{ Parameter } & \multicolumn{1}{c|}{ Value } \\
Solvent & CD2C12 \\
Temperature & 298.0 \\
Spectrometer Frequency & 471.21 \\
Nucleus & $19 \mathrm{~F}$ \\
\hline
\end{tabular}

Nucleus $19 \mathrm{~F}$

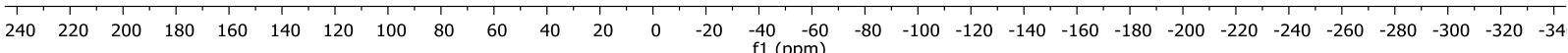


(R)-tert-butyldimethyl((2'-methyl-1,2,3,6-tetrahydro-[1,1'-biphenyl]-4-yl)oxy)silane 3j:

赵政

\begin{tabular}{|ll|}
\hline \multicolumn{1}{|c}{ Parameter } & Value \\
Solvent & CD2C12 \\
Temperature & 297.9 \\
Spectrometer Frequency & 500.81 \\
Nucleus & $1 \mathrm{H}$ \\
\hline
\end{tabular}

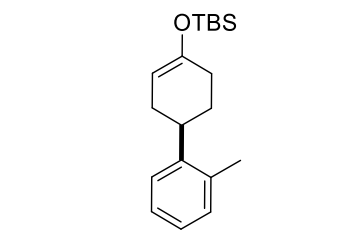

3j
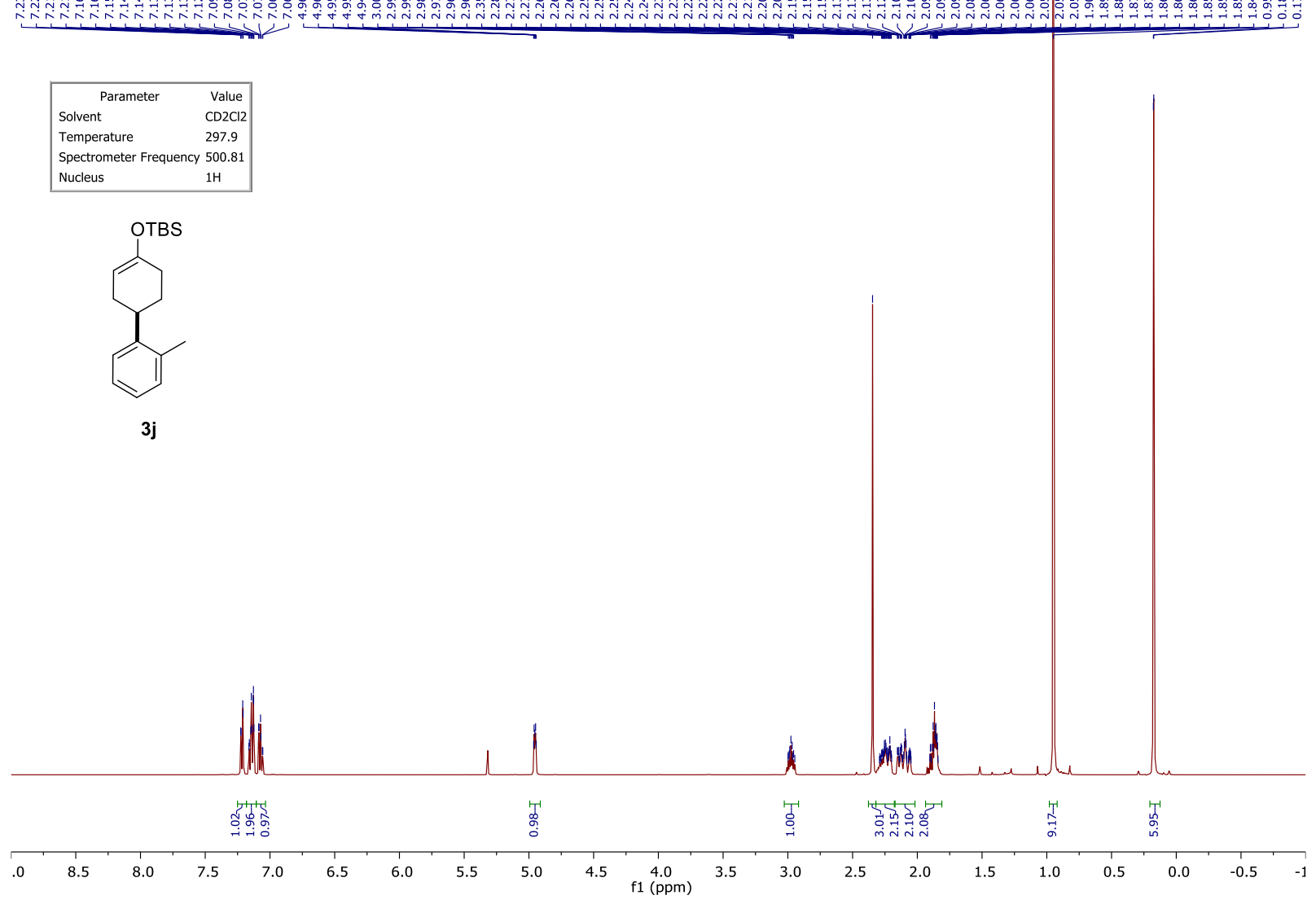

\begin{tabular}{|ll|}
\hline \multicolumn{1}{|c|}{ Parameter } & Value \\
Solvent & $\mathrm{CD} 2 \mathrm{Cl} 2$ \\
Temperature & 298.0 \\
Spectrometer Frequency & 125.94 \\
Nucleus & $13 \mathrm{C}$ \\
\hline
\end{tabular}

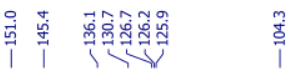

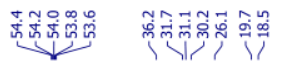

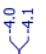

Nucleus $3 \mathrm{C}$

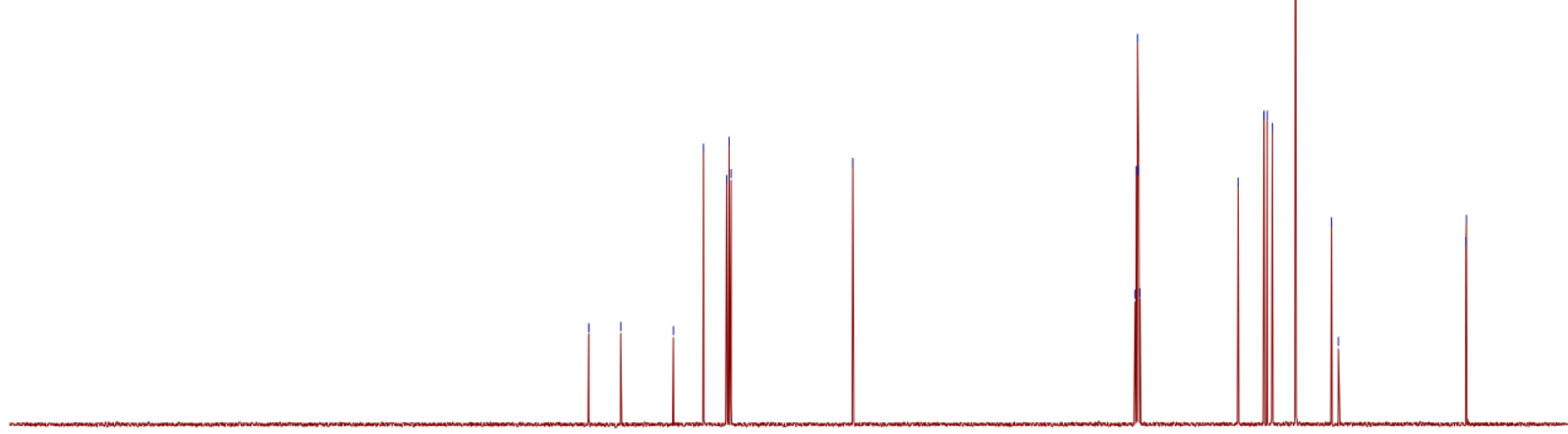

$\begin{array}{llllllllllllllllllllllllllllll}250 & 240 & 230 & 220 & 210 & 200 & 190 & 180 & 170 & 160 & 150 & 140 & 130 & \begin{array}{l}120 \\ \mathrm{f} 1(\mathrm{ppm})\end{array} & 100 & 90 & 80 & 70 & 60 & 50 & 40 & 30 & 20 & 10 & 0 & -10 & -20\end{array}$ 
(R)-tert-butyl((3',5'-dimethyl-1,2,3,6-tetrahydro-[1,1'-biphenyl]-4-yl)oxy)dimethylsilane 3k:

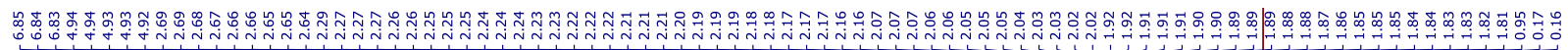
\begin{tabular}{|ll|}
\hline \multicolumn{1}{|c}{ Parameter } & \multicolumn{1}{c|}{ Value } \\
Solvent & CD2Cl2 \\
Temperature & 298.0 \\
Spectrometer Frequency & 500.81 \\
\hline
\end{tabular}

Nucleus

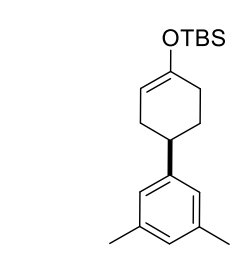

$3 \mathbf{k}$ $1 \mathrm{H}$

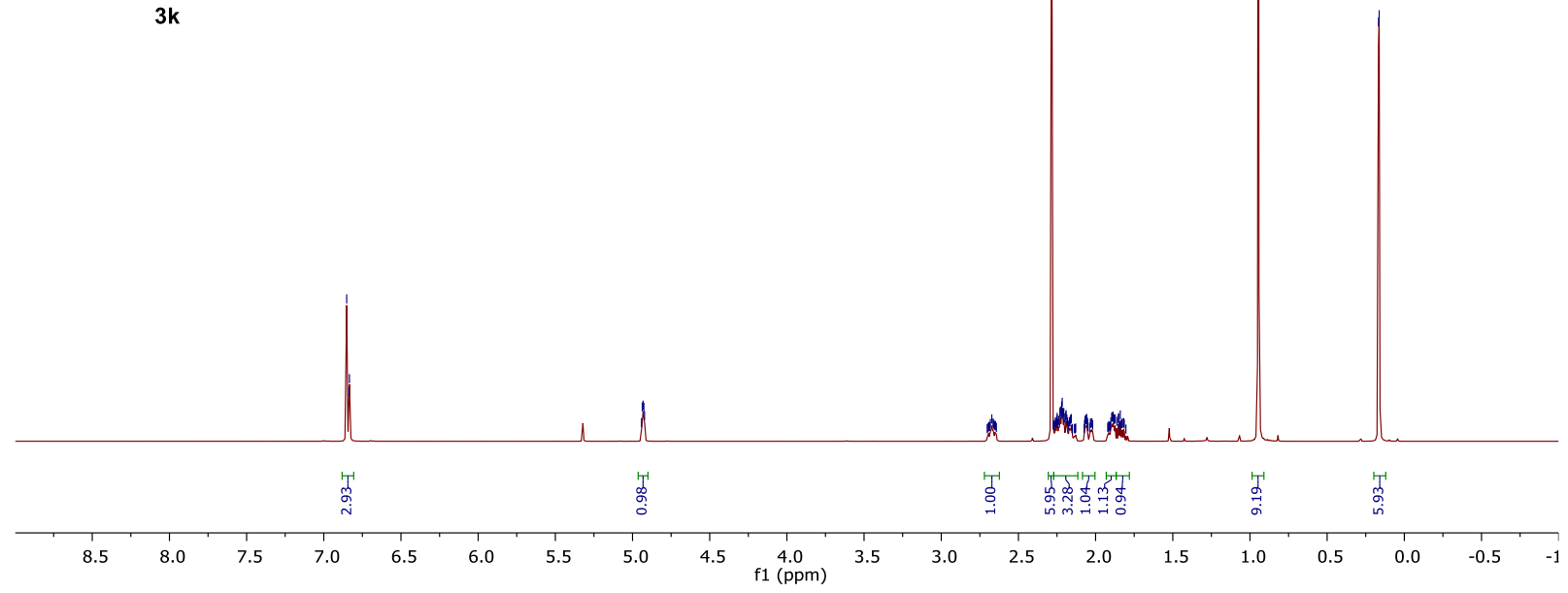

\begin{tabular}{|lc|}
\hline \multicolumn{1}{|c|}{ Parameter } & \multicolumn{1}{c|}{ Value } \\
Solvent & $\mathrm{CD} 2 \mathrm{Cl}$ \\
Temperature & 298.0 \\
Spectrometer Frequency & 125.94 \\
Nucleus & $13 \mathrm{C}$ \\
\hline
\end{tabular}

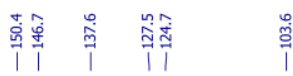

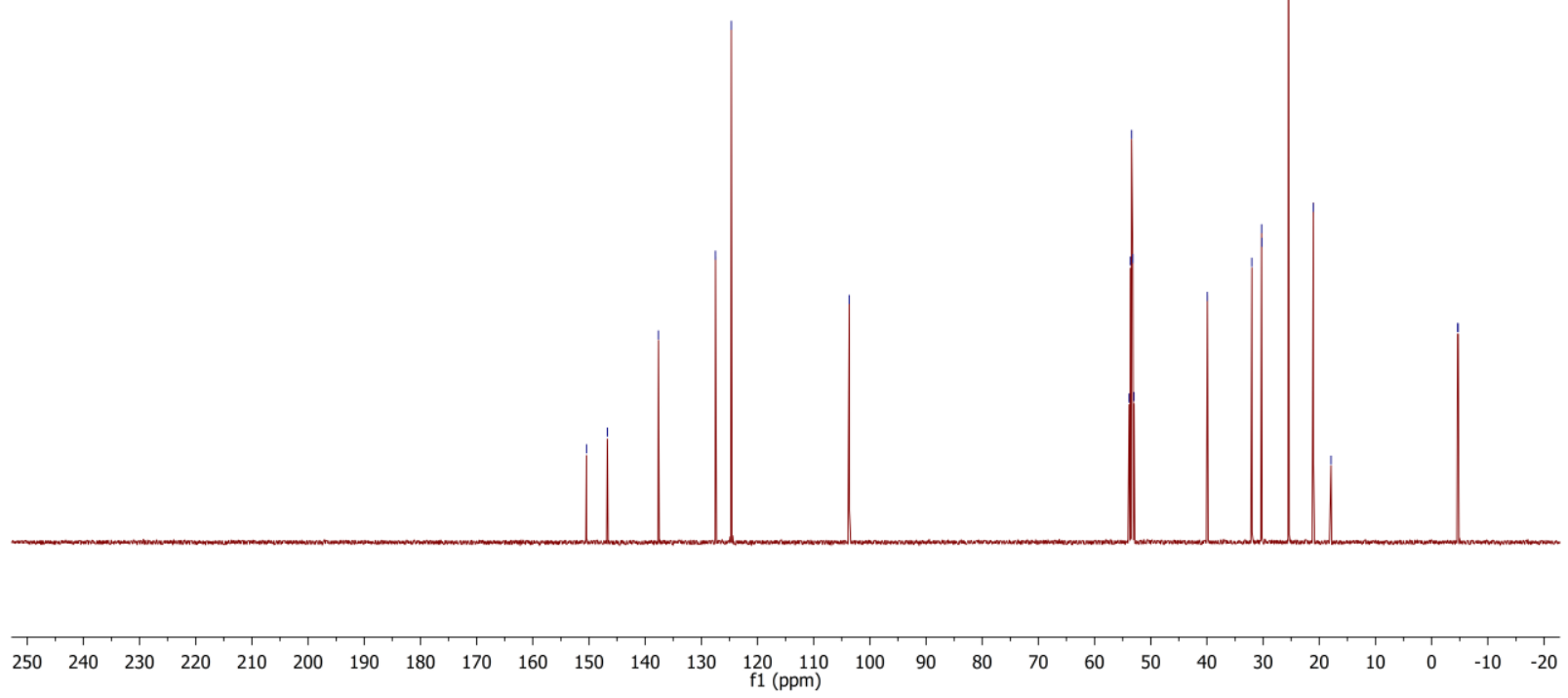


(R)-tert-butyl((3',5'-difluoro-1,2,3,6-tetrahydro-[1,1'-biphenyl]-4-yl)oxy)dimethylsilane 31:

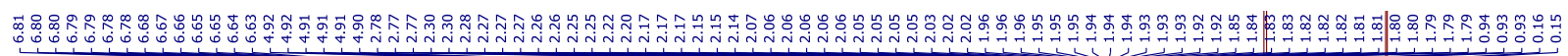

\begin{tabular}{|ll|}
\hline \multicolumn{1}{|c}{ Parameter } & \multicolumn{1}{c|}{ Value } \\
Solvent & CD2C12 \\
Temperature & 297.8 \\
Spectrometer Frequency & 500.81 \\
Nucleus & $1 \mathrm{H}$ \\
\hline
\end{tabular}

OTBS<smiles>CC1=CCC(c2cc(C)cc(F)c2)CC1</smiles>

3!

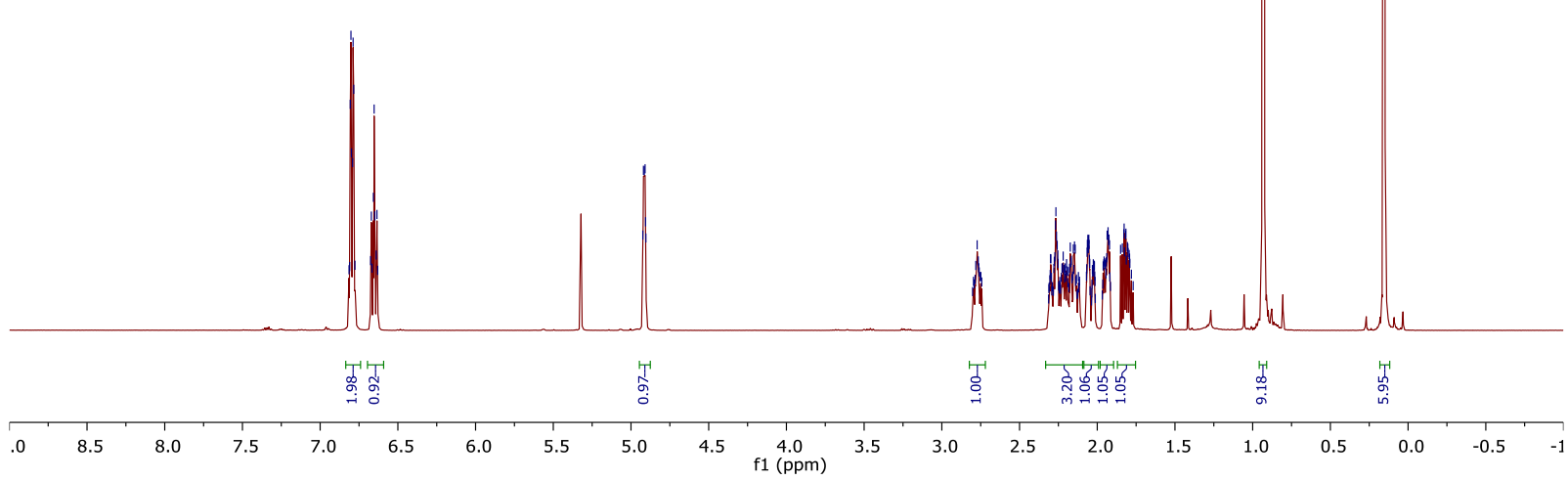

\begin{tabular}{|ll|}
\hline \multicolumn{1}{|c|}{ Parameter } & \multicolumn{1}{c|}{ Value } \\
Solvent & CD2C12 \\
Temperature & 298.0 \\
Spectrometer Frequency & 125.94 \\
Nucleus & $13 \mathrm{C}$ \\
\hline
\end{tabular}

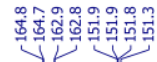

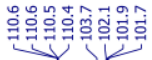

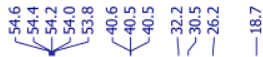

$\stackrel{\infty}{\text { ஸें }}$

Nucleus $13 \mathrm{C}$

$\begin{array}{lllllllllllllllllllllllllllllll}250 & 240 & 230 & 220 & 210 & 200 & 190 & 180 & 170 & 160 & 150 & 140 & 130 & 120 & 110 & 100 & 90 & 80 & 70 & 60 & 50 & 40 & 30 & 20 & 10 & 0 & -10 & -20\end{array}$ 


\begin{tabular}{|lc|}
\hline \multicolumn{1}{|c|}{ Parameter } & \multicolumn{1}{c|}{ Value } \\
Solvent & CD2CI2 \\
Temperature & 298.0 \\
Spectrometer Frequency & 471.21 \\
Nucleus & $19 \mathrm{~F}$ \\
\hline
\end{tabular}

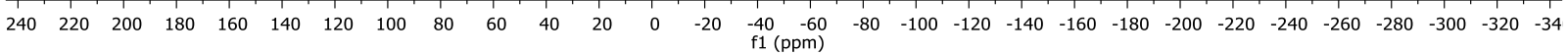

\section{(R)-tert-butyldimethyl((4-(naphthalen-1-yl)cyclohex-1-en-1-yl)oxy)silane 3m:}

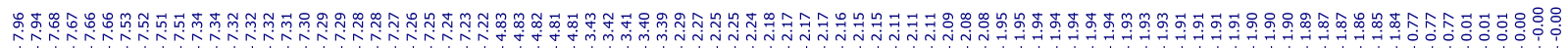

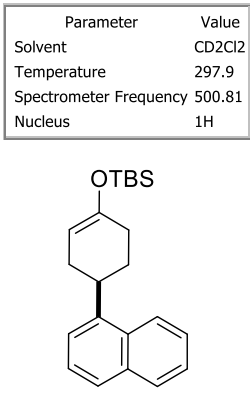

$3 \mathrm{~m}$

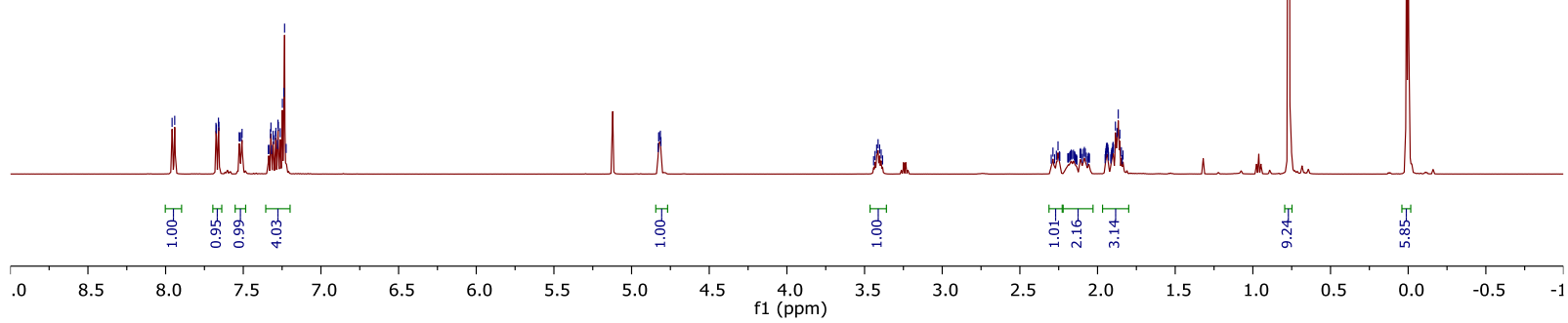




\begin{tabular}{|ll|}
\hline \multicolumn{1}{|c|}{ Parameter } & \multicolumn{1}{c|}{ Value } \\
Solvent & CD2C12 \\
Temperature & 298.0 \\
Spectrometer Frequency & 125.94 \\
Nucleus & $13 \mathrm{C}$ \\
\hline
\end{tabular}

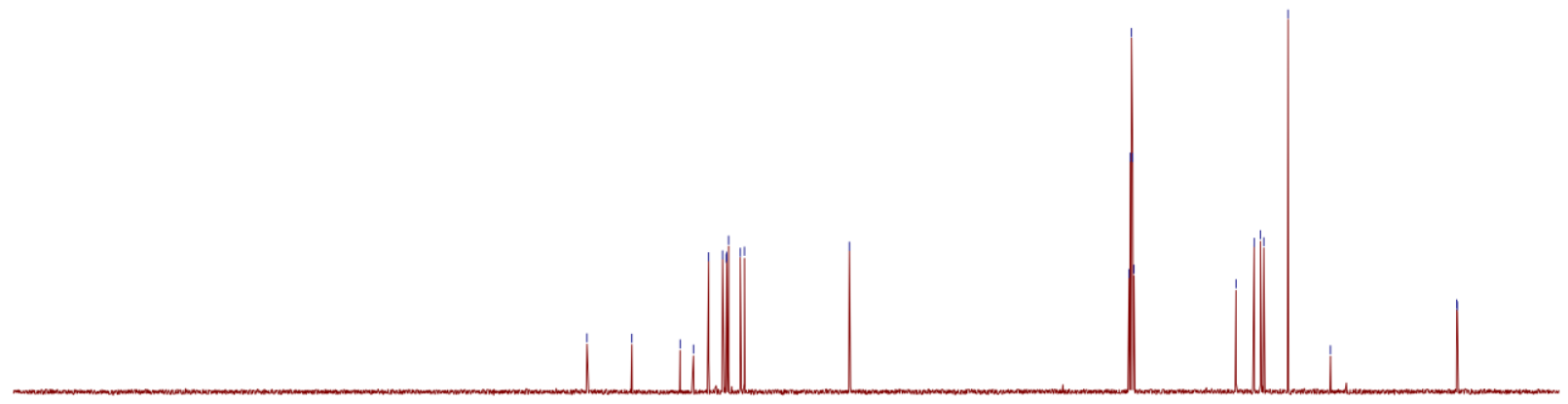

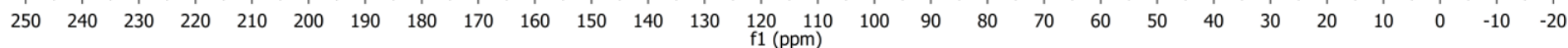

\section{(R)-tert-butyldimethyl((4-(naphthalen-2-yl)cyclohex-1-en-1-yl)oxy)silane 3n:}

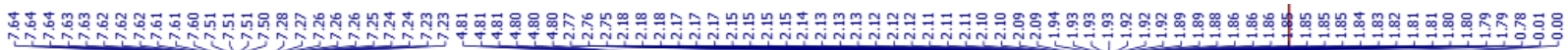

\begin{tabular}{|ll|}
\hline \multicolumn{1}{|c|}{ Parameter } & \multicolumn{1}{c|}{ Value } \\
1 Solvent & $\mathrm{CD} 2 \mathrm{Cl} 2$ \\
2 Temperature & 297.9 \\
3 Spectrometer Frequency & 500.81 \\
4 Nucleus & $1 \mathrm{H}$ \\
\hline
\end{tabular}<smiles>COc1ccc(C2CC=C(CO)CC2)cc1</smiles>

$3 n$

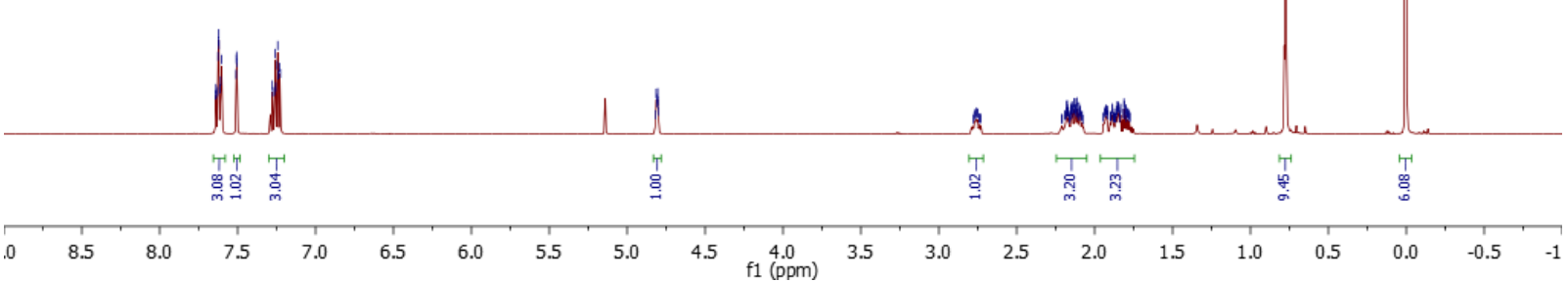




\begin{tabular}{|ll|}
\hline \multicolumn{1}{|c|}{ Parameter } & \multicolumn{1}{c|}{ Value } \\
1 Solvent & $\mathrm{CD} 2 \mathrm{C} 12$ \\
2 Temperature & 298.0 \\
3 Spectrometer Frequency & 125.94 \\
4 Nucleus & $13 \mathrm{C}$ \\
\hline
\end{tabular}

\section{(R)-tert-butyldimethyl((1,2,3,6-tetrahydro-[1,1':4',1''-terphenyl]-4-yl)oxy)silane 3o:}

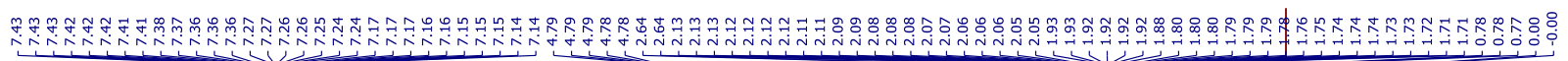

\begin{tabular}{|ll|}
\hline \multicolumn{1}{|c}{ Parameter } & \multicolumn{1}{c}{ Value } \\
Solvent & CD2Cl2 \\
Temperature & 297.9 \\
Spectrometer Frequency & 500.81 \\
Nucleus & $1 \mathrm{H}$ \\
\hline
\end{tabular}
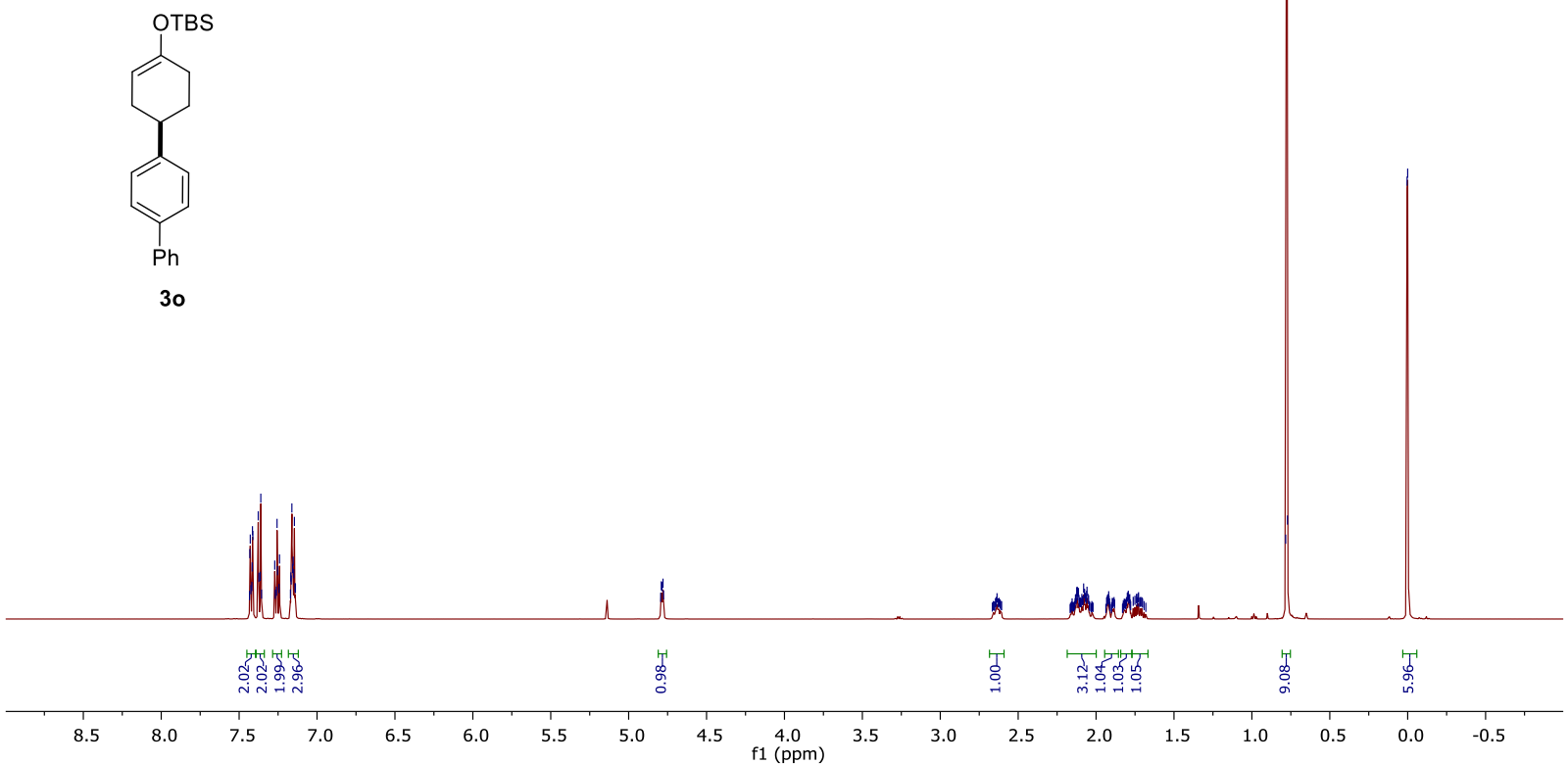


\begin{tabular}{|ll|}
\hline \multicolumn{1}{|c|}{ Parameter } & \multicolumn{1}{c|}{ Value } \\
Solvent & CD2C12 \\
Temperature & 298.0 \\
Spectrometer Frequency & 125.94 \\
Nucleus & $13 \mathrm{C}$ \\
\hline
\end{tabular}

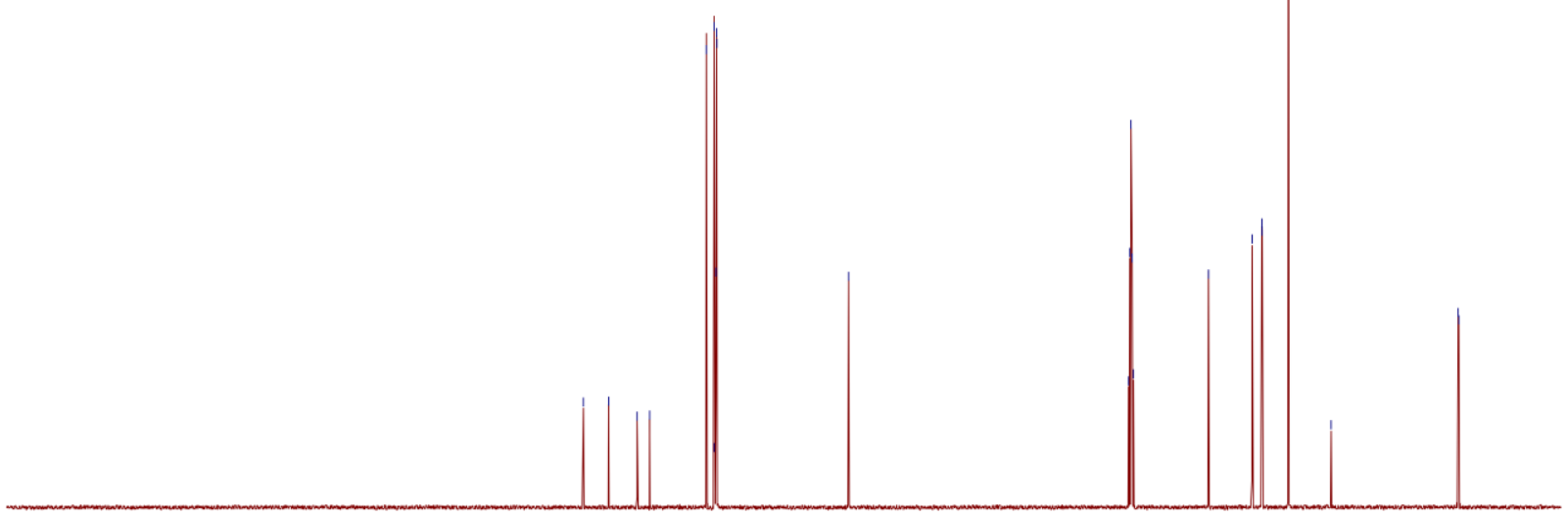

$\begin{array}{lllllllllllllllllllllllllllll}250 & 240 & 230 & 220 & 210 & 200 & 190 & 180 & 170 & 160 & 150 & 140 & 130 & \begin{array}{c}120 \\ \mathrm{f} 1(\mathrm{ppm})\end{array} & 100 & 90 & 80 & 70 & 60 & 50 & 40 & 30 & 20 & 10 & 0 & -10 & -20\end{array}$

(R)-tert-butyl((4-(tert-butyl)cyclohex-1-en-1-yl)oxy)dimethylsilane 3p:

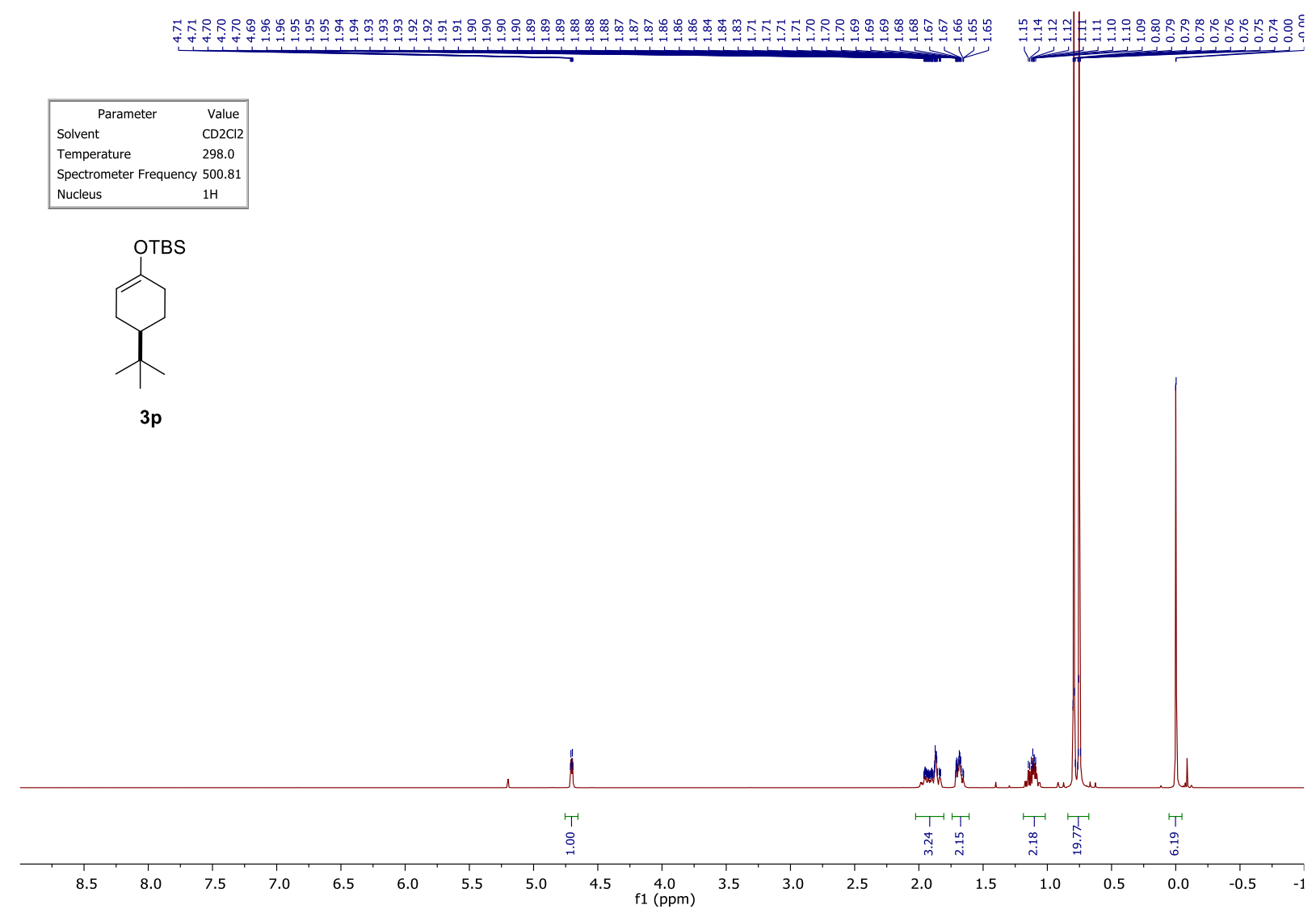




\begin{tabular}{|ll|}
\hline \multicolumn{1}{|c|}{ Parameter } & \multicolumn{1}{c|}{ Value } \\
Solvent & CD2C12 \\
Temperature & 298.0 \\
Spectrometer Frequency & 125.94 \\
Nucleus & $13 \mathrm{C}$ \\
\hline
\end{tabular}

\section{(R)-([1,1'-bi(cyclohexan)]-3-en-4-yloxy)(tert-butyl)dimethylsilane 3q:}

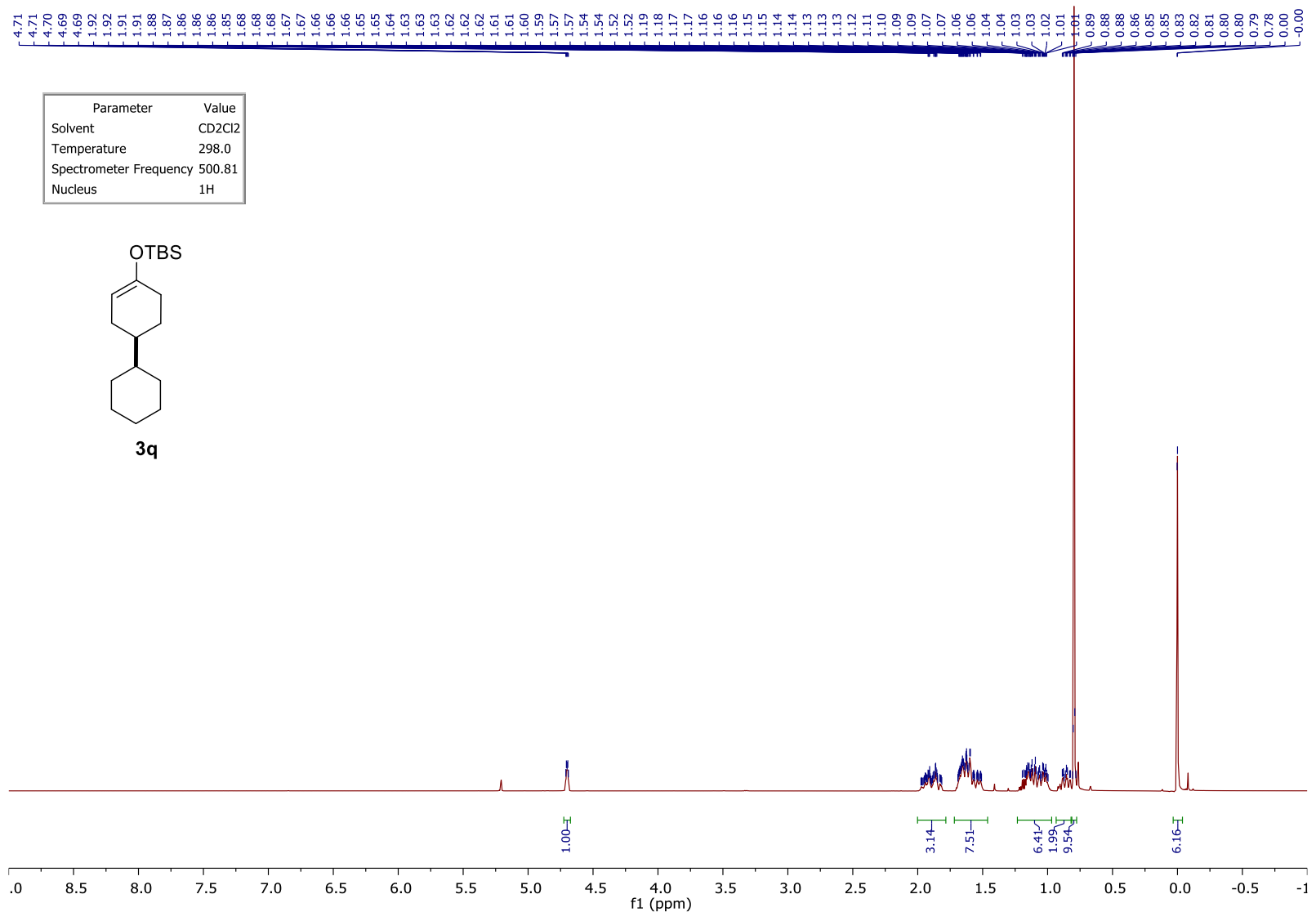




\begin{tabular}{|ll|}
\hline \multicolumn{1}{|c|}{ Parameter } & \multicolumn{1}{c|}{ Value } \\
Solvent & CD2C12 \\
Temperature & 298.0 \\
Spectrometer Frequency & 125.94 \\
Nucleus & $13 \mathrm{C}$ \\
\hline
\end{tabular}

\section{(R)-((4-benzylcyclohex-1-en-1-yl)oxy)(tert-butyl)dimethylsilane $3 r$}

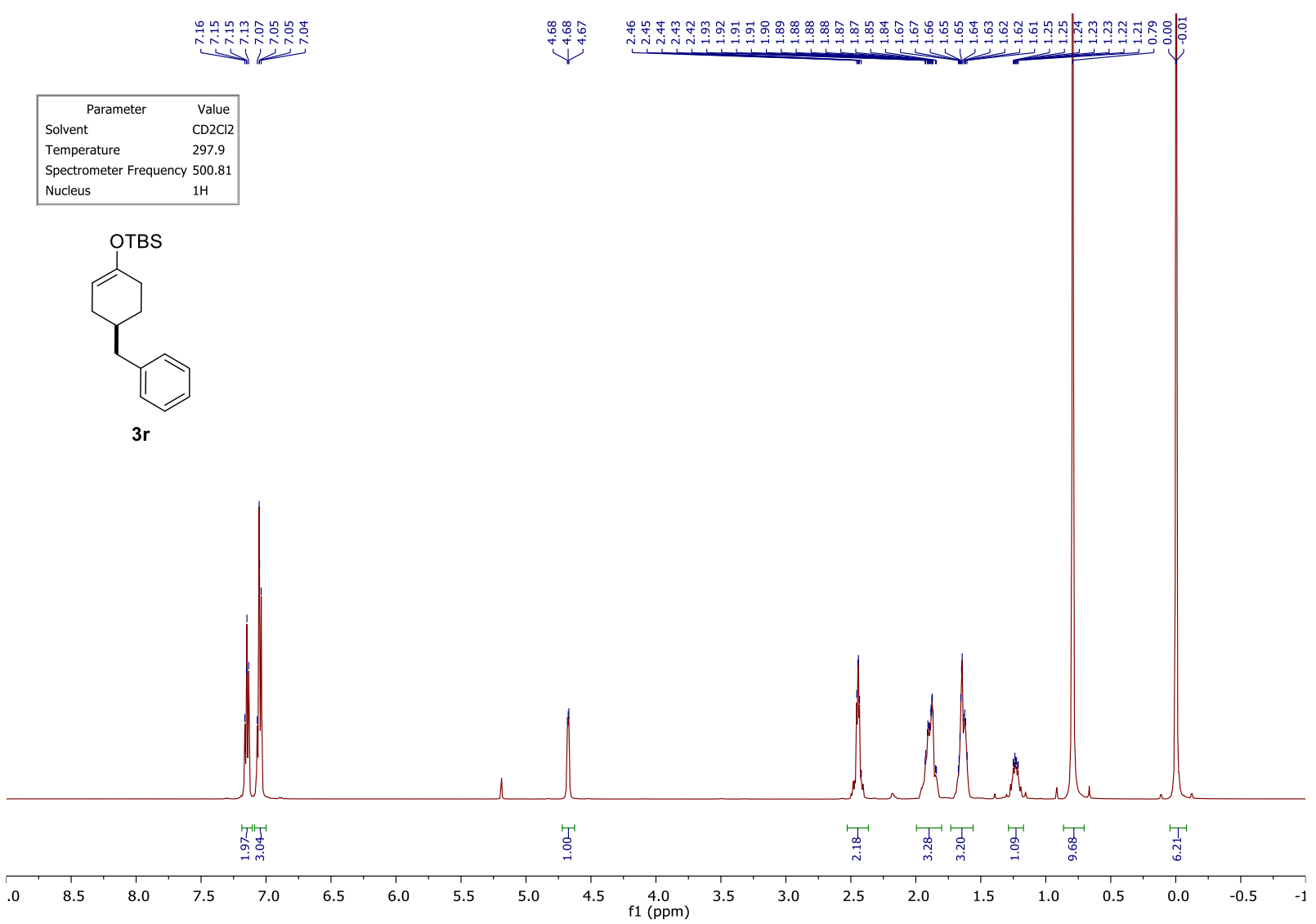




\begin{tabular}{|lc|}
\hline \multicolumn{1}{|c|}{ Parameter } & \multicolumn{1}{c|}{ Value } \\
Solvent & $\mathrm{CD} 2 \mathrm{C12}$ \\
Temperature & 298.0 \\
Spectrometer Frequency & 125.94 \\
Nucleus & $13 \mathrm{C}$ \\
\hline
\end{tabular}

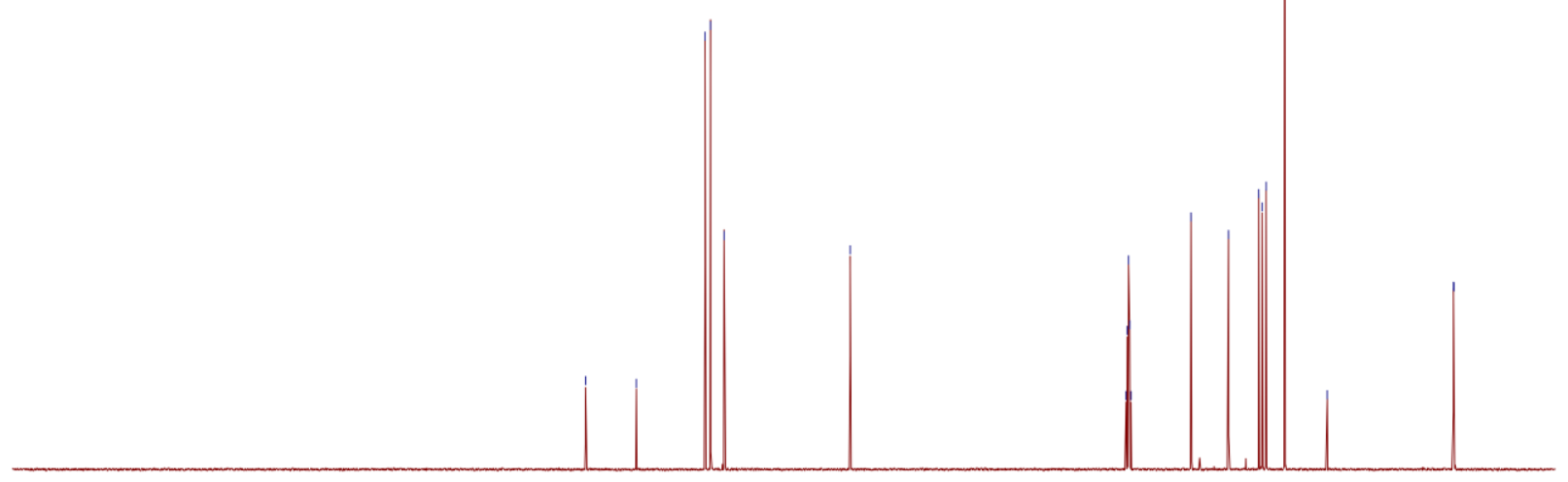

$\begin{array}{lllllllllllll}250 & 240 & 230 & 220 & 210 & 200 & 190 & 180 & 170 & 160 & 150 & 140 & 130\end{array}$

120
$\mathrm{f} 1(\mathrm{ppm})$

(R)-tert-butyldimethyl((1-methyl-1,2,3,6-tetrahydro-[1,1'-biphenyl]-4-yl)oxy)silane 3s:

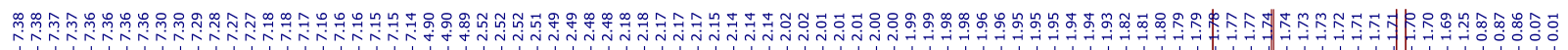

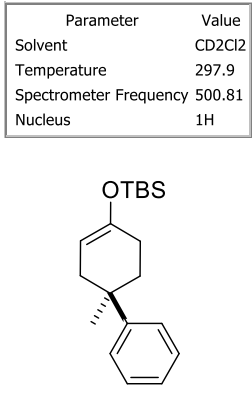

3s

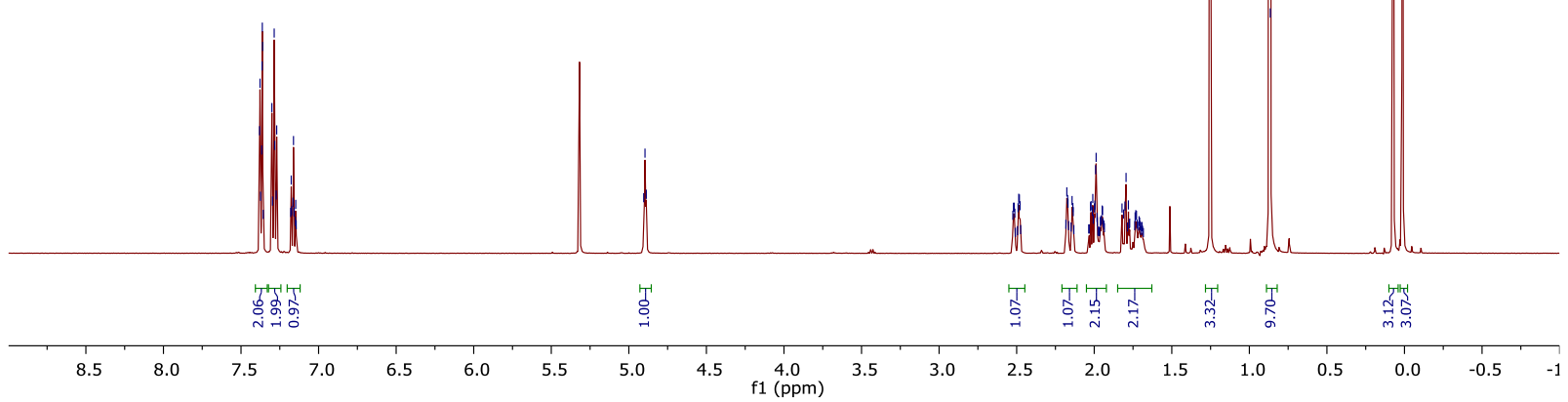




\begin{tabular}{|ll|}
\hline \multicolumn{1}{|c|}{ Parameter } & \multicolumn{1}{c|}{ Value } \\
Solvent & CD2C12 \\
Temperature & 298.0 \\
Spectrometer Frequency & 125.94 \\
Nucleus & $13 \mathrm{C}$ \\
\hline
\end{tabular}
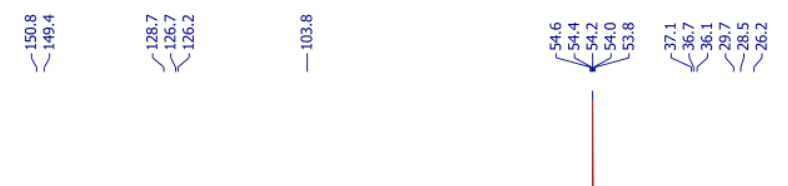

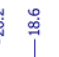

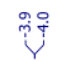

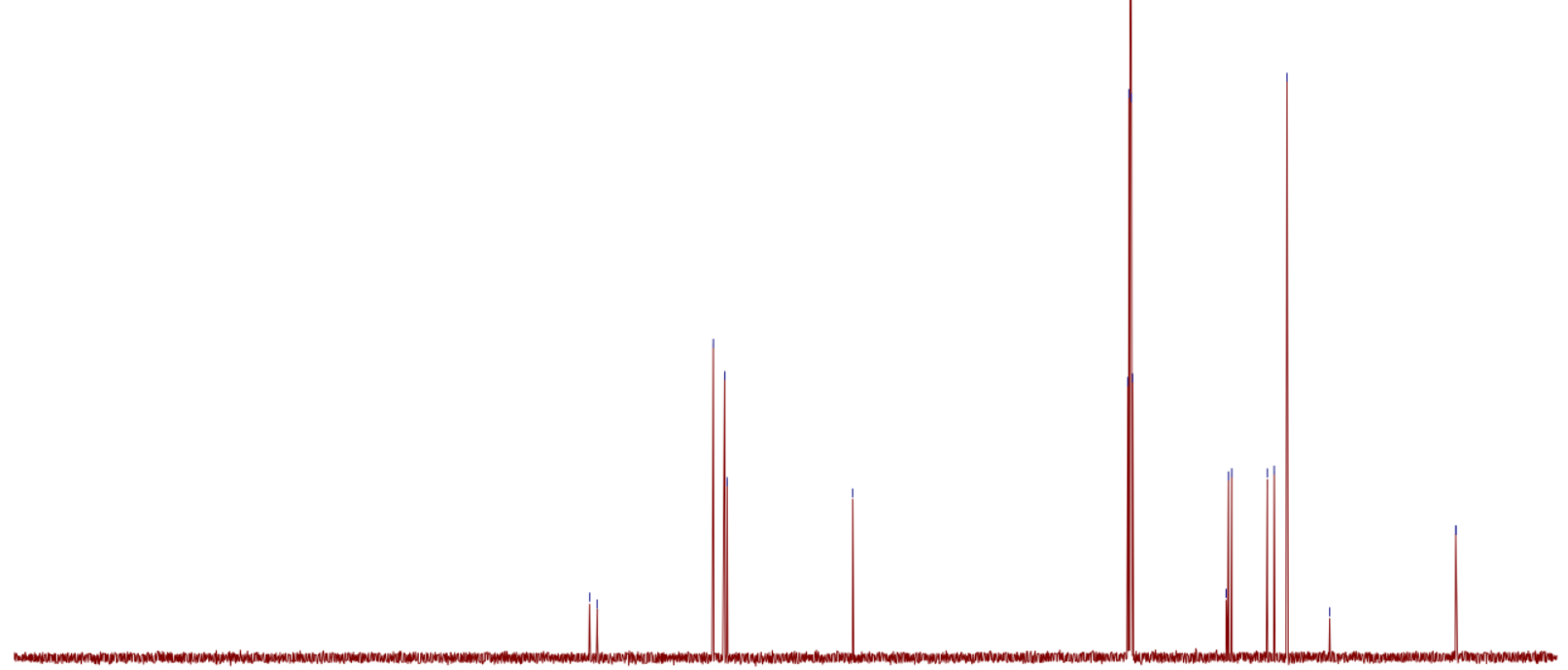

$\begin{array}{rllllllllllllllllllllllllllllllllll}250 & 240 & 230 & 220 & 210 & 200 & 190 & 180 & 170 & 160 & 150 & 140 & 130 & 120 & 110 & 100 & 90 & 80 & 70 & 60 & 50 & 40 & 30 & 20 & 10 & 0 & -10 & -20\end{array}$

(R)-4-((tert-butyldimethylsilyl)oxy)cyclohex-3-ene-1-carboxylate 3t:

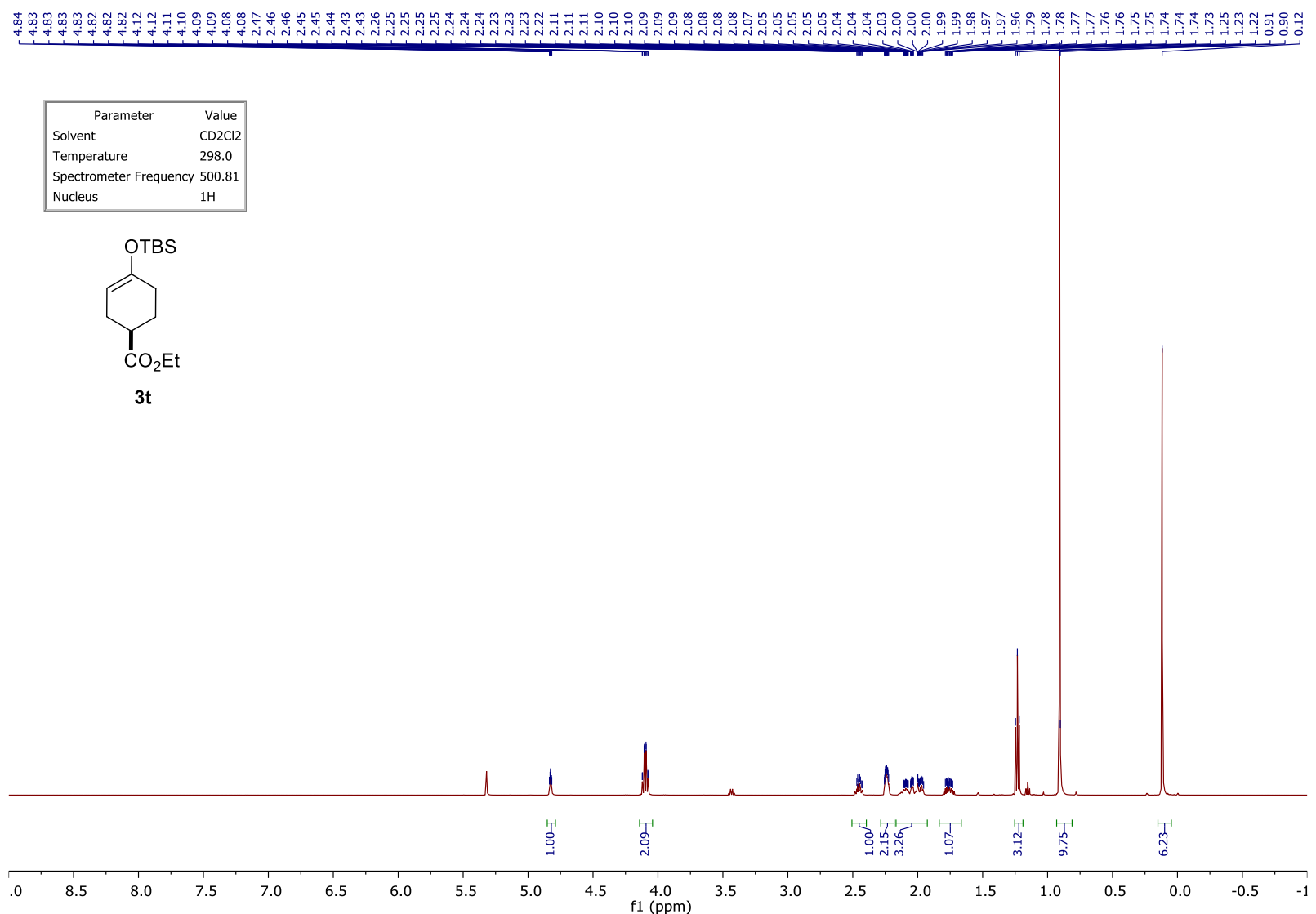




\begin{tabular}{|ll|}
\hline \multicolumn{1}{|c|}{ Parameter } & \multicolumn{1}{c|}{ Value } \\
Solvent & CD2C12 \\
Temperature & 298.0 \\
Spectrometer Frequency & 125.94 \\
Nucleus & $13 \mathrm{C}$ \\
\hline
\end{tabular}

Nucleus $13 \mathrm{C}$

$\begin{array}{lllllllllllllll}250 & 240 & 230 & 220 & 210 & 200 & 190 & 180 & 170 & 160 & 150 & 140 & 130 & \begin{array}{l}120 \\ \mathrm{f} 1(\mathrm{ppm})\end{array}\end{array}$

(((3aS,6aS)-1,3a,4,5,6,6a-hexahydropentalen-2-yl)oxy)triethylsilane 3u:

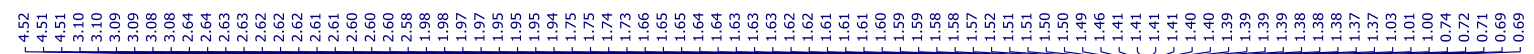

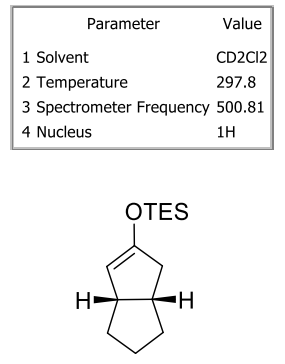

$3 \mathbf{u}$

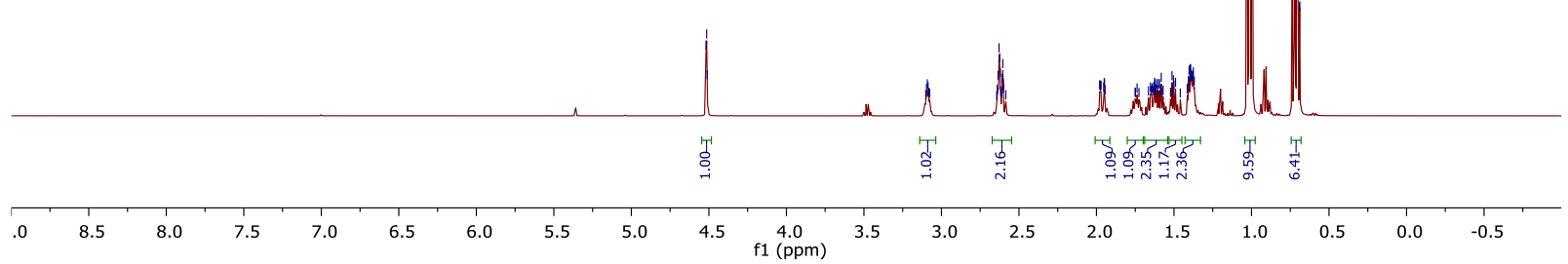




\begin{tabular}{|ll|}
\hline \multicolumn{1}{|c|}{ Parameter } & Value \\
1 Solvent & $\mathrm{CD} 2 \mathrm{Cl}$ \\
2 Temperature & 298.0 \\
3 Spectrometer Frequency & 125.94 \\
4 Nucleus & $13 \mathrm{C}$ \\
\hline
\end{tabular}

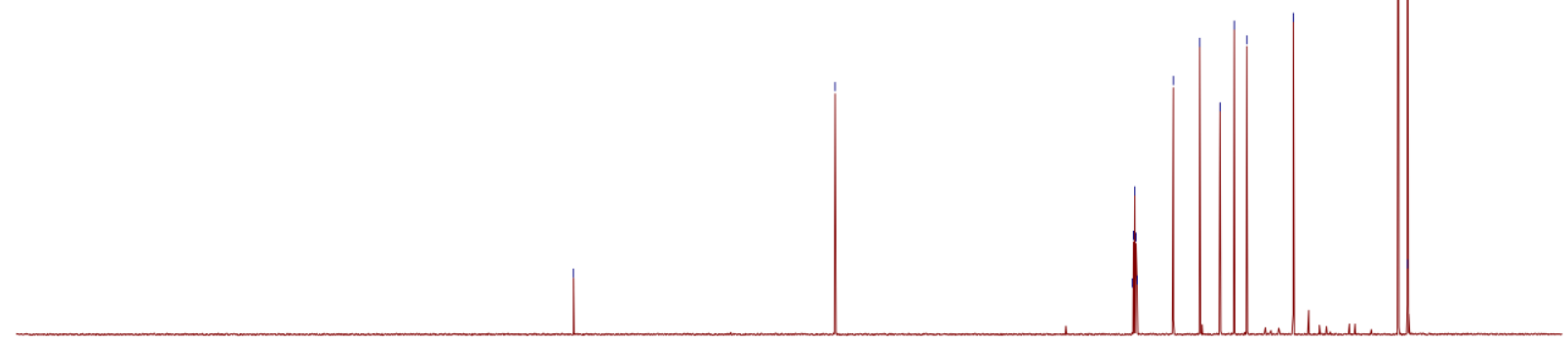

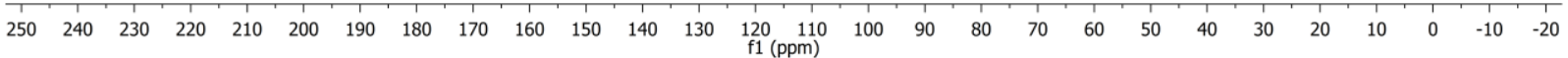

(R)-tert-butyldimethyl((3-phenylcyclobut-1-en-1-yl)oxy)silane 3v:
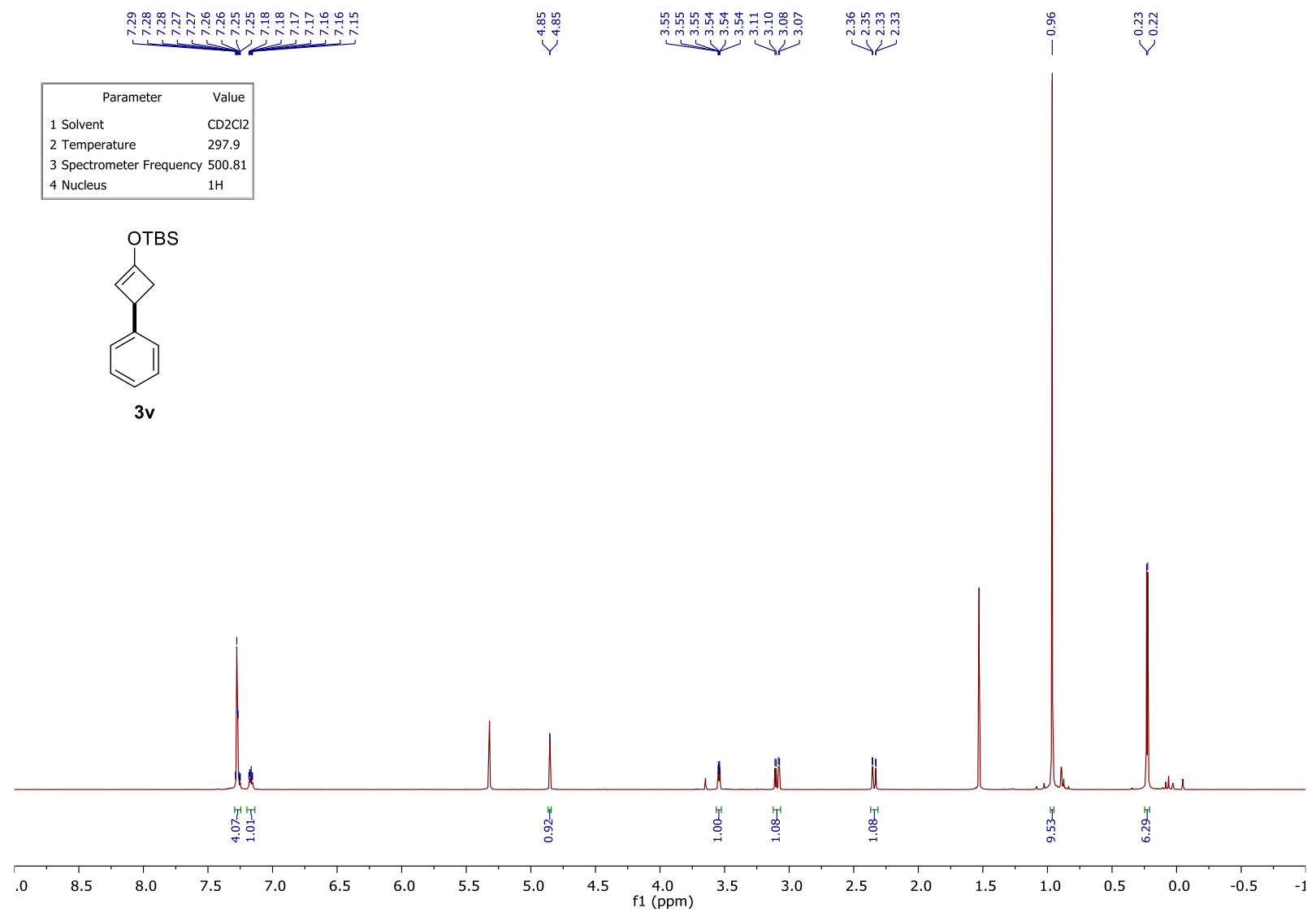

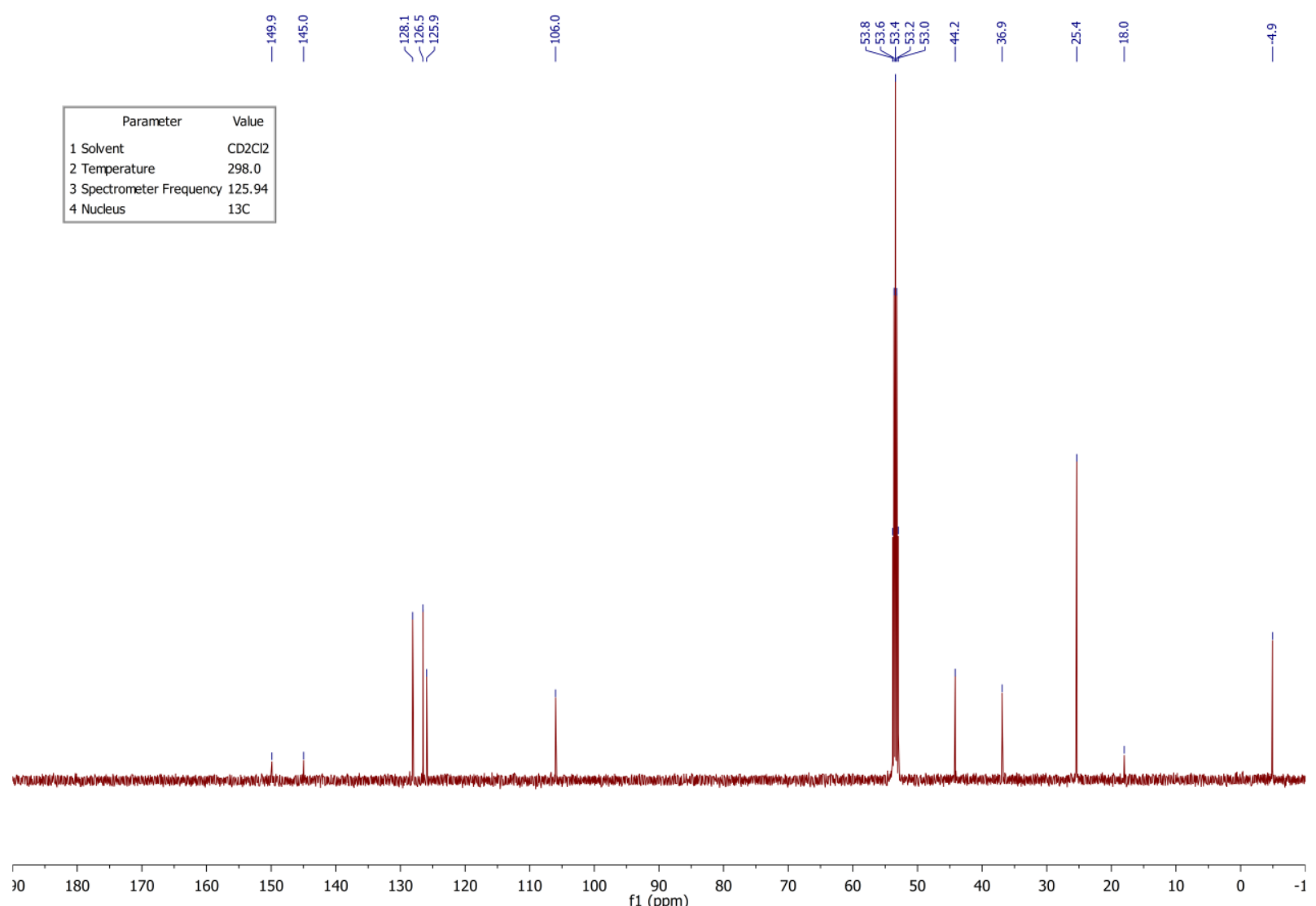

(((1R,3S)-3-bromo-1,2,3,6-tetrahydro-[1,1'-biphenyl]-4-yl)oxy)(tert-butyl)dimethylsilane 5:

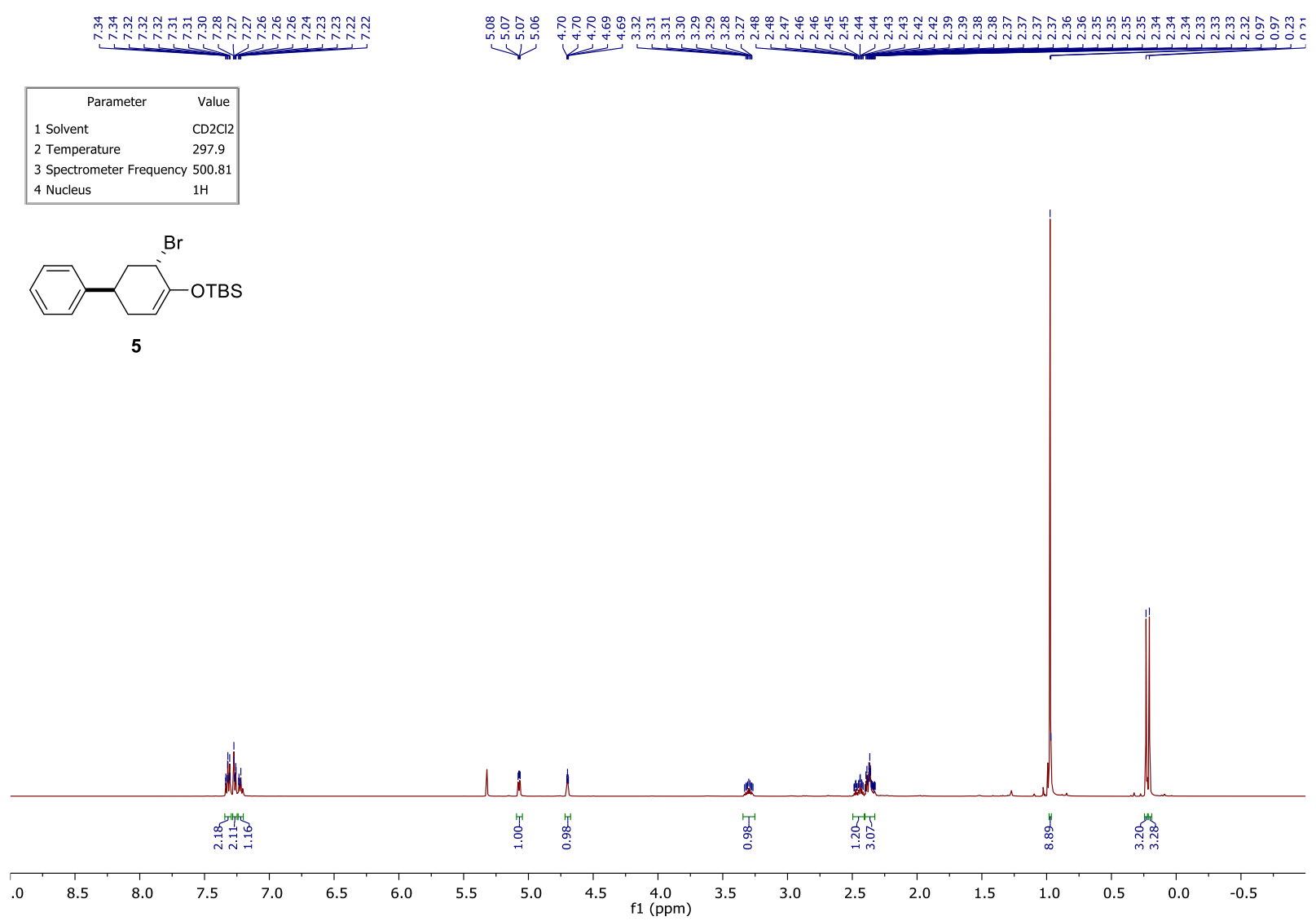




\begin{tabular}{|lc|}
\hline \multicolumn{1}{|c|}{ Parameter } & Value \\
1 Solvent & $\mathrm{CD} 2 \mathrm{Cl2}$ \\
2 Temperature & 298.0 \\
3 Spectrometer Frequency & 125.94 \\
4 Nucleus & $13 \mathrm{C}$ \\
\hline
\end{tabular}

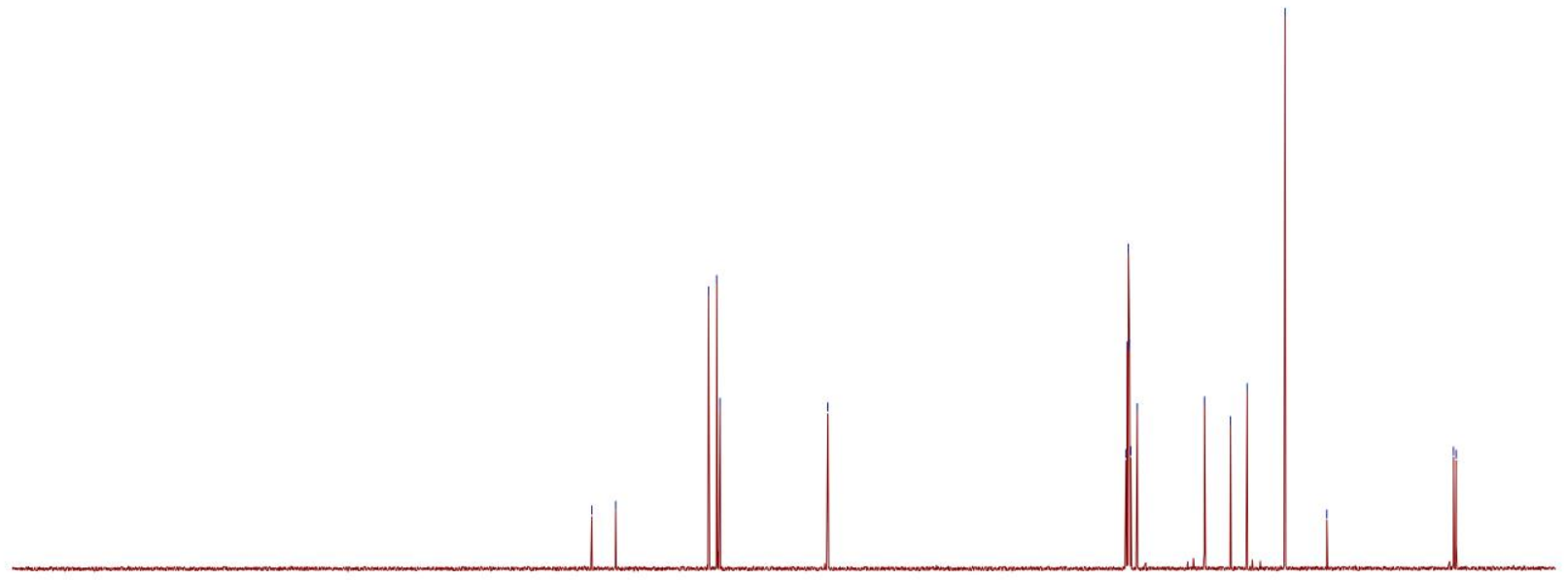

$\begin{array}{llllllllllllllllllllllllllll}250 & 240 & 230 & 220 & 210 & 200 & 190 & 180 & 170 & 160 & 150 & 140 & 130 & \begin{array}{c}120 \\ \mathrm{f} 1(\mathrm{ppm})\end{array} & 100 & 90 & 80 & 70 & 60 & 50 & 40 & 30 & 20 & 10 & 0 & -10 & -20\end{array}$

\section{(2R,4R)-2-fluoro-4-phenylcyclohexan-1-one 6:}<smiles>O=C1CS[C@@H](c2ccncc2)C[C@@H]1F</smiles>

\section{User Report ARK-AB-076-01}

NMR data support the formation of the 2,4-cis compound.

Important structural evidences are: NOEs between $\mathrm{H} 2, \mathrm{H} 4$ and one proton at $\mathrm{C} 6$ (cant be destiguished due to similar chemical shifts.) Strong couplings of $\mathrm{H}^{3}$ " with H2. and $\mathrm{H} 4(\sim 12.5 \mathrm{~Hz}) \rightarrow$ both trans-positioned

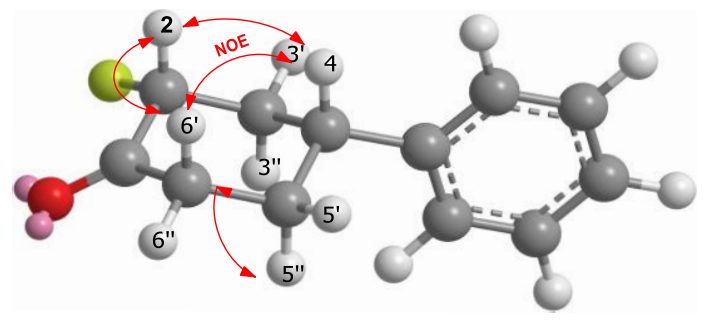

P-ID: ML00000

Measured on: $15 / 05 / 2019$

CHIFFRE:ARK-AB-076-01

ELNA\#:2878

Client: Jonas Aronow

Croup: List

Analysed on: $15 / 05 / 2019$

Analysed by: Leutzsch

Amount: $15 \mathrm{mg}$
Solvent: $\mathrm{CD} 2 \mathrm{Cl} 2$

eference: solvent

Semperature: $298 \mathrm{~K}$

Probe: 5 mm PABBO BB-1H/D Z-GRD Z119470/0004

C $1 \mathrm{H}]$-noesygpph, [13C, 1H]-hmbcgpl2ndqf, $1 \mathrm{H}$-zghfigqn 30

\begin{tabular}{|c|c|c|c|c|c|c|}
\hline \multicolumn{7}{|c|}{ Assignments } \\
\hline Atom & Chemical Shift & $\mathbf{J}$ & $\cos Y$ & HSQC & HMBC & NOESY \\
\hline $1 \mathrm{C}$ & 204.42 & $13.70\left(2^{\prime}\right)$ & & & $2,3 ", 6$ & \\
\hline $2 \mathrm{C}$ & 91.83 & $192.00\left(2^{\prime}\right)$ & & 2 & 3", 6 & \\
\hline $\mathrm{H}$ & 5.10 & $\begin{array}{l}48.30\left(2^{\prime} \mathrm{F}\right), \\
12.50\left(3^{\prime \prime}\right), 6.70\left(3^{\prime}\right)\end{array}$ & 3', 3" & 2 & $1,3,6$ & $3^{\prime}, 4,6$ \\
\hline 2' F & -189.39 & $\begin{array}{l}9.70(4), \\
192.00(2), \\
13.70(1), \\
17.80(3), 1.30(5), \\
48.30(2 \mathrm{H}), \\
1.80(4 \mathrm{H}), \\
9.70\left(3^{\prime \prime}\right), 4.30\left(3^{\prime}\right)\end{array}$ & & & & \\
\hline $3 \mathrm{C}$ & 40.66 & $17.80\left(2^{\prime}\right)$ & & $3^{\prime \prime}, 3^{\prime \prime}$ & 2 & \\
\hline $\mathrm{H}^{\prime}$ & 2.62 & $\begin{array}{l}3.40(4), \\
12.20\left(3^{\prime \prime}\right), \\
6.70(2), 4.30\left(2^{\prime} F\right)\end{array}$ & $2,3^{\prime \prime}, 4,5^{\prime}$ & 3 & & $2,3 ", 4$ \\
\hline $\mathrm{H}^{\prime \prime}$ & 2.10 & $\begin{array}{l}12.50(4), \\
12.50(2), \\
9.70\left(2^{\prime}\right)^{\prime} \\
12.20\left(3^{\prime}\right)\end{array}$ & $2,3^{\prime}, 4$ & 3 & $1,2,4,5,7$ & $3^{\prime}, 8,8^{\prime}$ \\
\hline $4 \mathrm{C}$ & 41.13 & $9.70\left(2^{\prime}\right)$ & & 4 & $3^{\prime \prime}, 5^{\prime}, 5^{\prime \prime}, 6,8,8^{\prime}$ & \\
\hline $\mathrm{H}$ & 3.19 & $\begin{array}{l}1.80\left(2^{\prime} \mathrm{F}\right), \\
12.50\left(3^{\prime \prime}\right), 3.40\left(3^{\prime}\right)\end{array}$ & $\begin{array}{l}\text { 3', 3", 5', 5", } \\
8,8^{\prime}\end{array}$ & 4 & $5,7,8,8^{\prime}$ & $2,3^{\prime}, 5^{\prime}, 6,8,8^{\prime}$ \\
\hline $5 \mathrm{C}$ & 34.18 & $1.30\left(2^{\prime}\right)$ & & $5^{\prime}, 5^{\prime \prime}$ & $3 ", 4,6$ & \\
\hline $\mathrm{H}^{\prime}$ & 2.20 & & 3', 4, 5", 6 & 5 & 4,6 & $4,5 ", 6$ \\
\hline $\mathrm{H}^{\prime \prime}$ & 1.89 & & $4,5^{\prime}, 6$ & 5 & $4,6,7$ & $5^{\prime}, 8,8^{\prime}$ \\
\hline $6 \mathrm{C}$ & 39.38 & & & 6 & $2,5^{\prime}, 5^{\prime \prime}$ & \\
\hline $\mathrm{H} 2$ & 2.54 & & $5^{\prime}, 5^{\prime \prime}$ & 6 & $1,2,4,5$ & $2,4,5^{\prime}$ \\
\hline $7 \mathrm{C}$ & 142.99 & & & & $3^{\prime \prime}, 4,5 ", 9,9^{\prime}$ & \\
\hline $8 \mathrm{C}$ & 126.58 & & & 8 & $4,8^{\prime}, 10$ & \\
\hline $\mathrm{H}$ & 7.25 & & 4 & 8 & $4,8^{\prime}, 10$ & $3^{\prime \prime}, 4,5^{\prime \prime}$ \\
\hline $8^{\prime} \mathrm{C}$ & 126.58 & & & $8^{\prime}$ & $4,8,10$ & \\
\hline $\mathrm{H}$ & 7.25 & & 4 & $8^{\prime}$ & $4,8,10$ & 3", 4, 5" \\
\hline $9 \mathrm{C}$ & 128.66 & & & 9 & $9^{\prime}$ & \\
\hline $\mathrm{H}$ & 7.34 & & & 9 & $7,9^{\prime}$ & \\
\hline $9^{\prime} \mathrm{C}$ & 128.66 & & & $9^{\prime}$ & 9 & \\
\hline H & 7.34 & & & $9^{\prime}$ & 7,9 & \\
\hline $\ln r$ & 12688 & & & $1 n$ & $88^{\prime}$ & \\
\hline
\end{tabular}




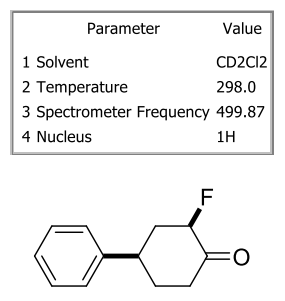

6
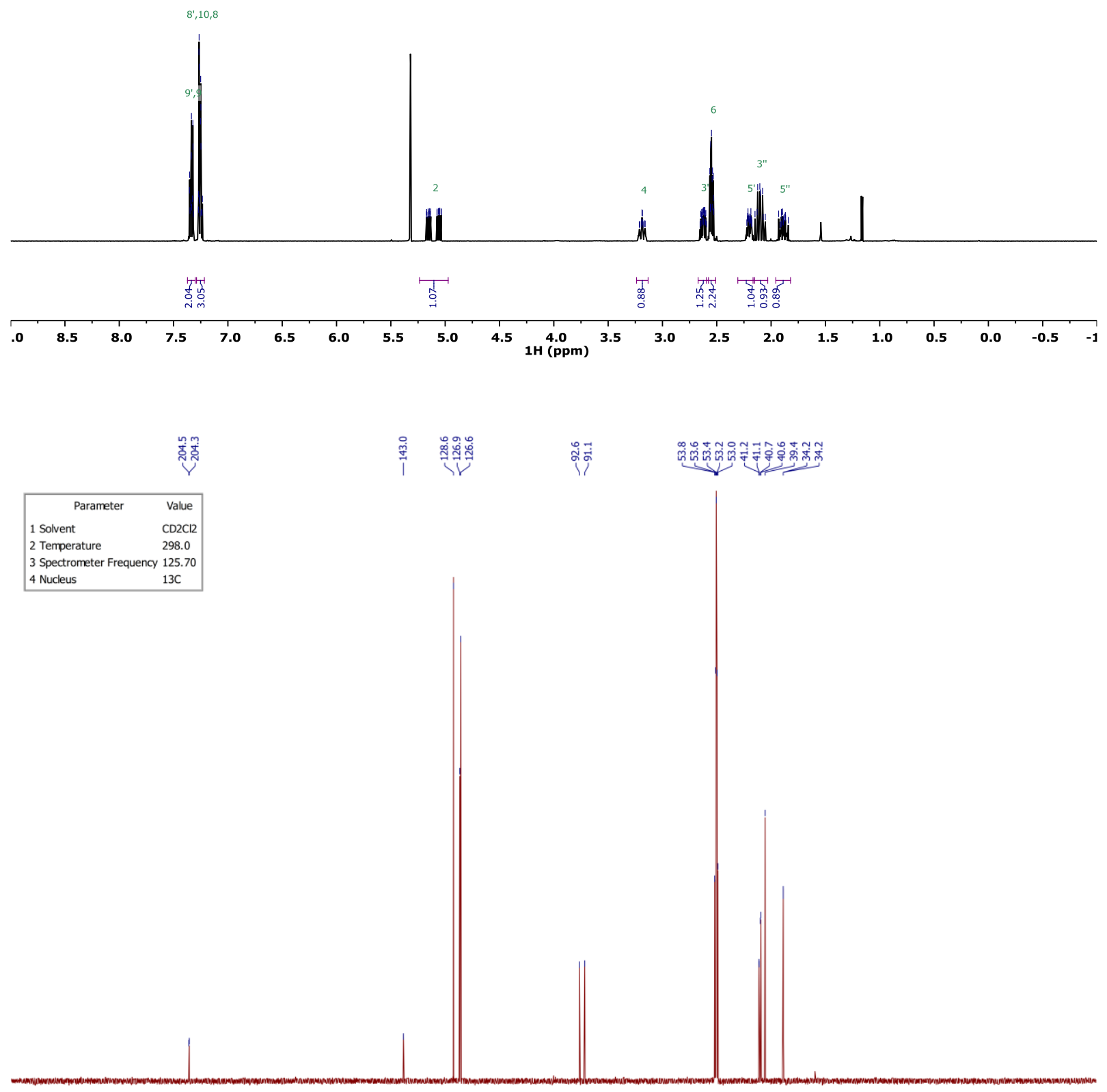

\begin{tabular}{lllllllllllllllllllllllllllllllllllllllll}
\hline 250 & 240 & 230 & 220 & 210 & 200 & 190 & 180 & 170 & 160 & 150 & 140 & 130 & 120 & 110 & 100 & 90 & 80 & 70 & 60 & 50 & 40 & 30 & 20 & 10 & 0 & -10 & -20 & -30 & -40 & -50
\end{tabular} 


\begin{tabular}{|lc|}
\hline \multicolumn{1}{|c|}{ Parameter } & Value \\
1 Solvent & $\mathrm{CD} 2 \mathrm{Cl} 2$ \\
2 Temperature & 298.0 \\
3 Spectrometer Frequency & 470.31 \\
4 Nucleus & $19 \mathrm{~F}$ \\
\hline
\end{tabular}

\begin{tabular}{rlllllllllllllllllll}
\hline 110 & 90 & 70 & 50 & 30 & 10 & -10 & -30 & -50 & -70 & -90 & -110 & -130 & -150 & -170 & -190 & -210 & -230 & -250 & -270
\end{tabular}

\section{${ }^{1} \mathrm{H}-{ }^{1} \mathrm{H} \operatorname{COSY}$}

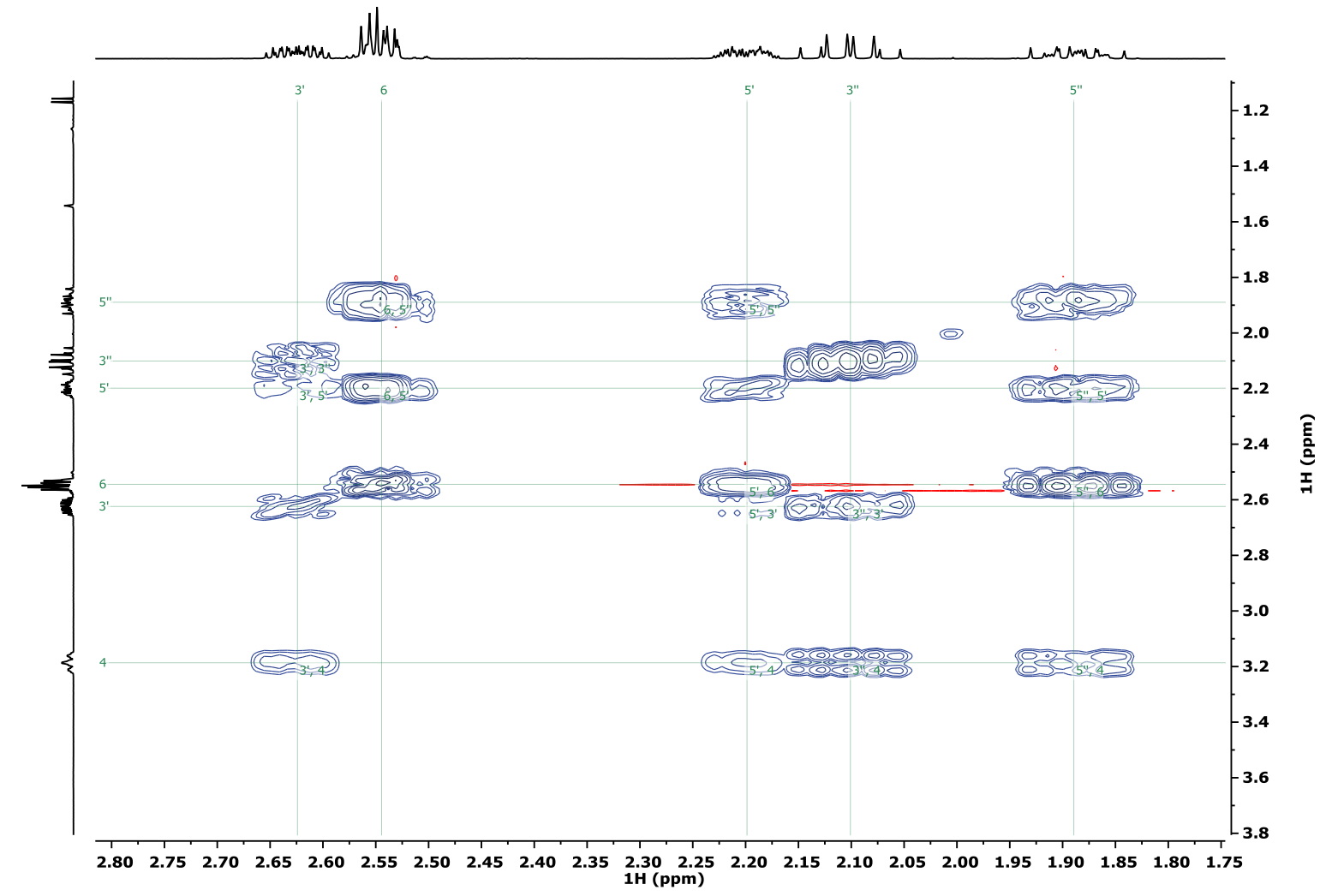


${ }^{1} \mathrm{H}-{ }^{13} \mathrm{C}$ HSQC

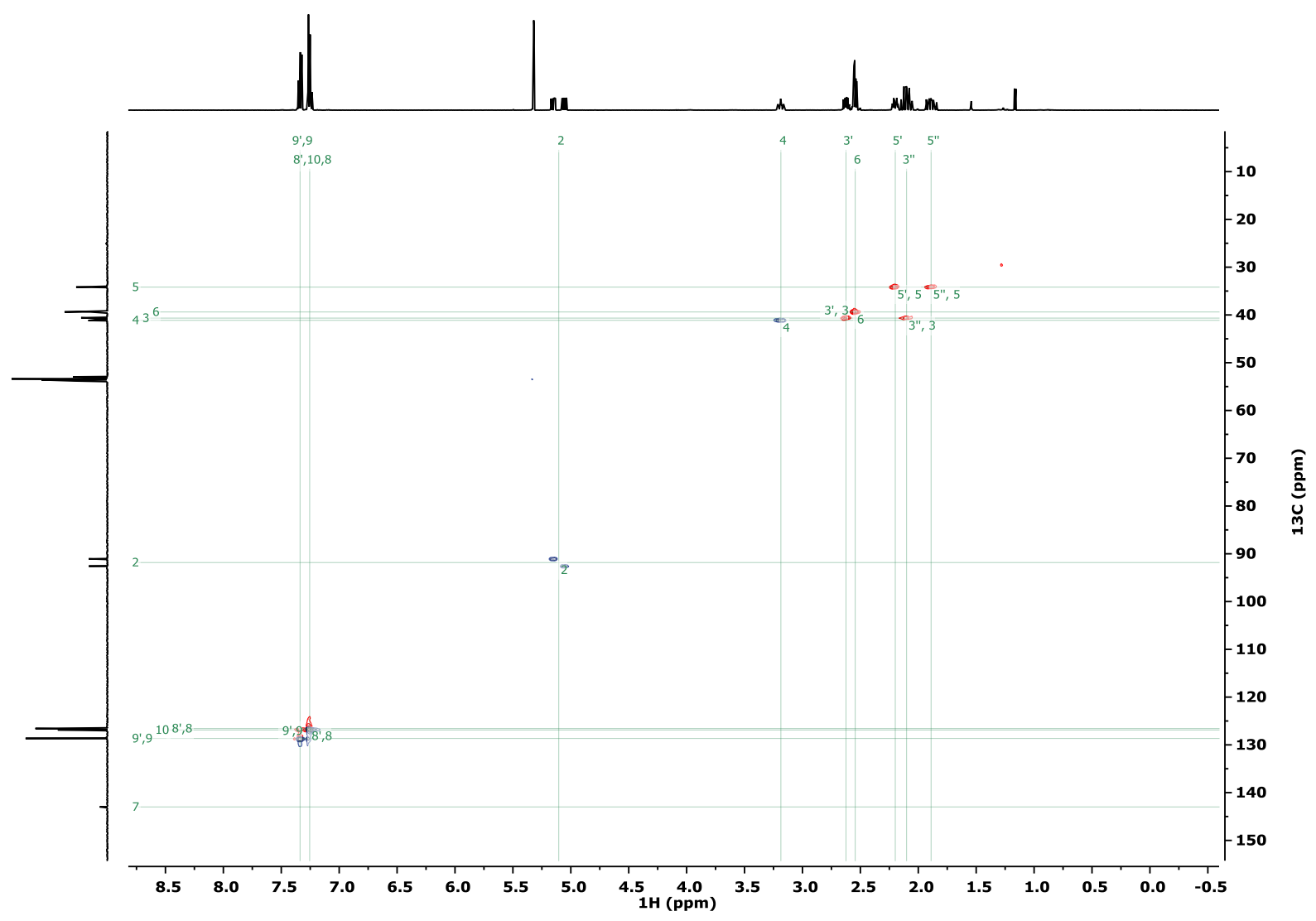

${ }^{1} \mathrm{H}^{-13} \mathrm{C}$ HMBC

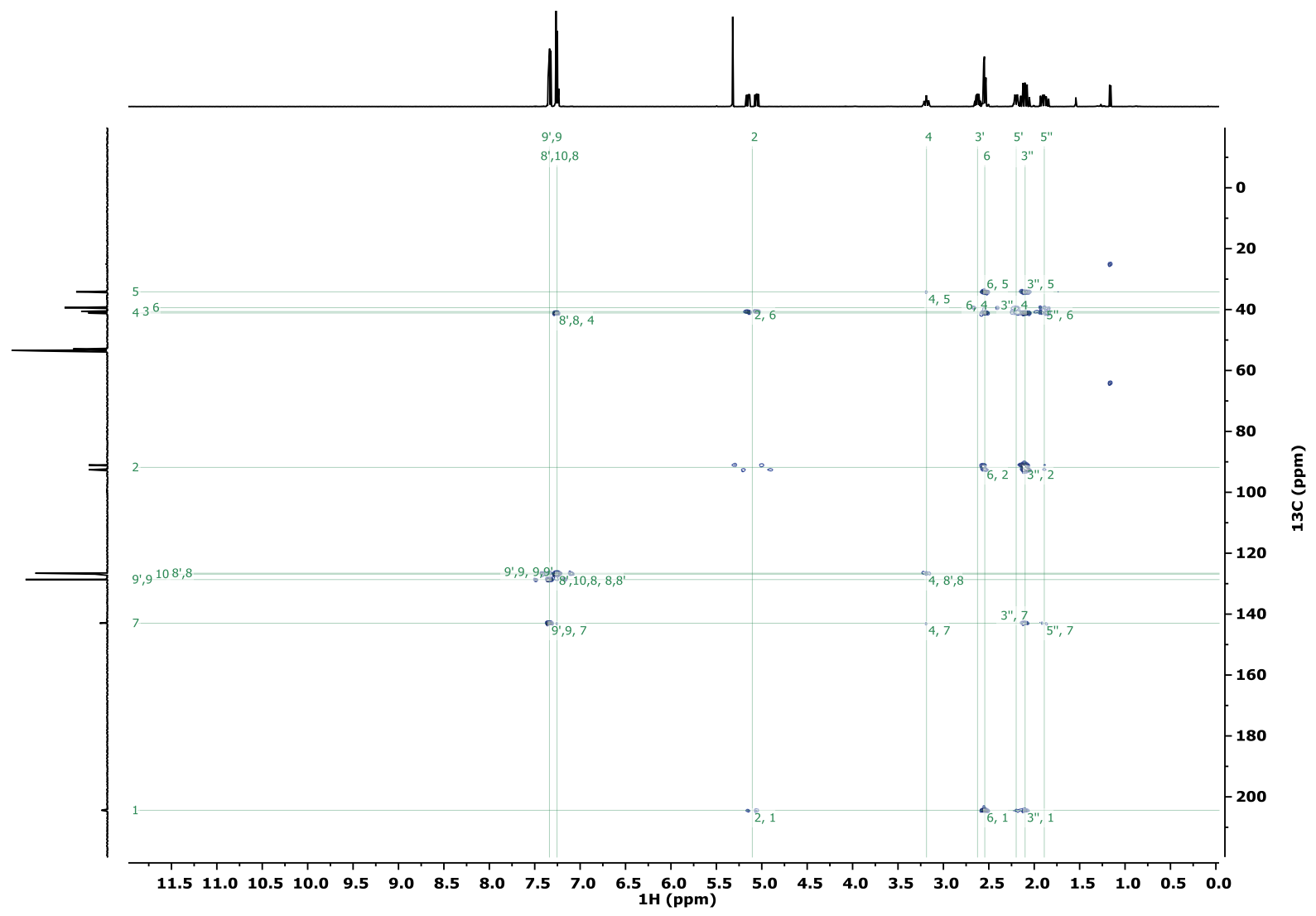

${ }^{1} \mathrm{H}-{ }^{1} \mathrm{H}$ NOESY 


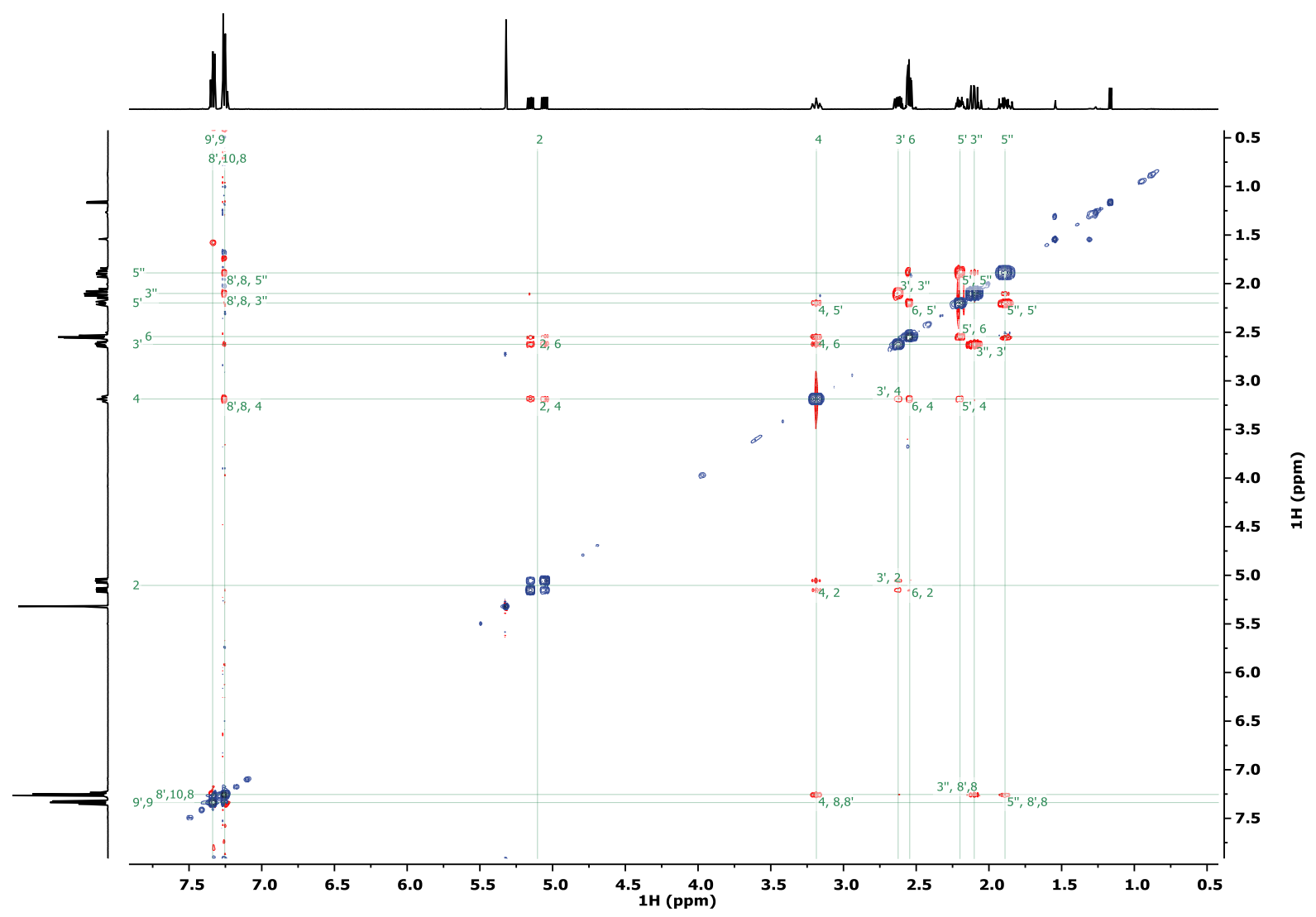

(R)-2,3-dihydro-[1,1'-biphenyl]-4(1H)-one 7:

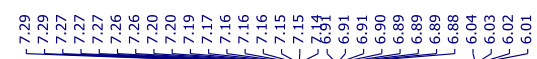

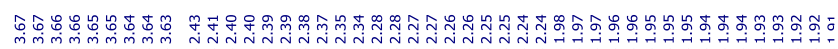
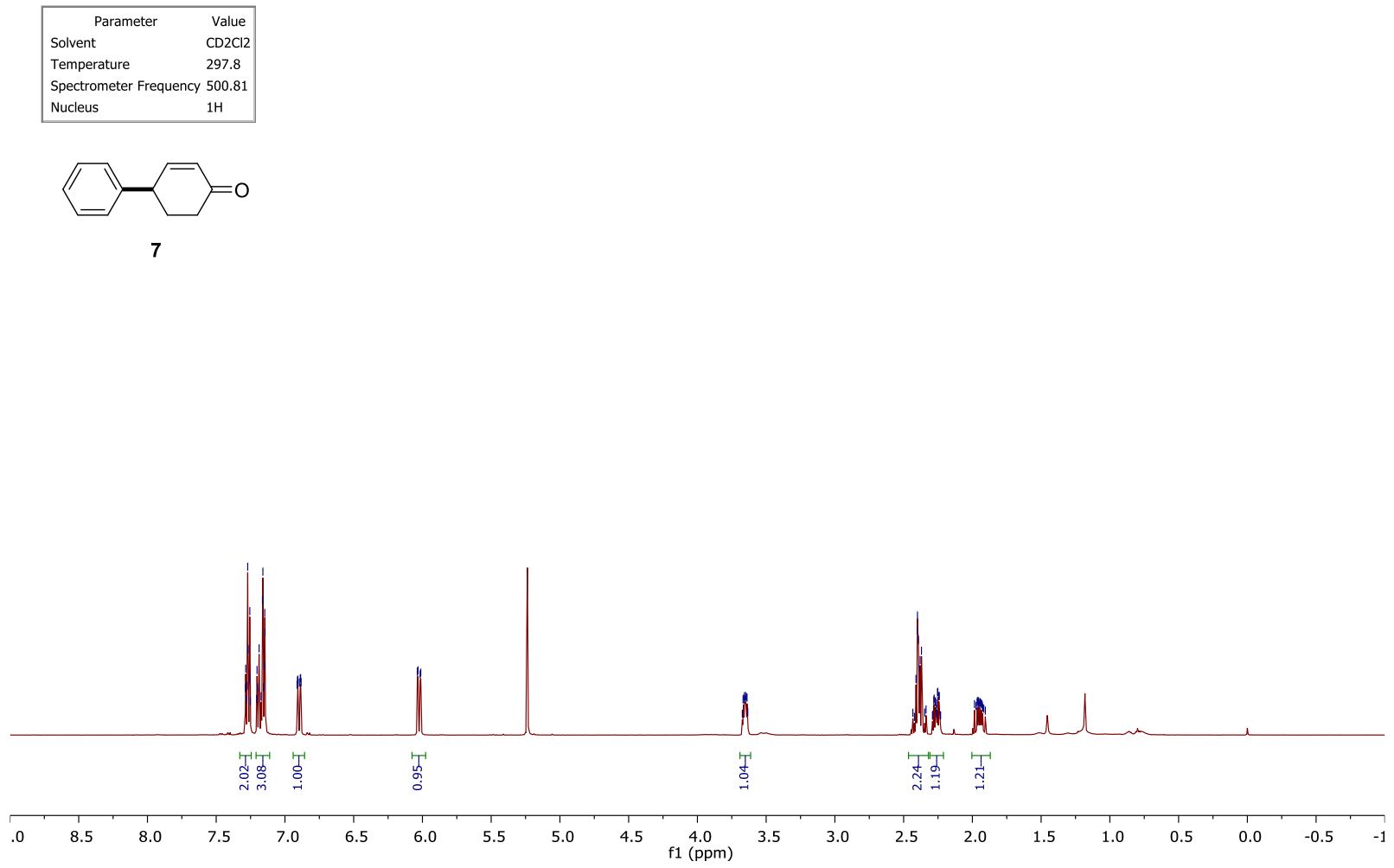


\begin{tabular}{|ll|}
\hline \multicolumn{1}{|c|}{ Parameter } & Value \\
Solvent & $\mathrm{CD} 2 \mathrm{Cl} 2$ \\
Temperature & 298.0 \\
Spectrometer Frequency & 125.94 \\
Nucleus & $13 \mathrm{C}$ \\
\hline
\end{tabular}

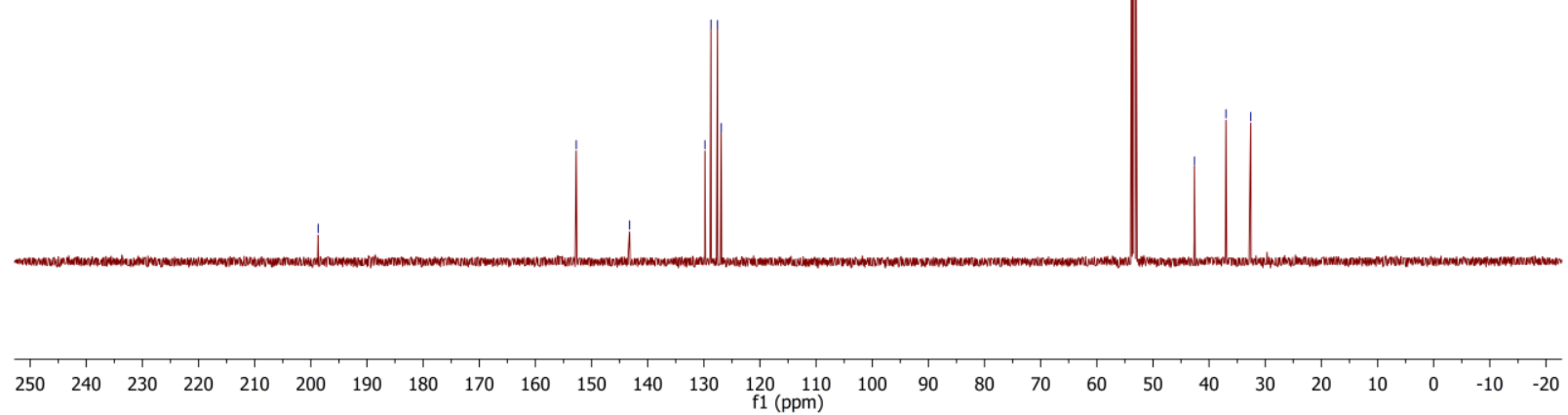

$(1 S, 3 S, 6 R, 7 R)-6-(($ tert-butyldimethylsilyl)oxy)-3 phenylbicyclo[4.2.0]octane-7-carboxylate 8 
<smiles>COC(=O)N1PC2CC(c3ccccc3)CC[C@]21OS(C)(C)N(C)C(C)(C)C</smiles>

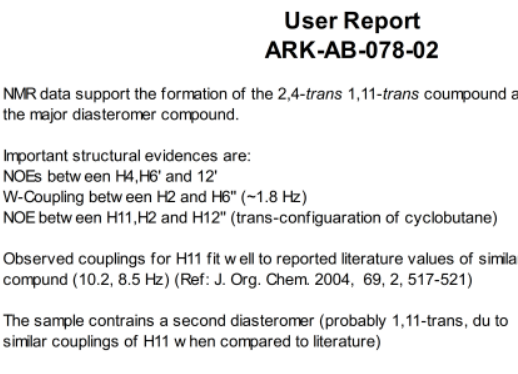

Due to big overlap of $\mathrm{H}^{\prime 2}$ and $\mathrm{H}^{\prime \prime}$, the sample was also measured in $\mathrm{C6D} 6$

3D Model of Major Component

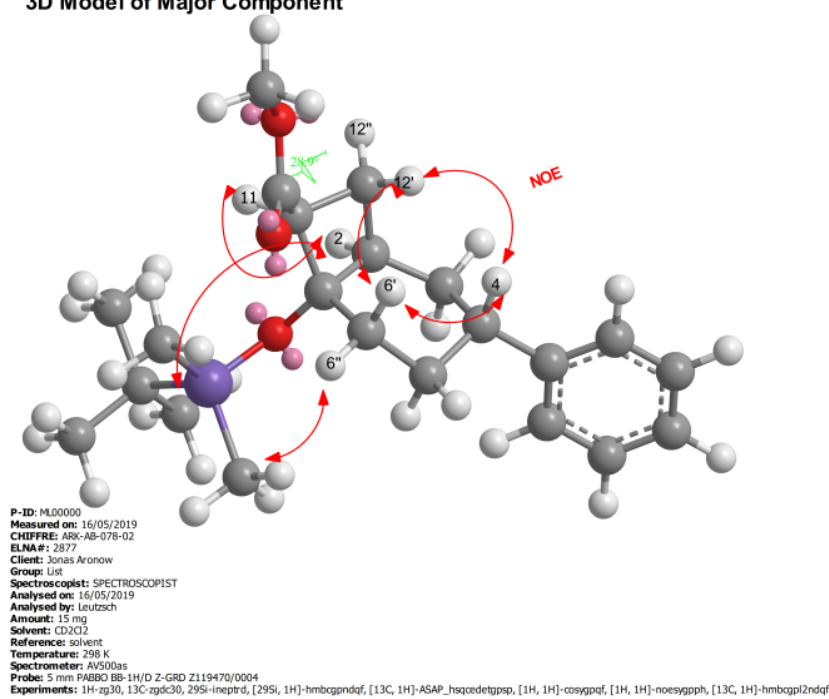

\begin{tabular}{|c|c|c|c|c|c|c|c|c|c|c|c|c|c|}
\hline \multicolumn{7}{|c|}{ A ssignments } & \multicolumn{7}{|c|}{ A ssignments } \\
\hline A tom & $\begin{array}{c}\text { chem } \\
\text { ical } \\
\text { shiff }\end{array}$ & $\mathrm{J}$ & $\cos \gamma$ & HSQ & $\underset{\mathrm{C}}{\text { HMB }}$ & $\underset{y}{\text { NoES }}$ & Atom & $\begin{array}{c}\text { chem } \\
\text { ical } \\
\text { shiff }\end{array}$ & $\mathrm{J}$ & $\cos Y$ & $\begin{array}{c}\text { HSQ } \\
\text { C }\end{array}$ & $\begin{array}{c}\text { HMB } \\
\text { C }\end{array}$ & NOES \\
\hline 1c & 75.55 & & & & $\begin{array}{l}3,11, \\
12,\end{array}$ & & $11 \mathrm{C}$ & 499.46 & & & 11 & $\begin{array}{l}12, " \\
12^{\prime \prime}\end{array}$ & \\
\hline $2 \mathrm{C}$ & 41.82 & & & 2 & $\begin{array}{l}3,11, \\
12^{2}, \\
2^{\prime \prime} \\
3,4,4\end{array}$ & & H & 2.97 & $\begin{array}{l}10.10) \\
\left.(121)^{2}\right) \\
8.30( \\
\left.12^{\prime \prime}\right)\end{array}$ & $\begin{array}{l}121 \\
122^{\prime \prime}\end{array}$ & 11 & $\begin{array}{l}1,2, \\
3,6, \\
12,13\end{array}$ & $\begin{array}{l}2,12, \\
101\end{array}$ \\
\hline H & & 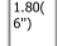 & $\begin{array}{l}3,1,12, \\
12^{2}\end{array}$ & $2^{2} x-10$ & $\begin{array}{l}3,4, \\
12\end{array}$ & $\begin{array}{l}\text { 3, 11, } \\
\text { 12", } \\
101\end{array}$ & $12 \mathrm{C}$ & 19.17 & & & $\begin{array}{l}l_{12 !}^{\prime \prime \prime} \\
12^{\prime \prime}\end{array}$ & 2,11 & \\
\hline $3 \mathrm{c}$ & 32.29 & & & 3 & $\begin{array}{l}2,5, \\
5^{\prime \prime},{ }^{\prime 1} \\
12^{\prime}\end{array}$ & & $\mathrm{H}^{\prime}$ & 1.76 & $\begin{array}{l}10.10 \\
(11)\end{array}$ & $\begin{array}{l}2,11, \\
122^{\prime \prime}\end{array}$ & 12 & $\begin{array}{l}1,2, \\
3,11 \\
13\end{array}$ & 4,11 \\
\hline H2 & 1.68 & & 2,4 & 3 & $\begin{array}{l}1,2,2 \\
4,5\end{array}$ & $\begin{array}{l}2,4 ; \\
8,8 ; \\
101\end{array}$ & $\mathrm{H}^{\prime \prime}$ & 1.83 & $\begin{array}{l}8.30( \\
11)\end{array}$ & $\begin{array}{l}2,11, \\
12^{\prime}\end{array}$ & 12 & 2,11 & 2 \\
\hline $4 \mathrm{C}$ & 39.77 & & & 4 & 2,3 & & $\mid 13 c$ & 172.37 & & & & $\begin{array}{l}11 ; \\
12 ;, 14\end{array}$ & \\
\hline & & 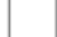 & & 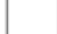 & 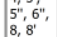 & & $\frac{14 \mathrm{C}}{\mathrm{H} 3}$ & 50.91 & & & 14 & 13 & \\
\hline H & 2.64 & & $\begin{array}{l}3,5, \\
5^{\prime \prime}\end{array}$ & 4 & $\begin{array}{l}4,6,6 \\
7,8,8^{\prime}\end{array}$ & 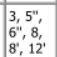 & $100 \mathrm{si}$ & 11.19 & & & & $\begin{array}{l}101, \\
102, \\
104\end{array}$ & \\
\hline $5 \mathrm{C}$ & 27.95 & & & $5^{\prime}, 5^{\prime \prime}$ & $3,6^{\prime \prime}$ & & & & & & & $\begin{array}{l}104 ; \\
104^{4}\end{array}$ & \\
\hline $\mathrm{H}^{\prime}$ & 1.57 & & $\mid \begin{array}{l}44^{4}, 5^{\prime} \\
5^{\prime \prime}, 6^{\prime}\end{array}$ & $5^{5}$ & 3,4 & $\begin{array}{l}8,8, \\
101\end{array}$ & $101 \mathrm{C}$ & -3.15 & & & 101 & & \\
\hline $\mathrm{H}^{\prime \prime}$ & 1.62 & & $\begin{array}{l}66^{\prime \prime}, 5^{\prime} \\
x^{\prime}, 6^{\prime \prime}\end{array}$ & 5 & 3,4 & 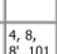 & H3 & 0.18 & & & 101 & $\begin{array}{l}100, \\
103\end{array}$ & $\begin{array}{l}2,3,3 \\
5,5, \\
6,11\end{array}$ \\
\hline $6 \mathrm{C}$ & 32.50 & & & 6', 6" & 4,11 & & $102 \mathrm{C}$ & -3.15 & & & 102 & & \\
\hline$H^{\prime}$ & 1.79 & $\begin{array}{l}14.50 \\
\left(6^{2}\right)\end{array}$ & $\begin{array}{l}5^{5,}, 5^{\prime \prime} \\
6^{\prime \prime}\end{array}$ & 6 & & 101 & $\mathrm{H3}$ & 0.18 & & & 102 & $\begin{array}{l}100, \\
103\end{array}$ & \\
\hline $\mathrm{H}^{\mathrm{N}}$ & 1.90 & 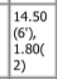 & $\begin{array}{l}5^{5,5 "}, \\
6^{\prime \prime}\end{array}$ & 6 & 4,5 & 4 & $103 \mathrm{C}$ & 17.83 & & & & $\begin{array}{l}101, \\
102, \\
102, \\
104, \\
104,\end{array}$ & \\
\hline $\begin{array}{l}7 \mathrm{C} \\
8 \mathrm{C}\end{array}$ & \begin{tabular}{|l|l}
147.39 \\
12.83
\end{tabular} & & & & $4,9,9^{\prime}$ & & $104 \mathrm{C}$ & 25.42 & & & 104 & & \\
\hline$\frac{8 \mathrm{C}}{\mathrm{H}}$ & $\mid \frac{126.83}{7.20}$ & & 9 & $\frac{8}{8} \cdot x \cdot y$ & $\begin{array}{l}4,8^{\prime} \\
4,8^{\prime}\end{array}$ & & $\mathrm{H3}$ & 0.92 & & & 104 & 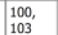 & \\
\hline sic & & & & & 10 & $5,5^{\prime \prime}$ & $104^{4} \mathrm{C}$ & 25.42 & & & 104' & & \\
\hline$\frac{8 \mathrm{C} C}{\mathrm{H}}$ & $\begin{array}{l}126.83 \\
7.20\end{array}$ & & $g^{\prime}$ & $\frac{8^{\prime}}{8^{\prime}}$ & \begin{tabular}{|l|}
4,8 \\
4,8,
\end{tabular} & $3,4, \quad, \quad \geqslant$ & H3 & 0.92 & & & $104^{\prime}$ & $\begin{array}{l}100, \\
103,\end{array}$ & \\
\hline${ }^{2}$ & & & & & 10 & $5,5^{\prime \prime}$ & $104^{\circ \mathrm{C}}$ & 25.42 & & & $104^{\prime \prime}$ & & \\
\hline$\frac{9 \mathrm{C}}{\mathrm{H}}$ & \begin{tabular}{|l}
128.28 \\
7.28
\end{tabular} & & 8,10 & 9 & $\frac{9^{\prime}}{7,9^{\prime}}$ & & $\mathrm{H}^{3}$ & & & & 104" & 罗00, & \\
\hline$g^{\prime} \mathrm{C}$ & 128.25 & & & $9^{\prime}$ & 9 & & 2000 & & & & & & \\
\hline $\mathrm{H}$ & 7.28 & & 88,10 & $g^{\prime}$ & 7,9 & & 2010 & & & & & & \\
\hline $10 \mathrm{C}$ & 125.86 & & & 10 & $8,8^{\prime}$ & & 2020 & & & & & & \\
\hline $\mathrm{H}$ & 7.17 & & $9,9^{\prime}$ & 10 & & & & & & & & & \\
\hline
\end{tabular}

Vั
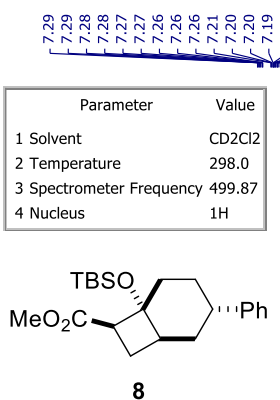

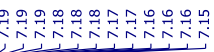

Value
CD2C12
298.0
499.87

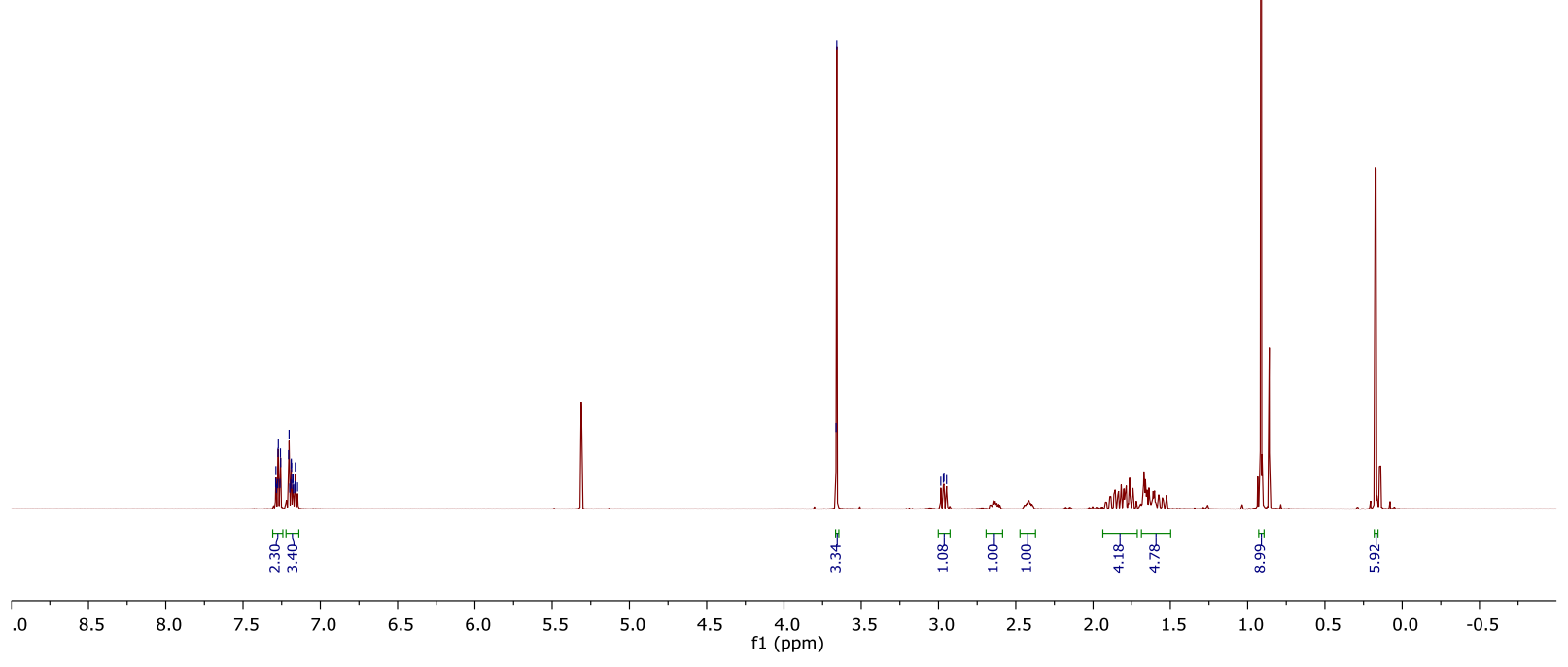




\begin{tabular}{|ll|}
\hline \multicolumn{1}{|c|}{ Parameter } & \multicolumn{1}{c|}{ Value } \\
1 Solvent & CD2C12 \\
2 Temperature & 298.0 \\
3 Spectrometer Frequency & 125.70 \\
4 Nucleus & $13 \mathrm{C}$ \\
\hline
\end{tabular}

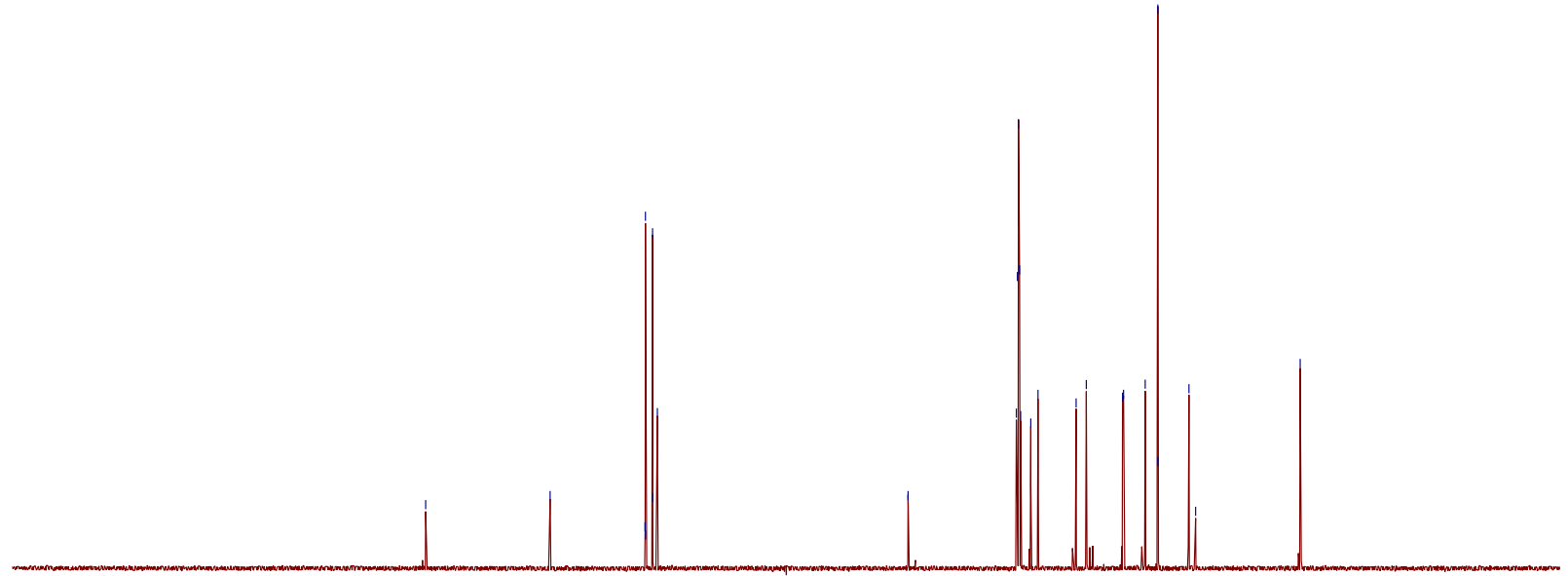

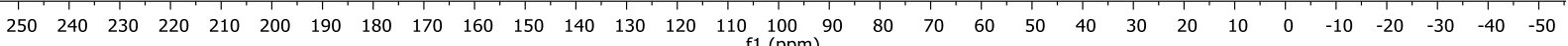

\section{${ }^{1} \mathrm{H}-{ }^{1} \mathrm{H} \operatorname{COSY}$}

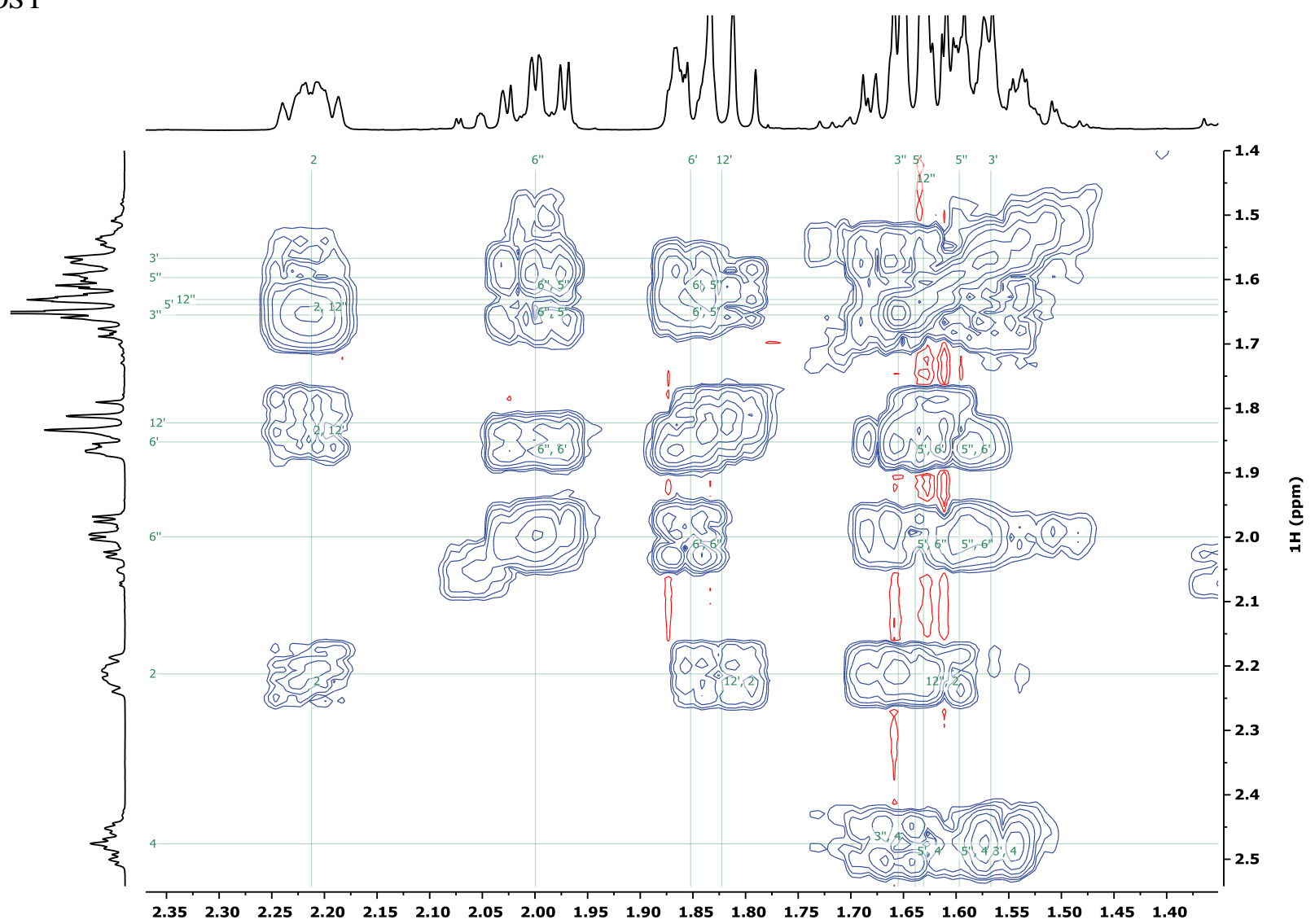


${ }^{1} \mathrm{H}-{ }^{13} \mathrm{C}$ HSQC

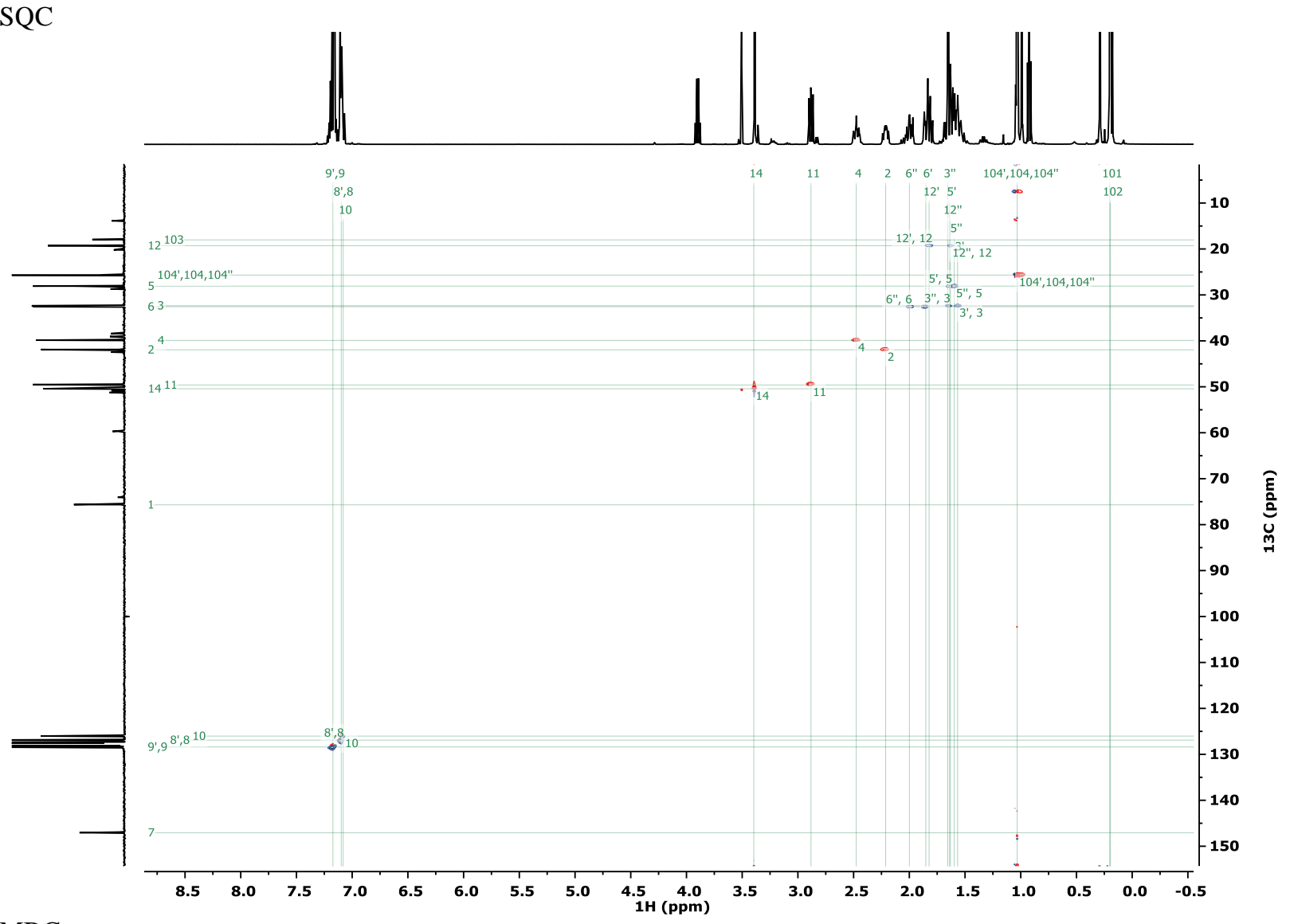

\section{${ }^{1} \mathrm{H}-{ }^{13} \mathrm{C}$ HMBC}

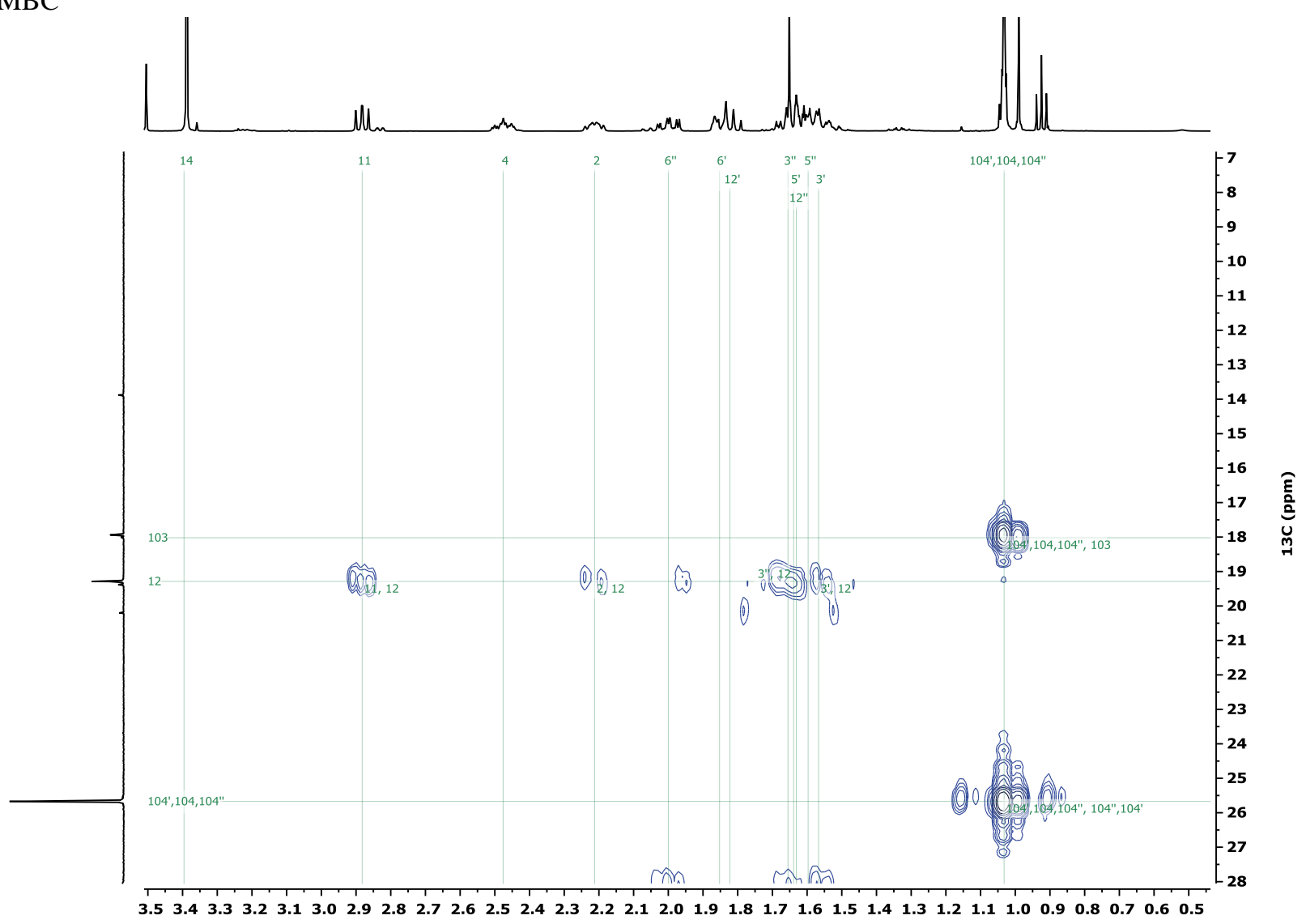




\section{${ }^{1} \mathrm{H}-{ }^{1} \mathrm{H}$ NOESY}

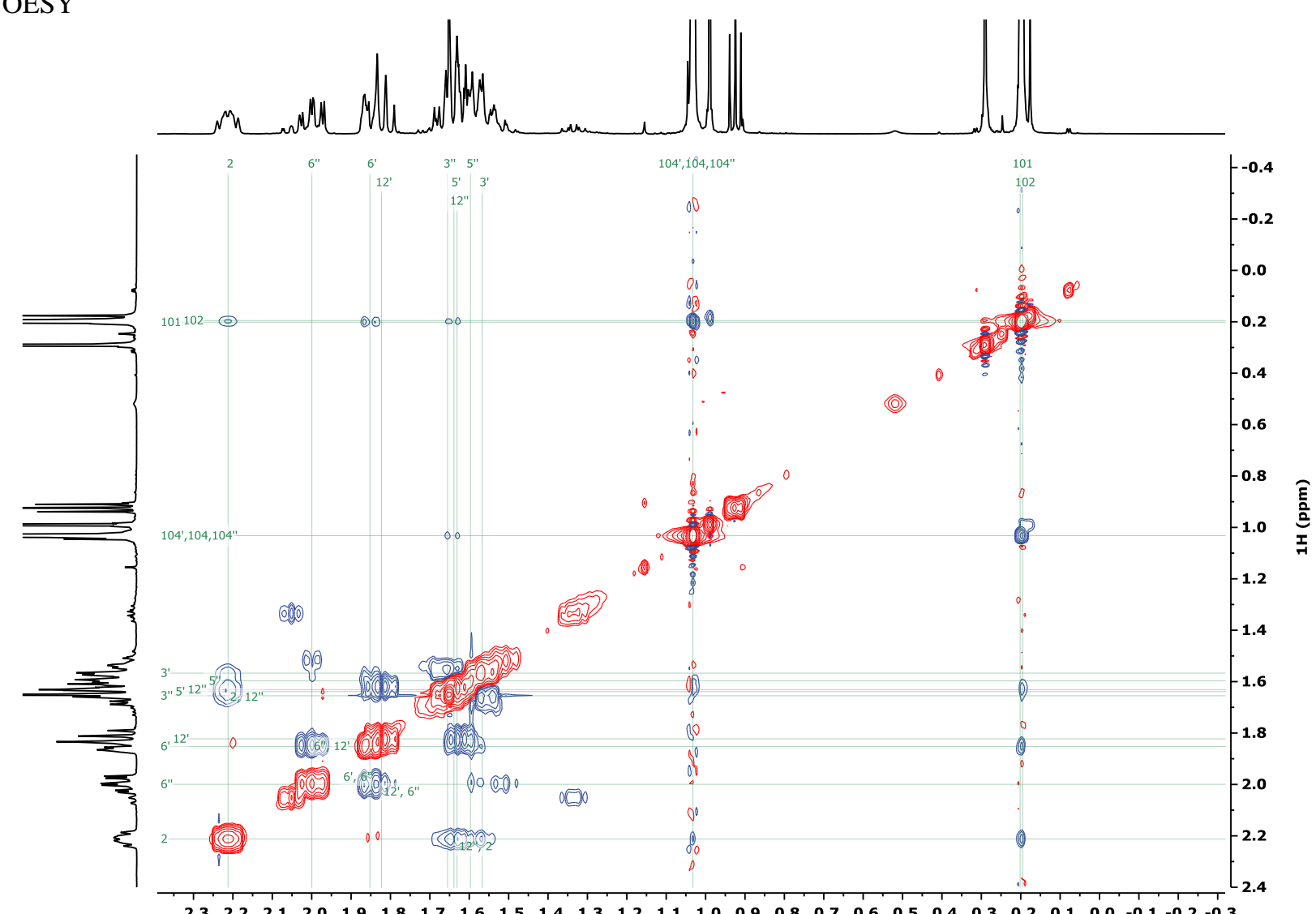

\section{(2S,4R)-2-benzyl-4-phenylcyclohexan-1-one 9:}

The data from $1 \mathrm{H} .13 \mathrm{C}$ and $2 \mathrm{D}$-correlations supports the following structure:

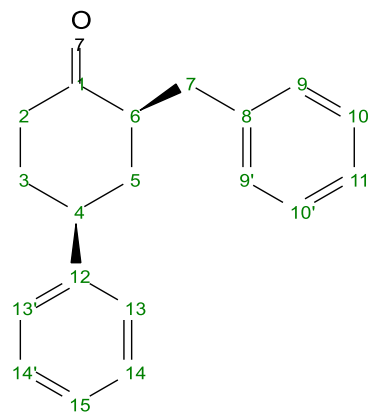

\section{User Report \\ $\mathrm{ZHH}-$}

ZB-324-01

For NMR assignment table please see the next page of this report.

H NMR $\left(500 \mathrm{MHz}\right.$ CDC $\left.7.33-7.12(\mathrm{~m}, 10 \mathrm{H}), 3.32(\mathrm{dd}, 14.1,4.6 \mathrm{~Hz}, 1 \mathrm{H}), 3.03 a_{t=1} .4 .4 .5 \mathrm{~Hz}, 1 \mathrm{H}\right)$, $2.85-2.71(\mathrm{~m}, 1 \mathrm{H}), 2.59-2.51(\mathrm{~m}, 2 \mathrm{H}), 2.42 \mathrm{~J}(\mathrm{dd}, 4.1,8.9 \mathrm{~Hz}, 1 \mathrm{H}), 2.27-2.21(\mathrm{~m}, 1 \mathrm{H}), 2.23-2.13(\mathrm{~m}, 1 \mathrm{H})$ $2.03-1.90(\mathrm{~m}, 1 \mathrm{H}), 1.64\left(\operatorname{app} J_{\mathrm{q}}, 12.8 \mathrm{~Hz}, 1 \mathrm{H}\right)$.

13C NMR (126 MHz, CDG6 211.60, 144.57, 140.11, 129.13, 128.56, 128.34, 126.70, 126.59, 126.01, 51.81, 43. $41.78,40.53,35.23,35.15$

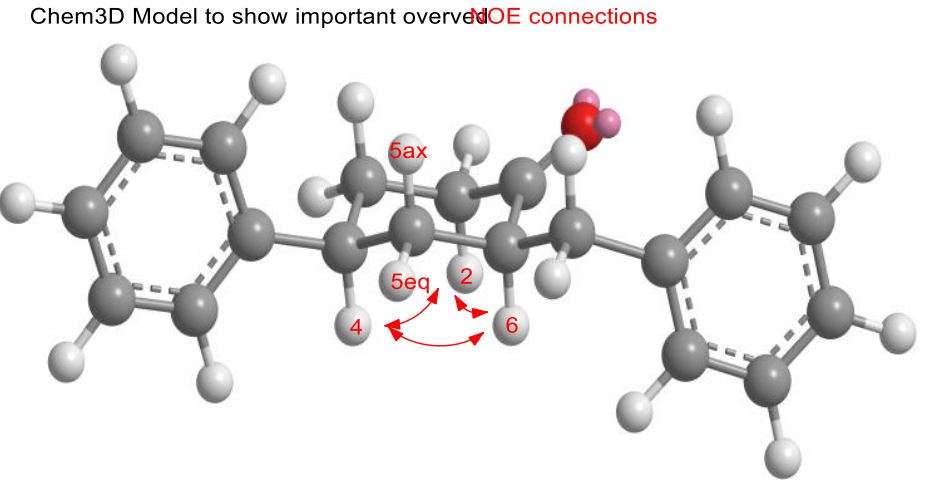

$\mathrm{H} 4$ hast 2 large $(12.4 \mathrm{~Hz})$ and 2 small couplings, that suggest it is in the axial position in ring. The coupling constant between $\mathrm{H} 5 \mathrm{ax}$ and $\mathrm{H} 4$ and $\mathrm{H} 5$ are both large $(>11 \mathrm{~Hz})$ this supports the fact that both of the $\mathrm{H}$ are in the axial position and so cis to eachother
P-ID: ML00000
Measured on: 30/09/2019
CHIFFRE:ZHH-ZB-324-01

ELNA\#: 3270

Client: Dr. Hui Zhou

Spectroscopist: Leutzsch

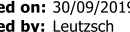

Analysed by: $L$
Amount: $9 \mathrm{mg}$

Solvent: $\mathrm{CDCl}$

Reference: solvent
Temperature: $298 \mathrm{~K}$

Experiments: 1H-zg30, 13C-zgdc30, [13C, 1H]-ASAP hsqcedetgpsp, [13C, 1H]-ASAP hsqcedetgpsp, [13C, 1H]-hmbcgpl2ndqf, [1H, 1H]-noesygpph, [1H, 1H]-clipcosysp.cf 


\begin{tabular}{|c|c|c|c|c|c|c|c|c|c|c|c|c|c|}
\hline \multicolumn{7}{|c|}{ Assignments } & \multicolumn{7}{|c|}{ Assignments } \\
\hline Atom & $\begin{array}{c}\text { Chemical } \\
\text { Shift }\end{array}$ & $\mathbf{J}$ & cosY & HSQC & HMBC & NOESY & Atom & $\begin{array}{c}\text { Chemical } \\
\text { Shift }\end{array}$ & J & cosy & HSQC & HMBC & NOESY \\
\hline \multirow[t]{2}{*}{$1 \mathrm{C}$} & \multirow[t]{2}{*}{211.596} & & & & \multirow{2}{*}{$\begin{array}{l}\text { 2, 3eq, } \\
\text { 5ax, 5eq, } \\
6,7^{\prime}, 7^{\prime \prime}\end{array}$} & & $9^{\prime} \mathrm{C}$ & 129.131 & & & $9^{\prime}$ & 7', 7", 9, 11 & \\
\hline & & & & & & & $\mathrm{H}$ & 7.150 & & & $9^{\prime}$ & 7,9 & $6,7^{\prime}, 7^{\prime \prime}$ \\
\hline $2 \mathrm{C}$ & 41.777 & & & 2 & $\begin{array}{l}3 a x, 3 e q, \\
4\end{array}$ & & $10 \mathrm{C}$ & 128.344 & & & 10 & & \\
\hline $\mathrm{H} 2$ & 2.564 & & $3 a x, 3 e q$ & 2 & $1,3,4,6$ & 4,6 & $\mathrm{H}$ & 7.253 & & & 10 & 8 & \\
\hline $3 \mathrm{C}$ & 35.147 & & & $3 a x, 3 e q$ & 2,4 & & $10^{\prime} \mathrm{C}$ & 128.344 & & & $10^{\prime}$ & & \\
\hline Hax & 1.955 & $12.40(4)$ & 2,4 & 3 & $2,4,5,12$ & $5 a x, 13$ & $\mathrm{H}$ & 7.253 & & & $10^{\prime}$ & 8 & \\
\hline Heq & 2.237 & $3.50(4)$ & 2,4 & 3 & $\begin{array}{l}1,2,4,5, \\
12\end{array}$ & 4 & $11 \mathrm{C}$ & 126.006 & & & 11 & & \\
\hline \multirow[t]{2}{*}{$4 \mathrm{C}$} & \multirow[t]{2}{*}{43.418} & & & \multirow[t]{2}{*}{4} & \multirow{2}{*}{$\begin{array}{l}\text { 2, 3ax, } \\
\text { 3eq, 5ax, } \\
\text { 5eq, 13, } \\
13^{\prime}\end{array}$} & & $\mathrm{H}$ & 7.160 & & & 11 & 9, 9' & \\
\hline & & & & & & & $12 \mathrm{C}$ & 144.567 & & & & $\begin{array}{l}\text { 3ax, 3eq, 4, 5ax, } \\
5 \text { eq, 14, 14' }\end{array}$ & \\
\hline \multirow[t]{3}{*}{$\mathrm{H}$} & \multirow[t]{3}{*}{3.030} & \multirow{3}{*}{$\begin{array}{l}\text { 12.40(5ax), } \\
3.50(3 \mathrm{eq}), \\
3.50(5 \mathrm{eq}), \\
12.40(3 \mathrm{ax})\end{array}$} & \multirow{3}{*}{$\begin{array}{l}\text { 3ax, 3eq, } \\
5 a x, 5 \text { eq }\end{array}$} & \multirow[t]{3}{*}{4} & \multirow{3}{*}{$\begin{array}{l}2,3,5,6 \\
12,13,13\end{array}$} & \multirow{3}{*}{$\begin{array}{l}\text { 2, 3eq, } \\
5 \text { eq, } 6,13\end{array}$} & $13 \mathrm{C}$ & 126.699 & & & 13 & $4,13 ', 15$ & \\
\hline & & & & & & & $\mathrm{H}$ & 7.181 & & & 13 & $4,13^{\prime}, 15$ & $3 a x, 4,5 a x$ \\
\hline & & & & & & & $13^{\prime} \mathrm{C}$ & 126.699 & & & $13^{\prime}$ & $4,13,15$ & \\
\hline $5 \mathrm{C}$ & 40.535 & & & $5 a x, 5 e q$ & $\begin{array}{l}\text { 3ax, 3eq, } \\
4,6,7^{\prime}, 7^{\prime \prime}\end{array}$ & & $\mathrm{H}$ & 7.181 & & & $13^{\prime}$ & $4,13,15$ & \\
\hline Hax & 1.643 & $\begin{array}{l}12.40(4), \\
12.80(6)\end{array}$ & 4,5 eq, 6 & 5 & $\begin{array}{l}1,4,6,7, \\
12\end{array}$ & $\begin{array}{l}3 a x, 7 ', 7 ", \\
13\end{array}$ & $14 \mathrm{C}$ & 128.562 & & & 14 & & \\
\hline Heq & 2.194 & $3.50(4)$ & 4, 5ax, 6 & 5 & $\begin{array}{l}1,4,6,7, \\
12\end{array}$ & 4,6 & $\mathrm{H}$ & 7.288 & & & 14 & 12 & \\
\hline $6 \mathrm{C}$ & 51.812 & & & 6 & $2,4,5 \mathrm{ax}$ & & $14^{\prime} \mathrm{C}$ & 128.562 & & & $14^{\prime}$ & & \\
\hline & & & & & 5eq, 7', 7" & & $\mathrm{H}$ & 7.288 & & & $14^{\prime}$ & 12 & \\
\hline H & 2.762 & $\begin{array}{l}12.80(5 \mathrm{ax}), \\
4.60\left(7^{\prime}\right),\end{array}$ & $\begin{array}{l}\text { 5ax, 5eq, } \\
7^{\prime}, 7^{\prime \prime}\end{array}$ & 6 & $1,5,7,8$ & $\begin{array}{l}2,4,5 \mathrm{eq} \\
9,9^{\prime}\end{array}$ & $15 \mathrm{C}$ & 126.585 & & & ק & 13,1 & \\
\hline 70 & & & & & 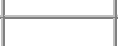 & & $\mathrm{H}$ & 7.200 & & & 15 & $13^{\prime} 13^{\prime}$ & \\
\hline $7 \mathrm{C}$ & 35.230 & & & 7', 7" & $\begin{array}{l}\text { 5ax, 5eq, } \\
6,9,9^{\prime}\end{array}$ & & & & & & & & \\
\hline $\mathrm{H}^{\prime}$ & 3.322 & $\begin{array}{l}14.10\left(7^{\prime \prime}\right), \\
4.60(6)\end{array}$ & $6,7^{\prime \prime}$ & 7 & $\begin{array}{l}1,5,6,8 \\
9,9^{\prime}\end{array}$ & $\begin{array}{l}\text { 5ax, 7", 9, } \\
9^{\prime}\end{array}$ & & & & & & & \\
\hline $\mathrm{H}^{\prime \prime}$ & 2.419 & $\begin{array}{l}14.10\left(7^{\prime}\right) \\
8.90(6)\end{array}$ & $6,7^{\prime}$ & 7 & $\begin{array}{l}1,5,6,8, \\
9,9^{\prime}\end{array}$ & $\begin{array}{l}5 a x, 7 ', 9, \\
9^{\prime}\end{array}$ & & & & & & & \\
\hline $8 \mathrm{C}$ & 140.112 & & & & $\begin{array}{l}6,7^{\prime}, 7^{\prime \prime} \\
10,10^{\prime}\end{array}$ & & & & & & & & \\
\hline $9 \mathrm{C}$ & 129.131 & & & 9 & $\begin{array}{l}\text { 7', 7", 9', } \\
11\end{array}$ & & & & & & & & \\
\hline $\mathrm{H}$ & 7.150 & & & 9 & $7,9^{\prime}$ & $6,7^{\prime}, 7^{\prime \prime}$ & & & & & & & \\
\hline
\end{tabular}



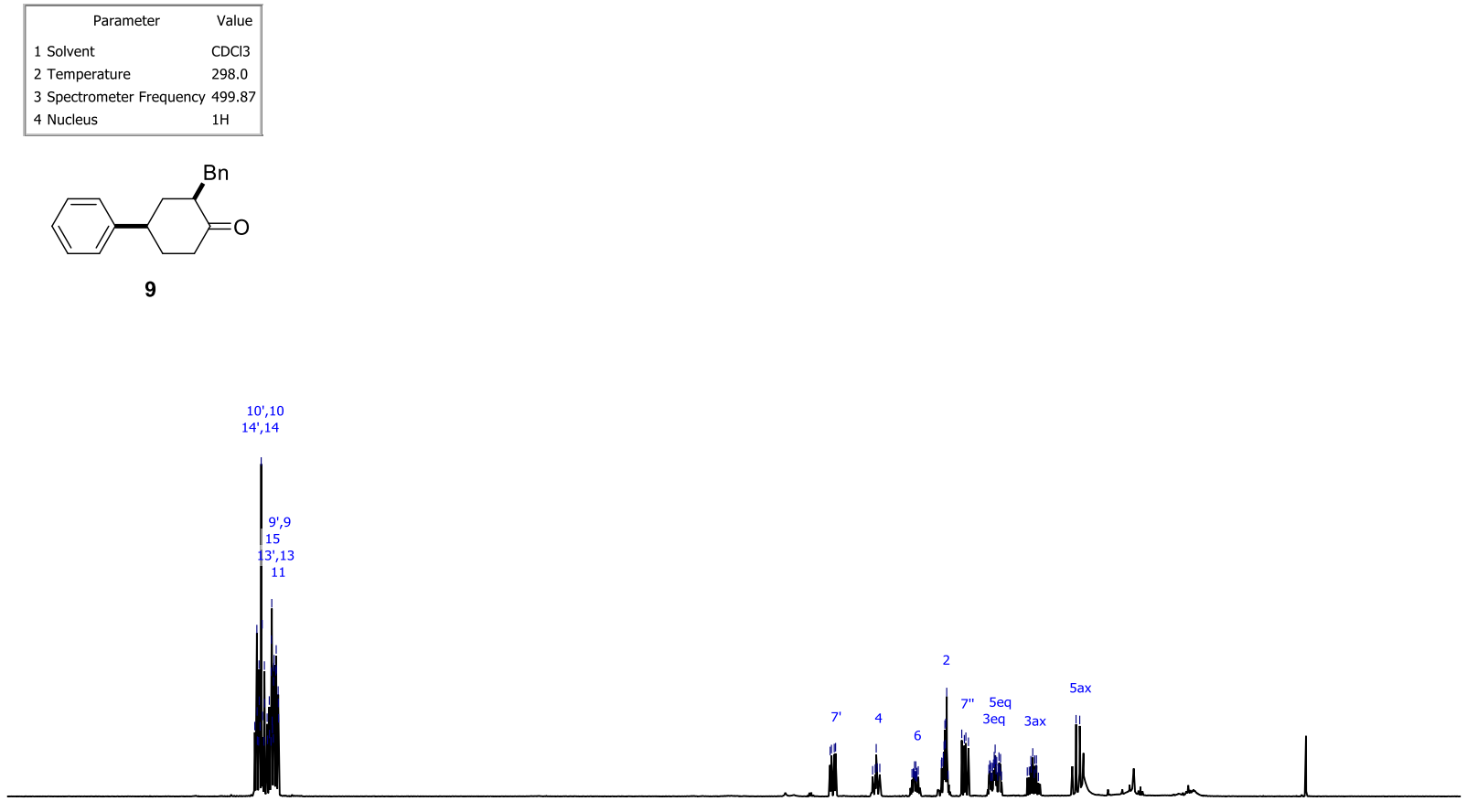

.0

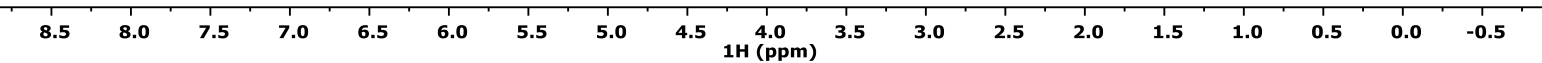

\begin{tabular}{|ll|}
\hline \multicolumn{1}{|c|}{ Parameter } & \multicolumn{1}{c|}{ Value } \\
1 Solvent & CDCl3 \\
2 Temperature & 298.0 \\
3 Experiment & $1 \mathrm{D}$ \\
4 Spectrometer Frequency & 125.70 \\
5 Nucleus & $13 \mathrm{C}$ \\
\hline
\end{tabular}
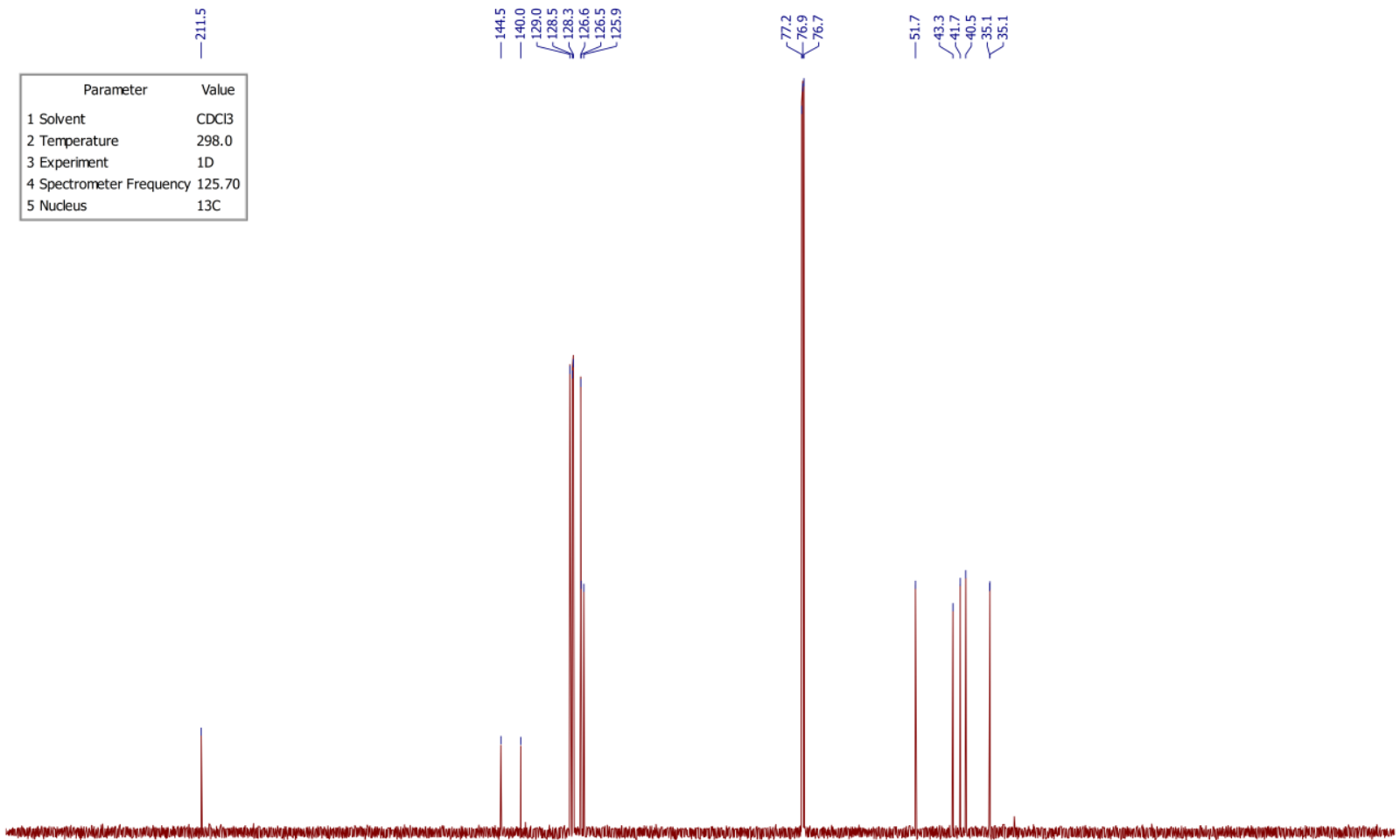

$\begin{array}{llllllllllllllllllllllllllllllllll}250 & 240 & 230 & 220 & 210 & 200 & 190 & 180 & 170 & 160 & 150 & 140 & 130 & 120 & 110 & 100 & 90 & 80 & 70 & 60 & 50 & 40 & 30 & 20 & 10 & 0 & -10 & -20 & -30 & -40 & -50\end{array}$ 


\section{${ }^{1} \mathrm{H}-{ }^{1} \mathrm{H}$ COSY}

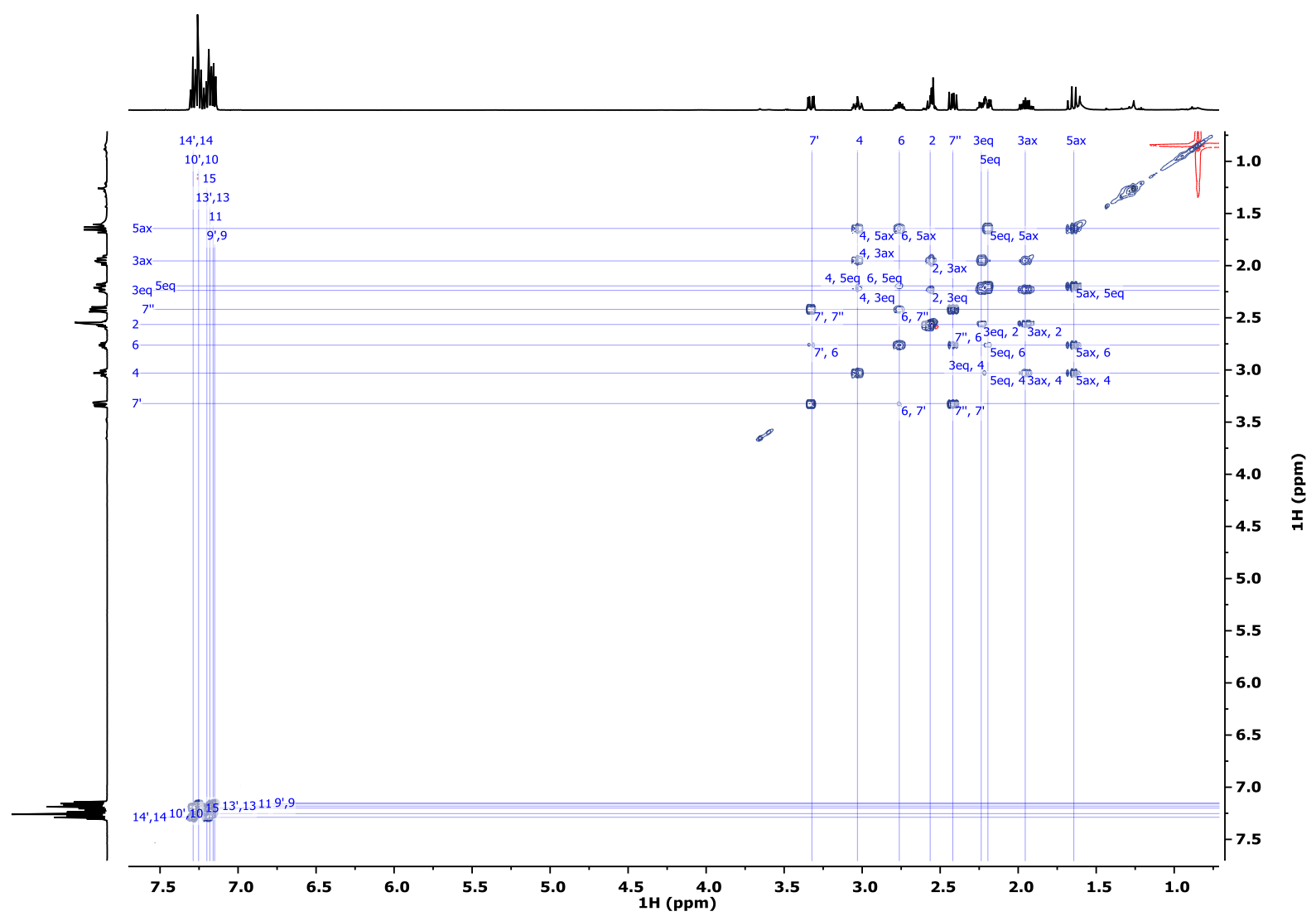

${ }^{1} \mathrm{H}-{ }^{13} \mathrm{C}$ HSQC

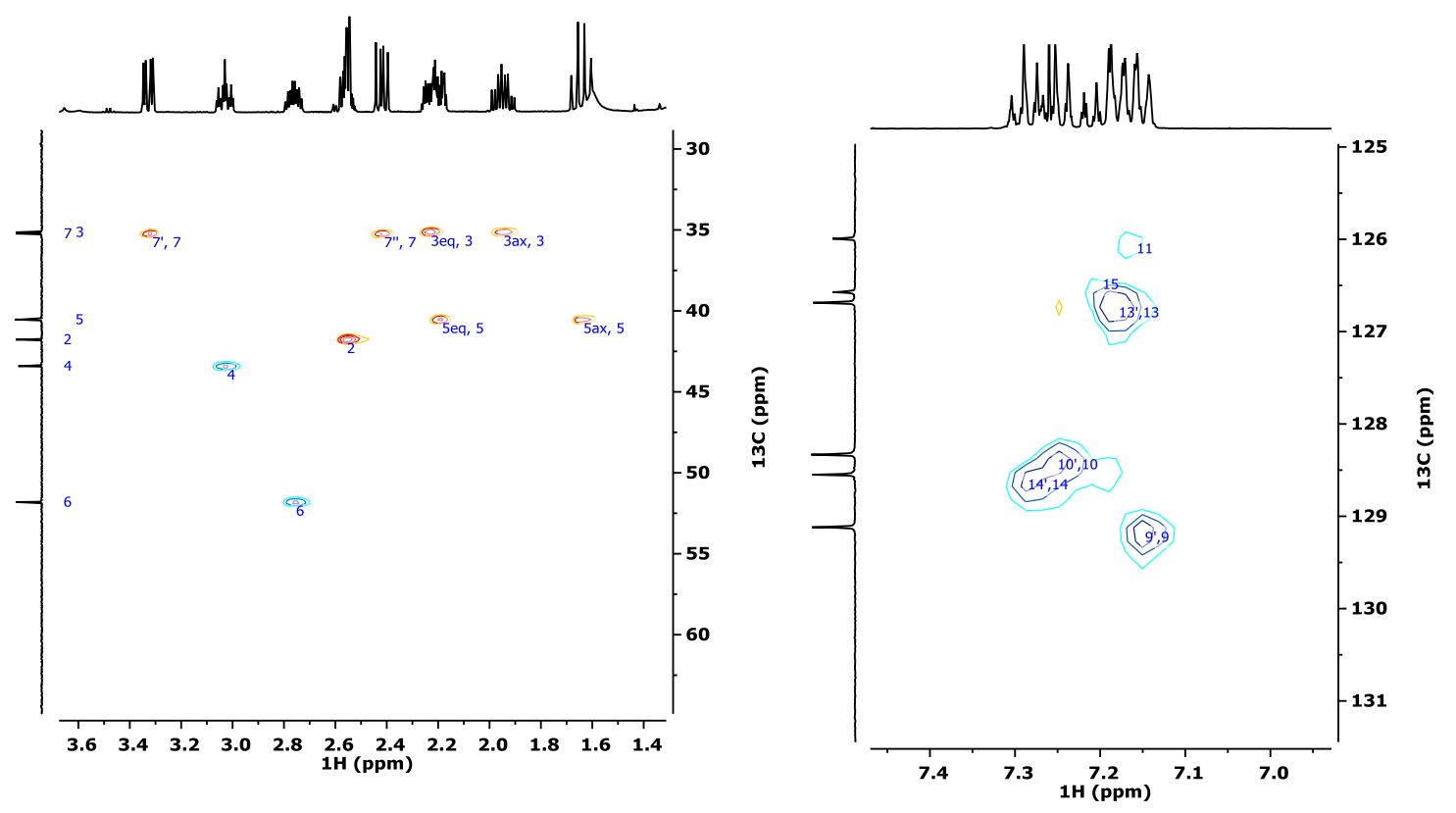

\section{${ }^{1} \mathrm{H}-{ }^{13} \mathrm{C}$ HMBC}




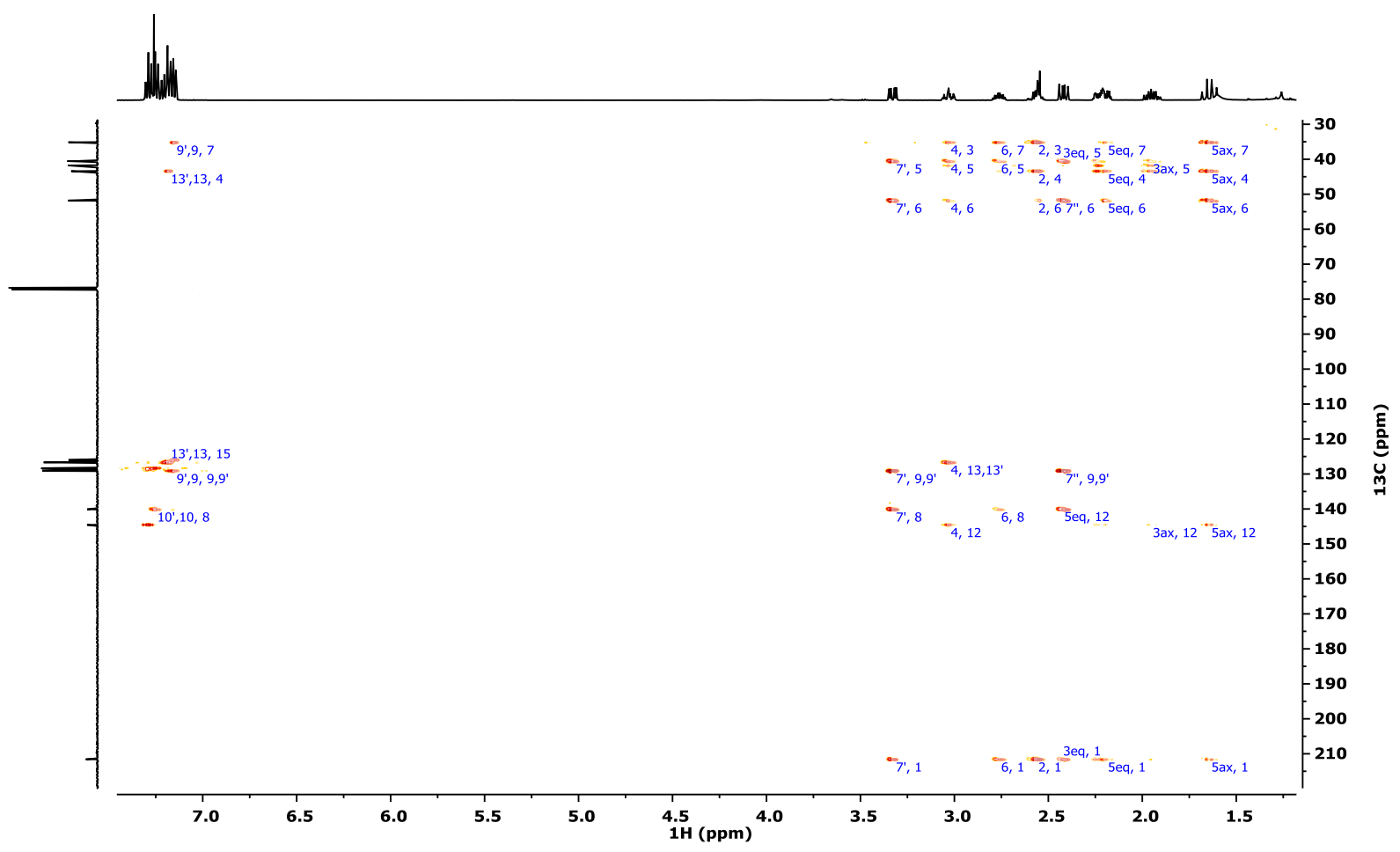

${ }^{1} \mathrm{H}-{ }^{1} \mathrm{H}$ NOESY

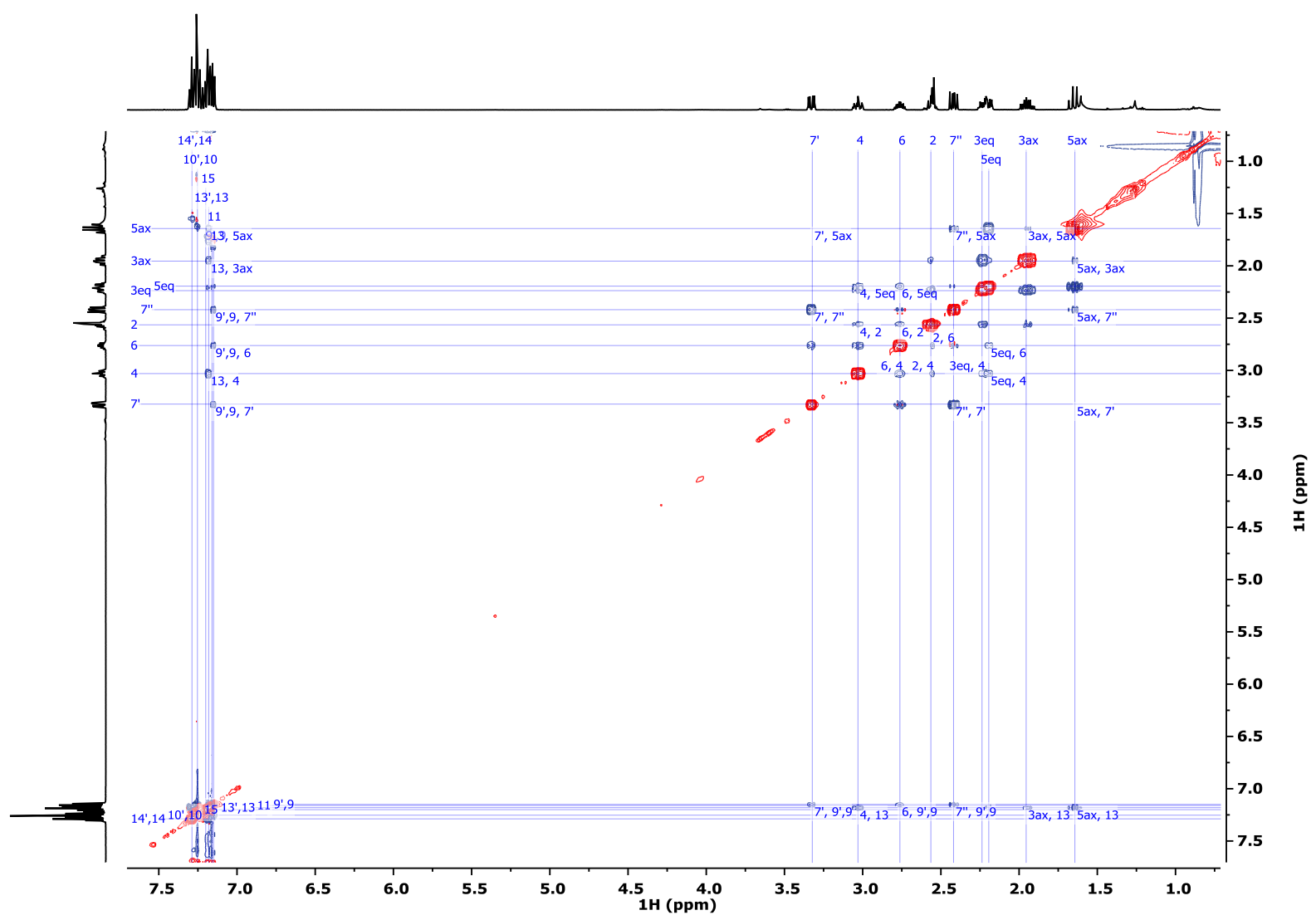




\section{$(2 S, 4 S)$-2-benzyl-4-phenylcyclohexan-1-one}

The data from $1 \mathrm{H},{ }^{13} \mathrm{C}$ and $2 \mathrm{D}$-correlations supports the following User Report structure:

$\mathrm{ZHH}-$

ZB-324-02

H NMR (500 MHz, CDG6 $7.34-7.27(\mathrm{~m}, 4 \mathrm{H}), 7.24-7.17(\mathrm{~m}, 6 \mathrm{H}), 3.25 \neq t \mathrm{tt}, 0.3,4.0 \mathrm{~Hz}, 1 \mathrm{H}), 3.12(\mathrm{dd}=13.0,5.2 \mathrm{~Hz}$, $1 \mathrm{H}), 2.84(\mathrm{dd} J=13.0,9.6 \mathrm{~Hz}, 1 \mathrm{H}), 2.84-2.75(\mathrm{~m}, 1 \mathrm{H}), 2.66$ (ddd, $4.9,11.4,5.9 \mathrm{~Hz}, 1 \mathrm{H}), 2.46(\mathrm{dtd}, 14.9,4.8,1.3 \mathrm{~Hz}$ $1 \mathrm{H}), 2.27-2.18(\mathrm{~m}, 1 \mathrm{H}), 2.18-2.05(\mathrm{~m}, 1 \mathrm{H}), 2.09$ ( $(\mathrm{dd} \mathbb{3} 3.8,10.3,5.2 \mathrm{~Hz}, 2 \mathrm{H}), 1.95(\mathrm{dtd}, 13.8,4.4,4.0,2.2 \mathrm{~Hz}, 1 \mathrm{H})$.

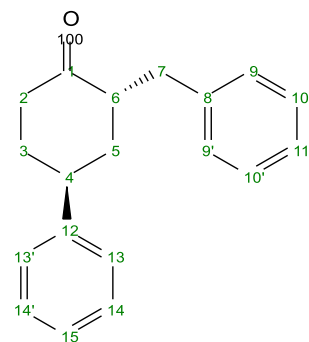

P-ID: ML00000

Measured on: 30/09/2019

CHIFFRE:ZHH-ZB-324-02

Clinat:3270

Client: Dr. Hui Zho
roup: List

Spectroscopist: SPECTROSCOPIST

Analysed on: 30/09/2019

Analysed by: Leutzsch

13C NMR (126 MHz, CDQ6 213.35, 144.09, 138.84, 128.93, 128.61, 128.56, 126.68, 126.48, 126.47, 50.62, 38.60, 3 $36.79,36.57,33.01$

For full assignment table please see the next page.

Chem3D Model to show important overveldOE connections.

Amount: $9 \mathrm{mg}$

Reference: solvent

Temperature: $298 \mathrm{~K}$

Spectrometer: AV500as

-GRD Z119470/0004

Experiments: $1 \mathrm{H}$-zg30, 13C-zgdc30, [13C, $1 \mathrm{H}]$-ASAP_hsqcedetgpsp, [13C, 1H]-hmbcgpl2ndqf, [1H, 1H]-noesygpph, [1H, $1 \mathrm{H}]$-clipcosysp.cf

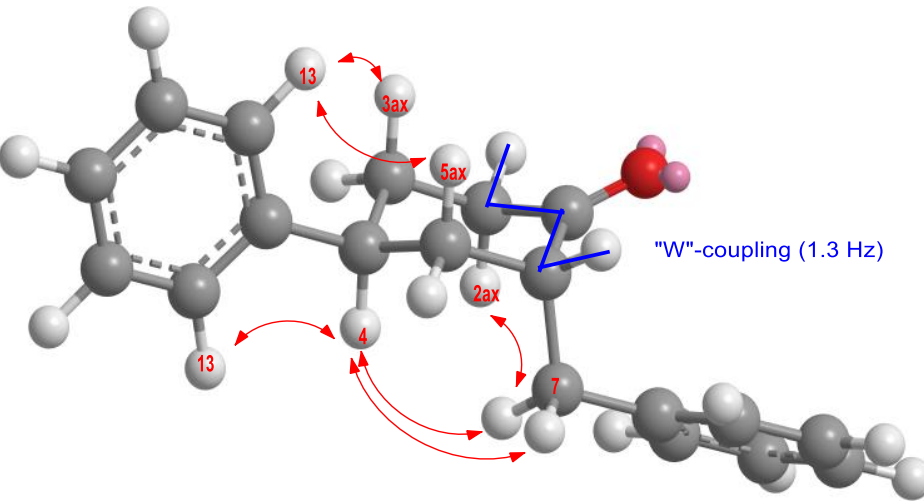

H4 hast 2 large $(12.4 \mathrm{~Hz})$ and 2 small couplings, that suggest it is dominantly in the axia position in the ring. $\mathrm{H} 5$ ax has two different coupling constants to $\mathrm{H} 4$ (10.3; larger) and $\mathrm{H}^{\mathrm{H}}$ (5.2; small). This indecates that $\mathrm{H} 4$ and $\mathrm{H} 5$ atms to eachother<smiles></smiles>

\begin{tabular}{|c|c|c|c|c|c|c|}
\hline \multicolumn{7}{|c|}{ Assignments } \\
\hline Atom & Chemical Shift & $\mathbf{J}$ & $\cos Y$ & HSQC & HMBC & NOESY \\
\hline $1 \mathrm{C}$ & 213.347 & & & & $\begin{array}{l}2 \mathrm{ax}, 2 \mathrm{eq}, 3 \mathrm{ax}, 5 \mathrm{ax}, \\
5 \mathrm{eq}, 6,7^{\prime}, 7^{\prime \prime}\end{array}$ & \\
\hline $2 \mathrm{C}$ & 38.596 & & & $2 \mathrm{ax}, 2 \mathrm{eq}$ & $3 a x, 4$ & \\
\hline Hax & 2.656 & $\begin{array}{l}14.90(2 \mathrm{eq}), \\
11.40(3 \mathrm{xa}), \\
5.90(3 \mathrm{eq})\end{array}$ & 3ax, 3eq & 2 & $1,3,4$ & 2eq, 3eq, 4, 7', 7"' \\
\hline Heq & 2.460 & $\begin{array}{l}1.30(6), 14.90(2 a x), \\
4.85(3 \mathrm{ax}), \\
4.85(3 \mathrm{eq})\end{array}$ & $3 a x, 3 e q$ & 2 & $1,3,4$ & $2 a x$ \\
\hline $3 \mathrm{C}$ & 33.009 & & & $3 a x, 3 e q$ & $2 a x, 2 e q, 4,5 a x$ & \\
\hline Hax & 2.123 & $\begin{array}{l}10.30(4), \\
11.40(2 \mathrm{zax}), \\
4.85(2 \mathrm{eq})\end{array}$ & $\begin{array}{l}\text { 2ax, 2eq, 3eq, } \\
4\end{array}$ & 3 & $1,2,4,5$ & $13,13^{\prime}$ \\
\hline Heq & 2.217 & $\begin{array}{l}4.00(4), 2.20(5 \mathrm{eq}), \\
5.90(2 \mathrm{ax}), \\
4.85(2 \mathrm{eq})\end{array}$ & 2 & 3 & & $2 a x, 4$ \\
\hline $4 \mathrm{C}$ & 37.078 & & & 4 & $\begin{array}{l}2 \mathrm{aax}, 2 \mathrm{eq}, 3 \mathrm{ax}, 5 \mathrm{ax}, \\
6,13,13^{\prime}\end{array}$ & \\
\hline $\mathrm{H}$ & 3.251 & $\begin{array}{l}4.00(5 \mathrm{eq}), \\
10.30(5 \mathrm{ax}), \\
10.30(3 \mathrm{ax}), \\
4.00(3 \mathrm{eq})\end{array}$ & $\begin{array}{l}\text { 3ax, 3eq, 5ax, } \\
\text { 5eq }\end{array}$ & 4 & $2,3,5,12,13,13^{\prime}$ & $\begin{array}{l}\text { 2ax, 3eq, 5ax, 5eq, } \\
7^{\prime}, 7^{\prime \prime}, 13,13^{\prime}\end{array}$ \\
\hline $5 \mathrm{C}$ & 36.566 & & & $5 \mathrm{ax}, 5 \mathrm{eq}$ & $3 a x, 4,7^{\prime}, 7^{\prime \prime}$ & \\
\hline Hax & 2.087 & $\begin{array}{l}10.30(4), \\
13.80(5 \text { eq }), 5.20(6)\end{array}$ & $4,5 e q$ & 5 & $1,3,4,6,7,12$ & $4,6,13,13^{\prime}$ \\
\hline Heq & 1.950 & $\begin{array}{l}4.00(4), 4.40(6), \\
13.80(5 \mathrm{ax}), \\
2.20(3 \mathrm{eq})\end{array}$ & $4,5 a x, 6$ & 5 & 1,12 & 4,6 \\
\hline $6 \mathrm{C}$ & 50.616 & & & 6 & $5 a x, 7^{\prime}, 7^{\prime \prime}$ & \\
\hline H & 2.792 & $\begin{array}{l}5.20\left(7^{\prime}\right), 9.60\left(7^{\prime \prime}\right), \\
4.40(5 \mathrm{eq}), \\
5.20(5 \mathrm{ax}), \\
1.30(2 \mathrm{eq})\end{array}$ & 5eq, 7', 7" & 6 & $1,4,7,8$ & $\begin{array}{l}5 a x, 5 \text { eq, 7', 7", 9, } \\
9^{\prime}\end{array}$ \\
\hline 7c & 36.794 & & & 7', 7" & $5 a x, 6,9,9^{\prime}$ & \\
\hline $\mathrm{H}^{\prime}$ & 3.117 & $13.00\left(7^{\prime \prime}\right), 5.20(6)$ & 6 & 7 & $1,5,6,8,9,9^{\prime}$ & $2 a x, 4,6,9,9^{\prime}$ \\
\hline $\mathrm{H}^{\prime \prime}$ & 2.844 & $13.00\left(7^{7}\right), 9.60(6)$ & 6 & 7 & $1,5,6,8,9,9^{\prime}$ & $2 a x, 4,6,9,9^{\prime}$ \\
\hline $8 \mathrm{C}$ & 138.839 & & & & $6,7^{\prime}, 7^{\prime \prime}, 10,10^{\prime}$ & \\
\hline $9 \mathrm{C}$ & 128.932 & & & 9 & 7', 7", 11 & \\
\hline $\mathrm{H}$ & 7.186 & & 10 & 9 & 7,11 & $6,7^{\prime}, 7^{\prime \prime}$ \\
\hline
\end{tabular}

\begin{tabular}{|c|c|c|c|c|c|}
\hline \multicolumn{6}{|c|}{ Assignments } \\
\hline Atom & $\begin{array}{l}\text { Chemical } \\
\text { Shift }\end{array}$ & \begin{tabular}{|l|l|}
$\mathrm{J}$ & $\cos \mathrm{Y}$
\end{tabular} & HSQC & HMBC & NOESY \\
\hline $9^{\prime} \mathrm{C}$ & 128.932 & & $9^{\prime}$ & $7 ', 7 ", 11$ & \\
\hline $\mathrm{H}$ & 7.186 & $10^{\prime}$ & $9^{\prime}$ & 7,11 & $6,7^{\prime}, 7^{\prime \prime}$ \\
\hline $10 \mathrm{C}$ & 128.562 & & 10 & & \\
\hline $\mathrm{H}$ & 7.292 & 9,11 & 10 & 8 & \\
\hline $10^{\prime} \mathrm{C}$ & 128.562 & & $10^{\prime}$ & & \\
\hline $\mathrm{H}$ & 7.292 & 9', 11 & $10^{\prime}$ & 8 & \\
\hline $11 \mathrm{C}$ & 126.475 & & 11 & $9,9^{\prime}$ & \\
\hline $\mathrm{H}$ & 7.210 & $10,10^{\circ}$ & 11 & $9,9^{\prime}$ & \\
\hline $12 \mathrm{C}$ & \begin{tabular}{|l|l|}
144.087 \\
\end{tabular} & & & $4,5 a x, 5 e q, 14,14$ & \\
\hline $13 \mathrm{C}$ & 126.685 & & 13 & 4 & \\
\hline $\mathrm{H}$ & 7.225 & 14 & 13 & 4,15 & $3 a x, 4,5 a x$ \\
\hline $13^{\prime} \mathrm{C}$ & 126.685 & & $13^{\prime}$ & 4 & \\
\hline $\mathrm{H}$ & 7.225 & & $13^{\prime}$ & 4,15 & $3 a x, 4,5 a x$ \\
\hline $14 \mathrm{C}$ & 128.610 & & 14 & & \\
\hline $\mathrm{H}$ & 7.309 & 13 & 14 & 12 & \\
\hline $14^{\prime} \mathrm{C}$ & 128.610 & & $14^{\prime}$ & & \\
\hline $\mathrm{H}$ & 7.309 & & $14^{\prime}$ & 12 & \\
\hline $15 \mathrm{C}$ & 126.468 & & 15 & $13,13^{\prime}$ & \\
\hline $\mathrm{H}$ & 7.210 & & 15 & & \\
\hline 1000 & & & & & \\
\hline
\end{tabular}




\begin{tabular}{|lc|}
\hline \multicolumn{1}{|c|}{ Parameter } & Value \\
1 Solvent & $\mathrm{CDCl} 3$ \\
2 Temperature & 298.0 \\
3 Spectrometer Frequency & 499.87 \\
4 Nucleus & $1 \mathrm{H}$ \\
\hline
\end{tabular}<smiles>O=C1CCC(c2ccccc2)CC1Cc1ccccc1</smiles>
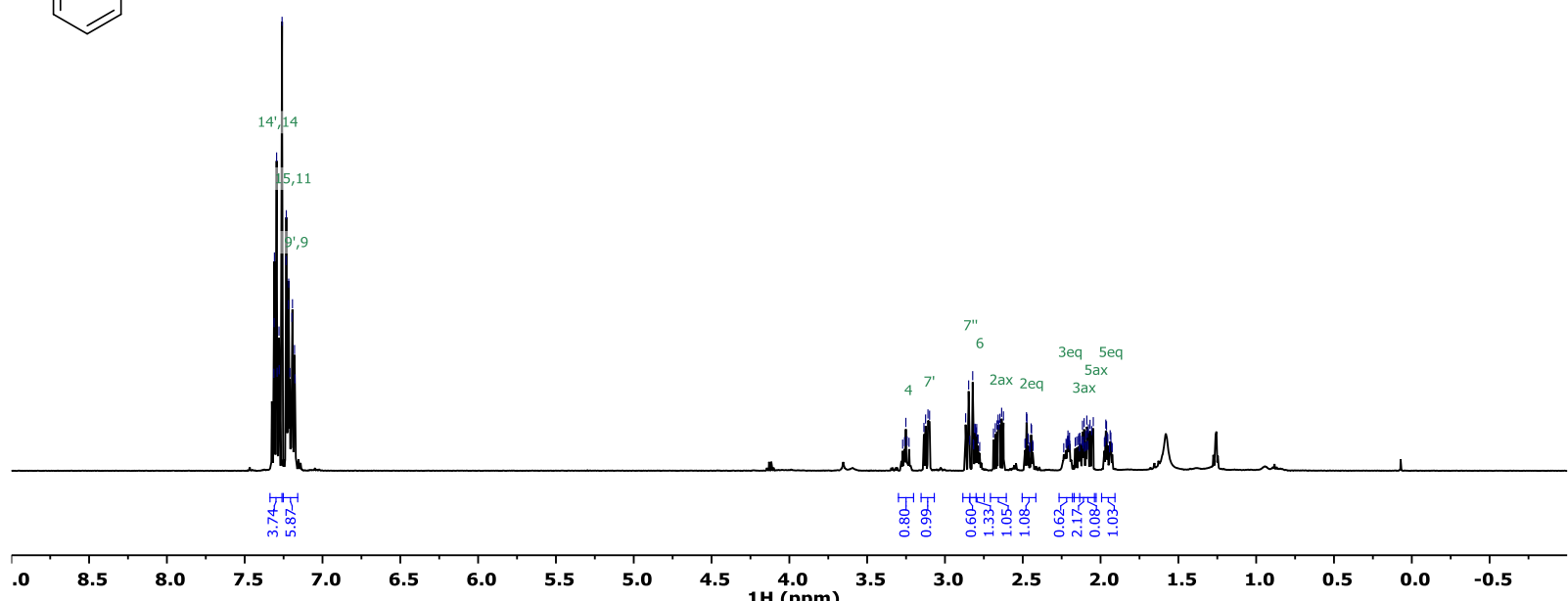

\begin{tabular}{|ll|}
\hline \multicolumn{1}{|c|}{ Parameter } & Value \\
1 Solvent & $\mathrm{CDCl3}$ \\
2 Temperature & 298.0 \\
3 Spectrometer Frequency & 125.70 \\
4 Nucleus & $13 \mathrm{C}$ \\
\hline
\end{tabular}
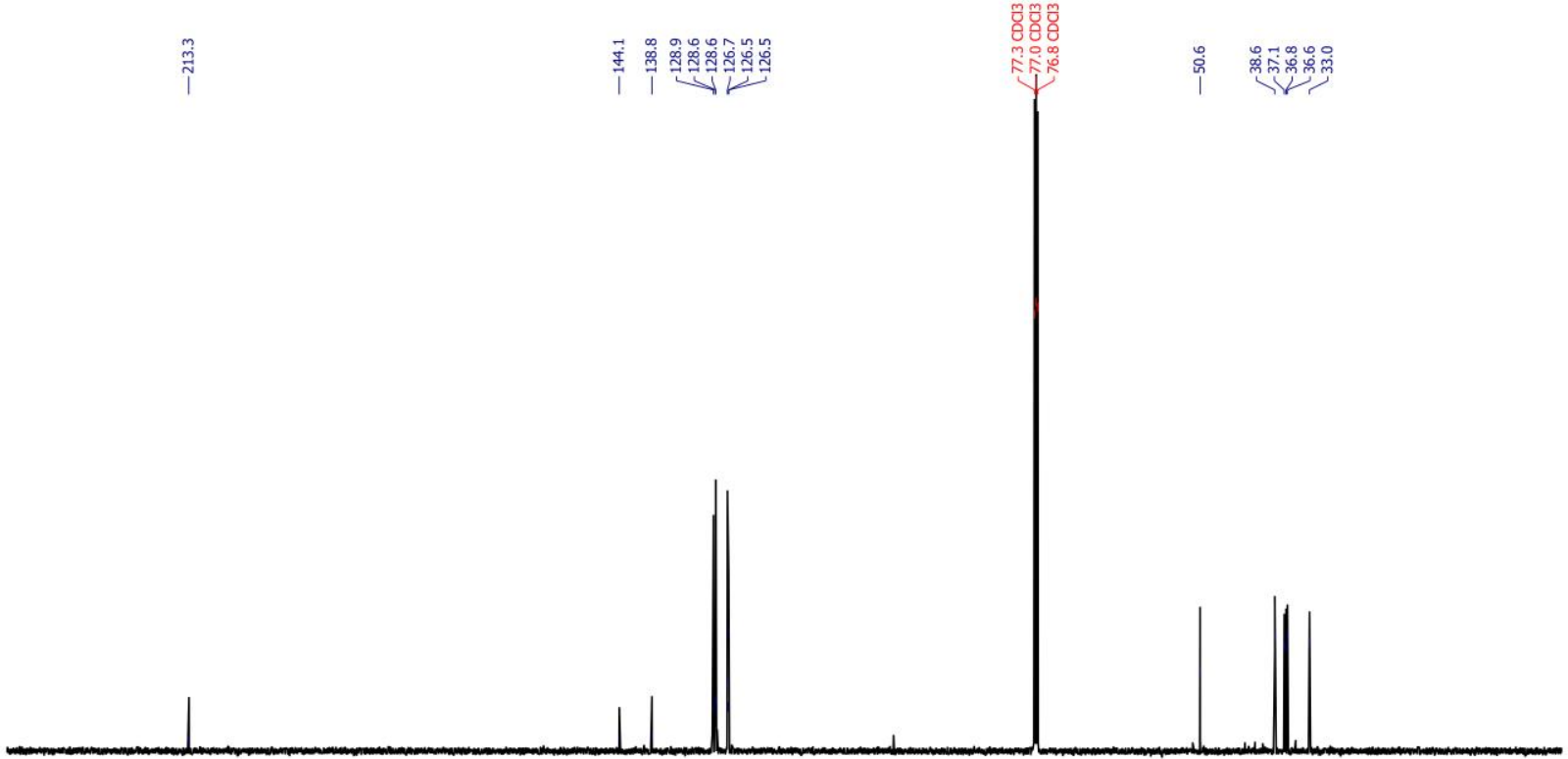

$\begin{array}{llllllllllllllllllllllll}240 & 230 & 220 & 210 & 200 & 190 & 180 & 170 & 160 & 150 & 140 & 130 & \begin{array}{l}120 \\ 13 \mathrm{cpm})\end{array} & 100 & 90 & 80 & 70 & 60 & 50 & 40 & 30 & 20 & 10 & 0\end{array}$ 


\section{${ }^{1} \mathrm{H}-{ }^{1} \mathrm{H}$ COSY}

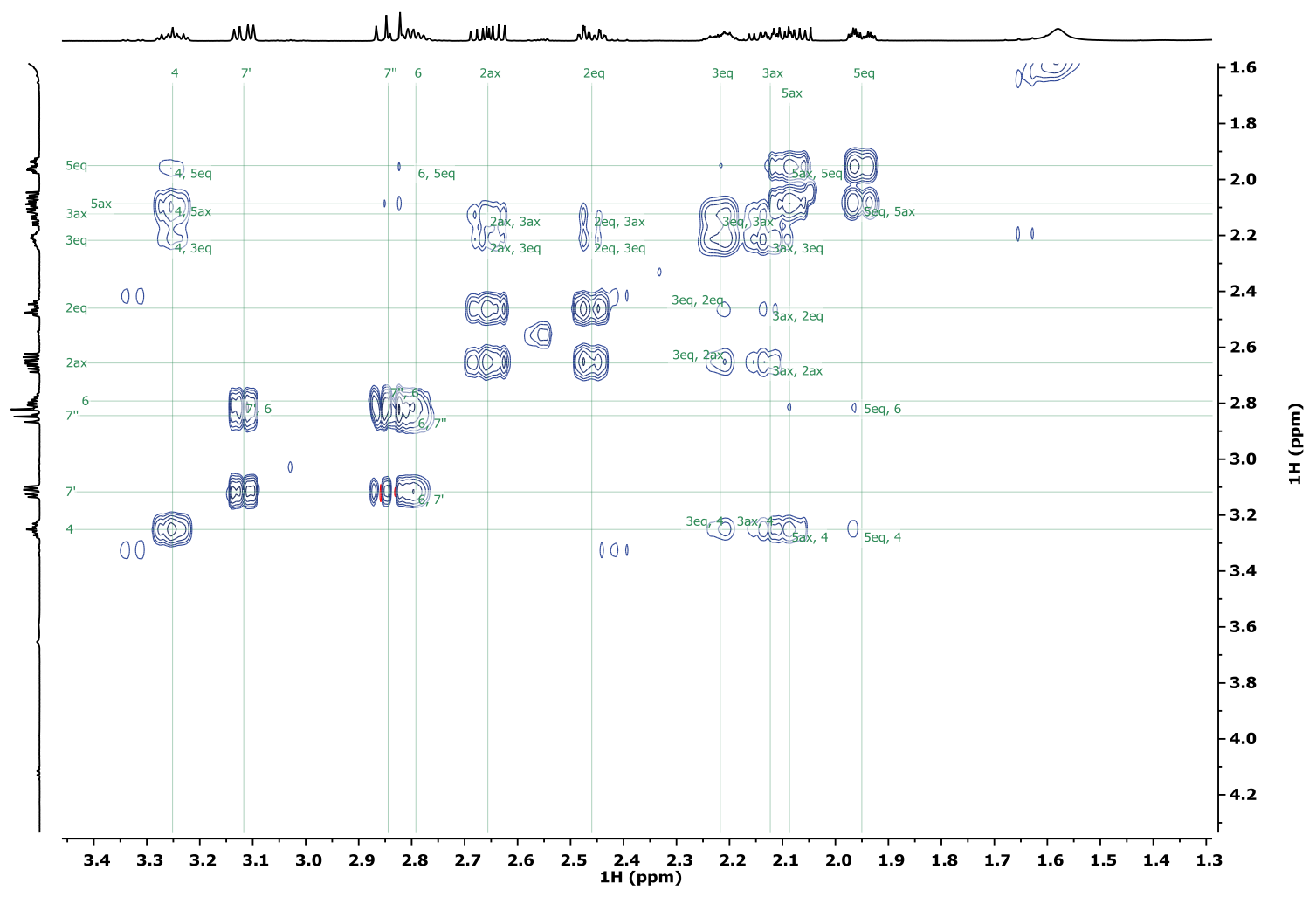

${ }^{1} \mathrm{H}-{ }^{13} \mathrm{C}$ HSQC

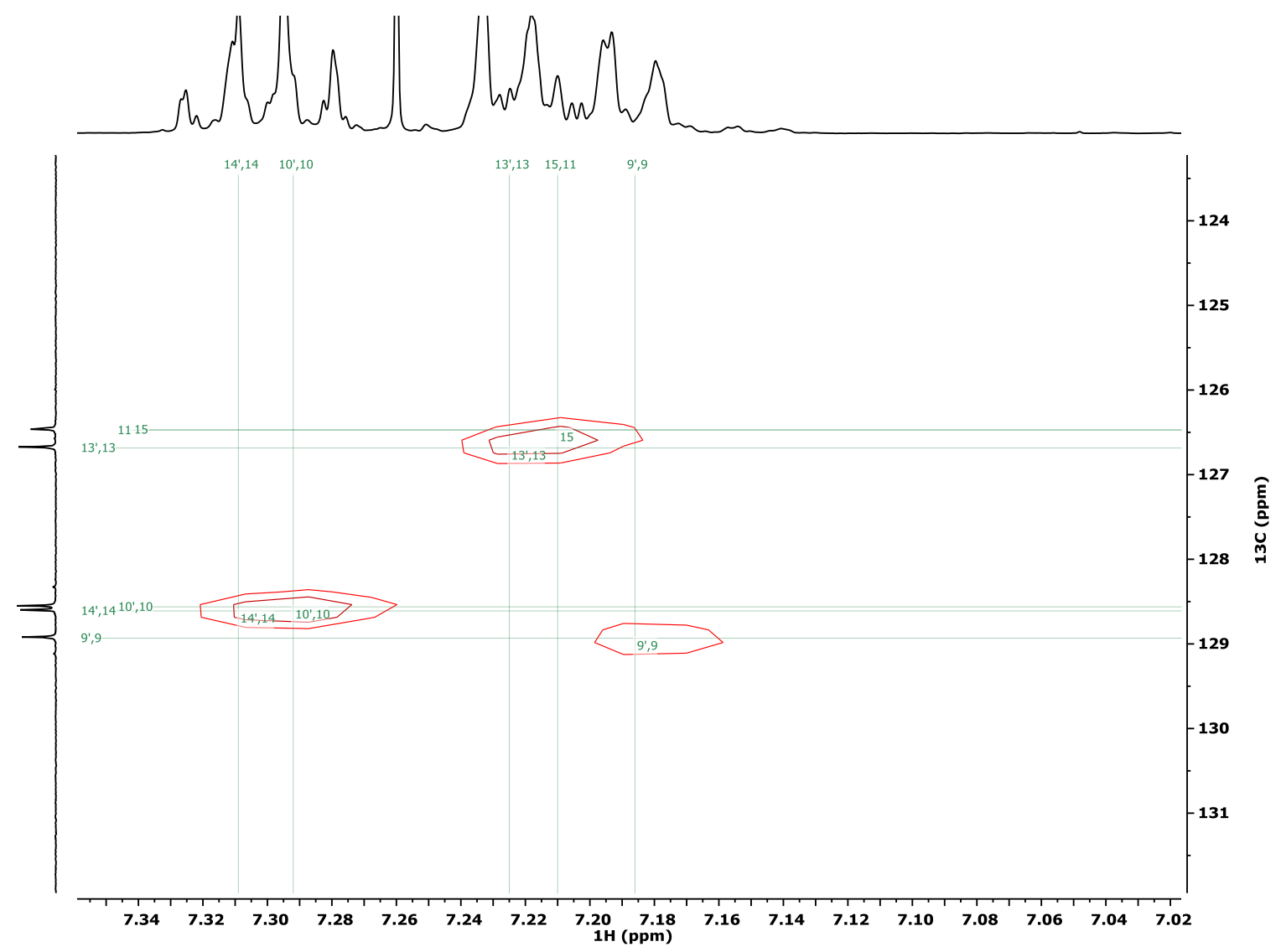




\section{${ }^{1} \mathrm{H}^{-13} \mathrm{C}$ HMBC}

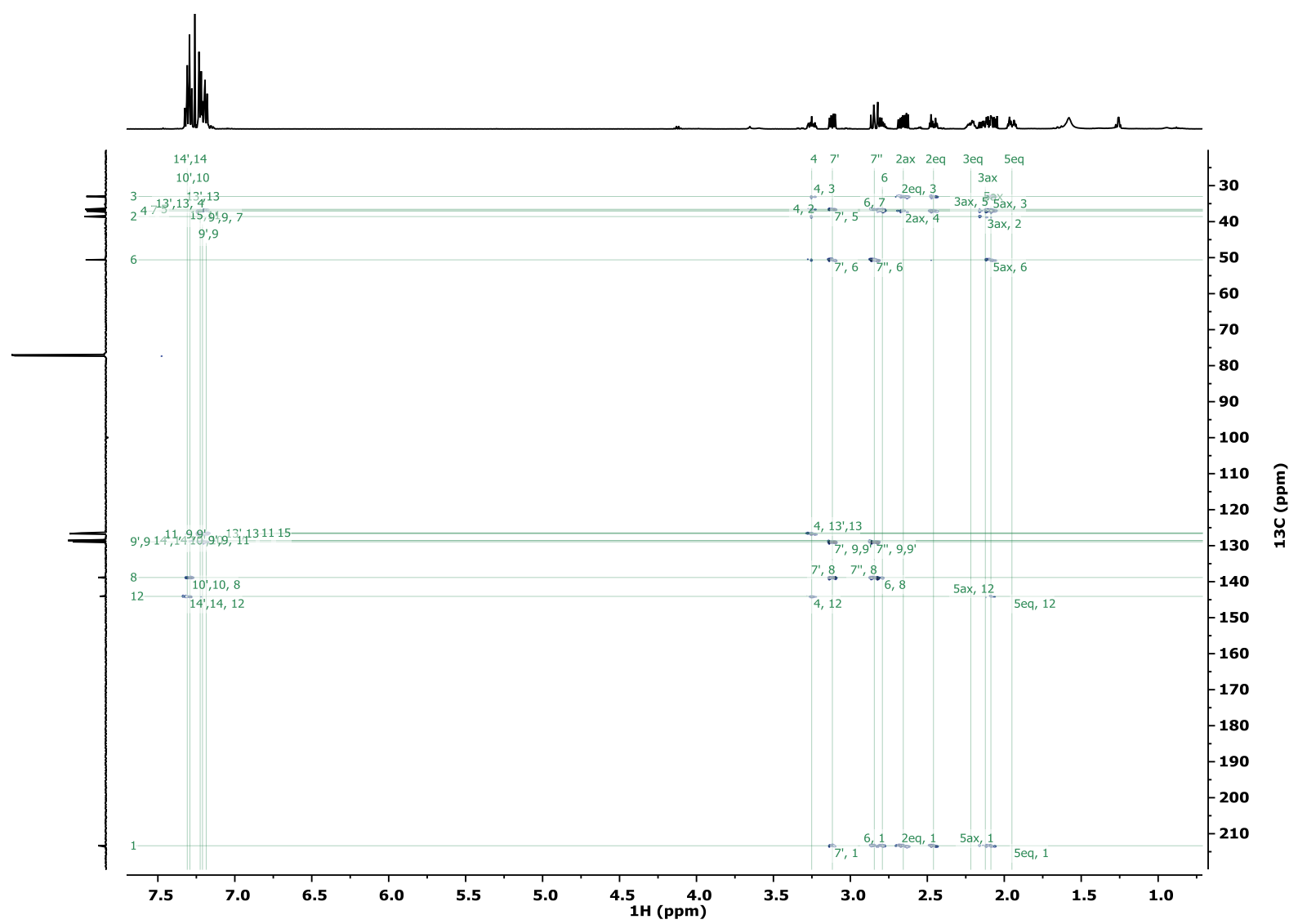

\section{${ }^{1} \mathrm{H}-{ }^{1} \mathrm{H}$ NOESY}

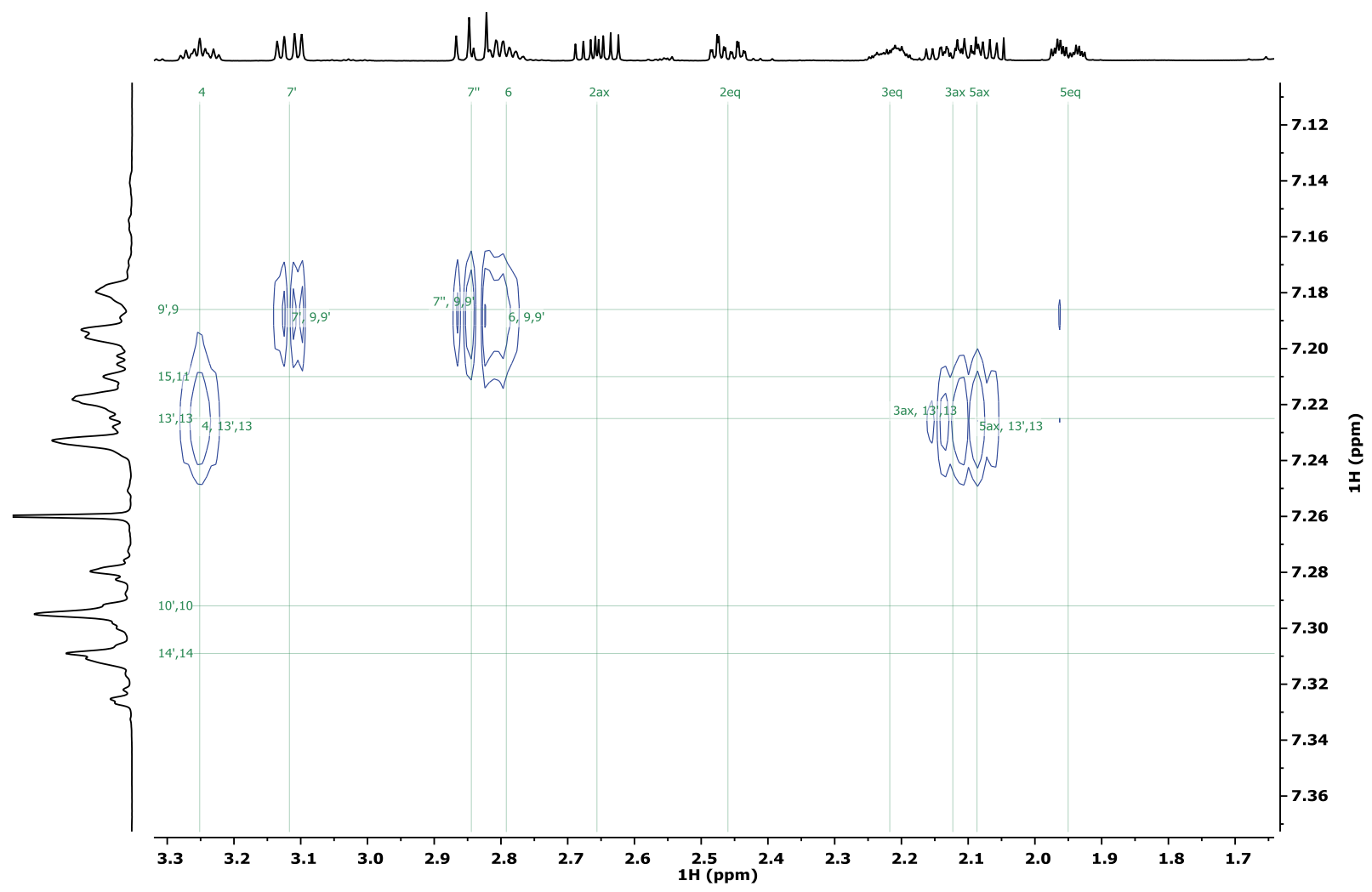


(((3aR,6aS)-5',5'-dimethyl-3,3a,4,6a-tetrahydro-1H-spiro[pentalene-2,2'-[1,3]dioxan]-5-yl)oxy)trimethylsilane 3w:

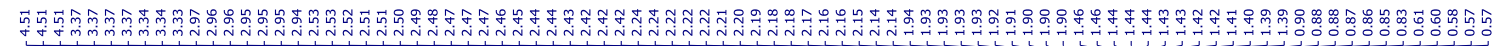

\begin{tabular}{|lc|}
\hline \multicolumn{1}{|c|}{ Parameter } & Value \\
1 S Solvent & CDCC12 \\
2 Temperature & 297.9 \\
3 Spectrometer Frequency 500.81 & 50. \\
4 Nucleus & $1 \mathrm{H}$ \\
\hline
\end{tabular}

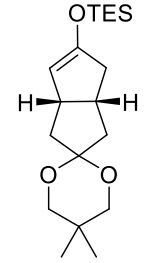

$3 w$

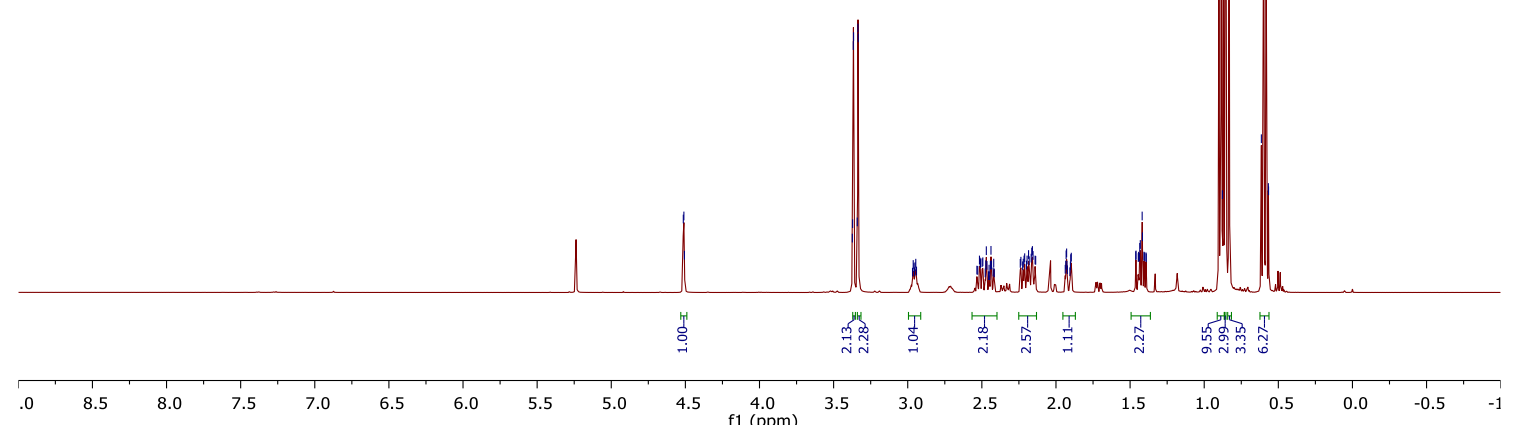

\begin{tabular}{|ll|}
\hline \multicolumn{1}{|c|}{ Parameter } & \multicolumn{1}{c|}{ Value } \\
1 Solvent & $\mathrm{CD} 2 \mathrm{Cl} 2$ \\
2 Temperature & 298.0 \\
3 Spectrometer Frequency & 125.94 \\
4 Nucleus & $13 \mathrm{C}$ \\
\hline
\end{tabular}

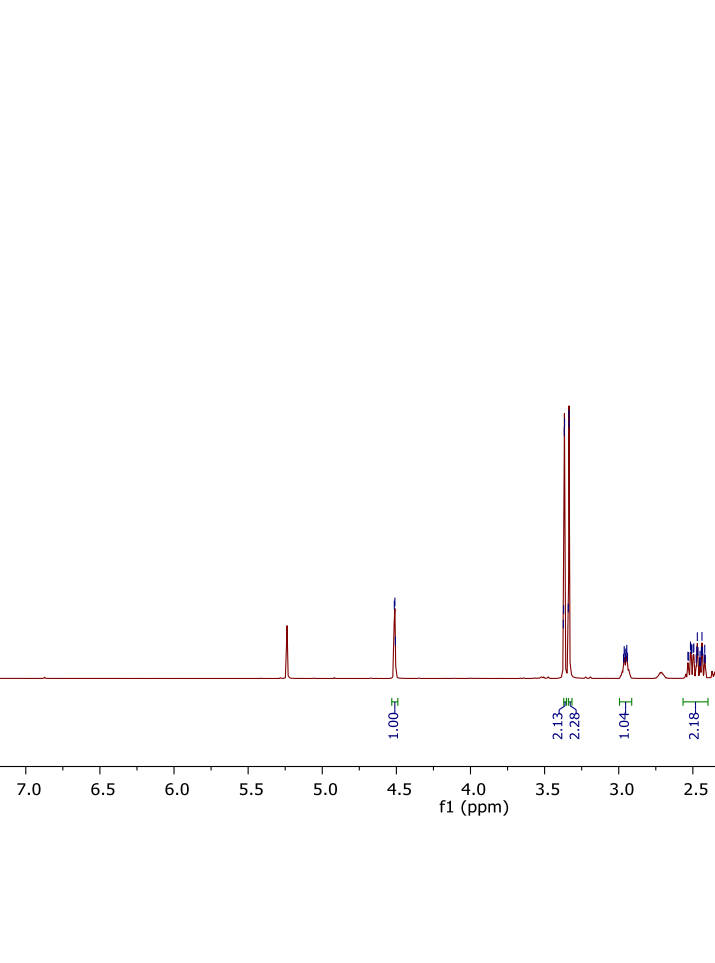

.


(S)-tert-butyl((4-isopropylcyclohex-1-en-1-yl)oxy)dimethylsilane 3x:

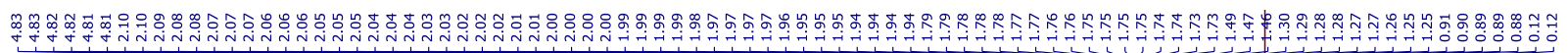

\begin{tabular}{|lc|}
\hline \multicolumn{1}{|c|}{ Parameter } & \multicolumn{1}{c|}{ Value } \\
Solvent & CD2Cl2 \\
Temperature & 297.9 \\
Spectrometer Frequency & 500.81 \\
Nucleus & $1 \mathrm{H}$ \\
\hline
\end{tabular}

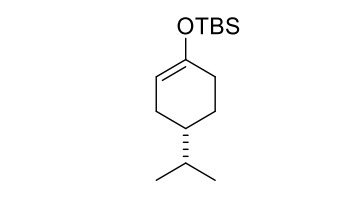

$3 \mathbf{x}$

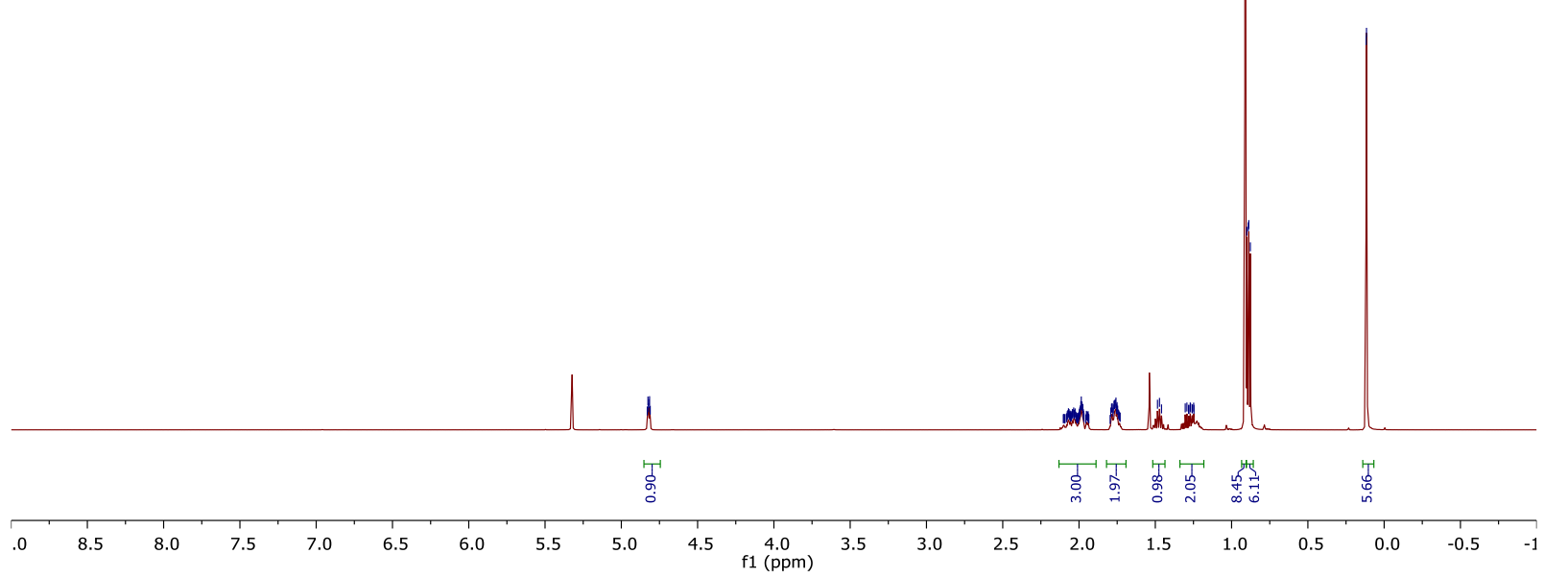

\begin{tabular}{|ll|}
\hline \multicolumn{1}{|c|}{ Parameter } & \multicolumn{1}{c|}{ Value } \\
Solvent & CD2C12 \\
Temperature & 298.0 \\
Spectrometer Frequency & 125.94 \\
Nucleus & $13 \mathrm{C}$ \\
\hline
\end{tabular}

।

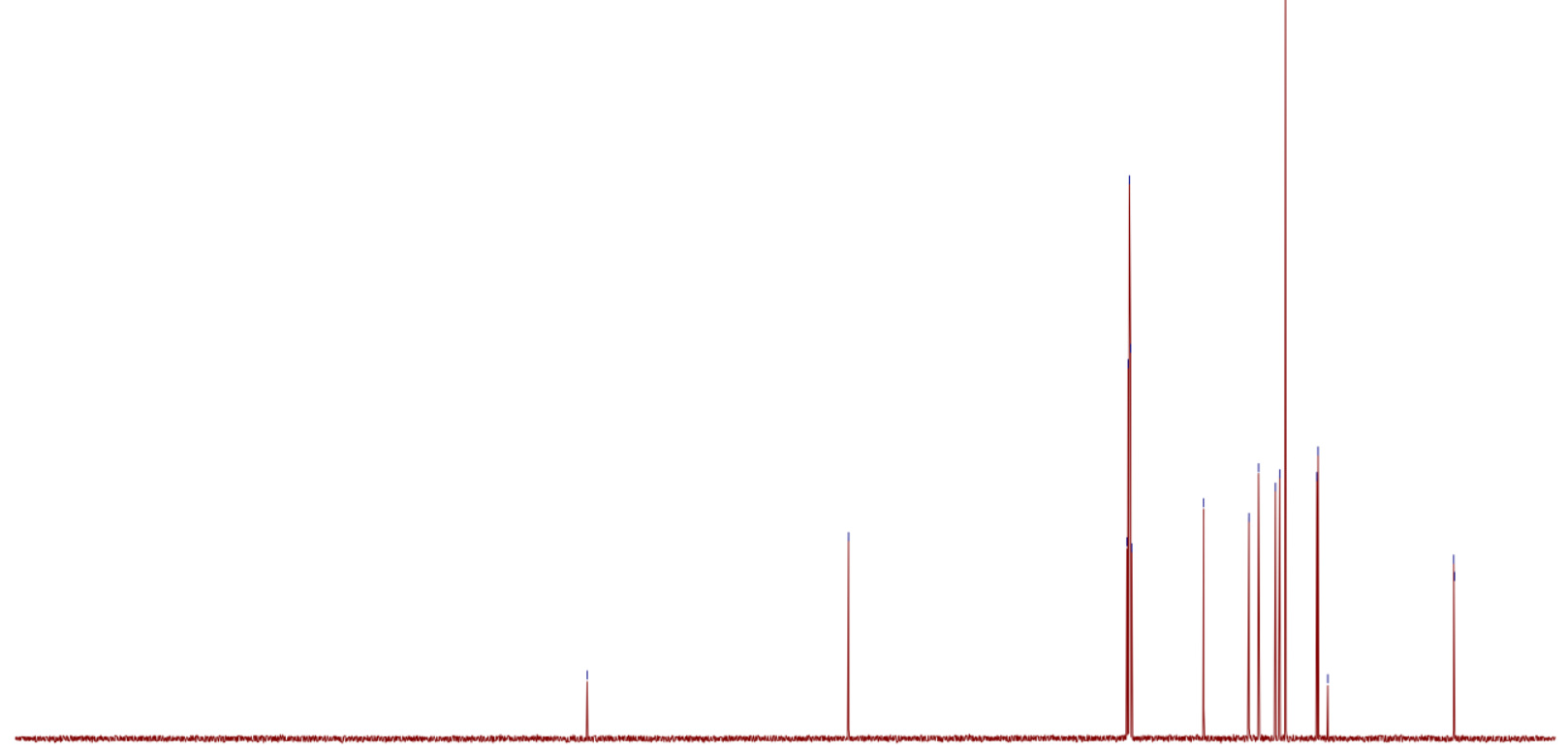
$\begin{array}{lllllllllllllllllllllllllllllllll}250 & 240 & 230 & 220 & 210 & 200 & 190 & 180 & 170 & 160 & 150 & 140 & 130 & \begin{array}{l}120 \\ \mathrm{f} 1(\mathrm{ppm})\end{array} & 100 & 90 & 80 & 70 & 60 & 50 & 40 & 30 & 20 & 10 & 0 & -10 & -20\end{array}$

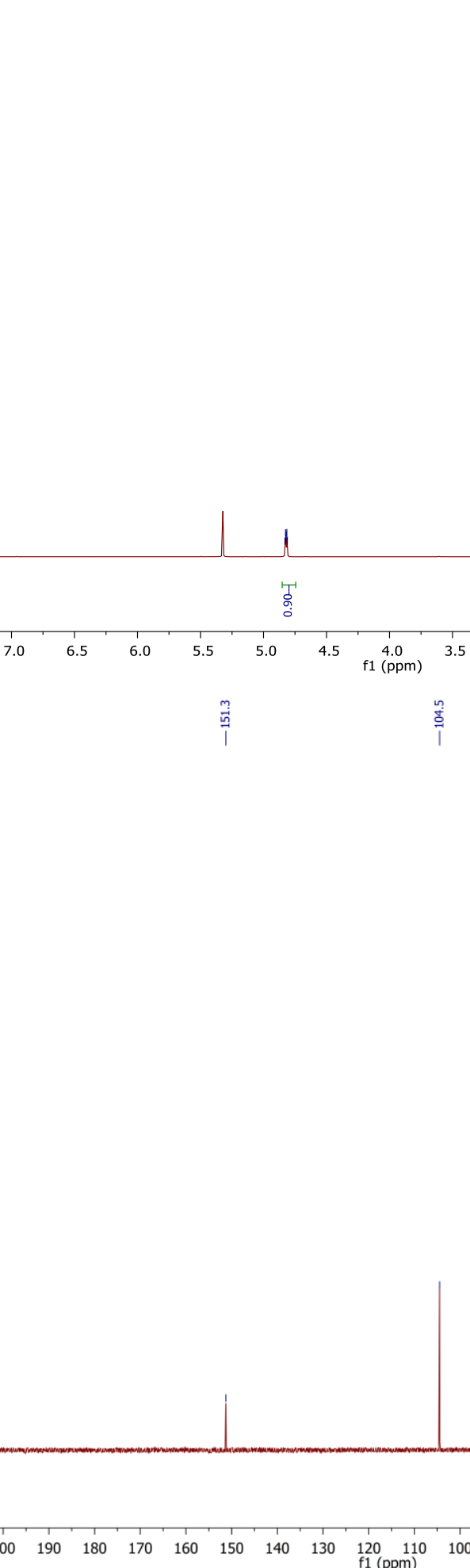




\section{(S)-tert-butyldimethyl((4-ethylcyclohex-1-en-1-yl)oxy)silane 3y:}

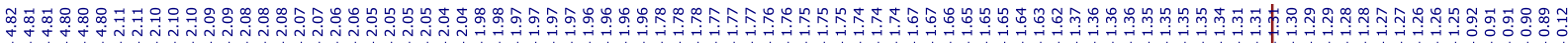 \\ (1)}

\begin{tabular}{|ll|}
\hline \multicolumn{1}{|c}{ Parameter } & \multicolumn{1}{c|}{ Value } \\
Solvent & CD2C12 \\
Temperature & 298.0 \\
Spectrometer Frequency & 500.81 \\
Nucleus & $1 \mathrm{H}$ \\
\hline
\end{tabular}

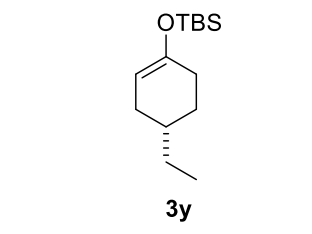

3y

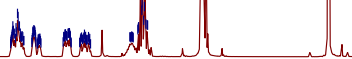

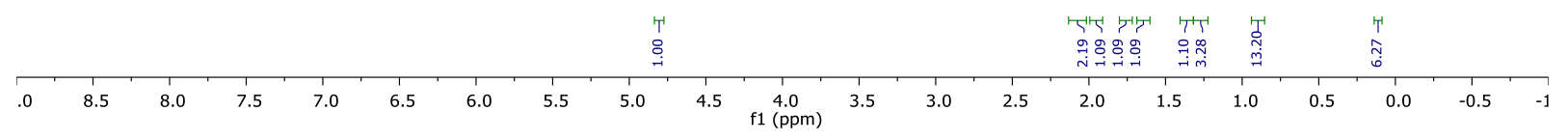

$\stackrel{m}{\underline{m}}$


(S)-tert-butyldimethyl((4-methylcyclohex-1-en-1-yl)oxy)silane 3z:

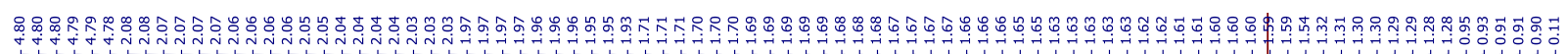

\begin{tabular}{|ll|}
\hline \multicolumn{1}{|c}{ Parameter } & \multicolumn{1}{c}{ Value } \\
Solvent & CD2Cl2 \\
Temperature & 297.9 \\
Spectrometer Frequency & 500.81 \\
Nucleus & $1 \mathrm{H}$ \\
\hline
\end{tabular}

Nucleus $\quad 1 \mathrm{H}$

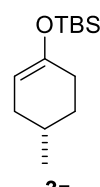

$3 z$

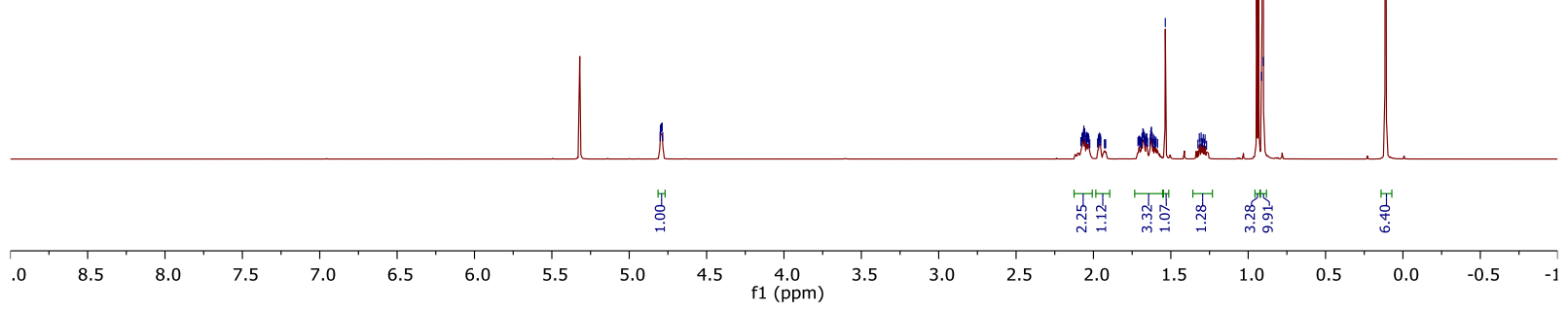

\begin{tabular}{|ll|}
\hline \multicolumn{1}{|c}{ Parameter } & \multicolumn{1}{c|}{ Value } \\
Solvent & CD2C12 \\
Temperature & 298.0 \\
Spectrometer Frequency & 125.94 \\
Nucleus & $13 \mathrm{C}$ \\
\hline
\end{tabular}

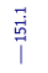

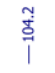

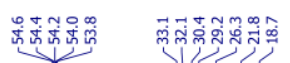

Nucleus $13 \mathrm{C}$

$\begin{array}{llllllllllllll}250 & 240 & 230 & 220 & 210 & 200 & 190 & 180 & 170 & 160 & 150 & 140 & 130 & 120 \\ \mathrm{f} 1(\mathrm{ppm})\end{array}$ 
(S)-tert-butyldimethyl((3,4,5,6-tetrahydro-[1,1'-biphenyl]-2-yl)oxy)silane 3aa:

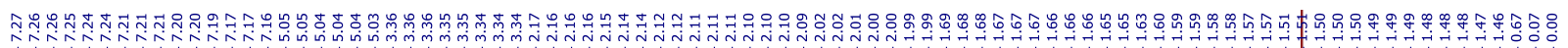
\begin{tabular}{|lc|}
\hline \multicolumn{1}{|c|}{ Parameter } & Value \\
1 Solvent & $\mathrm{CD} 2 \mathrm{Cl} 12$ \\
2 Temperature & 297.9 \\
3 Spectrometer Frequency & 500.81 \\
4 Nucleus & $1 \mathrm{H}$ \\
\hline
\end{tabular}
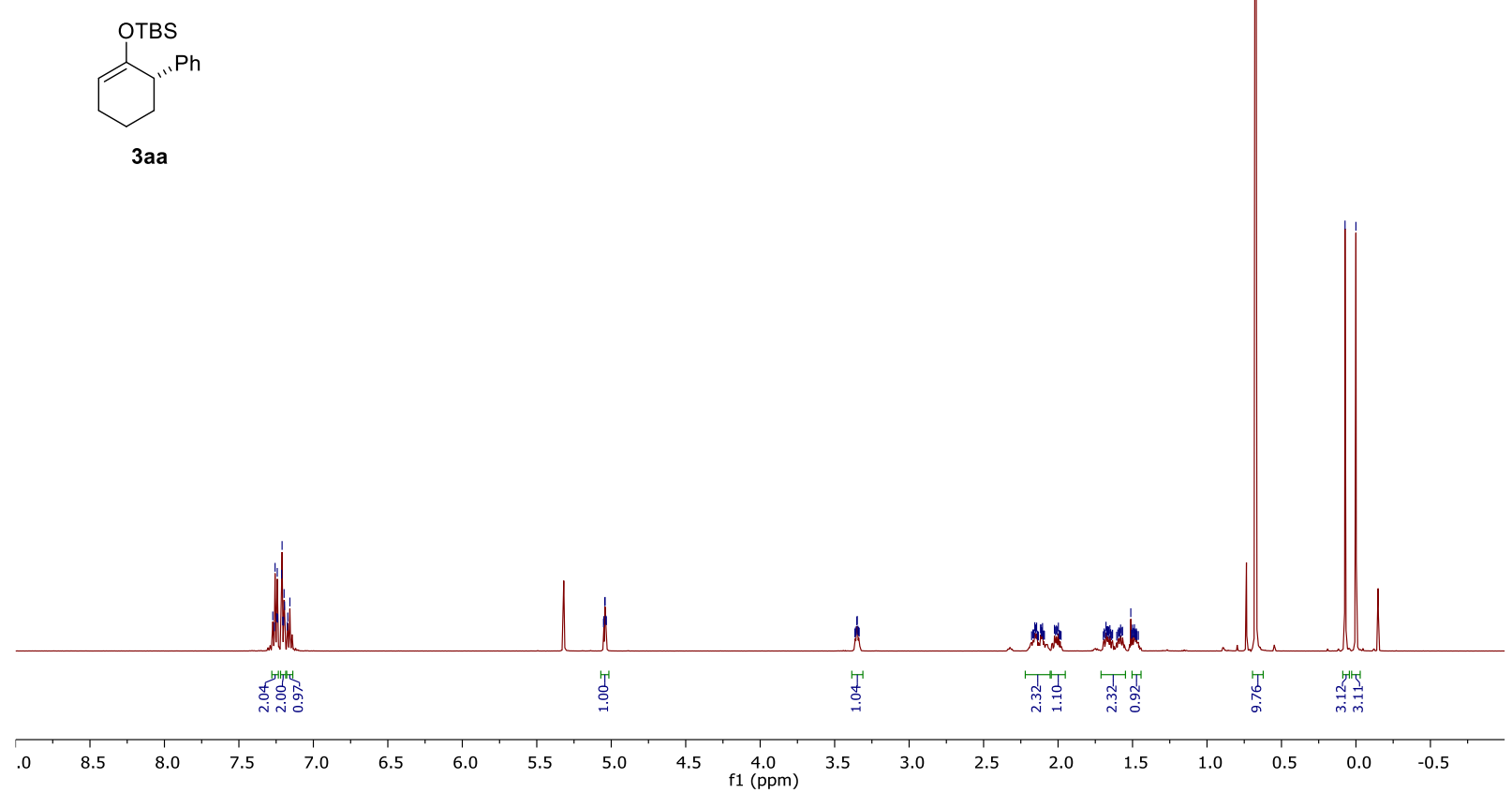

\begin{tabular}{|ll|}
\hline \multicolumn{1}{|c|}{ Parameter } & Value \\
Solvent & CD2C12 \\
Temperature & 298.0 \\
Spectrometer Frequency & 125.94 \\
Nucleus & $13 \mathrm{C}$ \\
\hline
\end{tabular}

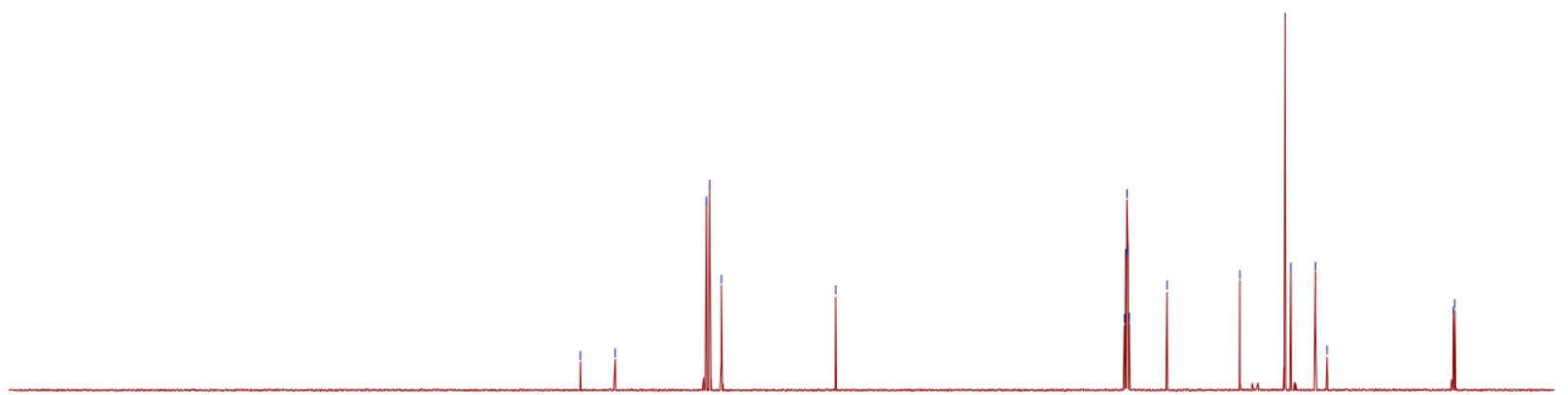

$\begin{array}{lllllllllllllllllllllllllllll}250 & 240 & 230 & 220 & 210 & 200 & 190 & 180 & 170 & 160 & 150 & 140 & 130 & \begin{array}{c}120 \\ \mathrm{f} 1(\mathrm{ppm})\end{array} & 100 & 90 & 80 & 70 & 60 & 50 & 40 & 30 & 20 & 10 & 0 & -10 & -20\end{array}$ 
(S)-([1,1'-bi(cyclohexan)]-2-en-2-yloxy)(tert-butyl)dimethylsilane 3ab:

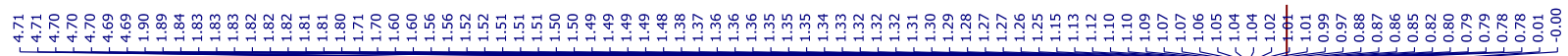

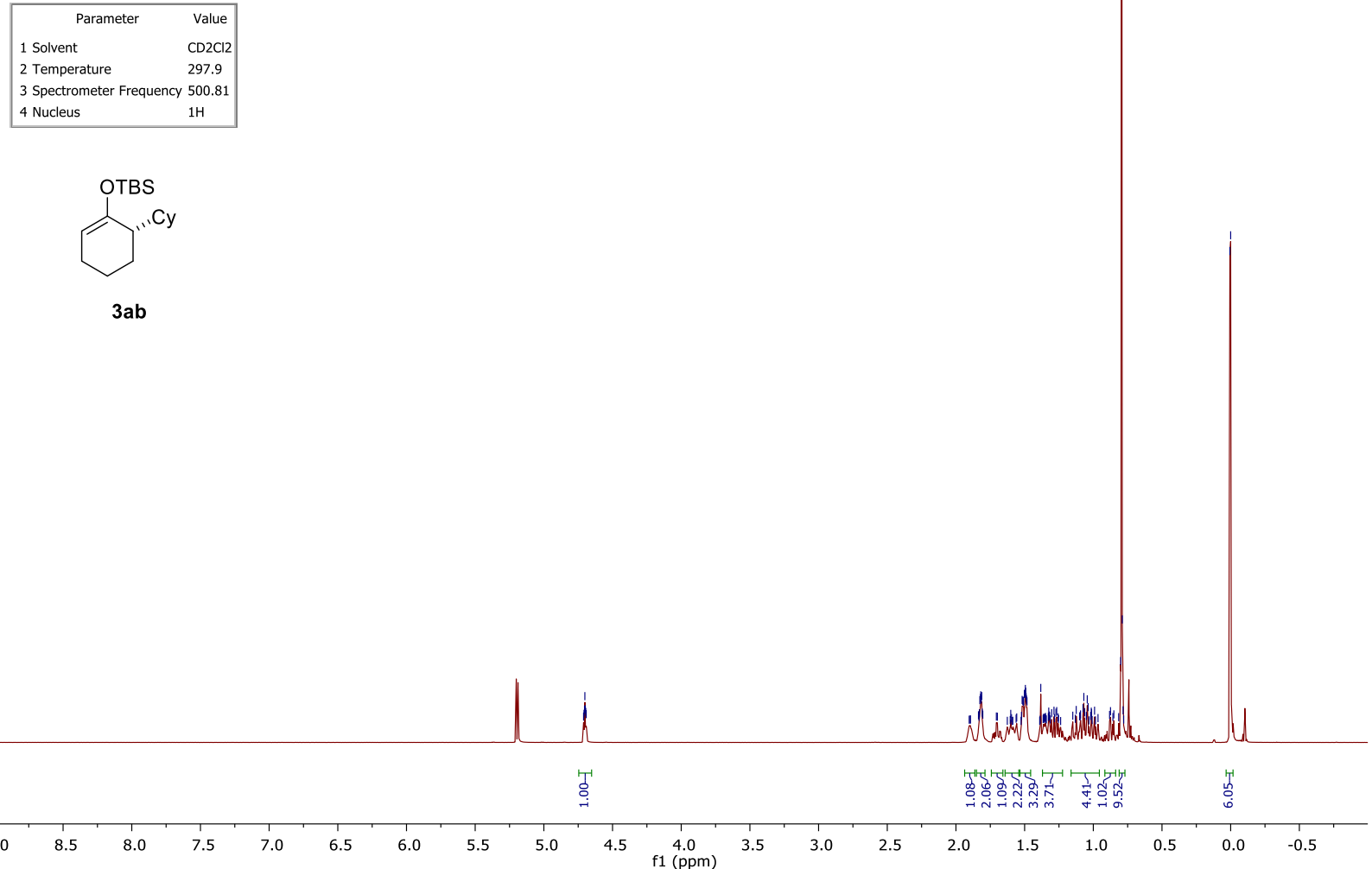

\begin{tabular}{|ll|}
\hline \multicolumn{1}{|c|}{ Parameter } & \multicolumn{1}{c|}{ Value } \\
1 Solvent & $\mathrm{CD2C12}$ \\
2 Temperature & 298.0 \\
3 Spectrometer Frequency & 125.94 \\
4 Nucleus & $13 \mathrm{C}$ \\
\hline
\end{tabular}

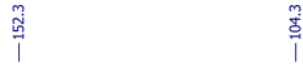

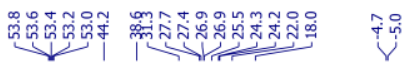

$\begin{array}{llllllllllllllllllllllllllllllllll}250 & 240 & 230 & 220 & 210 & 200 & 190 & 180 & 170 & 160 & 150 & 140 & 130 & 120 & 110 & 100 & 90 & 80 & 70 & 60 & 50 & 40 & 30 & 20 & 10 & 0 & -10 & -20\end{array}$ 
(S)-tert-butyldimethyl((4-methylcyclohex-1-en-1-yl)oxy)silane 3ac

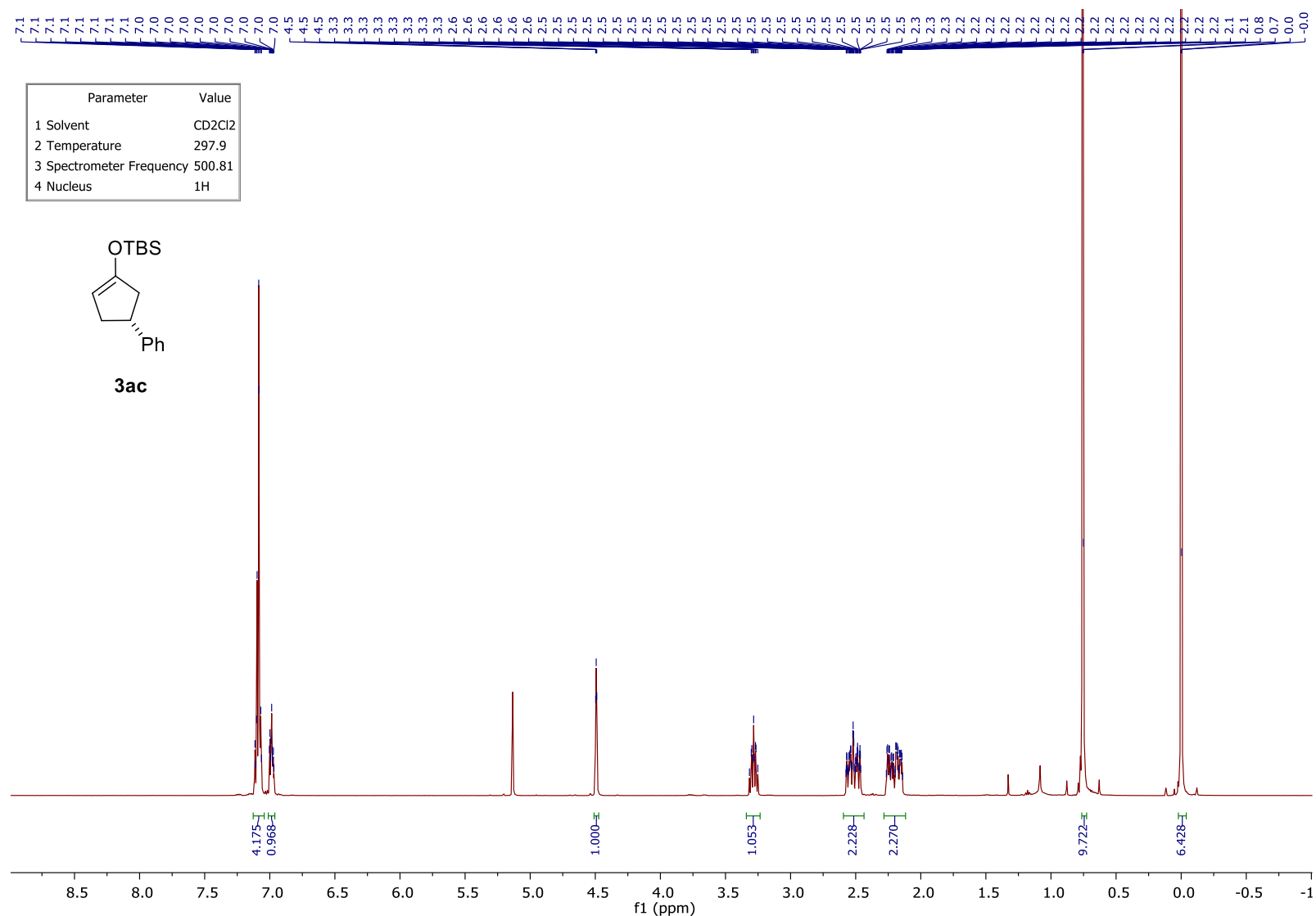

\begin{tabular}{|ll|}
\hline \multicolumn{1}{|c|}{ Parameter } & \multicolumn{1}{c|}{ Value } \\
1 Solvent & $\mathrm{CD} 2 \mathrm{C} 12$ \\
2 Temperature & 298.0 \\
3 Spectrometer Frequency & 125.94 \\
4 Nucleus & $13 \mathrm{C}$ \\
\hline
\end{tabular}

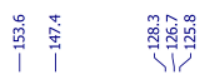

เัติำ

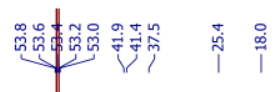

过

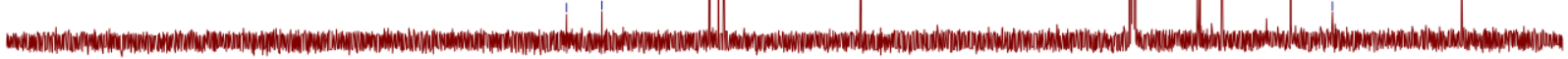

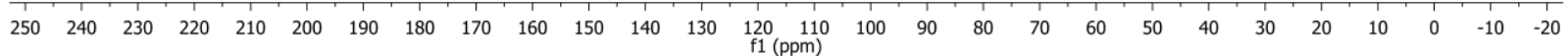


From the crude NMR, the remained product 3ac was a mixture of regiomers, the r.r. $=18: 1$ :

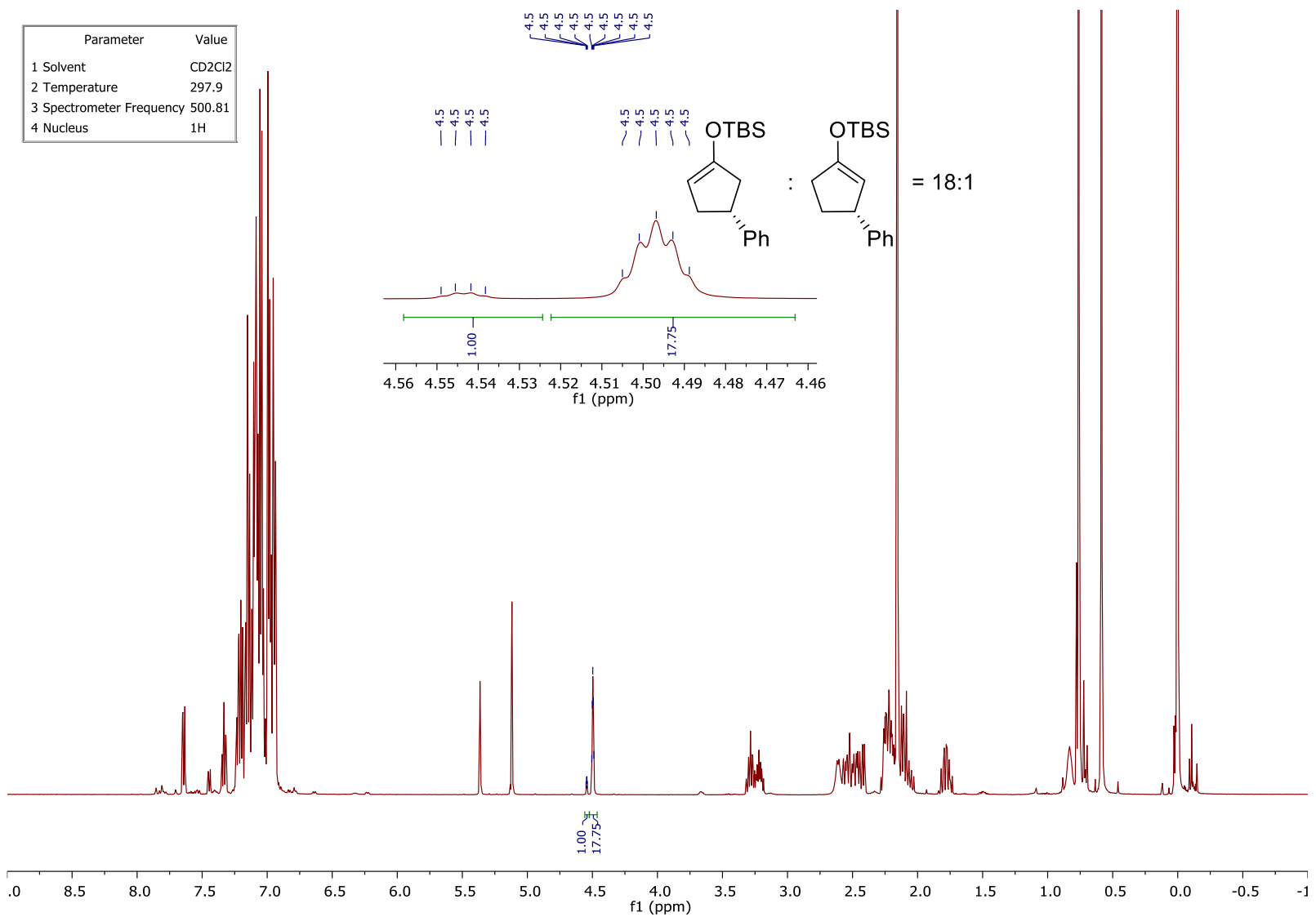

tert-butyldimethylsilyl [1,1'-biphenyl]-2-carboxylate

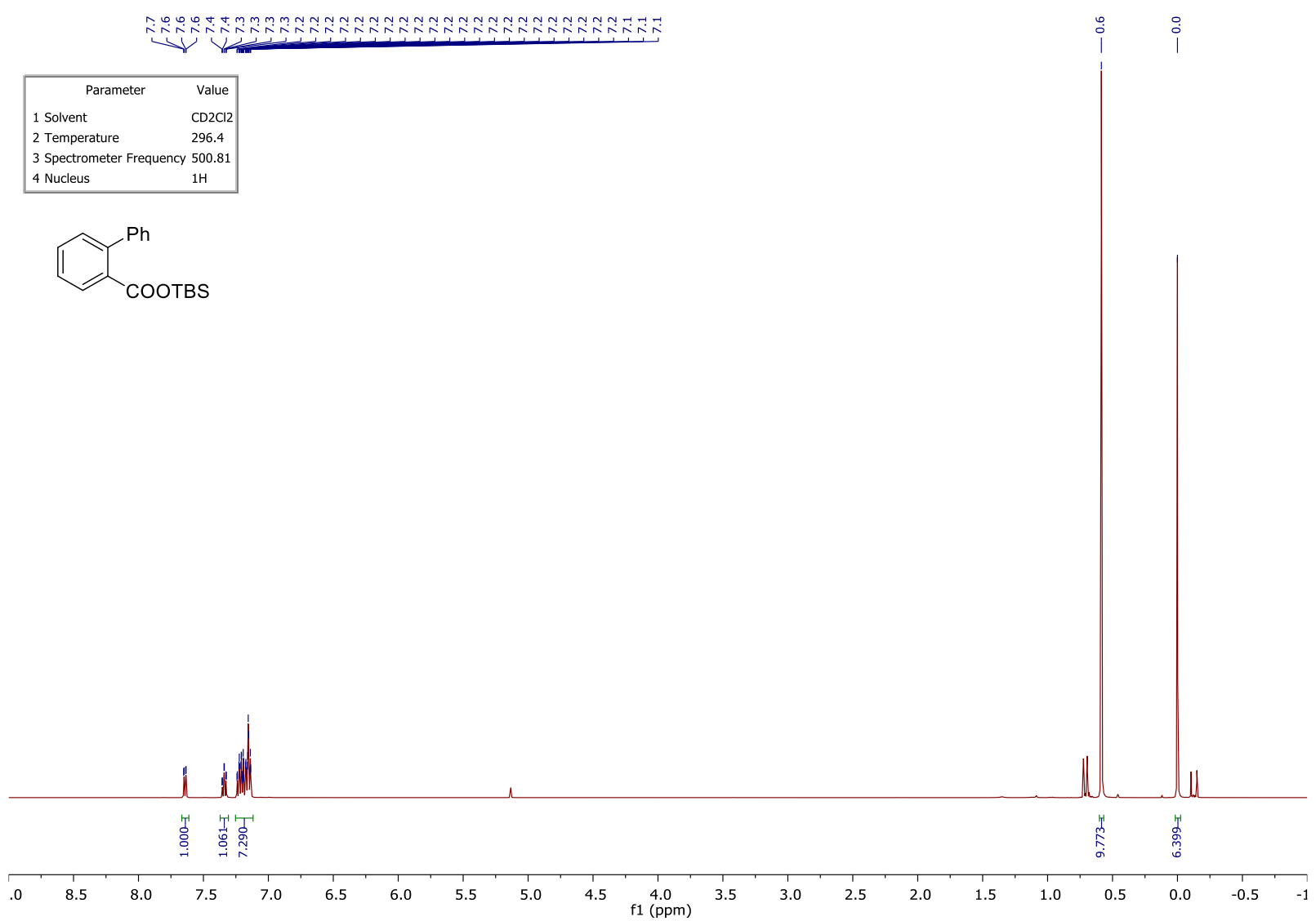




\begin{tabular}{|ll|}
\hline \multicolumn{1}{|c|}{ Parameter } & \multicolumn{1}{c|}{ Value } \\
1 Solvent & CD2C12 \\
2 Temperature & 297.7 \\
3 Spectrometer Frequency & 125.94 \\
4 Nucleus & $13 \mathrm{C}$ \\
\hline
\end{tabular}

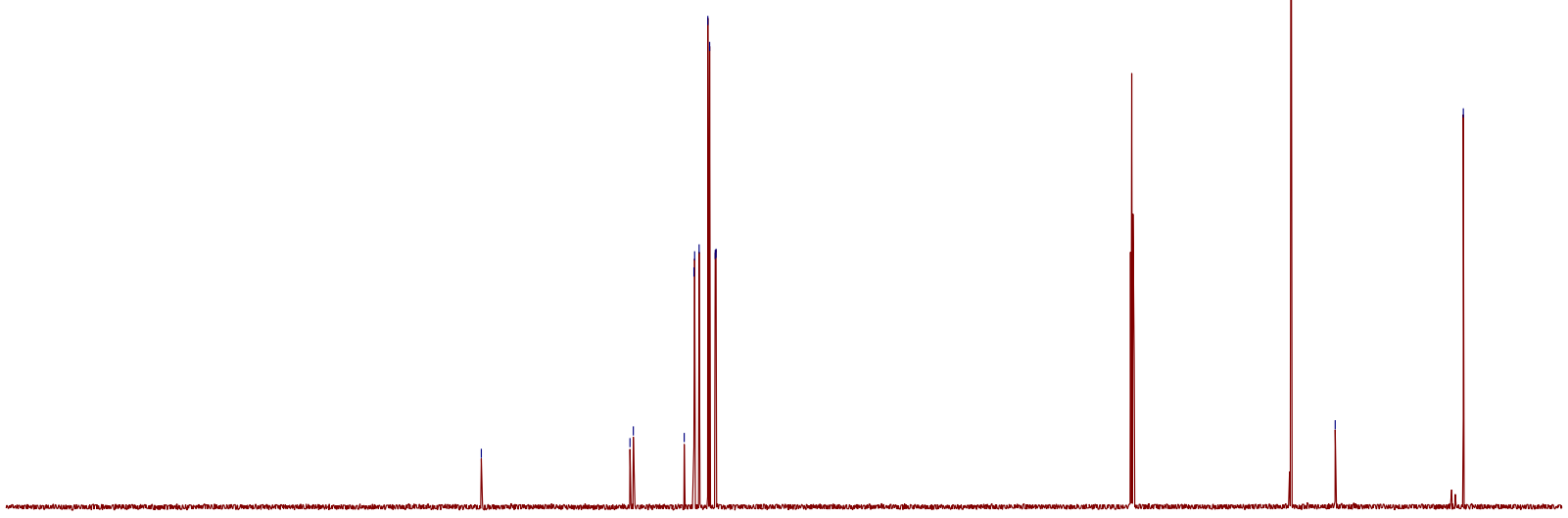

$\begin{array}{llllllllllllllllllllllllllll}250 & 240 & 230 & 220 & 210 & 200 & 190 & 180 & 170 & 160 & 150 & 140 & 130 & 120 & 110 & 100 & 90 & 80 & 70 & 60 & 50 & 40 & 30 & 20 & 10 & 0 & -10 & -20\end{array}$

Spectra for homo aldol product 11-5 (4-(2-oxo-5-phenylcyclohexyl)-1,2,3,6-tetrahydro-1,1'-biphenyl)

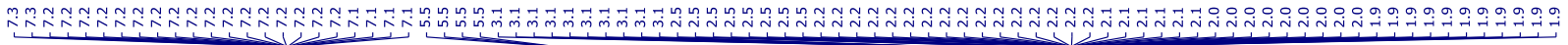

\begin{tabular}{|lc|}
\hline \multicolumn{1}{|c|}{ Parameter } & Value \\
1 Solvent & $\mathrm{CDCl} 3$ \\
2 Temperature & 297.8 \\
3 Spectrometer & Frequency \\
500.81 \\
4 Nucleus & $1 \mathrm{H}$ \\
\hline
\end{tabular}<smiles>O=C1CCC(c2ccccc2)CC1C1=CCC(c2ccccc2)CC1</smiles>

$11-5$

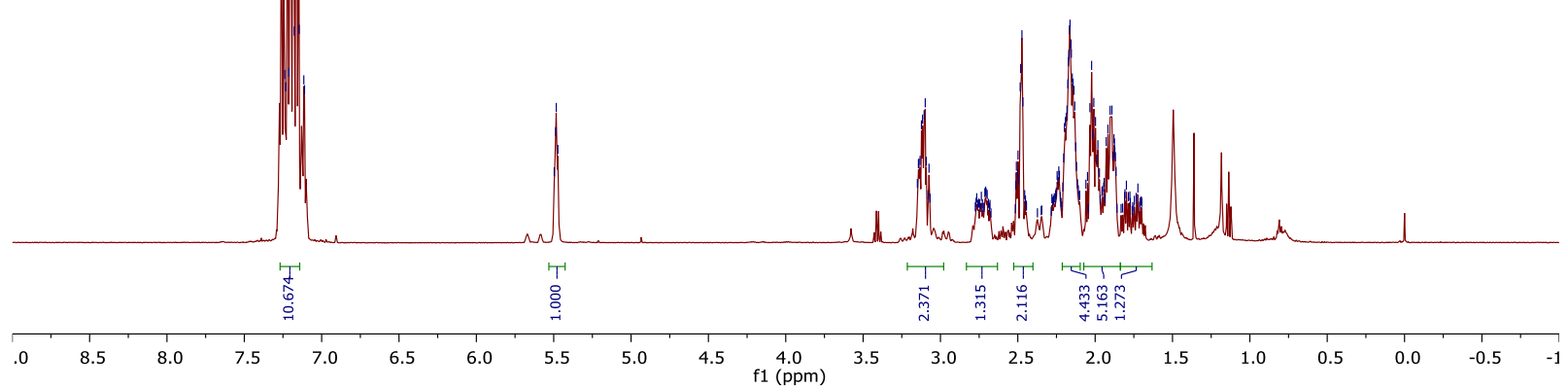




\begin{tabular}{|ll|}
\hline \multicolumn{1}{|c|}{ Parameter } & Value \\
1 Solvent & $\mathrm{CDCl}$ \\
2 Temperature & 298.0 \\
3 Spectrometer Frequency & 125.94 \\
4 Nucleus & $13 \mathrm{C}$ \\
\hline
\end{tabular}

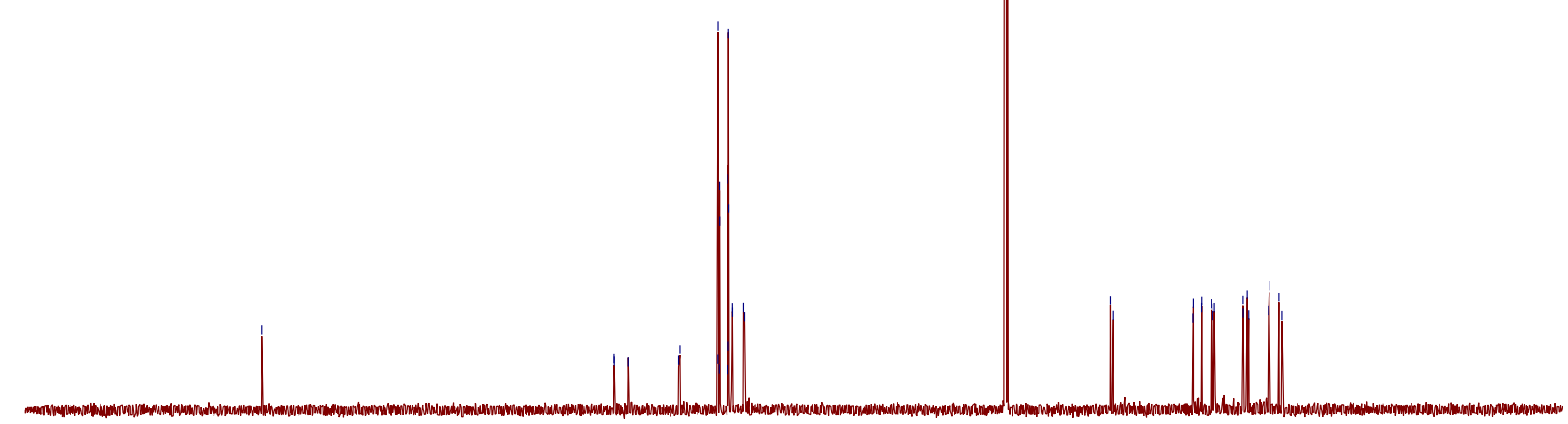

\begin{tabular}{rlllllllllllllllllllllllllllllllllllllll}
\hline 250 & 240 & 230 & 220 & 210 & 200 & 190 & 180 & 170 & 160 & 150 & 140 & 130 & 120 & 110 & 100 & 90 & 80 & 70 & 60 & 50 & 40 & 30 & 20 & 10 & 0 & -10 & -20
\end{tabular}

\begin{tabular}{|ll|}
\hline \multicolumn{1}{|c|}{ Parameter } & \multicolumn{1}{c|}{ Value } \\
1 Solvent & CDCl3 \\
2 Temperature & 298.0 \\
3 Experiment & DEPT-45 \\
4 Spectrometer Frequency & 125.94 \\
5 Nucleus & $13 \mathrm{C}$ \\
\hline
\end{tabular}

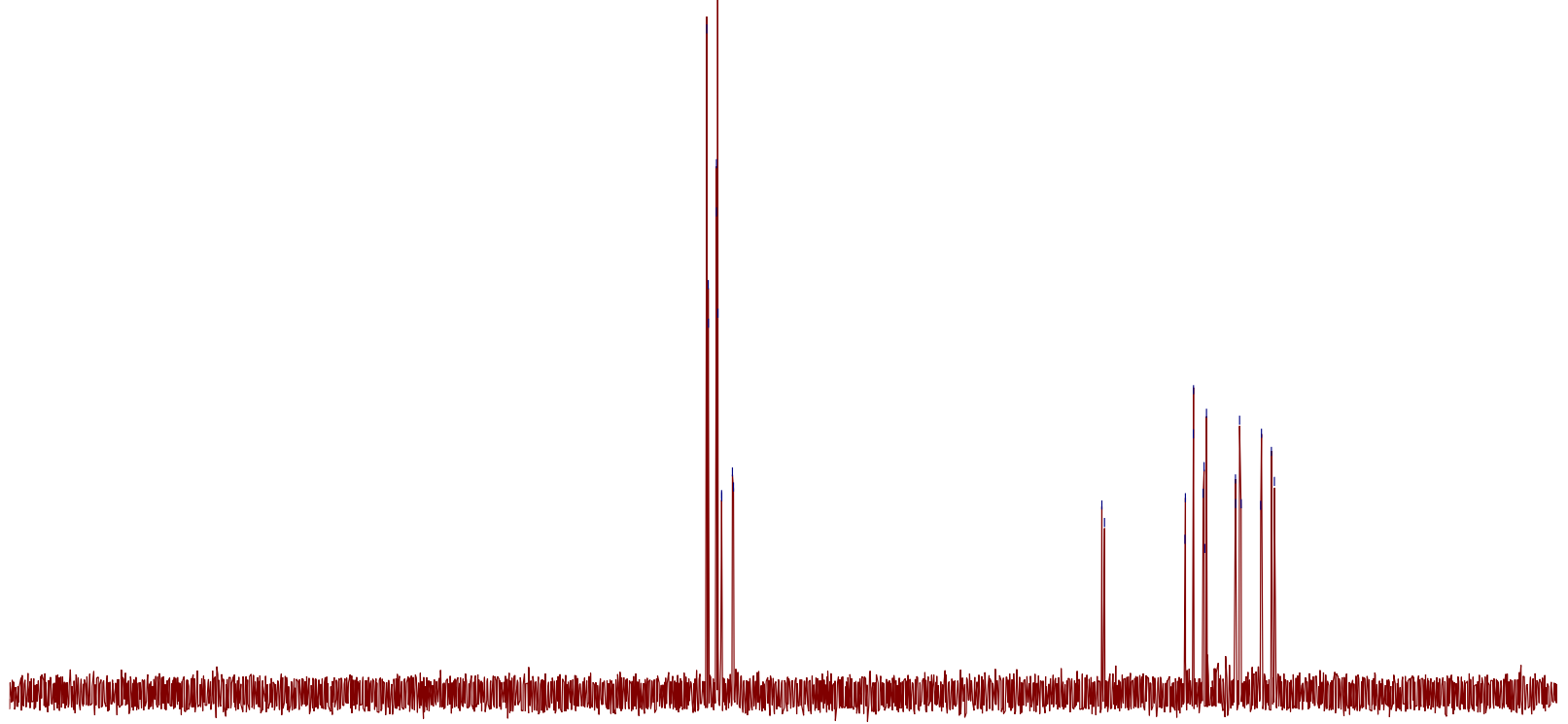

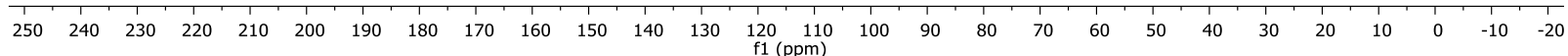




\begin{tabular}{|ll|}
\hline \multicolumn{1}{|c|}{ Parameter } & \multicolumn{1}{c|}{ Value } \\
1 Solvent & CDCl3 \\
2 Temperature & 298.0 \\
3 Experiment & DEPT-90 \\
4 Spectrometer Frequency & 125.94 \\
5 Nucleus & $13 \mathrm{C}$ \\
\hline
\end{tabular}

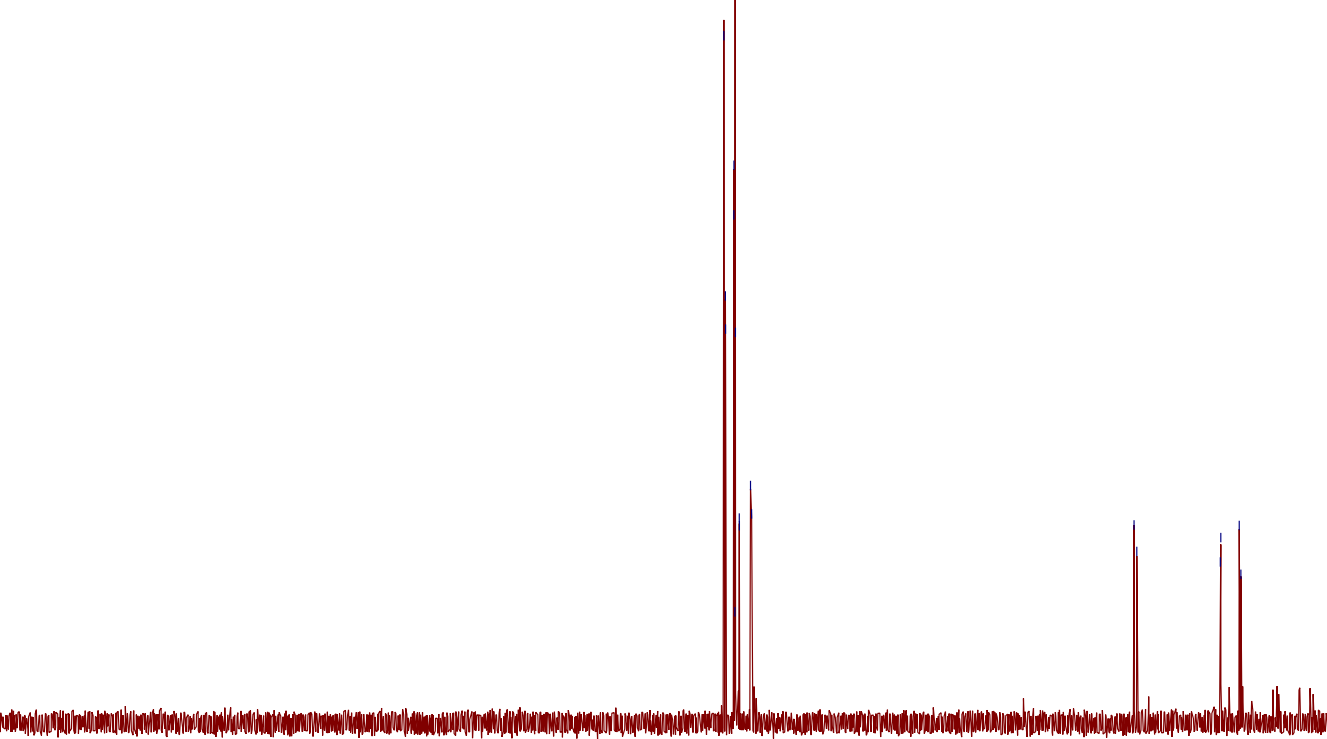

\begin{tabular}{|ll|}
\hline \multicolumn{1}{|c|}{ Parameter } & \multicolumn{1}{c|}{ Value } \\
1 Solvent & CDCl3 \\
2 Temperature & 298.0 \\
3 Experiment & DEPT-135 \\
4 Spectrometer Frequency & 125.94 \\
5 Nucleus & $13 \mathrm{C}$ \\
\hline
\end{tabular}

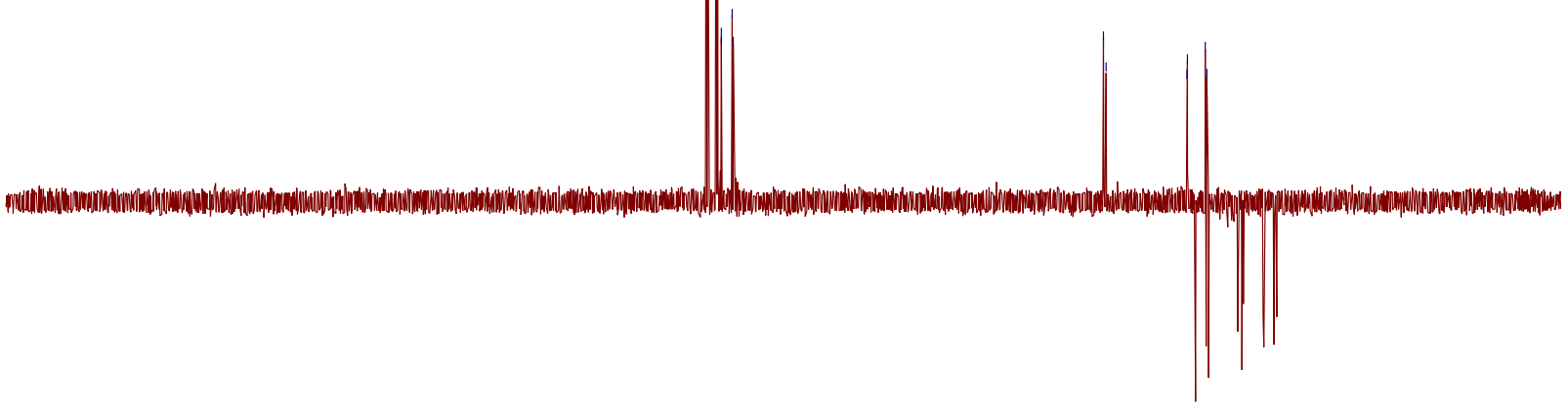

$\begin{array}{llllllllllllllllllllllllllllll}250 & 240 & 230 & 220 & 210 & 200 & 190 & 180 & 170 & 160 & 150 & 140 & 130 & \begin{array}{c}120 \\ \mathrm{f} 1(\mathrm{ppm})\end{array} & 100 & 90 & 80 & 70 & 60 & 50 & 40 & 30 & 20 & 10 & 0 & -10 & -20\end{array}$ 


\section{LC-MS spectrum of IDPi $\mathbf{4 c}$}

HPLC column: $50 \mathrm{~mm}$ YMC TriArt Bio C4, $3.0 \mathrm{~mm}$ i.D., 0.1\% TFA/MeCN 15:85, $1.0 \mathrm{~mL} / \mathrm{min}, 5.0 \mathrm{MPa}, 308 \mathrm{~K}, \lambda=254 \mathrm{~nm}$
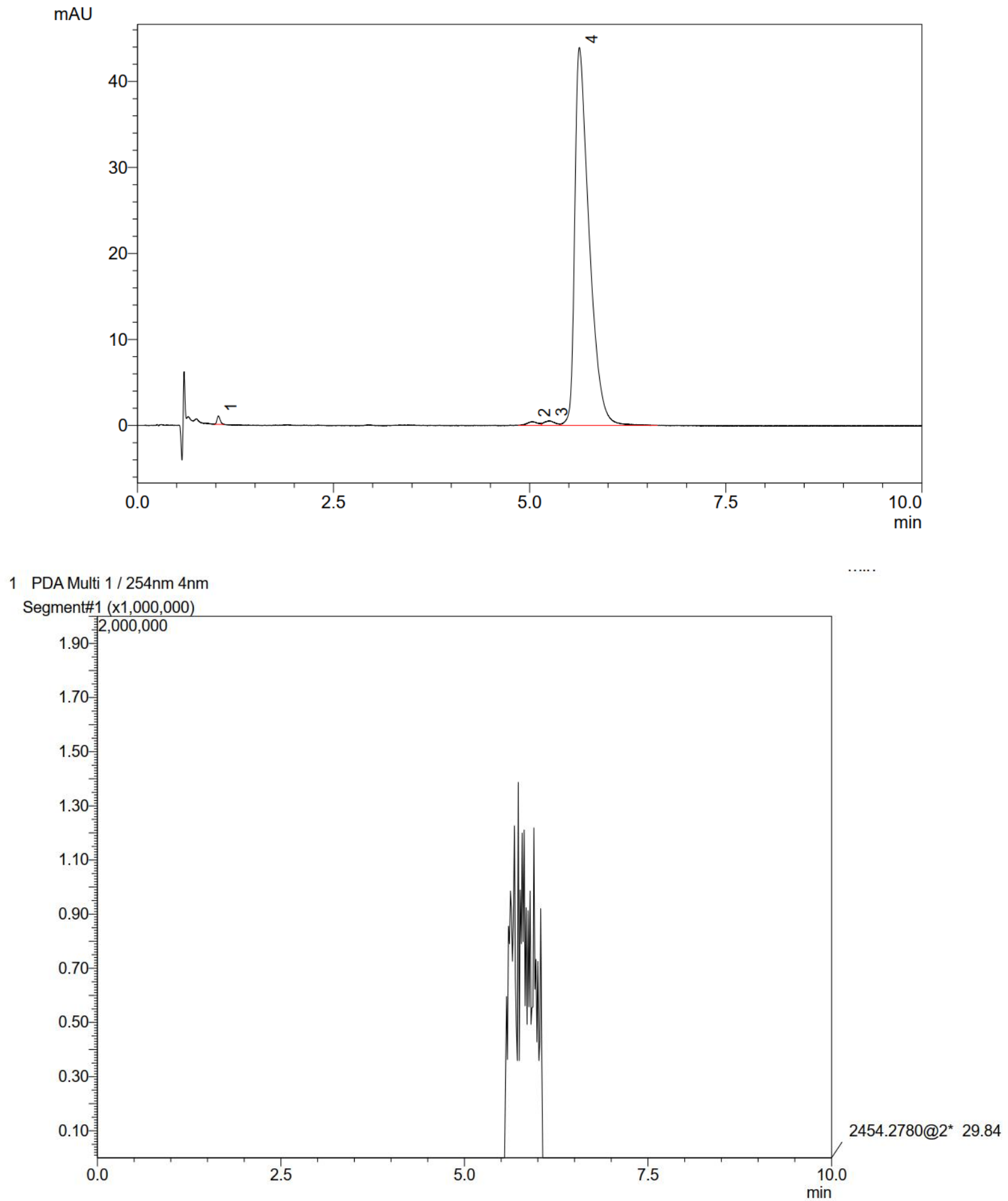

\begin{tabular}{|c|c|c|}
\hline $\mathrm{t}_{\mathrm{R}} / \mathrm{min}$ & \% peak area & $\mathrm{m} / \mathrm{z}$ found \\
\hline 5.63 & 98.1 & $2454\left([\mathrm{M}-\mathrm{H}]^{-}\right)$ \\
\hline Total & \multicolumn{2}{|c|}{100} \\
\hline
\end{tabular}




\section{LC-MS spectrum of IDPi $4 d$}
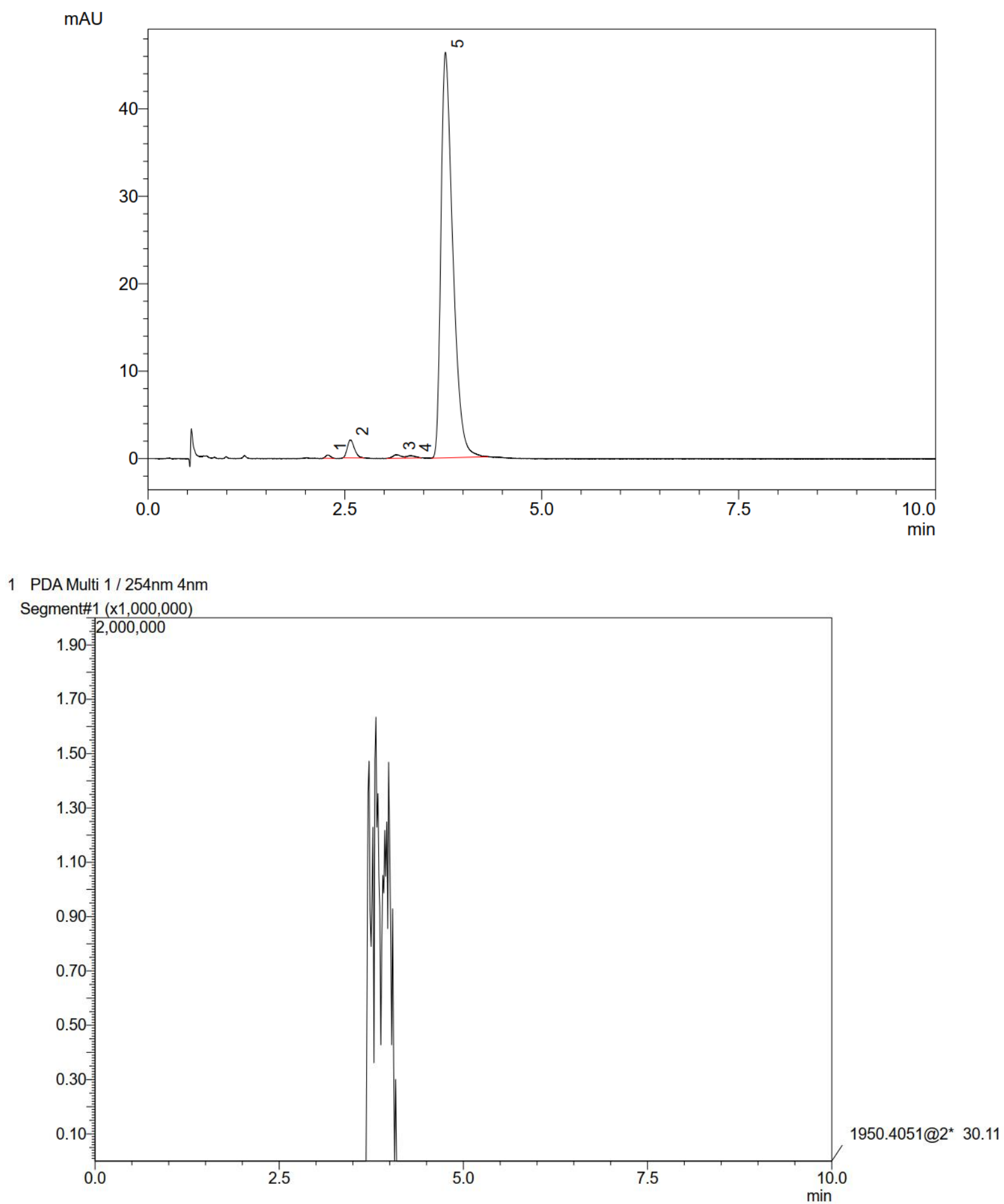

\begin{tabular}{|c|c|c|}
\hline $\mathrm{t}_{\mathrm{R}} / \mathrm{min}$ & \% peak area & $\mathrm{m} / \mathrm{z}$ found \\
\hline 3.78 & 96.2 & $1950\left([\mathrm{M}-\mathrm{H}]^{-}\right)$ \\
\hline Total & \multicolumn{2}{|c|}{100} \\
\hline
\end{tabular}




\section{Copies of HPLC and GC traces}

HPLC column: OJ-3R, Acetonitrile: Water = 70:30, $1.0 \mathrm{~mL} / \mathrm{min}, 298 \mathrm{~K}, 224 \mathrm{~nm}$.

mAU

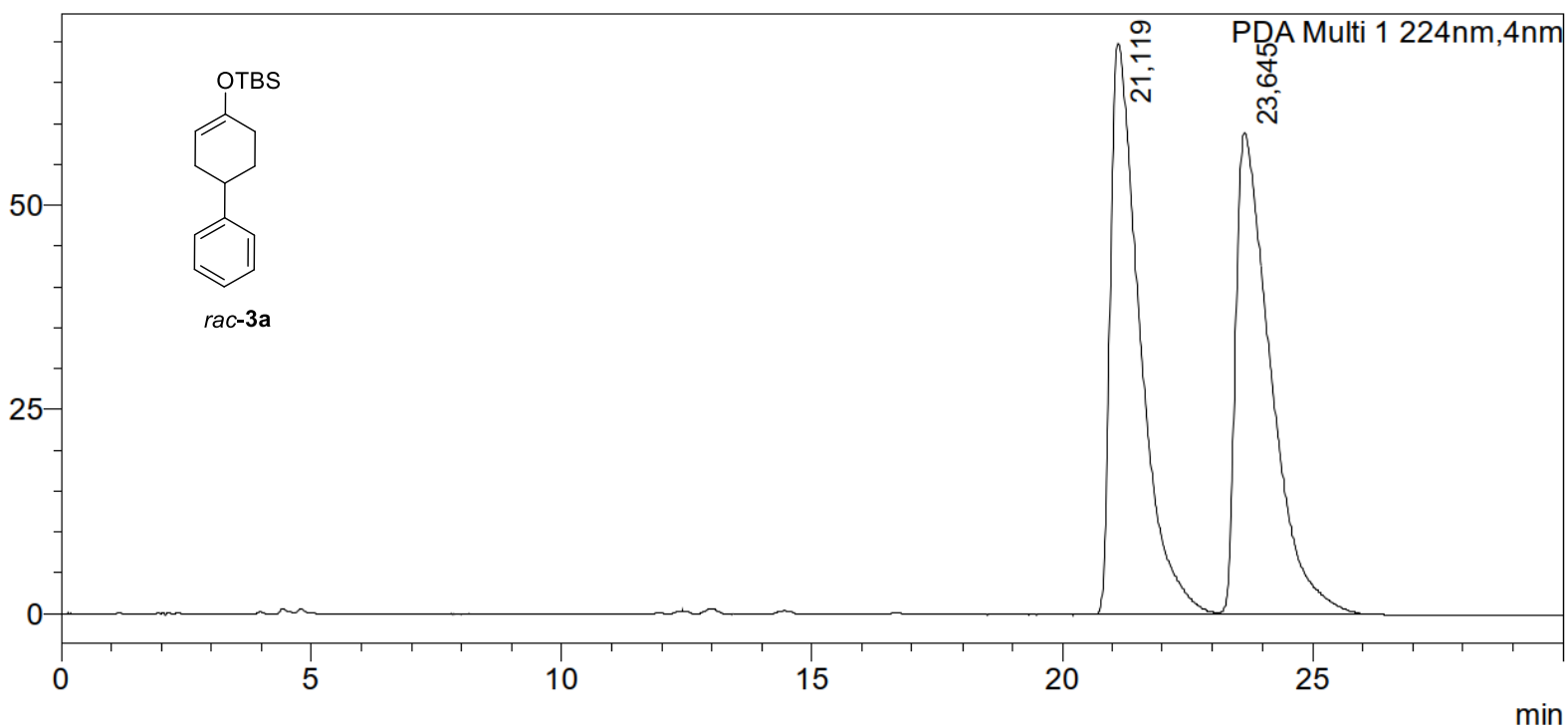

\begin{tabular}{|c|c|c|}
\hline Peak \# & $\mathrm{t}_{\mathrm{R}} / \mathrm{min}$ & $\%$ peak area \\
\hline 1 & 21.1 & 49.99 \\
\hline 2 & 23.6 & 50.01 \\
\hline \multicolumn{2}{|c|}{ Total } & 100 \\
\hline
\end{tabular}

mAU

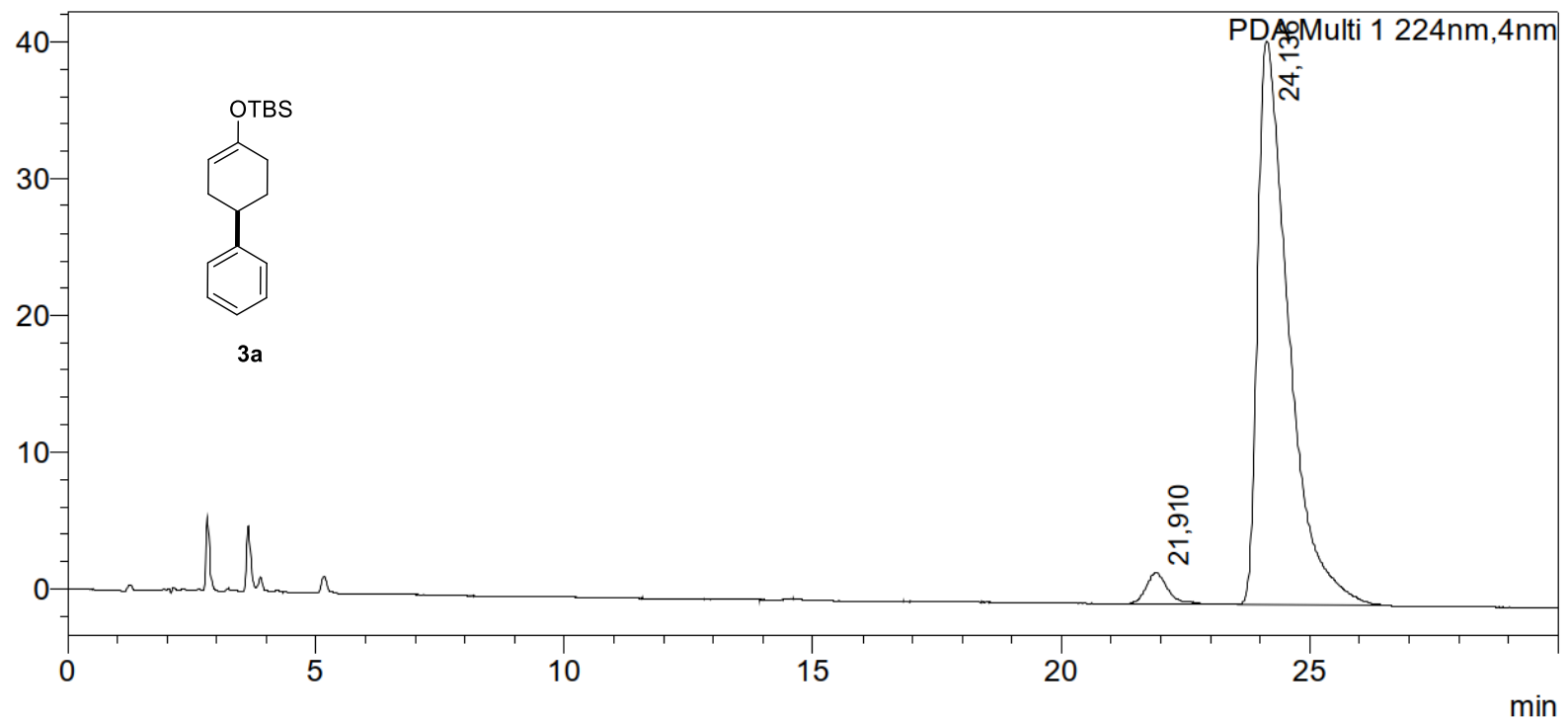

\begin{tabular}{|c|c|c|}
\hline Peak \# & $\mathrm{t}_{\mathrm{R}} / \mathrm{min}$ & $\%$ peak area \\
\hline 1 & 21.9 & 3.05 \\
\hline 2 & 24.1 & 96.95 \\
\hline \multicolumn{2}{|c|}{ Total } & 100 \\
\hline
\end{tabular}


HPLC column: OJ-3R, Acetonitrile: Water = 70:30, $1.0 \mathrm{~mL} / \mathrm{min}, 298 \mathrm{~K}, 220 \mathrm{~nm}$.

mAU

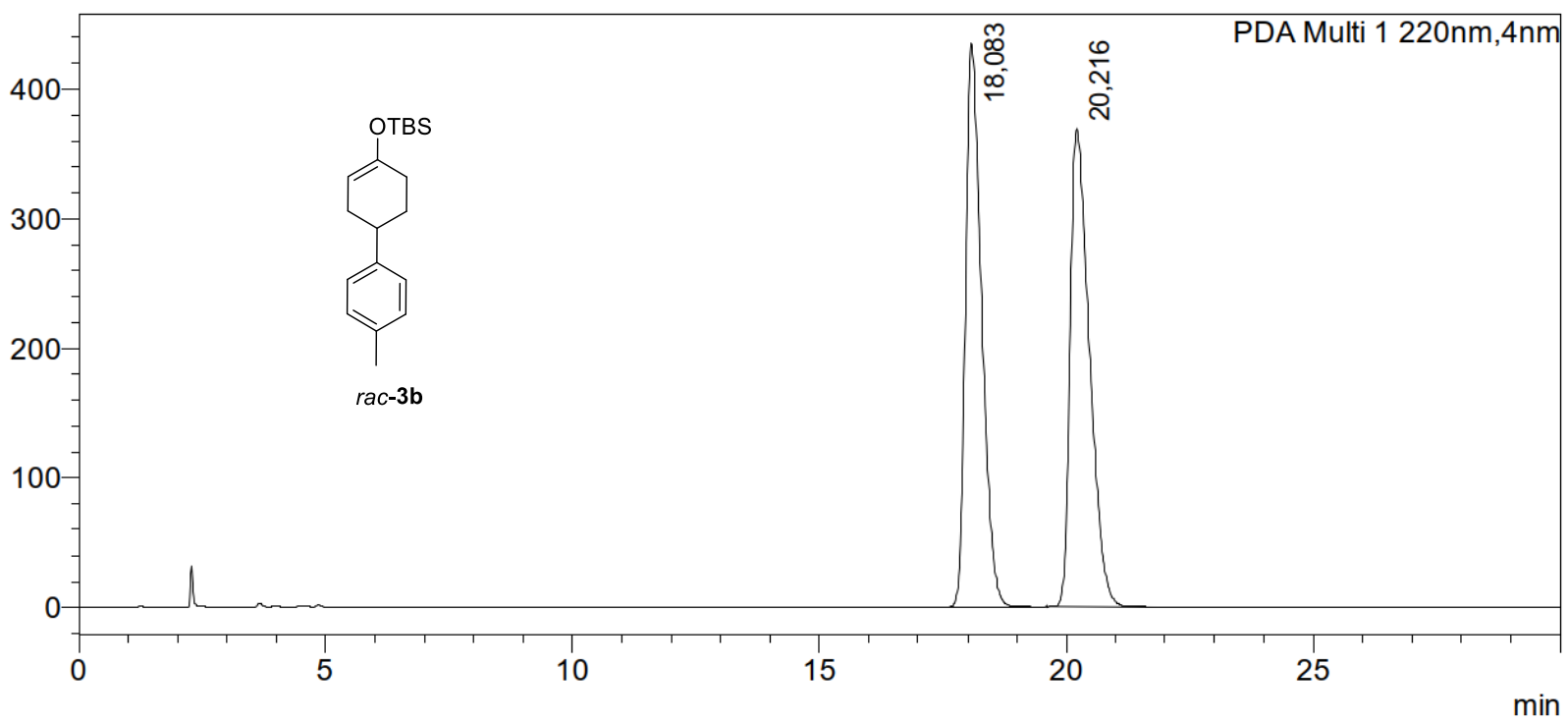

\begin{tabular}{|c|c|c|}
\hline Peak \# & $\mathrm{t}_{\mathrm{R}} / \mathrm{min}$ & \% peak area \\
\hline 1 & 18.0 & 49.91 \\
\hline 2 & 20.2 & 50.09 \\
\hline \multicolumn{2}{|c|}{ Total } & 100 \\
\hline
\end{tabular}

mAU

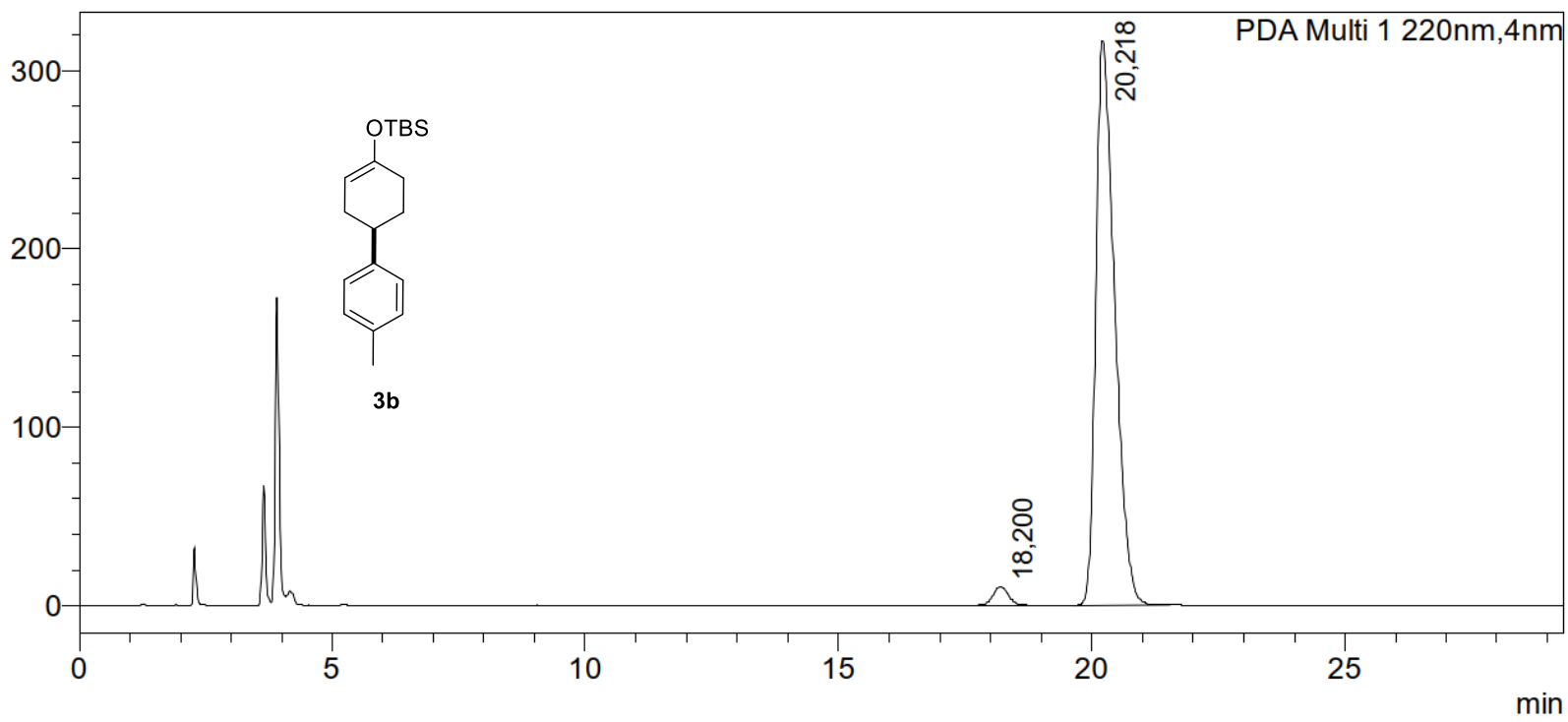

\begin{tabular}{|c|c|c|}
\hline Peak \# & $\mathrm{t}_{\mathrm{R}} / \mathrm{min}$ & \% peak area \\
\hline 1 & 18.2 & 2.62 \\
\hline 2 & 20.2 & 97.38 \\
\hline \multicolumn{2}{|c|}{ Total } & 100 \\
\hline
\end{tabular}


HPLC column: AD-3R, Acetonitrile: Water = 70:30, $1.0 \mathrm{~mL} / \mathrm{min}, 298 \mathrm{~K}, 220 \mathrm{~nm}$.

mAU

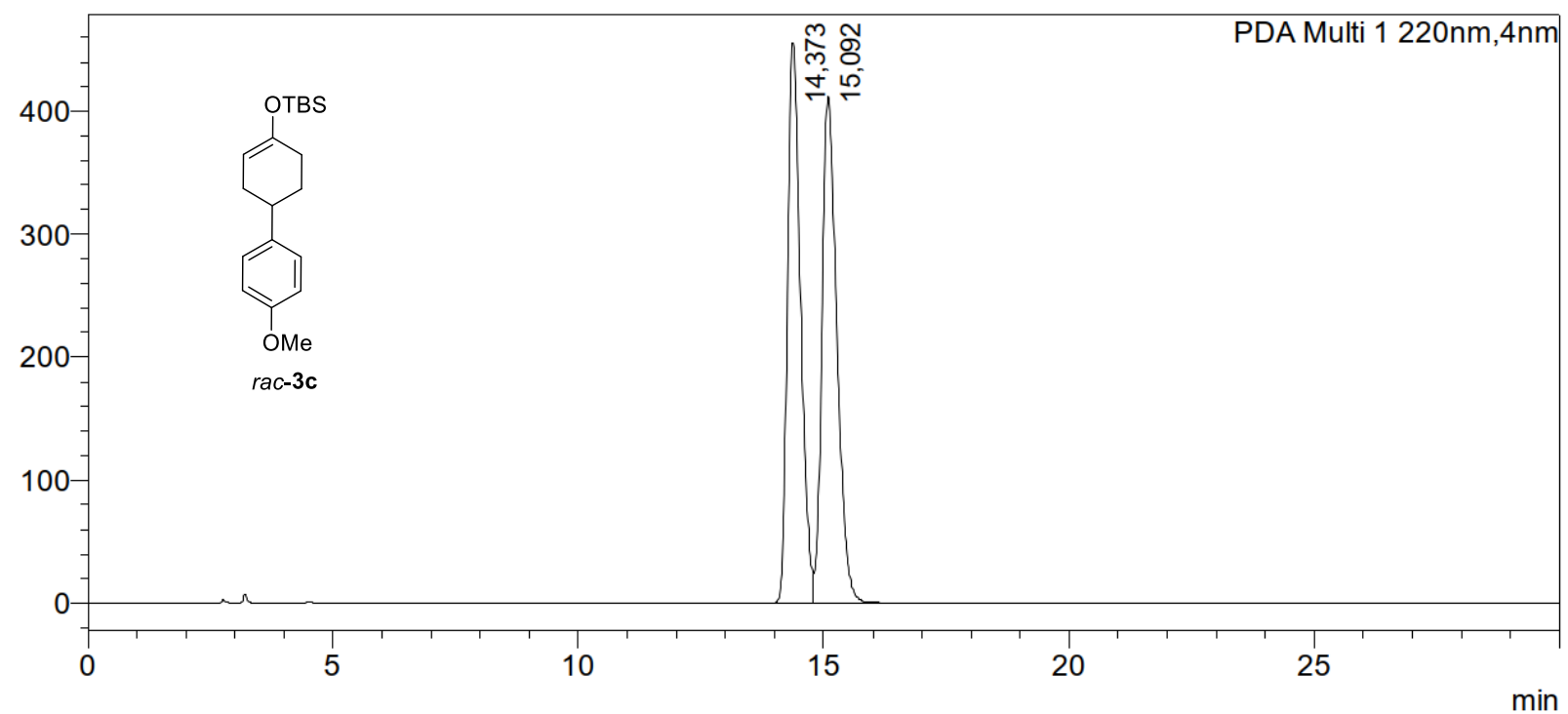

\begin{tabular}{|c|c|c|}
\hline Peak \# & $\mathrm{t}_{\mathrm{R}} / \mathrm{min}$ & $\%$ peak area \\
\hline 1 & 14.4 & 49.46 \\
\hline 2 & 15.1 & 50.54 \\
\hline \multicolumn{2}{|c|}{ Total } & 100 \\
\hline
\end{tabular}

mAU

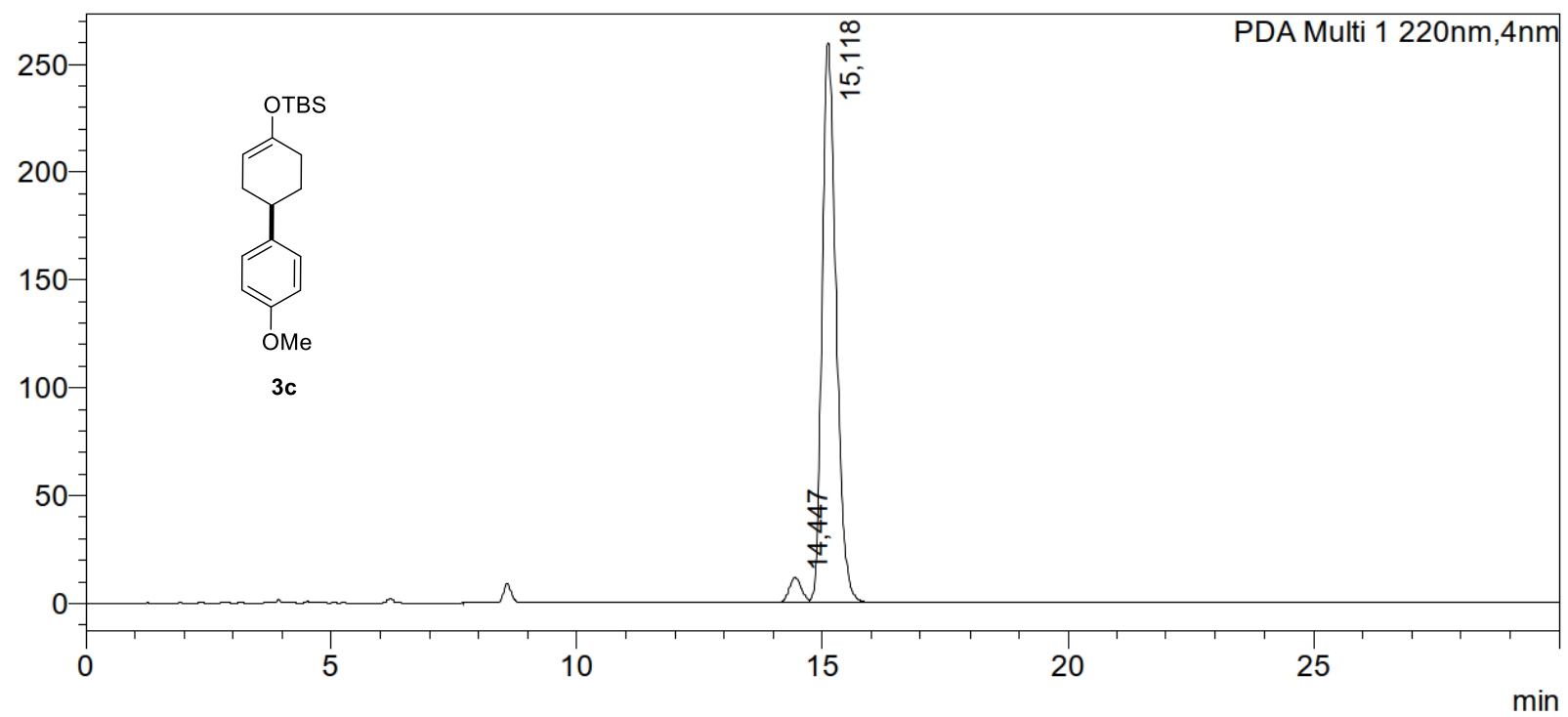

\begin{tabular}{|c|c|c|}
\hline Peak \# & $\mathrm{t}_{\mathrm{R}} / \mathrm{min}$ & $\%$ peak area \\
\hline 1 & 14.4 & 3.87 \\
\hline 2 & 15.1 & 96.13 \\
\hline \multicolumn{2}{|c|}{ Total } & 100 \\
\hline
\end{tabular}


HPLC column: OJ-3R, Acetonitrile: Water $=65: 35,1.0 \mathrm{~mL} / \mathrm{min}, 298 \mathrm{~K}, 220 \mathrm{~nm}$. mAU

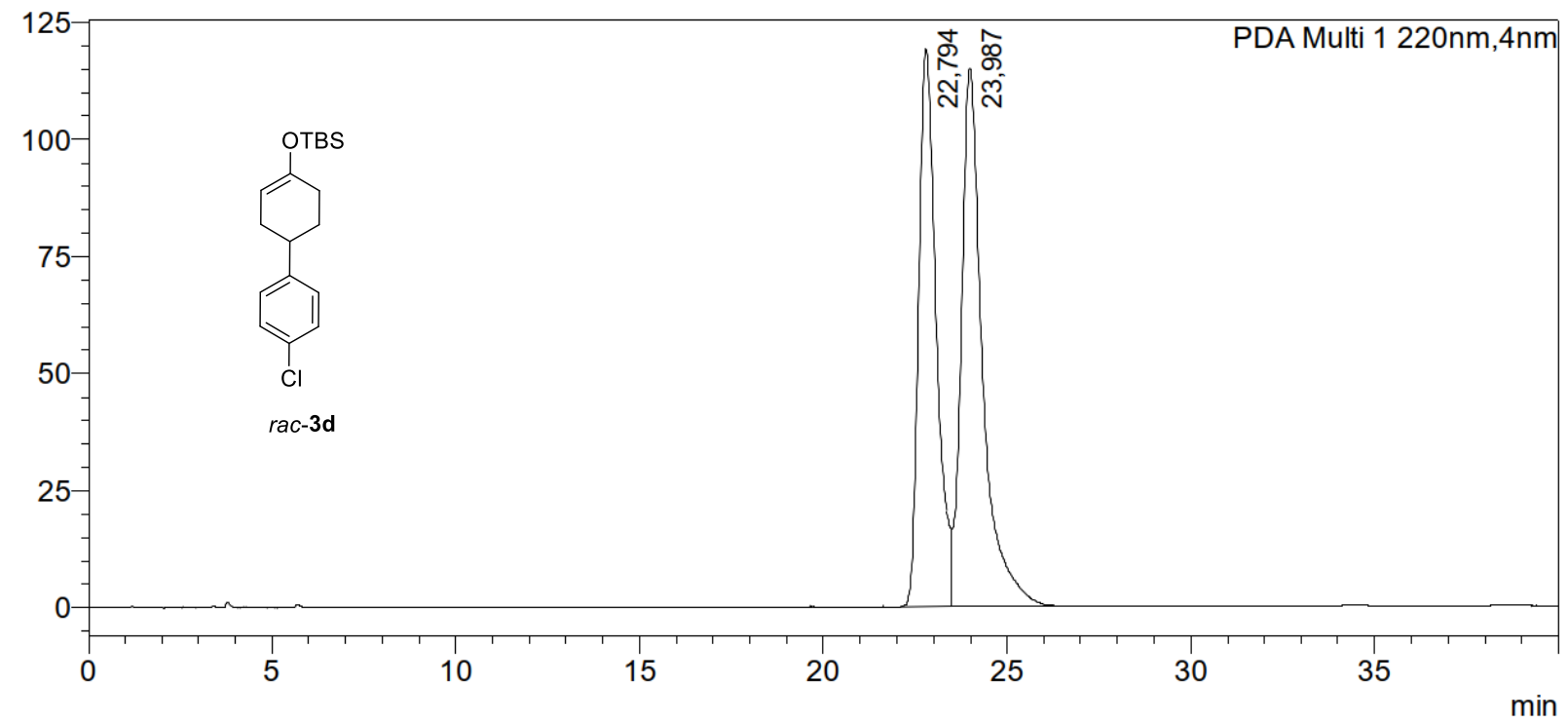

\begin{tabular}{|c|c|c|}
\hline Peak \# & $\mathrm{t}_{\mathrm{R}} / \mathrm{min}$ & \% peak area \\
\hline 1 & 22.7 & 45.91 \\
\hline 2 & 24.0 & 54.09 \\
\hline \multicolumn{2}{|c|}{ Total } & 100 \\
\hline
\end{tabular}

mAU

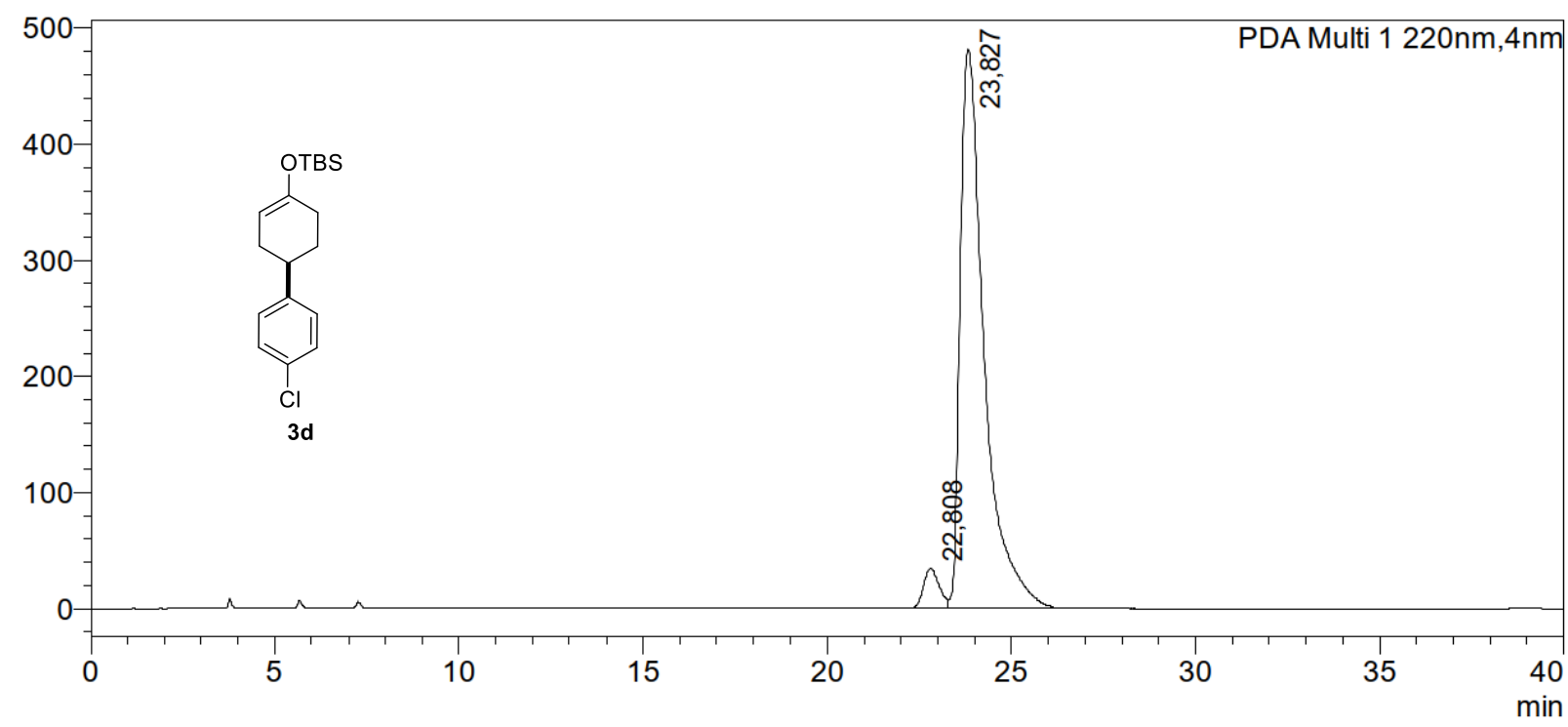

\begin{tabular}{|c|c|c|}
\hline Peak \# & $\mathrm{t}_{\mathrm{R}} / \mathrm{min}$ & \% peak area \\
\hline 1 & 22.8 & 4.55 \\
\hline 2 & 23.8 & 95.45 \\
\hline \multicolumn{2}{|c|}{ Total } & 100 \\
\hline
\end{tabular}


HPLC column: OJ-3R, Acetonitrile: Water =60:40, $1.0 \mathrm{~mL} / \mathrm{min}, 298 \mathrm{~K}, 220 \mathrm{~nm}$.

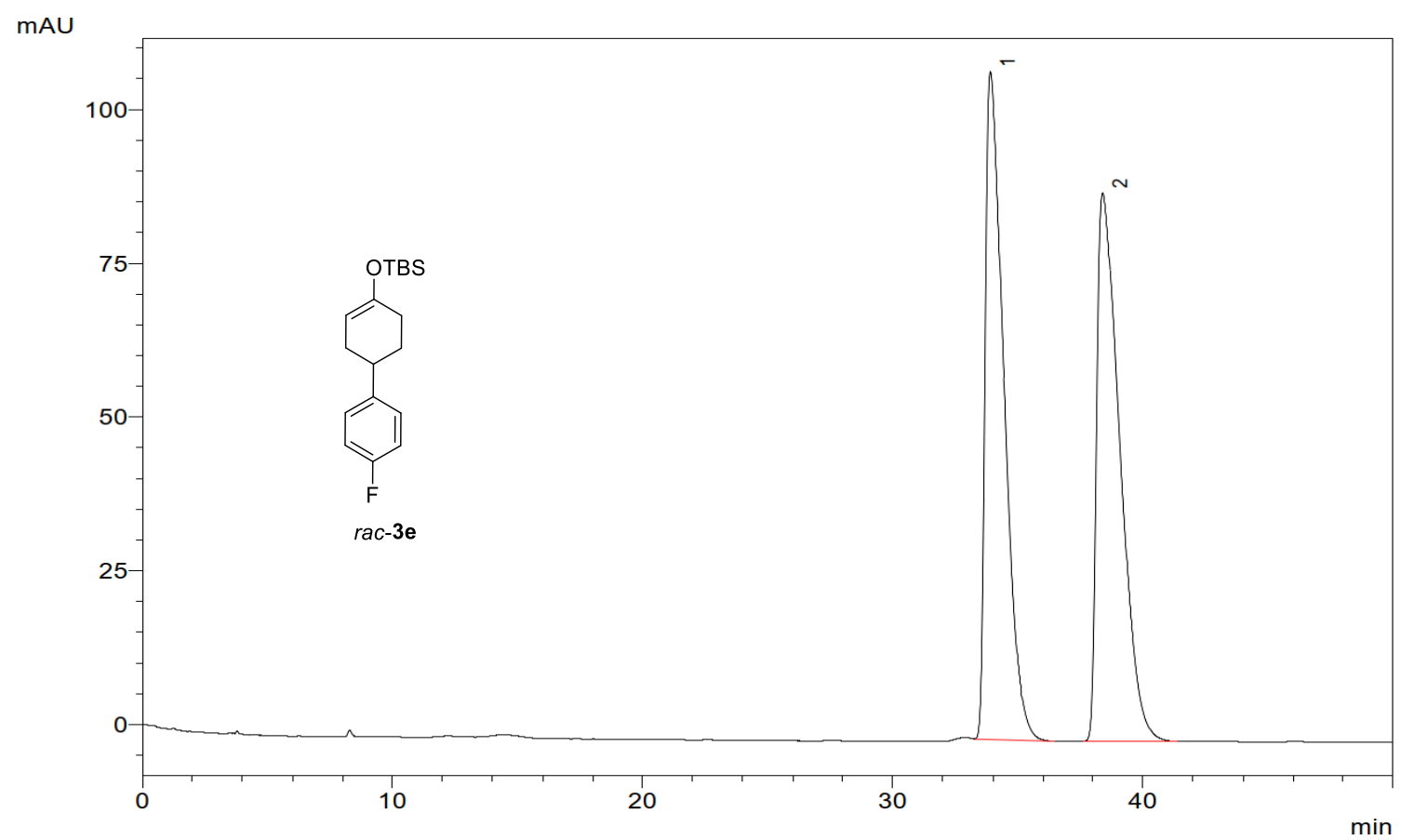

\begin{tabular}{|c|c|c|}
\hline Peak \# & $\mathrm{t}_{\mathrm{R}} / \mathrm{min}$ & $\%$ peak area \\
\hline 1 & 33.9 & 49.80 \\
\hline 2 & 38.4 & 50.20 \\
\hline \multicolumn{2}{|c|}{ Total } & 100 \\
\hline
\end{tabular}

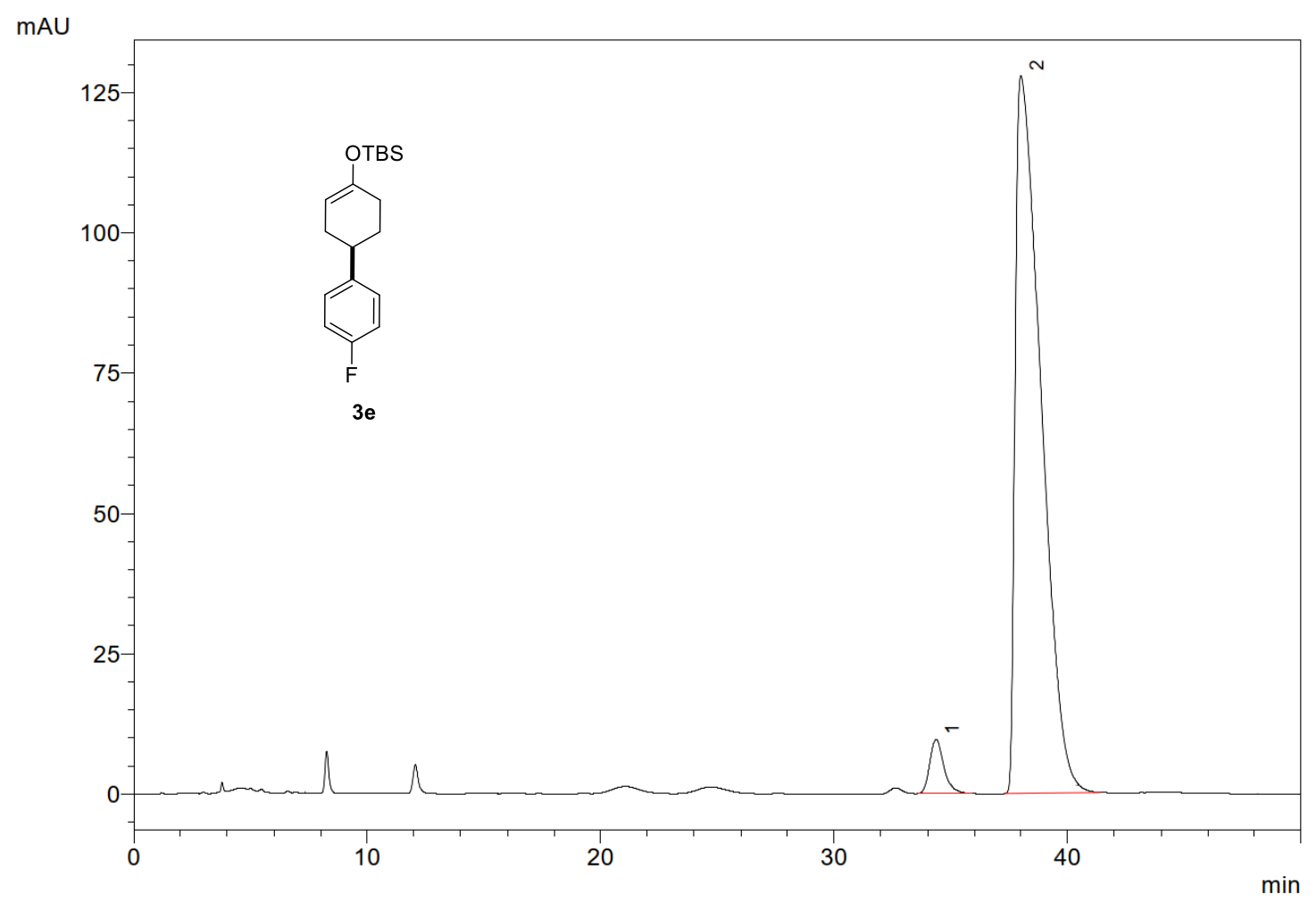

\begin{tabular}{|c|c|c|}
\hline Peak \# & $\mathrm{t}_{\mathrm{R}} / \mathrm{min}$ & $\%$ peak area \\
\hline 1 & 34.3 & 3.96 \\
\hline 2 & 38.0 & 96.04 \\
\hline
\end{tabular}




\begin{tabular}{|c|c|}
\hline Total & 100 \\
\hline
\end{tabular}

HPLC column: AD-3R, Acetonitrile: Water =60:40, $1.0 \mathrm{~mL} / \mathrm{min}, 298 \mathrm{~K}, 237 \mathrm{~nm}$.

mAU

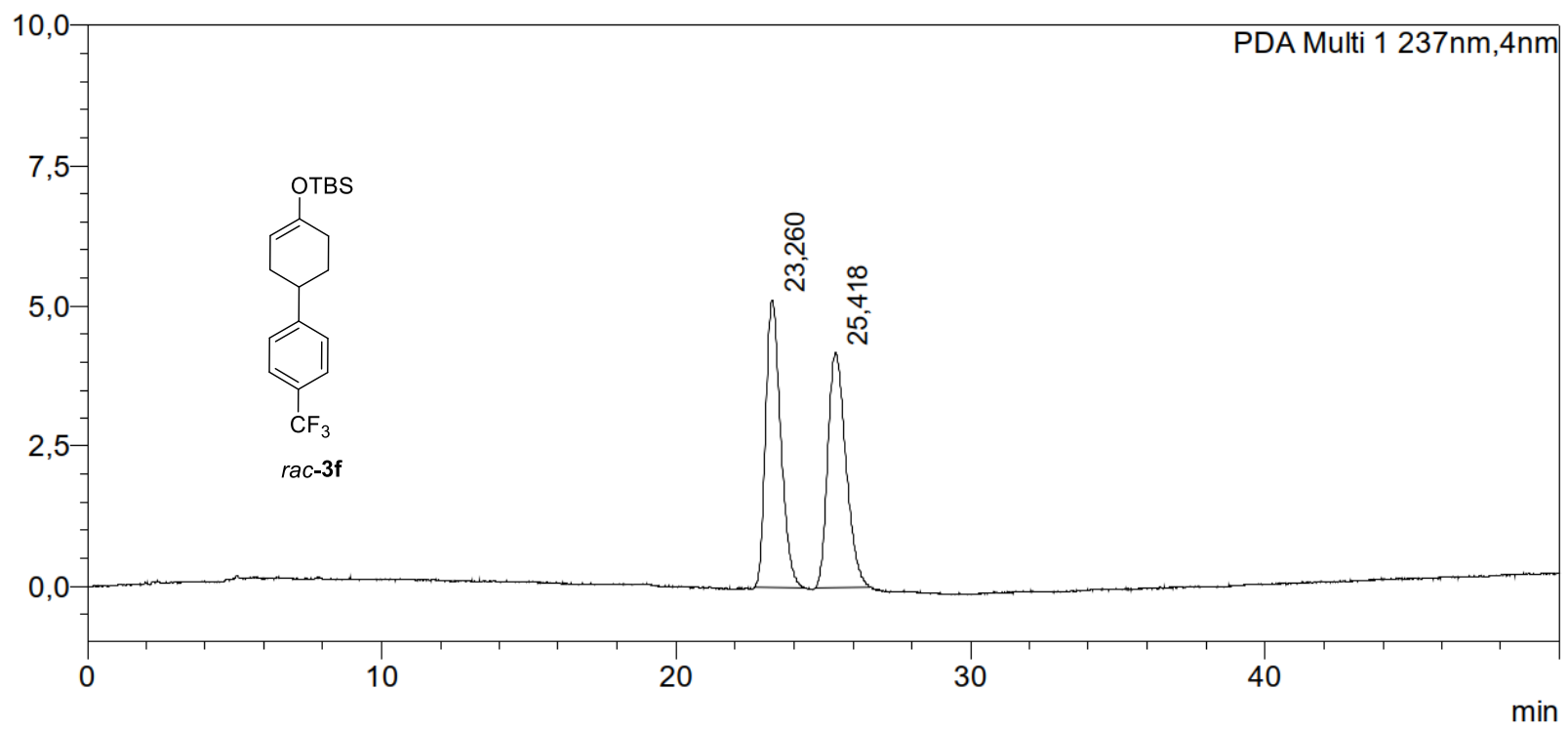

\begin{tabular}{|c|c|c|}
\hline Peak \# & $\mathrm{t}_{\mathrm{R}} / \mathrm{min}$ & $\%$ peak area \\
\hline 1 & 23.3 & 50.44 \\
\hline 2 & 25.4 & 49.56 \\
\hline \multicolumn{2}{|c|}{ Total } & 100 \\
\hline
\end{tabular}

mAU

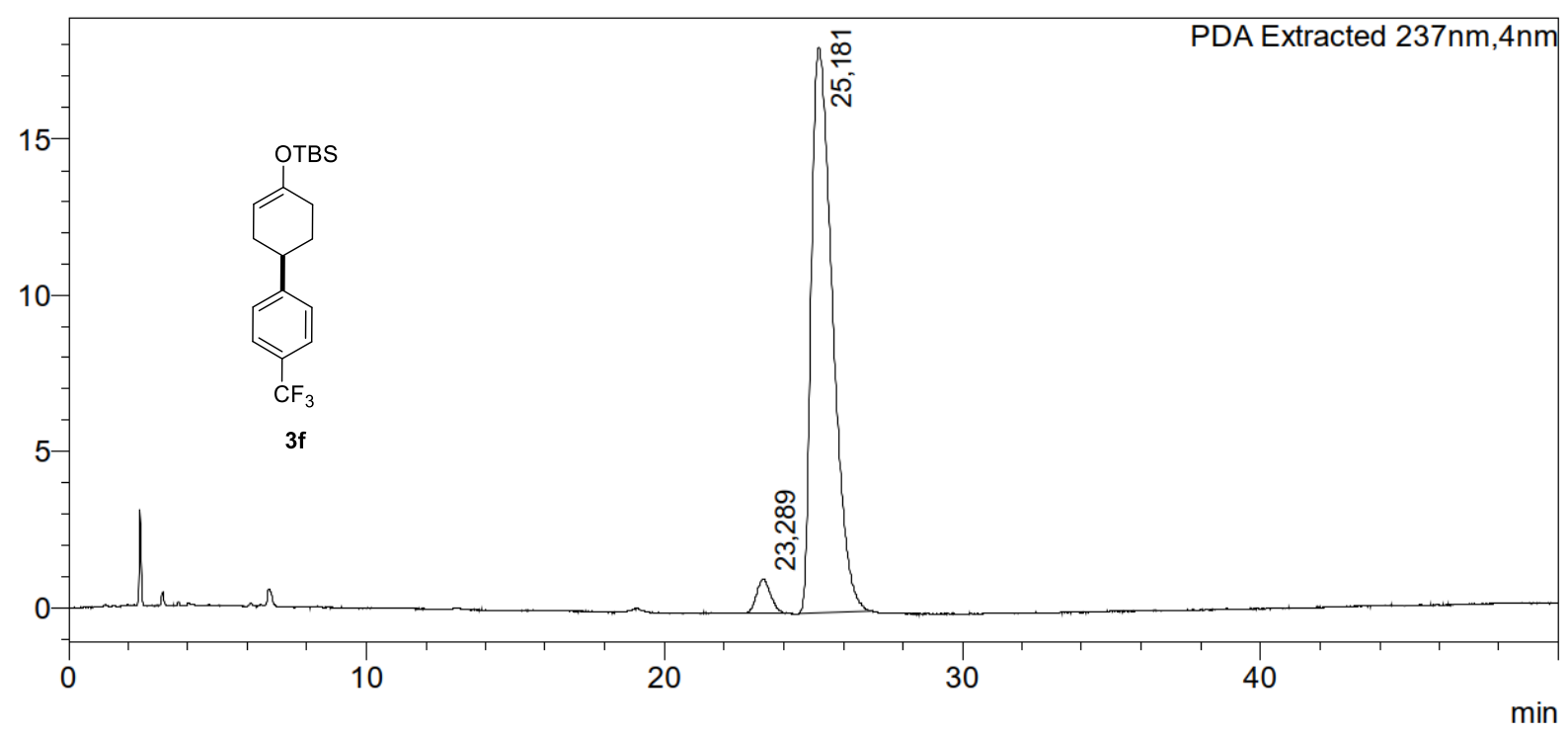

\begin{tabular}{|c|c|c|}
\hline Peak \# & $\mathrm{t}_{\mathrm{R}} / \mathrm{min}$ & $\%$ peak area \\
\hline 1 & 23.3 & 5.76 \\
\hline 2 & 25.2 & 94.24 \\
\hline \multicolumn{2}{|c|}{ Total } & 100 \\
\hline
\end{tabular}


HPLC column: OD-3R, Acetonitrile: Water $=65: 35,1.0 \mathrm{~mL} / \mathrm{min}, 298 \mathrm{~K}, 220 \mathrm{~nm}$

mAU

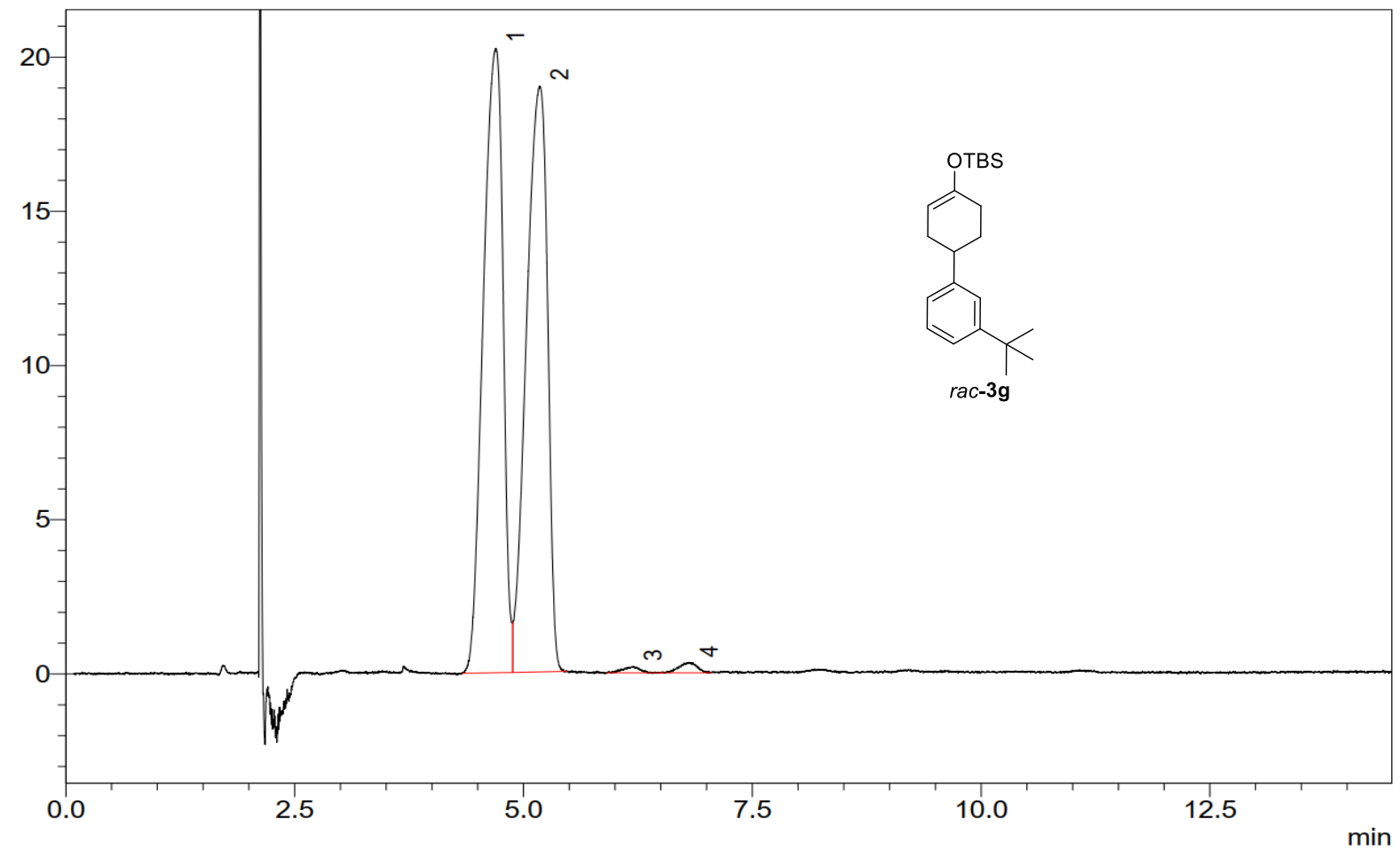

\begin{tabular}{|c|c|c|}
\hline Peak \# & $t_{R} / \min$ & $\%$ peak area \\
\hline 1 & 4.7 & 49.85 \\
\hline 2 & 5.2 & 50.15 \\
\hline \multicolumn{2}{|c|}{ Total } & 100 \\
\hline
\end{tabular}

mAU

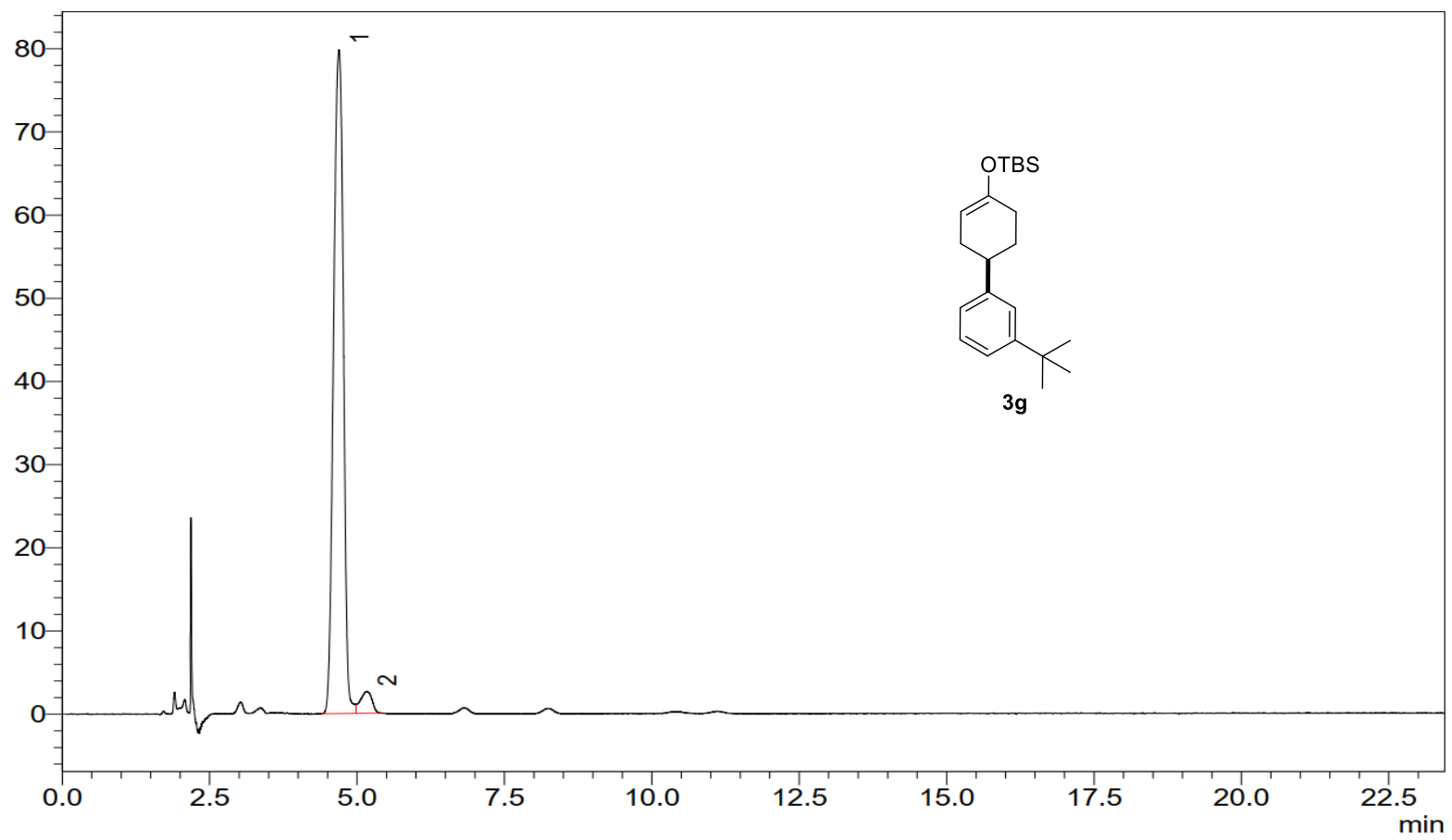

\begin{tabular}{|c|c|c|}
\hline Peak \# & $\mathrm{t}_{\mathrm{R}} / \mathrm{min}$ & \% peak area \\
\hline 1 & 4.5 & 96.24 \\
\hline 2 & 5.2 & 3.76 \\
\hline \multicolumn{3}{|c|}{ Total } \\
\hline
\end{tabular}


HPLC column: AD-3R, Acetonitrile: Water =60:40, $1.0 \mathrm{~mL} / \mathrm{min}, 298 \mathrm{~K}, 220 \mathrm{~nm}$.

mAU

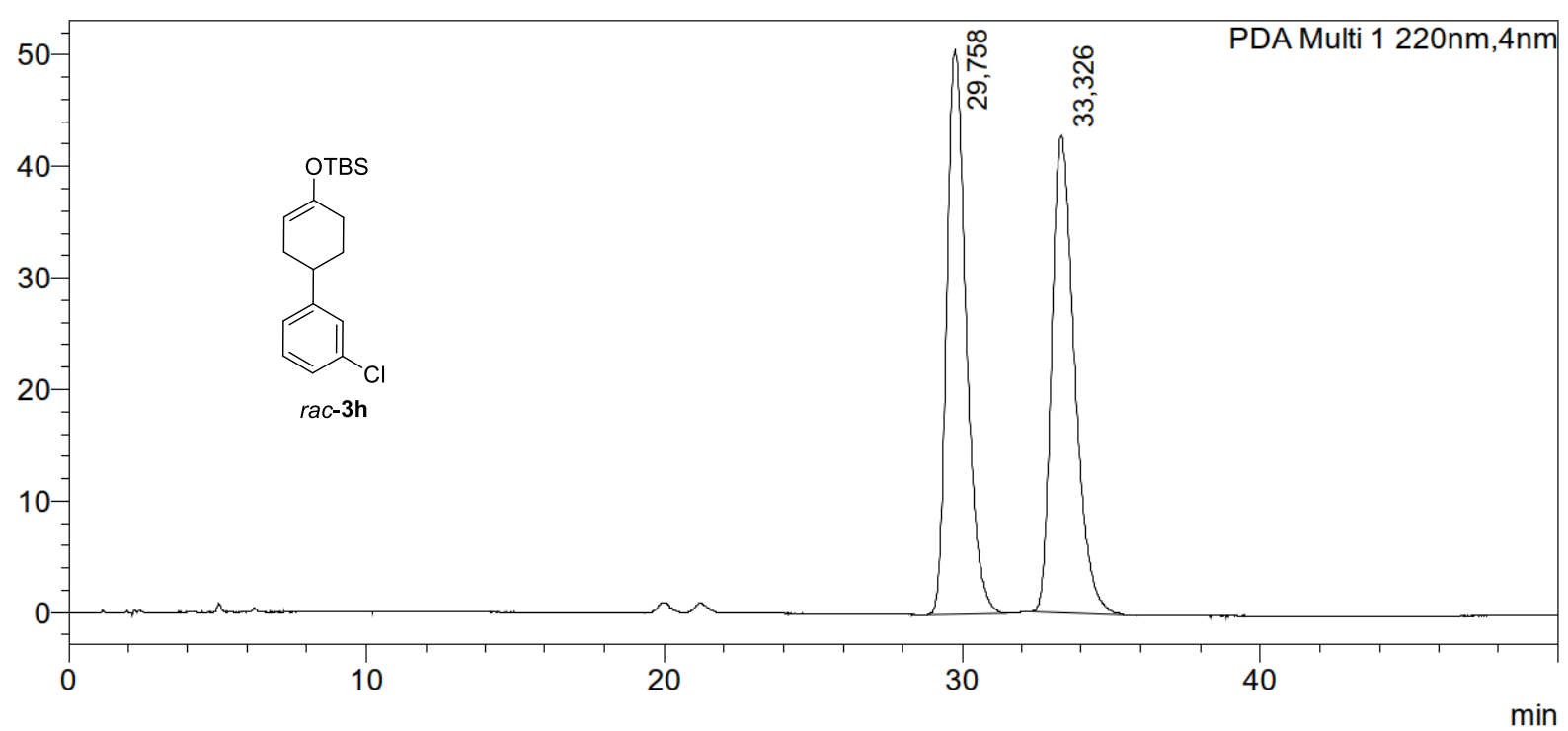

\begin{tabular}{|c|c|c|}
\hline Peak \# & $\mathrm{t}_{\mathrm{R}} / \mathrm{min}$ & $\%$ peak area \\
\hline 1 & 29.8 & 50.11 \\
\hline 2 & 33.3 & 49.89 \\
\hline \multicolumn{2}{|c|}{ Total } & 100 \\
\hline
\end{tabular}

mAU

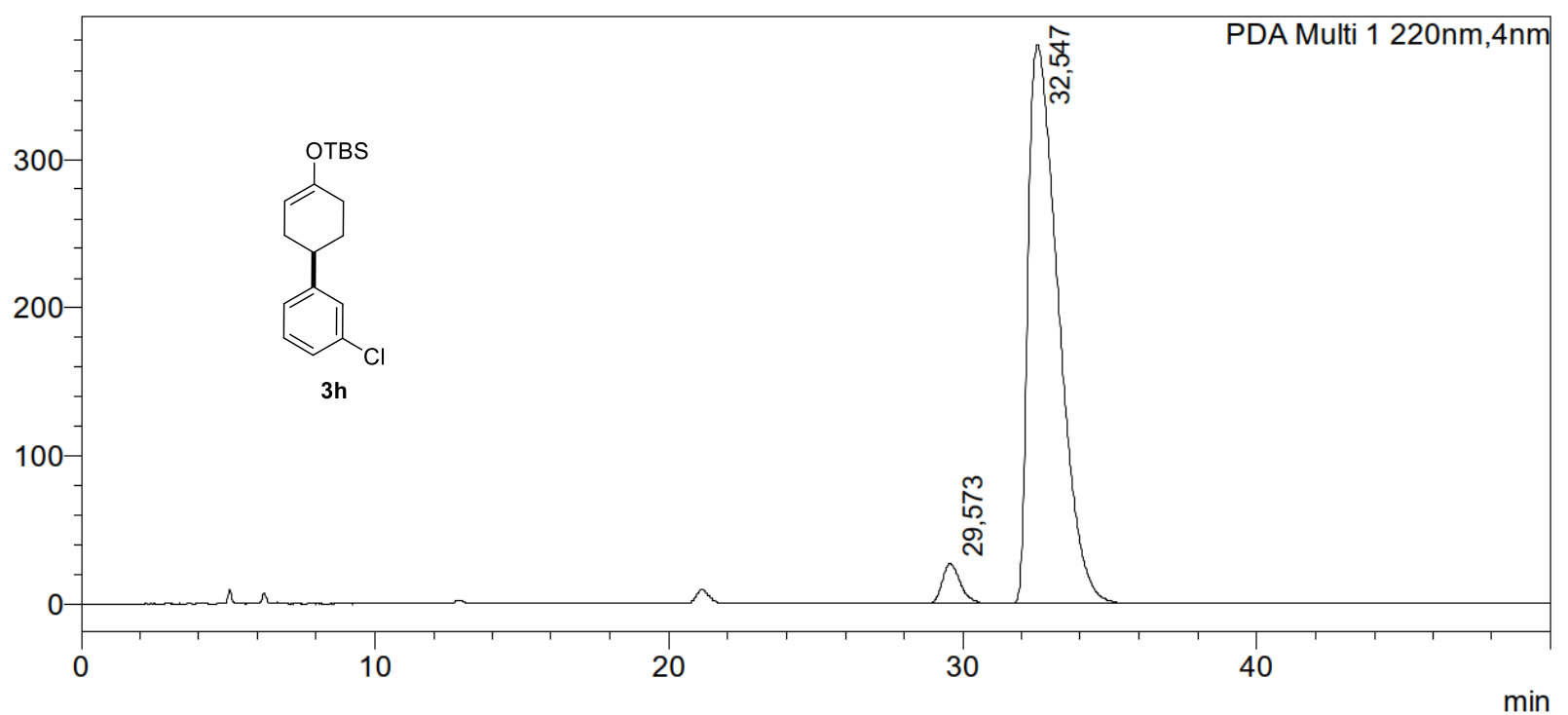

\begin{tabular}{|c|c|c|}
\hline Peak \# & $t_{R} / \min$ & $\%$ peak area \\
\hline 1 & 29.6 & 4.10 \\
\hline 2 & 32.5 & 95.90 \\
\hline \multicolumn{2}{|c|}{ Total } & 100 \\
\hline
\end{tabular}


HPLC column: OJ-3R, Acetonitrile: Water $=60: 40,1.0 \mathrm{~mL} / \mathrm{min}, 298 \mathrm{~K}, 244 \mathrm{~nm}$.

mAU

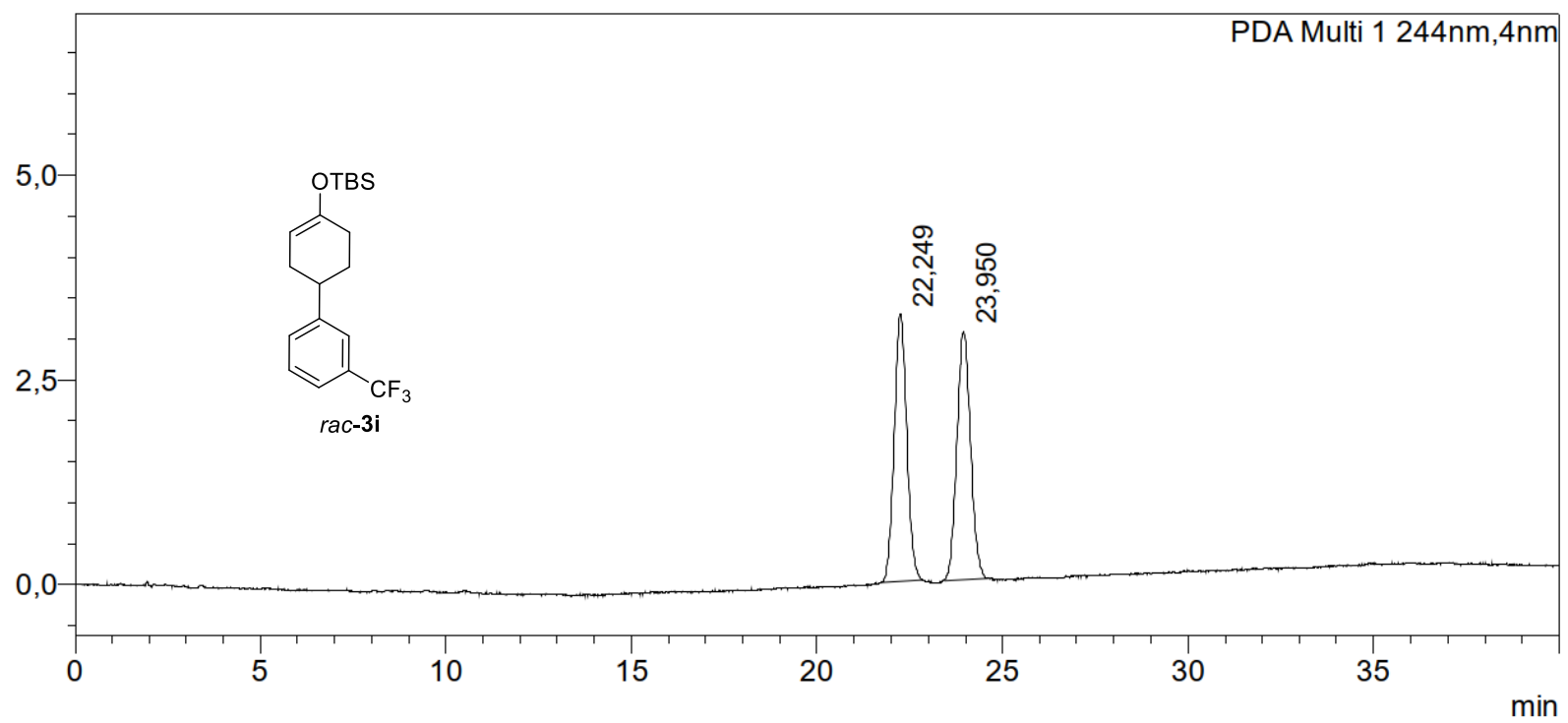

\begin{tabular}{|c|c|c|}
\hline Peak \# & $\mathrm{t}_{\mathrm{R}} / \mathrm{min}$ & \% peak area \\
\hline 1 & 22.2 & 49.92 \\
\hline 2 & 24.0 & 50.08 \\
\hline \multicolumn{2}{|c|}{ Total } & 100 \\
\hline
\end{tabular}

mAU

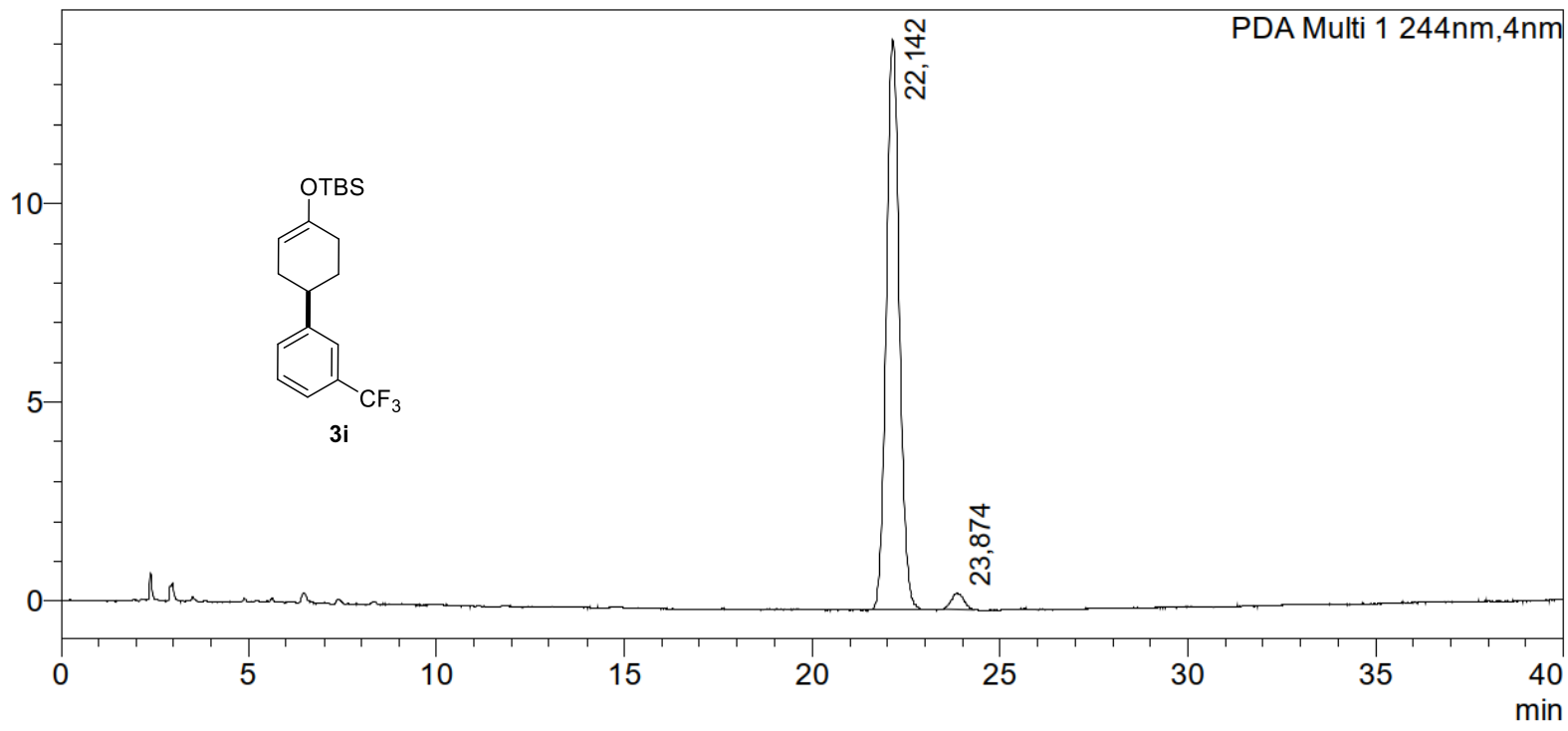

\begin{tabular}{|c|c|c|}
\hline Peak \# & $t_{R} / \min$ & $\%$ peak area \\
\hline 1 & 22.2 & 97.38 \\
\hline 2 & 23.9 & 2.62 \\
\hline \multicolumn{2}{|c|}{ Total } & 100 \\
\hline
\end{tabular}


HPLC column: OD-3R, Acetonitrile: Water $=65: 35,1.0 \mathrm{~mL} / \mathrm{min}, 298 \mathrm{~K}, 220 \mathrm{~nm}$

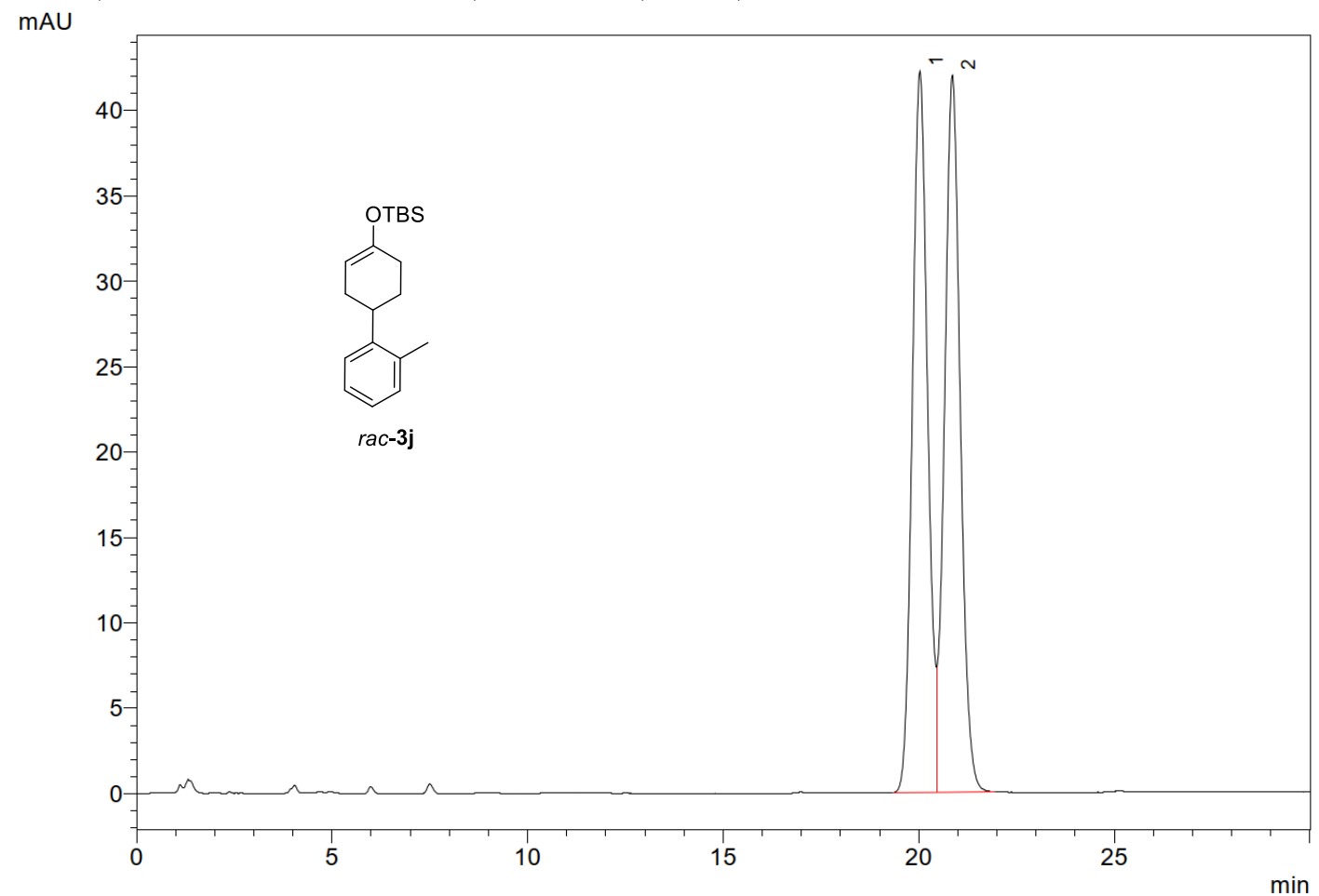

\begin{tabular}{|c|c|c|}
\hline Peak \# & $\mathrm{t}_{\mathrm{R}} / \mathrm{min}$ & $\%$ peak area \\
\hline 1 & 20.0 & 49.40 \\
\hline 2 & 20.9 & 50.60 \\
\hline \multicolumn{2}{|c|}{ Total } & 100 \\
\hline
\end{tabular}

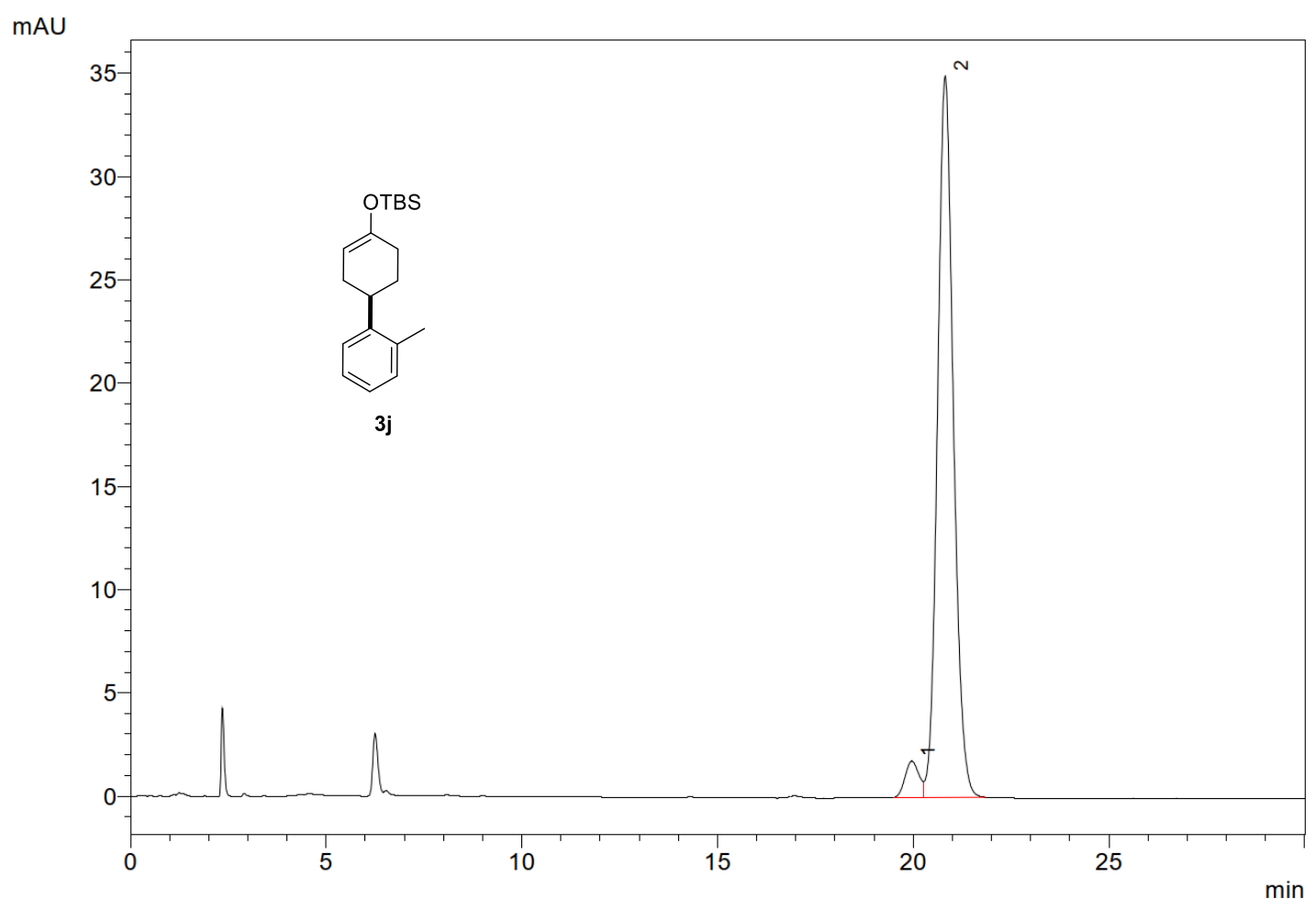

\begin{tabular}{|c|c|c|}
\hline Peak \# & $\mathrm{t}_{\mathrm{R}} / \mathrm{min}$ & $\%$ peak area \\
\hline 1 & 20.0 & 4.32 \\
\hline 2 & 20.8 & 95.68 \\
\hline \multicolumn{2}{|c|}{ Total } & 100 \\
\hline
\end{tabular}


HPLC column: OJ-3R, Acetonitrile: Water $=70: 30,1.0 \mathrm{~mL} / \mathrm{min}, 298 \mathrm{~K}, 220 \mathrm{~nm}$

mAU

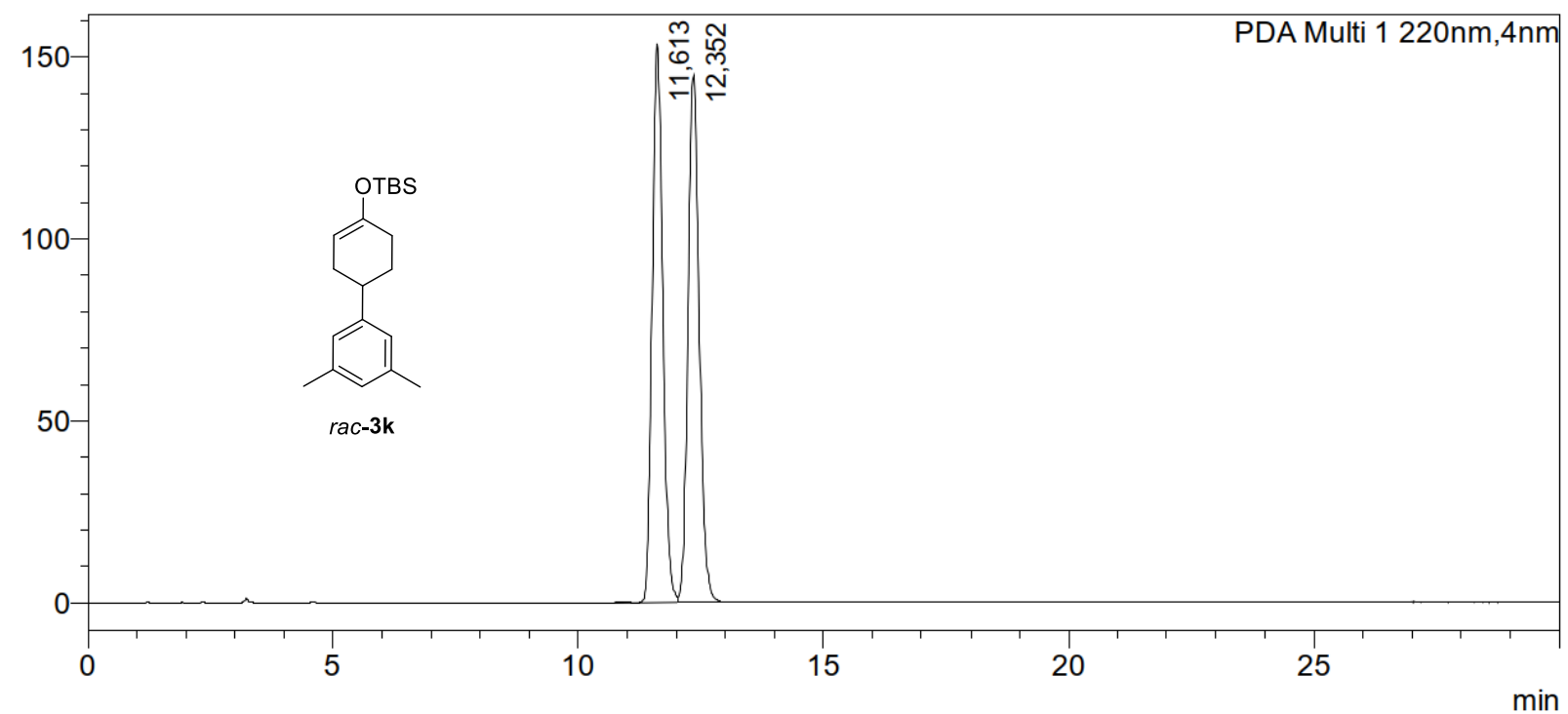

\begin{tabular}{|c|c|c|}
\hline Peak \# & $\mathrm{t}_{\mathrm{R}} / \mathrm{min}$ & \% peak area \\
\hline 1 & 11.6 & 51.49 \\
\hline 2 & 12.4 & 48.51 \\
\hline \multicolumn{2}{|c|}{ Total } & 100 \\
\hline
\end{tabular}

$\mathrm{mAU}$

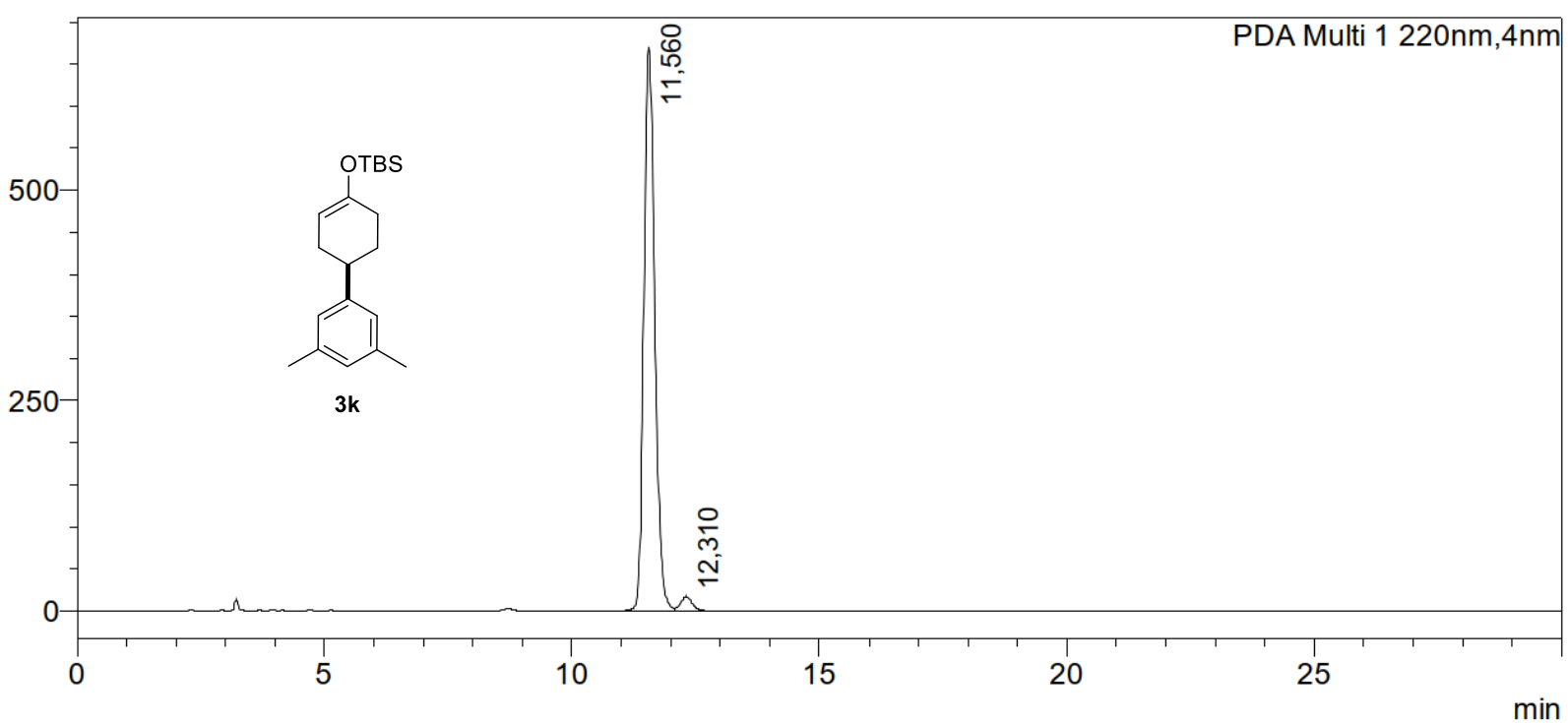

\begin{tabular}{|c|c|c|}
\hline Peak \# & $\mathrm{t}_{\mathrm{R}} / \mathrm{min}$ & \% peak area \\
\hline 1 & 11.6 & 97.53 \\
\hline 2 & 12.3 & 2.47 \\
\hline \multicolumn{2}{|c|}{ Total } & 100 \\
\hline
\end{tabular}


HPLC column: OJ-3R, Acetonitrile: Water $=60: 40,1.0 \mathrm{~mL} / \mathrm{min}, 298 \mathrm{~K}, 220 \mathrm{~nm}$.

mAU

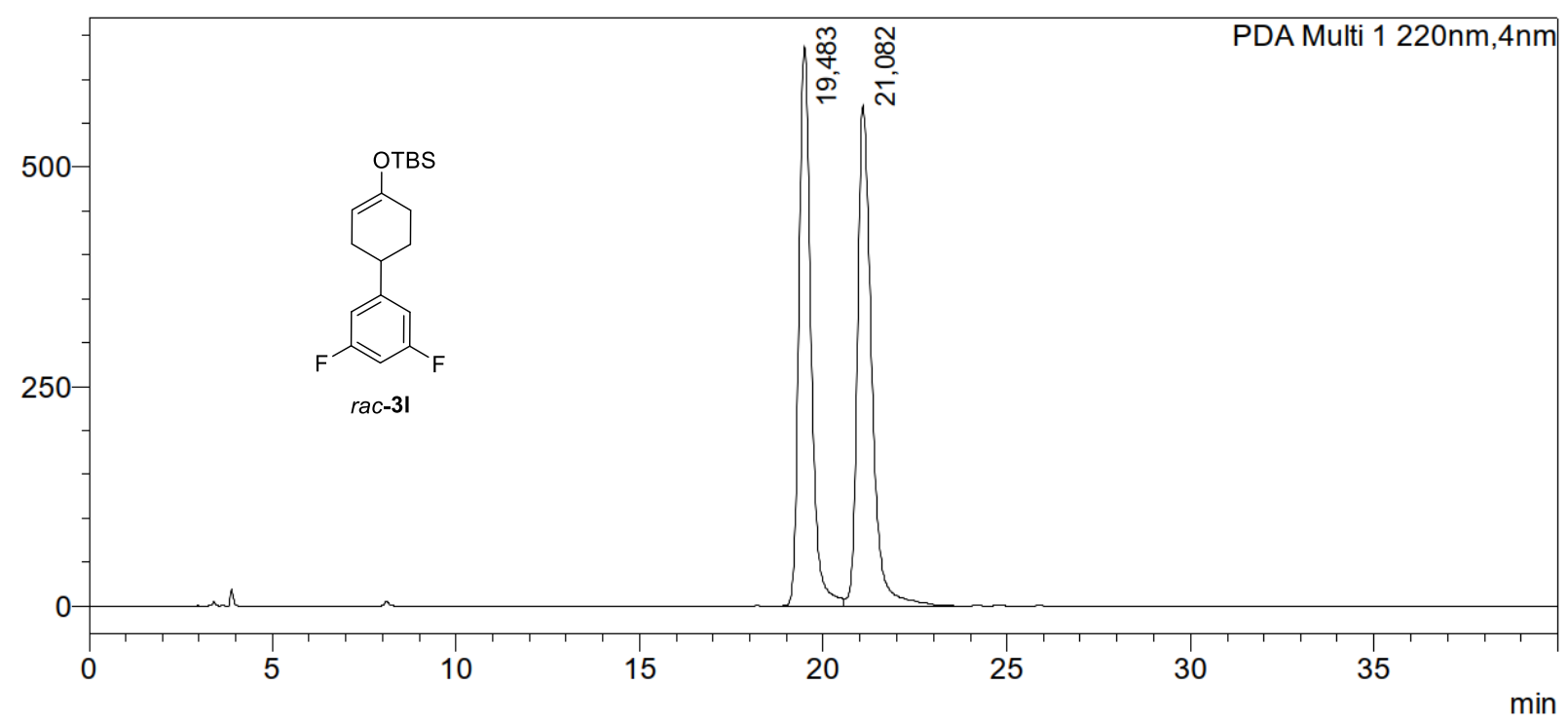

\begin{tabular}{|c|c|c|}
\hline Peak \# & $\mathrm{t}_{\mathrm{R}} / \mathrm{min}$ & \% peak area \\
\hline 1 & 19.5 & 52.80 \\
\hline 2 & 21.1 & 47.20 \\
\hline \multicolumn{2}{|c|}{ Total } & 100 \\
\hline
\end{tabular}

mAU

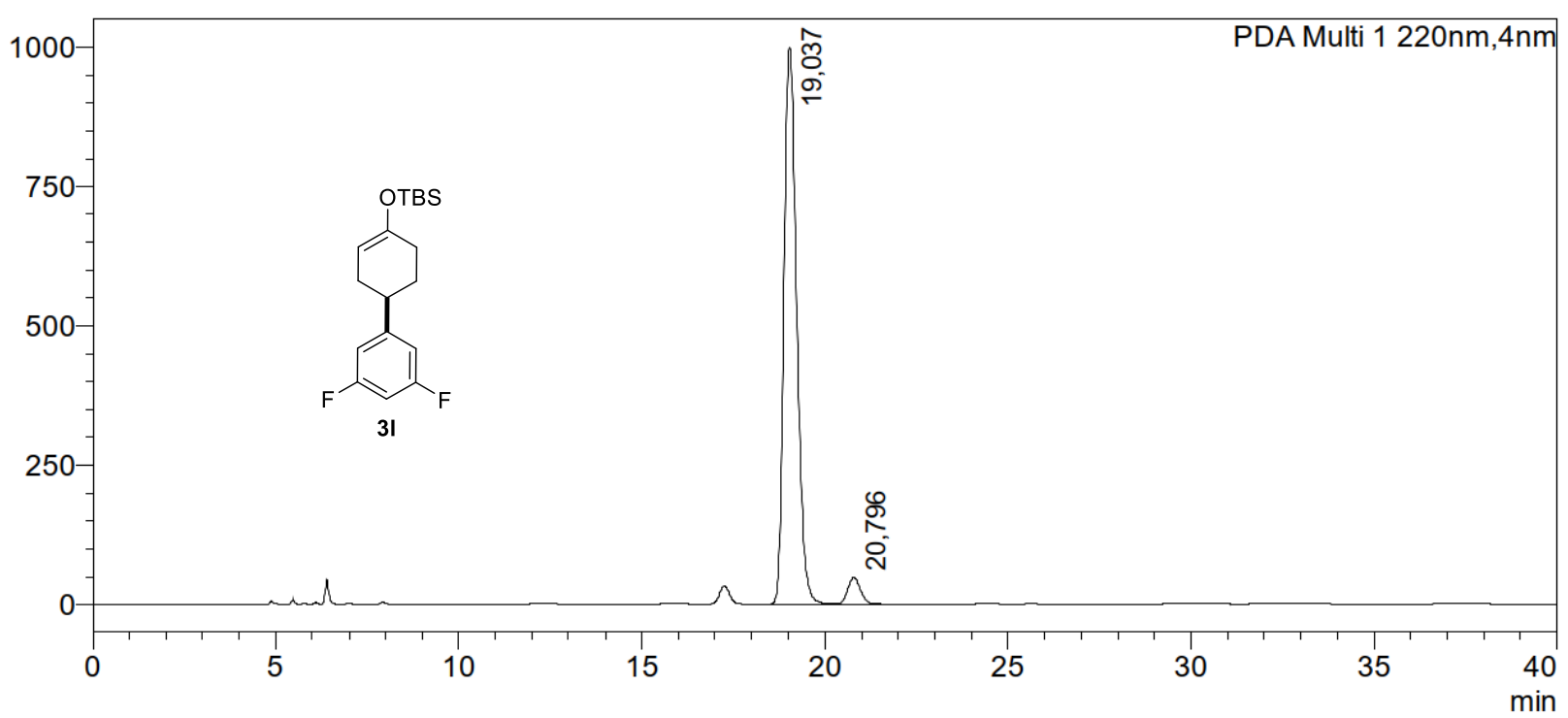

\begin{tabular}{|c|c|c|}
\hline Peak \# & $t_{\mathrm{R}} / \mathrm{min}$ & $\%$ peak area \\
\hline 1 & 19.0 & 95.42 \\
\hline 2 & 20.8 & 4.58 \\
\hline \multicolumn{2}{|c|}{ Total } & 100 \\
\hline
\end{tabular}


HPLC column: OD-3R, Acetonitrile: Water $=65: 35,1.0 \mathrm{~mL} / \mathrm{min}, 298 \mathrm{~K}, 220 \mathrm{~nm}$.

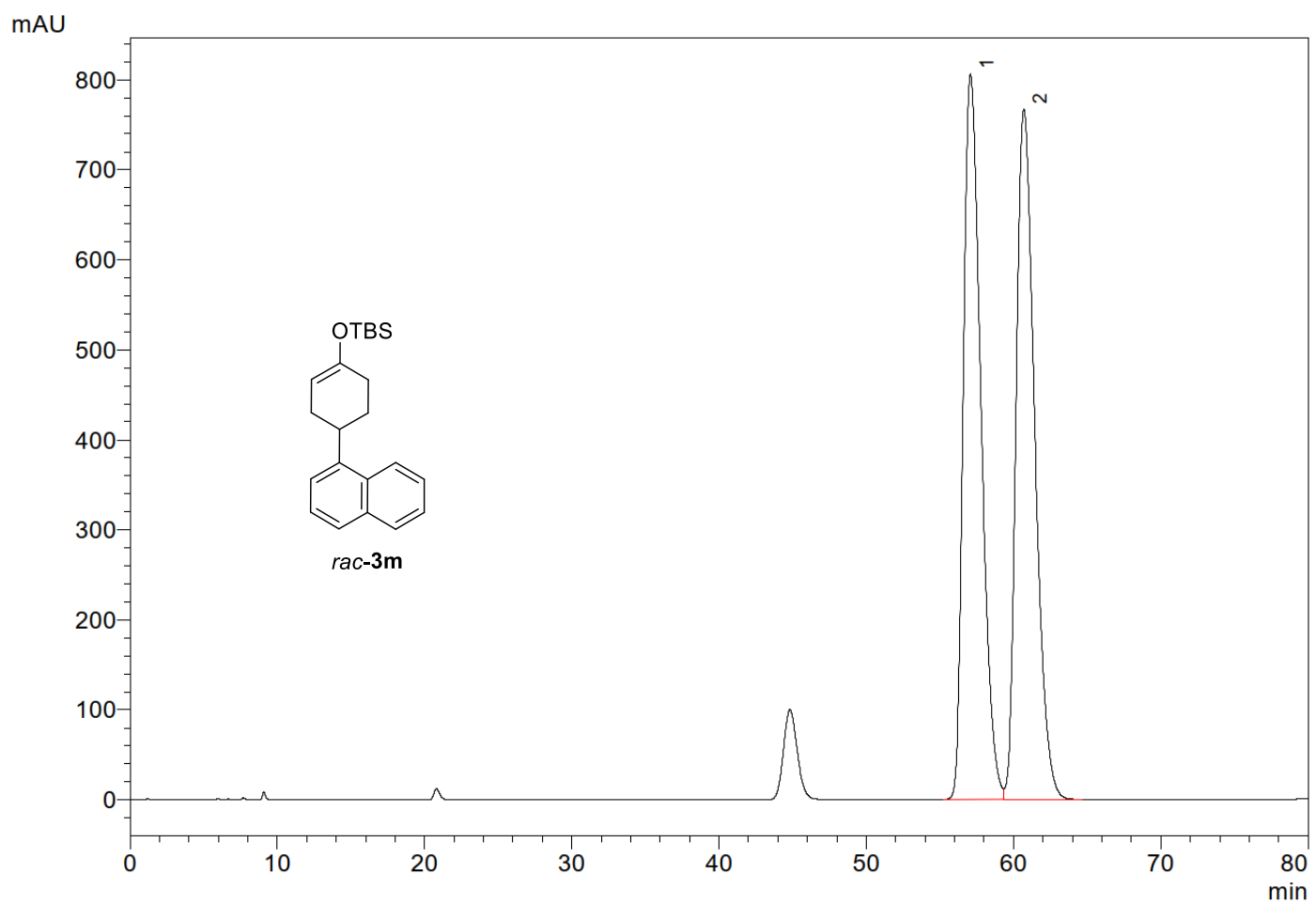

\begin{tabular}{|c|c|c|}
\hline Peak \# & $\mathrm{t}_{\mathrm{R}} / \mathrm{min}$ & $\%$ peak area \\
\hline 1 & 57.1 & 49.86 \\
\hline 2 & 60.7 & 50.14 \\
\hline \multicolumn{2}{|c|}{ Total } & 100 \\
\hline
\end{tabular}

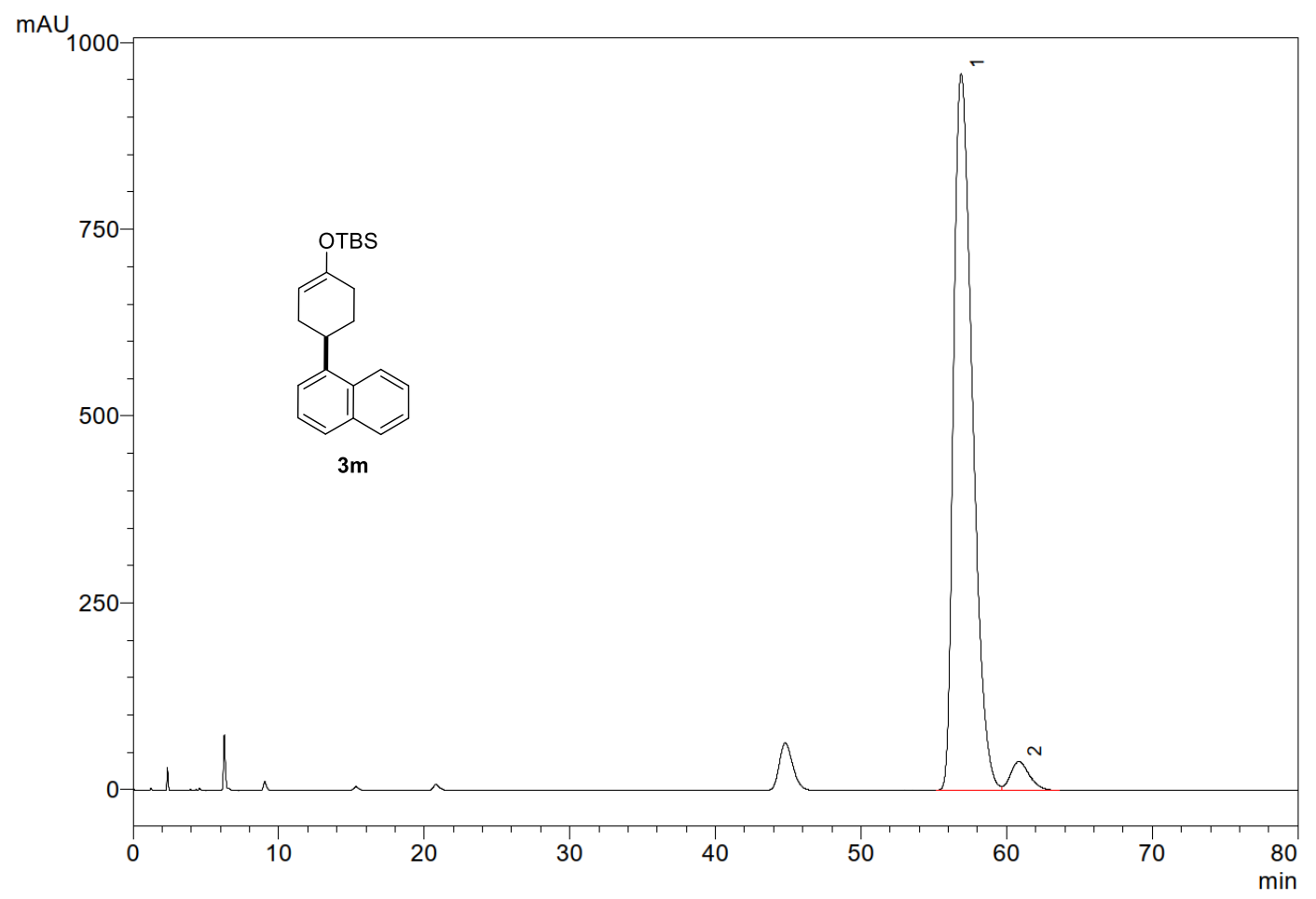

\begin{tabular}{|c|c|c|}
\hline Peak \# & $\mathrm{t}_{\mathrm{R}} / \mathrm{min}$ & \% peak area \\
\hline 1 & 56.9 & 96.16 \\
\hline 2 & 60.8 & 3.84 \\
\hline
\end{tabular}


HPLC column: OJ-3, CO $2 / 2-$ Propanol = 90:10, $1.0 \mathrm{~mL} / \mathrm{min}, 323 \mathrm{~K}, 220 \mathrm{~nm}$.

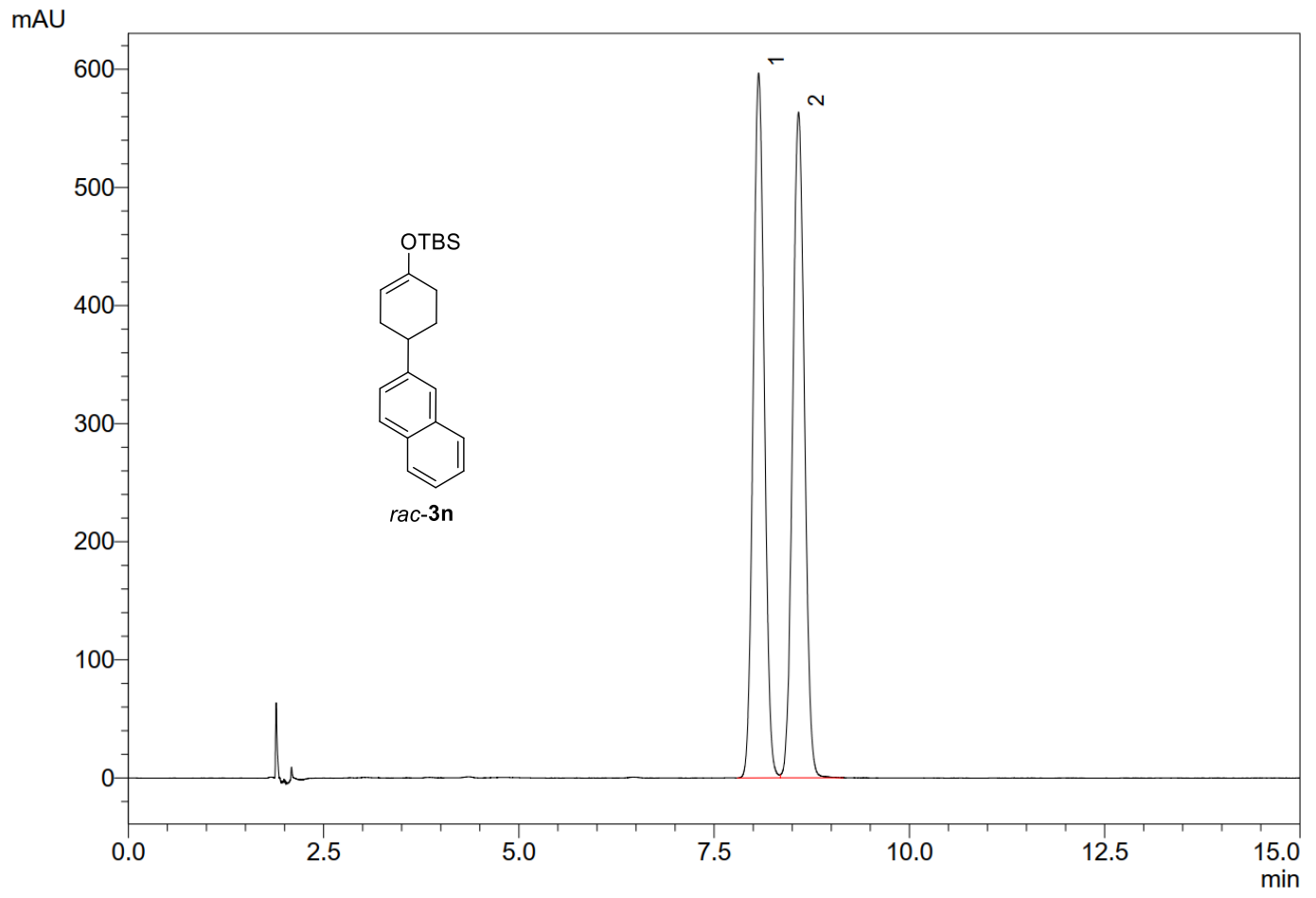

\begin{tabular}{|c|c|c|}
\hline Peak \# & $\mathrm{t}_{\mathrm{R}} / \mathrm{min}$ & $\%$ peak area \\
\hline 1 & 8.1 & 49.87 \\
\hline 2 & 8.6 & 50.13 \\
\hline \multicolumn{2}{|c|}{ Total } & 100 \\
\hline
\end{tabular}

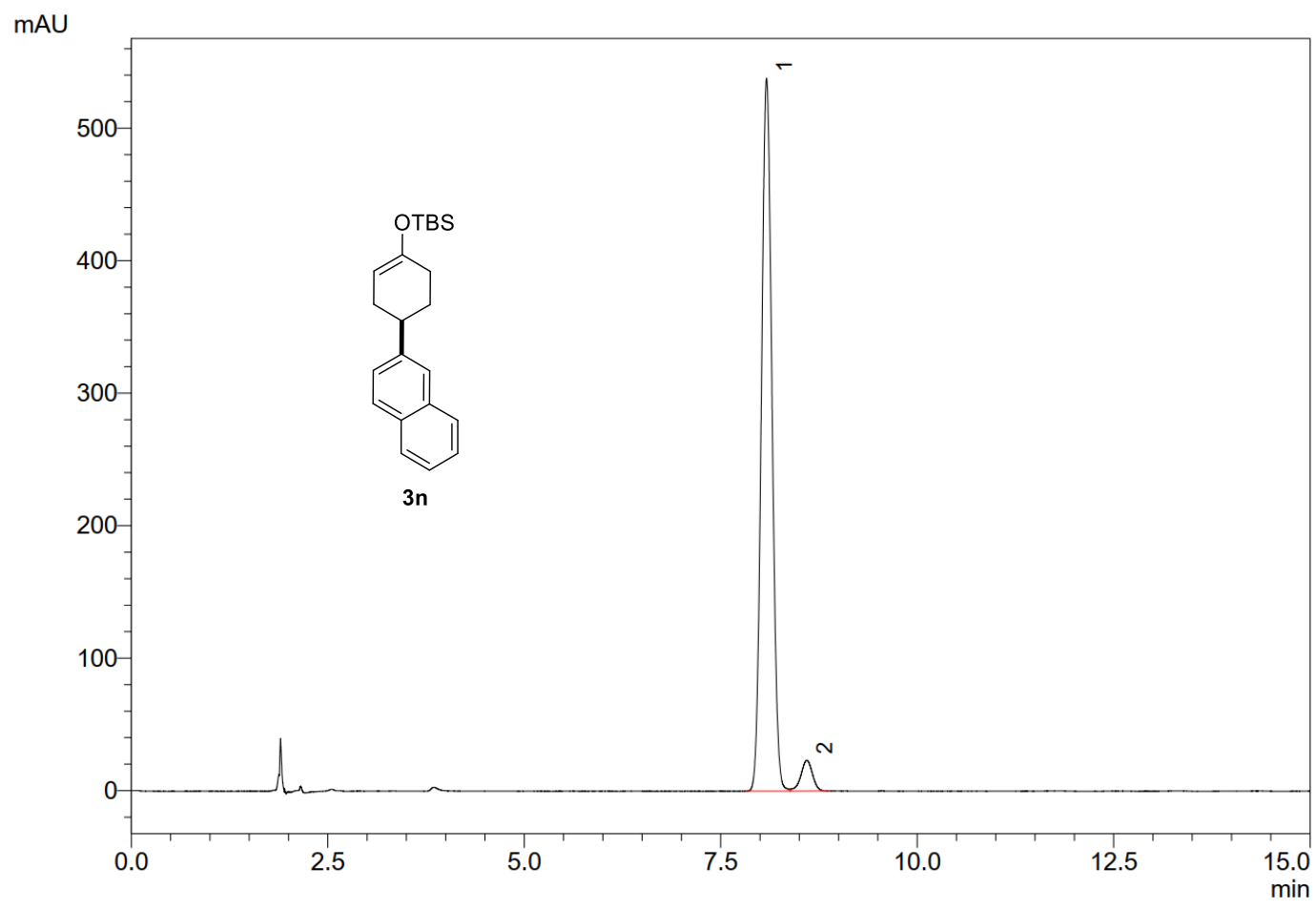

\begin{tabular}{|l|l|l|} 
Peak \# & $\mathrm{t}_{\mathrm{R}} / \mathrm{min}$ & $\%$ peak area \\
\hline
\end{tabular} 


\begin{tabular}{|c|c|c|}
\hline 1 & 8.1 & 95.49 \\
\hline 2 & 8.6 & 4.51 \\
\hline \multicolumn{2}{|c|}{ Total } & 100 \\
\hline
\end{tabular}

HPLC column: Amycoat RP, Acetonitrile/MeOH/Water = 45:45:10, $1.0 \mathrm{~mL} / \mathrm{min}, 298 \mathrm{~K}, 220 \mathrm{~nm}$.

Additional Info : Peak(s) manually integrated

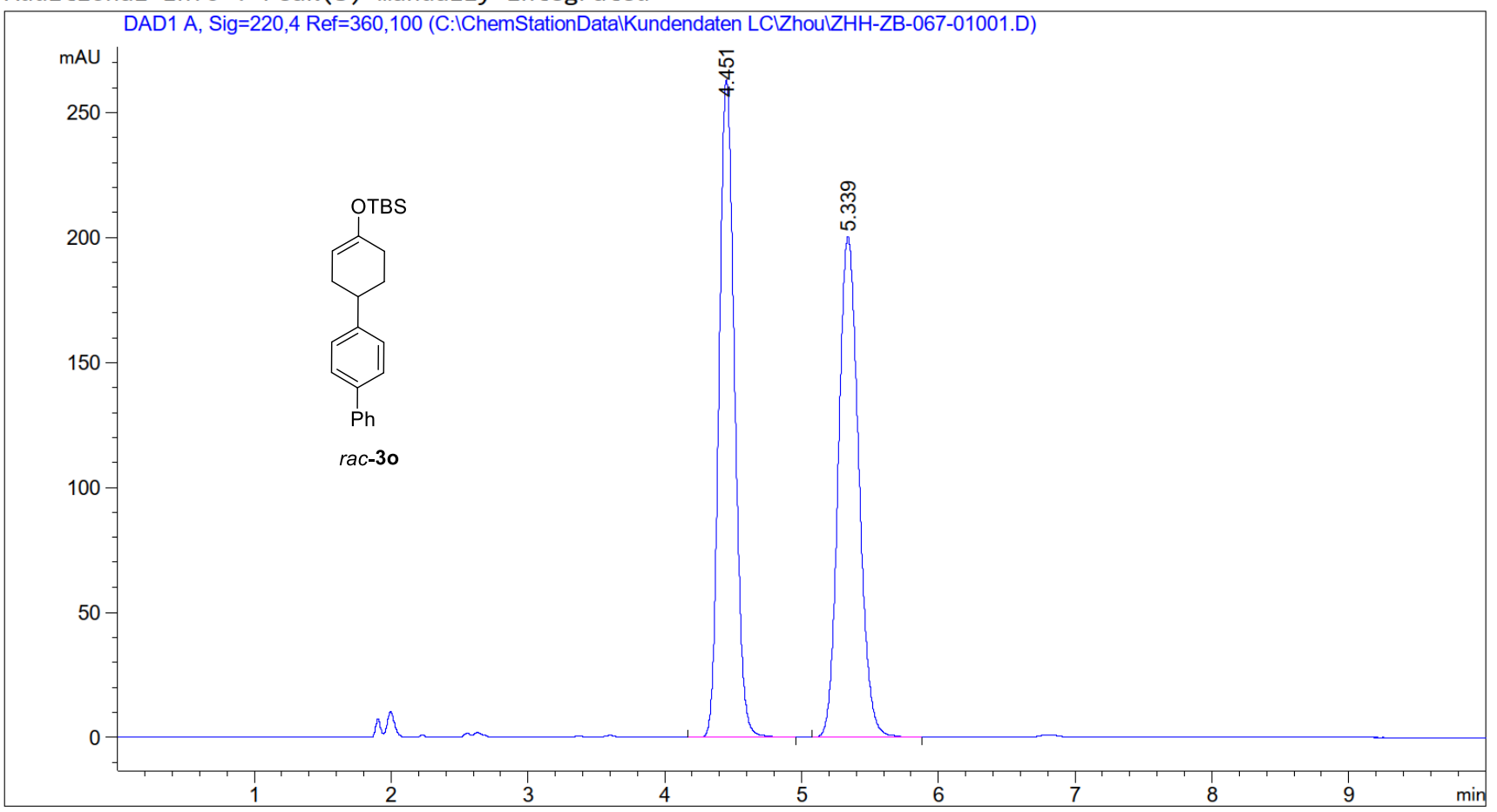

\begin{tabular}{|c|c|c|}
\hline Peak \# & $\mathrm{t}_{\mathrm{R}} / \mathrm{min}$ & \% peak area \\
\hline 1 & 4.4 & 49.91 \\
\hline 2 & 5.3 & 50.09 \\
\hline \multicolumn{2}{|c|}{ Total } & 100 \\
\hline
\end{tabular}


Additional Info : Peak(s) manually integrated

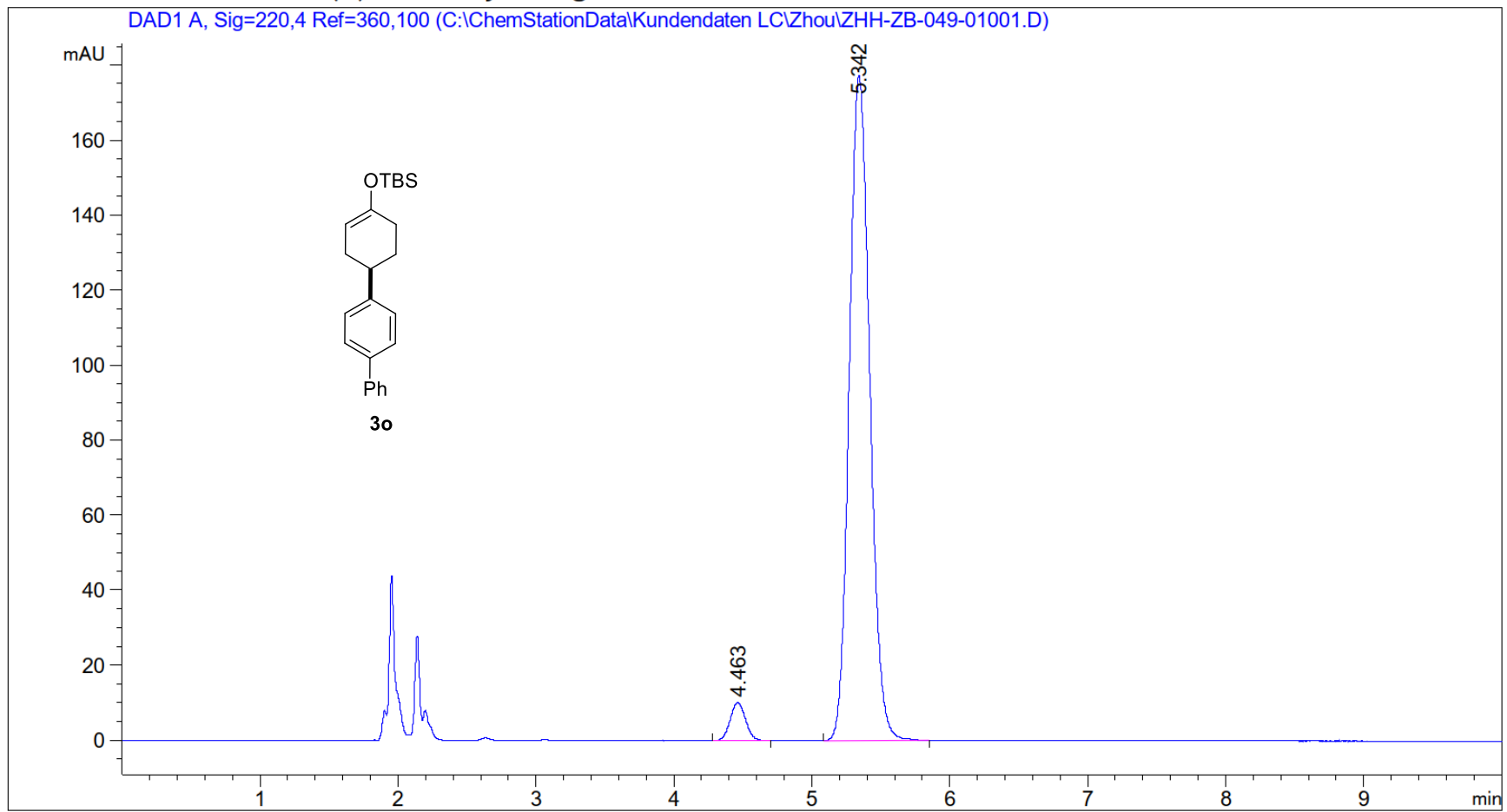

\begin{tabular}{|c|c|c|}
\hline Peak \# & $\mathrm{t}_{\mathrm{R}} / \mathrm{min}$ & $\%$ peak area \\
\hline 1 & 4.5 & 4.11 \\
\hline 2 & 5.3 & 95.89 \\
\hline \multicolumn{2}{|c|}{ Total } & 100 \\
\hline
\end{tabular}

HPLC column: OJ-3R, MeOH: Water = 75:25, $1.0 \mathrm{~mL} / \mathrm{min}, 298 \mathrm{~K}, 214 \mathrm{~nm}$. mAU

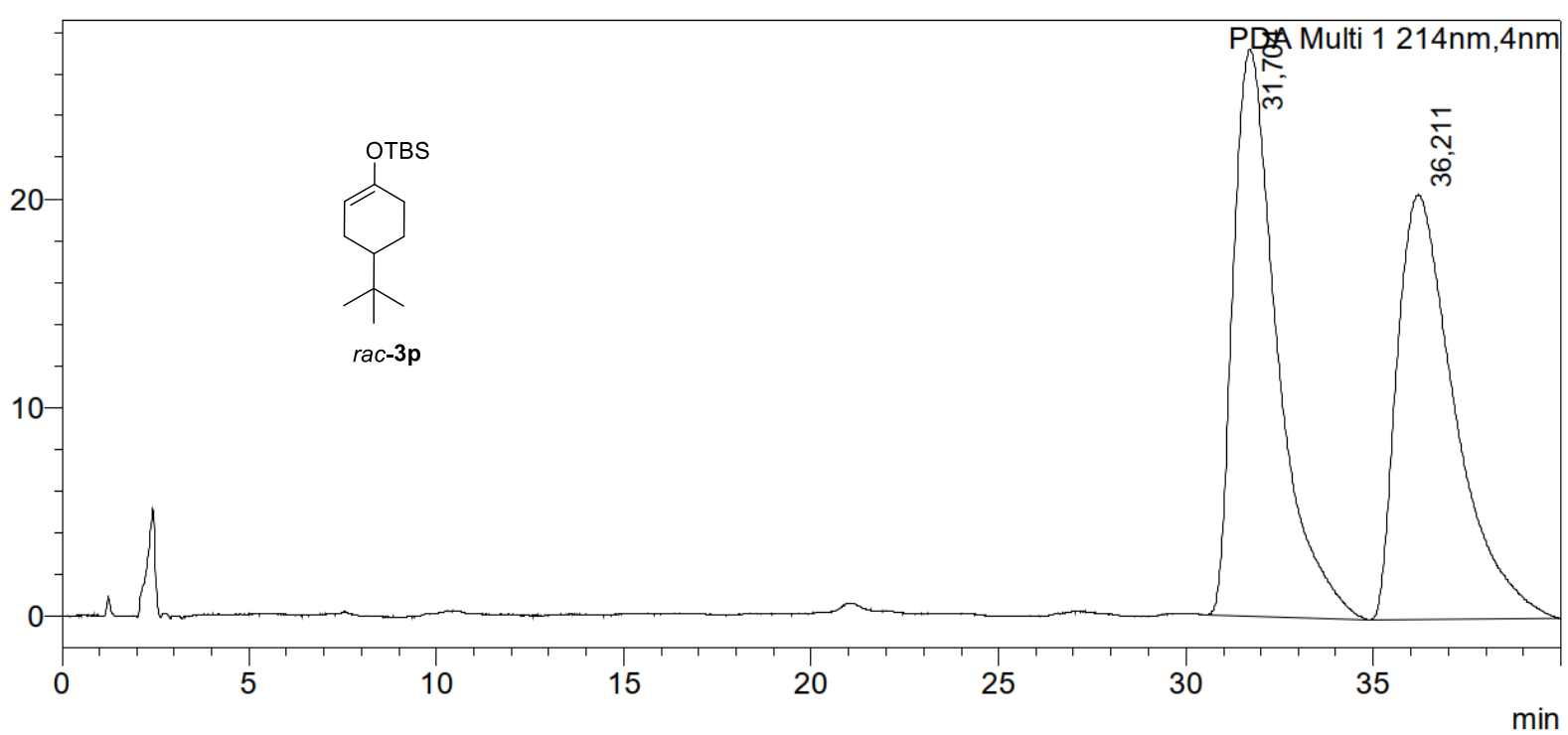

\begin{tabular}{|c|c|c|}
\hline Peak \# & $t_{R} / \min$ & $\%$ peak area \\
\hline 1 & 31.7 & 50.16 \\
\hline 2 & 36.2 & 49.84 \\
\hline \multicolumn{2}{|c|}{ Total } & 100 \\
\hline
\end{tabular}


mAU

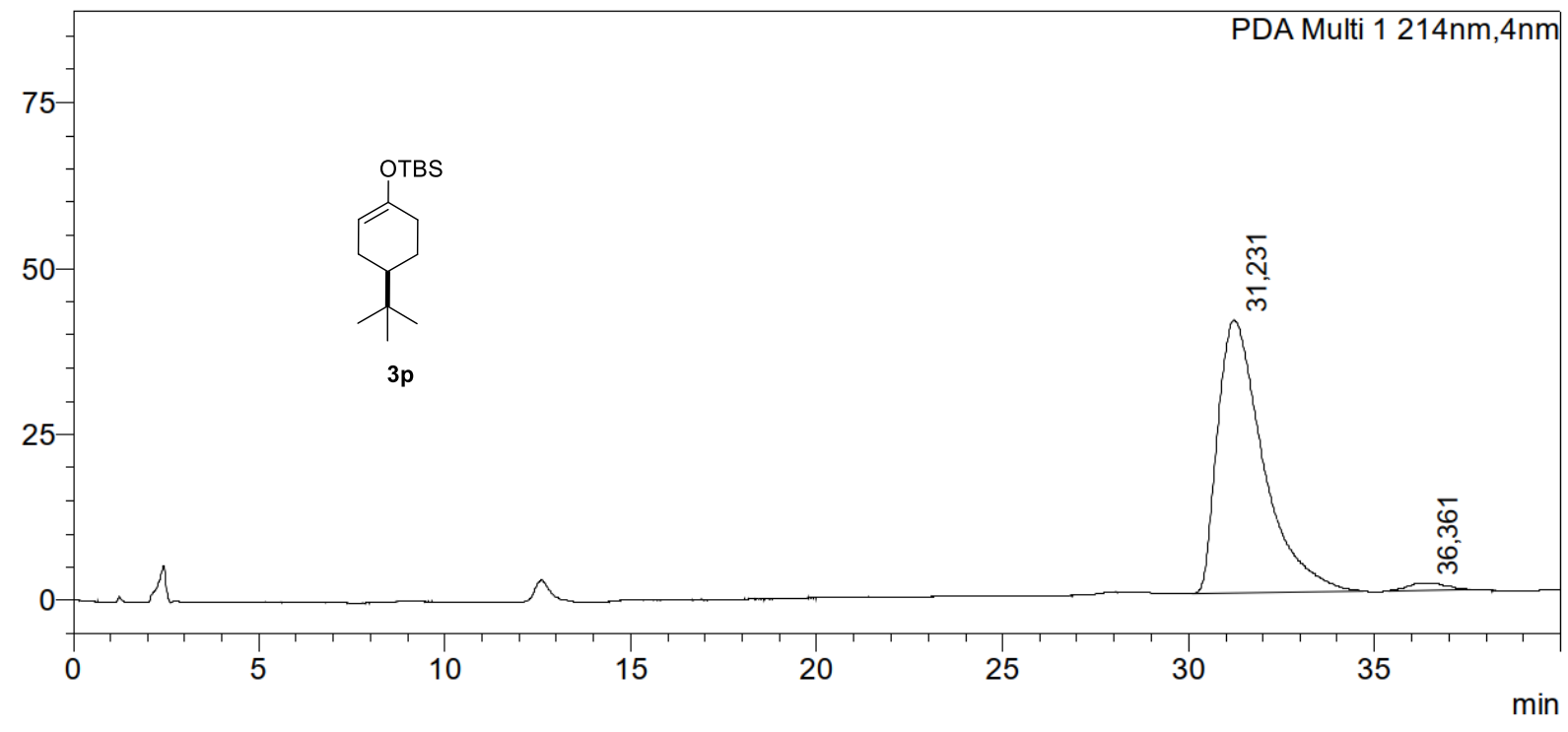

\begin{tabular}{|c|c|c|}
\hline Peak \# & $\mathrm{t}_{\mathrm{R}} / \mathrm{min}$ & \% peak area \\
\hline 1 & 31.2 & 97.28 \\
\hline 2 & 36.4 & 2.72 \\
\hline \multicolumn{2}{|c|}{ Total } & 100 \\
\hline
\end{tabular}

HPLC column: OJ-3R, Acetonitrile: Water = 70:30, $1.0 \mathrm{~mL} / \mathrm{min}, 298 \mathrm{~K}, 220 \mathrm{~nm}$.

mAU

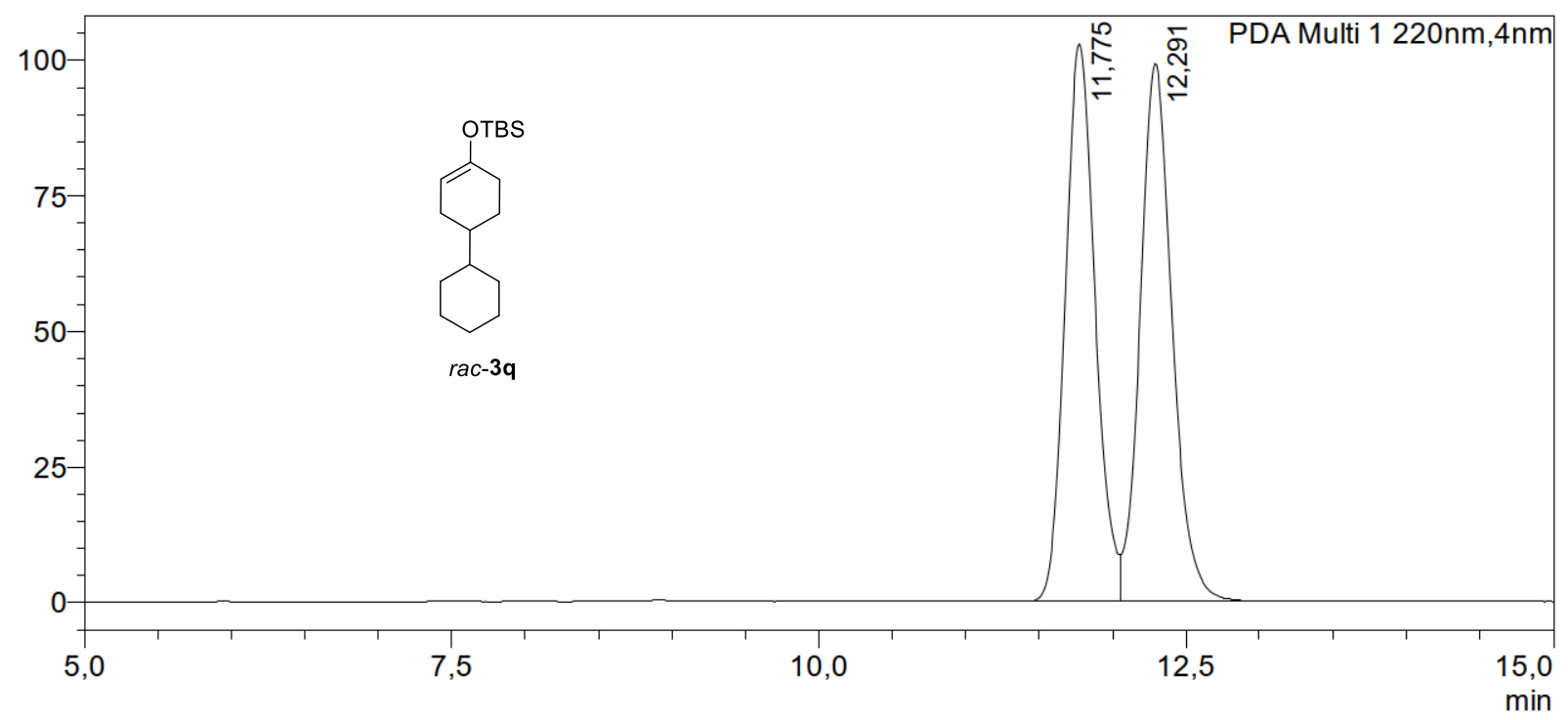

\begin{tabular}{|c|c|c|}
\hline Peak \# & $\mathrm{t}_{\mathrm{R}} / \mathrm{min}$ & \% peak area \\
\hline 1 & 11.8 & 50.89 \\
\hline 2 & 12.3 & 49.11 \\
\hline \multicolumn{2}{|c|}{ Total } & 100 \\
\hline
\end{tabular}


mAU

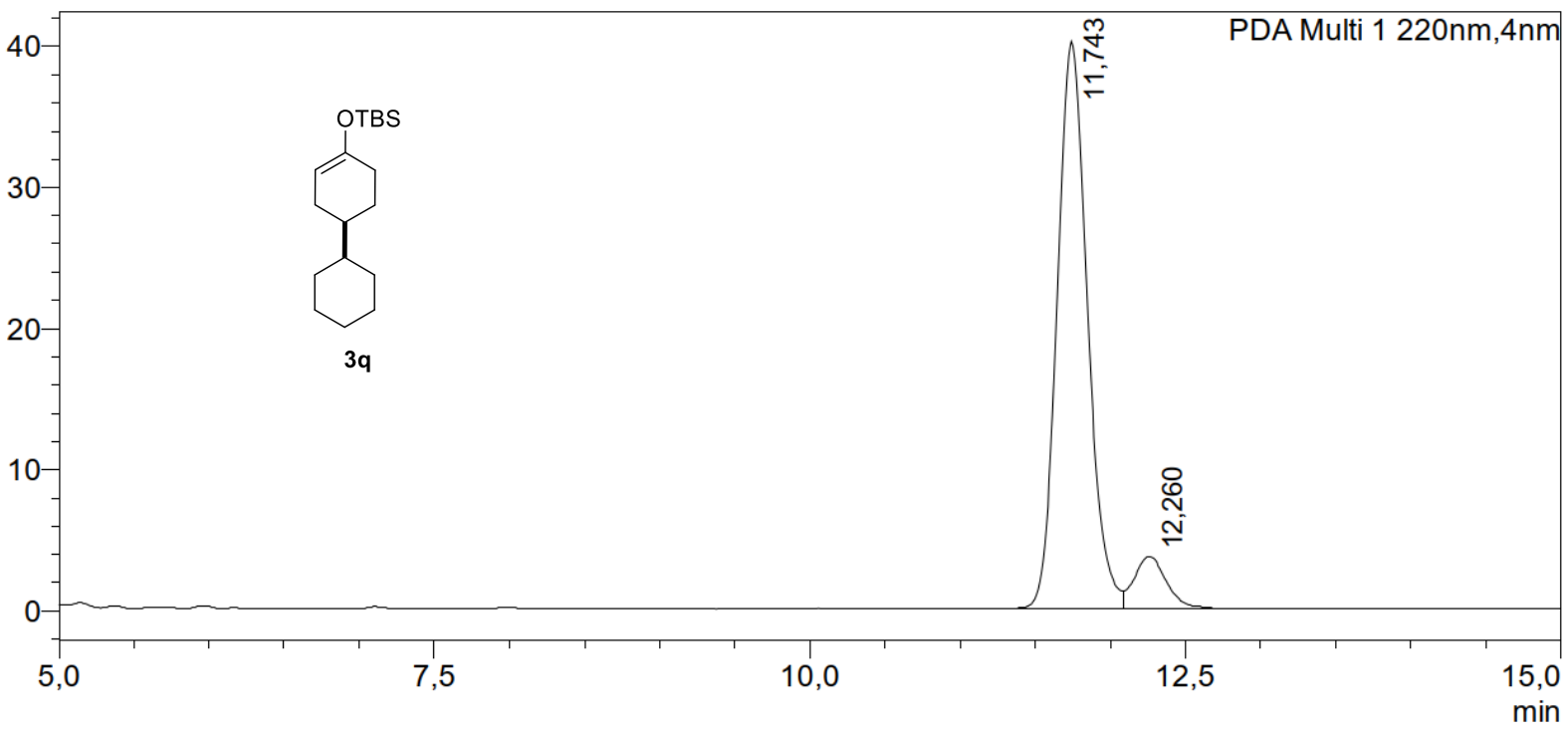

\begin{tabular}{|c|c|c|}
\hline Peak \# & $\mathrm{t}_{\mathrm{R}} / \mathrm{min}$ & $\%$ peak area \\
\hline 1 & 11.7 & 91.64 \\
\hline 2 & 12.3 & 8.36 \\
\hline \multicolumn{2}{|c|}{ Total } & 100 \\
\hline
\end{tabular}

HPLC column: OJ-3R, Acetonitrile: Water =70:30, $1.0 \mathrm{~mL} / \mathrm{min}, 298 \mathrm{~K}, 259 \mathrm{~nm}$. mAU

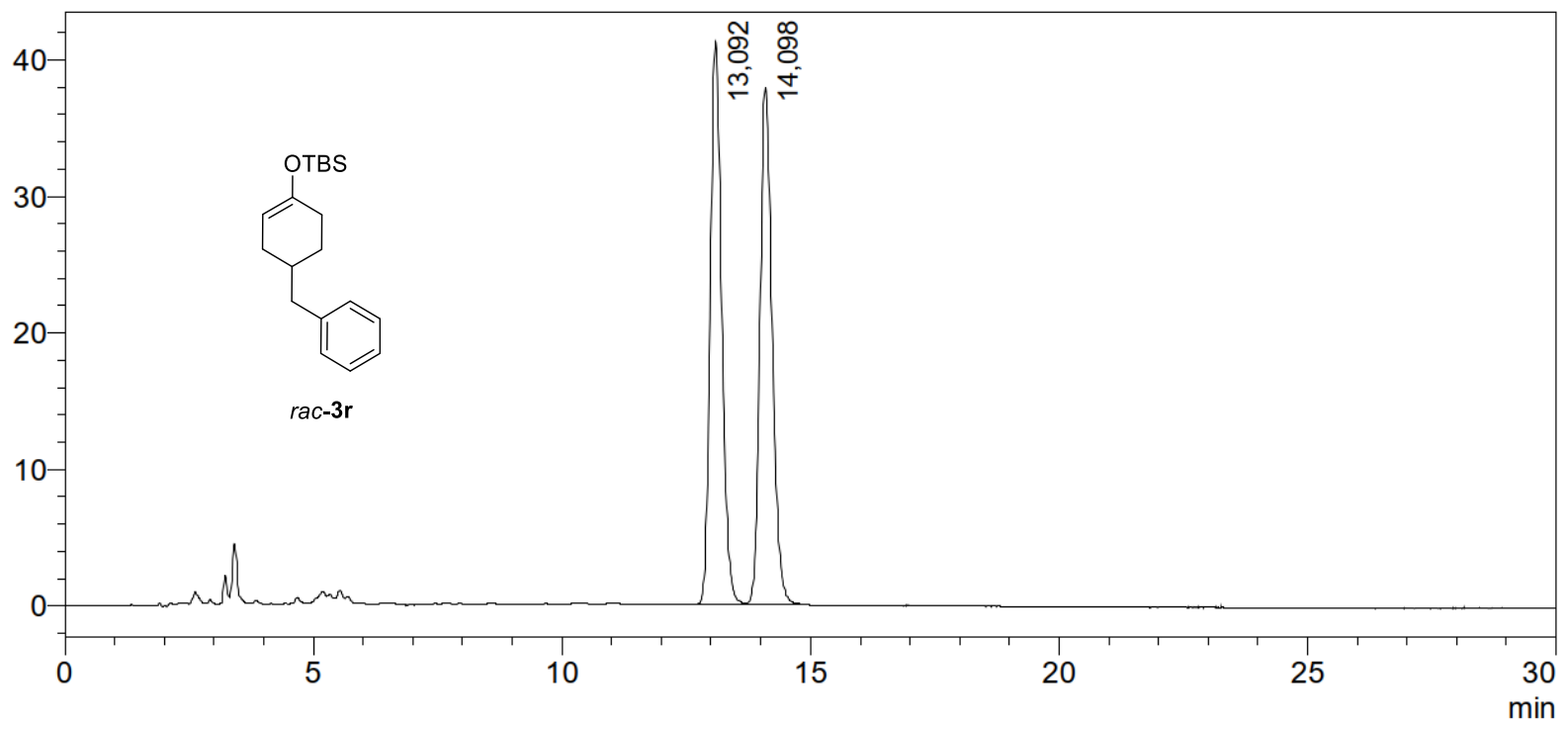

\begin{tabular}{|c|c|c|}
\hline Peak \# & $\mathrm{t}_{\mathrm{R}} / \mathrm{min}$ & $\%$ peak area \\
\hline 1 & 13.1 & 50.13 \\
\hline 2 & 14.1 & 49.87 \\
\hline \multicolumn{2}{|c|}{ Total } & 100 \\
\hline
\end{tabular}


mAU

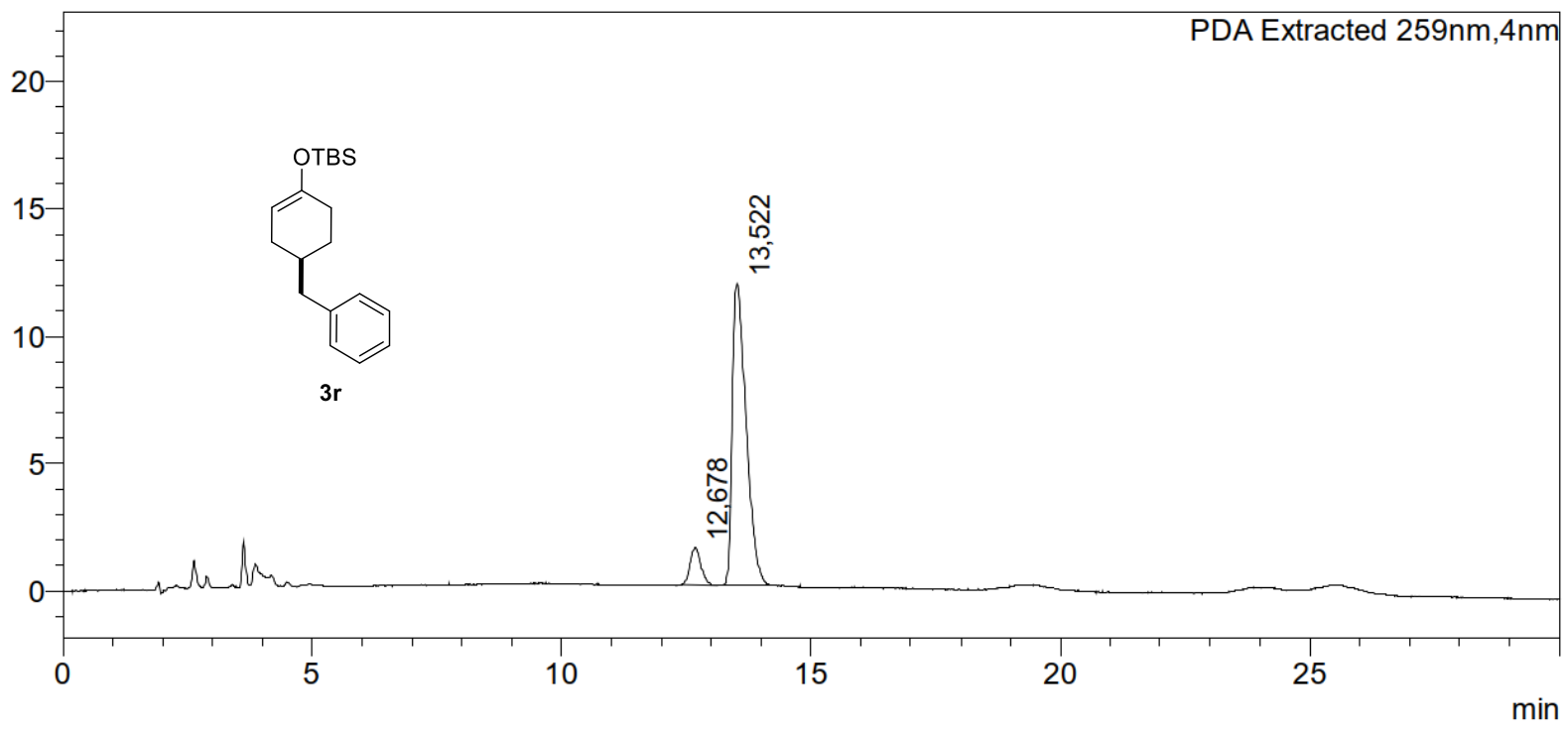

\begin{tabular}{|c|c|c|}
\hline Peak \# & $\mathrm{t}_{\mathrm{R}} / \mathrm{min}$ & $\%$ peak area \\
\hline 1 & 12.6 & 8.13 \\
\hline 2 & 13.5 & 91.87 \\
\hline \multicolumn{2}{|c|}{ Total } & 100 \\
\hline
\end{tabular}

HPLC column: OJ-3R, Acetonitrile: Water = 70:30, $1.0 \mathrm{~mL} / \mathrm{min}, 298 \mathrm{~K}, 220 \mathrm{~nm}$. mAU

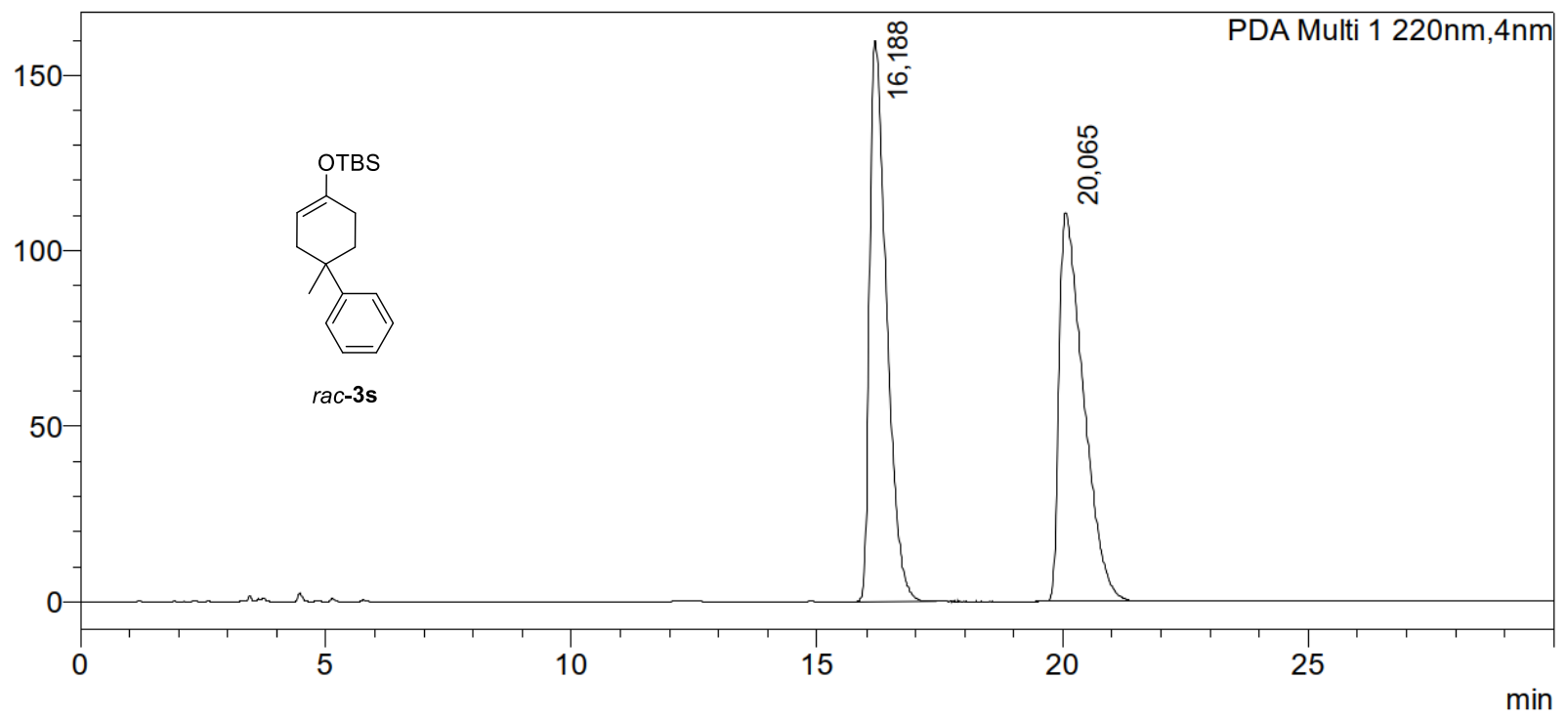

\begin{tabular}{|c|c|c|}
\hline Peak \# & $\mathrm{t}_{\mathrm{R}} / \mathrm{min}$ & $\%$ peak area \\
\hline 1 & 16.2 & 49.98 \\
\hline 2 & 20.1 & 50.02 \\
\hline \multicolumn{2}{|c|}{ Total } & 100 \\
\hline
\end{tabular}


mAU

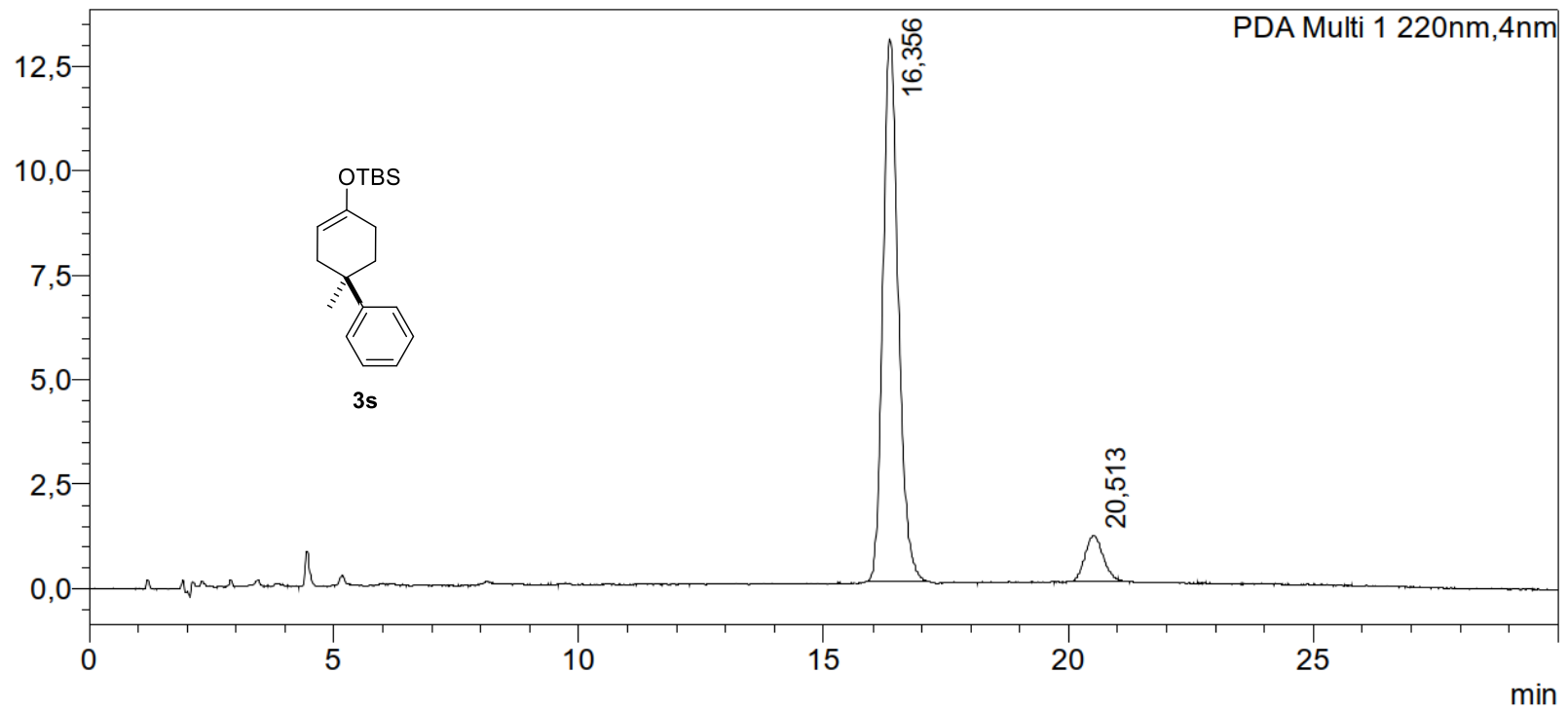

\begin{tabular}{|c|c|c|}
\hline Peak \# & $\mathrm{t}_{\mathrm{R}} / \mathrm{min}$ & $\%$ peak area \\
\hline 1 & 16.4 & 94.25 \\
\hline 2 & 20.5 & 5.75 \\
\hline \multicolumn{2}{|c|}{ Total } & 100 \\
\hline
\end{tabular}

HPLC column: AD-3R, Acetonitrile: Water = 50:50, $1.0 \mathrm{~mL} / \mathrm{min}, 298 \mathrm{~K}, 220 \mathrm{~nm}$.

MAU

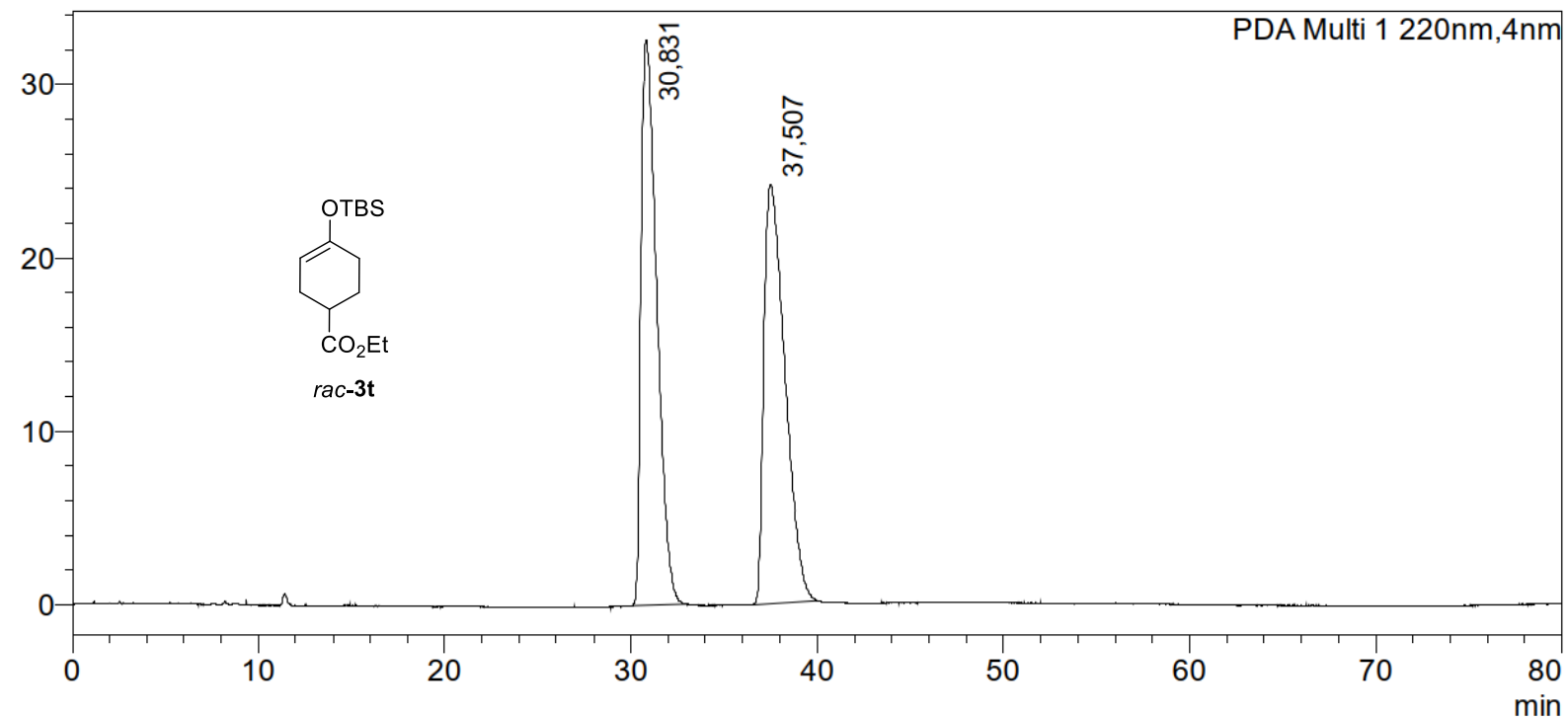

\begin{tabular}{|c|c|c|}
\hline Peak \# & $\mathrm{t}_{\mathrm{R}} / \mathrm{min}$ & $\%$ peak area \\
\hline 1 & 30.8 & 50.09 \\
\hline 2 & 37.5 & 49.91 \\
\hline \multicolumn{2}{|c|}{ Total } & 100 \\
\hline
\end{tabular}


mAU

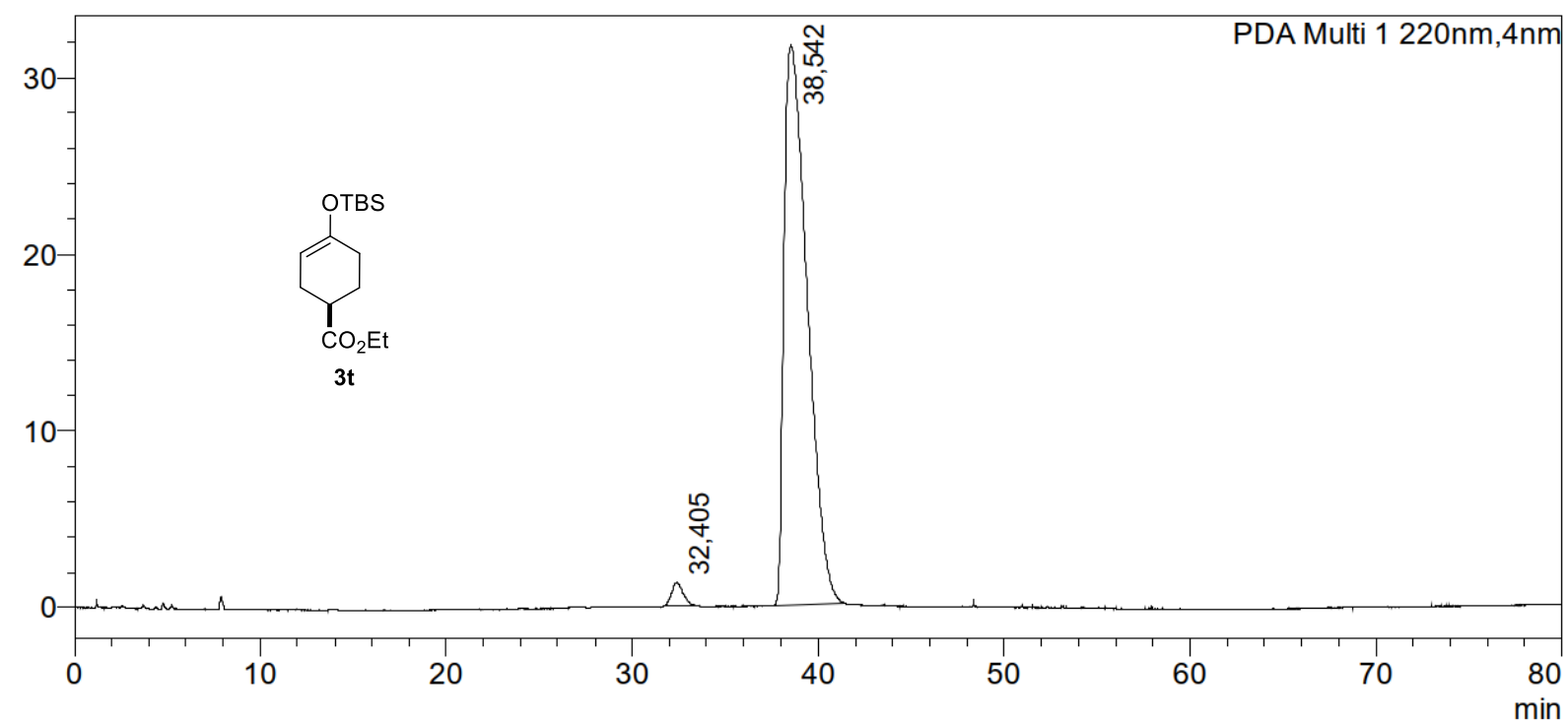

\begin{tabular}{|c|c|c|}
\hline Peak \# & $\mathrm{t}_{\mathrm{R}} / \mathrm{min}$ & $\%$ peak area \\
\hline 1 & 32.4 & 2.07 \\
\hline 2 & 38.5 & 97.93 \\
\hline \multicolumn{2}{|c|}{ Total } & 100 \\
\hline
\end{tabular}

HPLC column: AD-3R, Acetonitrile: Water = 50:50, 1.0 mL/min, 298 K, 215 nm. mAU

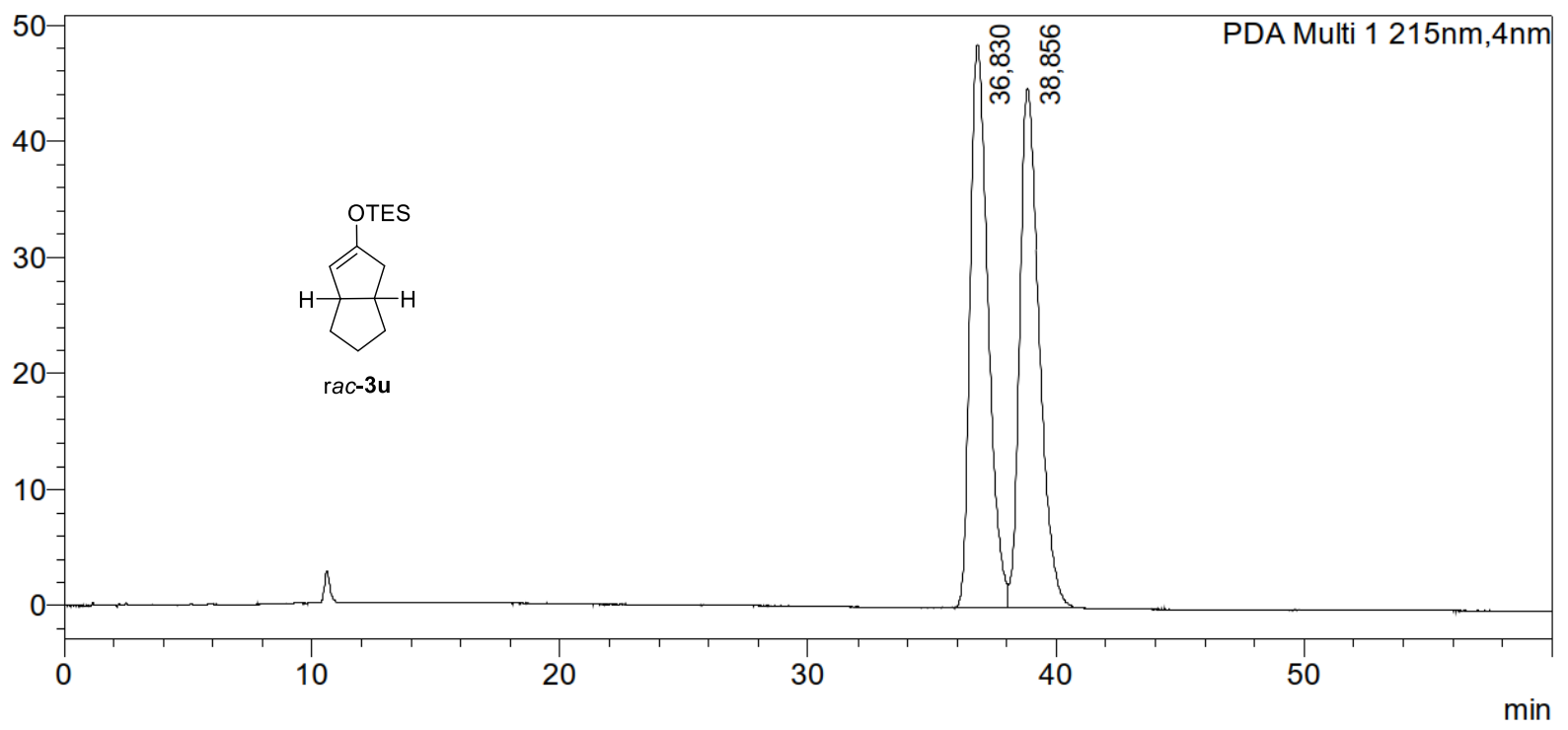

\begin{tabular}{|c|c|c|}
\hline Peak \# & $\mathrm{t}_{\mathrm{R}} / \mathrm{min}$ & $\%$ peak area \\
\hline 1 & 36.8 & 49.75 \\
\hline 2 & 38.8 & 50.25 \\
\hline \multicolumn{2}{|c|}{ Total } & 100 \\
\hline
\end{tabular}


mAU

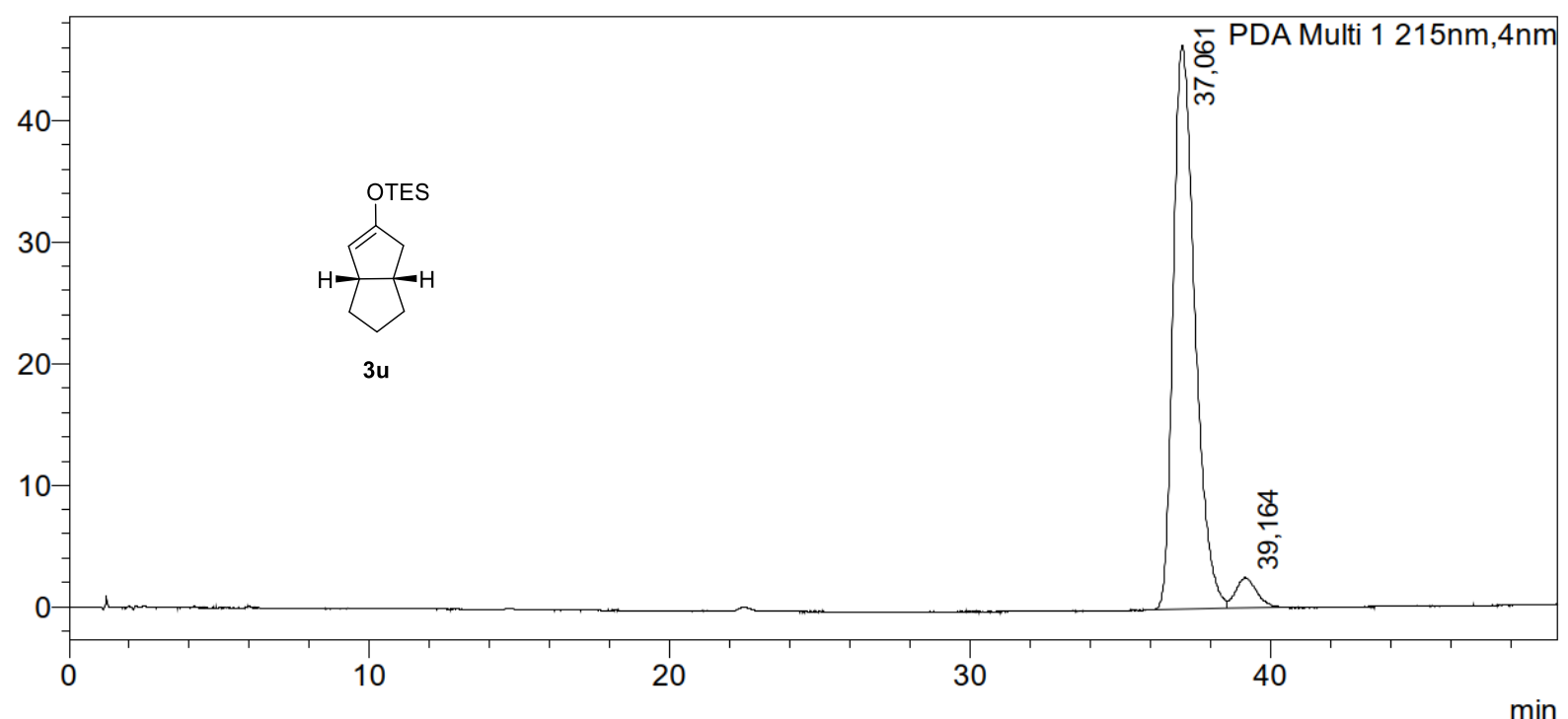

\begin{tabular}{|c|c|c|}
\hline Peak \# & $\mathrm{t}_{\mathrm{R}} / \mathrm{min}$ & $\%$ peak area \\
\hline 1 & 37.1 & 95.11 \\
\hline 2 & 39.2 & 4.89 \\
\hline \multicolumn{2}{|c|}{ Total } & 100 \\
\hline
\end{tabular}

HPLC column: OJ-3R, Acetonitrile: Water =60:40, $1.0 \mathrm{~mL} / \mathrm{min}, 298 \mathrm{~K}, 220 \mathrm{~nm}$.

mAU

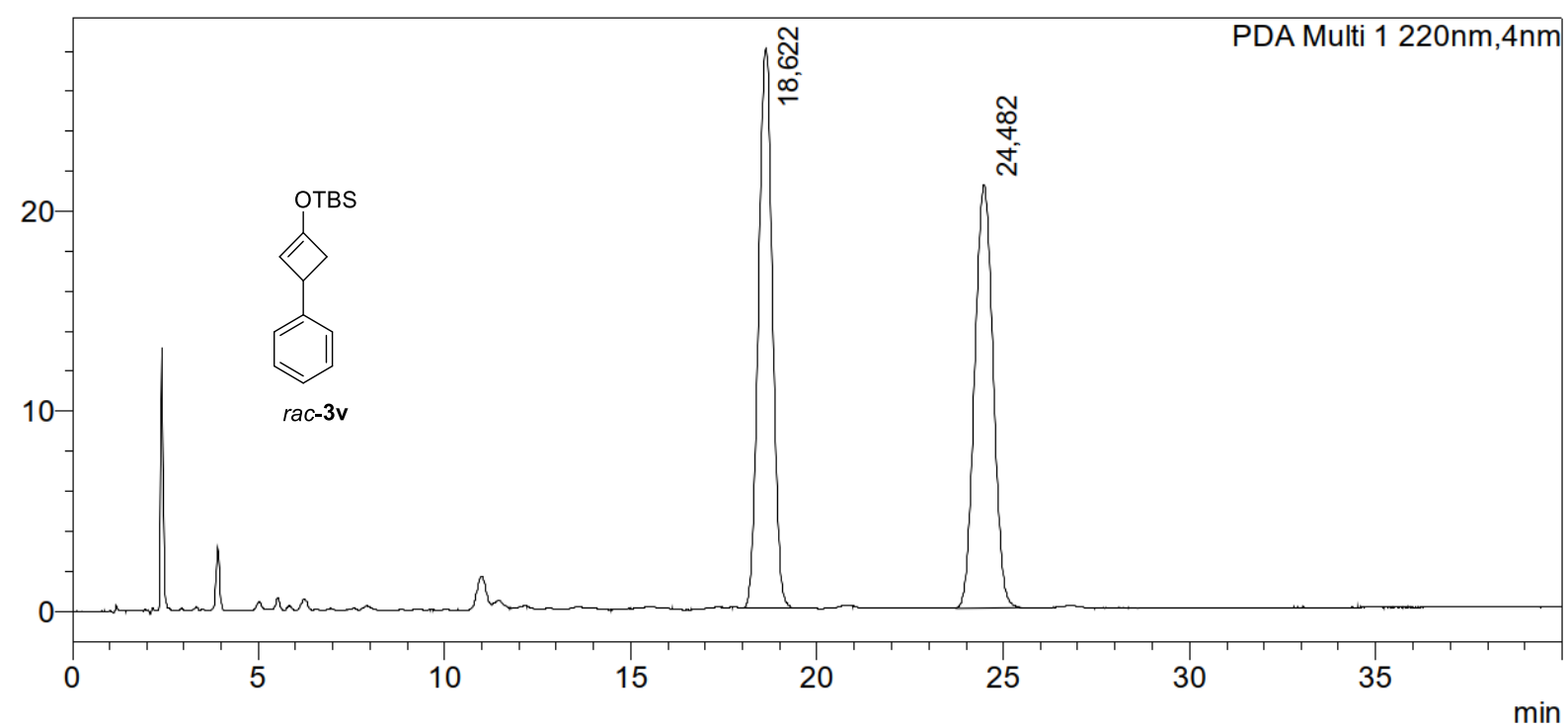

\begin{tabular}{|c|c|c|}
\hline Peak \# & $\mathrm{t}_{\mathrm{R}} / \mathrm{min}$ & $\%$ peak area \\
\hline 1 & 18.6 & 50.54 \\
\hline 2 & 24.5 & 49.46 \\
\hline \multicolumn{2}{|c|}{ Total } & 100 \\
\hline
\end{tabular}


mAU

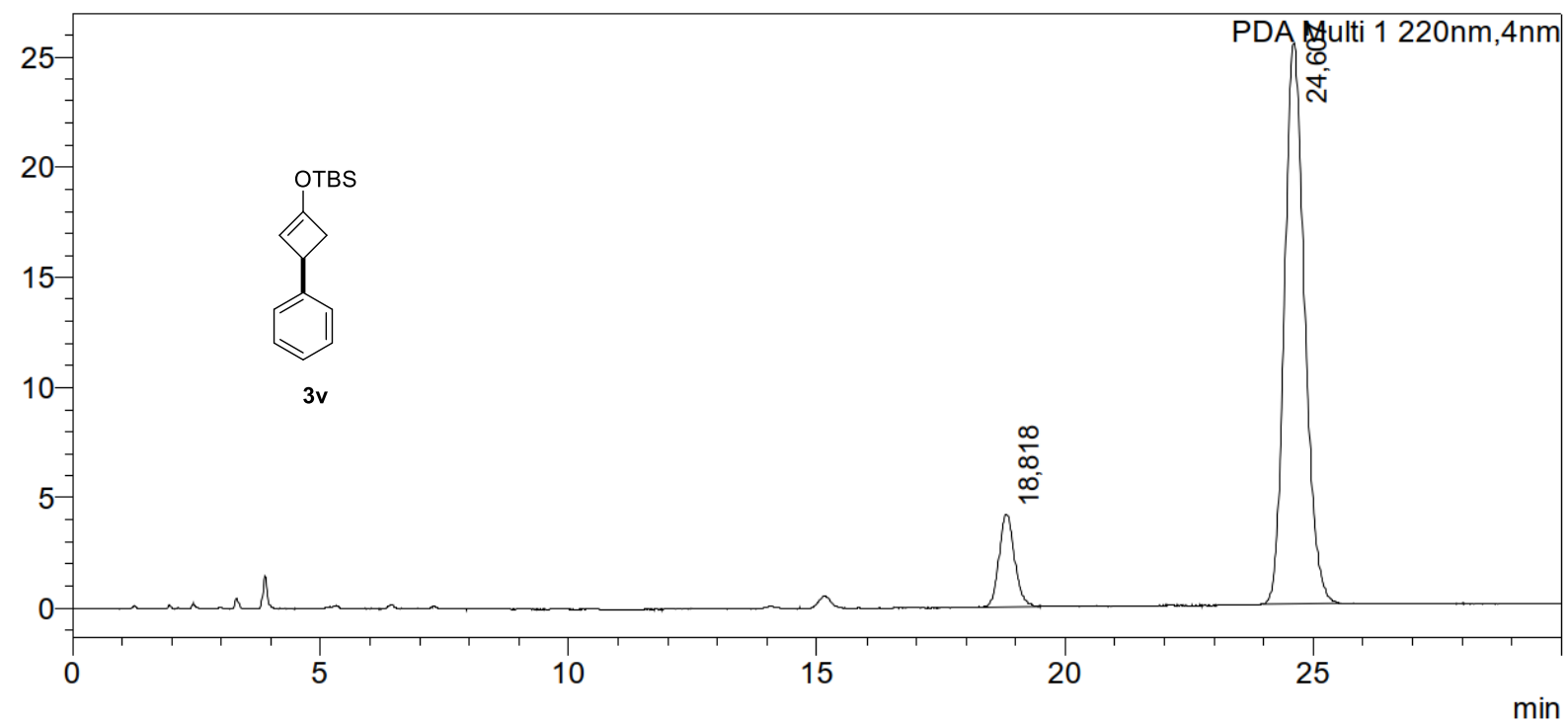

\begin{tabular}{|c|c|c|}
\hline Peak \# & $\mathrm{t}_{\mathrm{R}} / \mathrm{min}$ & $\%$ peak area \\
\hline 1 & 18.8 & 11.18 \\
\hline 2 & 24.6 & 88.82 \\
\hline \multicolumn{2}{|c|}{ Total } & 100 \\
\hline
\end{tabular}

HPLC column: OJ-3R, Acetonitrile: Water = 80:20, $1.0 \mathrm{~mL} / \mathrm{min}, 298 \mathrm{~K}, 254 \mathrm{~nm}$. mAU

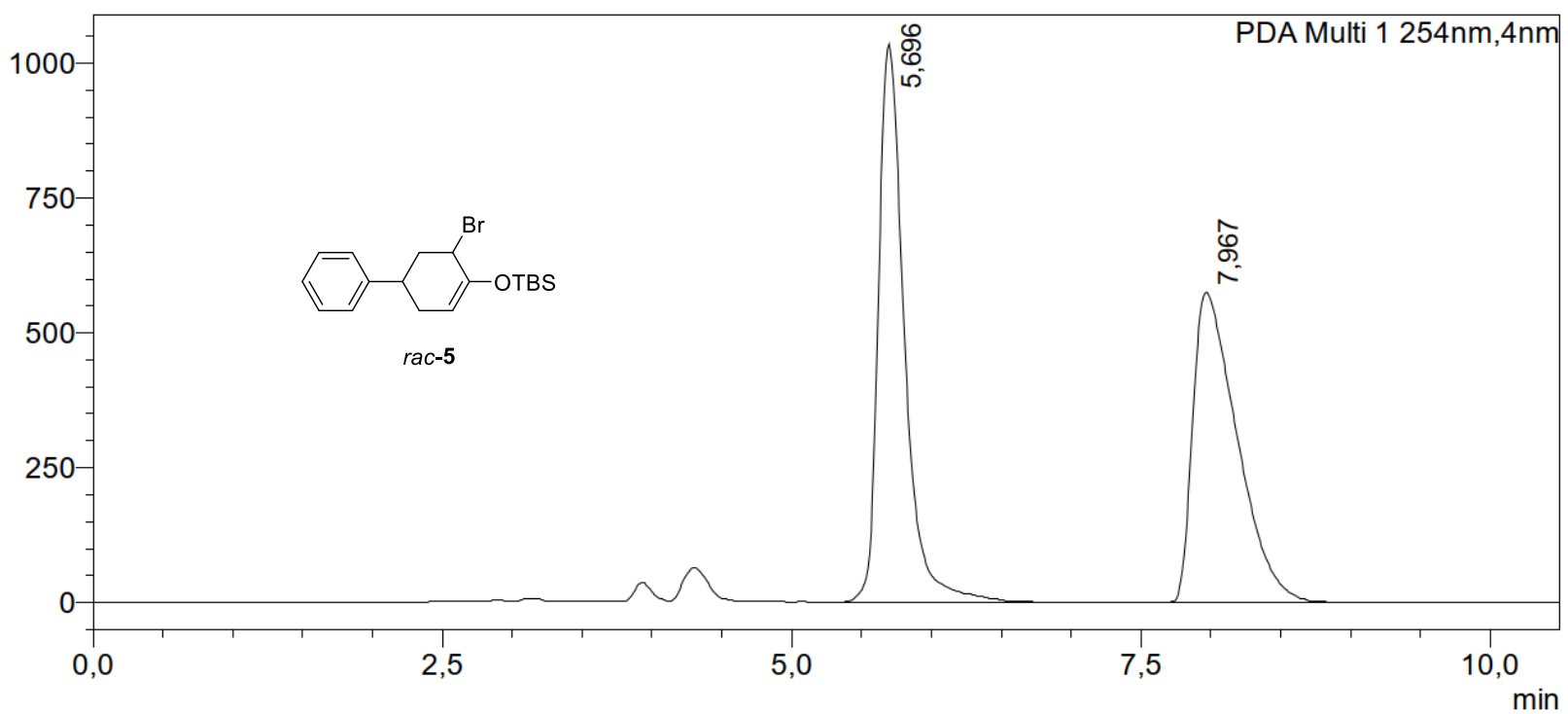

\begin{tabular}{|c|c|c|}
\hline Peak \# & $\mathrm{t}_{\mathrm{R}} / \mathrm{min}$ & \% peak area \\
\hline 1 & 5.7 & 51.53 \\
\hline 2 & 8.0 & 48.46 \\
\hline
\end{tabular}




\begin{tabular}{|l|l|}
\hline Total & 100 \\
\hline
\end{tabular}

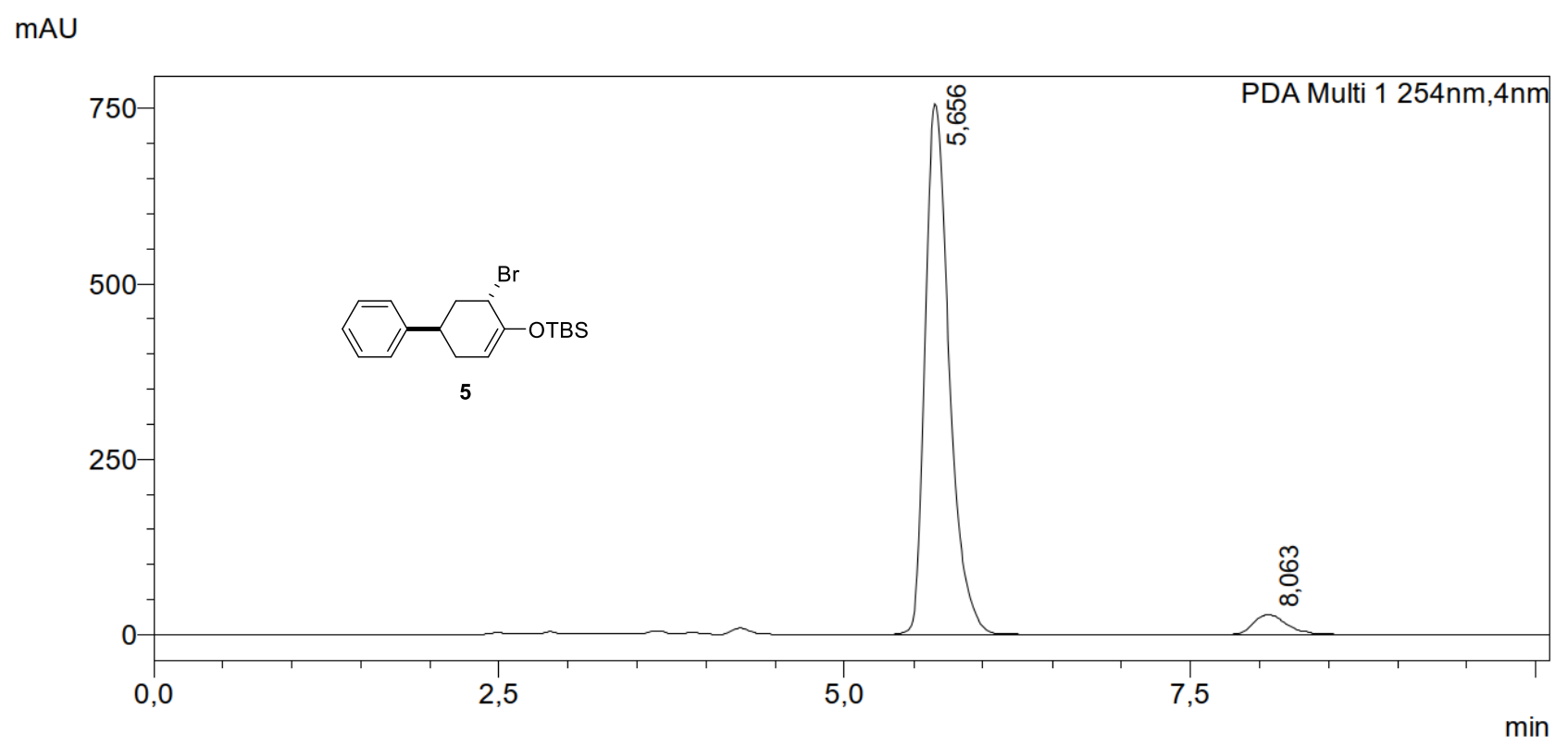

\begin{tabular}{|c|c|c|}
\hline Peak \# & $\mathrm{t}_{\mathrm{R}} / \mathrm{min}$ & \% peak area \\
\hline 1 & 5.6 & 94.94 \\
\hline 2 & 8.1 & 5.06 \\
\hline \multicolumn{2}{|c|}{ Total } & 100 \\
\hline
\end{tabular}

HPLC column: AD-3, $n$-heptane/ $i$-PrOH = 99:1, $1.0 \mathrm{~mL} / \mathrm{min}, 298 \mathrm{~K}, 254 \mathrm{~nm}$. 


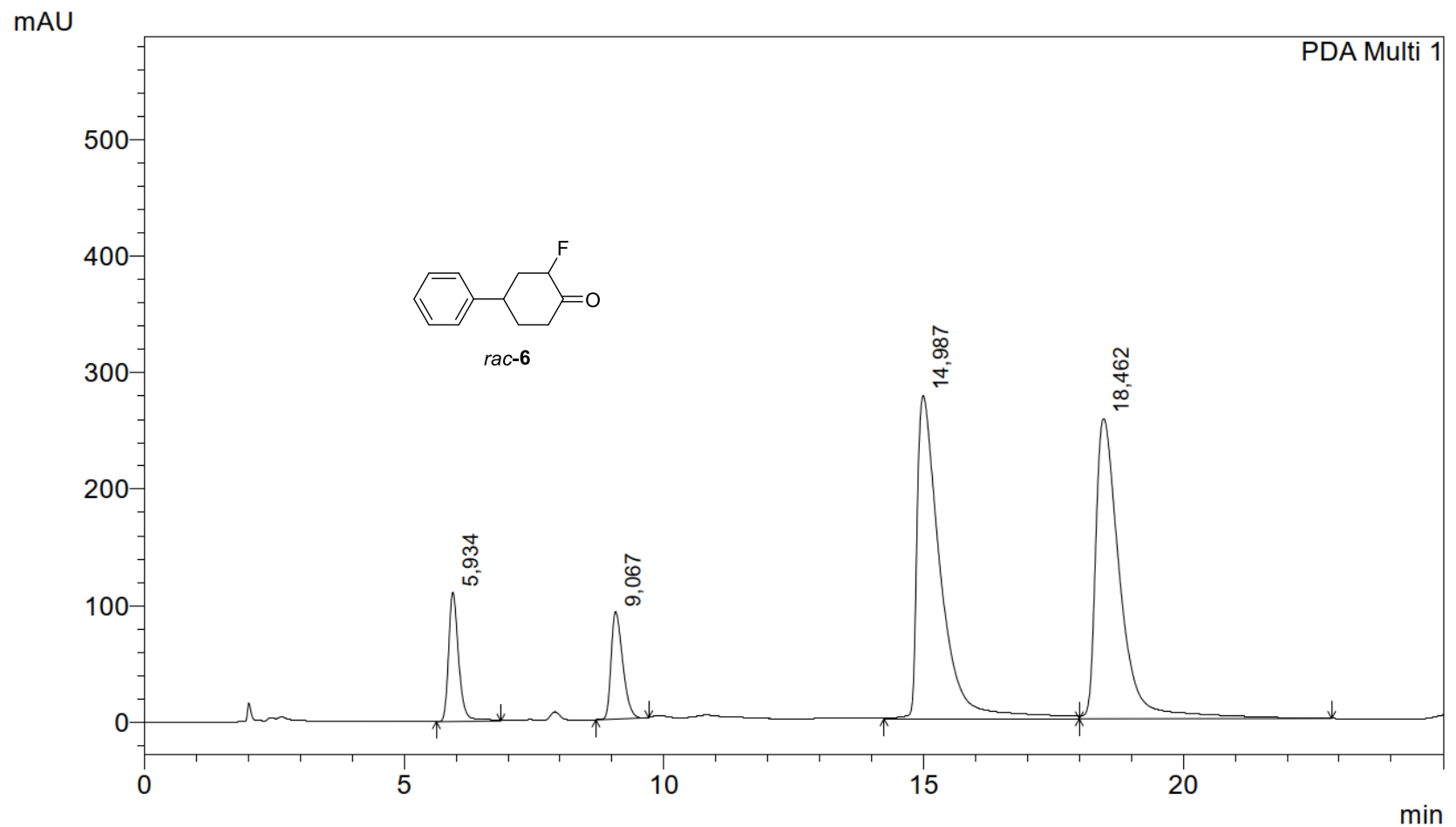

\begin{tabular}{|c|c|c|}
\hline Peak \# & $\mathrm{t}_{\mathrm{R}} / \mathrm{min}$ & \% peak area \\
\hline 1 & 5.9 & 7.53 \\
\hline 2 & 9.1 & 7.08 \\
\hline 3 & 15.0 & 43.30 \\
\hline 4 & 18.5 & 42.09 \\
\hline \multicolumn{2}{|c|}{ Total } & 100 \\
\hline
\end{tabular}

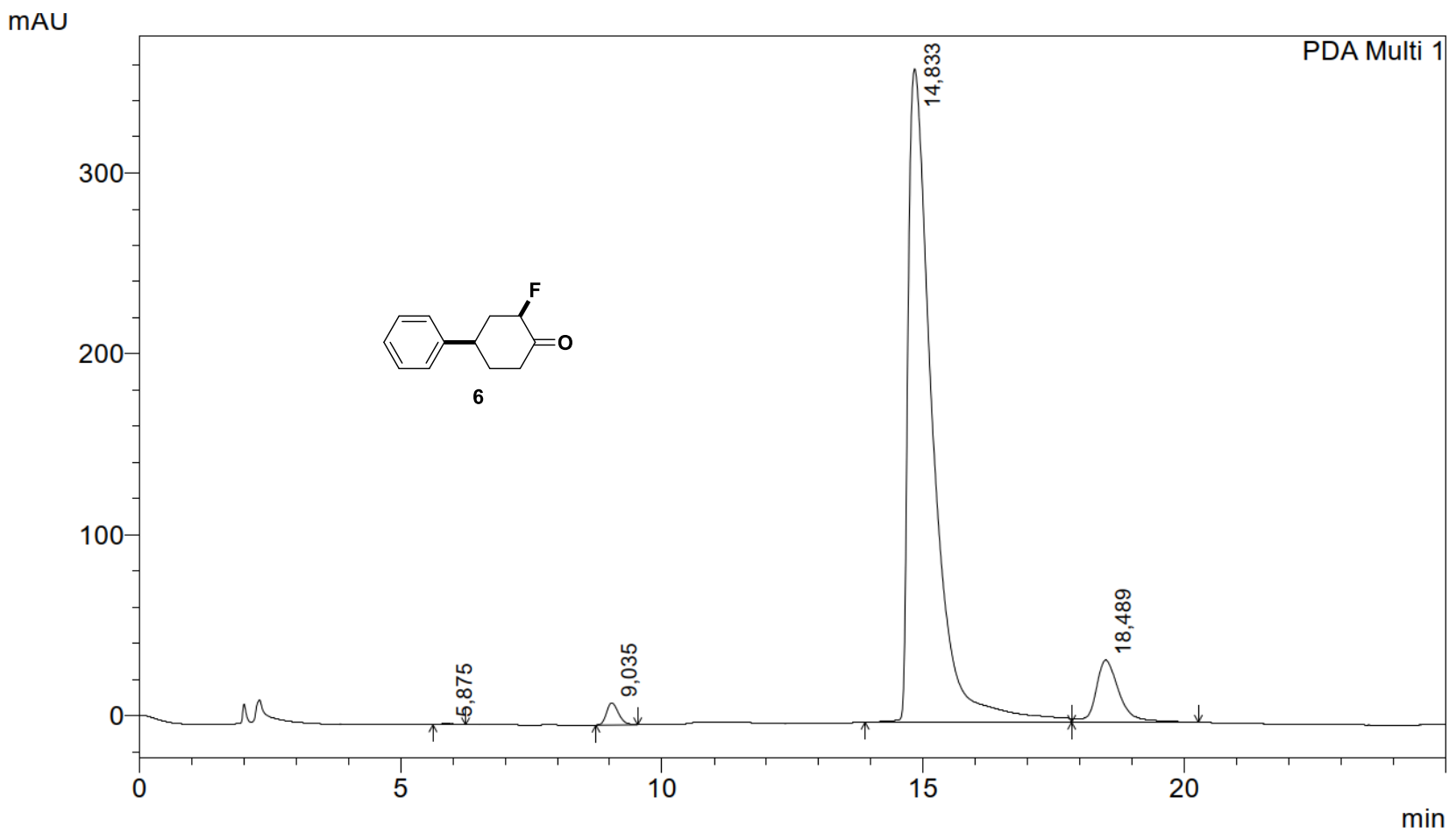

\begin{tabular}{|c|c|c|}
\hline Peak \# & $\mathrm{t}_{\mathrm{R}} / \mathrm{min}$ & \% peak area \\
\hline 1 & 5.9 & 0.10 \\
\hline
\end{tabular}




\begin{tabular}{|c|c|c|}
\hline 2 & 9.0 & 1.50 \\
\hline 3 & 14.8 & 90.13 \\
\hline 4 & 18.5 & 8.26 \\
\hline \multicolumn{2}{|c|}{ Total } & 100 \\
\hline
\end{tabular}

HPLC column: OJ-3, $n$-heptane/i-PrOH = 99:1, $1.0 \mathrm{~mL} / \mathrm{min}, 298 \mathrm{~K}, 220 \mathrm{~nm}$. mAU

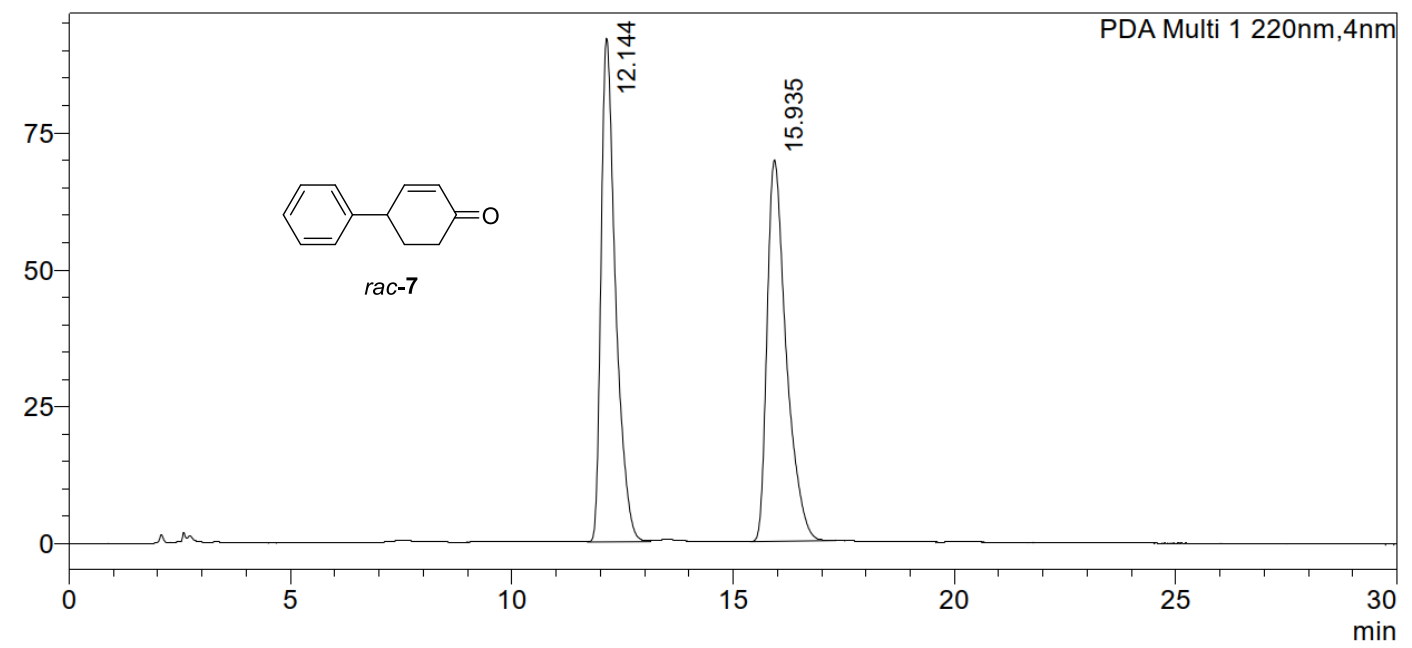

\begin{tabular}{|c|c|c|}
\hline Peak \# & $\mathrm{t}_{\mathrm{R}} / \mathrm{min}$ & $\%$ peak area \\
\hline 1 & 12.1 & 50.08 \\
\hline 2 & 15.9 & 49.92 \\
\hline \multicolumn{2}{|c|}{ Total } & 100 \\
\hline
\end{tabular}

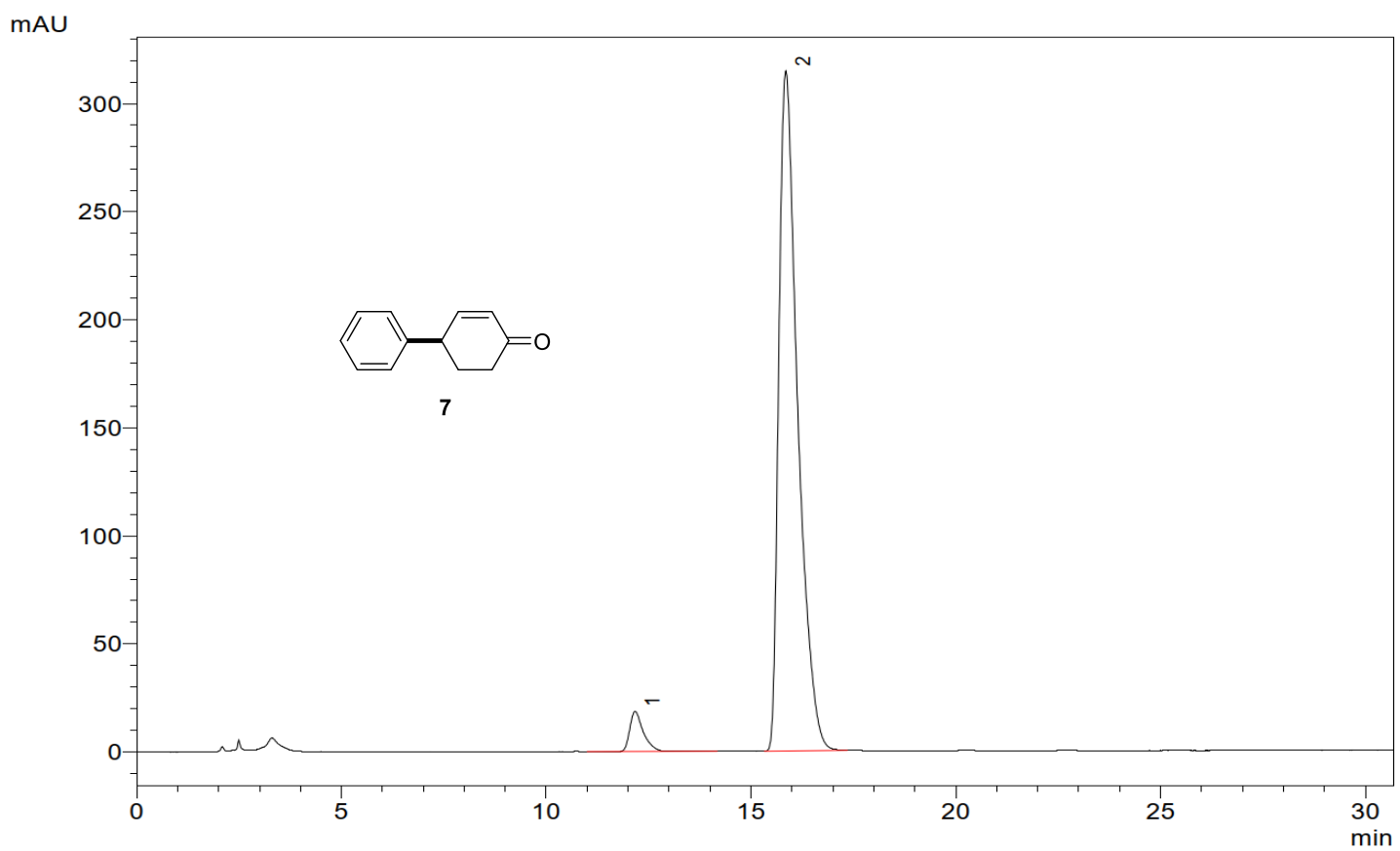

\begin{tabular}{|c|c|c|}
\hline Peak \# & $\mathrm{t}_{\mathrm{R}} / \mathrm{min}$ & $\%$ peak area \\
\hline 1 & 12.2 & 4.45 \\
\hline 2 & 15.8 & 95.55 \\
\hline \multicolumn{2}{|c|}{ Total } & 100 \\
\hline
\end{tabular}


HPLC column: OJ-3R, Acetonitrile: Water $=60: 40,1.0 \mathrm{~mL} / \mathrm{min}, 298 \mathrm{~K}, 220 \mathrm{~nm}$.

mAU

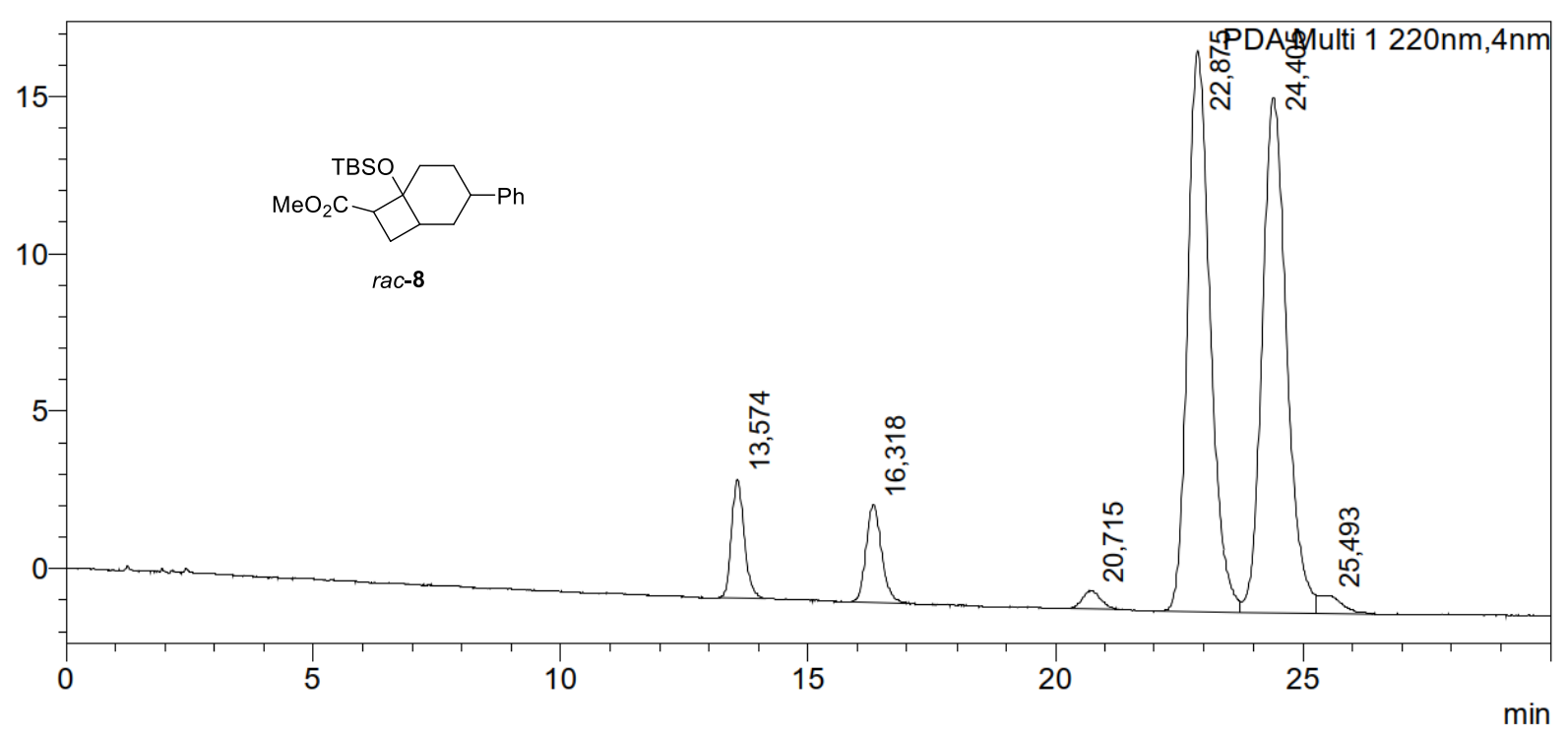

\begin{tabular}{|c|c|c|}
\hline Peak \# & $\mathrm{t}_{\mathrm{R}} / \mathrm{min}$ & \% peak area \\
\hline 1 & 13.6 & 5.13 \\
\hline 2 & 16.3 & 5.18 \\
\hline 3 & 20.7 & 1.14 \\
\hline 4 & 22.9 & 43.40 \\
\hline 5 & 24.4 & 43.60 \\
\hline 6 & 25.5 & 1.55 \\
\hline \multicolumn{2}{|c|}{ Total } & 100 \\
\hline
\end{tabular}

mAU

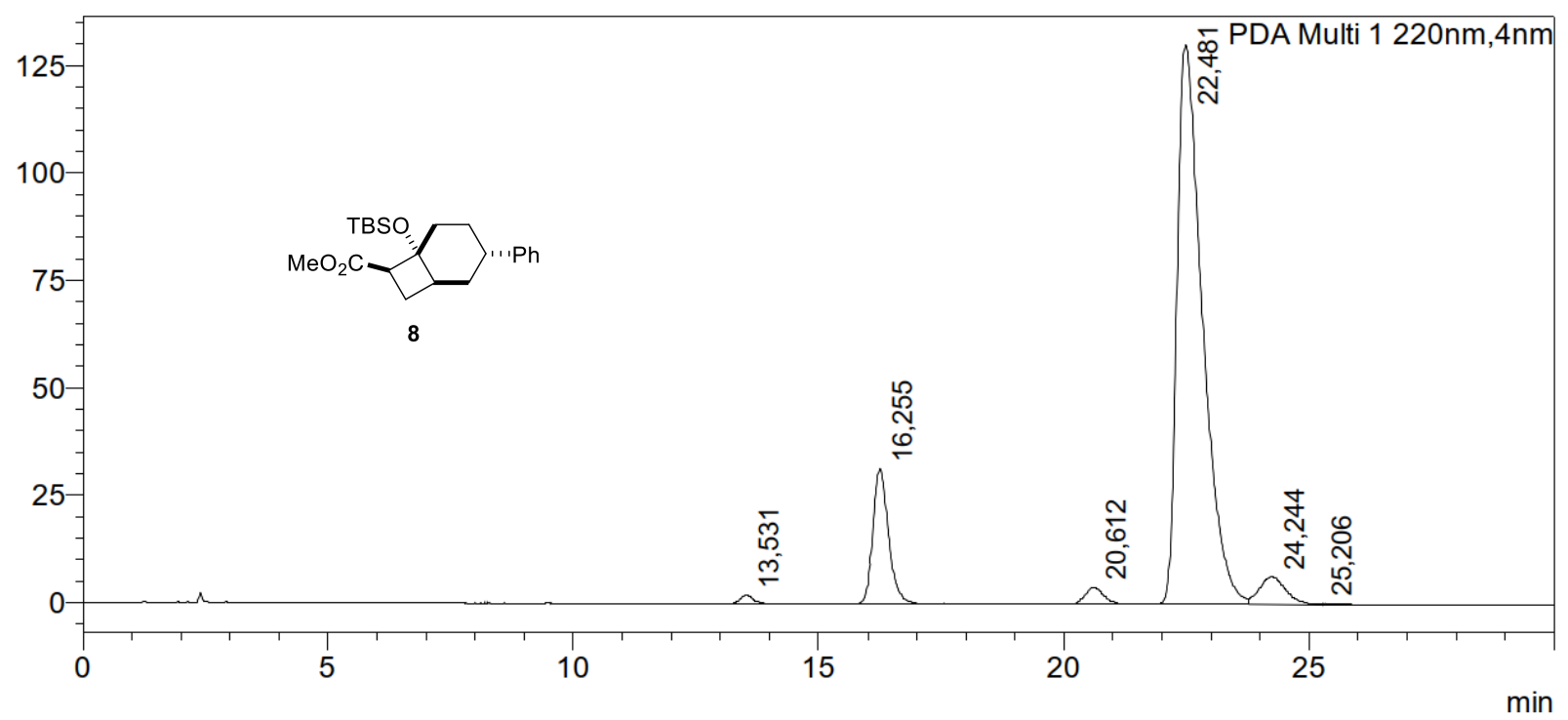

\begin{tabular}{|c|c|c|}
\hline Peak \# & $\mathrm{t}_{\mathrm{R}} / \mathrm{min}$ & \% peak area \\
\hline 1 & 13.5 & 0.57 \\
\hline 2 & 16.2 & 11.92 \\
\hline 3 & 20.6 & 1.83 \\
\hline
\end{tabular}




\begin{tabular}{|l|l|l|}
\hline 4 & 22.5 & 81.3 \\
\hline 5 & 24.2 & 4.23 \\
\hline 6 & 25.2 & 0.15 \\
\hline \multicolumn{2}{|c|}{ Total } & 100 \\
\hline
\end{tabular}

HPLC column: AD-3, $n$-heptane/ $i$-PrOH = 99:1, $1.0 \mathrm{~mL} / \mathrm{min}, 298 \mathrm{~K}, 220 \mathrm{~nm}$. $\mathrm{mAU}$

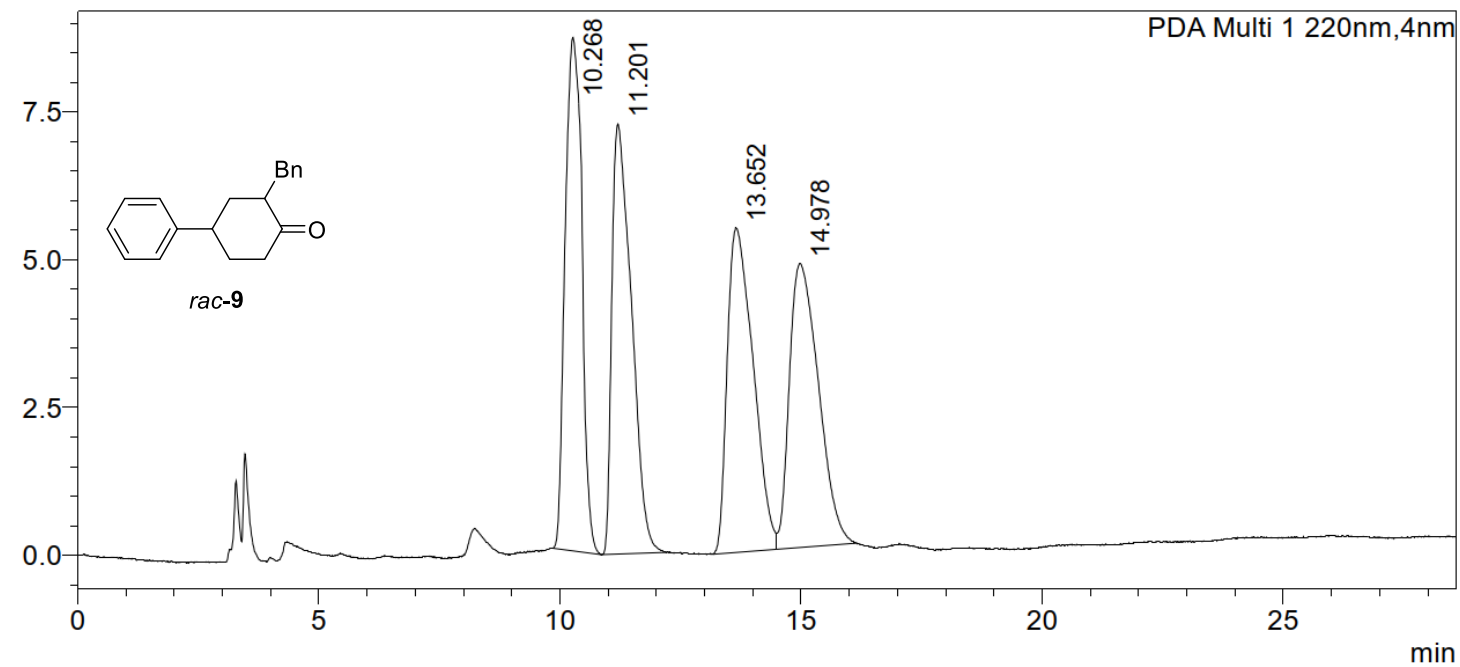

\begin{tabular}{|c|c|c|}
\hline Peak \# & $\mathrm{t}_{\mathrm{R}} / \mathrm{min}$ & $\%$ peak area \\
\hline 1 & 10.3 & 25.51 \\
\hline 2 & 11.2 & 25.04 \\
\hline 3 & 13.6 & 24.90 \\
\hline 4 & 15.0 & 24.55 \\
\hline \multicolumn{2}{|c|}{ Total } & 100 \\
\hline
\end{tabular}

mAU

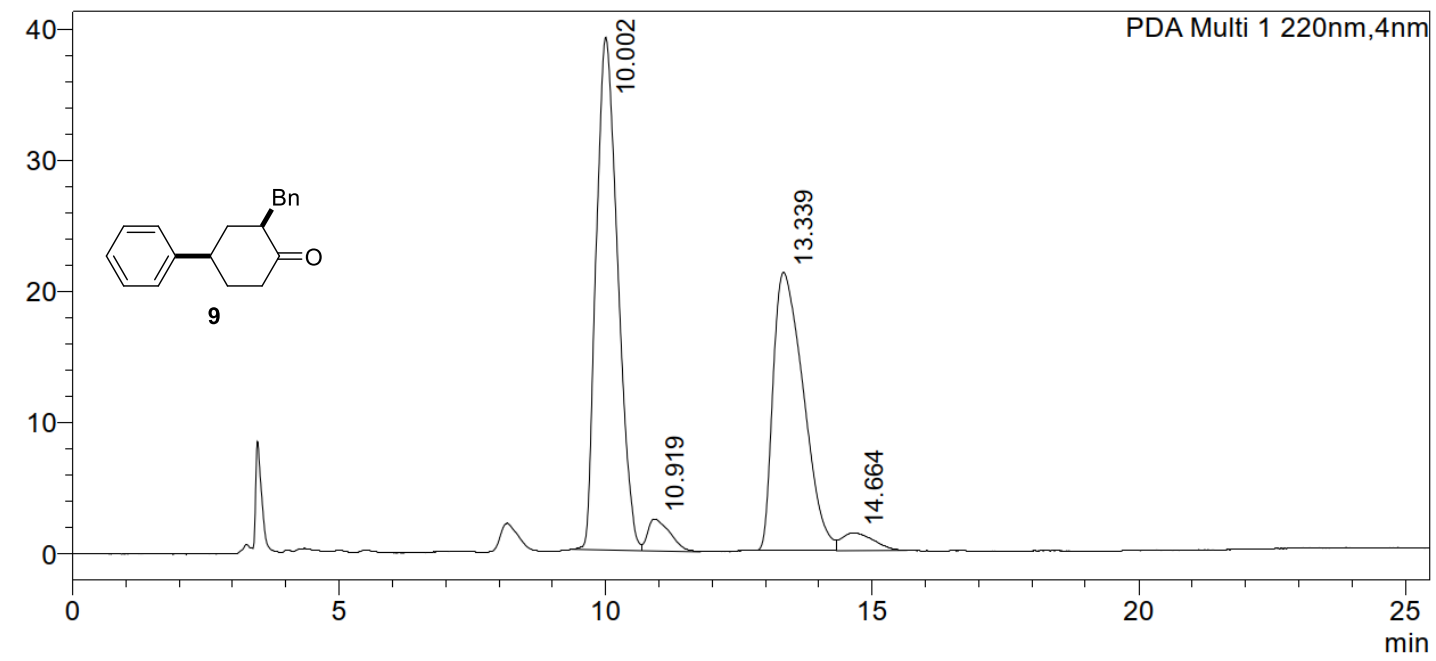

\begin{tabular}{|c|c|c|}
\hline Peak \# & $\mathrm{t}_{\mathrm{R}} / \mathrm{min}$ & $\%$ peak area \\
\hline 1 & 10.0 & 53.23 \\
\hline 2 & 10.9 & 3.40 \\
\hline 3 & 13.3 & 40.62 \\
\hline 4 & 14.7 & 2.75 \\
\hline \multicolumn{2}{|c|}{ Total } & 100 \\
\hline
\end{tabular}


HPLC column: OJ-3R, Acetonitrile: Water $=70: 30,1.0 \mathrm{~mL} / \mathrm{min}, 298 \mathrm{~K}, 220 \mathrm{~nm}$.

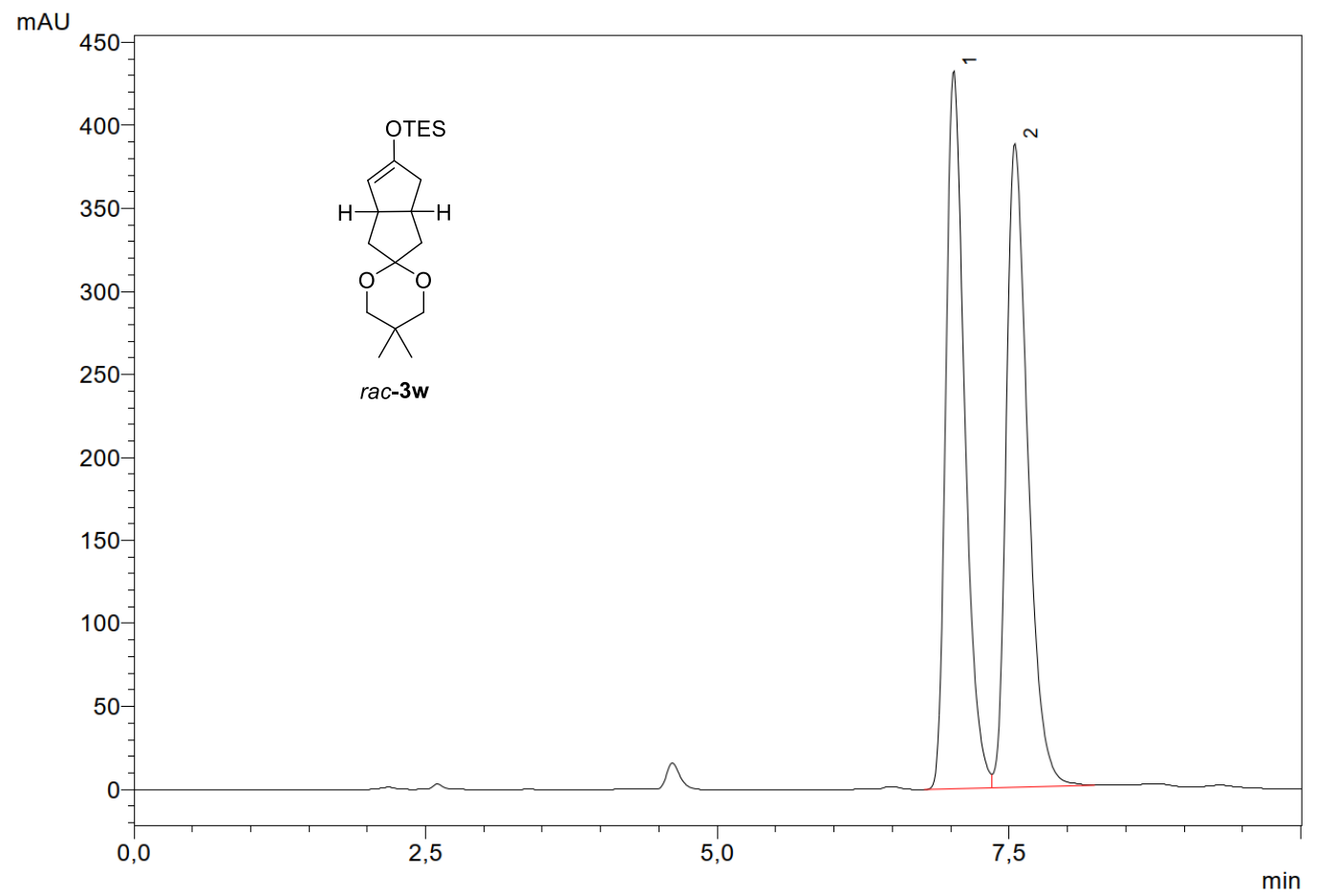

\begin{tabular}{|c|c|c|}
\hline Peak \# & $\mathrm{t}_{\mathrm{R}} / \mathrm{min}$ & \% peak area \\
\hline 1 & 7.0 & 49.51 \\
\hline 2 & 7.6 & 50.49 \\
\hline \multicolumn{2}{|c|}{ Total } & 100 \\
\hline
\end{tabular}

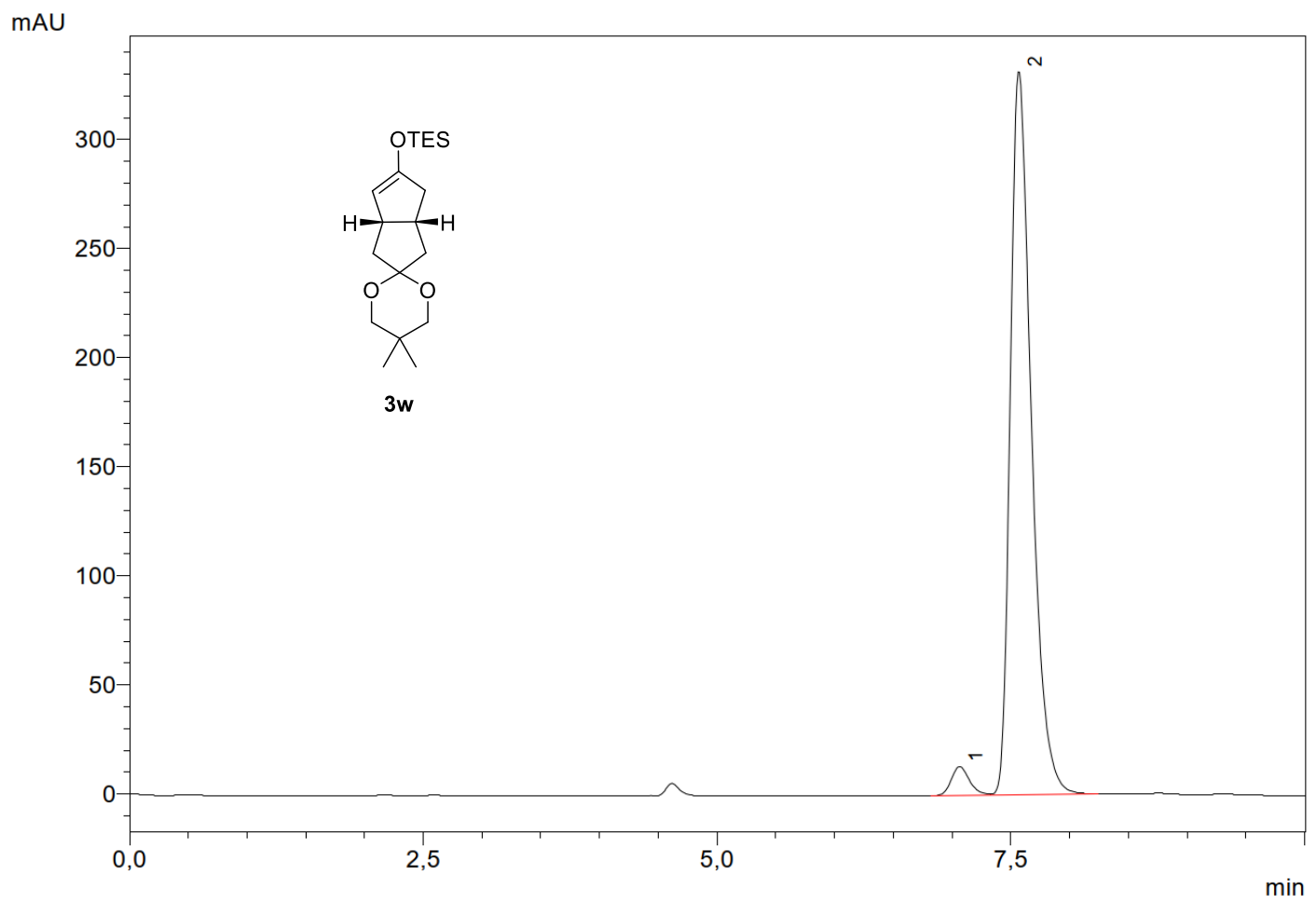

\begin{tabular}{|c|c|c|}
\hline Peak \# & $\mathrm{t}_{\mathrm{R}} / \mathrm{min}$ & \% peak area \\
\hline 1 & 7.1 & 3.31 \\
\hline 2 & 7.6 & 96.69 \\
\hline \multicolumn{2}{|c|}{ Total } & 100 \\
\hline
\end{tabular}


HPLC column: OJ-3R, Acetonitrile: Water $=70: 30,1.0 \mathrm{~mL} / \mathrm{min}, 298 \mathrm{~K}, 220 \mathrm{~nm}$.

mAU

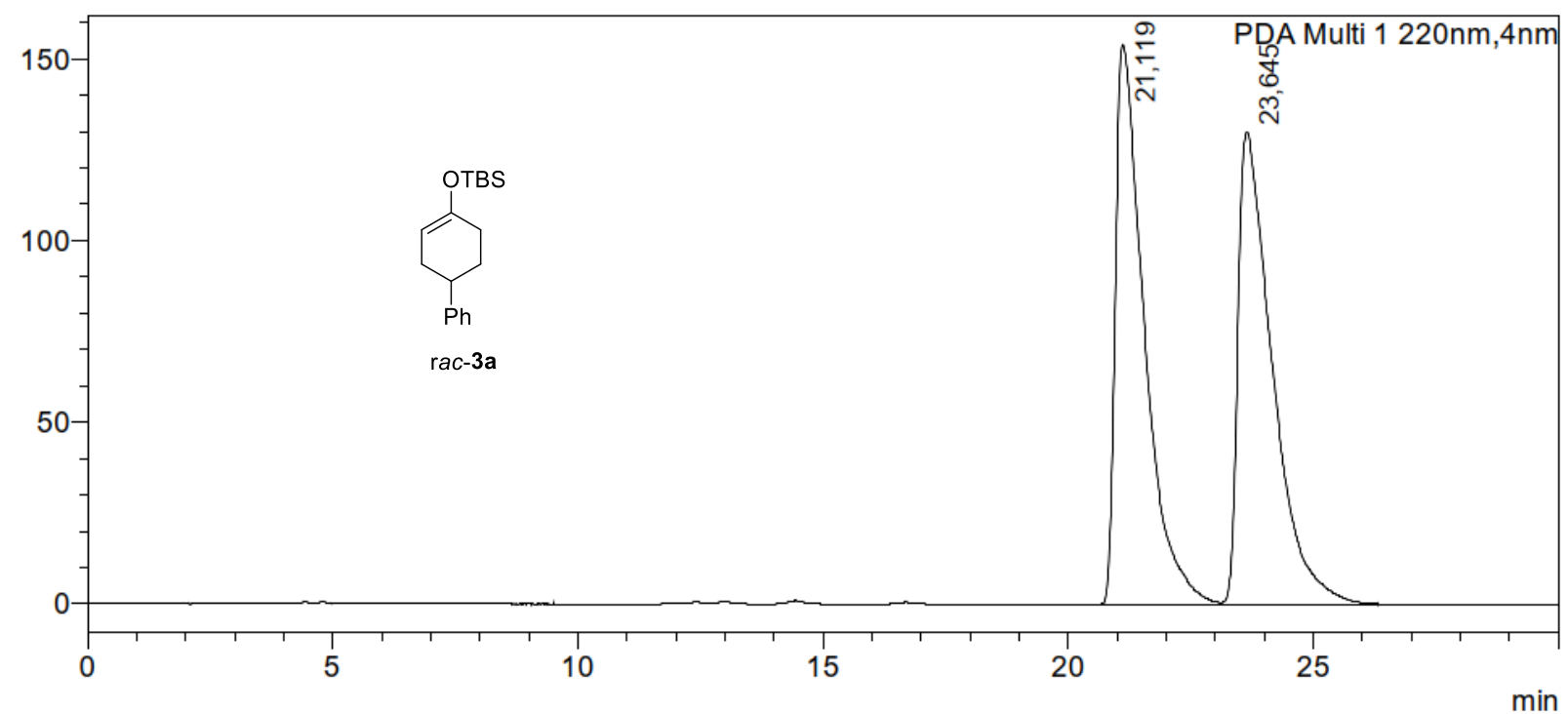

\begin{tabular}{|c|c|c|}
\hline Peak \# & $\mathrm{t}_{\mathrm{R}} / \mathrm{min}$ & \% peak area \\
\hline 1 & 21.1 & 49.99 \\
\hline 2 & 23.6 & 50.01 \\
\hline \multicolumn{3}{|c|}{ Total } \\
\hline
\end{tabular}

mAU

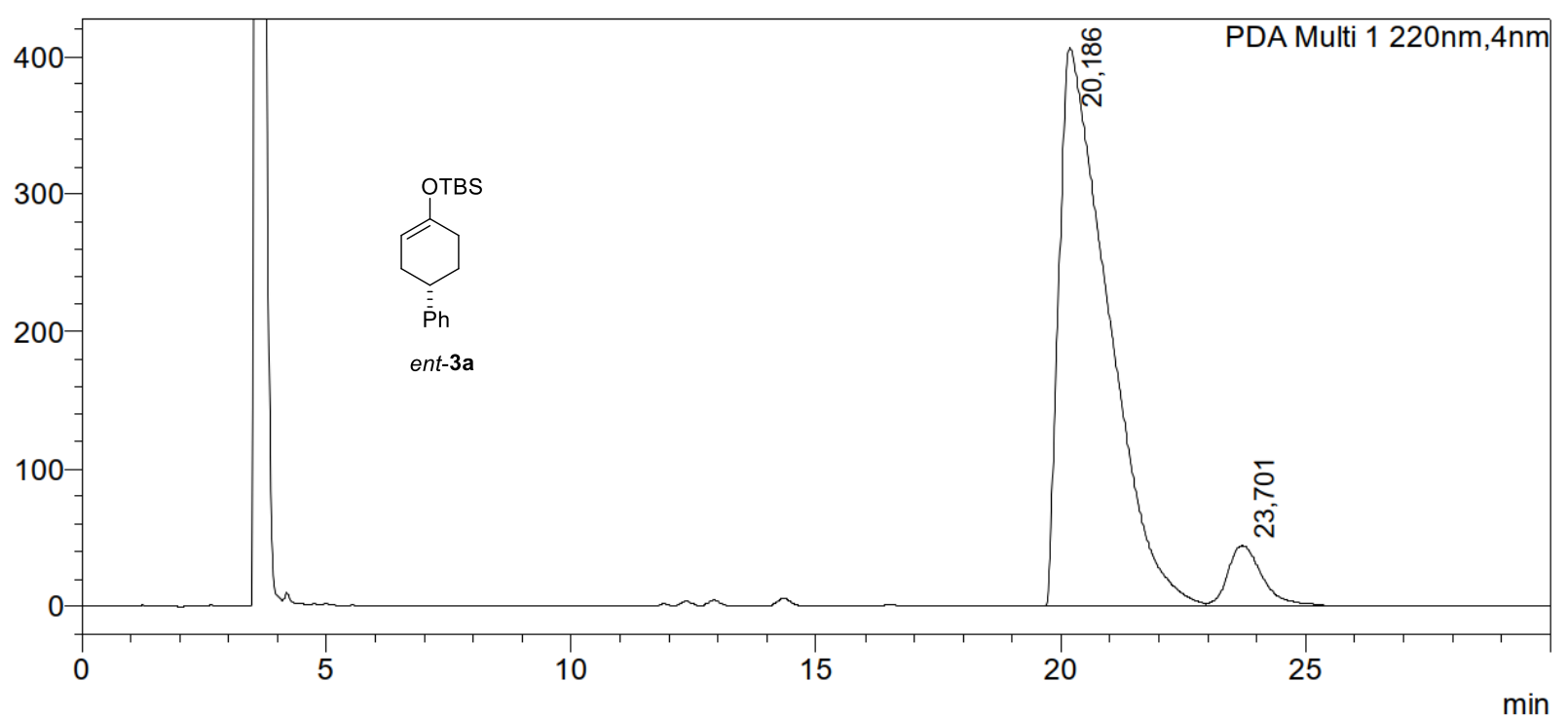

\begin{tabular}{|c|c|c|}
\hline Peak \# & $\mathrm{t}_{\mathrm{R}} / \mathrm{min}$ & $\%$ peak area \\
\hline 1 & 20.2 & 93.87 \\
\hline 2 & 23.7 & 6.13 \\
\hline \multicolumn{3}{|c|}{ Total } \\
\hline
\end{tabular}


HPLC column: OJ-3R, MeOH: Water = 80:20, $1.0 \mathrm{~mL} / \mathrm{min}, 298 \mathrm{~K}, 220 \mathrm{~nm}$.

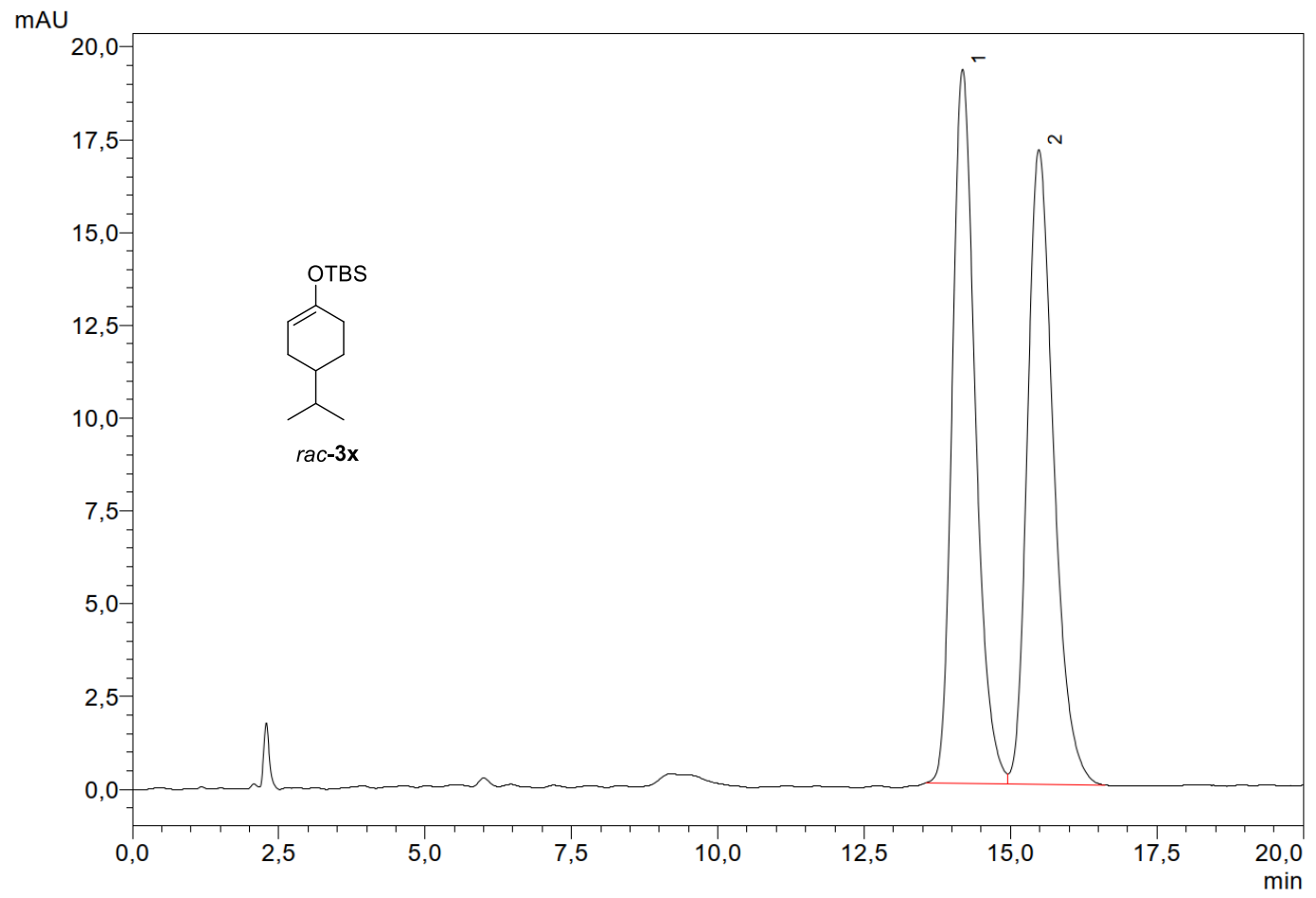

\begin{tabular}{|c|c|c|}
\hline Peak \# & $\mathrm{t}_{\mathrm{R}} / \mathrm{min}$ & $\%$ peak area \\
\hline 1 & 14.2 & 49.81 \\
\hline 2 & 15.5 & 50.19 \\
\hline \multicolumn{2}{|c|}{ Total } & 100 \\
\hline
\end{tabular}

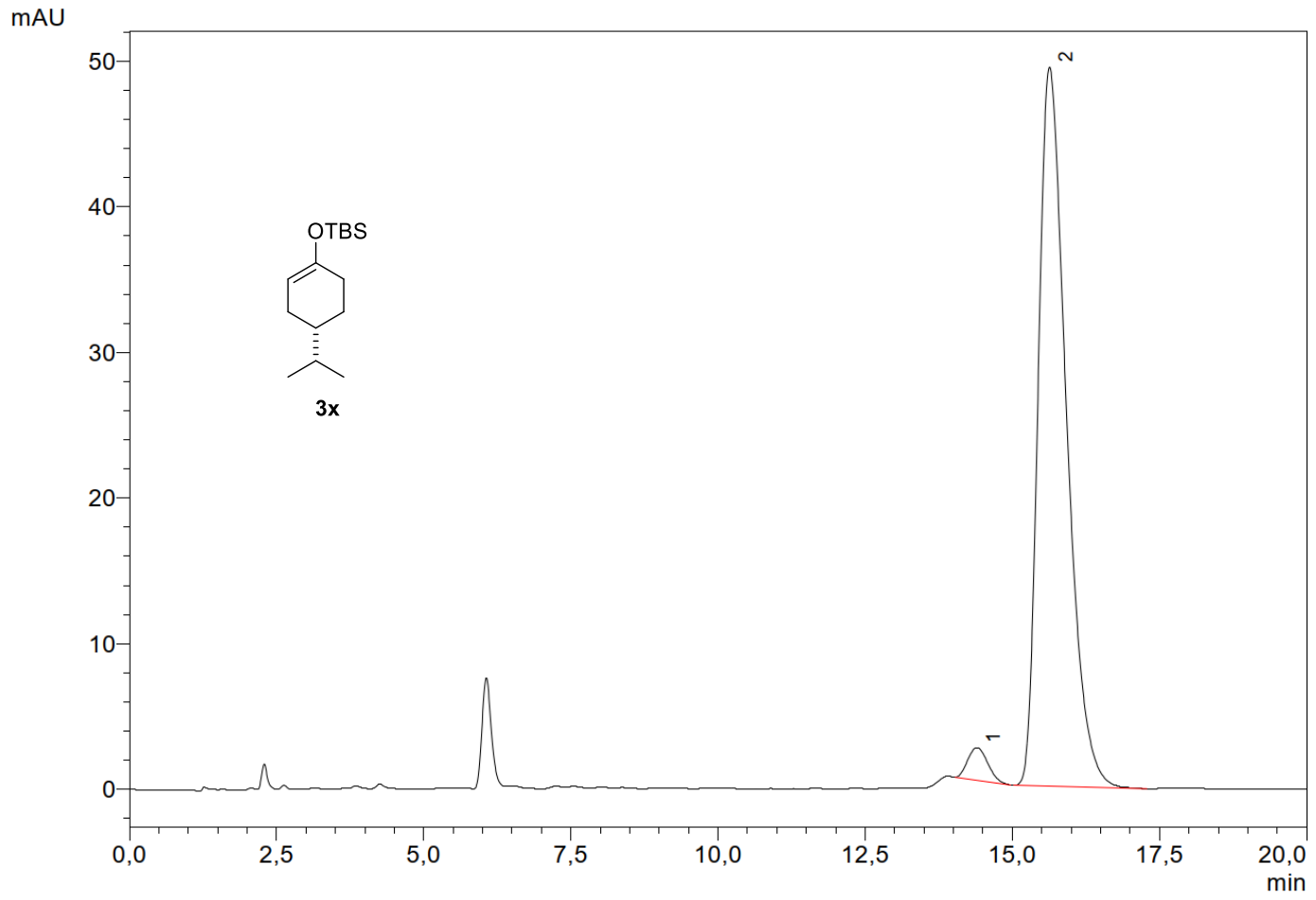

\begin{tabular}{|c|c|c|}
\hline Peak \# & $\mathrm{t}_{\mathrm{R}} / \mathrm{min}$ & $\%$ peak area \\
\hline 1 & 14.4 & 3.15 \\
\hline 2 & 15.6 & 96.85 \\
\hline \multicolumn{2}{|c|}{ Total } & 100 \\
\hline
\end{tabular}


The forward asymmetric silylation was conducted with IDPi $\mathbf{4} \mathbf{c}$ to afford $(R)-\mathbf{3 x}$ under standard reaction conditions, and the e.r. of $(R)$ 3x was determined to be 74:26.

Note: because the HPLC traces were acquired at different time, so calibration of racemate needed.

$\mathrm{mAU}$

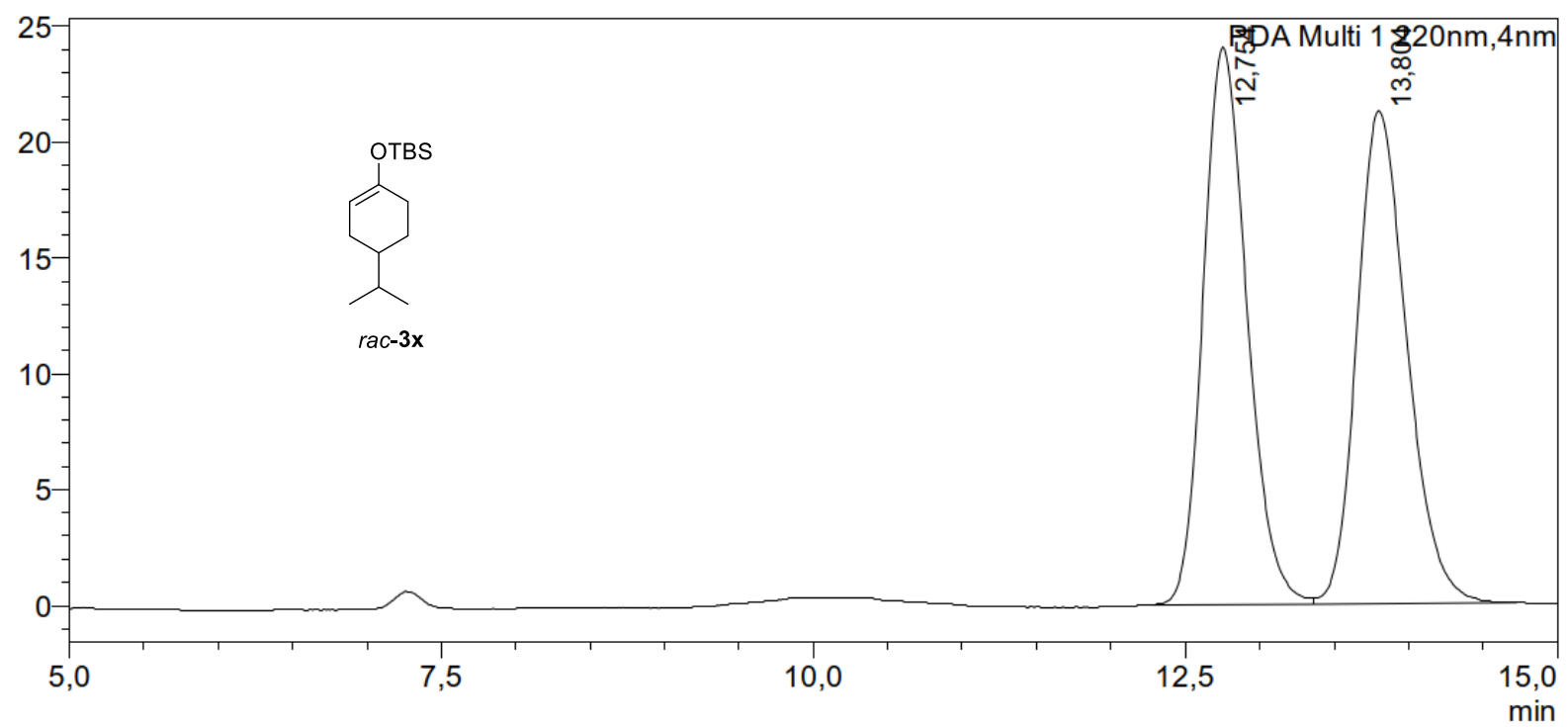

\begin{tabular}{|c|c|c|}
\hline Peak \# & $\mathrm{t}_{\mathrm{R}} / \mathrm{min}$ & $\%$ peak area \\
\hline 1 & 12.8 & 49.85 \\
\hline 2 & 13.8 & 50.15 \\
\hline \multicolumn{2}{|c|}{ Total } & 100 \\
\hline
\end{tabular}

mAU

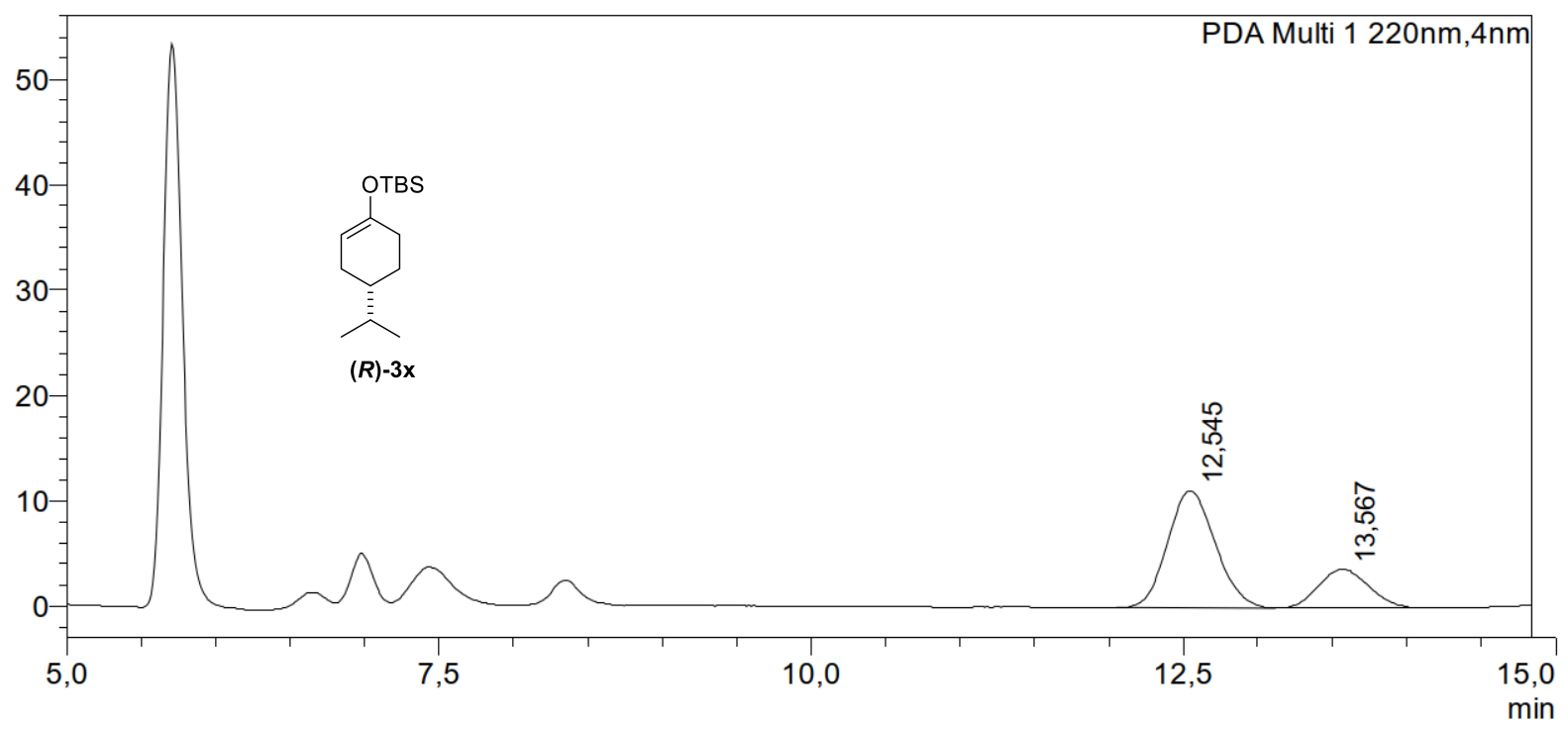

\begin{tabular}{|c|c|c|}
\hline Peak \# & $\mathrm{t}_{\mathrm{R}} / \mathrm{min}$ & $\%$ peak area \\
\hline 1 & 12.5 & 73.84 \\
\hline 2 & 13.6 & 26.16 \\
\hline \multicolumn{2}{|c|}{ Total } & 100 \\
\hline
\end{tabular}


HPLC column: OJ-3R, MeOH: Water $=80: 20,1.0 \mathrm{~mL} / \mathrm{min}, 298 \mathrm{~K}, 220 \mathrm{~nm}$.

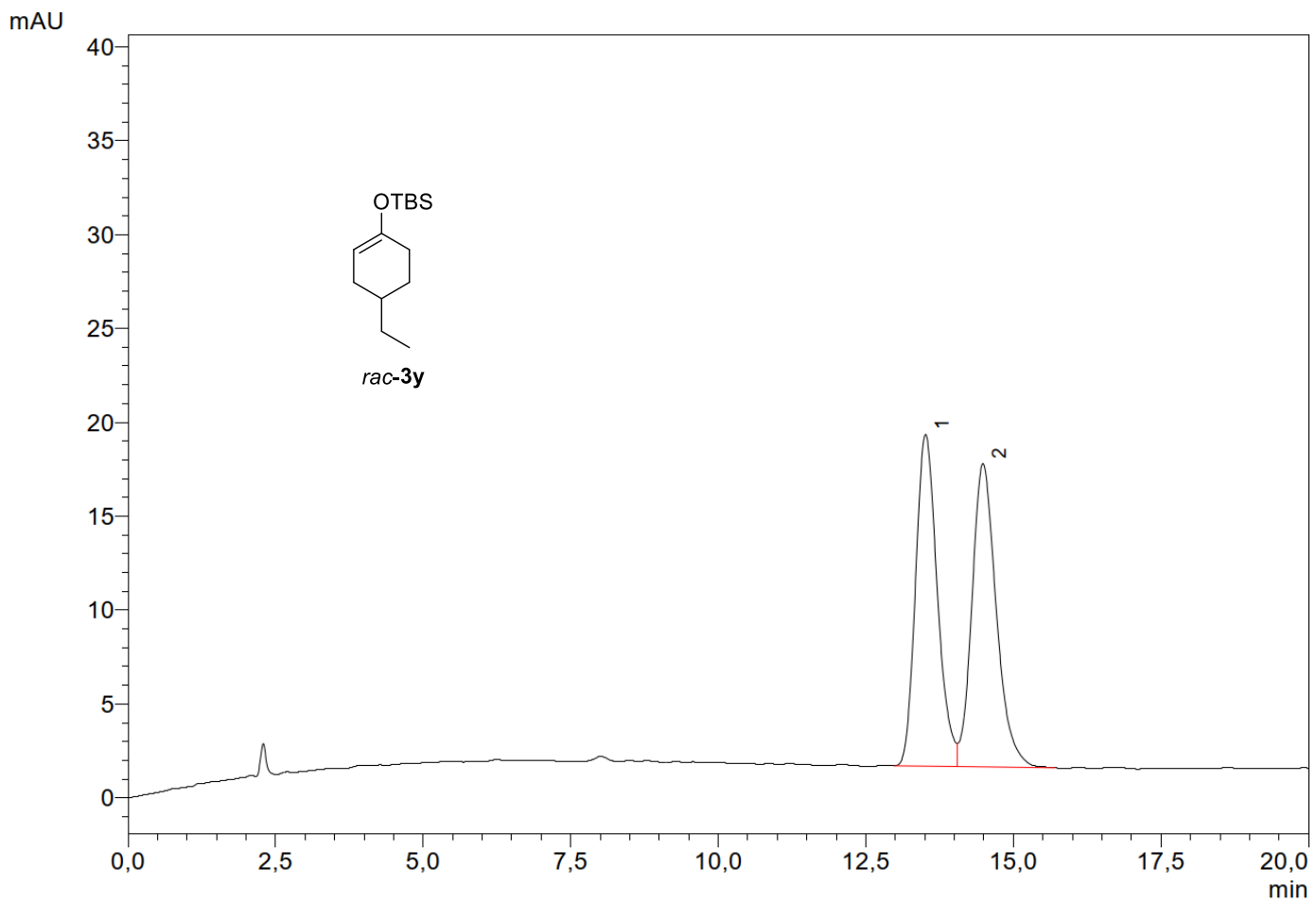

\begin{tabular}{|c|c|c|}
\hline Peak \# & $\mathrm{t}_{\mathrm{R}} / \mathrm{min}$ & \% peak area \\
\hline 1 & 13.5 & 49.33 \\
\hline 2 & 14.5 & 50.67 \\
\hline \multicolumn{2}{|c|}{ Total } & 100 \\
\hline
\end{tabular}

$\mathrm{mAU}$

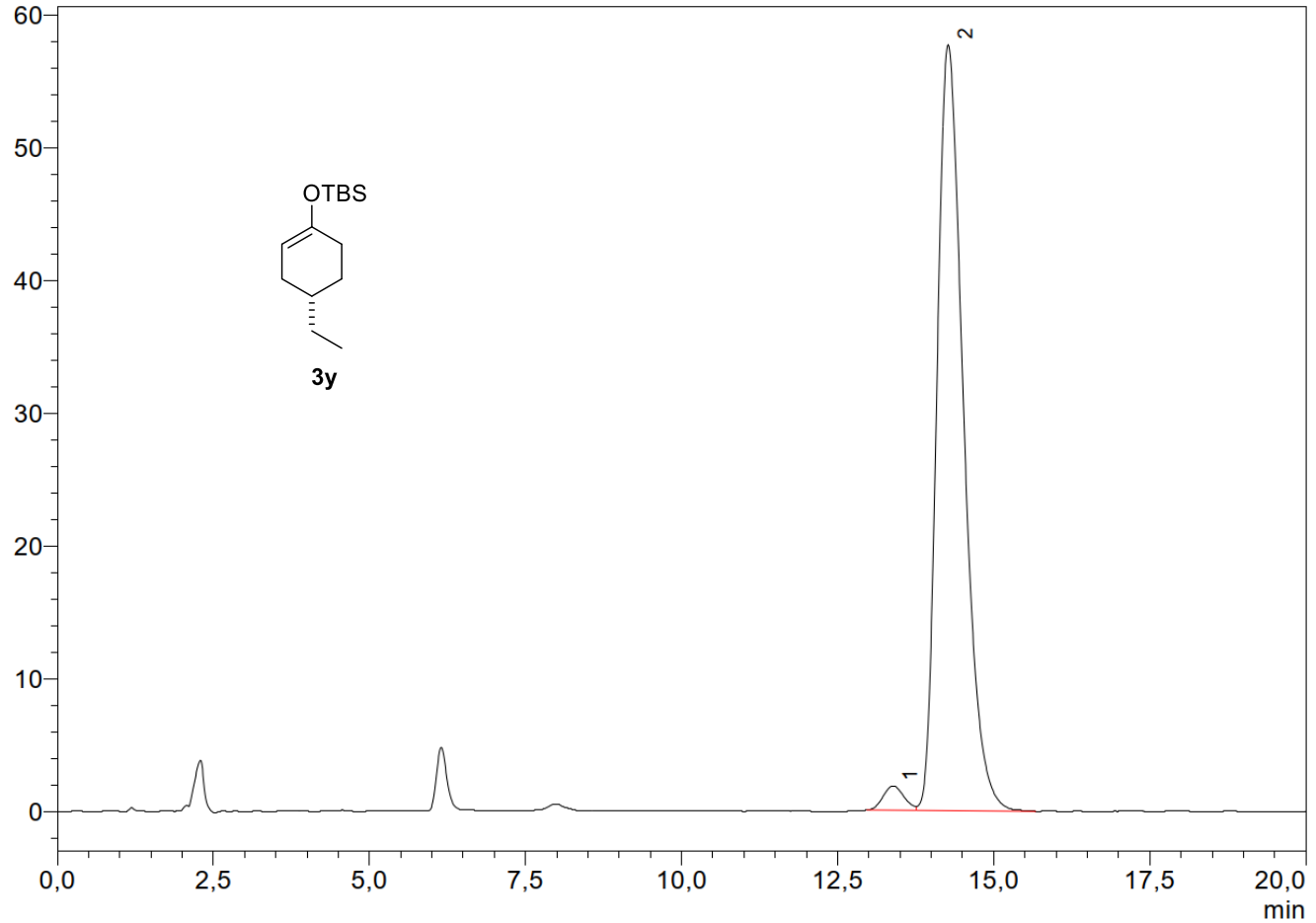

\begin{tabular}{|c|c|c|}
\hline Peak \# & $\mathrm{t}_{\mathrm{R}} / \mathrm{min}$ & \% peak area \\
\hline 1 & 13.4 & 2.46 \\
\hline 2 & 14.3 & 97.54 \\
\hline \multicolumn{2}{|c|}{ Total } & 100 \\
\hline
\end{tabular}


HPLC column: OJ-3R, MeOH: Water = 75:25, $1.0 \mathrm{~mL} / \mathrm{min}, 298 \mathrm{~K}, 220 \mathrm{~nm}$.

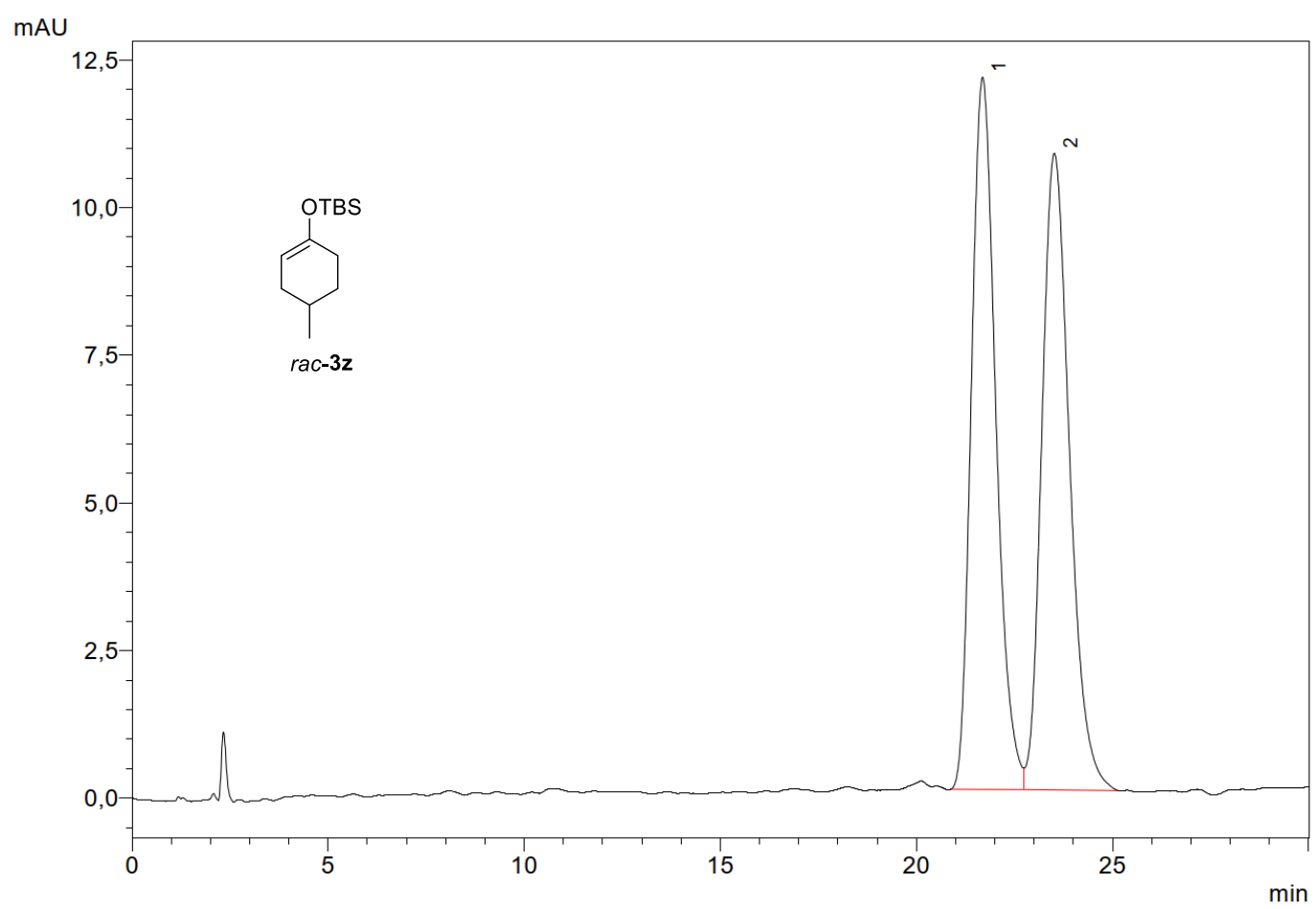

\begin{tabular}{|c|c|c|}
\hline Peak \# & $\mathrm{t}_{\mathrm{R}} / \mathrm{min}$ & $\%$ peak area \\
\hline 1 & 21.7 & 49.73 \\
\hline 2 & 23.5 & 50.27 \\
\hline \multicolumn{2}{|c|}{ Total } & 100 \\
\hline
\end{tabular}

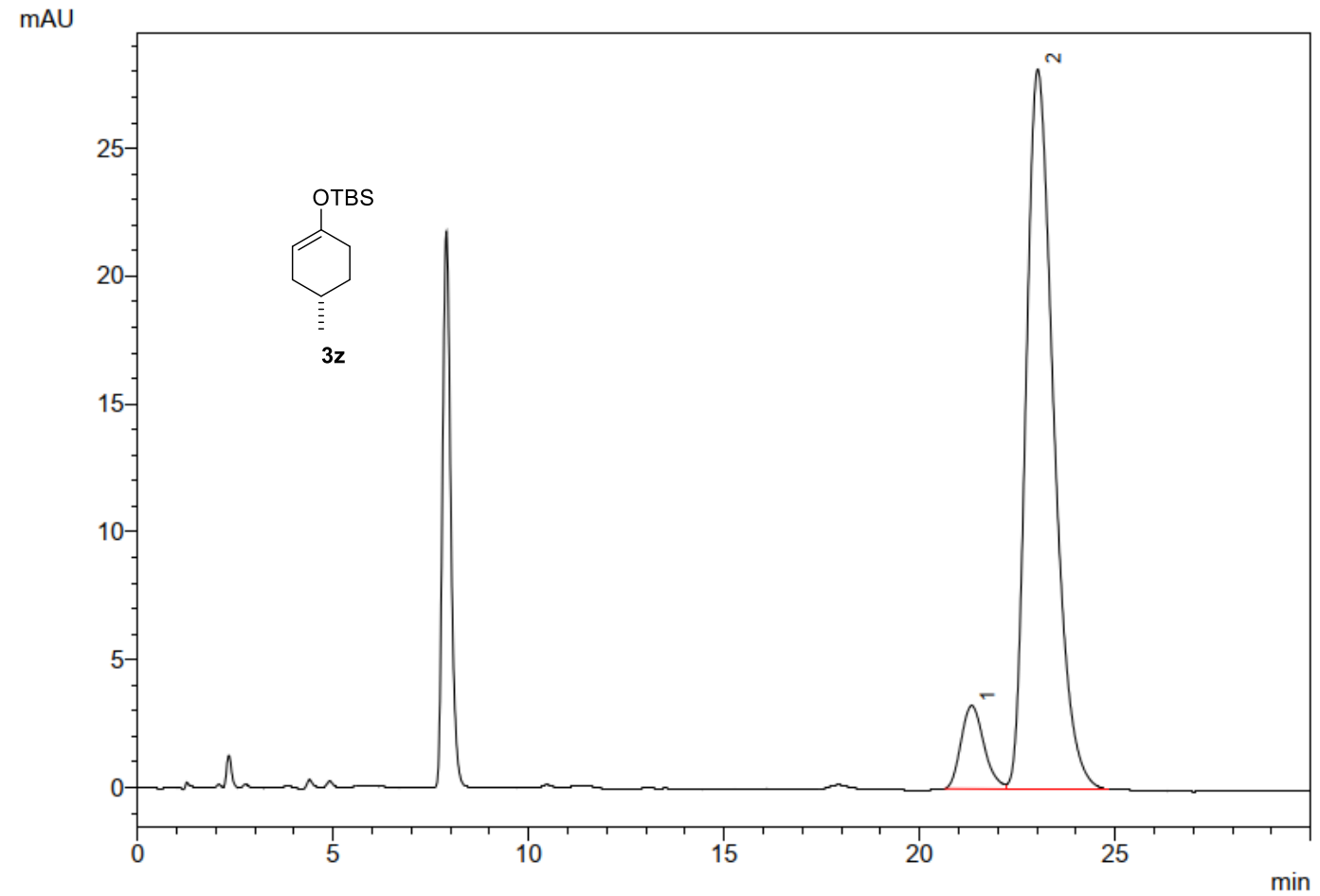

\begin{tabular}{|c|c|c|}
\hline Peak \# & $\mathrm{t}_{\mathrm{R}} / \mathrm{min}$ & $\%$ peak area \\
\hline 1 & 21.3 & 8.81 \\
\hline 2 & 23.0 & 91.19 \\
\hline \multicolumn{2}{|c|}{ Total } & 100 \\
\hline
\end{tabular}


HPLC column: AD-3R, Acetonitrile: Water $=50: 50,1.0 \mathrm{~mL} / \mathrm{min}, 298 \mathrm{~K}, 220 \mathrm{~nm}$.

Note: because the HPLC traces were acquired at different time, so calibration of rac-3t was needed again.

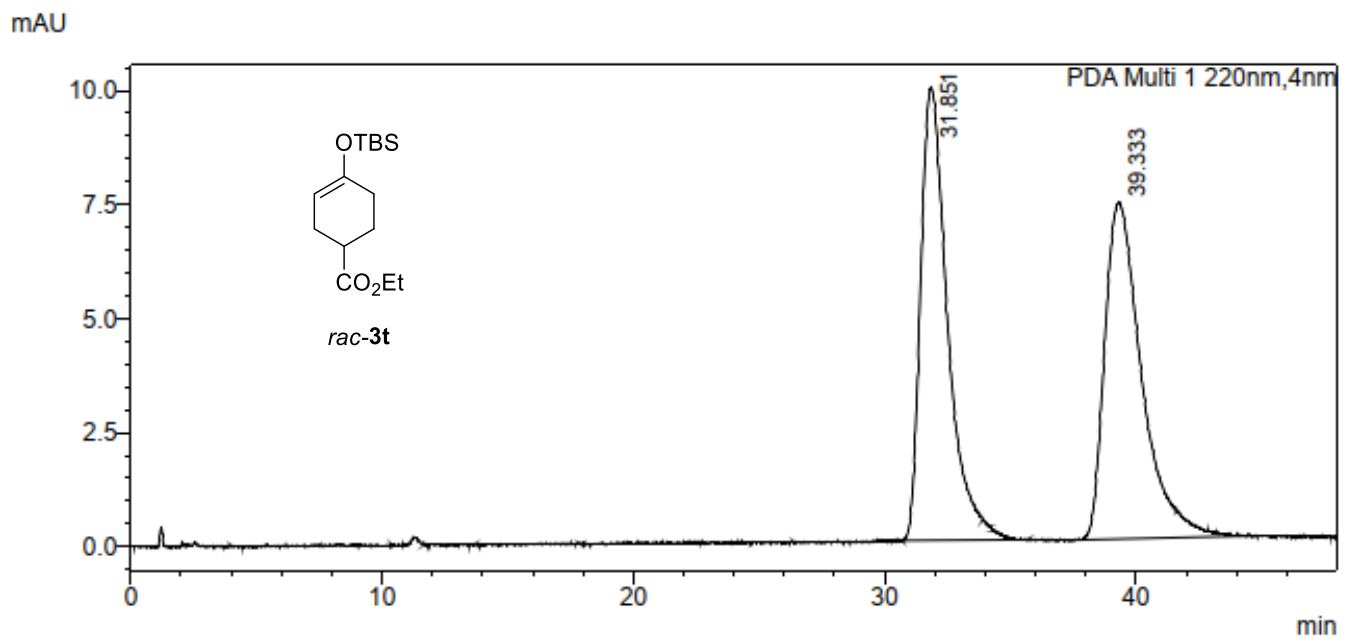

\begin{tabular}{|c|c|c|}
\hline Peak \# & $t_{R} / \min$ & $\%$ peak area \\
\hline 1 & 31.8 & 49.67 \\
\hline 2 & 39.3 & 50.33 \\
\hline \multicolumn{2}{|c|}{ Total } & 100 \\
\hline
\end{tabular}

mAU

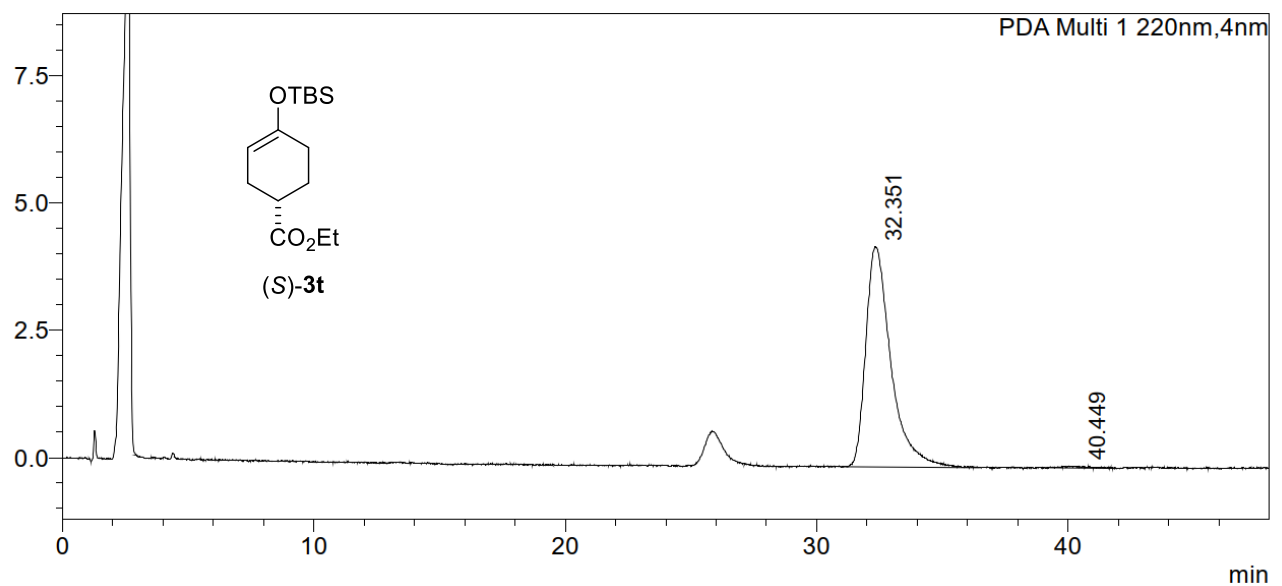

\begin{tabular}{|c|c|c|}
\hline Peak \# & $\mathrm{t}_{\mathrm{R}} / \mathrm{min}$ & $\%$ peak area \\
\hline 1 & 32.3 & 99.22 \\
\hline 2 & 40.4 & 0.78 \\
\hline \multicolumn{2}{|c|}{ Total } & 100 \\
\hline
\end{tabular}


HPLC column: OD-3R, Acetonitrile: Water $=50: 50,1.0 \mathrm{~mL} / \mathrm{min}, 298 \mathrm{~K}, 220 \mathrm{~nm}$.

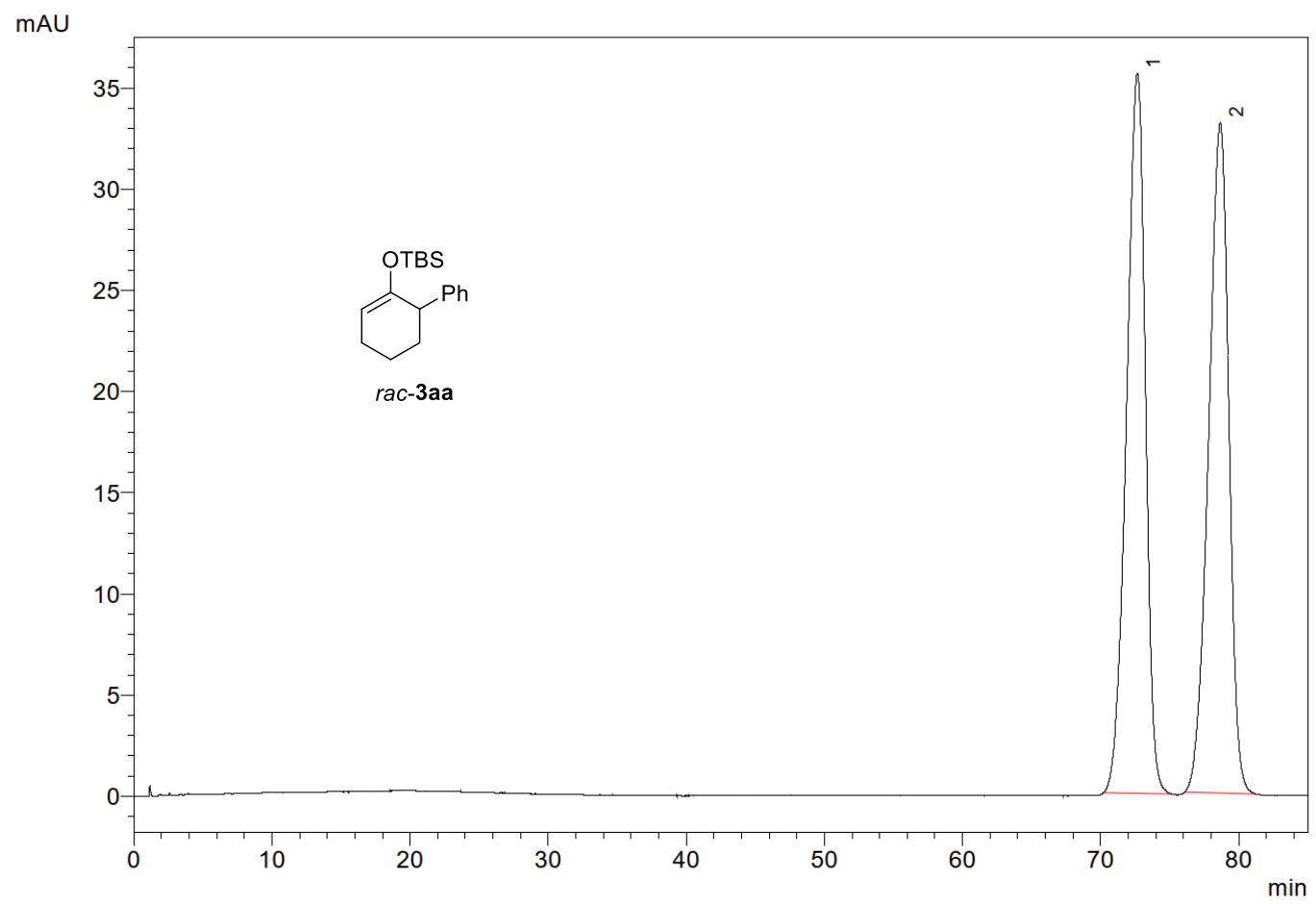

\begin{tabular}{|c|c|c|}
\hline Peak \# & $\mathrm{t}_{\mathrm{R}} / \mathrm{min}$ & \% peak area \\
\hline 1 & 72.7 & 50.06 \\
\hline 2 & 78.7 & 49.94 \\
\hline \multicolumn{2}{|c|}{ Total } & 100 \\
\hline
\end{tabular}

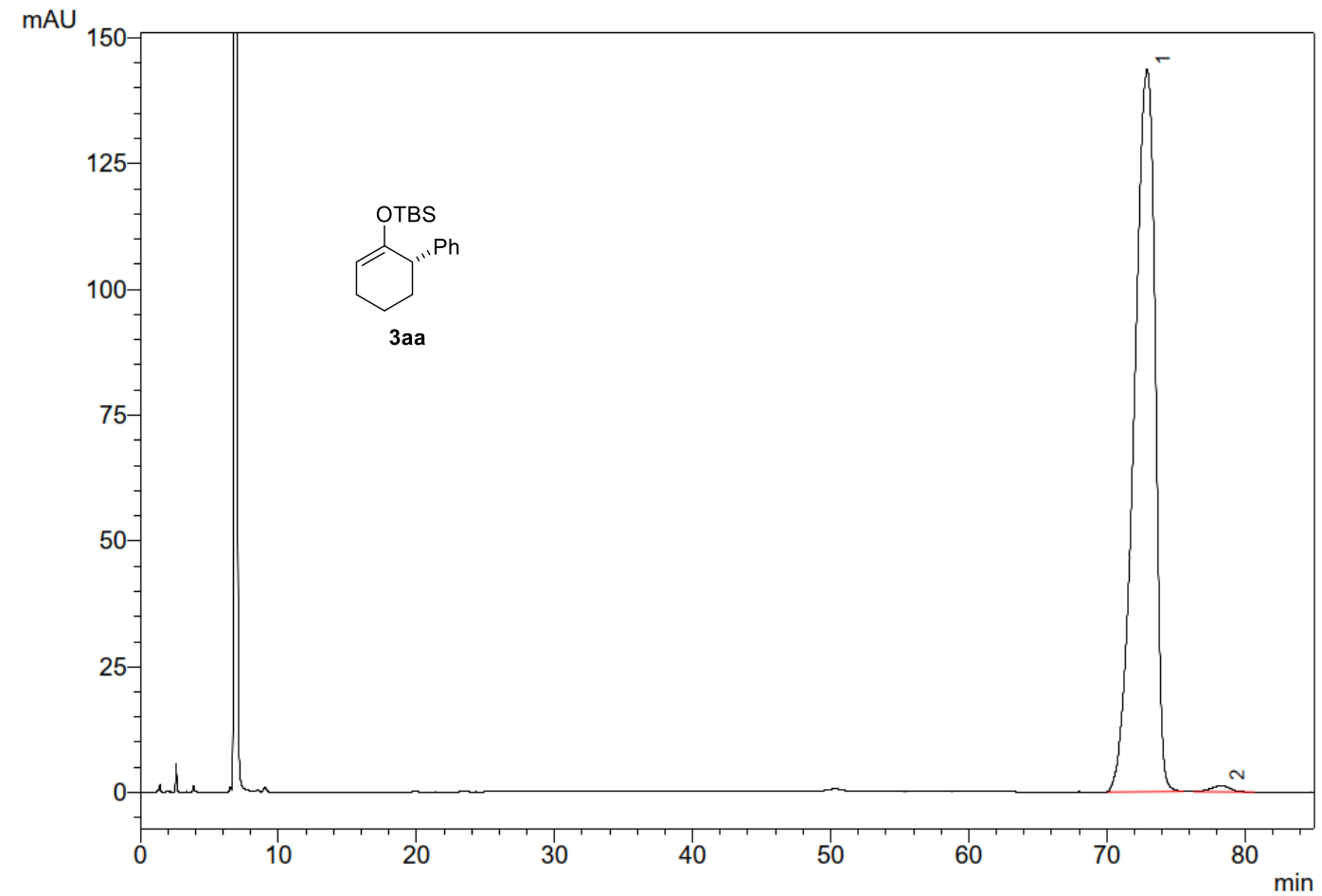

\begin{tabular}{|c|c|c|}
\hline Peak \# & $\mathrm{t}_{\mathrm{R}} / \mathrm{min}$ & \% peak area \\
\hline 1 & 72.9 & 99.25 \\
\hline 2 & 78.3 & 0.75 \\
\hline
\end{tabular}


HPLC column: OD-3, $n$-heptane $/ i-\mathrm{PrOH}=98: 2,1.0 \mathrm{~mL} / \mathrm{min}, 298 \mathrm{~K}, 220 \mathrm{~nm}$. $\mathrm{mAU}$

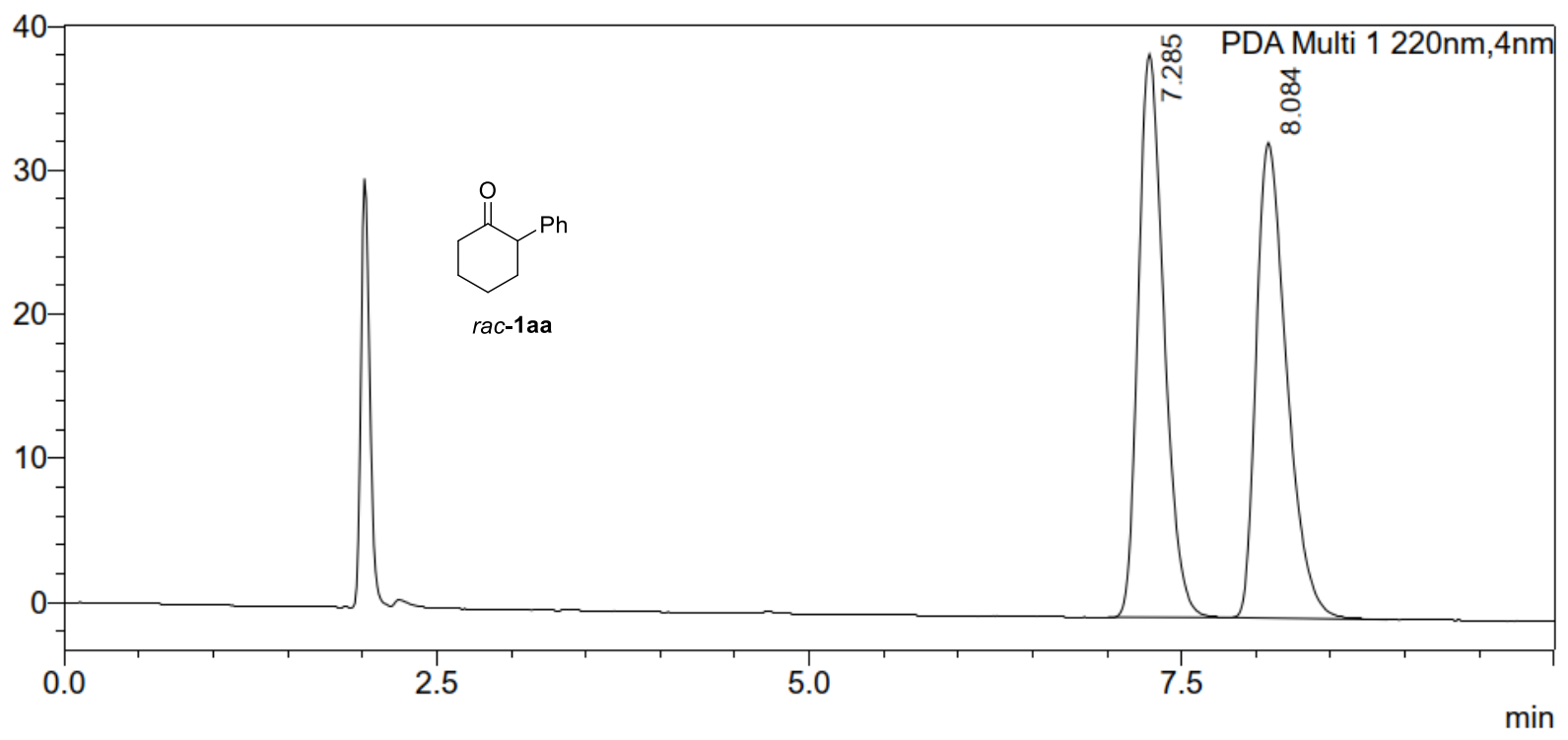

\begin{tabular}{|c|c|c|}
\hline Peak \# & $\mathrm{t}_{\mathrm{R}} / \mathrm{min}$ & $\%$ peak area \\
\hline 1 & 7.3 & 49.99 \\
\hline 2 & 8.1 & 50.01 \\
\hline \multicolumn{2}{|c|}{ Total } & 100 \\
\hline
\end{tabular}

$\mathrm{mAU}$

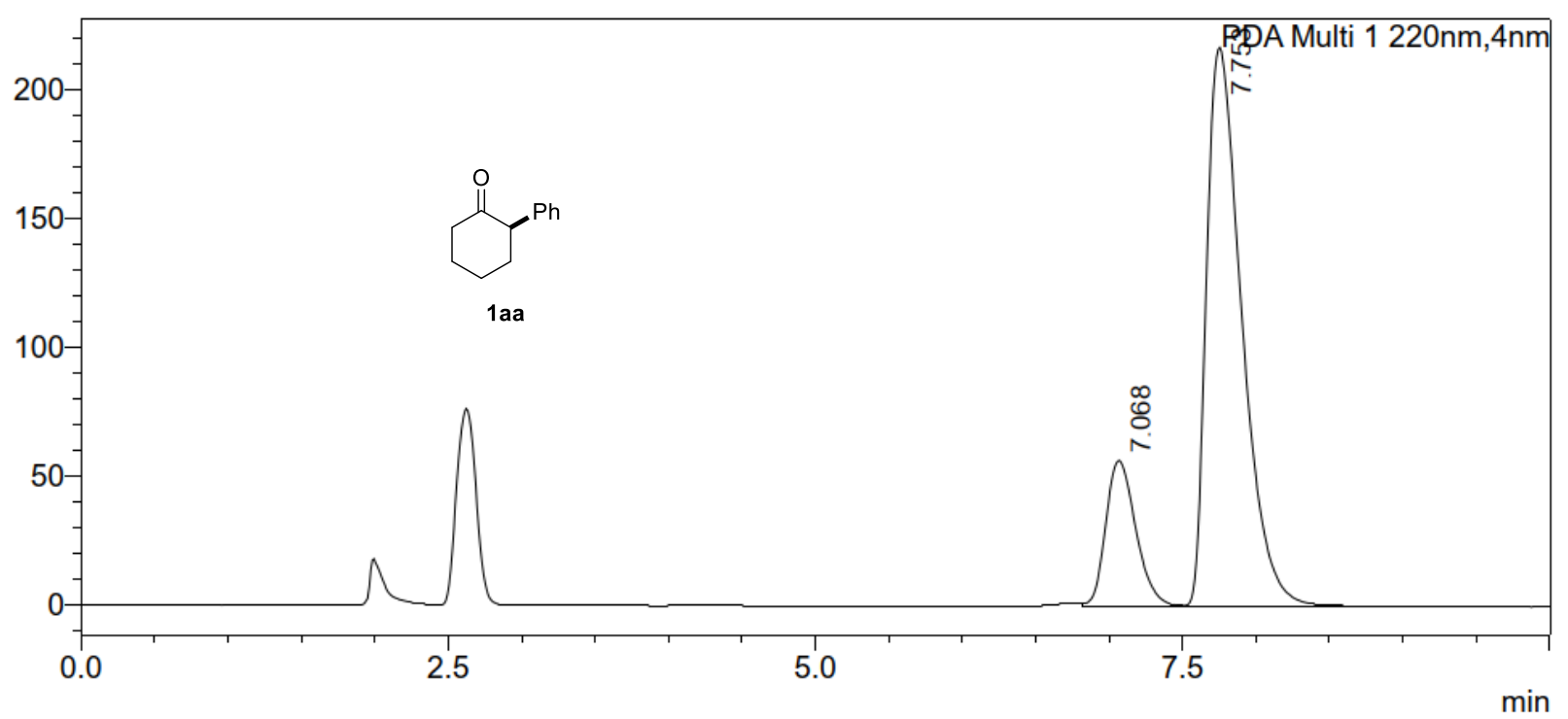

\begin{tabular}{|c|c|c|}
\hline Peak \# & $\mathrm{t}_{\mathrm{R}} / \mathrm{min}$ & $\%$ peak area \\
\hline 1 & 7.1 & 17.45 \\
\hline 2 & 7.8 & 82.55 \\
\hline \multicolumn{2}{|c|}{ Total } & 100 \\
\hline
\end{tabular}


HPLC column: OJ-3R, MeOH: Water= 80:20, $1.0 \mathrm{~mL} / \mathrm{min}, 298 \mathrm{~K}, 220 \mathrm{~nm}$.

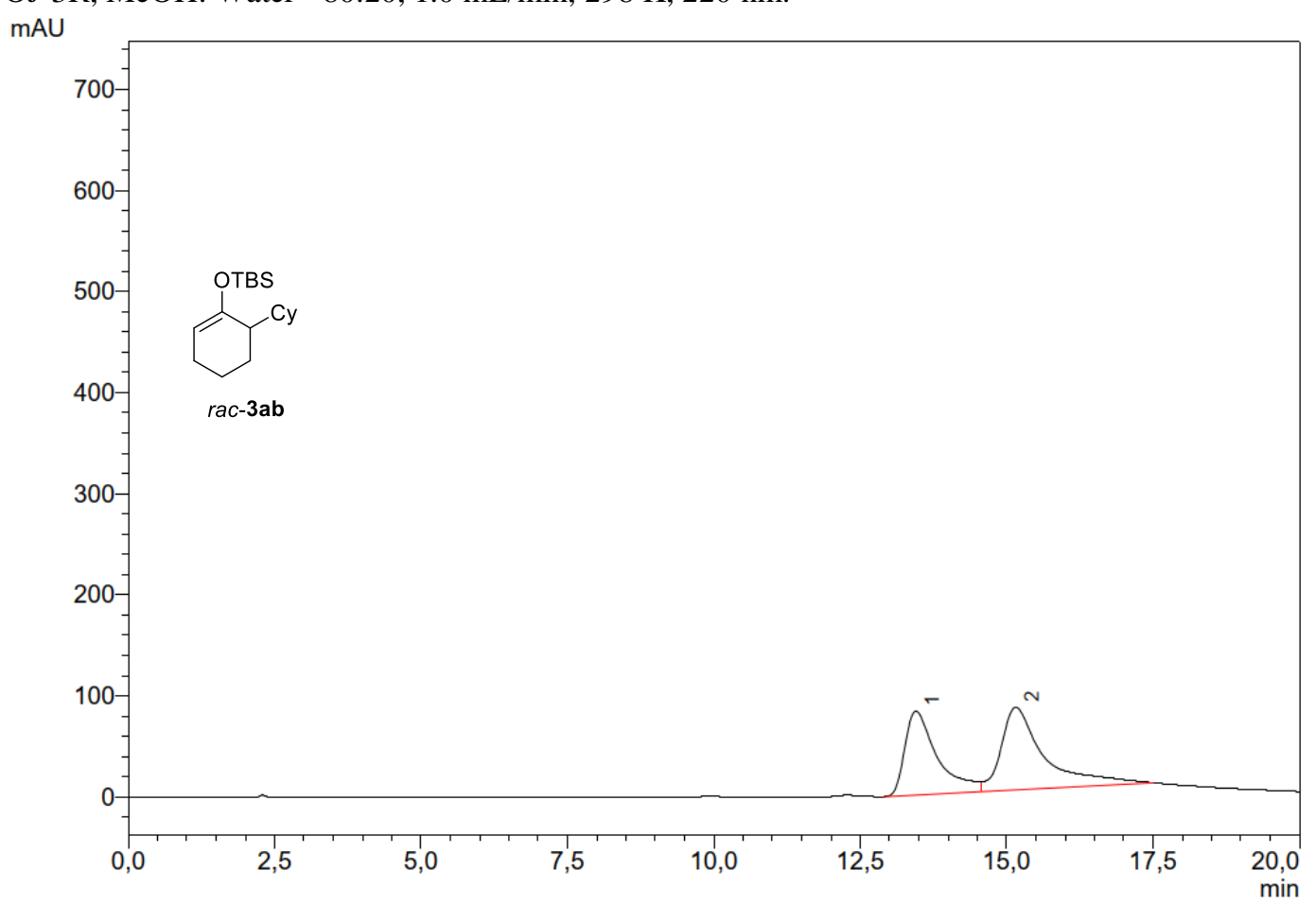

\begin{tabular}{|c|c|c|}
\hline Peak \# & $t_{R} / \min$ & $\%$ peak area \\
\hline 1 & 13.4 & 49.17 \\
\hline 2 & 15.2 & 50.83 \\
\hline \multicolumn{2}{|c|}{ Total } & 100 \\
\hline
\end{tabular}




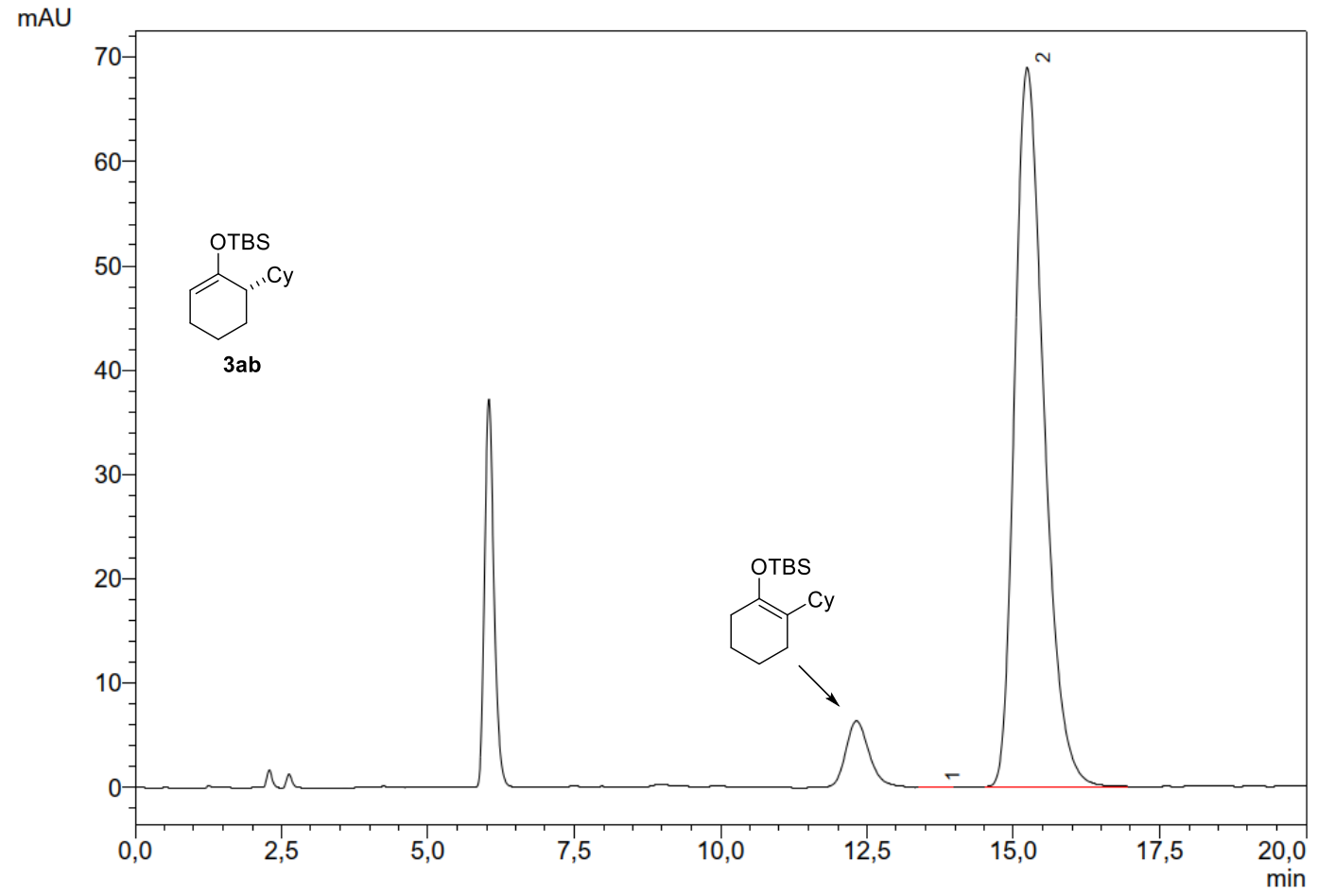

\begin{tabular}{|c|c|c|}
\hline Peak \# & $\mathrm{t}_{\mathrm{R}} / \mathrm{min}$ & $\%$ peak area \\
\hline 1 & 13.7 & 0.03 \\
\hline 2 & 15.2 & 99.97 \\
\hline \multicolumn{2}{|c|}{ Total } & 100 \\
\hline
\end{tabular}

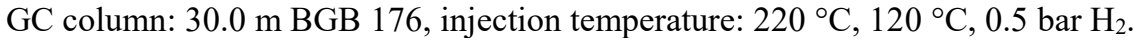

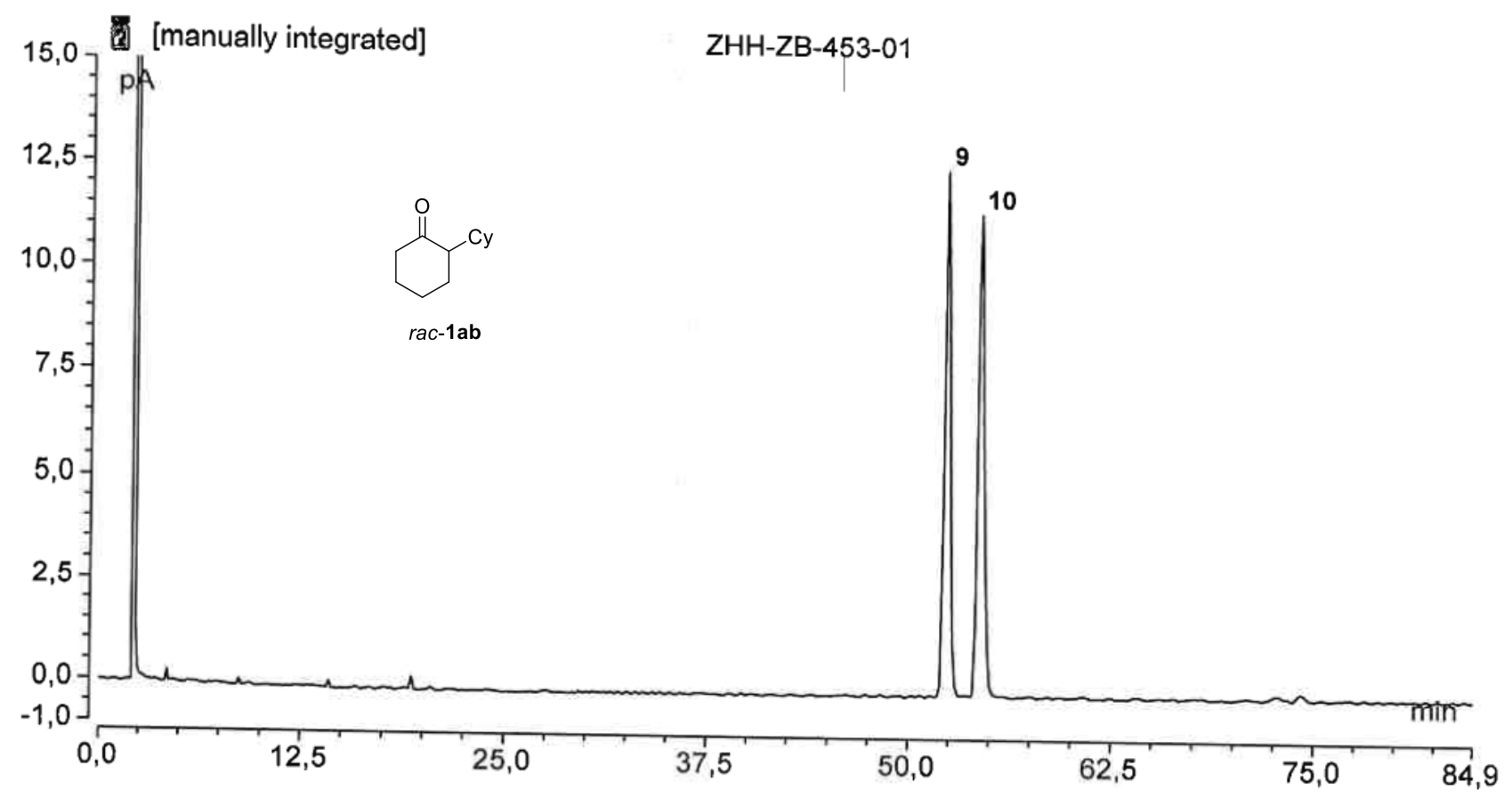

\begin{tabular}{|c|c|c|}
\hline Peak \# & $\mathrm{t}_{\mathrm{R}} / \mathrm{min}$ & \% peak area \\
\hline 1 & 52.3 & 49.99 \\
\hline 2 & 54.4 & 50.01 \\
\hline \multicolumn{2}{|c|}{ Total } & 100 \\
\hline
\end{tabular}




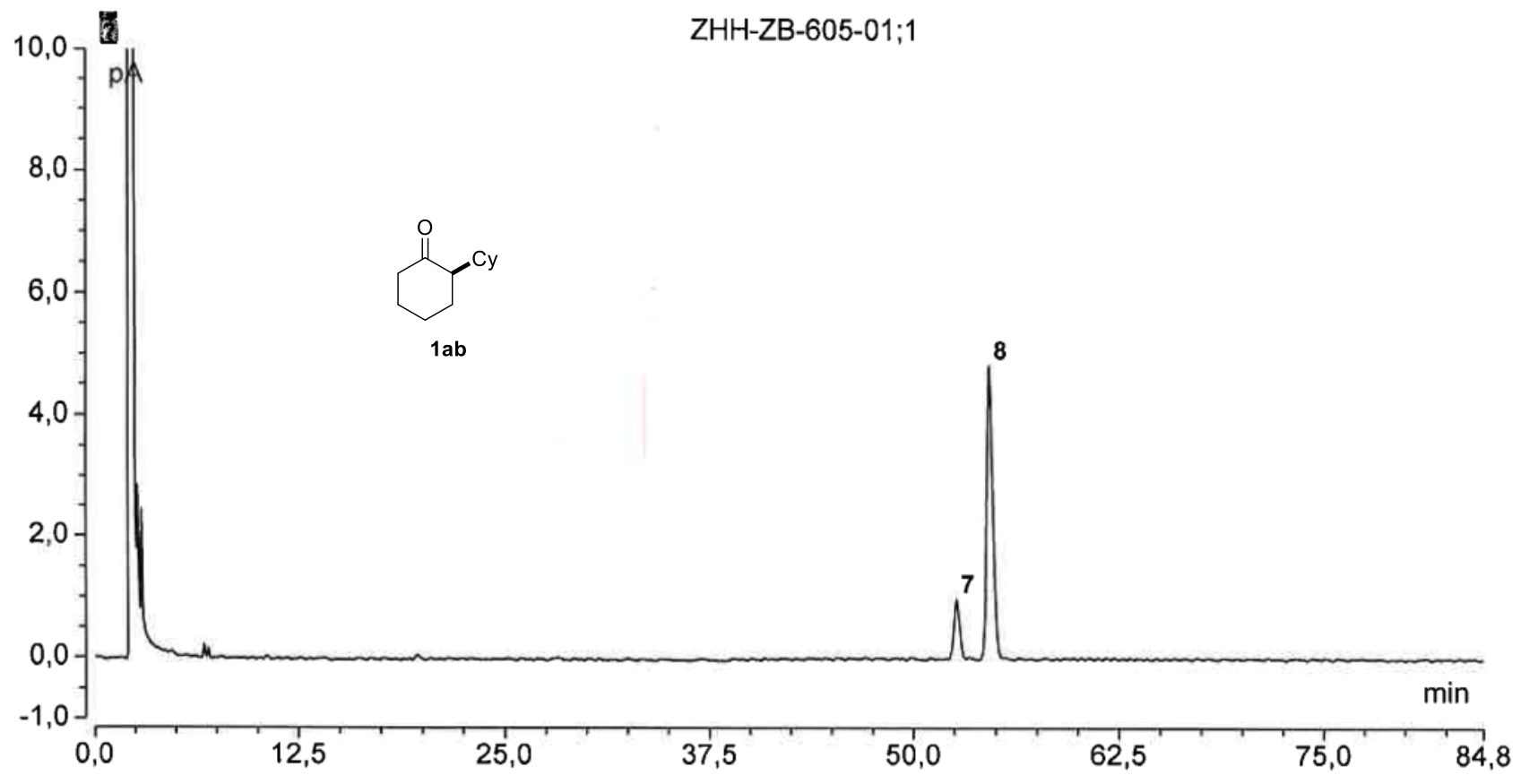

\begin{tabular}{|c|c|c|}
\hline Peak \# & $\mathrm{t}_{\mathrm{R}} / \mathrm{min}$ & $\%$ peak area \\
\hline 1 & 52.6 & 15.43 \\
\hline 2 & 54.6 & 84.57 \\
\hline \multicolumn{2}{|c|}{ Total } & 100 \\
\hline
\end{tabular}

HPLC column: OJ-3R, Acetonitrile: Water $=80: 20,1.0 \mathrm{~mL} / \mathrm{min}, 298 \mathrm{~K}, 220 \mathrm{~nm}$.

mAU

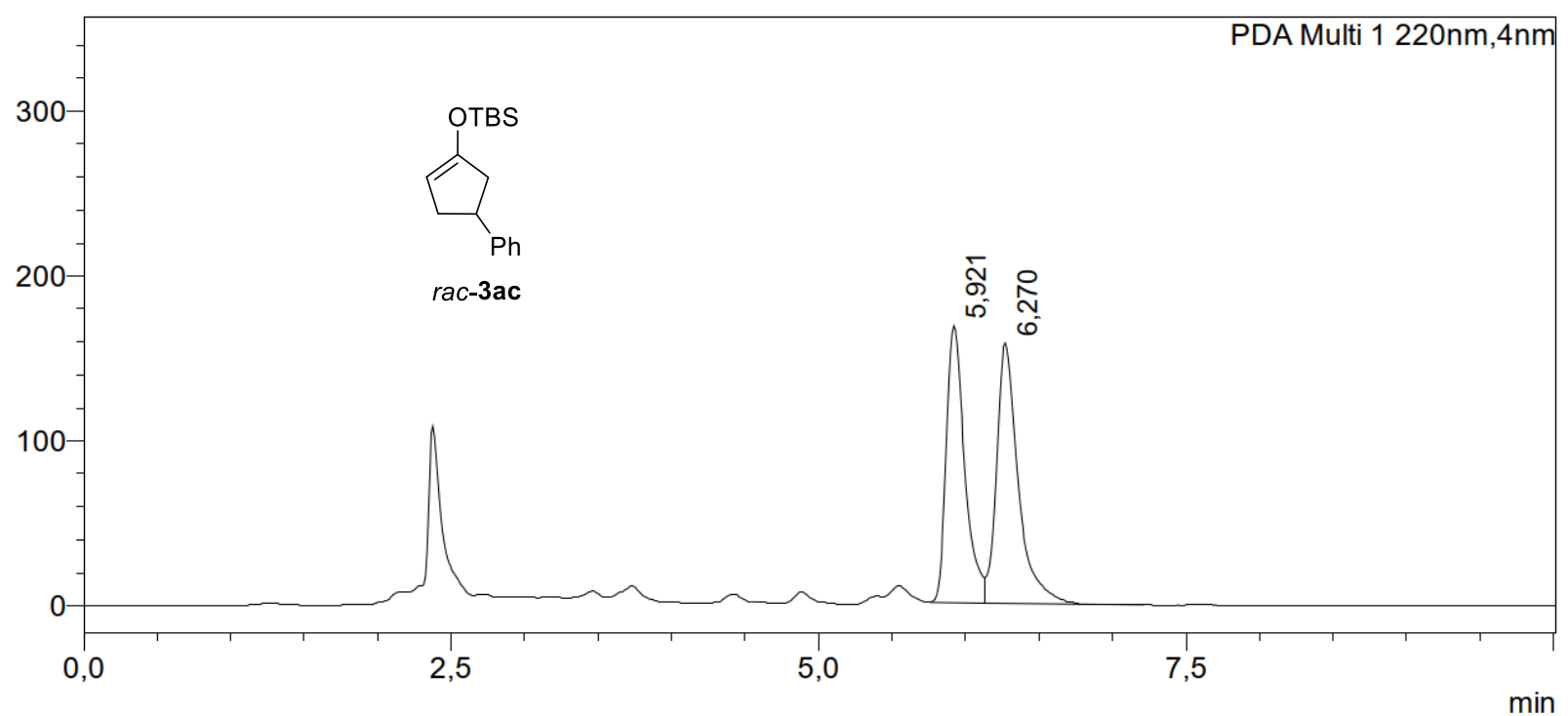

\begin{tabular}{|c|c|c|}
\hline Peak \# & $\mathrm{t}_{\mathrm{R}} / \mathrm{min}$ & $\%$ peak area \\
\hline 1 & 5.9 & 47.63 \\
\hline 2 & 6.3 & 52.37 \\
\hline \multicolumn{2}{|c|}{ Total } & 100 \\
\hline
\end{tabular}


mAU

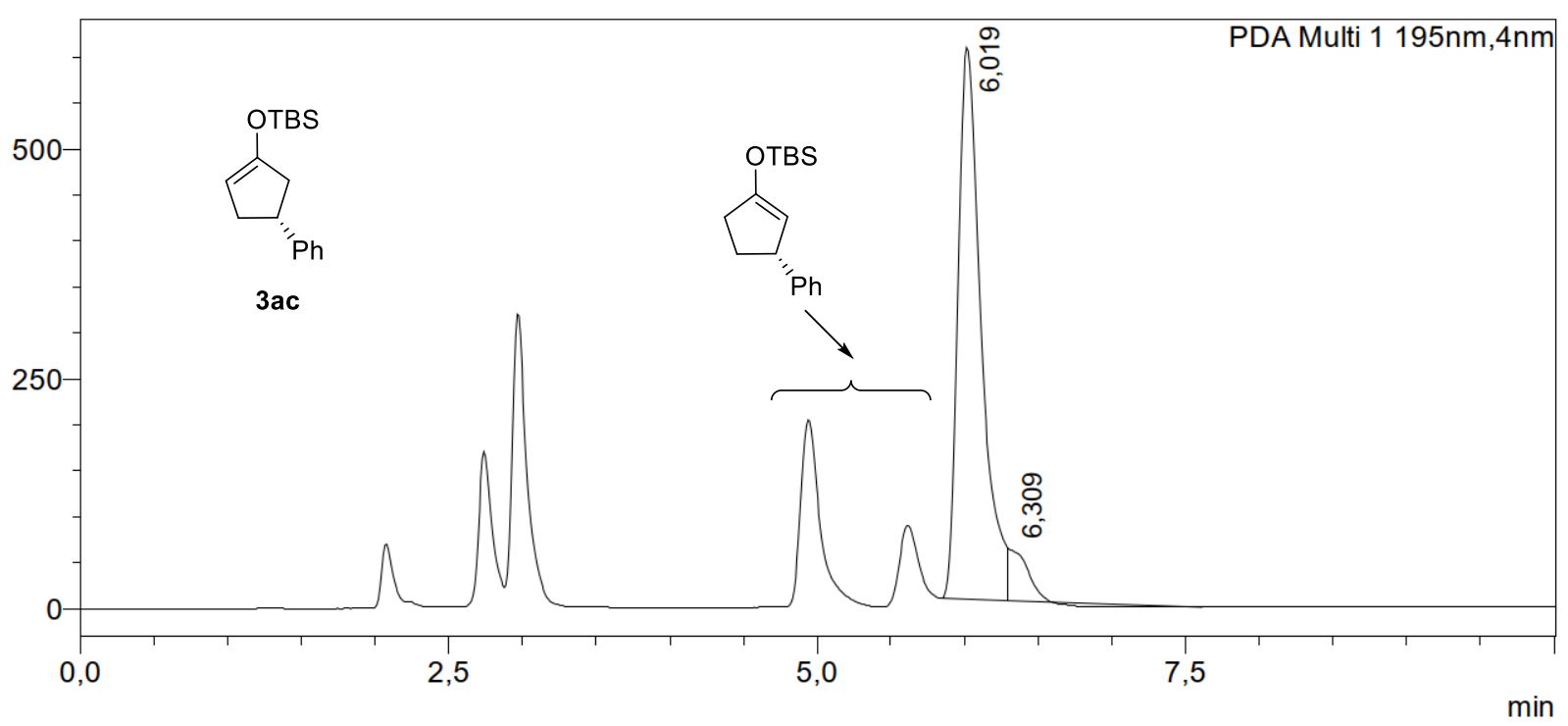

\begin{tabular}{|c|c|c|}
\hline Peak \# & $\mathrm{t}_{\mathrm{R}} / \mathrm{min}$ & $\%$ peak area \\
\hline 1 & 6.0 & 94.78 \\
\hline 2 & 6.3 & 5.22 \\
\hline \multicolumn{2}{|c|}{ Total } & 100 \\
\hline
\end{tabular}

mAU

HPLC column: AS-3, n-heptane/ $i$-PrOH = 99:1, $1.0 \mathrm{~mL} / \mathrm{min}, 298 \mathrm{~K}, 220 \mathrm{~nm}$.

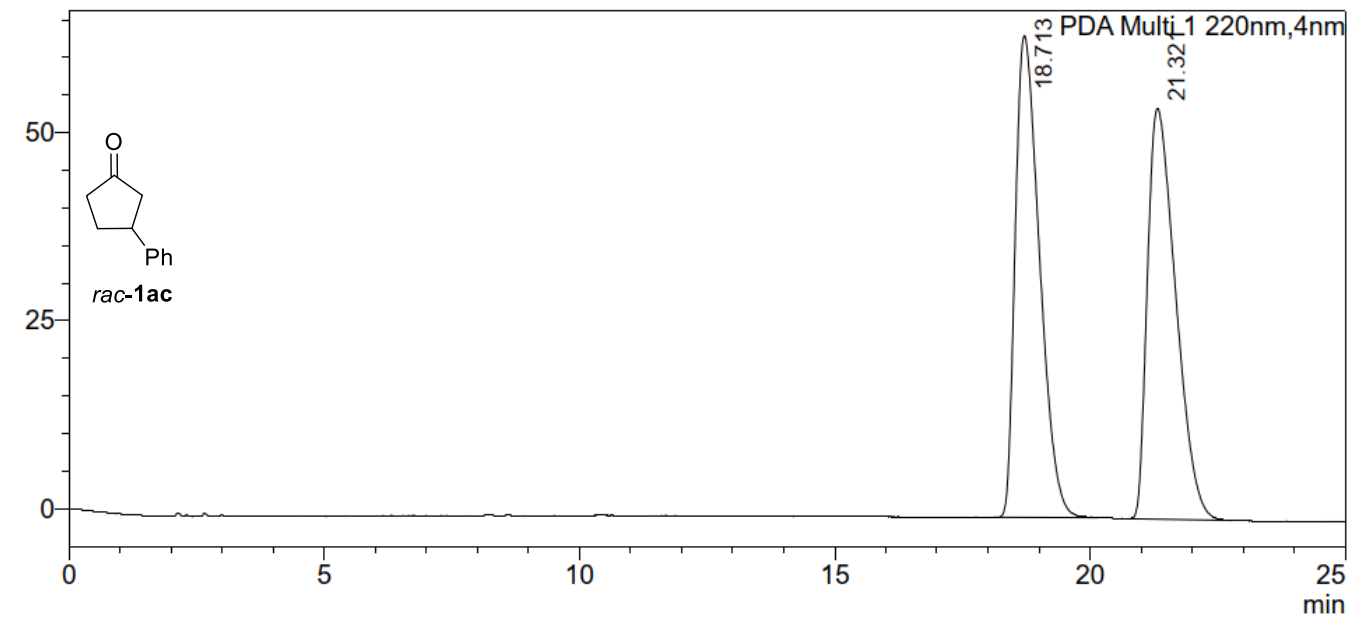

\begin{tabular}{|c|c|c|}
\hline Peak \# & $\mathrm{t}_{\mathrm{R}} / \mathrm{min}$ & $\%$ peak area \\
\hline 1 & 18.7 & 50.17 \\
\hline 2 & 21.3 & 49.83 \\
\hline \multicolumn{2}{|c|}{ Total } & 100 \\
\hline
\end{tabular}




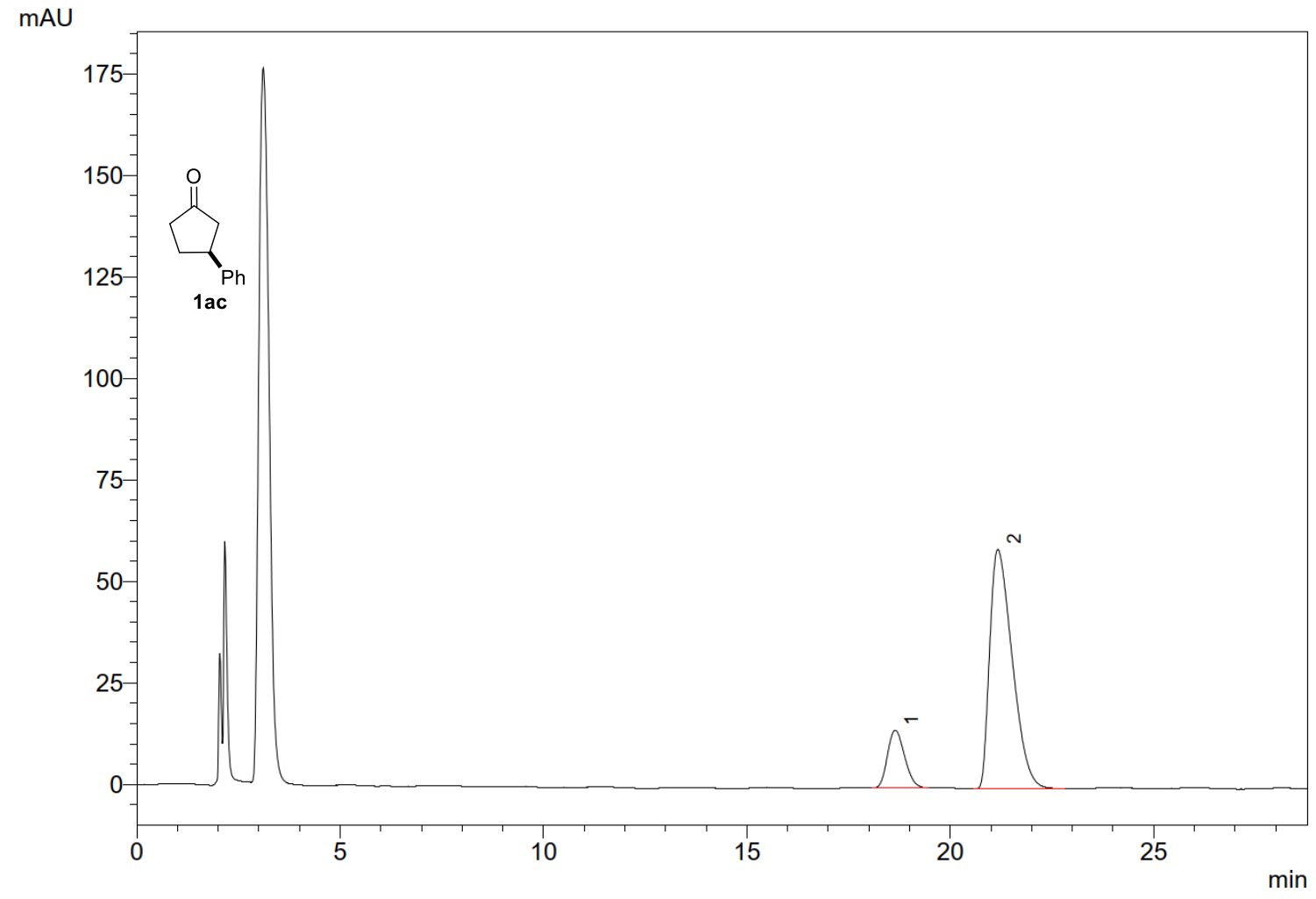

\begin{tabular}{|c|c|c|}
\hline Peak \# & $\mathrm{t}_{\mathrm{R}} / \mathrm{min}$ & $\%$ peak area \\
\hline 1 & 18.6 & 15.44 \\
\hline 2 & 21.2 & 84.56 \\
\hline \multicolumn{2}{|c|}{ Total } & 100 \\
\hline
\end{tabular}

Enantiomers of 1aa was made by prep LC. And the HPLC showed below:

HPLC column: IB-3, $n$-heptane $/ i-\mathrm{PrOH}=98: 2,1.0 \mathrm{~mL} / \mathrm{min}, 308 \mathrm{~K}, 210 \mathrm{~nm}$. 


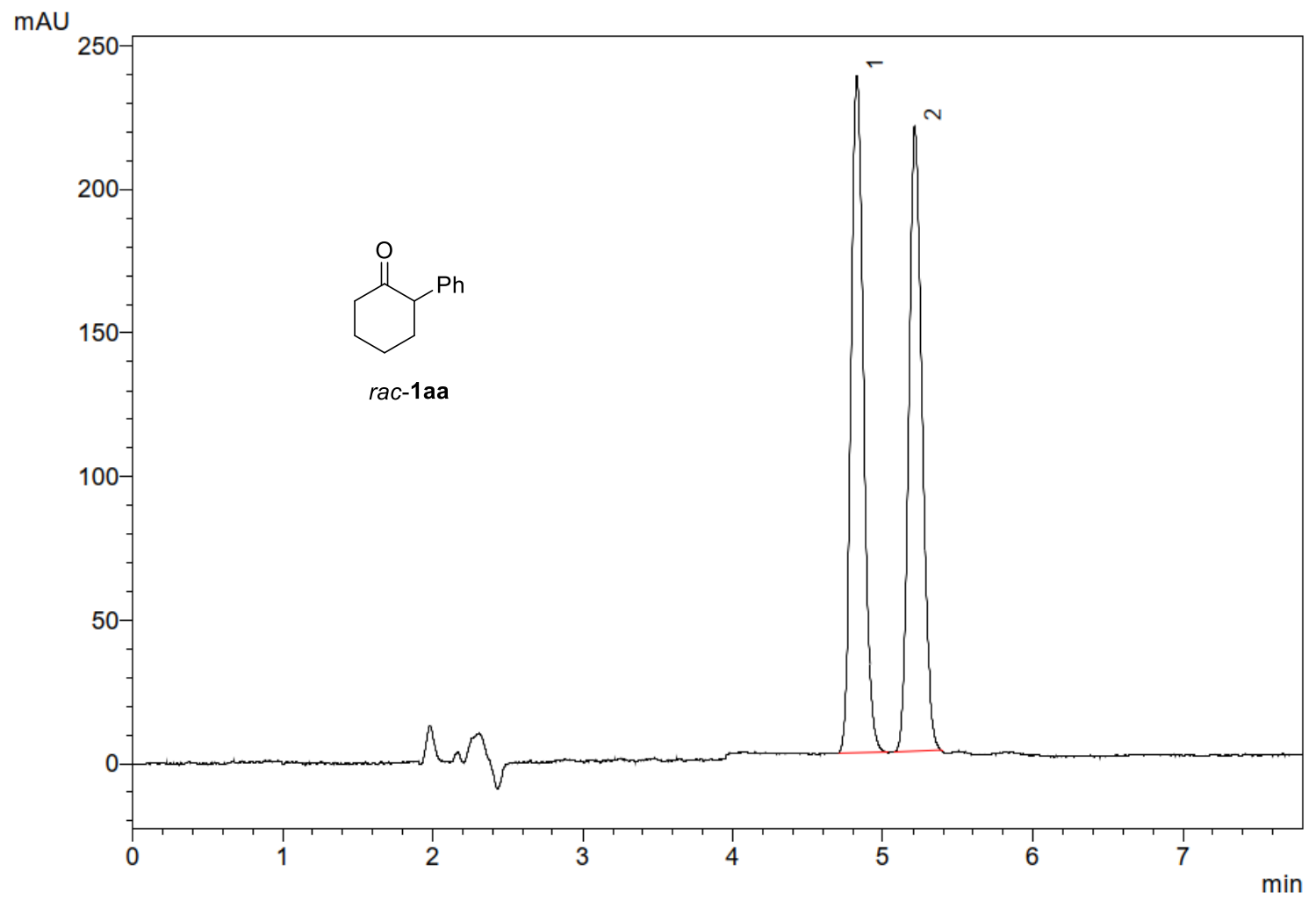

\begin{tabular}{|c|c|c|}
\hline Peak \# & $\mathrm{t}_{\mathrm{R}} / \mathrm{min}$ & \% peak area \\
\hline 1 & 4.8 & 50.28 \\
\hline 2 & 5.2 & 49.72 \\
\hline \multicolumn{2}{|c|}{ Total } & 100 \\
\hline
\end{tabular}

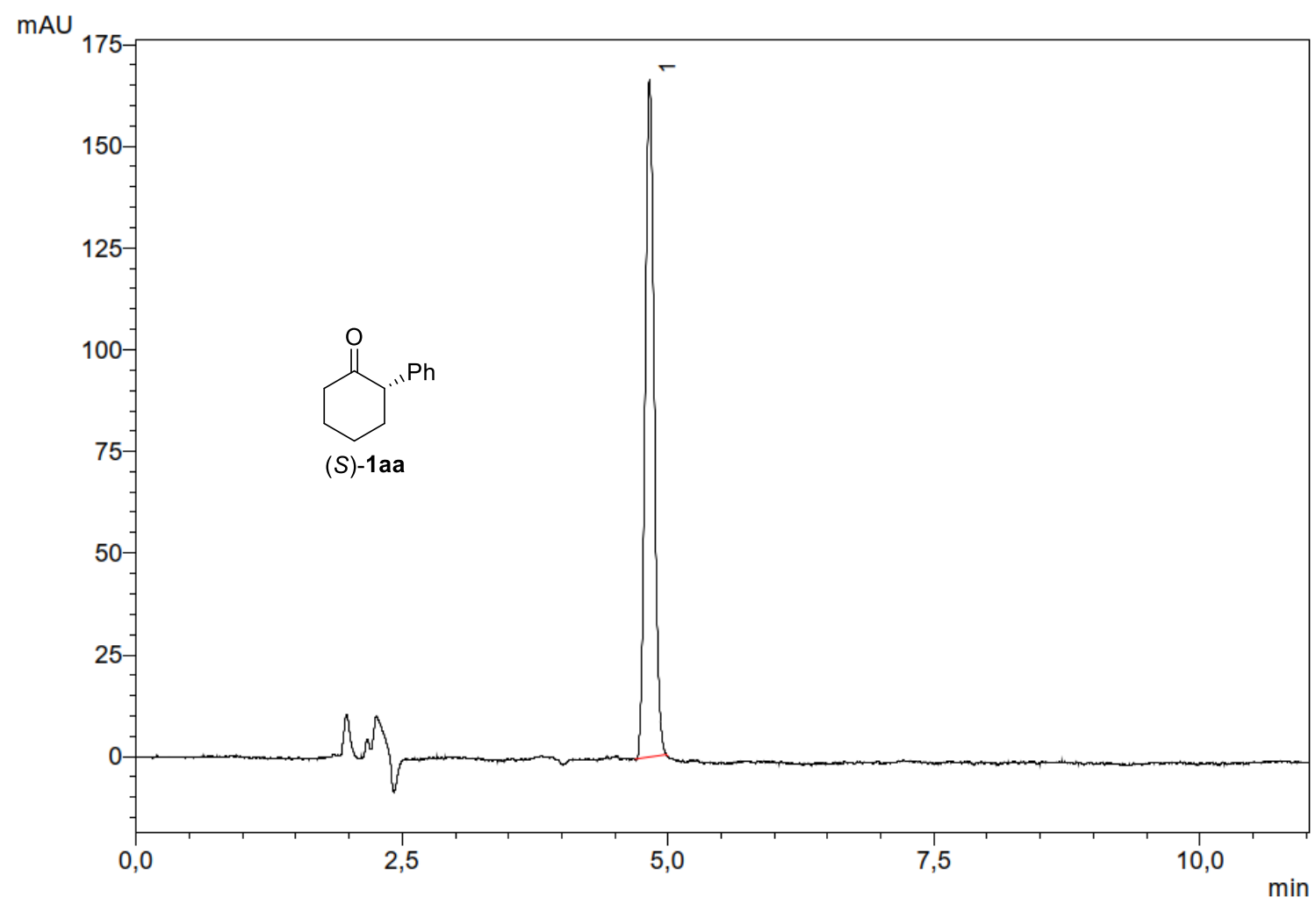

\begin{tabular}{|c|c|c|}
\hline Peak \# & $\mathrm{t}_{\mathrm{R}} / \mathrm{min}$ & \% peak area \\
\hline 1 & 4.8 & 100 \\
\hline
\end{tabular}




\begin{tabular}{|cc|c|c|}
\hline 2 & 5.2 & 0 \\
\hline \multicolumn{2}{|c|}{ Total } & 100 \\
\hline
\end{tabular}

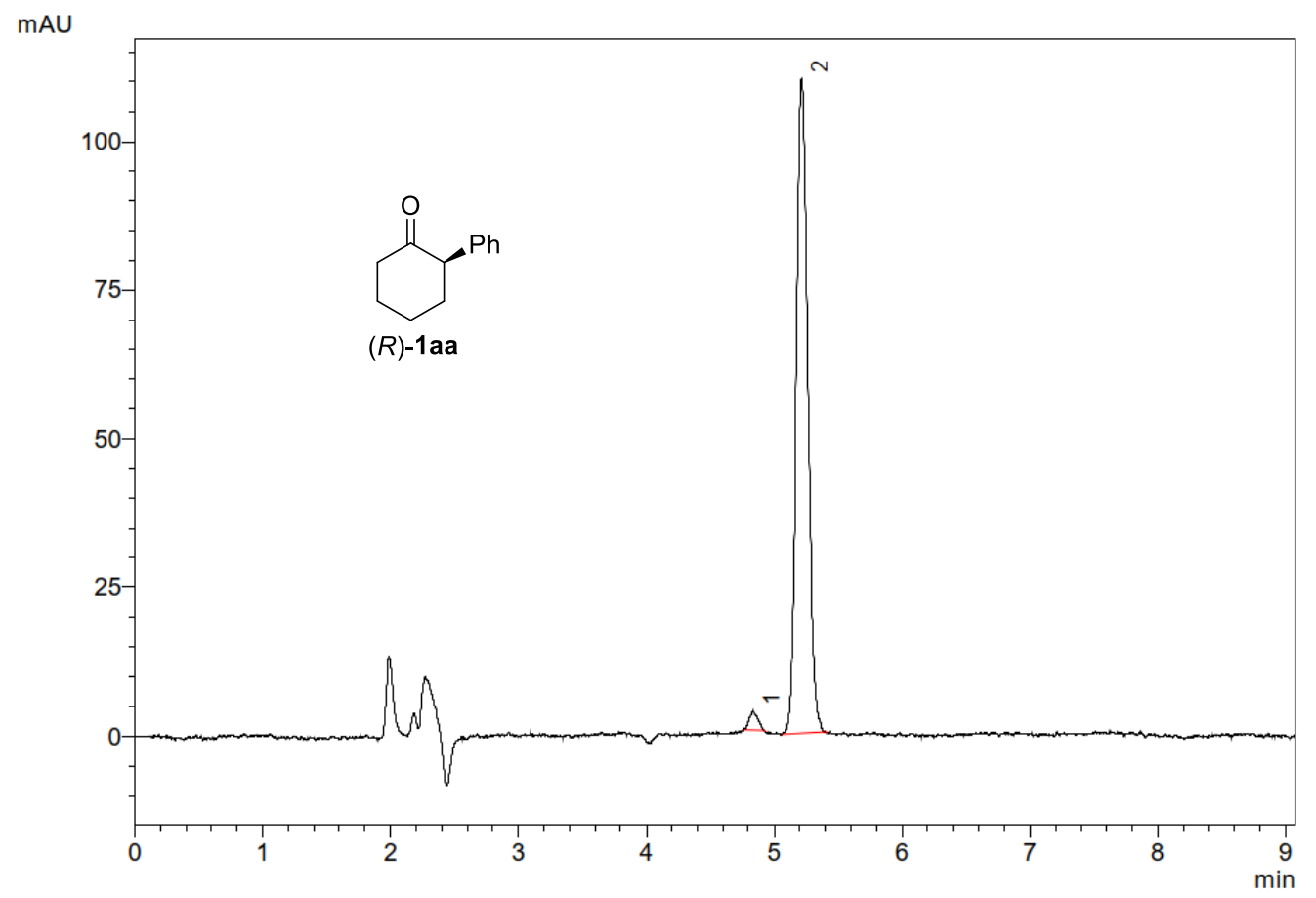

\begin{tabular}{|c|c|c|}
\hline Peak \# & $\mathrm{t}_{\mathrm{R}} / \mathrm{min}$ & $\%$ peak area \\
\hline 1 & 4.8 & 2 \\
\hline 2 & 5.2 & 98 \\
\hline \multicolumn{2}{|c|}{ Total } & 100 \\
\hline
\end{tabular}

Starting material of regiomer of racemic 3ac was separated by prep LC. And the HPLC traces are given below:

HPLC column: 3-Amycoat RP, Acetonitrile: Water = 75:25, $1.0 \mathrm{~mL} / \mathrm{min}, 298 \mathrm{~K}, 220 \mathrm{~nm}$. 


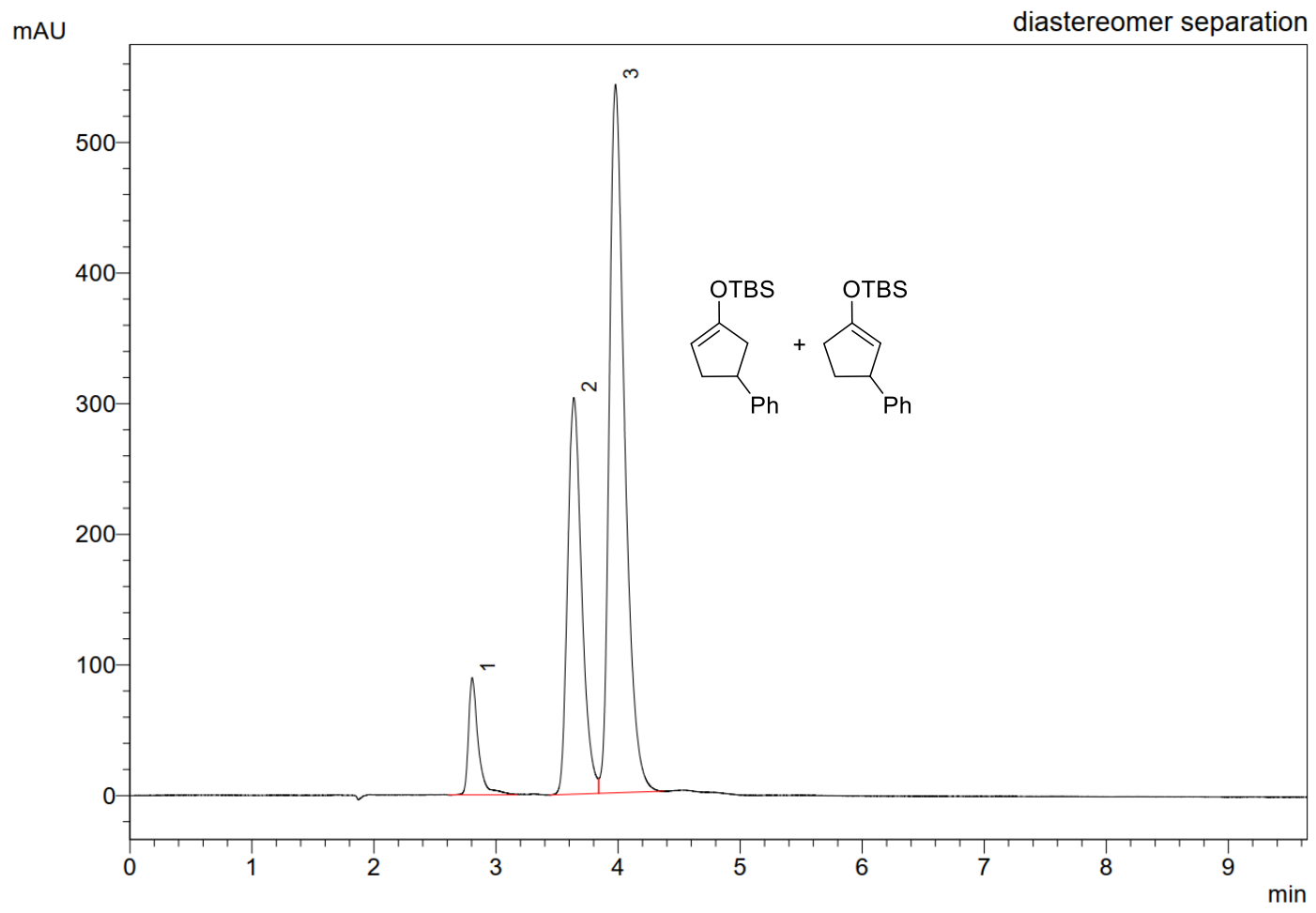

\begin{tabular}{|c|c|c|}
\hline Peak \# & $\mathrm{t}_{\mathrm{R}} / \mathrm{min}$ & $\%$ peak area \\
\hline 1 & 2.8 & 6.45 \\
\hline 2 & 3.6 & 31.77 \\
\hline 3 & 4.0 & 61.78 \\
\hline \multicolumn{2}{|c|}{ Total } & 100 \\
\hline
\end{tabular}

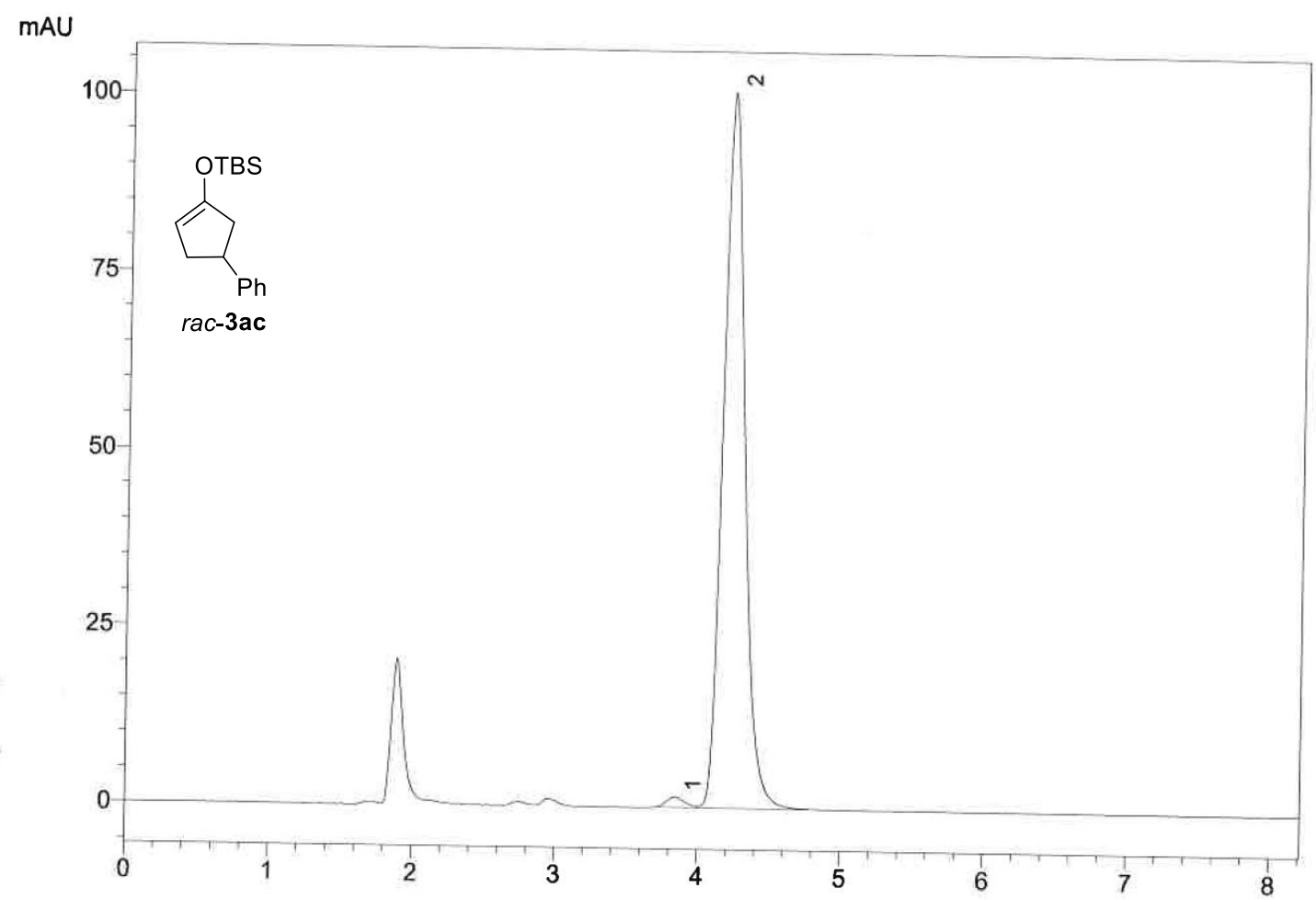

\begin{tabular}{|c|c|c|}
\hline Peak \# & $\mathrm{t}_{\mathrm{R}} / \mathrm{min}$ & $\%$ peak area \\
\hline 1 & 3.8 & 1.24 \\
\hline 2 & 4.2 & 98.76 \\
\hline \multicolumn{2}{|c|}{ Total } & 100 \\
\hline
\end{tabular}

